\title{
Statistical Evaluation of PACSTAT Random Number Generation Capabilities
}
G. F. Piepel
M. J. Budden
M. R. Toland
C. L. Bartley
H. Harty

May 1988

Prepared for the U.S. Department of Energy under Contract DE-AC06-76RLO 1830

Pacific Northwest Laboratory Operated for the U.S. Department of Energy by Battelle Memorial Institute 


\section{DISCLAIMER}

This report was prepared as an account of work sponsored by an agency of the United States Government. Neither the United States Government nor any agency thereof, nor Battelle Memorial Institute, nor any or their employees, makes any warranty, expressed or implied, or assumes any legal liability or responsibility for the accuracy, completeness, or usefulness of any information, apparatus, product, or process disclosed, or represents that its use would not infringe privately owned rights. Reference herein to any specific commercial product, process, or service by trade name, trademark, manufacturer, or otherwise does not necessarily constitute or imply its endorsement, recommendation, or favoring by the United States Government or any agency thereof, or Battelle Memorial Institute. The views and opinions of authors expressed herein do not necessarily state or reflect those of the United States Government or any agency thereof, or Battelle Memorial Institute.

\section{PACIFIC NORTHWEST LABORATORY operated by \\ BATTELLE MEMORIAL INSTITUTE for the UNITED STATES DEPARTMENT OF ENERGY under Contract DE-AC06-76RLO 1830}

\begin{tabular}{|c|c|}
\hline \multicolumn{2}{|c|}{ Printed in the United Slates of America } \\
\hline \multicolumn{2}{|c|}{$\begin{array}{c}\text { Available from } \\
\text { National Technical Information Service }\end{array}$} \\
\hline \multirow{4}{*}{\multicolumn{2}{|c|}{$\begin{array}{c}\text { National Technical Information Service } \\
\text { United States Department of Commerce } \\
5285 \text { Port Royal Road } \\
\text { Springfield. Virginia } 22167\end{array}$}} \\
\hline & \\
\hline & \\
\hline & \\
\hline \multirow{2}{*}{\multicolumn{2}{|c|}{$\begin{array}{l}\text { NIIS Price Codes } \\
\text { Microfiche A01 }\end{array}$}} \\
\hline & \\
\hline \multicolumn{2}{|c|}{ Printed Copy } \\
\hline & Price \\
\hline Pages & Codes \\
\hline $001-025$ & $\mathrm{~A} 02$ \\
\hline $026-050$ & $\mathrm{~A} 03$ \\
\hline $051-075$ & $\mathrm{AO4}$ \\
\hline $076-100$ & $\mathrm{~A} 05$ \\
\hline 101.125 & $A 06$ \\
\hline $126-150$ & $A 07$ \\
\hline$\uparrow 51-175$ & $A 0 B$ \\
\hline $176-200$ & $A 09$ \\
\hline $201-225$ & A010 \\
\hline $226-250$ & $\mathrm{~A} 011$ \\
\hline $251-275$ & $\mathrm{~A} 012$ \\
\hline $276-300$ & $\mathrm{~A} 013$ \\
\hline
\end{tabular}


PNL -6434

UC -70

STATISTICAL EVALUATION OF PACSTAT

RANDOM NUMBER GENERATION

CAPABILITIES

G. F. Piepel

M. R. Toland

H. Harty

M. J. Budden

C. L. Bartley

May 1988

Prepared for the U.S. Department of Energy under Contract DE-AC06-76RLO 1830

Pacific Northwest Laboratory Richland, Washington 99352 
SUMMARY

This report summarizes the work performed in verifying the general purpose Monte Carlo driver-program PACSTAT. The main objective of the work was to verify the performance of PACSTAT's random number generation capabilities. Secondary objectives were to document (using controlled configuration management procedures) changes made in PACSTAT at Pacific Northwest Laboratory, and to assure that PACSTAT jnput and output files satisfy quality assurance traceability constraints.

Upon receipt of the PRIME version of the PACSTAT code from the Basalt Waste Isolation Project, Pacific Northwest Laboratory staff converted the code to run on Digital Equipment Corporation (DEC) VAXs. The modifications to PACSTAT were implemented using the WITNESS configuration management system, with the modifications themselves intended to make the code as portable as possible. Certain modifications were made to make the PACSTAT input and output files conform to quality assurance traceability constraints.

The verification aspects of the work focused on checking PACSTAT's random number generation capabilities for the following distributions: uniform, normal, exponential, loguniform, lognormal, truncated exponential, truncated normal, and truncated lognormal. The highest level of effort went into checking the performance of the uniform random number generator (RNG), since it is used as the basis for generating random numbers from the other seven distributions. A variety of statistical tests and graphical methods were used to check the performance of the unjform RNG, with subsets of these techniques used to check the other distributions. Also, for each distribution, the programining was checked, and a few variates generated by PACSTAT were also computed by hand to check for agreement.

The verification investigations led to the discovery of several minor errors in PACSTAT. Upon correcting these, all eight RNGs appeared to perform adequately. The errors found (and corrected) were such that previous applications using PACSTAT may have been adversely affected. Actual impacts of the errors on previous applications depend on the specifics of each application. 
The uniform RNG used by PACSTAT is a multiplicative congruential type with modulus (2**31)-1 and multiplier 16807. Two comprehensive papers by Fishman and Moore $(1982,1986)$ consider the performance of various multipliers for uniform RNGs of this type. These papers indicate that the 16807 multiplier is a good one, but that better ones exist. 
CONTENTS

SUMMARY i i i

1.0 INTRODUCTION

1.1 VERIFICATION OF PACSTAT RANDOM NUMBER GENERATORS $\ldots \ldots \ldots \ldots \ldots .1 .1$

1.1 .1 Three-Stage Testing $\ldots \ldots \ldots \ldots \ldots \ldots \ldots \ldots \ldots \ldots \ldots \ldots \ldots . \ldots \ldots \ldots$

1.1.2 Statistical Tests for Evaluating Random Number

Generators ................................ 1.3

1.2 Pacstat MOdifications and CONFIgURATION Management $\ldots \ldots \ldots \ldots$.

1.3 QUal. ITY ASSURANCE CONSIDERATIONS $\ldots \ldots \ldots \ldots \ldots \ldots \ldots \ldots \ldots \ldots \ldots$

2.0 UNIFORM PSEUDO-RANDOM NUMBER GENERATOR $\ldots \ldots \ldots \ldots \ldots \ldots \ldots \ldots \ldots \ldots \ldots$

2.1 DESCRIPTION OF TESTS APPLIED TO THE UNIFORM RANDOM

NUMBER GENERATOR ............................... 2.1

2.2 RESULTS OF TESTS ON THE UNIFORM RANDOM NUMBER

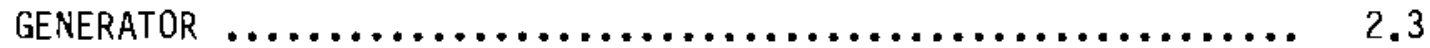

3.0 EXPONENTIAL AND NORMAL RANDOM NUMBER GENERATORS $\ldots \ldots \ldots \ldots \ldots \ldots \ldots . . . \ldots$

3.1 TESTS APPLIED TO THE NORMAL AND EXPONENTIAL RANDOM

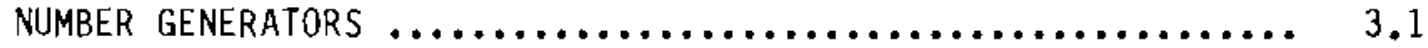

3.2 RESULTS OF TESTS ON THE NORMAL AND EXPONENTIAL RANDOM

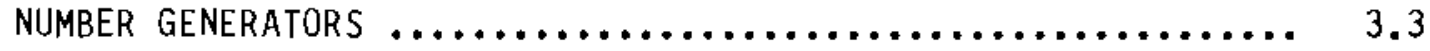

4.0 REMAINING RANDOM NUMBER GENERATORS $\ldots \ldots \ldots \ldots \ldots \ldots \ldots \ldots \ldots \ldots \ldots \ldots . . \ldots$

4.1 TESTS ON THE REMAINING FIVE RANDOM NUMBER GENERATORS $\ldots \ldots \ldots . .4 .1$

4.2 RESULTS OF TESTS ON THE REMAINING RANDOM NUMBER GENERATORS $\ldots 4.2$

5.0 CORRECTIONS AND LITERATURE FINDINGS $\ldots \ldots \ldots \ldots \ldots \ldots \ldots \ldots \ldots \ldots \ldots \ldots . . \ldots \ldots$

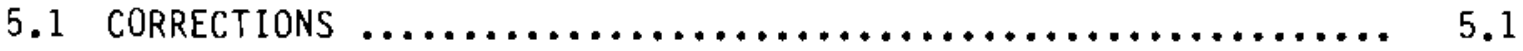

5.2 LiteratuRE Findings $\ldots \ldots \ldots \ldots \ldots \ldots \ldots \ldots \ldots \ldots \ldots \ldots \ldots \ldots \ldots \ldots \ldots$

6.0 QUALITY ASSURANCE AND CONFIGURATION CONTROL. OF PACSTAT $\ldots \ldots \ldots \ldots . . .6 .1$

6.1 CREATION OF PACSTAT VERSIONS A4 AND B4 $\ldots \ldots \ldots \ldots \ldots \ldots \ldots \ldots \ldots . . \ldots .2$

6.2 QUALITY ASSURANCE RECOMMENdATIONS $\ldots \ldots \ldots \ldots \ldots \ldots \ldots \ldots \ldots \ldots \ldots$ 
7.0 GENERATION OF RANDOM NUMBERS FROM PACSTAT FOR STATISTICAL TESTS

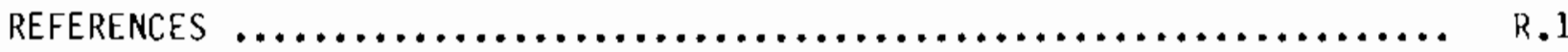

APPENDIX A - RANDOM NUMBER GENERATION IN PACSTAT ................. A.1

APPENDIX B - PACSTAT_AO.FOR (ORIGINAL INTERACTIVE VERSION,

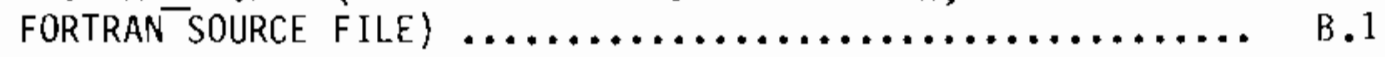

APPENDIX C - PACSTAT B4.FOR (FINAL BATCH VERSION, FORTRAN SOURCE

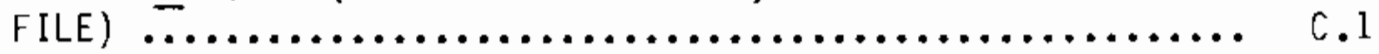

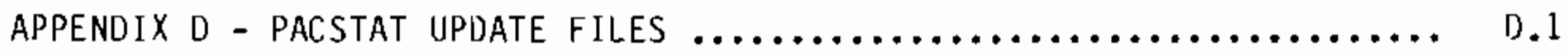

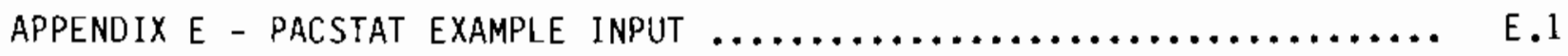

APPENDIX $F$ - PACSTAT EXAMPLE OUTPUT $\ldots \ldots \ldots \ldots \ldots \ldots \ldots \ldots \ldots \ldots \ldots \ldots \ldots \ldots$. 1

APPENDIX G - SOLN AND BLKDAT ROUTINES $\ldots \ldots \ldots \ldots \ldots \ldots \ldots \ldots \ldots \ldots \ldots \ldots \ldots . \ldots \ldots$

APPENDIX H - EXAMPLE VAX/VMS COMMAND FILE USED TO OBTAIN 100 SETS OF 10,000 UNIFDRM $(0,1)$ VARIATES FRDM PACSTAT $\ldots \ldots \ldots \ldots \ldots \ldots \ldots$ H.l

APPENDIX I - PROGRAM READ USED TO PREPARE PACSTAT OUTPUT FOR USE BY SAS FOR STATISTICAL TESTING PURPOSES $\ldots \ldots \ldots \ldots \ldots \ldots \ldots \ldots \ldots$ I. I 
FIGURES

1.1 Three-Stage Testing of the Pseudo-Random Number Generators in PACSTAT

2.1 Histogram of 10,000 Random Variates from the PACSTAT Uniform $(0,1)$ Random Number Generator ................... 2.8

2.2 Theoretical Quantile-Quantile Plot of 10,000 Random Variates from the PACSTAT Uniform $(0,1)$ Random Number Generator

3.1 Histogram of 500 Random Variates from the Exponential Distribution with Parameter $\theta=1$.

3.2 Theoretical Quantile-Quantile Plot of 500 Random Variates

from the Exponential Distribution with Parameter $\theta=1$

3.3 Histogram of 500 Random Variates from the Normal $(0,1)$

Distribution

3.4 Theoretical Quantile-Quantile Plot of 500 Random Variates

from the Normal $(0,1)$ Distribution

4.1 Histogram of 500 Random Variates from the Loguniform

Distribution Based on the Uniform $(0,1) \ldots \ldots \ldots \ldots \ldots \ldots \ldots$

4.2 Theoretical Quantile-Quantile Plot of 500 Random Variates

from the Loguniform Distribution Based on the Uniform

$(0,1)$

.........

4.3 Histogram of 500 Random Variates from the Truncated Exponential

Distribution.

4.4 Theoretical Quantile-Quantile Plot of 500 Random Variates

from the Truncated Exponential Distribution

4.5 Histogram of 500 Random Variates from the Lognormal

Distribution Based on the Normal $(0,1)$

4.6 Theoretical Quantile-Quantile Plot of 500 Random Variates

from the Lognormal Distribution Based on the Normal $(0,1) \ldots \ldots .4$

4.7 Histogram of 500 Random Variates from the Truncated Normal

Distribution Based on the Normal $(0,1)$

4.8 Theoretical Quantile-Quantile Plot of 500 Random Variates from the Truncated Normal Distribution Based on the Norma $1(0,1)$ 
4.9 Histogram of 500 Random Variates from the Truncated Lognormal Distribution Based on the Normal $(0,1) \ldots \ldots \ldots \ldots \ldots . . . . .4$

4.10 Theoretical Quantile-Quantile Plot of 500 Random Variates from the Truncated Lognormal Distribution Based on the Normal $(0,1)$ 


\section{TABLES}

2.1 Probabilities of Specified Numbers of Rejections Out of 91 lests, where the Probability of Incorrectly

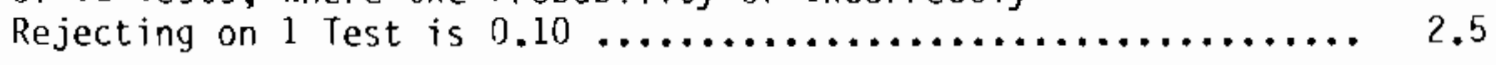

2.2. Probabilities of Specified Numbers of Rejections Out of 91 Tests, Where the Probability of Incorrectly

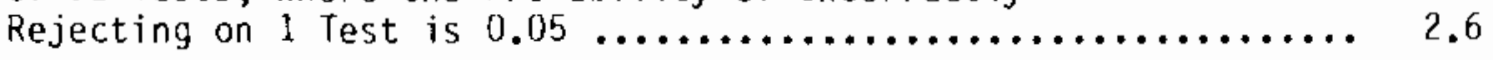

2.3 Number of Rejections from Goodness-of-Fit, Independence and Uniformity Tests $\ldots \ldots \ldots \ldots \ldots \ldots \ldots \ldots \ldots \ldots \ldots \ldots \ldots \ldots \ldots \ldots . \ldots \ldots$

3.1 Probabilities of Specified Numbers of Rejections Out of 100 Tests, Where the Probability of Incorrectly

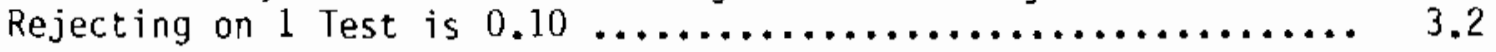

3.2 Probabilities of Specified Numbers of Rejections Out of 100 Tests, Where the Probability of Incorrectly

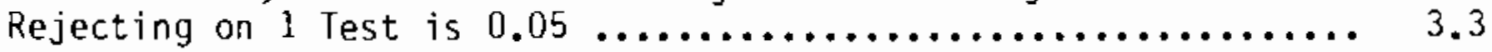

3.3 Number of Rejections in Each Tail in Goodness-of-Fit Tests for the Exponential and Normal Random Number Generators in PACSTAT 



\subsection{INTRODUCTION}

PACSTAT is a multipurpose "driver" routine that can be used to perform Monte Carlo simulations when connected to external deterministic codes. This report summarizes work performed at Pacific Northwest Laboratory (PNL) for the Basalt Waste Isolation Project (BWIP). The objectives of the work were

- to verify the random number generation capabilities of PACSTAT

- to document, via use of computer configuration management software, changes made to the PACSTAT code at PNL

- to assure that PACSTAT input and output files satisfy quality assurance traceability and reproducibility constraints.

Verifying the random number generation capabilities of PACSTAT was the primary objective of this work, with the other two aspects above relating to quality assurance goals.

Because PACSTAT is still "under development" and was not under formal configuration management at BWIP, it was not necessary to implement configuration management at PNL. The decision was made to do so as a means to document the changes made in PACSTAT at PNL. Changes were made to translate the original PRIME code to run on Digital Equipment Corporation (DEC) VAXs, and to correct errors found during verification efforts.

Although a user's guide for PACSTAT and a report on past verifjcation efforts exist, they cannot be formally referenced. Appendix A gives a brief overview of the methods used in PACSTAT for generating random numbers, but this is not intended as comprehensive documentation of PACSTAT.

Sections 2.0 through 5.0 are devoted to the results of verification tests performed on PACSTAT's random number generation capabilities. Sections 6.0 and 7.0 and Appendices $B$ through I relate to the quality assurance and configuration management aspects of the work.

\subsection{VERIFICATION OF PACSTAT RANDOM NUMBER GENERATORS}

The scope of work calls for verifying the eight continuous variable pseudo-random number generators in PACSTAT: uniform, normal, exponential, 
loguniform, lognormal, truncated exponential, truncated normal, and truncated lognormal. PACSTAT also has the capability of generating pseudo-random variates from the discrete Bernoulli probability distribution and can generate stratified variates from the eight continuous distributions. These capabilities were not investigated.

The word "pseudo" in the term pseudo-random number refers to the fact that generators can only attempt to mimic random behavior. The generators themselves are mathematical procedures that produce values in a structured, functionally related way. Our task was to ensure that the pseudo-random number generators in PACSTAT are implemented correctly and that the pseudo-random variates they produce have the correct properties. For simplicity in the remainder of the report, we drop the term "pseudo" and refer to random number generators (RNGs) and random variates.

\subsubsection{Three-Stage Testing}

The testing of the RNGs in PACSTAT was carried out in three stages. First, the uniform RNG was tested. Then the exponential and normal RNGs were tested. Lastly, the loguniform, lognormal, truncated exponential, truncated normal, and truncated lognormal RNGs were tested.

The three-stage testing of the RNGs is illustrated in Figure 1.1, and was suggested by the mathematical relationships among the RNGs. Figure 1.1 shows that the uniform RNG is the parent generator for the other PACSTAT random number generators considered. The exponential, normal, and loguniform random number generators produce their respective variates by mathematically transforming uniform random variates. Similarly, the truncated exponential, truncated normal, lognormal, and truncated lognormal RNGs produce their respective variates by mathematically manipulating variates from the exponential and normal random number generators. From a theoretical point of view, if the uniform RNG works and the appropriate mathematical transformations are programmed properly, the other RNGs will also work. The relationships among the RNGs are described in Appendix A. 


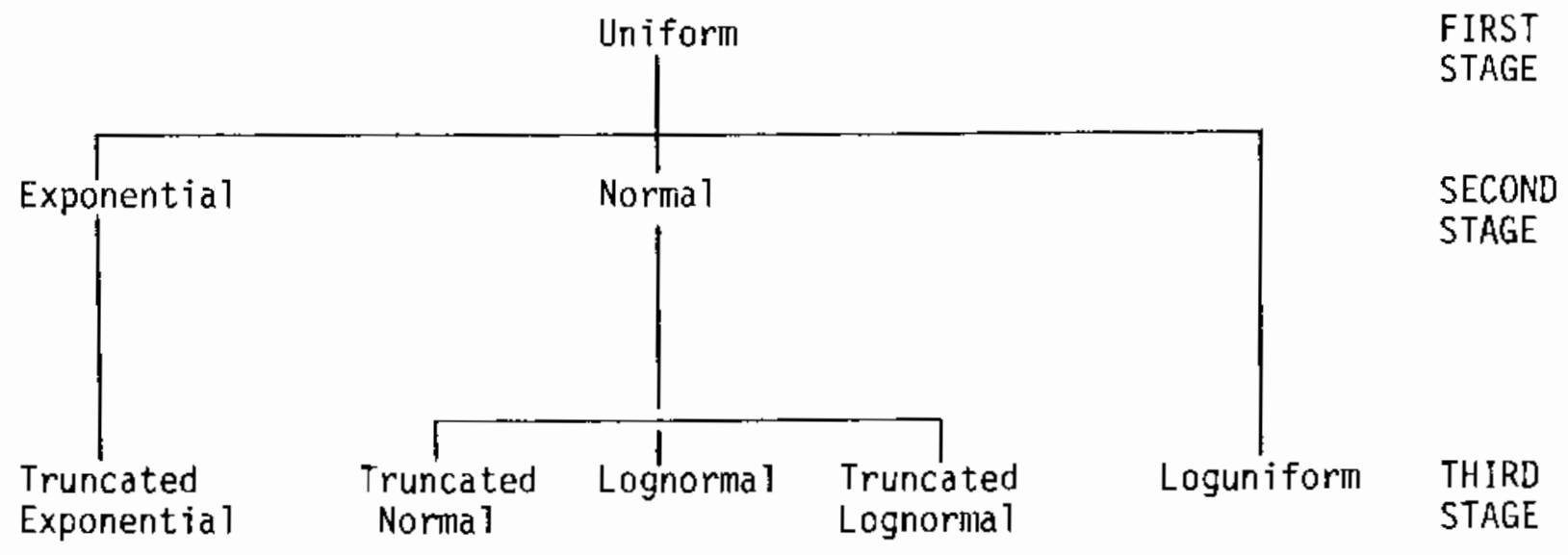

\section{FIGURE 1.1. Three-Stage Testing of the Pseudo-Random} Number Generators in PACSTAT

\subsubsection{Statistical Tests for Evaluating Randon Number Generators}

There are a great number of statistical tests in the literature for assessing the properties of a sequence of random numbers. The initial test plan contained a "shopping list" of some of these tests. A subset of tests was selected from the shopping list for the final test plan. The subset of tests was chosen to investigate the most important properties of the random variates, and was commensurate with the budget, computer resources, and time available.

The three test stages were hierarchical in terms of the extensiveness of testing. The variates from the uniform RNG were tested using goodness-of-fit, graphical, independence, and uniformity tests. The exponential and normal RNGs were tested using goodness-of-fit and graphical tests, and the remaining RNGs were tested using graphical tests. Summary statistics (mean, standard deviation, minimum, and maximum) were computed and compared to theoretical values for all eight distributions. Also, programming checks of the mathematical transformations and hand calculation checks of a few variates were performed for all distributions.

\subsection{PACSTAT MODIFICATIONS AND CONFIGURATION MANAGEMENT}

The original version of PACSTAT was delivered to PNL by BWIP on magnetic tape. PACSTAT had been running on a PRIME-750, and was not under configuration 
management. The PNL put PACSTAT under configuration management using the automated code version tracking tool, WITNESS, (Koontz and Stewart 1986) and proceeded to convert the code to run on the PNL VAX computer system. The PRIME-750 version was designated the $A 0$ version, and had many system-dependent features. These dependencies were removed and replaced with ANSI standard FORTRAN-77 implementation of the same features, so that PACSTAT is portable to VAXs, CRAYS, CDCS, or other PRIMEs. The final versions of PACSTAT used for verification were designated the $A 4$ (interactive) and $B 4$ (batch) versions. Additional details of the code conversion process, and the use of WITNESS to automatically track versions is contained in Section 6.0 .

\subsection{QUAL ITY ASSURANCE CONSIDERATIONS}

A11 work on PACSTAT was performed according to procedures described in the Statement of Work SOW-L1E2 and the Engineering Physics Department Quality Assurance Plan EPD-163-2. Other than the configuration management of PACSTAT, the major quality assurance (QA) additions to PACSIAT were to improve the traceability of input and output files. Additional details of the QA aspects of this work can be found in Sections 6.0 and 7.0 . 


\subsection{UNIFORM PSEUDO-RANDOM NUMBER GENERATOR}

The uniform RNG in PACSTAT is a multiplicative congruential RNG with multiplier 16807 and modulus (2**31)-1 (see Section A.1 of Appendix A). It produces pseudo-random variates which are uniformly distributed on the open interval $(0,1)$. This particular $R N G$ has been used in several computer programs, and its properties have been examined in the literature by Fishman and Moore $(1982,1986)$. Their results are given in Section 5.0 .

\subsection{DESCRIPTION OF TESTS APPLIED TO THE UNIFORM RANDOM NUMBER GENERATOR}

To test the uniform RNG, 100 strings of 10,000 variates each were generated. These 100 strings of variates were subjected to a battery of tests which can be grouped into four main categories: goodness-of-fit, graphical, independence, and uniformity. The tests were two-sided tests done at the 0.10 level of significance. Two-sided (or two-tailed) refers to the two potential types of anomalies that can be detected by the tests. For example, with the goodnessof-fit and uniformity tests, a string of variates may appear nonuniformly distributed, or it may appear to be superuniformly distributed (more uniform than expected by chance). For the independence (randomness) test, the variates in a string may not be independent, or they may be more independent than expected by chance.

Although variates being "too random" or "too uniform" do not sound like anomalies or defects, such findings are indicative of functional or nonrandom behavior in the generator. For example, consider the outcomes of 100 tosses of a fair coin (i.e., one for which the probability of getting a head is 0.5 ). If all of the tosses each resulted in a head, then one would suspect either the fairness of the coin or the fairness of the toss. Likewise, if the first 50 tosses resulted in heads and the last 50 tosses resulted in tails, the outcomes would not be considered random outcomes from tossing a fair coin. These two cases are examples of one extreme, lack of randomness. At the other extreme, if every other toss resulted in a head, either the coin's fairness or the toss would be suspect. This is an example of "too much" randomness. If a fair coin is tossed so that the outcome is just as likely to be a head as it is a tail, 
then one would expect the number of heads and tails in a row (size of runs) to be somewhere between the extremes given above.

A similar argument can be made for each of the other properties considered in this investigation. The uniform RNG can produce variates that are either not uniformly distributed or "too uniformly" distributed. For this reason twosided tests were performed.

The goodness-of-fit tests used were the Kolmogorov-Smirnov, Cramer-von Mises, Kuiper, and Watson tests. The Kolmogorov-Smirnov and Cramer-von Mises tests are sensitive to shifts in location, while the Kuiper and Watson tests are sensitive to shifts in variance. Too many rejections in either or both tails means the variates do not come from a uniform $(0,1)$ distribution, or come from a superuniform distribution. These goodness-of-fit tests are defined and compared by Stephens $(1969,1974)$.

The graphic tools employed were histograms and theoretical quantilequantile plots. A histogram shows the distribution of values in a string of variates, and should approximate the true probability density function (pdf). For uniform data, the pdf is a rectangle on the open interval $(0,1)$. A theoretical quantile-quantile plot graphs the quantiles of the hypothesized distribution versus the quantiles of a string of variates. When the data come from the hypothesized distribution, the plot looks like the plot of the line $y=x$. Theoretical quantile-quantile plots assess four areas: shift in location, shift in variance, symmetry, and lack-of-fit in the tails. The quantile-quantile plot is discussed by Chambers et al. (1983).

To test independence (randomness) of the variates, a runs test (Fishman and Moore 1982) was used to test the number of runs up (successively increasing variates) and the number of runs down (successively decreasing variates). Too many rejections in the lower tail indicates too much randomness and too many rejections in the upper tail indicates lack of randomness. In either case, departure from randomness probably is because of some functional behavior in the RNG.

Within each string of variates, uniformity on the unit line, unit square, and unit cube was tested (Fishman and Moore 1982) using the chi-square 
goodness-of-fit test. For the one-dimensional (unit line) test, the interval $(0,1)$ was divided into 1000 bins, and the counts of variates (of the 10,000 in a string) falling into each bin were obtained. For the two-dimensional test, the unit square was divided into $30 \times 30=900$ bins, with the counts of successive pairs of variates (from a string of 10,000) falling into each bin being obtained. The unit-cube was divided into $7 \times 7 \times 7=343$ bins, with the successive triples of variates used for the three-dimensional test. Too many rejections in the lower tail (for any test) indicates superuniformity (more uniform than expected by chance) which would be because of functional behavior. Too many rejections in the upper tail indicates the variates are not uniformly distributed.

Uniformity across strings of variates was also checked. Theoretically, the variates across strings should also be uniformly distributed. This was checked by performing tests on the means and variances of the strings. The individual string means and variances were checked for equality with their theoretical values. Then the variance of the string means was tested for equality with its theoretical value. These tests were performed at the 0.05 significance level.

\subsection{RESULTS OF TESTS ON THE UNIFURM RANDOM NUMBER GENERATOR}

This section summarizes the test results for the uniform random number generator. One hundred test statistics (corresponding to the 100 strings of 10,000 variates) were calculated for each test and compared against their corresponding distribution.

The tests for uniformity across strings of variates (discussed in Section 2.1) detected anomalous behavior beyond that expected by chance for the uniform random number generator in PACSTAT. It was found that only 91 distinct strings of variates had been generated because of some programming errors in PACSTAT. The errors and their corrections are discussed in Section 5.0. The long run time required by the SAS (1985a, 1985b) test software prevented the rerunning of the tests for 100 new strings. Hence, the remaining results in this section are for 91 strings of 10,000 variates. 
In performing the tests, some rejections of the hypothesis that the variates have the property under consideration are expected to occur by chance alone. Tables 2.1 and 2.2 give the cumulative and individual probabilities for given numbers of rejections under the assumption that the property being tested (e.g., goodness-of-fit, uniformity, or independence) holds. Tables 2.1 and 2.2 are based on 91 independent tests instead of 100. These tables do not depend on the distribution (uniform, normal, exponential, etc.) being tested. The only assumptions used in constructing Tables 2.1 and 2.2 are that the 91 tests are independent and that the significance level of each test is 0.10 (for Table 2.1) or 0.05 (for Table 2). The probabilities in Tables 2.1 and 2.2 are theoretical probabilities calculated from the binomial $B(0.10,91)$ and $B(0.05$, 91) distributions, respectively.

From Table 2.1 it can be seen that, when the property being tested holds, there is an $92 \%$ chance of observing between 5 and 14 rejections out of 91 tests. Although Table 2.1 shows that it is possible to observe more rejections than 14 or fewer than 5, the probability of doing so when the property being tested is true is about 0.08 . Therefore, if the total number of rejections in the goodness-of-fit, uniforinity (on the unit line, unit square, or unit cube), or independence tests are between 5 and 14 inclusive, then there is no reason to suspect that the variates have properties different than desired. On the other hand, since there is only a 3.6\% chance of observing more than 14 rejections and only a $4.4 \%$ chance of observing fewer than 5 rejections when the property holds, it is reasonable to decide (with an $8 \%$ change of being wrong) that the variates being considered do not have the property (goodness-of-fit, uniformity, independence) being tested. It may seem incongruous that too few rejections is unacceptable, but there is very little chance of such a thing occurring when a large number of independent tests are performed on truly random data. The idea behind all the tests in this report is that if the property of interest holds, then the probability of finding something anomalous should be very small. Therefore, if something with very little chance of occurring happens, then it is concluded that an anomaly has been found.

Recall that the goodness-of-fit, uniforinity, and independence tests are performed as two-tailed tests with 0.10 level of significance. However, in 
TABLE 2.1. Probabilities of Specified Numbers of Rejections Out of 91 Tests, Where the Probability of Incorrectly Rejecting on 1 Test is 0.10

\begin{tabular}{|c|c|c|}
\hline $\begin{array}{l}\text { Number of } \\
\text { Rejections } \\
\end{array}$ & $\begin{array}{l}\text { Individual } \\
\text { Probability }\end{array}$ & $\begin{array}{l}\text { Cumulative } \\
\text { Probability }\end{array}$ \\
\hline 0 & 0.000069 & 0.000069 \\
\hline 1 & 0.000693 & 0.000762 \\
\hline 2 & 0.003466 & 0.004228 \\
\hline 3 & 0.011425 & 0.015653 \\
\hline 4 & 0.027928 & 0.043581 \\
\hline 5 & 0.053995 & 0.097576 \\
\hline 6 & 0.085991 & 0.183567 \\
\hline 7 & 0.116020 & 0.299587 \\
\hline 8 & 0.135357 & 0.434944 \\
\hline 9 & 0.138699 & 0.573643 \\
\hline 10 & 0.126370 & 0.700014 \\
\hline 11 & 0.103394 & 0.803407 \\
\hline 12 & 0.076588 & 0.879995 \\
\hline 13 & 0.051713 & 0.931709 \\
\hline 14 & 0.032013 & 0.963722 \\
\hline 15 & 0.018259 & 0.981981 \\
\hline 16 & 0.009637 & 0.991618 \\
\hline 17 & 0.004724 & 0.996342 \\
\hline 18 & 0.002158 & 0.998499 \\
\hline 19 & 0.000921 & 0.999421 \\
\hline 20 & 0.000368 & 0.999789 \\
\hline 21 & 0.000138 & 0.999928 \\
\hline 22 & 0.000049 & 0.999976 \\
\hline 23 & 0.000016 & 0.999993 \\
\hline 24 & 0.000005 & 0.999998 \\
\hline 25 & 0.000002 & 0.999999 \\
\hline 26 & 0.000000 & 1.000000 \\
\hline
\end{tabular}


TABLE2.2. Probabilities of Specified Numbers of Rejections Out of 91 Tests, where the Probability of

Incorrectly Rejecting on 1 Test is 0.05

\begin{tabular}{|c|c|c|}
\hline $\begin{array}{l}\text { Number of } \\
\text { Rejections }\end{array}$ & $\begin{array}{l}\text { Individual } \\
\text { Probability } \\
\end{array}$ & $\begin{array}{l}\text { Cumulative } \\
\text { Probability }\end{array}$ \\
\hline 0 & 0.009394 & 0.0099394 \\
\hline 1 & 0.044992 & 0.054386 \\
\hline 2 & 0.106560 & 0.160946 \\
\hline 3 & 0.166383 & 0.327330 \\
\hline 4 & 0.192654 & 0.519984 \\
\hline 5 & 0.176431 & 0.696415 \\
\hline 6 & 0.133097 & 0.829512 \\
\hline 7 & 0.085062 & 0.914574 \\
\hline 8 & 0.047008 & 0.961582 \\
\hline 9 & 0.022817 & 0.984399 \\
\hline 10 & 0.009847 & 0.994246 \\
\hline 11 & 0.003816 & 0.998062 \\
\hline 12 & 0.001339 & 0.999401 \\
\hline 13 & 0.000428 & 0.999829 \\
\hline 14 & 0.000126 & 0.999955 \\
\hline 15 & 0.000034 & 0.999989 \\
\hline 16 & 0.000008 & 0.999997 \\
\hline 17 & 0.000002 & 0.999999 \\
\hline 18 & 0.000000 & 1.000000 \\
\hline
\end{tabular}

addition to recording the total number of rejections, we record the number in each "tail" of the test. These one-tailed numbers of rejections are what would be obtained if two separate one-tailed tests were performed at the 0.05 significance level. Therefore, the probabilities in Table 2.2 can be used to assess the number of rejections in either the lower or upper tail of any test. From Table 2.2 it is seen that 2 to 8 rejections are acceptable (probability of 0.91 of being in this range).

Table 2.3 contains the observed number of rejections (out of 91 tests) in each tail of the one-, two- and three-dimensional uniformity tests, goodnessof-fit tests, and independence test. 
TABLE 2.3. Number of Rejections from Goodness-of-Fit, Independence and Uniformity Tests

Number of Rejections (out of 91)

\begin{tabular}{|c|c|c|c|}
\hline Test & Lower-Tail (a) & Upper-Tail (b) & Total \\
\hline $\begin{array}{l}\text { Goodness-of-Fit: } \\
\text { Cramer-von Mises } \\
\text { Kolmogorov-Smirnov } \\
\text { Kuiper } \\
\text { Watson }\end{array}$ & $\begin{array}{l}4 \\
7 \\
5 \\
4\end{array}$ & $\begin{array}{l}4 \\
5 \\
3 \\
4\end{array}$ & $\begin{array}{r}8 \\
12 \\
8 \\
8\end{array}$ \\
\hline $\begin{array}{c}\text { Independence: } \\
\text { Runs Test }\end{array}$ & 6 & 5 & 11 \\
\hline $\begin{array}{l}\text { Uniformity: } \\
\text { One-dimensional } \\
\text { Two-dimensional } \\
\text { Three-dimensional }\end{array}$ & $\begin{array}{l}4 \\
3 \\
5\end{array}$ & $\begin{array}{l}4 \\
5 \\
4\end{array}$ & $\begin{array}{l}8 \\
8 \\
9\end{array}$ \\
\hline
\end{tabular}

(a) Lower-tail rejections indicate that the variates: (i) fit the uniform distribution too well (goodness-of-fit test), (ii) were too random (independence test), or ( $i i i)$ were too uniform (one-, two-, and threedimensional uniformity tests).

(b) Upper-tail rejections indicate that the variates: (i) had a significant lack-of-fit from the uniform distribution (goodness-of-fit test), (ii) had a lack of randomness (independence test), or (iii) had a lack of uniformity (one-, two-, and three-dimensional uniformity tests).

The theoretical mean and variance of uniform $(0,1)$ random variates are 0.5 and 0.08333333 respectively. The string means were assumed to be normally distributed, and when compared to 0.5 at the 0.05 significance level, three rejections occurred (which is in the acceptable range of rejections, based on Table 2.2). The string variances had a range of 0.08126699 to 0.08451434 and an average of 0.08343388 . Based on these results, there is no evidence that the true mean and variance of the strings of variates are not 0.5 and 0.08333333 respectively.

Figures 2.1 and 2.2 are typical of the histograms and theoretical quantile-quantile plots produced from the strings of uniform $(0,1)$ pseudo-random variates. In Figure 2.1 , the true uniform probability density function is superimposed on the histogram for comparison purposes. The histogram compares very well with the theoretical density function given that some differences are 


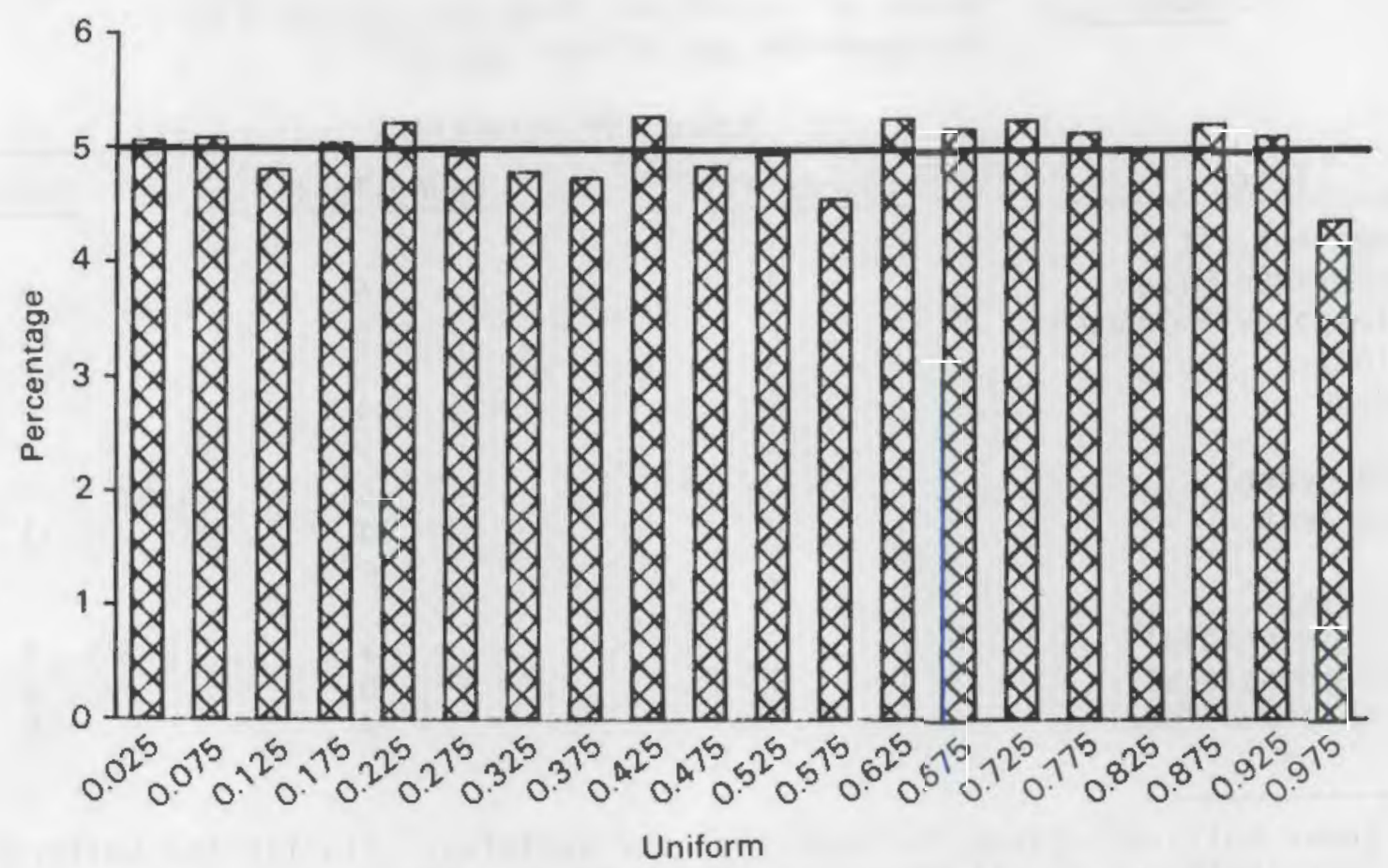

FIGURE 2.1. Histogram of 10,000 Random Variates from the PACSTAT Uniform $(0,1)$ Random Number Generator. The straight line is the theoretical ideal.

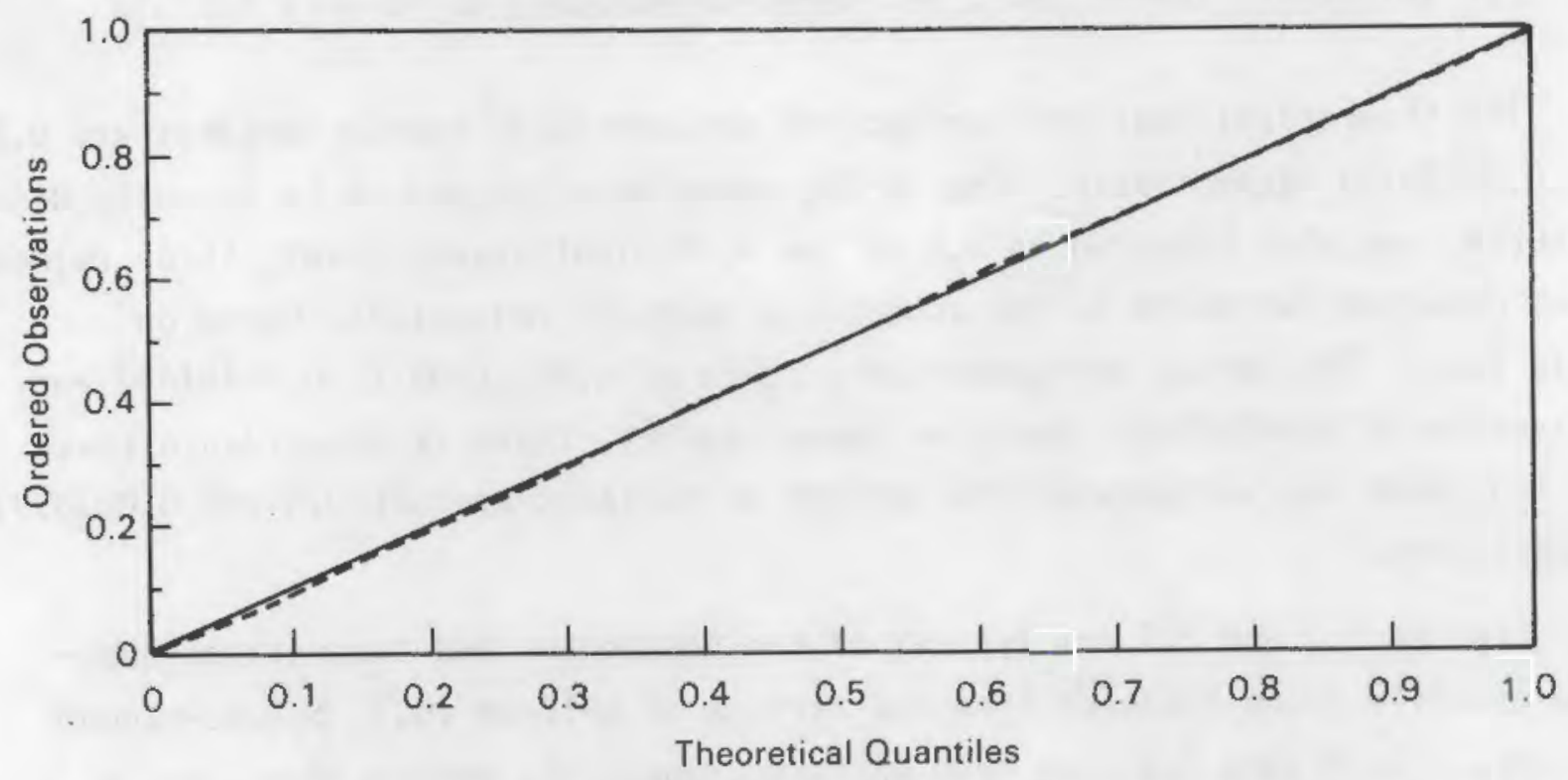

FIGURE 2.2. Theoretical Quantile-Quantile Plot of 10,000 Random Variates from the PACSTAT Uniform $(0,1)$ Random Number Generator. The straight line is the theoretical ideal. 
anticipated because of chance and the choice of bin width for plotting the histogram. In the theoretical quantile-quantile plot (Figure 2.2) the dashed line is the plot of the ordered data versus the theoretical quantiles. The solid line is the expected line if the distribution of the data matches the hypothesized distribution. The two lines coincide almost exactly, showing the good performance of the uniform RNG.

Based on the above results, there is no evidence that the variates within a string are not a random sample from a uniform $(0,1)$ distribution. In view of the probabilities in Tables 2.1 and 2.2, the numbers of rejections obtained with the tests are acceptable. Each string of variates appears to have the correct true mean and variance. The histograms and theoretical quantilequantile plots also did not show anything anomalous in the strings of variates. Hence, provided the corrections noted in Section 5 are made in PACSTAT, it appears to satisfactorily produce pseudo-random uniform $(0,1)$ variates. However, we briefly discuss in Section 5.0 the findings from a literature review that indicate a simple modification to the uniform random number generator used by PACSTAT would improve its performance. 


\subsection{EXPONENTIAL AND NORMAL RANDOM NUMBER GENERATORS}

The second stage of testing the PACSTAT RNGs focused on variates from the exponential and normal distributions. Variates from these distributions are generated in PACSTAT from uniform $(0,1)$ variates via the probability integral transformation method (see Appendix A). The transformation is theoretically exact for the exponential distribution, but involves a numerical approximation for the normal distribution.

\subsection{TESTS APPLIED TO THE NORMAL AND EXPONENTIAL RANDOM NUMBER GENERATORS}

In our tests, PACSTAT was used to produce variates from an exponential distribution with parameter $\theta=1$ and a normal distribution with the mean equal to zero and the standard deviation equal to one. The goodness-of-fit tests and the plots used to examine the uniform $(0,1)$ RNG were also used to check the exponential and normal RNGs. As for all distributions, summary statistics were computed for each string of variates for each distribution and were compared to theoretical values, and programming and hand calculation checks were made.

To test these two RNGs, 100 strings of 500 variates were generated from each distribution, and were subjected to the above mentioned tests. Only 500 variates were generated for these tests to reduce the long run times ( 8 to 12 hours) required to perform the statistical tests for 10,000 variates. Also, as discussed in Section 1.0, less extensive testing of the exponential and normal RNGs is required (because they are obtained using the uniform RNG, which was previously validated).

Prior to generating the 100 strings of 500 variates, the appropriate corrections (see Section 5.0) were made to the uniform RNG. Tables 3.1 and 3.2 correspond to Tables 2.1 and 2.2 except the probabilities are based on 100 independent tests instead of 91. From Tables 3.1 and 3.2 it is seen that 5 to 15 rejections are acceptable (probability of 0.94 ) when testing at a 0.10 significance level, and 2 to 8 rejections are acceptable (probability of 0.90 ) when testing at a 0.05 significance level. 
TABLE 3.1. Probabilities of Specified Numbers of Rejections Out of 100 Tests, Where the Probability of Incorrectly Rejecting on 1 Test is 0.10

\begin{tabular}{|c|c|c|}
\hline $\begin{array}{l}\text { Number of } \\
\text { Rejections }\end{array}$ & $\begin{array}{l}\text { Individual } \\
\text { Probability } \\
\end{array}$ & $\begin{array}{l}\text { Cumulative } \\
\text { Probability } \\
\end{array}$ \\
\hline 0 & 0.000027 & 0.000027 \\
\hline 1 & 0.000295 & 0.000322 \\
\hline 2 & 0.001623 & 0.001945 \\
\hline 3 & 0.005892 & 0.007836 \\
\hline 4 & 0.015875 & 0.023711 \\
\hline 5 & 0.033866 & 0.057577 \\
\hline 6 & 0.059579 & 0.117156 \\
\hline 7 & 0.088895 & 0.206051 \\
\hline 8 & 0.114823 & 0.320874 \\
\hline 9 & 0.130416 & 0.451290 \\
\hline 10 & 0.131865 & 0.583156 \\
\hline 11 & 0.119878 & 0.703033 \\
\hline 12 & 0.098788 & 0.801821 \\
\hline 13 & 0.074302 & 0.876123 \\
\hline 14 & 0.051304 & 0.927427 \\
\hline 15 & 0.032682 & 0.960109 \\
\hline 16 & 0.019292 & 0.979401 \\
\hline 17 & 0.010592 & 0.989993 \\
\hline 18 & 0.005427 & 0.995419 \\
\hline 19 & 0.002602 & 0.998021 \\
\hline 20 & 0.001171 & 0.999192 \\
\hline 21 & 0.000496 & 0.999688 \\
\hline 22 & 0.000198 & 0.999886 \\
\hline 23 & 0.000075 & 0.999960 \\
\hline 24 & 0.000027 & 0.999987 \\
\hline 25 & 0.000009 & 0.999996 \\
\hline 26 & 0.000003 & 0.999999 \\
\hline 27 & 0.000001 & 1.000000 \\
\hline 28 & 0.000000 & 1.000000 \\
\hline
\end{tabular}


TABLE 3.2. Probabilities of Specified Numbers of Rejections Out of 100 Tests, Where the Probability of Incorrectly Rejecting on 1 Test is 0.05

\begin{tabular}{|c|c|c|}
\hline $\begin{array}{l}\text { Number of } \\
\text { Rejections } \\
\end{array}$ & $\begin{array}{l}\text { Individual } \\
\text { Probability } \\
\end{array}$ & $\begin{array}{l}\text { Cumulative } \\
\text { Probability } \\
\end{array}$ \\
\hline 0 & 0.005921 & 0.005921 \\
\hline 1 & 0.031161 & 0.037081 \\
\hline 2 & 0.081182 & 0.118263 \\
\hline 3 & 0.139576 & 0.257839 \\
\hline 4 & 0.178143 & 0.435981 \\
\hline 5 & 0.180018 & 0.615999 \\
\hline 6 & 0.150015 & 0.766014 \\
\hline 7 & 0.106026 & 0.872040 \\
\hline 8 & 0.064871 & 0.936910 \\
\hline 9 & 0.034901 & 0.971812 \\
\hline 10 & 0.016716 & 0.988528 \\
\hline 11 & 0.007198 & 0.995726 \\
\hline 12 & 0.002810 & 0.998536 \\
\hline 13 & 0.001001 & 0.999537 \\
\hline 14 & 0.000327 & 0.999864 \\
\hline 15 & 0.000099 & 0.999963 \\
\hline 16 & 0.000028 & 0.999991 \\
\hline 17 & 0.000007 & 0.999998 \\
\hline 18 & 0.000002 & 0.999999 \\
\hline 19 & 0.000000 & 1.000000 \\
\hline
\end{tabular}

3.2 RESULTS OF TESTS ON THE NORMAL ANO EXPONENTIAL RANDOM NUMBER GENERATORS

Table 3.3 summarizes the results of the goodness-of-fit tests. Figures $3.1,3.2,3.3$, and 3.4 show typical histograms and theoretical quantilequantile plots for exponential and normal variates.

Table 3.3 gives no reason to question the fit of the exponential $(\theta=1)$ and normal $(0,1)$ distributions to their corresponding sets of variates. Also, the comparison of string means and variances to theoretical values gave no reason to doubt the performance of the two RNGs. Figures 3.1 and 3.3 are 
TABLE 3.3. Number of Rejections in Each Tail in Goodness-of-Fit Tests for the Exponential and Normal Random Number Generators in PACSTAT

\begin{tabular}{|c|c|c|c|}
\hline Tests & Lower-Tail (a) & Upper-Tail (b) & Total \\
\hline \multicolumn{4}{|l|}{ Exponential: } \\
\hline Cramer-von Mises & 5 & 4 & 9 \\
\hline Kolmogorov-Smirnov & 5 & 6 & 11 \\
\hline Kuiper & 6 & 5 & 11 \\
\hline Watson & 5 & 5 & 10 \\
\hline \multicolumn{4}{|l|}{ Normal: } \\
\hline Cramer-von Mises & 5 & 4 & 9 \\
\hline Kolmogorov-Smirnov & 5 & 5 & 10 \\
\hline Kuiper & 6 & 4 & 10 \\
\hline Watson & 5 & 5 & 10 \\
\hline
\end{tabular}

(a) Lower-tail rejections indicate that the RNG variates fit the distribution (normal or exponential) too well.

(b) Upper-tail rejections indicate that the RNG variates do not fit the distribution (normal or exponential) of interest.

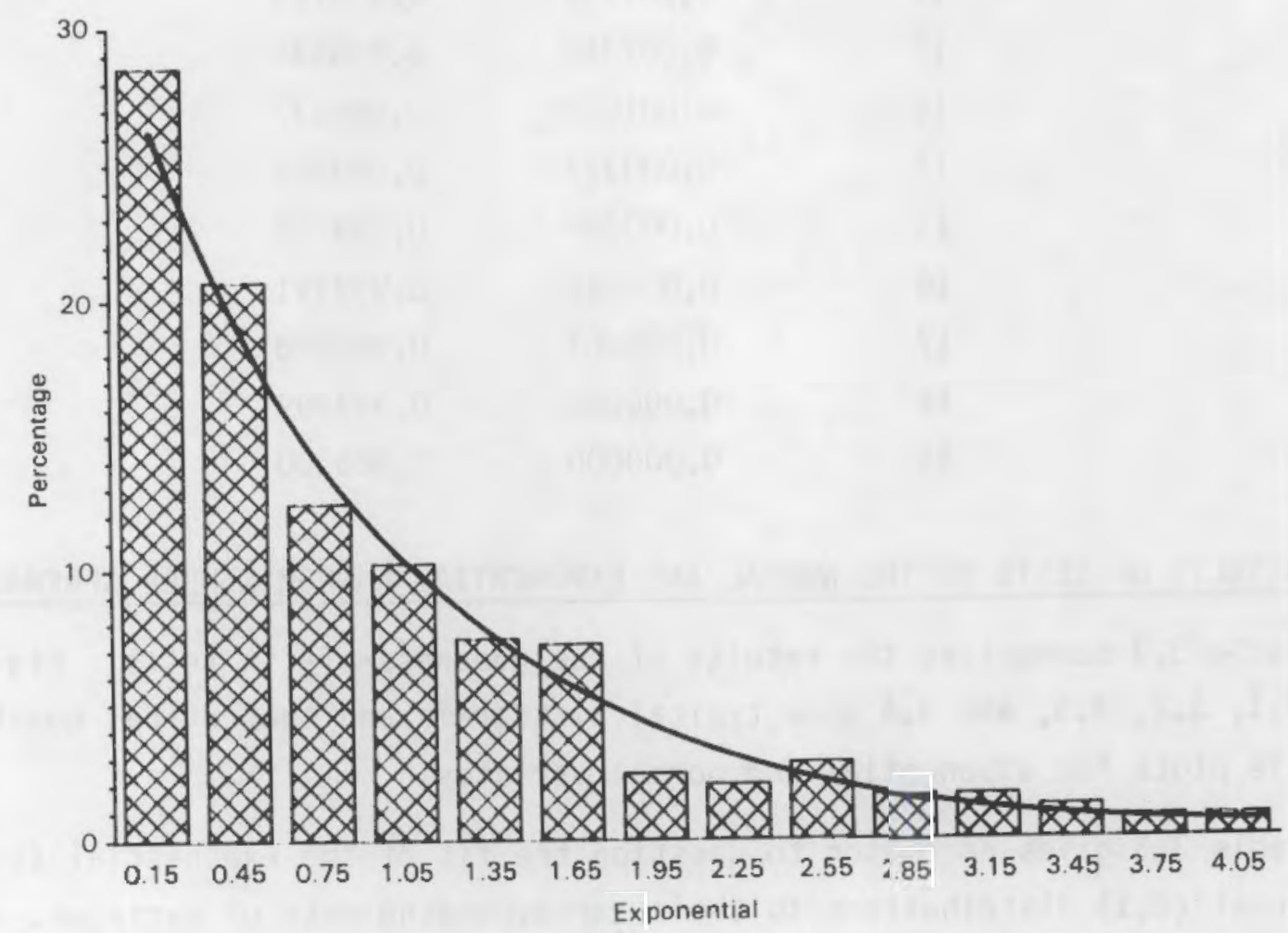

FIGURE 3.1. Histogram of 500 Random Variates from the Exponential Distribution with Parameter $\theta=1$. The solid curve is the theoretical ideal. 


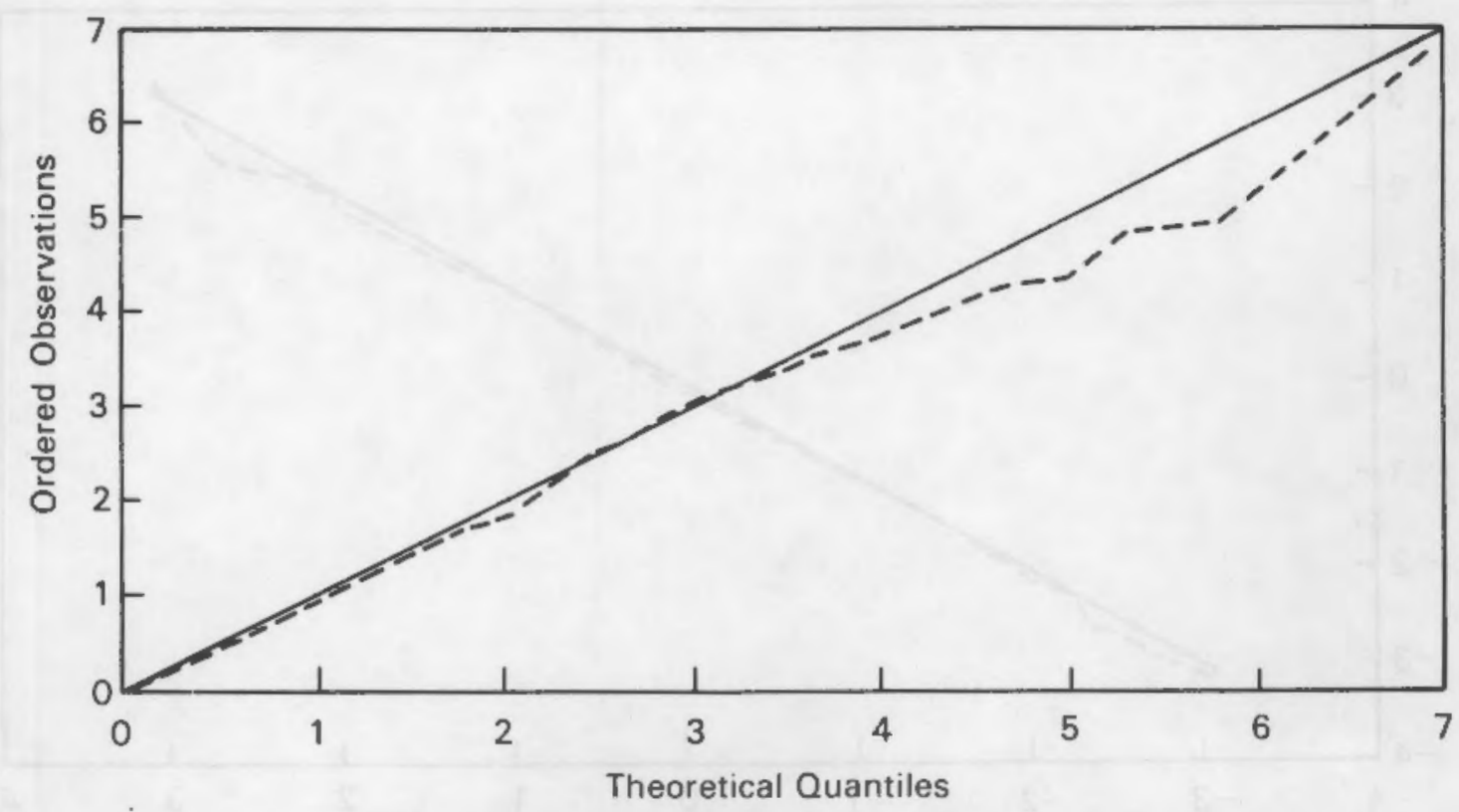

FIGURE 3.2. Theoretical Quantile-Quantile Plot of 500 Random Variates from the Exponential Distribution with Parameter $\theta=1$. The straight line is the theoretical ideal.

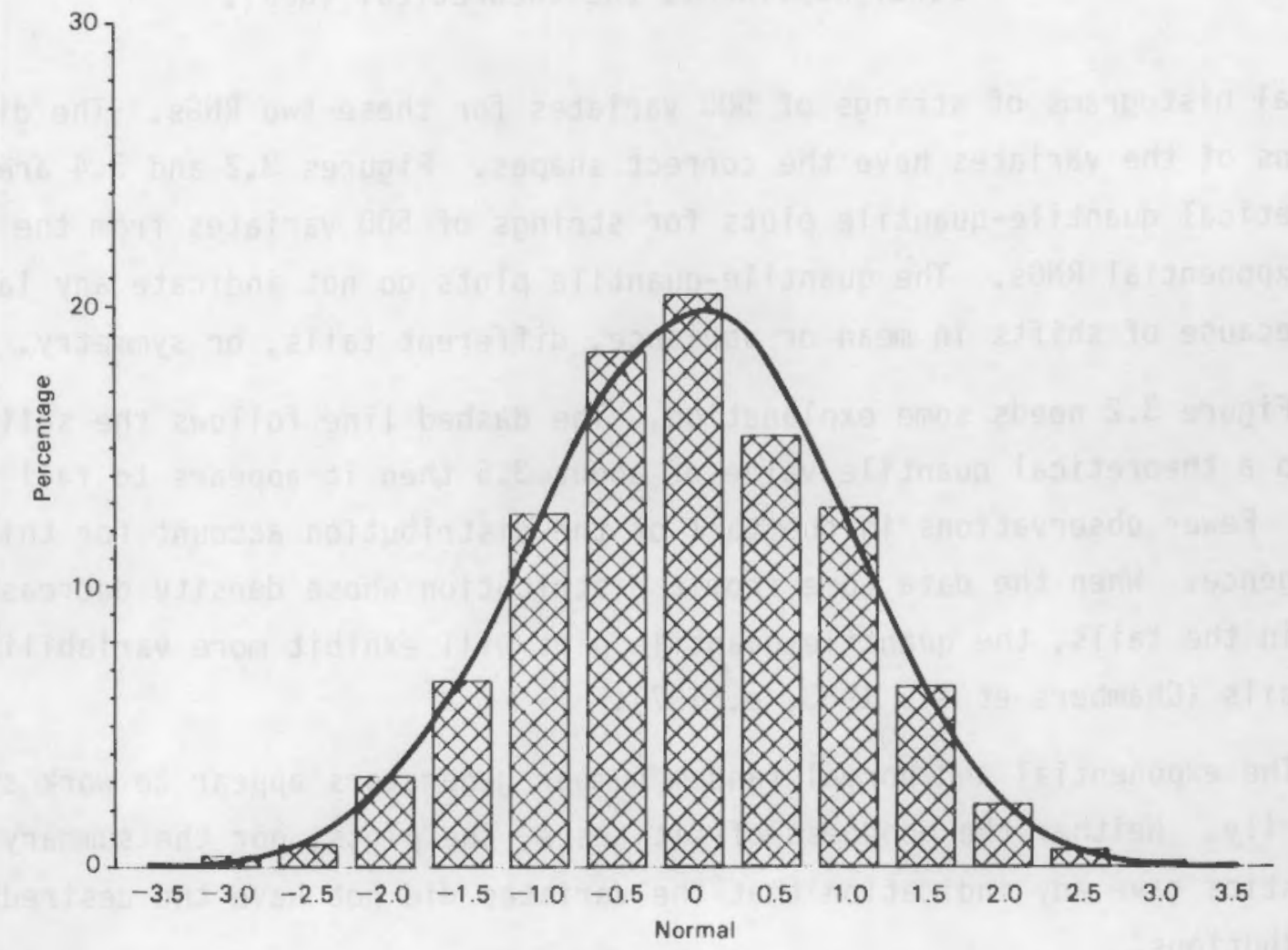

FIGURE 3.3. Histogram of 500 Randorn Variates from the Normal $(0,1)$ Distribution. The solid curve is the theoretical ideal. 


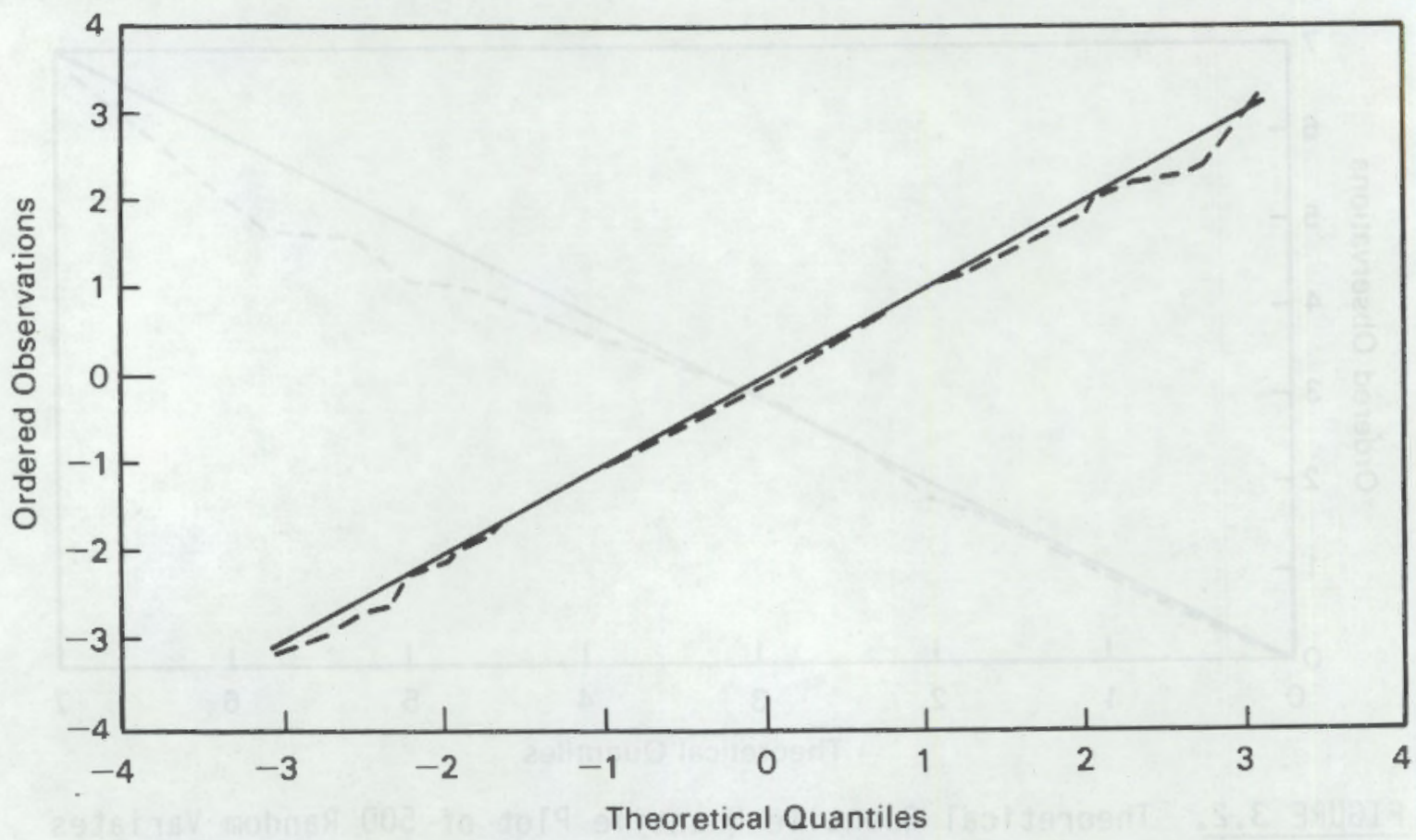

FIGURE 3.4. Theoretical Quantile-Quantile Plot of 500 Random Variates from the Normal $(0,1)$ Distribution. The straight line is the theoretical ideal.

typical histograms of strings of 500 variates for these two RNGs. The distributions of the variates have the correct shapes. Figures 3.2 and 3.4 are the theoretical quantile-quantile plots for strings of 500 variates from the normal and exponential RNGs. The quantile-quantile plots do not indicate any lack-offit because of shifts in mean or variance, different tails, or symmetry.

Figure 3.2 needs some explanation. The dashed line follows the solid line out to a theoretical quantile value of about 3.5 then it appears to fall away. Fewer observations in the tail of the distribution account for this divergence. When the data come from a distribution whose density decreases to zero in the tails, the quantile-quantile plot will exhibit more variability in the tails (Chambers et al. 1983, p. 197).

The exponential and normal random number generators appear to work satisfactorily. Neither the goodness-of-fit tests, the plots, nor the summary statistics gave any indication that the variates did not have the desired distributions. 


\subsection{REMAINING RANDOM NUMBER GENERATORS}

The five remaining PACSTAT random number generators were checked in the third stage of testing. PACSTAT was used to generate 100 strings of 500 variates for:

- the loguniform (common log) from a uniform $(0,1)$

- the truncated exponential from an exponential $(\theta=1)$ with limits 0.5 to 2.5

- the lognormal (natural log) from a normal $(0,1)$

- the truncated normal from a normal $(0,1)$ with limits -1.5 to 1.5

- the truncated lognormal (natural log) from a normal $(0,1)$ with limits -1.5 to 1.5 .

\subsection{TESTS ON THE REMAINING FIVE RANDOM NUMBER GENERATORS}

For each of the 100 strings of each distribution, summary statistics were computed and compared to theoretical values. The programing of the mathematical transformations used to produce variates from the five distributions was checked. Hand calculation checks were performed for a few variates from each of the five distributions. Histograms and theoretical quantile-quantile plots were constructed for 10 strings of 500 variates for each distribution.

Extensive testing of the loguniform, lognormal, truncated normal, truncated lognormal, and truncated exponential RNGs is not required at this stage. In the case of the lognormal and loguniform RNGs, variates are generated by simple exponentiation of normal and uniform variates, respectively. In the case of the truncated distributions, variates are essentially generated by sampling from the parent distribution but from within a restricted range. Since results of the first two stages of testing gave no reason to doubt the performance of the uniform, exponential, and normal random number generators, the five derivative generators should, aside from programming errors, work properly. See Section 1.0 for additional discussion of the hierarchial nature of the testing. 


\subsection{RESULTS OF TESTS ON THE REMAINING RANDOM NUMBER GENERATORS}

All five of the distributions mentioned above appeared to perform satisfactorily after some programming errors in PACSTAT were detected and corrected. The programming errors, which affected the generation of truncated normal and truncated lognormal variates, are discussed and corrections listed in Section 5.0. Figures 4.1 to 4.10 are typical of the histograms and theoretical quantile-quantile plots produced for strings of variates from each distribution. In the case of the loguniform, lognormal, and truncated lognormal distributions, the variates were back-transformed to uniform, normal, and truncated normal variates, respectively. Nothing atypical appeared in the plots.

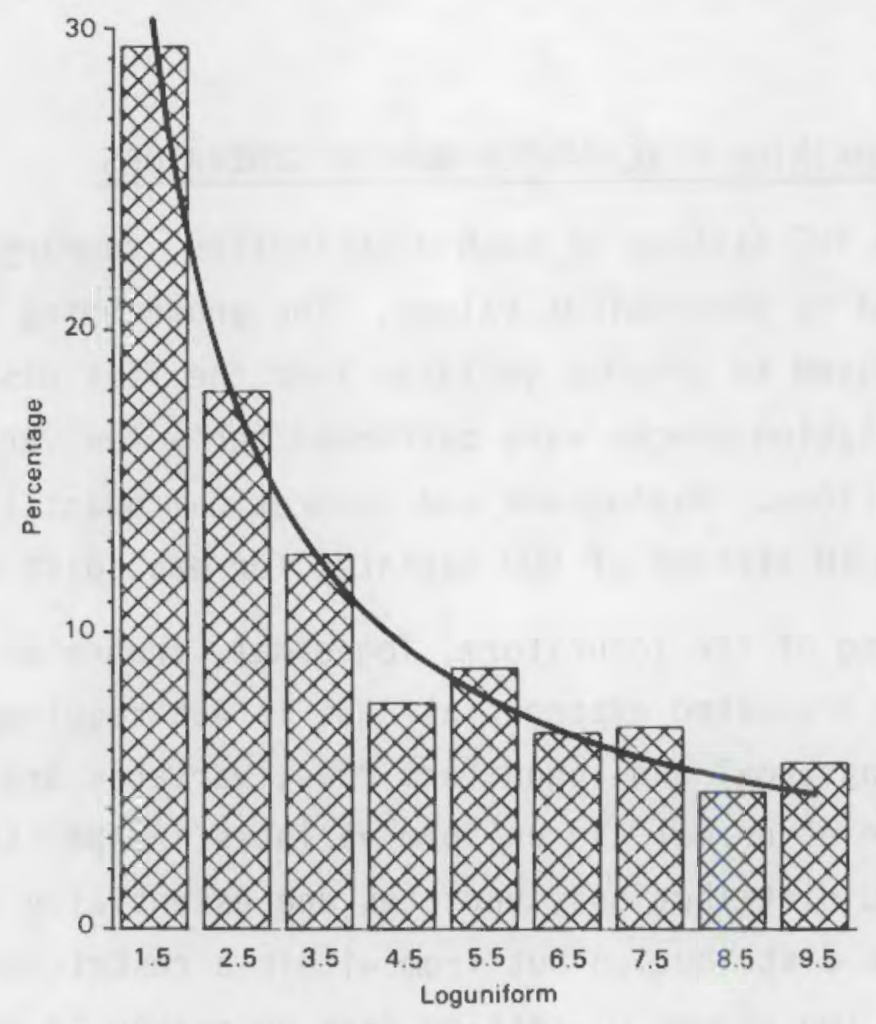

FIGURE 4.1. Histogram of 500 Random Variates from the Loguniform Distribution Based on the Uniform $(0,1)$. The solid curve is the theoretical ideal. 


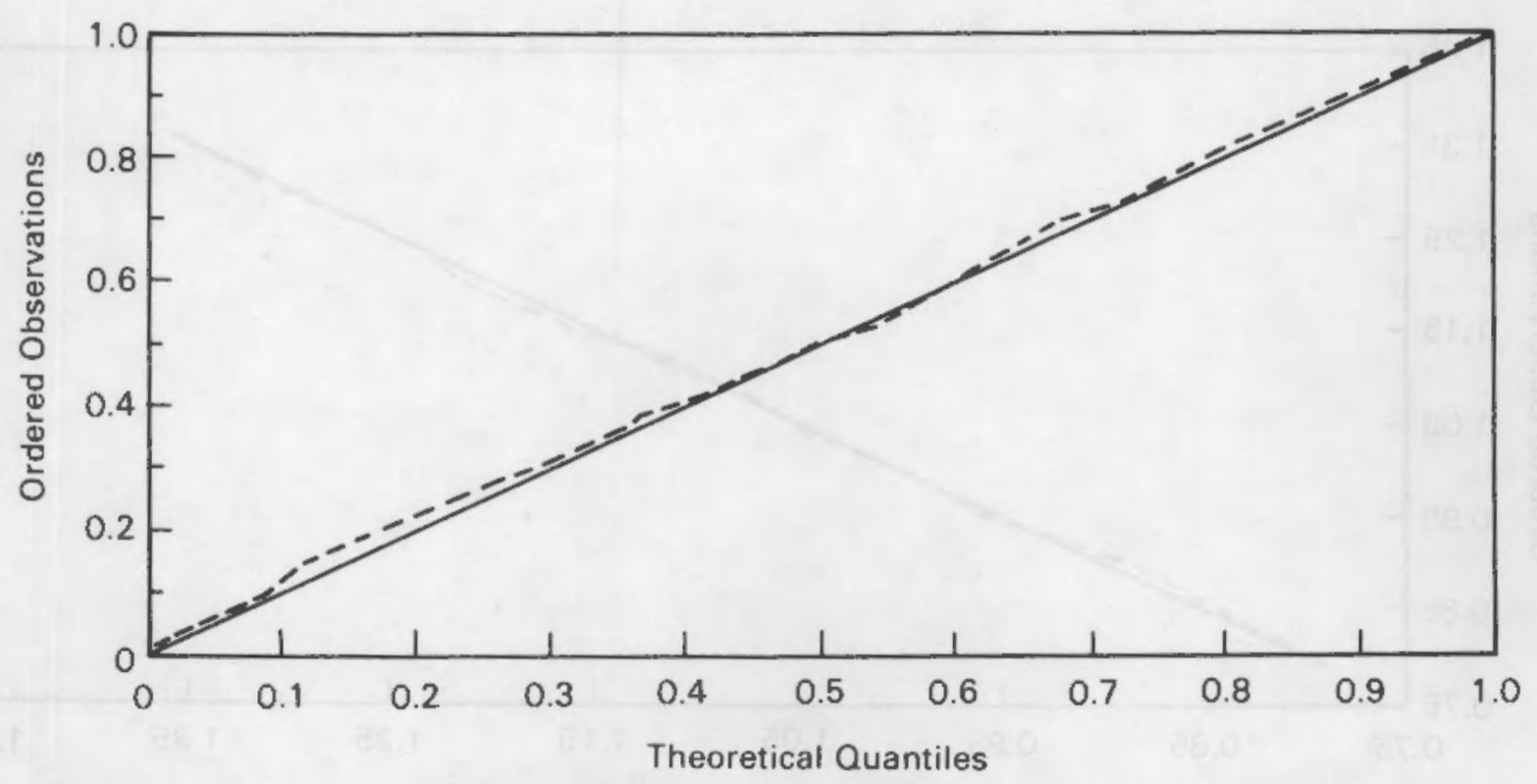

FIGURE 4.2. Theoretical Quantile-Quantile Plot of 500 Random Variates from the Loguniform Distribution Based on the Uniform $(0,1)$. The straight line is the theoretical ideal.

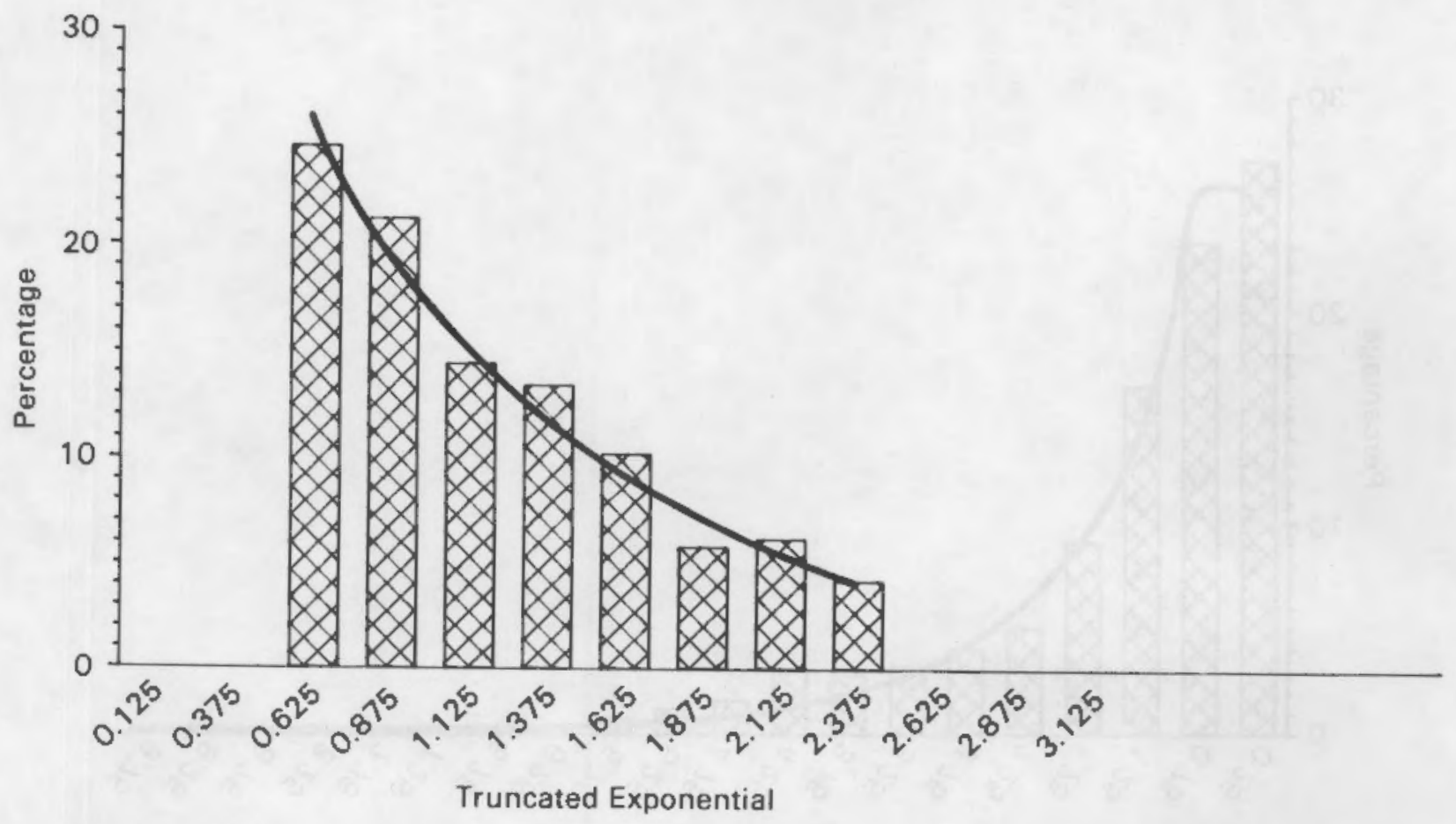

FIGURE 4.3. Histogram of 500 Random Variates from the Truncated Exponential Distribution. Truncation limits are 0.5 and 2.5. The solid curve is the theoretical ideal. 


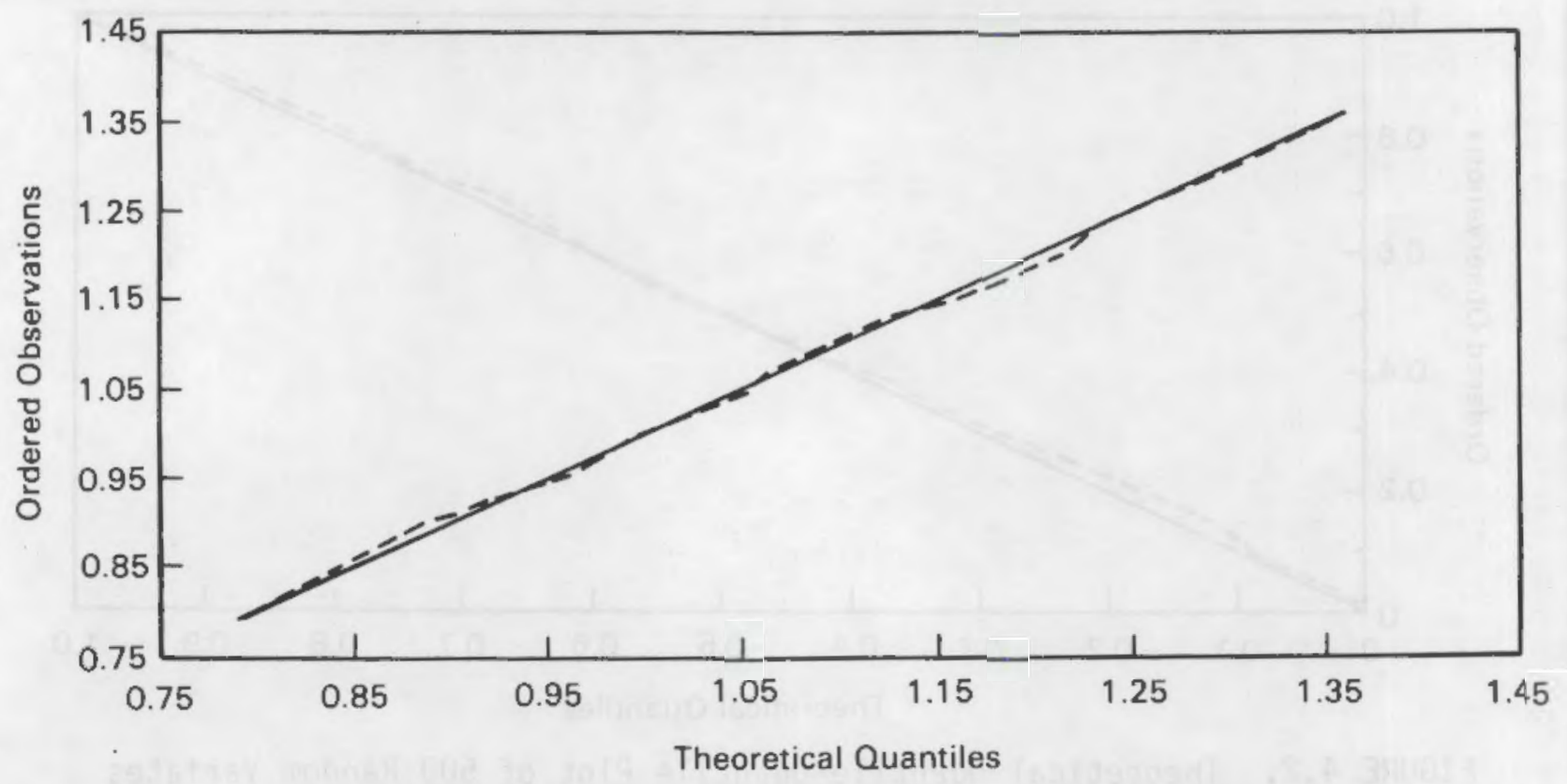

FIGURE 4.4. Theoretical Quantile-Quantile Plot of 500 Random Variates from the Truncated Exponential Distribution. Truncation limits are 0.5 and 2.5. The straight line is the theoretical ideal.

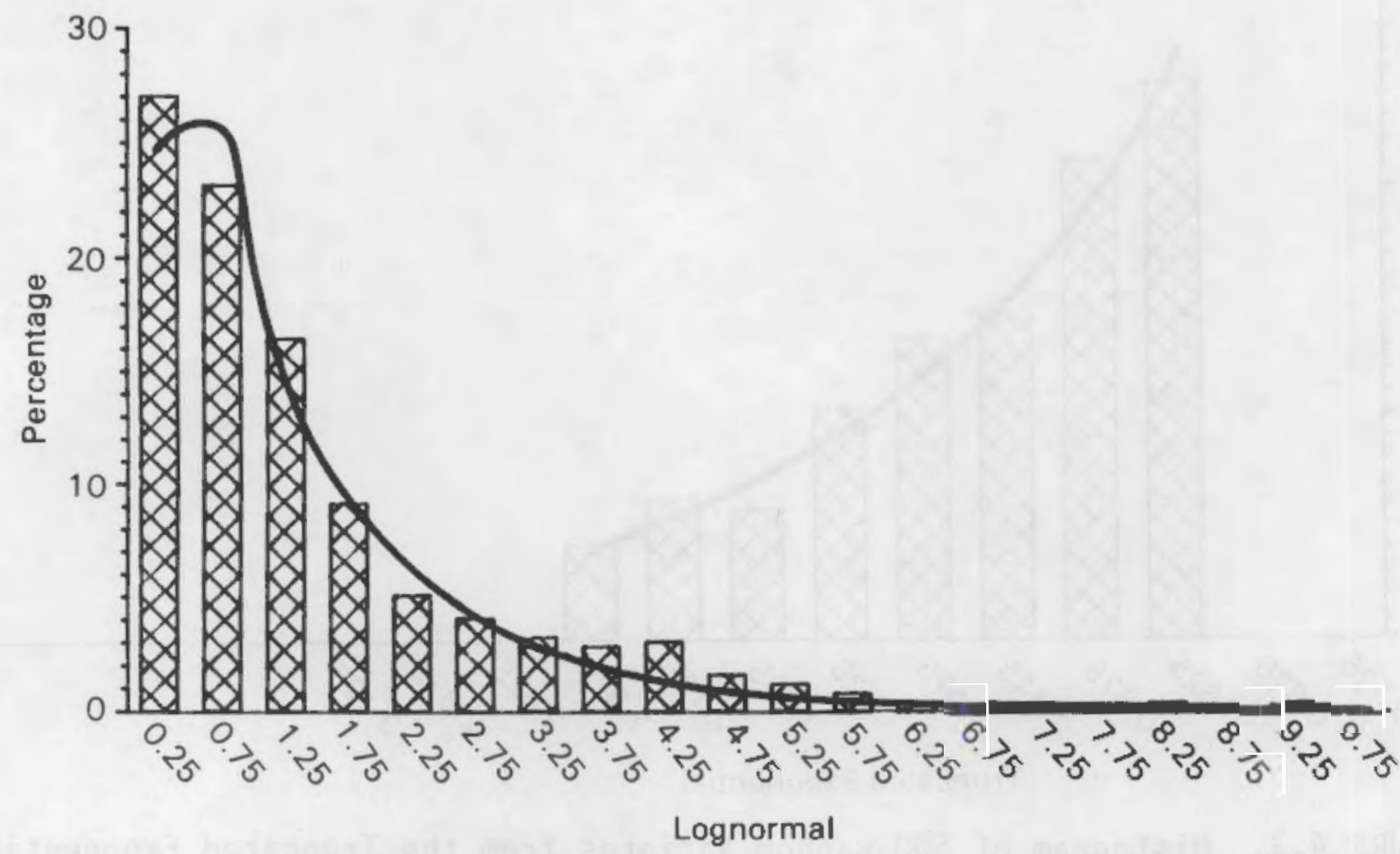

FIGURE 4.5. Histogram of 500 Random Variates from the Lognormal Distribution Based on the Normal $(0,1)$. The solid curve is the theoretical ideal. 


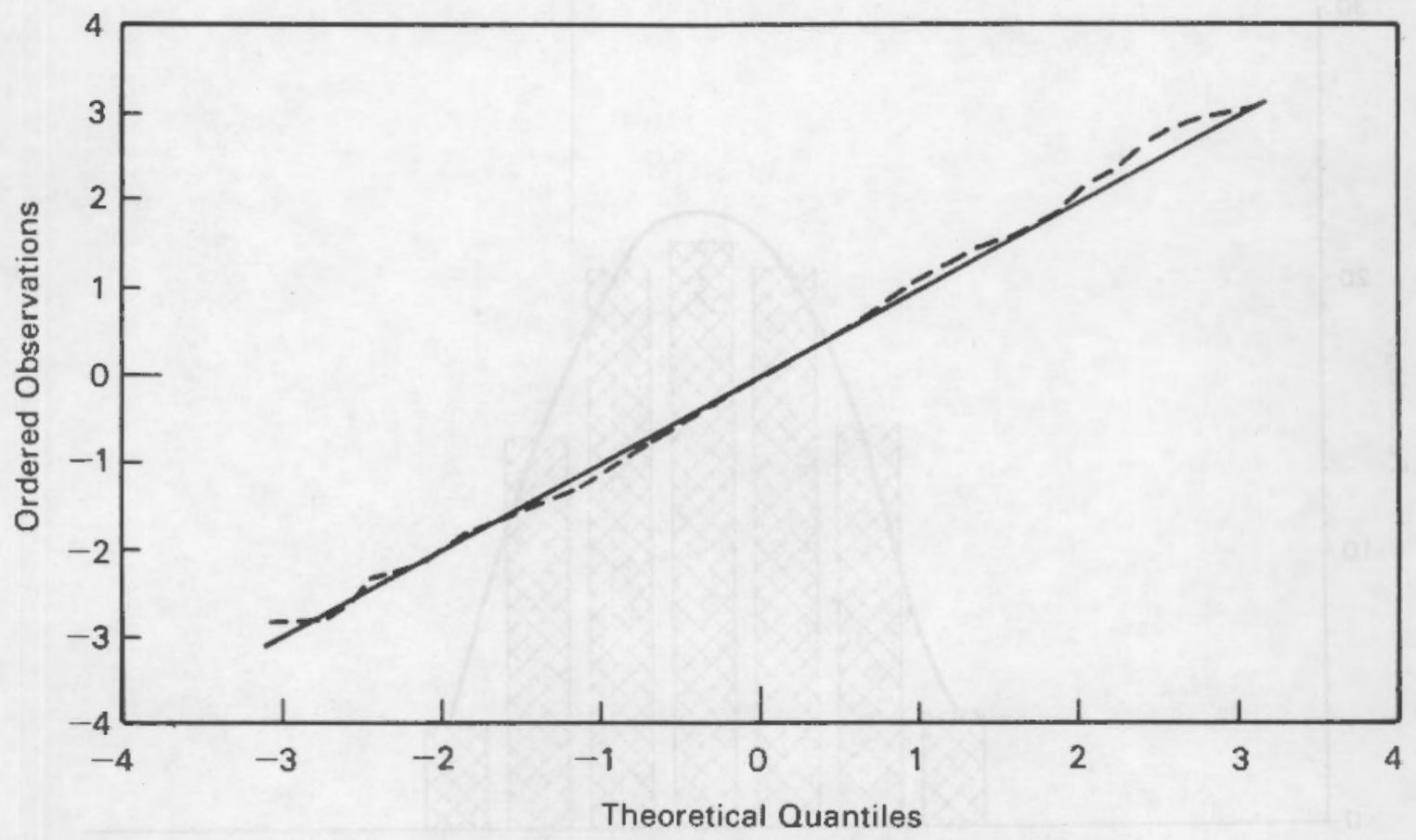

FIGURE 4.6. Theoretical Quantile-Quantile Plot of 500 Random Variates from the Lognormal Distribution Based on the Normal $(0,1)$. The straight line is the theoretical ideal. 


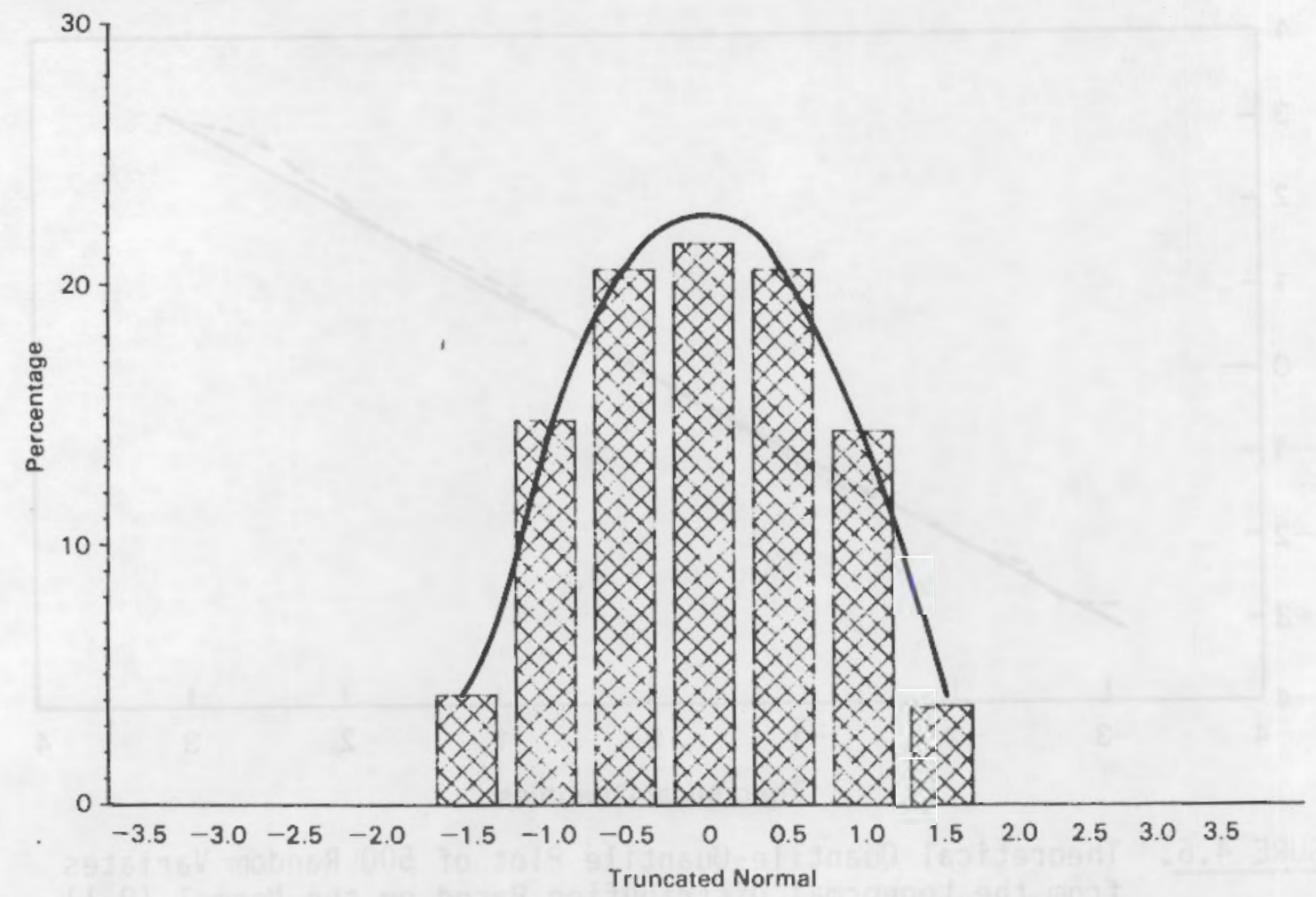

FIGURE 4.7. Histogram of 500 Random Variates from the Truncated Normal Distribution Based on the Normal $(0,1)$. Truncation limits are -1.5 and 1.5 . The solid curve is the theoretical ideal. 


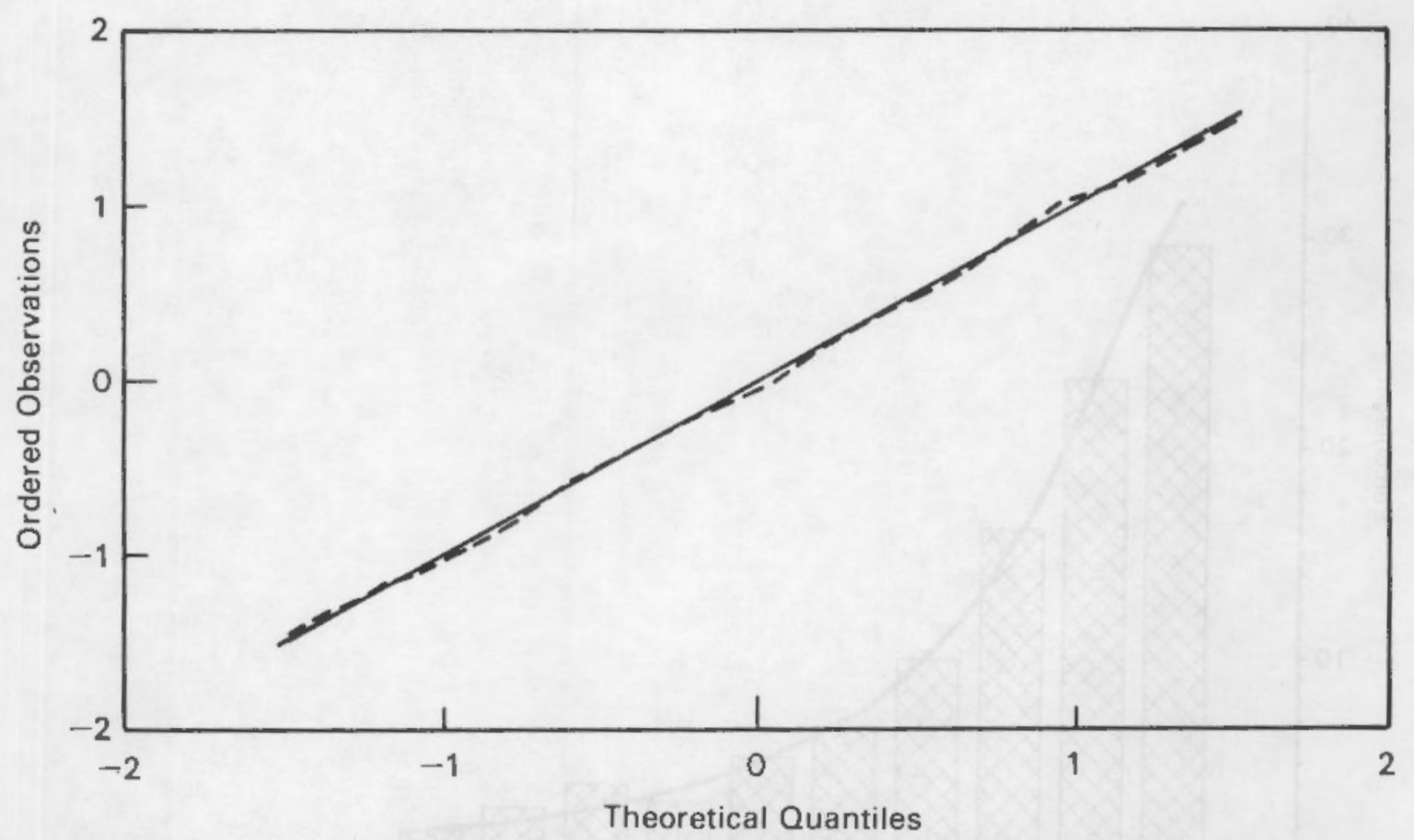

FIGURE 4.8. Theoretical Quantile-Quantile Plot of 500 Random Variates from the Truncated Normal Distribution Based on the Normal $(0,1)$. Truncation limits are -1.5 and 1.5 . The straight line is the theoretical ideal. 


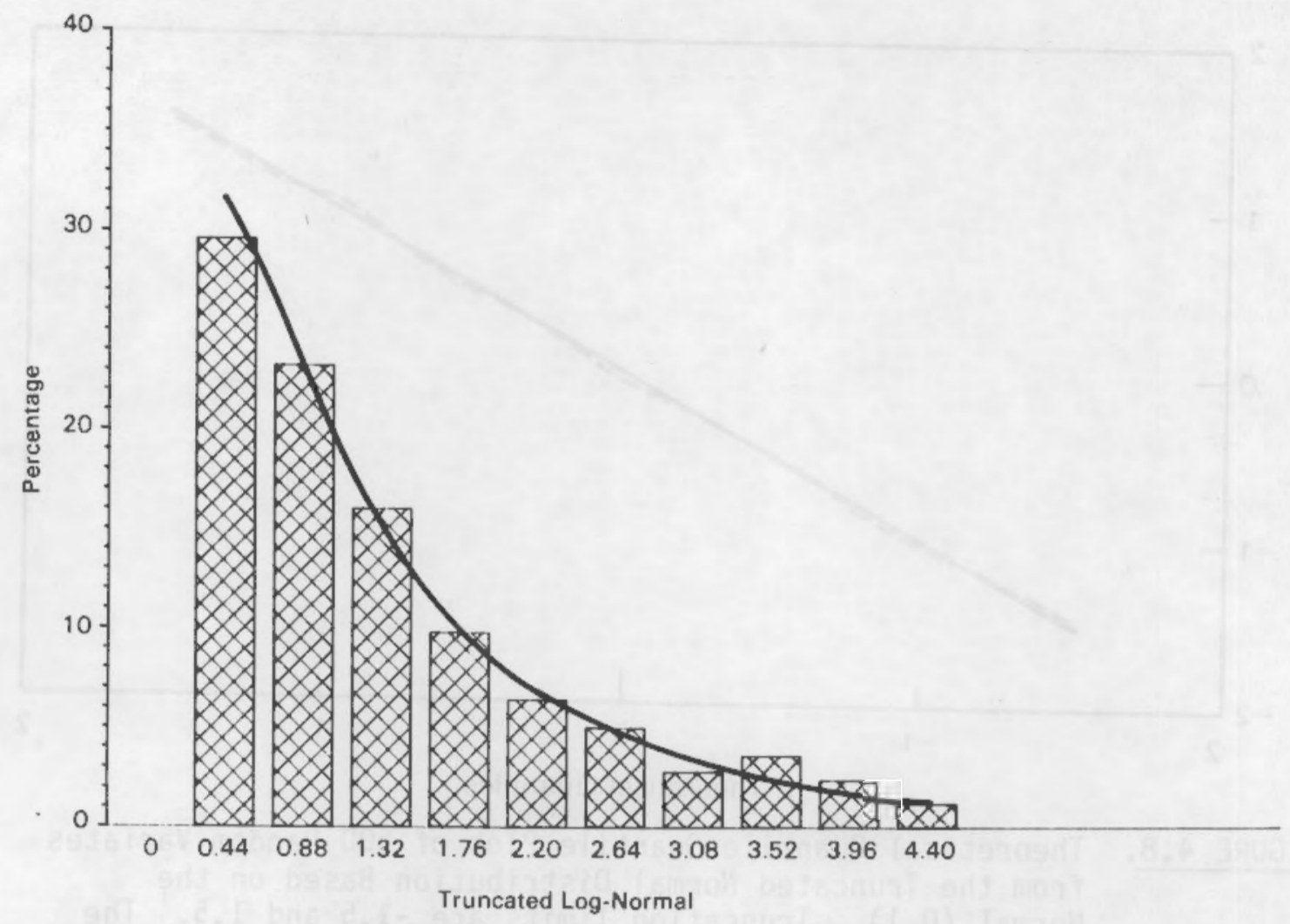

FIGURE 4.9. Histogram of 500 Random Variates from the Truncated Lognormal Distribution Based on the Normal $(0,1)$. The truncation limits are -1.5 and 1.5 . The solid curve is the theoretical ideal. 


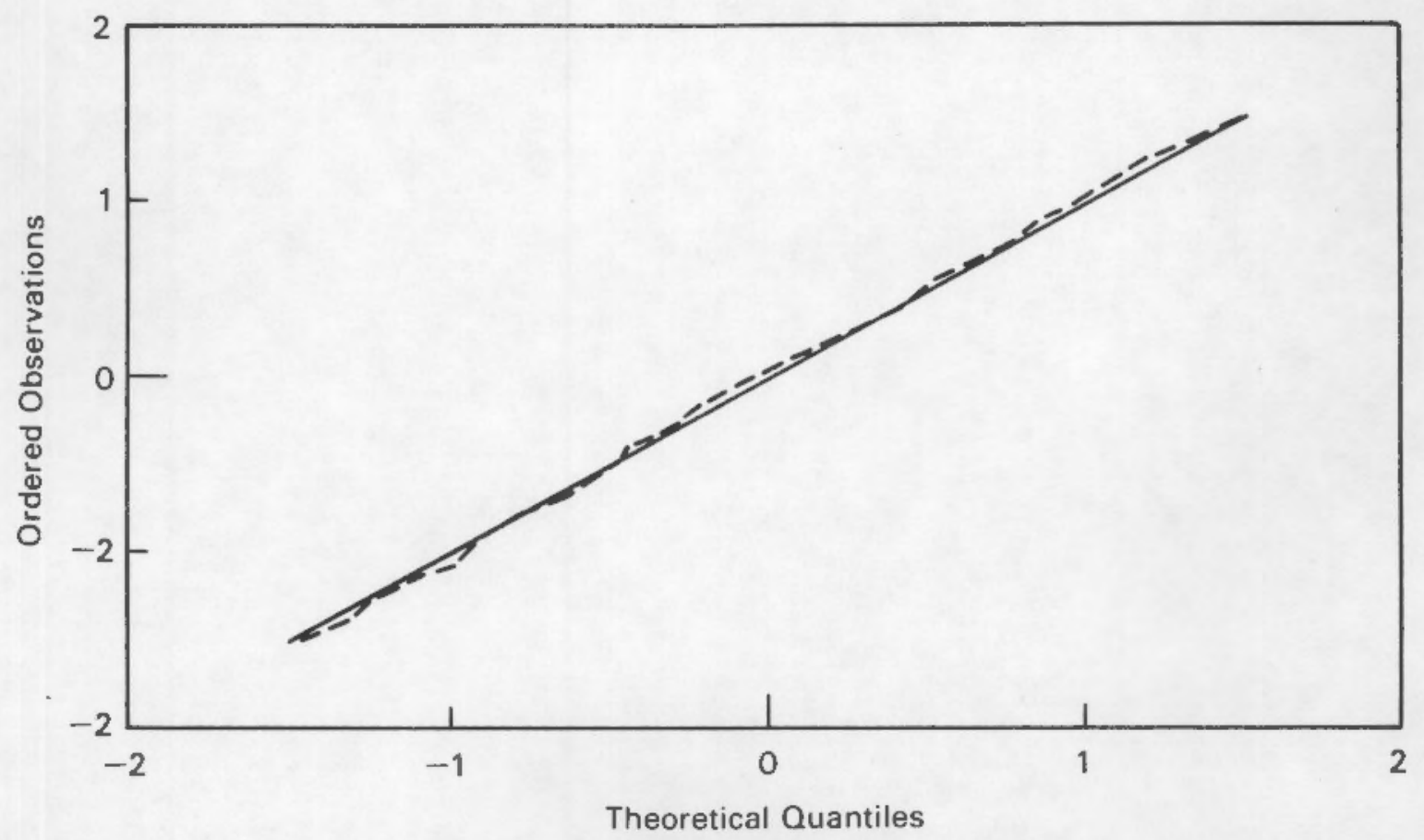

FIGURE 4.10. Theoretical Quantile-Quantile Plot of 500 Random Variates from the Truncated Lognormal Distribution Based on the Normal $(0,1)$. Truncation limits are -1.5 and 1.5 . The straight line is the theoretical ideal. 


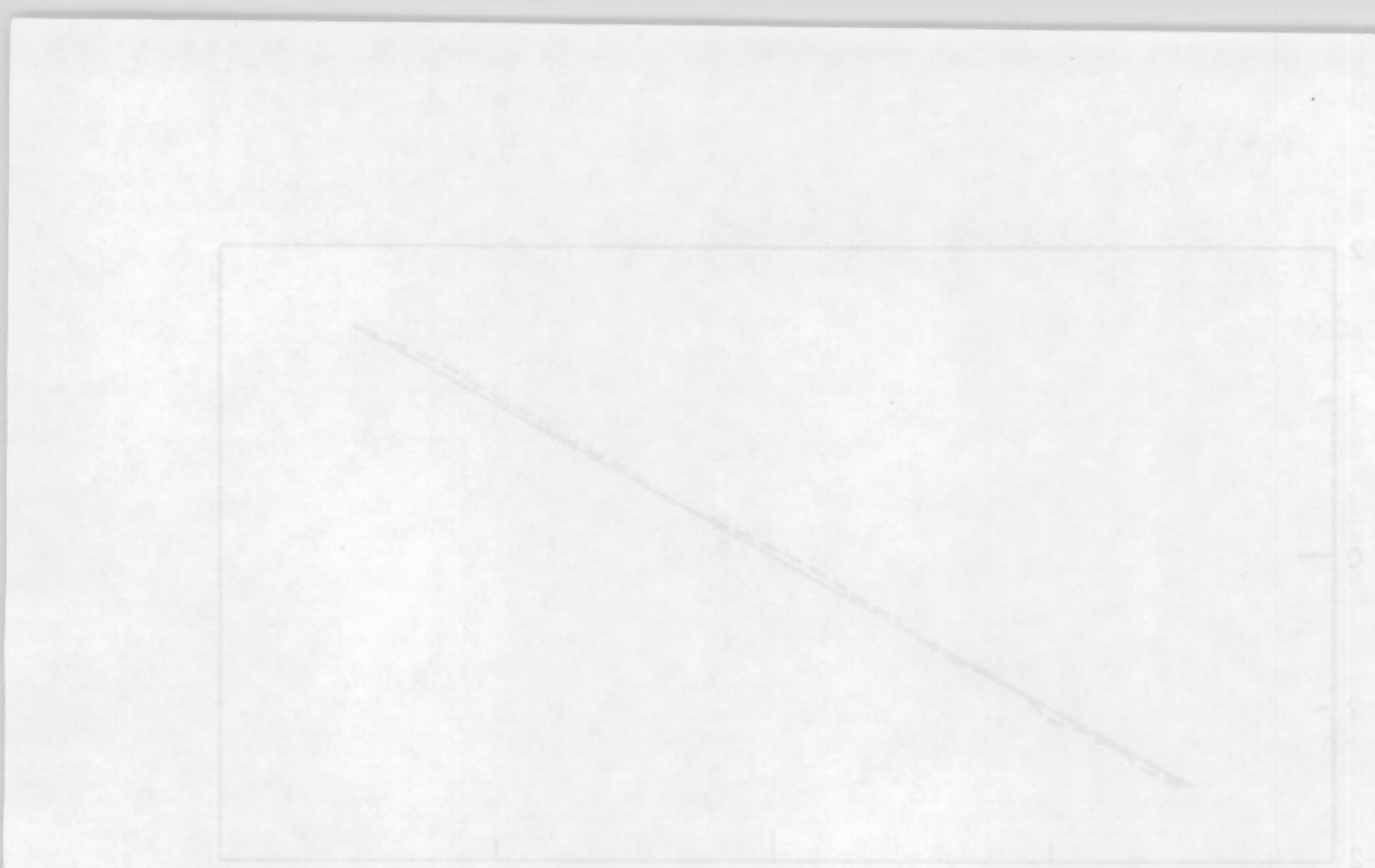

,

.

.

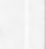

. 


\subsection{CORRECTIONS AND LITERATURE FINDINGS}

This section summarizes the corrections needed to make the RNGs in PACSTAT function as designed. Some minor errors were found that affected the uniform, truncated normal, and truncated lognormal RNGs. This section also contains a discussion of findings from a literature review.

\subsection{CORRECTIONS}

Two errors were found in the uniform RNG. First, the seeds were being read using single precision and then they were boosted to double precision. This caused the last 2 or 3 digits to be changed. The resulting seeds actually used were always even. Secondly, the range of permissible seed values was restricted to the closed interval [2, 2E+09] instead of the (correct) closed interval [1, (2**31)-2]. Values outside the interval [2, 2E+09] were set equal to $2 E+05$. The difference between the two intervals was the elimination of over 147 million permissible seed values. As the RNG started up, it iterated once and then checked the new seed, setting it to $2 E+05$ if the new seed was outside the interval [2, 2E+09]. This process produced 10 identical strings of variates when the uniform RNG was being tested. Hence, only 91 distinct strings of variates were tested instead of 100 . The first error was corrected by reading the input seed values in double precision and by eliminating the first call to the random number generator in the main module of PACSTAT. The specific corrections to the original version of PACSTAT ( $A 0$, contained in Appendix $B$ ) are:

Deleted

$\begin{array}{ll}\text { READ }(I N, *, E R R=190) \text { WD } & \text { PACSTAT.228 } \\ \text { DSEED }=D B L E(W D) & \text { PACSTAT.230 } \\ \text { A=U01(DSEED) } & \text { PACSTAT.231 }\end{array}$

Added

READ (IN, * , ERR $=190)$ DSEED

HEIDI3.6 
The second error was corrected by extending the range of seed values to the permissible range. The specific corrections to the AO version of PACSTAT are:

Deleted

$$
\begin{array}{ll}
\text { IF ((WD.LT.2.).OR. (WD.GT.2.E+9)) GO TO 19D } & \text { PACSTAT.229 } \\
\text { IF ((DSEED.LT.2.ODO).OR. (DSEED.GT.2.OE+9)) DSEED }=2.0 E+5 & \text { UO1S.39 } \\
\text { +NERATOR.' } / 1 X, '(\text { A REAL NUMBER BETWEEN } 1.0 \text { AND 2.0E+9)') } & \text { PACSTAT.335 }
\end{array}
$$

\begin{tabular}{|c|c|}
\hline DOUBLE PRECISION MXSEED & HEIDI3.1 \\
\hline REAL $\star 4$ MNSEED & HEIDI 3.2 \\
\hline$R E A L \star 8 \quad M X S E E D$ & HEIDI3.3 \\
\hline MNSEED $=1.0$ & HE ID 13.4 \\
\hline $\operatorname{MXSEED}=(2.0 \star \star 31)-2$ & HEIDI3.5 \\
\hline IF((DSEED.LT.MNSEED).OR. (DSEED.GT.MXSEED)) GO TO 190 & HEIDI3.8 \\
\hline NERATDR.' $/ 1 X, '($ A REAL NUMBER BETWEEN 1.0 AND $2 \wedge 31\rangle-2.)^{\prime}$ & BCSFIX.36 \\
\hline
\end{tabular}

Added

It is recommended that line U01S.39 be replaced with a check on the correct limits for a seed value, with a flag being set and returned in the subroutine U01S argument list. Further, the calling routine would then have to check the flag and appropriately handle an out-of-range seed. Because all seeds provided to PACSTAT during our testing were in the correct range, the lack of a check as recommended did not affect our runs. D. W. Langford at Boeing Computer Services (BCS) mentioned that routines other than PACSTAT also access subroutine U01S, so any corrections should be made keeping all uses in mind. It was for this reason that the PNL staff did not implement a correction, but merely deleted the line U01S.39.

There were two typographical errors in the subroutine ALNORM which affected the truncated normal and truncated lognormal random number generators. The specific corrections to the AO (original) version of PACSTAT are: 
Deleted

$+(Z+3.9806477940-4+1.9861538136400 /$

ALNORM. 38

$+(Z+4.838591280800+15.150897245100)$

ALNORM.40

Added

$+(Z+3.980647940-4+1.9861538136400)$

HEIDI 3.9

$+(Z+4.838591280800-15.150897245100)$

HE IDI 3.10

The A0 version of PACSTAT, the one received from BWIP, is listed in Appendix $B$. The line numbers listed above for deleted lines of code are from the AO version. The B4 version of PACSTAT (Appendix C) implements the deletions and additions noted above.

With the above corrections, and based on the strings of variates produced and tested, the RNGs in PACSTAT appear to work as intended. However, the fact that errors were found and corrected raises the question of the impact on previous applications of PACSTAT. The errors in subroutine ALNORM affect the truncated norma? and truncated lognormal RNGs, so any applications of PACSTAT which called these RNGs would be suspect and should be rerun. The errors in reading and checking seed values for the uniform RNG affect the output of all RNGs, since uniform variates are used by all RNGs. For applications where only one seed was used, the results are probably acceptable. For applications where more than one seed was used or where results were compared to other runs utilizing PACSTAT, the results should be considered faultly. In these latter cases, we recommend rerunning the application using the corrected version of PACSTAT.

\subsection{LITERATORE FINDINGS}

Fishman and Moore (1982) tested the uniform RNG used in PACSTAT along with 15 other such generators. The 16 uniform RNGs were all multiplicative congruential RNGs with modulus (2**31)-1 (see Appendix A, Section A.1). The difference between the RNGs was the value of the multiplier used (PACSTAT uses 16807). Fishman and Moore generated 100 strings of 200,000 variates to test and compare the 16 generators/multipliers. In addition to the goodness-of-fit 
tests, runs test, and tests of uniformity (on the unit line, unit square, and unit cube) used in our study, Fishman and Moore also performed several other tests, among them a test that uniformity on the unit line, unit square, and unit cube hold simultaneously. The generator with 16807 failed Fishman and Moore's test for two-dimensional uniformity and their simultaneous test for one-, two-, and three-dimensional uniformity. The fact that Fishman and Moore used strings of 200,000 variates where we only used 10,000 (because of computing limitations) may explain why they found problems that we did not. Another possible explanation is that their findings were due to chance, since some properties of an adequate RNG will occasionally seem invalid because of randomness.

In a recent paper, Fishman and Moore (1986) present the results of an exhaustive study of all possible multipliers for multiplicative congruential RNGs with modulus $(2 \star \star 31)-1$. Via several theoretical performance measures that characterize how well the RNG/multiplier "fills" $k$-dimensional unit cubes ( $k=2$, $\ldots, 6)$, they recommend the five best multipliers. Fishman and Moore compare these five best multipliers to 16807 and to two other comnonly used multipliers. They also perform the same statistical tests for the five best multipliers as in their 1982 article (again using 100 sets of 200,000 variates), concluding that the five multipliers show no empirical aberrations.

In comparing the statistical/empirical results of Fishman and Moore's 1982 and 1986 papers, only slight improvements in the five best multiplier results are seen relative to the 16807 multiplier results. However, the theoretical performance measures are clearly superior for the five new generators as compared to 16807. Since the statistical/empirical tests employed in this review are not designed to specifically test what Fishman and Moore's theoretical performance measures consider, it is not clear what the final evaluation should be.

In conclusion, the work of Fishman and Moore (1982, 1986) indicates that the PACSTAT random number generator could be improved theoretically by replacing the 16807 multiplier with one of their five best multipliers. However, it is not clear what the practical improvenent would be. Our belief is that the current uniform random number generator in PACSTAT (which is used to generate 
random numbers from all other distributions) is an adequate one, but there may be better ones. Changing the multiplier in the uniform random number generator in PACSTAT to one of the values recominended by Fishman and Moore would not require its retesting. 
. 


\subsection{QUALITY ASSURANCE AND CONFIGURATION CONTROL OF PACSTAT}

All work on PACSTAT was performed according to procedures described in the Statement of Work SOW-L1E2, and according to the Engineering Physics Department Quality Assurance (QA) Plan EPD-163-2. The operating environment for the computer work on PACSTAT was the PNL-BVAX (DEC VAX-11/780) running VMS-4.2. A11 files necessary to establish QA traceability are stored on DISK3 under the project name BWIP86. Additional files and backup files are stored on the user mountable disk labeled "BUDUEN", which is kept in the PNL-BVAX computer room. Tape copies of these files will be transmitted to BWIP at project completion. During FY86 through FY87 the following PNL staff members had access to the project and have established subdirectories under the main account [in brackets ]

$$
\begin{array}{ll}
\text { C. L. Bartley } & \text { [BWIP86.CLB] } \\
\text { M. J. Budden } & \text { [BWIP86.MJB] } \\
\text { H. Harty } & \text { [BWIP86.HH] } \\
\text { G. F. Piepel } & \text { [BWIP86.GFP] } \\
\text { M. R. Toland } & \text { [BWIP86.MRT] }
\end{array}
$$

The QA controlled version of PACSTAT used at the start of this work is called the $A 3$ version and is stored in its own subdirectory, [BWIP86.PACSTAT.A3].

During the verification process several minor errors were uncovered in the A3 version of PACSTAT and were fixed. Additionally, changes were made to enhance the QA traceability by writing the input file back to a data echo file, and writing multiple title/comments cards to the output file and the data echo file. This unambiguously ties a particular input file to the respective output file. This final augmented version is designated $A 4$ and is stored in its own subdirectory, [BWIP86.PACSTAT.A4].

The appropriate individual files on this subdirectory and their types are: 


$\begin{array}{ll}\text { PACSTAT_A4.SRC } & \text { WITNESS Source File } \\ \text { PACSTAT_A4.FOR } & \text { FORTRAN Source File } \\ \text { PACSTAT_A4.LIS } & \text { FORTRAN Compiler Listing File } \\ \text { PACSTAT_A4.OPL } & \text { WITNESS 01d Program Library } \\ \text { PACSTAT_A4.OLB } & \text { VAX Object Library (relocatable) } \\ \text { PACSTAT_A4.EXE } & \text { Executable Image File (absolute) } \\ \text { PACSTAT_A4.DXE } & \text { Debug Executable Image File (absolute) }\end{array}$

The PACSTAT_A4.SRC file should also serve as in input file to the BWIP utility HISTORIAN so that the identical version of PACSTAT that PNL has verified can be installed on the BWIP PRIME, or the CRAY at Los A1amos National Laboratory (LANL).

\subsection{CREATION OF PACSTAT VERSIONS A4 AND B4}

The PACSTAT source code and ancillary records were received on March 17, 1986. The format was a 9-track, 1600-bpi, EBCDIC, 80-cpr tape. This tape was copied with the MYTAPE utility that allows foreign tapes to be read on the VAX 11/780. One file was copied from this tape. This file contained, in the following order:

1. source program of PACSTAT

2. subroutine SOLN - sample input

3. prime job control language

4. block data

5. subroutine SOLN - sample input

6. sample output

7. block data

8. user's notes - variable definitions and general information on PACSTAT code.

After a file was created on the VAX, the source code was identified and separated from the other records. The PACSTAT code was then put under configuration management using the utility WITNESS (Koontz and Stewart 1986). 
WITNESS provides an automated and traceable means for installing, modifying, and documenting FORTRAN programs or other text files by assigning each line of source text a permanent unique identifier composed of a character string and an integer. WITNESS stores each line of text and its identifier in a program library. The program library can then be modified by referencing a line of text by its identifier. WITNESS allows the user to insert new lines and to delete existing lines. New lines are uniquely identified in the program library for future reference. Deleted lines remain in the program library (designated as inactive) and therefore may be restored. WITNESS keeps track of all changes to each line of text when they were made, and which "change ident" caused the change. All information may be retrieved by the "audit" feature. This unique and permanent identification of each line of text eliminates the possibility of the user accidentally destroying the integrity of the file (as sometimes happens with an interactive text editor). It also provides an orderly method of tracking code versions and identifying the various changes from version to version. This feature is indispensable for quality assurance, especially when multiple users require code modification privileges, as was the case for the FY86 work.

WITNESS is compatible with and provides most of the capabilities of Control Data Corporation's (CDC) UPDATE utility, which has been widely used for maintaining large simulation codes on CDC computers, and is similar to the HISTDRIAN utility offered by 0pcode, Inc. (available on the LANL computer system). WITNESS was written by PNL to provide UPDATE capabilities on other machines, specifically DEC-VAX-11/780 and IBM systems.

The first step in the configuration control procedure for PACSTAT was to use WITNESS in the "creation run" mode. This step requires a WITNESS source file as input, which is identical to a FORTRAN source file, except for the presence of $\star D E C K$ and ${ }^{\circ} C O M D E C K$ cards that immediately precede subroutines and common blocks, respectively. The *DECK and *CDMDECK cards provide the character part of the identifier for each line of FORTRAN source. WITNESS increments a counter for each card or record it encounters until it finds a new *DECK card. The counter provides the integer part of the unique identifier for each line of source. The identifier is written to the .FOR file into columns 
81 to 96 , which is then sent to the compiler. WITNESS also creates what is called a "Program Library" which contains all of the audit information as well as the identifiers. This is written to another file with the extension .OPL (01d Program Library) and is a randon access formatted file that cannot be edited. The only means of changing the 01d Program Library (OLDPL) is through the use of WITNESS so that the OLDPL becomes the primary archive file for maintaining versions of a code. The ${ }^{2} D E C K$ and ${ }^{*}$ COMDECK cards were inserted in the the appropriate location in the PACSTAT FORTRAN source file and it was run through WITNESS. This created the AO version of PACSTAT (1isted in Appendix $B$ ). The AO version of PACSTAT is essentially the version of PACSTAT that BWIP originally transferred to PNL, the only difference being the WITNESS generated "idents" in columns 81 to 96 . Because of the system dependent features of PACSTAT, the AO version would not compile on the PNL-VAX system computer. Thus, a series of changes were begun to convert PACSTAT into a VAX executable version, that was as much as possible, American National Standards Institute (ANSI) FORTRAN-77 compatible.

The initial set of updates (changes) consisted largely of deleting PRIME system-dependent functions and inserting coding to transform PACSTAT into WITNESS compatible form. Al1 "\$Insert *<Common> COMDECK name" commands were replaced with "*Call COMDECK name," and three subroutine calls were commented out and new subroutines written. Specifically for our purposes, subroutine ASGVAX replaced subroutine ASG, subroutine IDVAX took the place of subroutine IONUM, and subroutine RELEASE was modified. Subroutine VERSION was written to provide a banner page on the output file. VERSION's purpose was primarily for QA, providing traceability information on the version number of PACSTAT currently in use, update information, fixes and extensions, and applicable documentation.

The set of updates with the BCSFIX identifier was received from BCS during the PACSTAT conversion process, and included the addition of subroutine DISCLAIMER. This routine prints a verification/benchmarking disclaimer (required by the new $Q A$ procedures) and also makes some cosmetic changes to the output file. Coding with the "ECHO" identifier was added for the capability of having the input data echo to the output ECHO file (PACSTAT_A4.ECH). This 
echoing of input data provides a good, traceable QA record of the input when running interactively, and unambiguously ties a particular input file to a specific output file. Included in this set of updates was the added feature of being able to run PACSTAT interactively or batch. The final set of updates with the identifier "HEIOI3" implement the corrections noted in Section 5.1. These updates modify PACSTAT to correct errors involving the generation of random numbers. This includes typographical errors detected in routine ALNORM as well as data overflow errors caused by using a single precision variable to handle the double precision random number seed in the PACSTAT routine.

The following seven steps describe exactly how the final version of PACSTAT (the A4 version) was created from the tape given PNL by BWIP.

1. The tape from D. Langford with PRIME-750 version of PACSTAT source and data tapes was received.

2. The PACSTAT source was edited, ${ }^{\star D E C K}$ cards were put in appropriate places to create PACSTAT_AD.SRC. (Note that an EXTERNAL statement was added for BLKDAT.)

3. With the .SRC file from above as input, WITNESS was run in the creation mode, and it generated a file PACSTAT_AO.FOR ready to go to the FORTRAN compiler.

4. The .FOR file from 3 would not compile (PRIME system dependent constructs) so an update change file PACSTAT_AO.UPD was created to create a new PACSTAT Al.FOR.

5. PACSTAT_Al.FOR compiled but had many different corrections. The PACSTAT_A2.FOR version was created from the A1 version by resequencing all cards, making the A2 version "clean."

6. Version $A 3$ was created by taking the $A 2$ version above and adding PACSTAT_A2.UPD, which contains an entire subroutine called VERSION. VERSION is used for tracking the various PACSTAT versions by writing important version information to the OUTPUT file. The B3 (batch version) was created the same way. The final versions capable of running on the $V A X$ and satisfying acceptable traceability requirements are PACSTAT_A3.FOR and PACSTAT_B3.FOR. 
7. PACSTAT A3. UPD was used to change the $A 3$ version into the final $A 4$ version. Fixes included in this file were the changes to subroutine VERSION, the BCS fixes, a correction set called HEIDI which fixes some minor errors in the RNG; and some input echo fixes. The resulting interactive and batch versions are PACSTAT_A4.FOR and PACSTAT_B4.FOR.

A list of each of the .UPD update files described above is presented in Appendix $D$. All changes necessary to enable a person to go from the A0 version to the $A 4$ or $B 4$ version are contained in these files.

The final Configuration Controlled Code Versions are designated the A4 (interactive) and $B 4$ (batch) versions, and contain the changes described above. A listing of the $B 4$ version is provided in Appendix $C$. The character/integer identifiers in columns 81 to 96 of this file clearly show each change PNL has made to the original PACSTAT version. New sample input and output files are shown in Appendices $E$ and $F$. All changes have been made upward compatible; this means that old PACSTAT input decks should still run on both the A4 and B4 versions. The new features of input are multiple title/comment cards on the input file for additional QA identification and a mode switch to indicate batch or interactive execution.

\subsection{QUALITY ASSURANCE RECOMMENDATIONS}

Based on the experience with PACSTAT there are two recommendations that we feel would improve the $Q A$ features of the code. First, we recommend that the current version and all subsequent versions be put under configuration management with HISTORIAN or HITNESS, as we have done at PNL. This will make transportation of the code to different machines easy, and traceable. It will save significant time in any further efforts on the code where quality assurance is required.

Secondly, we recommend that a concerted effort be made to remove all system-dependent features and make the code transportable between the VAX, PRIME, and LANL CRAY machines. Most of the system-dependent features have been 
removed. Subroutine ASGVAX replaces subroutine ASG and will work on the PNL DEC VAX machine. It will probably work on the CRAY also, but this conjecture has not been tested.

Some system-dependent features still remain, but they could be easily changed. For instance, the date and time routines, and the run identification coding are system dependent and probably always will be. This feature can be made nearly transportable by requiring the user to specify as input data what machine he is running in. This information can then be used inside the code to branch to the proper system dependent calls, other calls being by-passed. This way the difference between truly system-dependent versions can be minimized. It would also be heipful if all the system-dependent features could be combined or organized into one or two subroutines. 
$\cdot$
+ 


\subsection{GENERATION OF RANDOM NUMBERS FROM PACSTAT FOR STATISTICAL TESTS}

The process for generating random numbers from PACSTAT for use in the statistical tests (see Sections 2.0 to 4.0) started with the creation of a new version of the SOLN routine to be linked with PACSTAT. The SOLN routine was originally intended to perform calculations on the variates generated with the RNGs, with the results of the calculations printed to both binary and formatted output files. The new version of SOLN provides data for the statistical tests by setting the output variable equal to the input data provided by the random number generator. The block data routine BLKDAT was also modified to reflect the number of solutions provided by $\operatorname{SOLN}$, and provide internal documentation for the output files. Copies of BLKDAT and SOLN are included in Appendix G.

Next, VAX/VMS command files were built to run the PACSTAT program and merge the data provided by each run. A separate run of PACSTAT was made for each random number string that was generated by PACSTAT. A11 the runs for one type of distribution were contained in one command file. An annotated copy of the command file (for one run of PACSTAT) which generated 100 sets of 10,000 uniform variates is included in Appendix $H$. The initial seeds for the random number generator used in the command files, are themselves random numbers generated by SAS.

The output that resuited from the PACSTAT runs was not in a form immediately usable to the SAS procedures that performed the statistical tests. A short program was written to read the output file from PACSTAT; strip off the internal documentation and identifying numbers to each value in each random number string; and sort each random number string. The output of this program was used directly by the SAS procedures that performed the statistical tests. An example of the program that performed these functions for the uniform variates is provided in Appendix I. 

REFERENCES

Abramowitz, M. and Stegun, 1. eds. (1974). Handbook of Mathematical Functions, 10th Printing, National Bureau of Standards, U.S. Department of Commerce.

Chambers, J. M., W. S. Cleveland, B. Kleiner, and P. A. Tukey. 1983. Graphical Methods for Data Analysis, Duxbury Press, Boston, MA.

Fishman, G. S. and L. R. Moore. 1982. "A Statistical Evaluation of Multiplicative Congruential Random Number Generators with Modulus (2**31)-1." Journal of the American Statistical Association 77(377):129-136.

Fjshman, G. S. and L. R. Moore, 1986. "An Exhaustive Analysis of Multiplicative Congruential Random Number Generators With Modulus $2^{31}-1 . "$ SIAM J. Sci. Stat. Computing, 7:24-45.

Koontz, A. S. and C. W. Stewart (1986). "WITNESS: A FORTRAN Utility for Maintenance and Modification of Text Files," BN-SA-2376, Battelle, Pacific Northwest Laboratories, Richland, Washington.

Mood, A.M., F. A. Graybill, and D. C. Boes. (1974). Introduction to the Theory of Statistics, Third Edition, McGraw-Hill Book Company.

SAS Institute Inc. 1985a. SAS User's Guide: Basics, Version 5 Edition. SAS Institute Inc., Cary, North Carolina.

SAS Institute Inc. 1985b. SAS User's Guide: Statistics, Version 5 Edition. SAS Institute Inc., Cary, North Carolina.

Stephens, M. A. 1969. "Use of Kolmogorov-Smirnov, Cramer-von Mises and Related Statistics without Extensive Tables." Journal of the Royal Statistical Society, Ser. B 32(1):115-122.

Stephens, M. A. 1974. "EDF Statistics for Goodness of Fit and Some Comparisons." Journal of the American Statistical Association $69(347): 730-737$. 


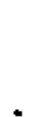


APPENDIX A

RANDOM NUMBER GENERATION IN PACSTAT 
APPENDIX A

RANDDM NUMBER GENERATION IN PACSTAT

This appendix discusses the mathematical methods used to define the uniform, loguniform, exponential, truncated exponential, normal, truncated normal, lognormal, and truncated lognormal random number generators (RNG) in PACSTAT, (a)

Considerable use is made of the "probability integral transform" method (Mood, Graybill, and Boes 1974, p. 202). The method states that for any random variable $X$ having cumulative distribution $F(X)$, the transformation

$$
F(X)=U
$$

yields a random variable $U$ having a uniform distribution on the interval $(0,1)$. Hence, given a random uniform variate $u$, a random variate $x$ with cumulative distribution function $F$ is given by

$$
x=F^{-1}(u)
$$

We now discuss the eight PACSTAT random number generators in turn.

\section{A.1 GENERATION OF UNIFORM VARIATES}

PACSTAT utilizes a multiplicative congruential generator with modulus $2^{31}-1$ and multiplier 16807 to produce uniform $(0,1)$ variates. It has the form

$$
z_{i}=16807 z_{i-1}\left(\bmod 2^{31}-1\right)
$$

\footnotetext{
(a) This discussion is based on an August 1985 draft report, "Verification of PACSTAT Monte Carlo Routines", by P. W. Eslinger and D. W. Langford of Boeing Computer Services Richland, Incorporated.
} 
with the normalization

$$
u_{i}=\frac{z_{i}}{2^{31}-1}
$$

producing uniform $(0,1)$ variates. A seed $z_{0}\left(1,2, \ldots, 2^{31}-2\right)$ is required to start the generator. Given a uniform $(0,1)$ variate y from Equation (A.2), a uniform variate $x$ on the interval $\left(u_{\ell}, u_{r}\right)$ is calculated as

$$
x=\left(u_{r}-u_{\ell}\right) y+u_{\ell}
$$

The probability density function (pdf) for the uniform distribution is

$$
f(x)=\left\{\begin{array}{cc}
\frac{1}{u_{r}-u_{\ell}} & u_{\ell} \leq x \leq u_{r} \\
0 & \text { otherwise }
\end{array}\right.
$$

A.2 GENERATION OF LOGUNIFORM VARIATES

Variates from a loguniform distribution can be generated using either the natural or common logarithm. The pdf is

$$
f(x)=\left\{\begin{array}{ccc}
\frac{\log _{a}(e)}{u_{r}-u_{\ell}} & \frac{1}{x} & u_{\ell} \leq x \leq u_{r} \\
0 & & \text { otherwise }
\end{array}\right.
$$

where $a$ is 10 for the common logarithm, and e for the natural logarithm.

Variates from a loguniform distribution are obtained by first obtaining a uniform $\left(u_{\ell}, u_{r}\right)$ variate $y$, and then transforming to a loguniform variate $x$ via $x=a^{y}$.

\section{A.3 GENERATION OF EXPONENTIAL VARIATES}

The exponential distribution with parameter $\theta$ has pdf 


$$
f(x)= \begin{cases}e^{-\theta x} & x>0 \\ 0 & \text { otherwise }\end{cases}
$$

cumulative distribution function (cdf)

$$
F(x)= \begin{cases}1-\mathrm{e}^{-\theta x} & x>0 \\ 0 & \text { otherwise }\end{cases}
$$

and inverse cdf

$$
\mathrm{F}^{-1}(u)=-\log _{e}(1-u) / \theta
$$

The probability integral transform method is used to produce exponential variates $x$ via

$$
x=F^{-1}(u)
$$

where $u$ is a random uniform $(0,1)$ variate, and $F^{-1}$ is the inverse cdf from Equation (A.7).

\section{A.4 GENERATION OF TRUNCATED EXPONENTIAL VARIATES}

The truncated exponential distribution with parameter $\theta$ takes values in the interval $\left(e_{\ell}, e_{r}\right)$, where $e_{\ell} \geq 0$ and $e_{\Gamma}>e_{\ell}$. The truncated exponential has pdf

$$
f(x)=\left\{\begin{array}{cl}
\frac{e^{-\theta x}}{e^{-\theta e} \ell-e^{-\theta e} r} & e_{\ell} \leq x \leq e_{r} \\
0 & \text { otherwise }
\end{array}\right.
$$

Variates from the truncated exponential are generated using the probability integral transform method. The first step is to compute the limits $u_{\ell}$ and $u_{r}$ 
on a uniform distribution that corresponds to the exponential limits $e_{\ell}$ and $e_{r}$. This is accomplished by solving $F\left(e_{\ell}\right)=u_{\ell}$ and $F\left(e_{r}\right)=u_{r}$, where $F$ is given by $(A .6)$ :

$$
u_{\ell}=1-e^{-\theta e_{\ell}} \quad \text { and } \quad u_{\ell}=1-e^{-\theta e_{r}}
$$

A truncated exponential variate is obtained by first generating a $\left(u_{\ell}, u_{r}\right)$ uniform variate $y$, and then computing

$$
x=-\log _{\mathrm{e}}(1-y) / \theta
$$

\section{A.5 GENERATION OF NORMAL VARIATES}

The normal distribution with mean $\mu$ and standard deviation o has pdf

$$
f(x)=\frac{1}{\sqrt{2 \pi} \sigma} \exp -\frac{(x-\mu)^{2}}{2 \sigma^{2}}-\infty \leq x \leq \infty
$$

Normal random variates are obtained via the probability integral transform method. An accurate numerical approximation (Abramowitz and Stegun 1974, Eq. 26.2.23, p. 933) for the inverse $c d f$ is used, since an analytical closed form does not exist.

The PACSTAT method for generating normal variates has the following five steps:

1. Generate a variate, $u$, from the uniform $(0,1)$ distribution.

2. If $u$ is less than 0.5 , the sign of the normal variate, $x$, will be positive. If $u$ is greater than 0.5 , set $u=1-u$ and the sign of $x$ will be negative.

3. Generate $x$ from $u$ using the following equations:

$$
t=[-2 \ln (u)]^{1 / 2}
$$




$$
x=t-\frac{c_{0}+c_{1}+c_{2} t^{2}}{1+d_{1} t+d_{2} t^{2}+d_{3} t^{3}}
$$

where

$c_{0}=2.515517 \quad d_{1}=1.432788$

$c_{1}=0.802853 \quad d_{2}=0.189269$

$c_{2}=0.010328 \quad d_{3}=0.001308$

The absolute value of the error in $x$ is less than $4.5 E-04$.

4. Determine the sign of $x$ from Step 2, the result is a variate from the normal distribution with mean 0 and standard deviation 1.

5. If a variate $y$ from a normal distribution with mean $\mu$ and standard deviation $\sigma$ is desired, use the expression $y=\sigma x+\mu$.

A.6 GENERATION OF TRUNCATED NORMAL VARIATES

A truncated normal distribution with parameters $\mu$ and $\sigma$ takes values in the interval $\left(n_{\ell}, n_{r}\right)$. The pdf is

$$
f(x)= \begin{cases}\frac{1}{\sqrt{2 \pi} \sigma} \frac{\exp \left(-\frac{(x-\mu)^{2}}{2 \sigma^{2}}\right)}{F\left(\frac{n_{r}-\mu}{\sigma}\right)-F\left(\frac{n_{\ell}-\mu}{\sigma}\right)} & n_{l} \leq x \leq n_{r} \\ 0 & \text { otherwise, }\end{cases}
$$

where $F$ is the cdf of the nontruncated standard normal distribution (i.e., the normal distribution with $\mu=0$ and $\sigma=1$ ).

Truncated normal variates are generated via the probability integral transform method using the numerical approximation for the inverse cdf given in Section A.5. First, the uniform limits $u_{\ell}$ and $u_{r}$ corresponding to the truncated normal limits $n_{\ell}$ and $n_{r}$ are determined. This is accomplished by using the inverse cdf approximation of Section A.5 in a mixed Newton's and 
bisection method to find the values $u_{\ell}$ and $u_{r}$ which satisfy $F\left[\left(n_{\ell}-\mu\right) / \sigma\right]=u_{\ell}$ and $F\left[\left(n_{r}-\mu\right) / \sigma\right]=u_{r}$. A truncated normal variate $x$ is then calculated from $a$ uniform $(0,1)$ variate using the inverse cdf approximation in Step 3 of Section A.5.

\section{A.7 GENERATION OF LOGNORMAL VARIATES}

Variates can be generated from a lognormal distribution for either common or natural logarithms. The pdf of the lognormal distribution with parameters $\mu$ and $\sigma$ is

$$
f(x)=\frac{\log _{a}(e)}{\sqrt{2 \pi} \sigma x} \exp \left(-\frac{\left(\log _{a}(x)-\mu\right)^{2}}{2 \sigma^{2}}\right) \quad x>0
$$

where $a$ is 10 for common logarithm and e for natural logarithm.

Lognormal variates $x$ are generated by first generating a variate $y$ from the normal distribution with mean $\mu$ and standard deviation $\sigma$, and then computing $\mathrm{x}=\mathrm{a}^{\mathrm{y}}$.

\section{A.8 GENERATION OF TRUNCATED LOGNORMAL VARIATES}

A truncated lognormal distribution with parameters $\mu$ and $\sigma$ takes values in the interval $\left(1 n_{\ell}, 1 n_{r}\right)$. Truncated lognormal variates are generated by the following three steps:

1. Calculate the limits $u_{\ell}$ and $u_{r}$ for the uniform distribution corresponding to the lognormal truncation limits $\ln _{\ell}$ and $1 n_{r}$ (using a combined Newton and bisection method).

2. Calculate a truncated normal variate y from a uniform variate u on the interval $\left(u_{\ell}, u_{r}\right)$.

3. Transform the normal variate $y$ to a lognormal (truncated) variate $x$ via $x=a^{y}$ (where a is 10 for common logarithm and e for natural logarithm). 
APPENDIX B

PACSTAT_AO.FOR (ORIGINAL INTERACTIVE VERSION, FORTRAN SOURCE FILE) 


\begin{tabular}{|c|c|c|c|}
\hline & FUNCTION NLNORU $(X)$ & ALNORM & 2 \\
\hline c & & ALNORM & . \\
\hline C & ALGORITHU FROU APPL. STATIST, (1973) VOL. 22, NO. 3, PP 424-427. & ALNORN & . \\
\hline C & & ALNORH & . \\
\hline C & EVALUATES THE AREA UNDER A STANDARD NORUAL CURVE (MEAN $=0.0_{1}$ & NLNORM & . \\
\hline c & STANDARD DEVIATION $=1.0$ ), INTEGRATING FROY UINUS INFINITY TO $X$. & N.NORN & . \\
\hline c & A POLYNOMIAL METHOD IS USED. & ALNORM & . \\
\hline c & & ALNORM & . \\
\hline & REAL $* 8$ & ALNDRM & 10 \\
\hline & $+\quad A$, & ALNORM & 11 \\
\hline & LBGICAL & ALNORM & 12 \\
\hline & - POSTIV & $A L N Q R M$ & 13 \\
\hline & DATA & ALNORM & 14 \\
\hline & $+\operatorname{CON} / 1.28 /$ & ALNDRM & 15 \\
\hline & $+\quad$ UTZERD / $9.58 /$ & ALNORH & 16 \\
\hline c & & ALNOPM & 17 \\
\hline & POSTIV=. TRUE. & ALNORN & 18 \\
\hline & IF (X.LT..$\overline{\text { f }}$ ) POSTIV=.FALSE. & ALNORN & 19 \\
\hline & $Z=\operatorname{DBLE}(A B S(X))$ & ALNORM & 26 \\
\hline c & & ALNORM & 21 \\
\hline & IF (X.LT.UTZERO) GQ TD 160 & ALNORM & 22 \\
\hline & NLNORN=6. & NLNORM & 23 \\
\hline & GO TO 130 & ALNORM & 24 \\
\hline 160 & CONTINUE & ALNORM & 25 \\
\hline & $Y=8.500 * Z * Z$ & ALNORN & $2 B$ \\
\hline & IF (X.GT.CON) GO TO 110 & ALNDRM & 27 \\
\hline$c$ & & ALNORM & 28 \\
\hline & $A=0.506-Z *(0.39894228644406-6.39998343850406 * Y /$ & ALNQRN & 29 \\
\hline & $+(Y+5.75886486458 \mathrm{DD}-29.821355786800 /$ & ALNORM & 38 \\
\hline & $+(Y+2.6243312167900+48.695993669200 /$ & ALNORN & 31 \\
\hline & $+(Y+5.92885724438 D())))$ & ALNORM & 32 \\
\hline & GO TO 120 & ALNORM & 33 \\
\hline $\mathrm{C}$ & & ALNORM & 34 \\
\hline 110 & CONTINUE & ALNORM & 35 \\
\hline & $A=6.398942286385 * \operatorname{DEXP}(-Y) /$ & ALNORM & 36 \\
\hline & $+(2-3.8052 D-8 \quad \rightarrow 1.6000661530200 /$ & ALNORM & 37 \\
\hline & $+(Z+3.98868477940-4+1.9861538136406 /$ & ALNORM & 38 \\
\hline & $+(Z-0.15187911683500+5.2933832492600)$ & ALNORN & 39 \\
\hline & $+(Z+4.838591280800+15.150897245100 /$ & $A L N Q R M$ & 46 \\
\hline & $+(Z+6.74238092482708+30.78993303400 /$ & ALNORM & 41 \\
\hline & $+((+3.9961941761100))))))$ & N.NORM & . \\
\hline c & & ALNORM & 43 \\
\hline 126 & B CONTINUE & ALNORM & 44 \\
\hline & ALNORM $=$ SNGL $(A)$ & ALNORM & 45 \\
\hline 136 & CONTINUE & ALNORA & 46 \\
\hline & IF (POSTIV) ALNORM $=1.0-A L N O R M$ & ALNORM & 47 \\
\hline & RETURN & ALNORN & 48 \\
\hline & END & ALNDRM & 49 \\
\hline & SUBROUTINE ASG (ITYP, IERR, IFIL, NLEN, INF) & ASG & . \\
\hline c & & ASG & 3 \\
\hline C & RDUTINE FOR INTERACTIVE ASSIGNMENT OF FILES DN PRIME & ASG & ' \\
\hline C & INPUT IS ITYP = 1 FOR WRITE FILE, 9 FOR READ ONLY & ASG & . \\
\hline $\mathrm{C}$ & $=$ OTHER FOR READ/WRITE FILE (MUST BE EXISTING FILE) & ASG & . \\
\hline c & IERR = ERRQR RETUJRN FLAG & ASG & 7 \\
\hline
\end{tabular}




\begin{tabular}{|c|c|c|c|}
\hline \multicolumn{2}{|r|}{ IFIL = UNIT NUPER TO USE } & ASG & 8 \\
\hline \multicolumn{2}{|r|}{ NLEN = DIUENSION OF INF } & ASG & 9 \\
\hline c & \multirow[t]{2}{*}{ INF $=$ NAME OF INPUT FILE (CHAR 4 , DIMENSION $=$ NLEN $)$} & ASG & 10 \\
\hline C & & ASG & 11 \\
\hline c & DESIGNED FOR PRIME 250 AND 750. FUNITS $1-4,46-42,51$, & ASG & 12 \\
\hline C & \multirow[t]{2}{*}{55 AND UNITS 21-28 ARE COWITTED ON THESE WACHINES. } & ASG & 13 \\
\hline C & & ASG & 14 \\
\hline \multicolumn{2}{|c|}{ SINSERT SYSCOUYABKEYS } & ASG & 15 \\
\hline \multicolumn{2}{|c|}{ SINSERT * SOUMN $>$ INOUT } & ASG & 16 \\
\hline \multicolumn{2}{|r|}{ LOGICN LOGIC } & ASG & 17 \\
\hline \multicolumn{2}{|r|}{ CHARACTER*1 } & ASG & 18 \\
\hline \multicolumn{2}{|r|}{$+\quad$ ANS } & ASG & 19 \\
\hline \multicolumn{2}{|r|}{ CHARACTER*4 } & ASG & 20 \\
\hline & $+\quad \operatorname{INF}($ MEE $)$ & ASG & 21 \\
\hline \multicolumn{2}{|r|}{$I E R R=\theta$} & ASG & 22 \\
\hline C & & ASG & 23 \\
\hline C & \multirow[t]{2}{*}{ CHECK FOR FILE NLREADY OPEN ON THIS UNIT NUNBER } & ASG & 24 \\
\hline C & & ASG & 25 \\
\hline \multicolumn{2}{|r|}{ REIIND（IFIL, ERR=100) } & ASG & 28 \\
\hline & WRITE (IOUT, 560) IFIL, (INF(I), I=1, NLEN) & ASG & 27 \\
\hline \multicolumn{2}{|r|}{ GO TO 180} & ASG & 28 \\
\hline \multicolumn{2}{|c|}{100 CONTINUE } & ASG & 28 \\
\hline \multicolumn{2}{|r|}{ NML4=4*NLEN } & ASG & 30 \\
\hline $\mathrm{C}$ & & ASG & 31 \\
\hline C & \multirow[t]{2}{*}{ CHECK FOR NLOTABLE UNIT MUMEER. SET PRIME OFFSET. } & ASG & 32 \\
\hline C & & ASO & 33 \\
\hline & IF (IFIL.LE.4) G0 TO 110 & ASG & 34 \\
\hline & IF ((IFIL.GE. 21).AND. (IFIL.LE. 28)) G0 TO 110 & ASO & 35 \\
\hline & IF ((IFIL. OE. 40).AND. (IFIL.LE. 42)) GD TO 110 & ASG & 38 \\
\hline & IF ((IFIL.EQ.51).OR. (IFIL.EQ.55)) GO TO 110 & ASG & 37 \\
\hline & IF (IFIL.GT.127) GO TO 110 & ASO & 38 \\
\hline & GO TO 126 & ASG & 39 \\
\hline 110 & CONTINUE & ASO & 40 \\
\hline & WRITE (IOUT, 510) IFIL & ASO & 41 \\
\hline 128 & CONTINUE & ASG & 42 \\
\hline & IF (IFIL.LT.21) THEN & ASG & 43 \\
\hline & IFUNIT=IFIL-4 & ASG & 44 \\
\hline & ESE & ASG & 45 \\
\hline & IFUNIT=IFIL-12 & ASG & 46 \\
\hline & ENDIF & ASG & 47 \\
\hline$c$ & & ASG & 48 \\
\hline c & CHECK TO SEE IF FILE NLREADY EXISTS & ASG & 49 \\
\hline C & & ASO & 50 \\
\hline 130 & CONTINUE & ASG & 51 \\
\hline & LOGIC $=$ EXST\$A (INF, INTS $($ NLL4) $)$ & ASG & 52 \\
\hline c & & ASG & 53 \\
\hline$c$ & READ GNLY OR READ/WRITE FILE & ASG & 54 \\
\hline$c$ & FILE WST NLREAYY EXIST & ASG & 55 \\
\hline$c$ & & ASO & 56 \\
\hline & IF (ITYP.NE. I) THEN & ASG & 57 \\
\hline & IF (.NDT.LOGIC) THEM & ASG & 58 \\
\hline 149 & CONTINUE & ASG & 59 \\
\hline & WRITE (IDUT, 520) (INF(I), I=1, NLEN) & ASG & 60 \\
\hline & WRITE (IOUT, 530) & ASO & . 61 \\
\hline
\end{tabular}




\begin{tabular}{|c|c|c|c|}
\hline & READ (IN,546) AKS & ASG & 62 \\
\hline & IF ((ANS. NE. 'Y') AND. (ANS. NE. 'N')) THEN & ASG & 63 \\
\hline & IF ((ANS. NE. ' ' $\left.y^{\prime}\right)$. AND. (ANS. NE. 'n')) GO TO 146 & ASG & 64 \\
\hline & ENDIF & ASG & 65 \\
\hline & IF ((ANS. EQ.'Y') .OR. (ANS.EQ. ' $\left.y^{\prime}\right)$ ) THEN & ASG & 66 \\
\hline & IRITE (IOUT, 55\%) IQB & ASG & 67 \\
\hline & $\operatorname{READ}(I N, 680)(\operatorname{INF}(I), I=1, N L E N)$ & ASG & 68 \\
\hline & GO TO 136 & ASG & 69 \\
\hline & 日SE & ASG & 76 \\
\hline & GO TO 188 & ASG & 71 \\
\hline & ENDIF & ASG & 72 \\
\hline & ESE & ASG & 73 \\
\hline & IF (ITYP.EQ.9) THEN & ASG & 74 \\
\hline & LOGIC = DPENSA (ASREAD+ASSALF,INF, INTS (NLL4),INTS (IFUNIT)) & ASG & 75 \\
\hline & 日SE & ASG & 76 \\
\hline & LOGIC = OPENSA (ASRDWR+ASSALF, INF, INTS (NUL4), INTS (IFUNIT)) & ASG & 77 \\
\hline & EMDIF & ASG & 78 \\
\hline & ENDIF & ASG & 79 \\
\hline \multicolumn{2}{|c|}{ 日一SE } & ASG & 80 \\
\hline C & & ASG & 81 \\
\hline C & WRITE DNLY FILE. CHECK WITK USER BEFORE & ASG & 82 \\
\hline \multirow{2}{*}{\multicolumn{2}{|c|}{ DELETING AN EXISTING FILE. }} & ASG & 83 \\
\hline & & ASG & 84 \\
\hline & IF (LOGIC) THEN & ASG & 85 \\
\hline \multirow[t]{8}{*}{150} & CONTINUE & ASG & 86 \\
\hline & WRITE (IOUT, 576) (INF (I), I=1, NLEN) & ASG & 87 \\
\hline & WRITE (IOUT, 580) IQB & ASG & 88 \\
\hline & READ (IN,546) ANS & ASG & 89 \\
\hline & IF ((ANS.NE. 'Y'). AND. (ANS.NE. 'N')) THEN & ASG & 90 \\
\hline & IF ((ANS.NE. ' $y$ ') , AND. (ANS. NE. 'n')) GO TO 150 & ASG & 91 \\
\hline & ENDIF & ASG & 92 \\
\hline & IF ((ANS.EQ.'N') .OR. (AKS.EQ.'n')) THEN & ASO & 93 \\
\hline \multirow[t]{19}{*}{160} & CONTINUE & ASO & 94 \\
\hline & WRITE (IOUT, 538) IQB & ASG & 96 \\
\hline & READ (IN, 549) ANS & ASG & 96 \\
\hline & IF ((ANS.NE. 'Y') AND. (ANS. NE. 'N')) THEN & ASG & 97 \\
\hline & IF ((ANS.NE. 'Y') AND. (ANS.NE. 'n')) GO TO 166 & ASO & 98 \\
\hline & ENDIF & ASG & 99 \\
\hline & IF ((ANS.EQ.'N') OR. (ANS.ER.' 'n')) GO TO 180 & ASG & .100 \\
\hline & $A N S=' N '$ & ASG & . 181 \\
\hline & ENDIF & ASG & . 102 \\
\hline & IF ((ANS.ER. 'Y'). DR. (ANS.ER. ' $\left.\left.y^{\prime}\right)\right)$ THEN & ASG & . 103 \\
\hline & LDGIC = DELEA(INF, INTS(NL4)) & ASG & . 104 \\
\hline & ESE & ASG & . 105 \\
\hline & WRITE (IOUT, 550) IQB & ASG & . 106 \\
\hline & READ (IN, 560) (INF (I), I=1, NLEN) & ASG & . 167 \\
\hline & GO TO 130 & ASG & . 168 \\
\hline & ENDIF & ASG & . 169 \\
\hline & ENDIF & ASG & . 116 \\
\hline & LOGIC = OPENSA (ASWRIT+ASSALF, INF, INTS (NL4), INTS(IFUNIT)) & ASG & . 111 \\
\hline & IDIF & ASG & . 112 \\
\hline \multirow[t]{2}{*}{ C } & & ASG & . 113 \\
\hline & (LDGIC) RETURN & ASO & . 114 \\
\hline$c$ & & ASG & 115 \\
\hline
\end{tabular}




\begin{tabular}{|c|c|c|c|}
\hline $17 \%$ & CONTINUE & ASG & . 116 \\
\hline & VRITE (IOUT, 590) & ASO & . 117 \\
\hline & WRITE (IOUT, 630) IQ8 & ASG & . 118 \\
\hline & READ (IN, 546) ANS & ASG & . 119 \\
\hline & IF ((AKS.NE. 'Y') AKD. (ANS. NE. 'N')) THEK & ASG & . 126 \\
\hline & IF ((ANS.NE.' 'y') AND. (ANS. NE. ' $\left.\left.n^{\prime}\right)\right)$ GO TO 176 & ASG & . 121 \\
\hline & ENDIF & ASG & . 122 \\
\hline & IF ((ANS.EQ. 'N').DR. (ANS.EQ.' $\left.n^{\prime}\right)$ ) GO TO 180 & ASG & . 123 \\
\hline & IRITE (IOUT, 550) IQB & ASG & 124 \\
\hline & READ (IN, 588) (INF(I), I=1, NLEN) & ASG & . 125 \\
\hline & GO TO 136 & ASG & . 126 \\
\hline C & & ASG & 127 \\
\hline 180 & CONTIMUE & ASG & . 128 \\
\hline & WRITE (IOUT, 606) & ASG & . 129 \\
\hline & IERR $=1$ & ASG & . 130 \\
\hline & RETURN & ASG & . 131 \\
\hline C & & ASG & . 132 \\
\hline 509 & FORWAT (/1X, 'FILE UNIT ',I5,' IS NLREADY ASSIGNED TO AN OPEN FILE. & ASG & . 133 \\
\hline & +'/1X,'ATISBPT TO ASSIGN FILE' ',28A4) & ASG & 134 \\
\hline 510 & FORUAT (/1X, 'FILE UNIT',I5,' IS IN POSSIBE CONFLICT ITH PRIWE SY & ASG & 135 \\
\hline & +TEY UNITS' $/$ ) & ASG & . 136 \\
\hline 526 & FDRUAT (/1X,'UNABLE TO LOCATE FILE',20A4) & ASG & . 137 \\
\hline 530 & FOREAT (IX, 'DO YOU IISH TO TRY AGIN $(Y / N)$ ? ',A1) & ASG & 138 \\
\hline 548 & FORUAT (A1) & ASG & 139 \\
\hline 550 & FORUAT (1X, 'ENTER FILE MAME ', & ASG & 140 \\
\hline 580 & FORUAT (20A4) & ASG & . 141 \\
\hline 578 & FORUAT (1X, 'FILE', 20A4) & ASG & . 142 \\
\hline 580 & FQRMAT ( $X$ X, 'NREADY EXISTS, NLL RIGHT TD DELTE $(Y / N)$ ? ',A1) & ASG & . 143 \\
\hline 590 & FORUAT (/1X, 'FILE ASSIGNWENT UNSUCCESSFLL. ') & ASG & . 144 \\
\hline 600 & FORWAT (IX,'PROGRAY ABCRTED IN RDUTINE ASG.') & ASG & . 145 \\
\hline & END & ASG & 146 \\
\hline & SUBROUTINE BERNLI ( $N$, RNDVAL,RP1) & BERNLI & . \\
\hline c & & BERNLI & . \\
\hline C & ROUTINE FOR TRANSFORIING A UNIFORKYY DISTRIBUTED DATA SET DN THE & BERNLI & . \\
\hline C & INTERVN 6,1 TO A BERNOULI DISTRIQUTED DATA SET. INPUT VALUES & BERNLI & . \\
\hline c & BECOME $\square$ IF THEY ARE LESS THAN RP1, AND 1 IF THEY ARE GREATER THAN & BERNLI & . \\
\hline c & RP1. & BERNLI & . \\
\hline C & & BERNLI & . \\
\hline c & N = WMBER OF VNLUES IN VECTOR RNDVN. & BERNLI & . \\
\hline c & RNDVN = ON INPUT, VECTOR OF UNIFORACY DISTRIBUTED VALUES. & BERNLI & 10 \\
\hline c & ON QUTPUT, VECTOR OF BERNOULII DISTRIBUTED VNUES. & BERNLI & 11 \\
\hline c & RP1 = PROBABILITY OF THE ZERO STATE OCCURRING. & BERNLI & 12 \\
\hline c & & BERNLI & 13 \\
\hline & DINENSION & BERNLI & 14 \\
\hline & - RNDVN_(N) & BERNLI & 15 \\
\hline$c$ & & BERNLI & 16 \\
\hline c & BERNOULI DISTRIBUTION & BERNLI & 17 \\
\hline c & & BERNLI & 18 \\
\hline & DO $160 I=1, N$ & BERNLI & 19 \\
\hline & $R V=1$ & BERNLI & 20 \\
\hline & IF (RNOVNL(I), LT. RPI) RV=o. & BERNLI & 21 \\
\hline & RNDVAL(I)=RY & BERNLI & 22 \\
\hline 180 & CONTINUE & BERNLI & 23 \\
\hline & RETURN & BERNLI & 24 \\
\hline
\end{tabular}




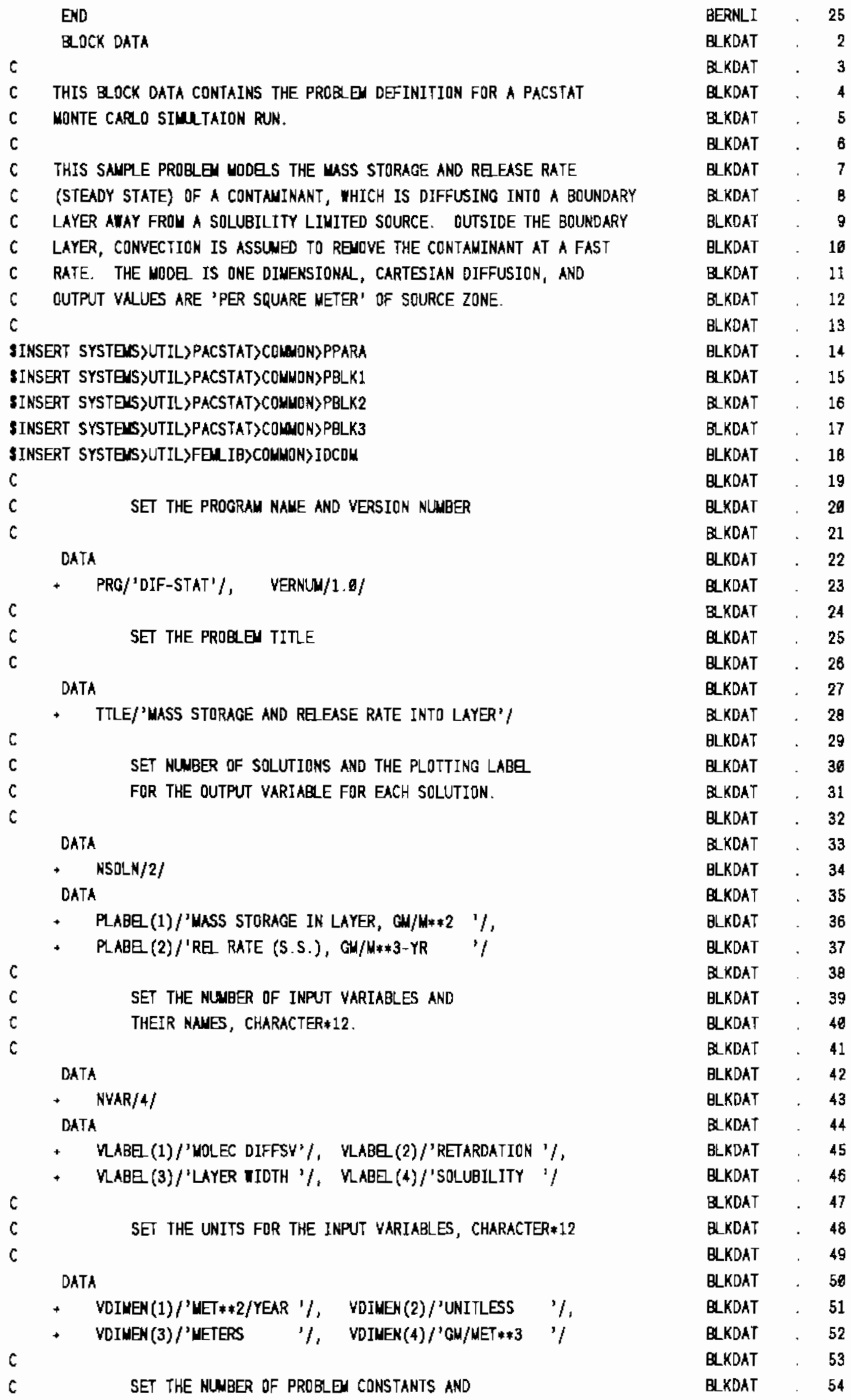

B. 5 


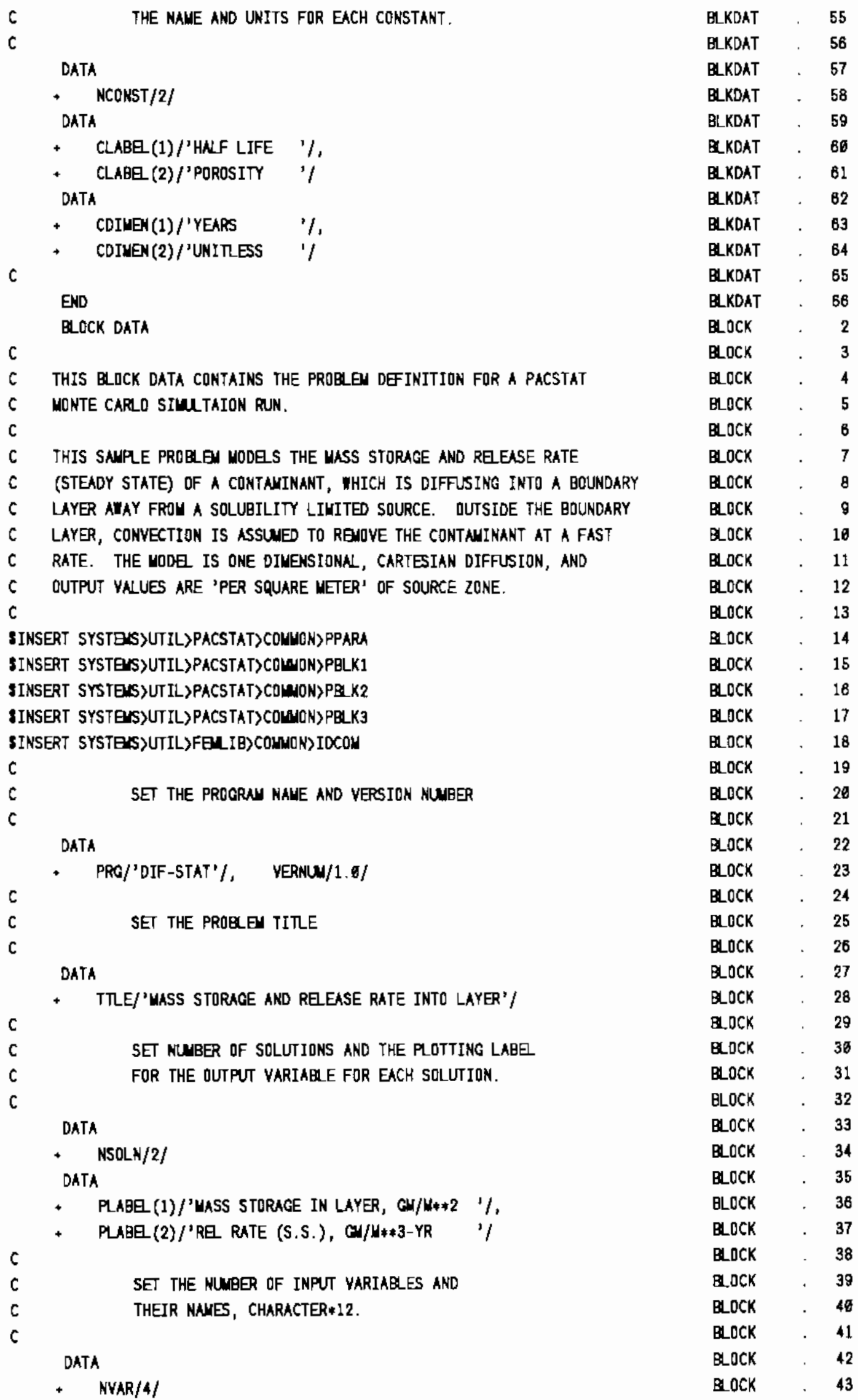




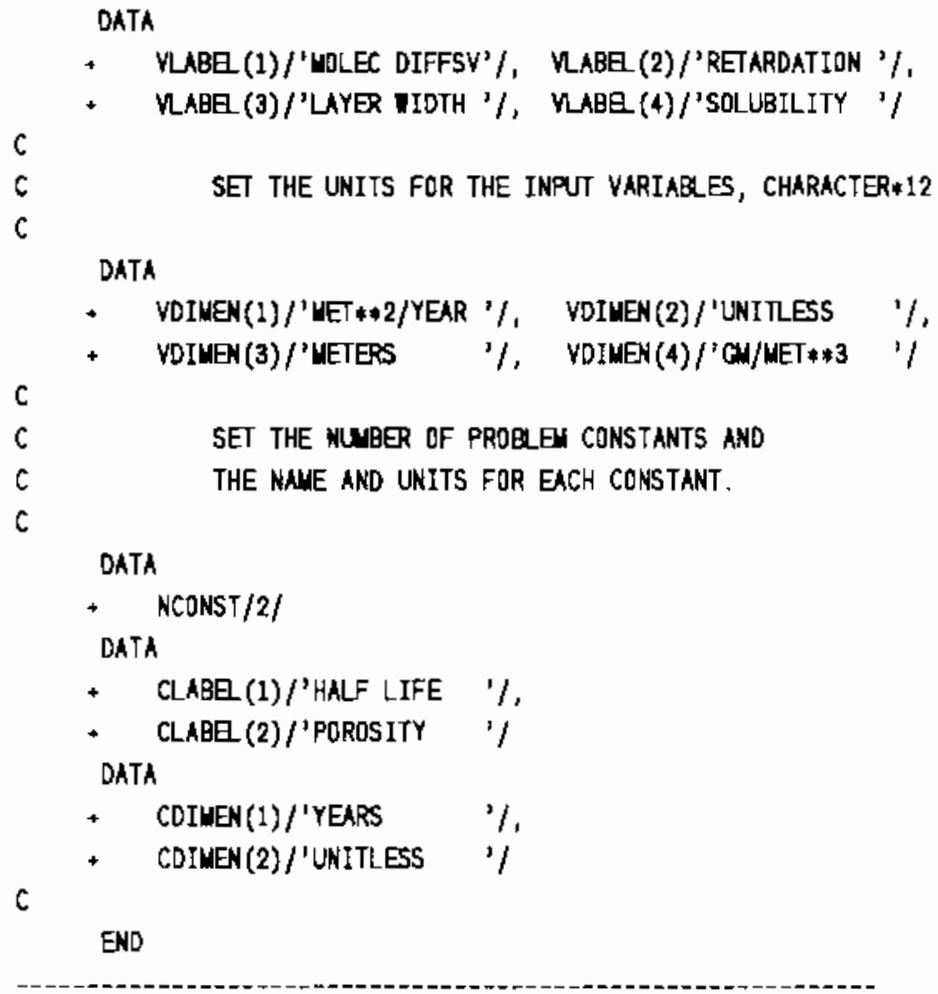

SAMPLE QLOCK DATA ROUTINE

THIS IS A SALPLE LISTING OF A BLOCK DATA ROUTINE. THIS ROUTINE DEINES THE INPUT AND OUTPUT YARIABLES WHICH ARE USED BY THE MODEL BEING IMPLENENTED.

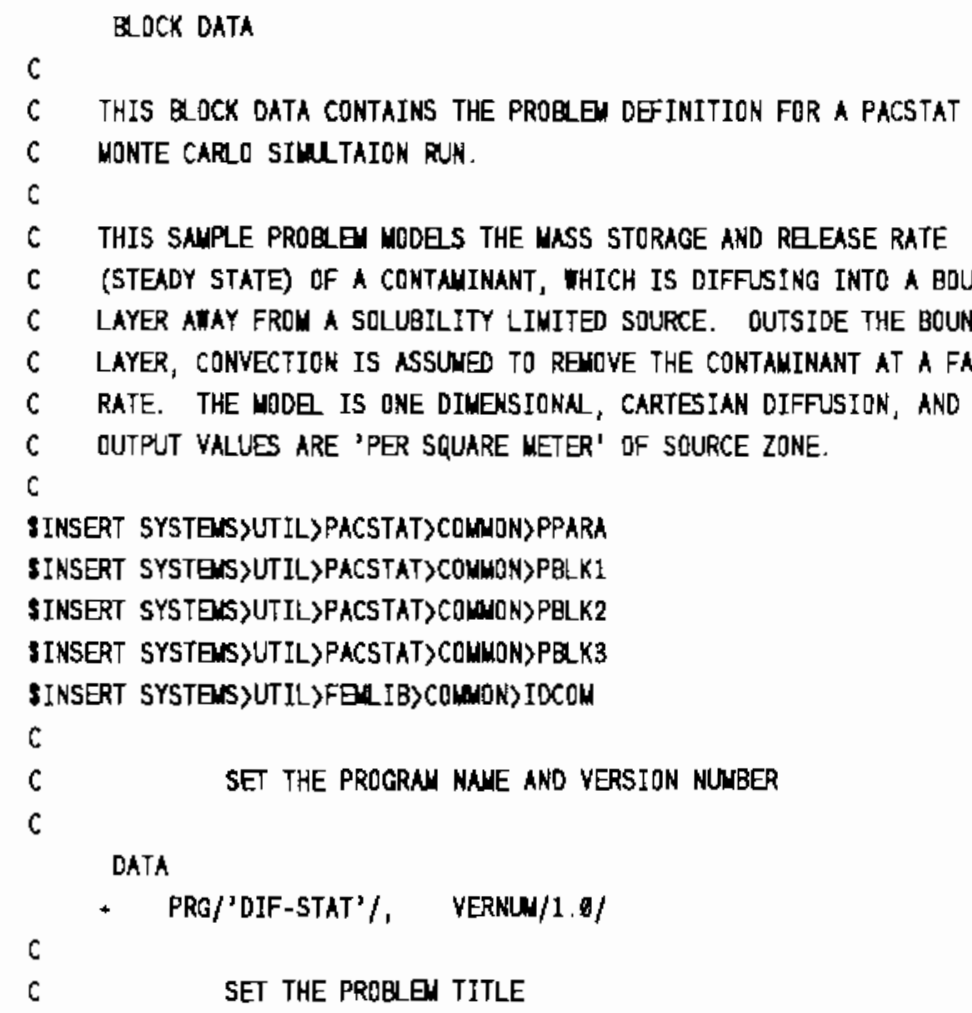

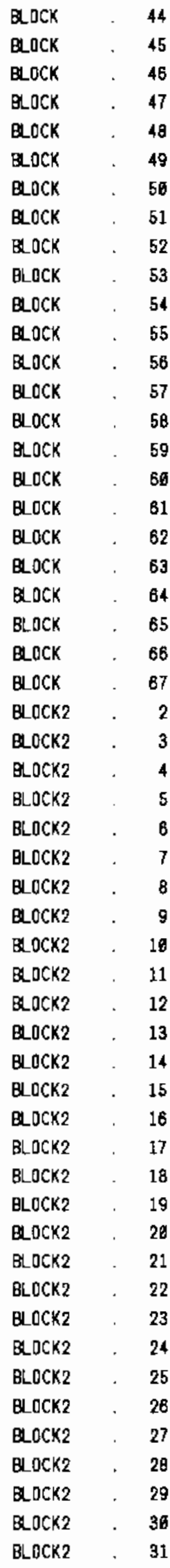




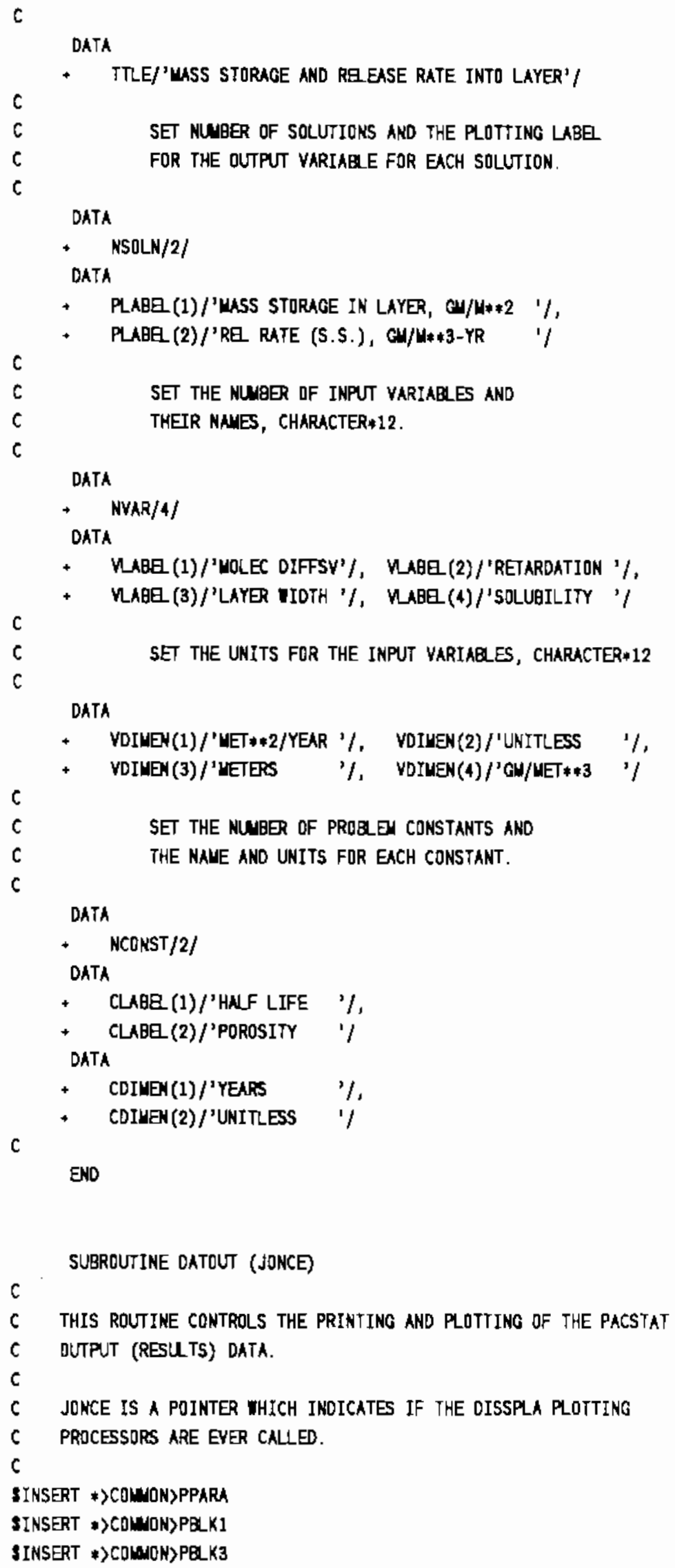




\begin{tabular}{|c|c|c|c|}
\hline \multicolumn{2}{|c|}{ SINSERT *)COIMON>PQK5 } & DATOUT & 13 \\
\hline \multicolumn{2}{|c|}{ SINSERT $\Rightarrow$ COMON>PQ X8 } & DATOUT & 14 \\
\hline \multicolumn{2}{|c|}{ IINSERT *>COWON>PQLK7 } & DATOUT & 15 \\
\hline \multicolumn{2}{|c|}{ IINSERT $*>$ CONDN>PQLX } & DATOUT & 18 \\
\hline \multicolumn{2}{|c|}{ IINSERT $\leadsto$ CONON>PQKK17 } & DATOUT & 17 \\
\hline \multicolumn{2}{|c|}{ SINSERT SYSTESS>UTIL>USRLIB>COLUON>INOUT } & DATDUT & 18 \\
\hline \multicolumn{2}{|r|}{ CHARACTER*1 } & DATOUT & 19 \\
\hline \multicolumn{2}{|r|}{$\begin{array}{c}\text { ANS } \\
\text { CHARACTER*32 }\end{array}$} & DATOUT & 20 \\
\hline \multicolumn{2}{|r|}{ CHARACTER*32 } & DATOUT & 21 \\
\hline & NOPTN & DATOUT & 22 \\
\hline \multicolumn{2}{|r|}{ DATA } & DATOUT & 23 \\
\hline - & NOPTN $/ \prime$ - ENTER A NEM PRINT INYERVAL - ' & DATOUT & 24 \\
\hline \multicolumn{2}{|r|}{ 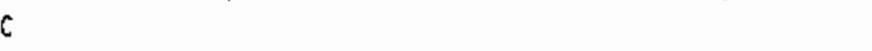 } & DATOUT & 25 \\
\hline \multicolumn{2}{|r|}{ IF ((NSNAC.LT.NSA) . AND. (NPRT.GT.1)) THEH } & DATOUT & 26 \\
\hline \multicolumn{2}{|r|}{$A=$ FLOAT (NPRT)/FLOAT (NSN) } & DATOUT & 27 \\
\hline \multicolumn{2}{|r|}{ NPRT $=\operatorname{IFIX}(\theta .5+F L D A T(N S N A C) * A)$} & DATOUT & 28 \\
\hline & IF (NPRT.LT.1) NPRT=1 & DATOUT & 29 \\
\hline \multicolumn{2}{|r|}{ EMDIF } & DATOUT & 36 \\
\hline \multicolumn{2}{|l|}{$c$} & DATOUT & 31 \\
\hline \multirow{2}{*}{\multicolumn{2}{|c|}{$\begin{array}{l}\text { C SET CONFIDENCE LEYES } \\
c\end{array}$}} & DATOUT & 32 \\
\hline & & DATDUT & 33 \\
\hline \multicolumn{2}{|r|}{$I C C=1$} & DATOUT & 34 \\
\hline \multicolumn{2}{|r|}{ C=FLOAT (NSAMC) } & DATOUT & 35 \\
\hline \multicolumn{2}{|r|}{ DO $160 \mathrm{I}=1$, NSAMC } & DATOUT & 38 \\
\hline & $\operatorname{CONF}(\mathrm{I})=\mathrm{FLOAT}(\mathrm{I}) / \mathrm{C}$ & DATOUT & 37 \\
\hline 108 & CONTINUE & DATOUT & 38 \\
\hline c & & DATOUT & 39 \\
\hline c & ENTER SOLUTION TO CHOSE FROM & DATOUT & 40 \\
\hline c & & DATOUT & 41 \\
\hline 110 & CONTINUE & DATOUT & 42 \\
\hline & WRITE (IOUT, 506) & DATDUT & 43 \\
\hline & WRITE (IOUT, 510) (I, PLABE (I), I=1, NSOLN) & DATOUT & 44 \\
\hline & $J=N S O L N+1$ & DATOUT & 45 \\
\hline & WRITE (IDUT, 516) J,NOPTN & DATOUT & 46 \\
\hline & NRITE (ICUT, 520) IQB & DATOUT & 47 \\
\hline & READ $(I N, *, E R R=118)$ ISOLN & DATOUT & 48 \\
\hline & IF (ISOLN.EA.9) RETURN & DATOUT & 49 \\
\hline & IF (ISOLN.LT.1) Go TO 110 & DATDUT & 56 \\
\hline & IF (ISOLN.GT, NSOLN) THEK & DATOUT & 51 \\
\hline 126 & CONTINUE & DATOUT & 52 \\
\hline & WRITE (IOUT, 530) NPRT & DATOUT & 53 \\
\hline & READ (IN, * ERR=129) NPRT & DATOUT & 54 \\
\hline & IF (NPRT.LT.1) NPRT=1 & DATOUT & 55 \\
\hline & IF (NPRT.GT.NSANC) NPRT=NSANC & DATOUT & 56 \\
\hline & GO TO 116 & DATOUT & 57 \\
\hline & ENDIF & DATDUT & 58 \\
\hline C & & DATOUT & 59 \\
\hline c & PRDMPT FDR PROBABLITY/CONPLENENTARY PROBABILITY & DATEUT & 60 \\
\hline C & & DATOUT & 61 \\
\hline 1300 & CONTINUE & DATOUT & 62 \\
\hline & WRITE (IOUT, 548) & DATOUT & 63 \\
\hline & READ (IN, *,ERR=139) ICCD & DATOUT & 64 \\
\hline & IF ((ICCD.NE.1).AND. (ICCD.NE. 2)) GO TD 139 & DATOUT & 65 \\
\hline C & & DATOUT & 66 \\
\hline
\end{tabular}




\begin{tabular}{|c|c|c|c|}
\hline c & TRANSFER SOLUTION TO PLOT/PRINT ARRAY & DATOUT & 67 \\
\hline c & & DATOUT & 88 \\
\hline & $I Z E=0$ & DATOUT & 69 \\
\hline & $N=N S L O C(I S O L N)-1$ & DATQUT & 79 \\
\hline & DO $140 \mathrm{I}=1, \mathrm{~N} 5 \mathrm{NAC}$ & DATDUT & 71 \\
\hline & $J=I \bullet N$ & DATOUT & 72 \\
\hline & $\operatorname{RPLT}(\mathrm{I})=\operatorname{RL}, T S(J)$ & DATOUT & 73 \\
\hline & IF (RPLT(I).EQ. 0.0$)$ IZE=1 & DATOUT & 74 \\
\hline 140 & CONTINUE & DATOUT & 75 \\
\hline & IF (RPLT(1).LT, 0 . $)$ IZE $=\varnothing$ & DATOUT & 76 \\
\hline C & & DATOUT & 77 \\
\hline & IF (ICC.NE.ICCD) THEN & DATOUT & 78 \\
\hline & $A=\operatorname{CONF}(1)$ & DATOUT & 79 \\
\hline & IF (ICC.EQ 2) $A=$ CONF (NSALC) & DATOUT & 80 \\
\hline & DO $150 \mathrm{I}=1$, NSALC & DATDUT & 81 \\
\hline & $\operatorname{CONF}(I)=1 . \theta-\operatorname{CONF}(1)+\lambda$ & DATOUT & 82 \\
\hline 150 & CONTINUE & DATOUT & B3 \\
\hline & $\mathrm{ICC}=\mathrm{ICCD}$ & DATOUT & 84 \\
\hline & ENDIF & DATOUT & 85 \\
\hline C & & DATOUT & 86 \\
\hline$c$ & PRINT RESUTS & DATOUT & 87 \\
\hline$c$ & & DATOUT & 88 \\
\hline c & OUTPUT DATA TABLE HEADERS & DATDUT & 89 \\
\hline C & & DATOUT & 96 \\
\hline & WRITE (IOUT, 558) PLABE (ISLLN) & DATQUT & 91 \\
\hline & IF (IDAT,GT.6) WRITE (IDAT, 650) PLABE (ISOLN) & DATOUT & 92 \\
\hline & IF (IZE.EQ.1) THEN & DATOUT & 93 \\
\hline & WRITE (IOUT, 568) & DATOUT & 94 \\
\hline & IF (IDAT.GT. ) WRITE (IDAT, 586) & DATOUT & 95 \\
\hline & ENDIF & OATOUT & 96 \\
\hline & IF (ICCD.EQ.2) THEN & DATOUT & 97 \\
\hline & WRITE (IDUT, 576) & DATDUT & 98 \\
\hline & IF (IDAT.GT, V) VRITE (IDAT, 57ø) & DATDUT & 99 \\
\hline & 日SE & DATQUT & .100 \\
\hline & WRITE (IOUT, 586) & OATOUT & . 101 \\
\hline & IF (IDAT.GT, W) WRITE (IDAT, 586) & DAFQUT & . 162 \\
\hline & ENDIF & DATOUT & . 103 \\
\hline C & & DATOUT & . 104 \\
\hline$c$ & FIRST DATA POINT, IF NDT PRINTED BY DEAUT & OATOUT & . 105 \\
\hline$c$ & & DATOUT & . 106 \\
\hline & NSTRT=NPRT & DATOUT & . 187 \\
\hline & IF (NPRT.GT.1) THEN & DATOUT & . 108 \\
\hline & RITE (IOUT, 590) 1, RPLT(1), CDNF(1) & DATQUT & . 169 \\
\hline & IF (IDAT, GT, D) WRITE (IDAT, 596) 1, RPLT(1), CONF(1) & DATOUT & - 110 \\
\hline & IF (ICCD.ER. 2) NSTRT=NSTRT+1 & DATOUT & . 111 \\
\hline & ENOIF & DATOUT & . $\quad 112$ \\
\hline c & & DATOUT & . 113 \\
\hline$c$ & PRINTING LDOP & DATDUT & . $\quad 114$ \\
\hline C & & DATOUT & . 115 \\
\hline & $J=6$ & DATOUT & . 118 \\
\hline & $K=\emptyset$ & DATOUT & . 117 \\
\hline & DO $160 I=N S T R T$, NSALC, NPRT & DATOUT & . 118 \\
\hline & $J=I$ & DATOUT & - $\quad 119$ \\
\hline & IF ((RPLT (I).EQ. E. & DATOUT & . 128 \\
\hline
\end{tabular}




\begin{tabular}{|c|c|c|}
\hline WRITE (IOUT, 598) I, RPLT(I), CONF (I) & DATOUT & . 121 \\
\hline IF (IDAT.GT.6) WRITE (IOAT, 590) I, RPLT(I), CONF(I) & DATOUT & . 122 \\
\hline$K=K+1$ & DATOUT & 123 \\
\hline 166 CONTINUE & DATOUT & . 124 \\
\hline c & DATOUT & . 125 \\
\hline LAST DATA POINT, IF NDT PRINTED BY DEALLT & DATOUT & . 126 \\
\hline 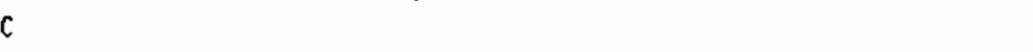 & DATOUT & 127 \\
\hline IF (J.NE. RSALC) THEV & DATOUT & . 128 \\
\hline WRITE (IOUT, 596) NSAL, RPLT(NSNAC), CONF (NSALC) & DATOUT & . 129 \\
\hline IF (IDAT.GT.8) WRITE (IDAT, 598) NSNC, RPLT(NSNC), CONF (NSALC) & DATOUT & . 130 \\
\hline ENDIF & DATOUT & 131 \\
\hline c & DATOUT & . 132 \\
\hline WARNING IF $\amalg$ DATA POINTS ARE ZERD & DATOUT & . 133 \\
\hline$c$ & DATDUT & . 134 \\
\hline IF (K.EQ. W) WITE (IOUT, 898) PLABEL(ISOLN) & DATOUT & . 135 \\
\hline c & DATDUT & . 136 \\
\hline PLOT RESULTS & DATOUT & . 137 \\
\hline c & DATOUT & . 138 \\
\hline IF ((K.GT. ). AND. (NSALC.GT.1)) THEN & DATOUT & 139 \\
\hline 170 CONTINUE & DATOUT & . 140 \\
\hline WRITE (IOUT, B10) IQB & DATOUT & . 141 \\
\hline READ (IN, 626) ANS & DATOUT & 142 \\
\hline IF ((ANS.NE. 'Y'). AND. (ANS. NE. 'N')) THEN & DATOUT & 143 \\
\hline IF ((ANS. NE. 'y'). AND. (ANS.NE. 'n')) GO TO 179 & DATOUT & . 144 \\
\hline ENDIF & DATOUT & . 145 \\
\hline IF ((ANS.EQ. 'Y') OR. (ANS.EQ. ' $y$ ')) THEN & DATOUT & . 146 \\
\hline IF (JONCE.EQ. G) THEN & DATOUT & . 147 \\
\hline$X\llcorner 2=14,2$ & DATOUT & . 148 \\
\hline$Y L 2=10.1$ & DATOUT & . 149 \\
\hline CALL PLINIT (XL2, YL2, IRUN) & DATOUT & . $\quad 156$ \\
\hline $\mathrm{X}=\mathrm{XL2}-2.2$ & DATOUT & . 151 \\
\hline$Y=Y L 2-2.8$ & DATOUT & . 152 \\
\hline ENDIF & DATOUT & . 153 \\
\hline CNL PPLT & DATOUT & . 154 \\
\hline JDNCE $=1$ & DATOUT & . 155 \\
\hline ENDIF & DATOUT & . 156 \\
\hline ENDIF & DATOUT & . 157 \\
\hline GD TO 110 & DATOUT & . $\quad 158$ \\
\hline c & DATQUT & . 159 \\
\hline 586 FDRMAT (/1X, 'AVAILABLE VARIABLES ARE'/7X, '0) EXIT LIST') & DATQUT & . 160 \\
\hline 510 FDRUAT $\left.\left(3 x, 15,{ }^{\prime}\right)^{\prime}, A 32\right)$ & DATQUT & . 161 \\
\hline 520 FORWAT (IX, 'THICH WOUD YOU LIKE (ENTER NUMBER)? ',$A 1$ ) & DATOUT & 162 \\
\hline 536 FORWAT ( $1 X$, 'THE CURRENT PRINT INTERVN IS ', I5,' '/1X,'ENTER THE $D$ & DATOUT & . 163 \\
\hline +ESIRED VALUE. ') & DATOUT & . 164 \\
\hline 540 FORMAT (/1X, 'NHICH WOUD YOU LIKE'/3X, '1) CUWULATIVE PROBABILITY'/ & DATOUT & 166 \\
\hline +3X, '2) CDHPLEUENTARY CUMATIVE PRDBABILITY'/1X, 'ENTER 1 OR 2') & DATOUT & . 166 \\
\hline 550 FORWAT $\left(/ / / 1 X,{ }^{\prime} *+\cdots\right.$ OUTPUT CUAMUATIVE PROBABILITIES'/7X, 'FOR STO & DATOUT & . 187 \\
\hline -CASTIC VARIABLE, ${ }^{\prime}, 16 \times,{ }_{1}$ A32) & DATOUT & . 168 \\
\hline 560 FORMAT (/8X, 'IN THE FDLONING TABLE, YNUES OF ZERO ARE OMITTED. ') & DATOUT & . 169 \\
\hline 570 FORWAT $(/ / 8 X$, 'SAMPLE',6X, 'YARIABLE',7X, 'COMPLENENTARY'/6X, 'NUMBER' & DATOUT & 176 \\
\hline$\rightarrow, 8 X$, 'YNUE', 10X, 'CONFIDENCE') & DATOUT & 171 \\
\hline 580 FORMAT (//BX, 'SALPLE', 6X, 'YARIABLE', 8X, 'CUMMULATIYE'/6X, 'NUMBER', & DATOUT & . 172 \\
\hline$+8 X$, 'YNUE', 16X, 'CONFIDENCE') & DATOUT & 173 \\
\hline 596 FORUAT $(1 X, I 10,5 X, 612.4,5 X, F 11.5)$ & DATOUT & . 174 \\
\hline
\end{tabular}




\begin{tabular}{|c|c|c|c|}
\hline 608 & FORUAT (/1X, 'ALL COMPUTED VALUES ARE ZERO FOR ', A32) & DATOUT & . 175 \\
\hline 810 & FORUAT (/1X, 'WOUL YOU LIKE THE DATA PLDTTED $\left.(Y / N) ?{ }^{\prime}, \wedge 1\right)$ & DATOUT & . 176 \\
\hline 628 & FORMAT (A1) & DATOUT & . 177 \\
\hline & END & DATOUT & . 178 \\
\hline & SUBROUTINE DATSET (IERR) & DATSET & 2 \\
\hline $\mathrm{c}$ & & DATSET & 3 \\
\hline C & THIS ROUTINE WOVES THE INPUT VARIABLES INTO ARRAY VINPT, AND & DATSET & 4 \\
\hline C & CALLS THE SOLUTION ROUTINE SOLN (IF NEEDED) TO COMPUTE THE & DATSET & 5 \\
\hline$c$ & QUTPUT VALUES FOR EACH SAUPLE. THESE DUTPUT VALUES ARE WOVED & DATSET & 6 \\
\hline$c$ & INTO ARRAY RLTS FOR LATER PROCESSING. & DATSET & 7 \\
\hline C & & DATSET & 8 \\
\hline SINSER & RT $\#>C O W O N>P P A R A$ & DATSET & 9 \\
\hline SINSER & RT *SCOLONOPBLK1 & DATSET & 10 \\
\hline SINSER & RT $\#$ CONDONDPBLA & DATSET & 11 \\
\hline SINSER & RT $\Rightarrow C O Q M D N>P B L K 5$ & DATSET & 12 \\
\hline \$INSER & RT $\#>C O M O N>P B C 7$ & DATSET & 13 \\
\hline SINSER & RT $\#>$ COLAON)PBEK8 & DATSET & 14 \\
\hline SINSERT & RT $*>$ COLANONYPBLK12 & DATSET & 15 \\
\hline I INSERT & RT $*>$ COLANON $>$ PQ $\times 17$ & DATSET & 16 \\
\hline C & & DATSET & 17 \\
\hline$c$ & & DATSET & 18 \\
\hline & IERR=6 & DATSET & 19 \\
\hline & NUMERR=ø & DATSET & 20 \\
\hline & DO 140 INDEX=1, NSAM & DATSET & 21 \\
\hline & NERR $($ IKDEX) $=0$ & DATSET & 22 \\
\hline C & & DATSET & 23 \\
\hline$c$ & SAVE INPUT VALUES IN ARRAY VINPT & DATSET & 24 \\
\hline$c$ & & DATSET & 25 \\
\hline & DO $100 \mathrm{I}=1$, NYAR & DATSET & 28 \\
\hline & $J=N V D O C(I)+I N D E X-1$ & DATSET & 27 \\
\hline & $\operatorname{VINPT}(\mathrm{I})=\operatorname{VAR}(\mathrm{J})$ & DATSET & 28 \\
\hline 160 & CONTINUE & DATSET & 29 \\
\hline C & & DATSET & 30 \\
\hline c & SOLUTION ROUTINE CAШED ONCE THEN NSOLN=ø & DATSET & 31 \\
\hline C & & DATSET & 32 \\
\hline & IF (NSOLN.GT. E) GO TD 110 & DATSET & 33 \\
\hline & IF (INDEX.EQ. I) CALL SOLN (IERR) & DATSET & 34 \\
\hline & GO TO 146 & DATSET & 35 \\
\hline 116 & CONTINUE & DATSET & 36 \\
\hline$c$ & & DATSET & 37 \\
\hline$c$ & COMPUTE OUTPUT VARIABLES & DATSET & 38 \\
\hline$c$ & & DATSET & 39 \\
\hline & CALL SOLN (IERR) & DATSET & 40 \\
\hline & IF (IERR.EQ. $\theta$ ) GO TO 126 & DATSET & 41 \\
\hline & IF (IQUIT.GT.E) GD TD 150 & DATSET & 42 \\
\hline & NUMERR=NUMERR +1 & OATSET & 43 \\
\hline & NERR $($ INDEX) $=$ INDEX & DATSET & 44 \\
\hline & IERR $=0$ & DATSET & 45 \\
\hline 120 & CONTINUE & DATSET & 46 \\
\hline$c$ & & DATSET & 47 \\
\hline C & SAVE VALUES IN OUTPUT ARRAY & DATSET & 48 \\
\hline C & & DATSET & 49 \\
\hline & DO $130 \mathrm{I}=1, \mathrm{NSOLN}$ & DATSET & 50 \\
\hline & $J=N S L O C(I)+I N D E X-1$ & DATSET & 51 \\
\hline
\end{tabular}




\begin{tabular}{|c|c|c|c|c|}
\hline \multicolumn{3}{|r|}{$\operatorname{RLTS}(\mathrm{J})=\operatorname{VALU}(\mathrm{I})$} & DATSET & 52 \\
\hline 139 & \multicolumn{2}{|c|}{ CONTINUE } & DATSET & 53 \\
\hline \multicolumn{3}{|c|}{246 CONTINUE } & DATSET & 54 \\
\hline \multicolumn{3}{|c|}{ RETURN } & DATSET & 55 \\
\hline \multicolumn{3}{|l|}{ c } & DATSET & 56 \\
\hline \multirow[t]{6}{*}{150} & \multicolumn{2}{|c|}{ CONTINUE } & DATSET & 57 \\
\hline & \multicolumn{2}{|c|}{ IERR=1 } & DATSET & 58 \\
\hline & \multicolumn{2}{|c|}{ NSAN=INDEX-1 } & DATSET & 59 \\
\hline & \multicolumn{2}{|c|}{ RETURN } & DATSET & Bo \\
\hline & \multicolumn{2}{|l|}{ END } & DATSET & 81 \\
\hline & \multicolumn{2}{|c|}{ SUBROUTINE DEXPF (N,RNDVAL,RP1) } & DEXPF & 2 \\
\hline \multicolumn{3}{|l|}{ C } & DEXPF & 3 \\
\hline$c$ & \multicolumn{2}{|r|}{ ROUTINE FOR TRANSFDRUING A UNIFORMYY DISTRIBUTED DATA SET ON THE } & DEXPF & 4 \\
\hline c & \multirow{2}{*}{\multicolumn{2}{|c|}{ INTERVAL G,1 TO AN EXPONENTIALLY DISTRIBUTED DATA SET. }} & DEXPF & 5 \\
\hline $\mathrm{C}$ & & & DEXPF & 6 \\
\hline C & N & $=$ THE NUMBER OF ITENS IN THE DATA SET. & DEXPF & 7 \\
\hline 6 & \multicolumn{2}{|r|}{ RNDVAL = YECTOR DF UNIFORLY OISTRIBUTED VALUES ON INPUT. EACH } & DEXPF & 8 \\
\hline C & \multicolumn{2}{|c|}{ VALUE WUST BE BETHEEN ZERO AND ONE. } & DEXPF & 9 \\
\hline c & \multirow{2}{*}{\multicolumn{2}{|c|}{$\begin{aligned} & \text { = VECTOR OF EXPONENTIALLY DISTRIBUTED VALUES ON OUTPUT. } \\
& \text { RP1 }=\text { MEAN VALUE FOR THE EXPONENTIAL DISTRIBNTION. }\end{aligned}$}} & DEXPF & 10 \\
\hline c & & & DEXPF & 11 \\
\hline \multicolumn{3}{|l|}{ c } & DEXPF & 12 \\
\hline \multicolumn{3}{|c|}{ DILENSION } & DEXPF & 13 \\
\hline & $+\quad R$ & NDVNL(N) & DEXPF & 14 \\
\hline c & & & DEXPF & 15 \\
\hline$c$ & & EXPONENTIAL DISTRIBUTION & DEXPF & 16 \\
\hline$c$ & & & DEXPF & 17 \\
\hline & DO 18 & $I=1, N$ & DEXPF & 18 \\
\hline & RV $=$ & $1.0-R N D V A(I)$ & DEXPF & 19 \\
\hline & IF & (RV.LT.1.6E-21) RV=1.6E-21 & DEXPF & 28 \\
\hline & RND & VNL $(I)=-N L O G(R V) / R P 1$ & DEXPF & 21 \\
\hline 108 & CONTI & NUE & DEXPF & 22 \\
\hline & RETUR & & DEXPF & 23 \\
\hline & END & & DEXPF & 24 \\
\hline & SUBRO & UTINE DEXPFL (N, RNDVAL, RP1, ANIN, ANAX) & DEXPFL & . \\
\hline$c$ & & & DEXPFL & . \\
\hline$c$ & RDUTIN & IE FOR TRANSFORUING A UNIFORMY DISTRIQUTED DATA SET ON THE & DEXPFL & . \\
\hline$c$ & INTERV & IAL D, I TO AK EXPONENTIALLY DISTRIBUTED DATA SET, WITH THE & DEXPFL & . \\
\hline c & CONSTR & RAINT THAT MLL DUTPUT VALUES LIE BETHEEN SPECIFIED LIMITS. & DEXPFL & . \\
\hline c & & & DEXPFL & . \\
\hline C & N & $=$ THE NUMBER DF ITESS IN THE RANDOM VARIABLE VECTOR RNDVAL. & DEXPFL & . \\
\hline c & RNDVAL & $=$ ON INPUT, A YECTOR OF N RANDOM YALUES, UNIFORALY & DEXPFL & . \\
\hline c & & DISTRIUTED, ON THE INTERVAL ZERD TO ONE. & DEXPFL & 16 \\
\hline c & & $=$ ON DUTPUT, A VECTOR OF N RANDON VALUES, EXPONENTIALLY & DEXPFL & 11 \\
\hline c & & DISTRIBUTED WITH 'MEAN' RP1, AND WITH VALUES BEOW ANIN & DEXPFL & 12 \\
\hline c & & AND ABOVE AMX DELETED FRON THE DISTRIBUTION. & DEXPF & 13 \\
\hline c & RP1 & $=$ MEAN VALUE FOR THE DESIRED EXPONENTIAL DISTRIBUTION. & DEXPFL & 14 \\
\hline c & AMIN & = MINIMU LIMIT VALUE FDR THE OUTPUT DISTRIBUTION. & DEXPFL & 15 \\
\hline$c$ & AMAX & $=$ MAXIMU LIMIT VNLUE FOR THE OUTPUT DISTRIBUTION. & DEXPFL & 16 \\
\hline c & & & DEXPFL & 17 \\
\hline c & UNIN, & AMX = LIMITS DN THE UNIFORM DISTRIBUTION. THESE ARE & DEXPFL & 18 \\
\hline$c$ & & AVALIABLE TO THE USER THROUGH COMMON BLOCK ULIMTS. & DEXPFL & 19 \\
\hline$c$ & & & DEXPFL & 26 \\
\hline SINSE & RT $\neq>C$ & DWON>ULIUTS & DEXPL & 21 \\
\hline$c$ & & & DEXPFL & 22 \\
\hline
\end{tabular}




\begin{tabular}{|c|c|c|c|}
\hline & DIMENSION & DEXPFL & 23 \\
\hline & - RNDVAL(N) & DEXPFL & 24 \\
\hline & DATA & DEXPFL & 25 \\
\hline & $\rightarrow \quad$ IONE $/ 1 /$ & DEXPFL & 26 \\
\hline c & & DEXPFL & 27 \\
\hline & IF (RP1.LT.6.) RP1=ABS(RP1) & DEXPFL & 28 \\
\hline & IF (AUIN.LE. $\mu M A X$ ) CO TO 100 & DEXPFL & 29 \\
\hline & MD=ANIN & DEXPFL & 30 \\
\hline & $A M I N=A M A X$ & DEXPFL & 31 \\
\hline & AUAX $=$ WD & DEXPFL & 32 \\
\hline 160 & CONTIRUE & DEXPFL & 33 \\
\hline & IF (ANIN.LT.AMX) GO TO 110 & DEXPFL & 34 \\
\hline & NIN $=0$. & DEXPFL & 35 \\
\hline & AWAX $=130, * R P 1$ & DEXPFL & 36 \\
\hline 110 & CONTINUE & DEXPFL & 37 \\
\hline C & & DEXPFL & 38 \\
\hline$c$ & UNIFORW DISTRIBUTION LIKITS & DEXPFL & 39 \\
\hline c & & DEXPFL & 46 \\
\hline & UNIN=1.g-EXP $(-R P 1 * A M I N)$ & DEXPFL & 41 \\
\hline & $U N A X=1.0-E X P(-R P 1 * A N A X)$ & DEXPFL & 42 \\
\hline & RV=UNIN & DEXPFL & 43 \\
\hline & CALL DEXPF (IONE, RY, RPI) & DEXPFL & 44 \\
\hline & $B=R V$ & DEXPFL & 45 \\
\hline & $R V=U M N X$ & DEXPFL & 46 \\
\hline & CALL DEXPF (IONE, RY, RP1) & DEXPFL & 47 \\
\hline & $T=R V$ & DEXPFL & 48 \\
\hline & IF (B.LE.T) GO TO 120 & DEXPFL & 49 \\
\hline & LMIN=1. D- LMIN & DEXPFL & 50 \\
\hline & $\operatorname{LNAX}=1.0-\operatorname{UNAX}$ & DEXPFL & 51 \\
\hline 120 & CONTINUE & DEXPFL & 52 \\
\hline c & & DEXPFL & 53 \\
\hline c & ADJUST UNIFOFN DISTRIQUTION TO NE LIHITS & DEXPFL & 54 \\
\hline c & & DEXPFL & 55 \\
\hline & WD=LMNX-LNIN & DEXPFL & 56 \\
\hline & $00230 I=1, N$ & DEXPFL & 57 \\
\hline & RNDVAL (I) $=U N I N+R N D V A L(I) * W D$ & DEXPFL & 58 \\
\hline 138 & CONTINUE & DEXPFL & 59 \\
\hline C & & DEXPFL & 60 \\
\hline c & CONPUTE EXPONENTIAL DISTRIBUTION & DEXPFL & 61 \\
\hline$c$ & & DEXPFL & 62 \\
\hline & CAL DEXPF (N,RNOVAL,RP1) & DEXPFL & 63 \\
\hline & RETURN & DEXPFL & 64 \\
\hline & ERO & DEXPFL & 65 \\
\hline & FUNCTIDN DNORU (RNDYAL, RP1, RP2) & DNORM & 2 \\
\hline c & & DNORM & 3 \\
\hline c & ROUTINE FOR TRANFQRUING A SINGE VALUE FROW A UNIFORMYY & DNORM & 4 \\
\hline C & DISTRIQUTED DATA SET ON THE INTERVAL 0,1 TO A SINGL VALUE IN A & DNORM & 5 \\
\hline C & NORUALLY DISTRIBUTED DATA SET. & DNORM & 6 \\
\hline c & & ONDRN & 7 \\
\hline$c$ & A POLYNOMIAL METHOD IS USED. & DNORM & 8 \\
\hline $\mathrm{C}$ & REFGREKCE ABRALOWITZ STECUN, PAGE 933. & DNDRM & 9 \\
\hline C & & DNORN & 10 \\
\hline c & RNOVAL = INPUT RANDDM VALUE BETWEEN OND 1. & DNDRM & 11 \\
\hline c & RP1 = WEAN VALUE FOR THE NORWAL DISTRIBUTION. & ONORM & 12 \\
\hline
\end{tabular}




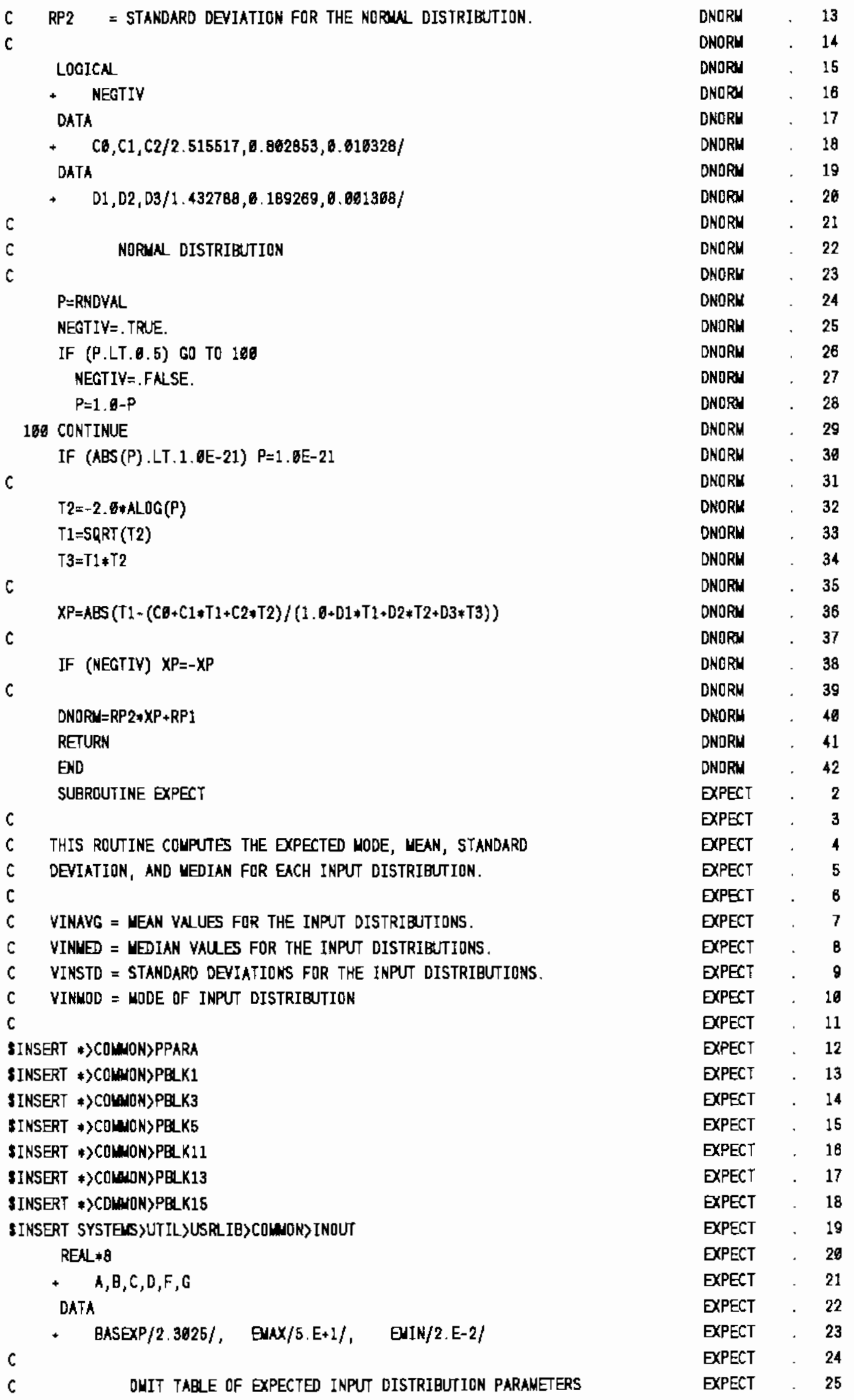




\begin{tabular}{|c|c|c|c|}
\hline$c$ & IF NL OF THE YARIABCES ARE CONSTANTS (NSTOC=1), ARE & EXPECT & 26 \\
\hline$c$ & ENDPOINT LINITED, OR ARE READ FROM AN EXTERNAL FILE. & EXPECT & 27 \\
\hline c & SET COUNTER 'LIMITS' TO INDICATE HOW MANY YARIABLES ARE & EXPECT & 28 \\
\hline c & ENDPOINT LIMITED. & EXPECT & 29 \\
\hline c & & EXPECT & 30 \\
\hline & LIMITS=6 & EXPECT & 31 \\
\hline & $\mathrm{J}=\varnothing$ & EXPECT & 32 \\
\hline & DO $100 \mathrm{~N}=1$, NYAR & EXPECT & 33 \\
\hline & IF (ILIU(N). NE.6) LIMITS=LIMITS+1 & EXPECT & 34 \\
\hline & IF ((ILIM(N).NE. B). OR. (ITYPE(N).GT.8)) J=J+1 & EXPECT & 35 \\
\hline 160 & CONTINUE & EXPECT & 36 \\
\hline & IF (LIMITS.EQ.NYAR) GO TO 240 & EXPECT & 37 \\
\hline & IF (J.EQ.NYAR) GO TO 280 & EXPECT & 38 \\
\hline & IF (NSTOC.EQ.1) GO TO 280 & EXPECT & 39 \\
\hline c & & EXPECT & 40 \\
\hline C & OUTPUT FILE UNIT & EXPECT & 41 \\
\hline c & & EXPECT & 42 \\
\hline & $I I I=I D A T$ & EXPECT & 43 \\
\hline & IF (IDAT.ER. E) III=IDUT & EXPECT & 44 \\
\hline c & & EXPECT & 45 \\
\hline C & TAQE HEADER & EXPECT & 46 \\
\hline c & & EXPECT & . 47 \\
\hline & IF (III.GT.ø) THEN & EXPECT & . 48 \\
\hline & WRITE (III, 500) & EXPECT & . 49 \\
\hline & WRITE (III, 510) & EXPECT & 50 \\
\hline & ENDIF & EXPECT & 51 \\
\hline C & & EXPECT & 52 \\
\hline C & IAPUT VARIABQE EXPECTED STATISTICS. OHIT ANY YARIABLE & EXPECY & 53 \\
\hline C & WHICH IS ENDPOINT LINITED. INDICATED BY ILIM(N) > $\theta$. & EXPECT & 54 \\
\hline C & & EXPECT & 55 \\
\hline & DO $230 \mathrm{~N}=1$, NYAR & EXPECT & 56 \\
\hline & IF (ILIU(N).GT.0) G0 TO 230 & EXPECT & 57 \\
\hline & $A=0$ & EXPECT & 58 \\
\hline & $\mathrm{B}=\mathrm{\theta}$ & EXPECT & 59 \\
\hline & $C=0$. & EXPECT & . 60 \\
\hline & $\mathrm{D}=\boldsymbol{\sigma}$ & EXPECT & 61 \\
\hline & IOHIT $=0$ & EXPECT & 62 \\
\hline & $R P 1=P A R(N, 1)$ & EXPECT & . 63 \\
\hline & $R P 2=\operatorname{PAR}(N, 2)$ & EXPECT & 64 \\
\hline C & & EXPECT & 85 \\
\hline C & DISTRIBUTION TYPE & EXPECT & 66 \\
\hline C & & EXPECT & 67 \\
\hline & IF (ITYPE(N) . GE. NTYPE) GO TO 230 & EXPECT & 68 \\
\hline & CD TO $(116,120,136,146,180,180,190,200,236,236)$ ITYPE(N) & EXPECT & . $\quad 69$ \\
\hline C & & EXPECT & . 79 \\
\hline C & NORIN DISTRIBUTION & EXPECT & . 71 \\
\hline C & & EXPECT & . 72 \\
\hline 110 & CONTINUE & EXPECT & . 73 \\
\hline & $A=\mathrm{DB} E(\mathrm{RP} I)$ & EXPECT & 74 \\
\hline & $\mathrm{B}=\mathrm{DBC}(\mathrm{RP} 2 * \mathrm{RP} 2)$ & EXPECT & 75 \\
\hline & C=DQE(RP1) & EXPECT & . 76 \\
\hline & $\mathrm{D}=\mathrm{DQ} E(\mathrm{RP} 1)$ & EXPECT & . 77 \\
\hline & Go TO 210 & EXPECT & . 78 \\
\hline C & & EXPECT & 79 \\
\hline
\end{tabular}




\begin{tabular}{|c|c|}
\hline DXPECT & 88 \\
\hline XPECT & 81 \\
\hline EXPECT & B2 \\
\hline EXPECT & 83 \\
\hline DXECT & 84 \\
\hline EXPECT & 85 \\
\hline EXPECT & 86 \\
\hline EXPECT & 87 \\
\hline EXPECT & 86 \\
\hline EXPECT & 89 \\
\hline EXPECT & 98 \\
\hline EXPECT & 91 \\
\hline EXPECT & 92 \\
\hline EXPECT & 93 \\
\hline EXPECT & 94 \\
\hline EXPECT & 95 \\
\hline EXPECT & 96 \\
\hline EXPECT & 97 \\
\hline EXPECT & 98 \\
\hline EXPECT & 99 \\
\hline EХPECT & . 100 \\
\hline EXPECT & 101 \\
\hline EXPECT & 102 \\
\hline EXРECT & . 103 \\
\hline EXPECT & 184 \\
\hline EXPECT & 186 \\
\hline EXPECT & . 106 \\
\hline EXPECT & . 167 \\
\hline EXPECT & 188 \\
\hline EXPECT & . 109 \\
\hline EXPECT & 116 \\
\hline EXPECT & 111 \\
\hline EXPECT & 112 \\
\hline EXРECT & 113 \\
\hline EXPECT & 114 \\
\hline ЕХРЕСТ & 115 \\
\hline EXPECT & 116 \\
\hline EXPECT & 117 \\
\hline EXPECT & 118 \\
\hline ЕХРЕСТ & 119 \\
\hline EXPECT & 120 \\
\hline EXPECT & 121 \\
\hline EXPECT & . 122 \\
\hline EXPECT & 123 \\
\hline EXPECT & 124 \\
\hline EXPECT & 125 \\
\hline EXPECT & 126 \\
\hline EXPECT & . 127 \\
\hline EXPECT & . 128 \\
\hline EXРECT & . 129 \\
\hline EXPECT & . 130 \\
\hline EXPECT & . $\quad 131$ \\
\hline EXPECT & . 132 \\
\hline EXPECT & . $\quad 133$ \\
\hline
\end{tabular}




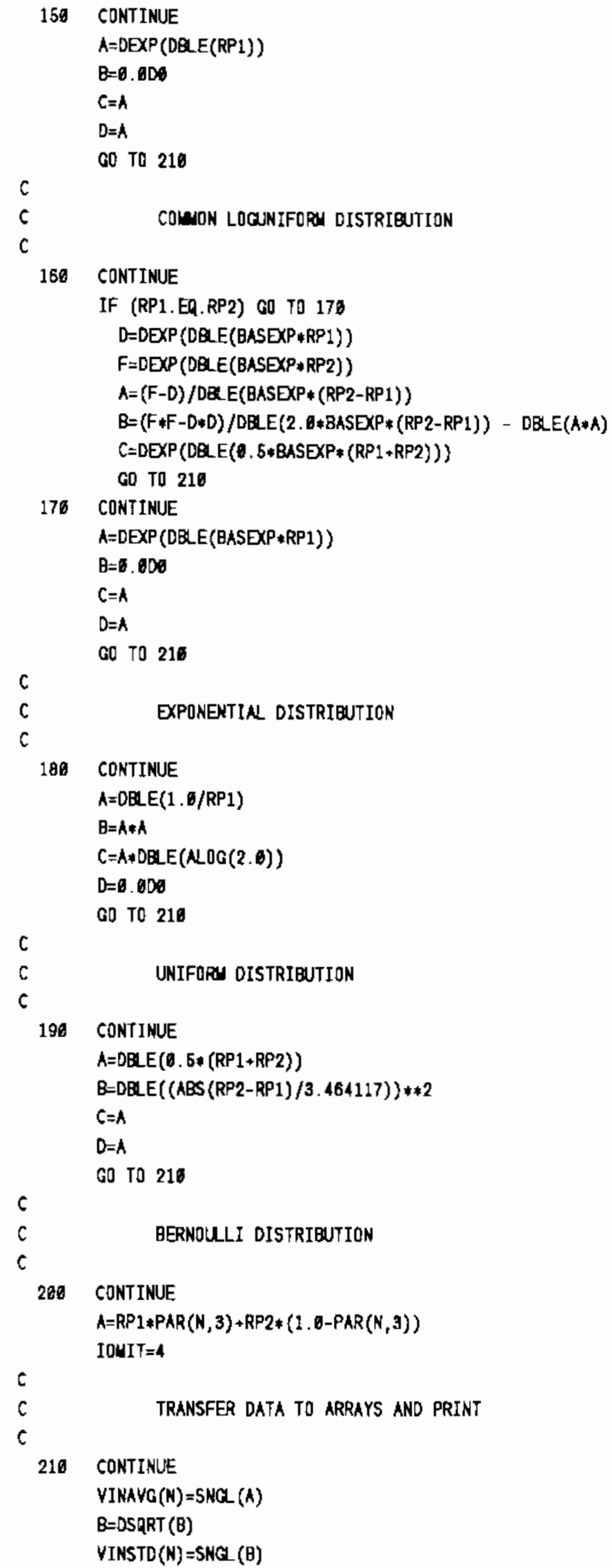




\begin{tabular}{|c|c|c|c|}
\hline & VINMED $(N)=S N G L(C)$ & EXPECT & . 188 \\
\hline & VINHOD $(N)=5 N G$ C $(D)$ & EXPECT & . 189 \\
\hline & IF (IOLIT. NE. $\theta$ ) GO TO 220 & EXPECT & -198 \\
\hline & URITE (III, 520) VABE (N), D, A,C,B & EXPECT & . 191 \\
\hline & GO TO 230 & EXPECT & . 192 \\
\hline 226 & CONTINUE & EXPECT & . 193 \\
\hline & IF (IOUIT.EQ.1) VRITE (III, 630) hABE $(N), D, C$ & EXPECT & . 194 \\
\hline & IF (IDUIT.EQ.2) VRITE (III, 54e) VABE (N), $A, C, B$ & EXPECT & . 195 \\
\hline & IF (IOUIT.EQ.3) VRITE (III, 55ø) VABE (N),C & EXPECT & . 198 \\
\hline & IF (IOUIT.EQ.4) VRITE (III, 546) VABE (N),A & EXPECT & . $\quad 197$ \\
\hline & CDNTINUE & EXPECT & . 198 \\
\hline & IF (IDAT.GT.ø) WRITE $(\mathrm{III}, 580)$ & EXPECT & . 199 \\
\hline c & & EXPECT & . 280 \\
\hline C & PRINT EFFETIVE DISTRIQUTION LIMITS & EXPECT & . $2 \pi 1$ \\
\hline C & & EXPECT & . 202 \\
\hline 248 & 8 CONTINUE & EXPECT & . 203 \\
\hline & IF (LIMITS.ER. G) CO TO 268 & EXPECT & . 204 \\
\hline & WRITE (III, 570) & EXPECT & . 205 \\
\hline & DO $258 H=1$, AYAR & EXPECT & . 296 \\
\hline & IF (ILIU(N).EQ.9) CO TO 250 & EXPECT & 207 \\
\hline & WRIFE (III, 586) $\operatorname{VABE}(N), \operatorname{VINT}(N, 1), \operatorname{VIMT}(N, 2), \operatorname{RLIM}(N, 1)$, & EXPECT & . 208 \\
\hline & $\operatorname{RLIN}(N, 2)$ & EXPECT & . 289 \\
\hline 250 & CONTINUE & EXPECT & . 210 \\
\hline & IF (IDAT.GT.ø) WRITE (III, 568) & EXPECT & . 211 \\
\hline 268 & 8 CONTIRUE & EXPECT & . 212 \\
\hline c & & EXPECT & . 213 \\
\hline & RETURN & EXPECT & . 214 \\
\hline 586 & 6 FORALT $(/ / 1 X, ' * *---$ EXPECTED INPUT VARIABLE STATISTICS') & EXPECT & . 215 \\
\hline 510 & 9 FORMAT (//1X, 'YARIABLE', T18, 'MODE', T2B, 'MEAN', T37, 'MEDIAK', T46, 'ST & EXPECT & . 216 \\
\hline & $\left.\rightarrow N D D E Y^{\prime}\right)$ & EXPECT & . 217 \\
\hline 520 & GORIAT $(1 X, A 12,1 X, 1$ PEg . 2, 3(1X,E9.2)) & EXPECT & . 218 \\
\hline 538 & FORUT $(1 X, A 12,1 X, 1$ PEg. 2, 11X, E9.2) & EXPECT & . 219 \\
\hline 540 & GORUT $(1 X, 112,16 \times, 3(1 X, 1$ PE9 . 2)) & EXPECT & . 226 \\
\hline 558 & FORUT $(1 X, A 12,21 X, 1 P E 9.2)$ & EXPECT & . 221 \\
\hline 566 & 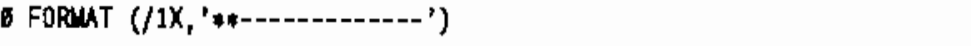 & EXPECT & . 222 \\
\hline 576 & FORUT (/1X,'EFECTIVE LIUITS ON THE INPUT DISTRIBUTION VARIABLES' & EXPECT & . 223 \\
\hline & $+/ 4 X$, 'VARIABLE', $16 \mathrm{X}$, 'VARIABLE LIMITS', 13X, 'INTERVAL LIMITS'/22X, 'LO & EXPECT & . 224 \\
\hline & $+W E R ', 7 X$, 'UPPER', 10X, 'LOWER', 7X, 'UPPER') & EXPECT & . 225 \\
\hline 580 & 6 FORUT $(1 X, A 12,5 X, 1 P E 10.3,2 X, E 16.3,5 X, 9 P F 10.4,2 X, F 10.4)$ & EXPECT & - 226 \\
\hline & END & EXPECT & . 227 \\
\hline & SUBROUTIKE IDNUA (IRUNID, USRNAM) & IDNUN & 2 \\
\hline $\mathrm{C}$ & & IDNUM & 3 \\
\hline c & THIS ROUTINE CREATES A UNIQUE RUN IDENTIFIER NUMBER, UTILIZING & IDNUA & 4 \\
\hline c & THE DATE AND TIME AT WHICH THIS ROUTINE IS CALLE. THE SYSTEN & IDNUN & 5 \\
\hline C & USER NANE IS ALSO PLACED INTO A CHARACTER VARIABLE. THIS & IDNUA & 6 \\
\hline C & INFORATION IS USED TD INSURE TRACEABILITY AND ACCDUNTABILITY & IONUN & 7 \\
\hline C & FOR DATA FILES FROH SIMLATION PROGRAMS. & IDNU: & 8 \\
\hline c & & IDNUN & 9 \\
\hline c & IRUNID = RUN IDENTIFIER, AS FOLLOWS: & IDNUA & 10 \\
\hline C & DIGITS $9-19=$ MONTH DF CURRENT DATE. & IDNUA & 11 \\
\hline C & DIGITS 7-8 = DAY OF THE MDNTH (1 TO 31). & IDNUN & 12 \\
\hline C & DIGITS $5-6=$ CURRENT YEAR. & IDNUM & 13 \\
\hline C & DIGITS 3-4 = CURRENT HDUR ( 6 TO 23). & IDNUM & 14 \\
\hline C & DIGITS 1-2 = CURRENT WINUTE (6 TO 59). & IDNUM & 15 \\
\hline
\end{tabular}



INTECER*4

- IRUNID

- JHR, JMIN

c

INTEGER*2

- LOCAL(15)

CHARACTER*6

- ASDATE

CHARACTER*B

- TIME,

CHARACTER*16

+ DATE

EQUIVRLENCE

- (ASDATE, LOCAL(1)),

- (USRN, LOCAL(13))

c

$c$

$c$

$c$

c

c

c

c

c

CAL TIMOAT (LOCAL(1), INTS(16))

CAL DATESA (DATE)

CALL TIMESA (TIME)

c

READ (ASDATE, 500) JWA, JDD, JYY

$J H R=\operatorname{LOCAL}(4) / 8 \theta$

JMIN $=$ LDCAL (4) $-J H R * 8 \theta$

$J W=J * 100060800$

$J D O=J D D * 1000680$

$J Y Y=J Y * 10060$

$J H R=J H R * 160$

IRUNID $=J \boldsymbol{J}+J D D+J Y Y+J H R+J U I N$

LSRNAM=USRN

c

RETURN

500 FORMAT (3I2)

END

SUBRDUTINE IOPLT (IRUNID, XID, YID)

c

C SUBROUTINE IDPLT TAGS A PLOT IITH AN INTEGER RUN ID IN A

C USER-SPECIFIED LOCATION. THIS IS DONE IN TWO PARTS, AS

c OISSPLA CANNOT HANDLE TEN DIGIT INTEGERS.

c

DATA

- IDATE/10000/

c

$\mathrm{c}$

c

JDD, JYY,

USRNAM

LSRN,

us

NOT TINE

JY,
HE CURRENT SYSTEN USER.

IDNUN

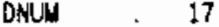

IDNUN $\quad 18$

IDNUM $\quad 19$

IONUN $\quad 20$

IDNUM - 21

IDNLN . 22

IDNUN $\quad 23$

IDNUN - 24

IDNUM . 25

IDNUW - 26

IDNUM - 27

IDNLW - 28

IDNLM . 29

IDNM - 30

IDNUM . 31

IDNUM - 32

IDNU - 33

IDNLM . 34

IDNLW . 35

IDNLW - 38

IDNLW - 37

IDNUW . 38

IDNUW . 39

IDNLM - 46

IDNLM . 41

IDNUN $\quad 42$

IDNUIN $\quad 43$

IDNUN $\quad 44$

IDNUN . 45

IDNLW . 46

IDNUM $\quad .47$

IDNUIM $\quad 48$

IDNUW $\quad 49$

IDNUM - 50

IDNUN $\quad 51$

IDNUM . 52

IONIM $\quad 53$

IDNUM - 54

IDNUN - 55

IDNUIN - 58

IDNUN . 57

IDNUM - 58

IDPLT - 2

IDPLT - 3

IOPLT - 4

IDPLT - 5

IDPLT - 8

IDPLT - 7

IOPLT - 8

IDPLT - 9

IDPLT $\quad 10$

IOPLI - 11

IDPLT $\quad 12$ 


\begin{tabular}{|c|c|c|c|}
\hline & IMDY=IRUKID/IDATE & IDPLT & 13 \\
\hline & IF (IMOY.GT.0) THEN & IDPLT & 14 \\
\hline & CALL INTNO (INOY,XID, YID) & IDPLT & 15 \\
\hline & ESSE & IDPLT & 16 \\
\hline & CALL MESSAG ('OEEG', 4, XID, YID) & IDPLT & 17 \\
\hline & ENDIF & IDPLT & 18 \\
\hline$c$ & & IDPLT & 19 \\
\hline C & TIUE OF DAY & IDPLT & 26 \\
\hline$c$ & & IDPLT & 21 \\
\hline & ITME $=$ IRUNID-IMDY *IDATE & IDPLT & 22 \\
\hline & DO $100 \quad \mathrm{I}=1,4$ & IDPLT & 23 \\
\hline & $J=4-I$ & IDPLT & 24 \\
\hline & IF (J.GT. $\theta$ ) THEN & IDPLT & 25 \\
\hline & $J=10 * * J$ & IDPLT & 26 \\
\hline & ESE & IDPLT & 27 \\
\hline & $\mathrm{J}=1$ & IDPLT & 28 \\
\hline & ENDIF & IDPLT & 29 \\
\hline & IF (ITME.GE.J) GO TO 110 & IDPLT & 39 \\
\hline & CNLL UESSAG ('O',1,'ADUT','ABUT') & IDPLT & 31 \\
\hline 106 & CONTINUE & IDPLT & 32 \\
\hline & GD TO 120 & IDPLT & 33 \\
\hline 116 & CONTINUE & IDPLT & 34 \\
\hline & CALL INTHO (ITHE, 'ABUT', 'ABUT') & IDPLT & 35 \\
\hline c & & IDPLT & 36 \\
\hline 120 & CONTINUE & IDPLT & 37 \\
\hline & RETURN & IDPLT & 38 \\
\hline & END & IDPLT & 39 \\
\hline & SUBROUTINE IINPUT & IINPUT & 2 \\
\hline$c$ & & IINPUT & . \\
\hline$c$ & ROUTINE FOR INPUTTING DISTRIQUTION TYPES AND PARMNETERS & IINPUT & . \\
\hline$c$ & FOR THE INPUT VARIABQES. & IINPUT & 5 \\
\hline$c$ & & IINPUT & 8 \\
\hline IINSEF & CRT $\because>C D M O N>P P A R A$ & IINPUT & 7 \\
\hline IINSE & BRT *>COMDKSPBL1 & IINPUT & . \\
\hline SINSES & ERT $*>C O M O N>P Q L K 2$ & IINPUT & 9 \\
\hline IINSES & ET *>COMON>PBL3 & IINPUT & 16 \\
\hline SINSE & RT * COMONSPBLA & IINPUT & 11 \\
\hline IINSEF & $R T * C$ CDMONDPBK5 & IINPUT & 12 \\
\hline IINSEF & RT $\Rightarrow>C O L O N>P B L K 13$ & IINPUT & 13 \\
\hline \$INSES & RT $\Rightarrow$ COLANON>PBLK14 & IINPUT & 14 \\
\hline SINSER & ERT $\#>C O M D N>P B C K 15$ & IINPUT & 15 \\
\hline SINSER & RT $\rightarrow$ CONON>PBLK17 & IINPUT & 16 \\
\hline INSSER & $R T *>C O N O N>P Q L \times 18$ & IINPUT & 17 \\
\hline SINSER & RT SYSTEAS>UTIL>RANDOH>COWON)STRATI & IINPUT & 18 \\
\hline IINSER & RT SYSTEMS $>U T I L>F E M$ IB>CONON>IDCOM & IINPUT & 19 \\
\hline SINSER & RT SYSTEAS)UTIL>USRLIE>CDLNON>INDUT & IINPUT & 26 \\
\hline & CHARACTER 1 & IINPUT & 21 \\
\hline & $\rightarrow \quad$ ANS & IINPUT & 22 \\
\hline & DATA & IINPUT & 23 \\
\hline & - LABEL (1) /'NORML & IINPUT & 24 \\
\hline & + LABE (2) /'NATURAL LOGNDRML $1 /$, & IINPUT & 25 \\
\hline & + LABE (3) /'COMON LOGNORUAL $1 /$, & IINPUT & 28 \\
\hline & + LABE (4) /'NATURAL LOCUNIFORM ' $/$, & IINPUT & 27 \\
\hline & + LABE (5) /'CONON LOGUNIFORN '/, & IINPUT & 28 \\
\hline
\end{tabular}




\begin{tabular}{|c|c|c|c|}
\hline & - LABE (6) /'EXPONENTIAL & IINPUT & 29 \\
\hline & $+\quad$ LABE (7) / UNIFORU & IINPUT & 30 \\
\hline & $+\quad$ LABE (B) / BERNOULI & IINPUT & 31 \\
\hline & + LABE (9) /'EXTERNAL CONF FUNCTN'/, & IINPUT & 32 \\
\hline & - LABE (10)/'EXTERNAL DATA PDINTS'/ & IINPUT & 33 \\
\hline & DATA & IINPUT & 34 \\
\hline & - LABE (NTYPE)/'CONSTANT & IINPUT & 35 \\
\hline C & & IINPUT & 36 \\
\hline C & - & IINPUT & 37 \\
\hline c & - NOTE THE CODE IS SET UP SO THAT DISTRIBUTIONS ONE TO (NTYPE-1) - & IINPUT & 38 \\
\hline C & - ARE EXTENDED DISTRIQUTIONS, AND DISTRIBUTION (NTYPE) IS A & IINPUT & 39 \\
\hline C & - CONSTANT YALUE. IF NEM DISTRIBUTIONS ARE REqUIRED, IT IS NDST - & IINPUT & 40 \\
\hline c & - EASILY INSERTE INSERTE JUST BEFORE \$ NTYPE. SU8ROUTINES & IINPUT & 41 \\
\hline c & - EXPECT AND RNGS UST ALSO BE MODIFIED IF NTYPE IS INCREASED. & IIMPUT & 42 \\
\hline c & - ROUTINE AND STATOT MST ALSO BE MIOIFIED IF THERE IS ANY & IINPUT & 43 \\
\hline c & - CHANGE IN THE OROERING OR TYPES OF THESE DISTRIBUTIONS. & IINPUT & 44 \\
\hline C & --1-1 & IINPUT & 45 \\
\hline & DATA & IINPUT & 46 \\
\hline & - NSTTL/0/, & IINPUT & 47 \\
\hline & $\rightarrow \quad$ STILE/' & IINPUT & 48 \\
\hline c & & IINPUT & 49 \\
\hline c & COUNTER INITIALIZATION & IINPUT & 50 \\
\hline C & & IINPUT & 51 \\
\hline & DO $160 \mathrm{I}=1$, NYAR & IINPUT & 52 \\
\hline & $\operatorname{NLI}(I, 1)=0$ & IINPUT & 53 \\
\hline & $\operatorname{NLIU}(1,2)=0$ & IINPUT & 54 \\
\hline 160 & CONTINUE & IINPUT & 55 \\
\hline c & & IINPUT & 56 \\
\hline C & PROMPT FOR SYSTESS VARIAQLE UPDATE, & IINPUT & 57 \\
\hline$c$ & EXCEPT FIRST TIUE THROUGH ROUTINE. & IINPUT & 58 \\
\hline C & & IINPUT & 59 \\
\hline & IUPDAT $=6$ & IINPUT & 60 \\
\hline & NDIST $=N T Y P E$ & IINPUT & 61 \\
\hline & IF (ICNT.GT.1) THEN & IINPUT & 62 \\
\hline 110 & CONTINUE & IINPUT & 63 \\
\hline & WRITE (IDUT, 500) IQB & IINPUT & 64 \\
\hline & READ (IN, 510) ANS & IINPUT & 65 \\
\hline & IF ((ANS.NE. 'Y') AND. (ANS.NE. 'N')) THEN & IINPUT & 66 \\
\hline & IF ((ANS.NE. 'y'), AND. (ANS.NE. 'n')) GO TO 110 & IINPUT & 67 \\
\hline & ENDIF & IINPUT & 68 \\
\hline & IF ((ANS.Eq. 'N'), OR. (ANS.Eq. 'n')) GO TO 270 & IINPUT & 69 \\
\hline & NDIST $=N T Y P E+1$ & IINPUT & 76 \\
\hline & ENDIF & IINPUT & 71 \\
\hline & IUPOAT $=1$ & IINPUT & 72 \\
\hline & NSTOC $=1$ & IINPUT & 73 \\
\hline C & & IINPUT & 74 \\
\hline C & LOOP UPDATES SYSTE VARIABLES & IINPUT & 75 \\
\hline C & & IINPUT & 76 \\
\hline & WRITE (IOUT, 529) & IINPUT & 77 \\
\hline & LPNT=1 & IINPUT & 78 \\
\hline & $N=0$ & IINPUT & 79 \\
\hline 120 & CONTINUE & IINPUT & 80 \\
\hline & $\mathrm{N}=\mathrm{N}+1$ & IINPUT & . $\quad 81$ \\
\hline & $\operatorname{ILI}(N)=0$ & IINPUT & 82 \\
\hline
\end{tabular}




\begin{tabular}{|c|c|c|c|}
\hline$c$ & & IINPUT & 83 \\
\hline c & INPUTS DISTRIBUTIONS/PARANETERS FDR SYSTEUS VARIAELES & IINPUT & 84 \\
\hline c & & IYNPUT & 85 \\
\hline \multirow[t]{10}{*}{130} & CONTIMUE & IINPUY & 86 \\
\hline & WRITE (IOUT, 530) N, VAABE (N), VDIMEN(N) & IINPUT & 87 \\
\hline & WRITE (IOUT, 548) (I,LABE (I), I=1, NTYPE) & IINPUT & 88 \\
\hline & IF (ICHT.GT.1) VRITE (IOUT, 558) NDIST & IINPUT & 89 \\
\hline & WRITE (IOUT, 580) IQB & IINPUT & 96 \\
\hline & READ (IN, * ERR=410) IT & IINPUT & 91 \\
\hline & IF ((IT.LT.1).OR. (IT.GT.NDIST)) GO TO 410 & IINPUT & 92 \\
\hline & IF (IT.GT.NTYPE) GQ TO 266 & IINPUT & 93 \\
\hline & WRITE (IDUT, 670) LABE (IT) & IINPUT & 94 \\
\hline & ITYPE $(N)=I T$ & IINPUT & 95 \\
\hline c & & IINPUT & 96 \\
\hline C & NORULL OISTRIBUTION & IINPUT & 97 \\
\hline \multirow[t]{4}{*}{ c } & & IINPUT & 98 \\
\hline & IF (IT.NE.1) CO TO 150 & IINPUT & 99 \\
\hline & WRITE (IOUT, 586) IQB & IINPUY & . 100 \\
\hline & READ $(I N, *, E R R=416) \operatorname{PAR}(N, 1)$ & IINPUT & . 101 \\
\hline \multirow[t]{8}{*}{140} & CONTINUE & IIAPUT & $=102$ \\
\hline & IRITE (IOUT, 580) IQB & IINPUT & . 103 \\
\hline & READ $\left(I X_{1} *, E R R=410\right) \operatorname{PAR}(N, 2)$ & IINPUT & . 164 \\
\hline & IF (PAR(N,2).LT.. ) THEN & IINPUT & . 105 \\
\hline & WRITE (IOUT,6日の) PAR(N,2) & IINPUT & . 186 \\
\hline & GO TO 146 & IINPUT & . 187 \\
\hline & ENDIF & IINPUT & . 168 \\
\hline & GO TO 256 & IINPUT & 169 \\
\hline c & & IINPUT & . 110 \\
\hline c & LOGNORUNL DISTRIBUTIONS & IINPUT & . 111 \\
\hline C & & IINPUT & . $\quad 112$ \\
\hline \multirow[t]{2}{*}{156} & CONTINUE & IINPUT & - $\quad 113$ \\
\hline & IF ((IT.NE. 2).AND. (IT.NE.3)) GO TO 1BO & IINPUT & . 114 \\
\hline \multirow[t]{9}{*}{166} & CONTINE & IINPUT & . 115 \\
\hline & WRITE (IOUT, B10) IQB & IINPUT & . 118 \\
\hline & $\operatorname{READ}(I N, *, E R R=410) \operatorname{PAR}(N, 1)$ & IINPUT & . 117 \\
\hline & IF (PAR(N,1).LE. $\boldsymbol{\theta} . \boldsymbol{\emptyset})$ THEN & IINPUT & . 118 \\
\hline & WRITE (IOUT, 606) PAR(N,1) & IINPUT & . 119 \\
\hline & GO TO 180 & IINPUT & . 126 \\
\hline & ENDIF & IINPUT & . 121 \\
\hline & $\operatorname{PAR}(N, 1)=N \operatorname{LG}(\operatorname{PAR}(K, 1))$ & IINPUT & . $\quad 122$ \\
\hline & IF (IT.EQ.3) $\operatorname{PAR}(N, 1)=\operatorname{PAR}(N, 1) / 2.382585$ & IINPUT & . 123 \\
\hline \multirow[t]{8}{*}{170} & CONTINUE & IINPUT & . 124 \\
\hline & WRITE (IOUT, 620) PAR(N,1),IQB & IINPUT & . 125 \\
\hline & READ (IN, * ERR=410) PAR $(N, 2)$ & IINPUT & . 126 \\
\hline & IF (PAR(N,2).LT. G.) THEN & IINPUT & . 127 \\
\hline & WRITE (IOUT, 666) PAR(N,2) & IINPUT & . 128 \\
\hline & GO TO 170 & IINPUT & . 129 \\
\hline & ENDIF & IINPUT & . 130 \\
\hline & GO TO 250 & IINPUT & . 131 \\
\hline C & & IINPUT & 132 \\
\hline c & UNIFORU LOGNORAA DISTRIBUTIONS & IINPUT & 133 \\
\hline c & & IINPUT & . 134 \\
\hline \multirow[t]{2}{*}{180} & CONTINUE & IINPUT & . 135 \\
\hline & IF ((IT.NE.4).AND. (IT.NE.5)) GO TO 196 & IINPUT & . 136 \\
\hline
\end{tabular}




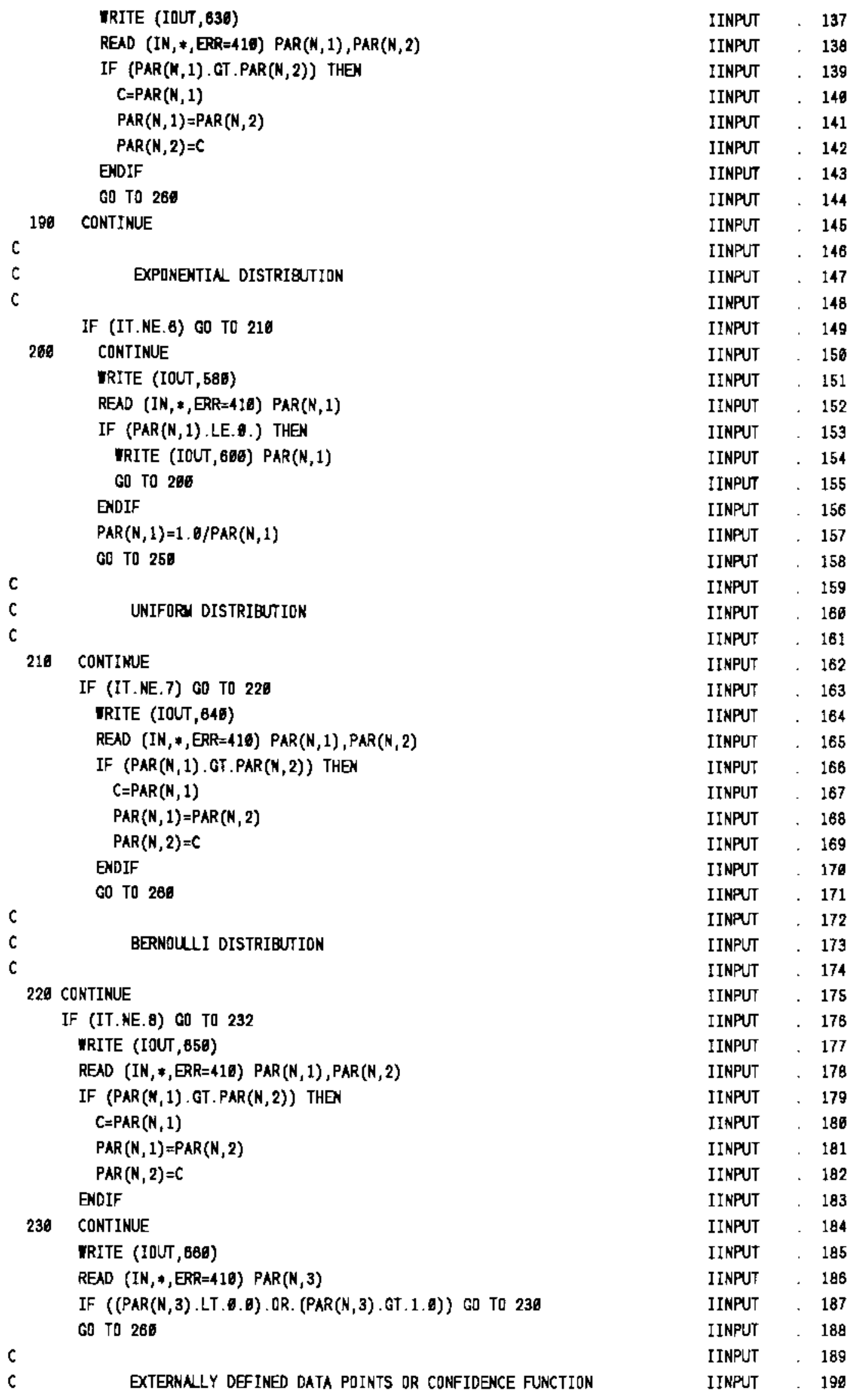


C

232 CONTINUE

IF ((IT. NE. 9) . AND. (IT. NE. 10) ) GO TO 240

IF (IT.EQ.9) WRITE (IOUT, 670)

IF (IT.EQ.18) "RITE (IOUT, 685)

READ (IN, 69\%) (JJNF( $I, N), I=1, N$ LEN)

GDTO 288

C

c

C

240 CONTINUE

IF (IT.NE. NTYPE) GO TO 260

TRITE (IOUT,706) IQ8

READ (IN, *, ERR=410) PAR(N,1)

GO TO 268

$c$

C SET UPPER/LOWER LIMITS OH DISTRIBUTION

C

250 CONTINUE

WRITE (IOUT,710) IQB

REAO (IN, 510) ANS

IF ((ANS. HE. 'Y') , AND. (ANS. NE. 'N')) THEN

IF ((ANS. NE. ' $y$ ') , AND. (ANS.NE. ' $n$ ')) GO TO 250 ENDIF

IF ((ANS.EQ.' 'Y') . OR. (ANS. EQ.' ' $y$ ')) THEN

$\operatorname{ILIU}(\mathrm{N})=1$

IRITE (IOUT, 720)

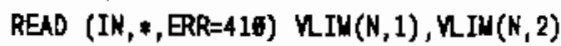

If ( $\operatorname{MIM}(N, 1) . G T . \operatorname{LIM}(N, 2))$ THEN

$C=\operatorname{LIM}(N, 1)$

$\operatorname{VIM}(N, 1)=\operatorname{MIM}(N, 2)$

$\operatorname{nIM}(N, 2)=C$

ENDIF

ENDIF

c

c

c

260 CONTINUE

NEXT VARIABLE

IF (IT. NE. NTYPE) NSTOC $=0$

IF (N.LT.NYAR) GO 129

c

C OPTION TO SKIP THE UPDATING OF SYSTEN CONSTANTS

C

270 CONTINUE

IF (NCONST.LE. O) GO 310

IF (ICNT.GT.1) THEN

280 CONTINUE

WRITE (IDUT, 739) IQB

REAO (IN, 510) ANS

IF ((ANS.NE. 'Y') . AND. (ANS. NE. 'N')) THEN

IF ((ANS. NE. ' $\left.y^{\prime}\right)$, AND. (ANS. NE. ' $n$ ')) CO TO 280 ENDIF

IF ((ANS.EQ. ' ${ }^{\prime}$ ) , OR. (ANS. Eq. ' $\left.n^{\prime}\right)$ ) GO TO 316 ENDIF

\begin{tabular}{|c|c|}
\hline IINPUT & 191 \\
\hline IINPUT & 192 \\
\hline IINPUT & 193 \\
\hline IINPUT & 194 \\
\hline I INPUT & 185 \\
\hline IINPUT & 196 \\
\hline IINPUT & . 197 \\
\hline IINPUT & . 198 \\
\hline IINPUT & . 199 \\
\hline IINPUT & 200 \\
\hline IINPUT & 201 \\
\hline IINPUT & 202 \\
\hline IINPUT & 203 \\
\hline IINPUT & . 204 \\
\hline IINPUT & 285 \\
\hline IINPUT & 288 \\
\hline IINPUT & 207 \\
\hline IINPUT & 208 \\
\hline IINPUT & 269 \\
\hline IINPUT & 210 \\
\hline IINPUT & 211 \\
\hline IINPUT & 212 \\
\hline IINPUT & 213 \\
\hline IINPUT & 214 \\
\hline IINPUT & 215 \\
\hline IINPUT & 216 \\
\hline IINPUT & . 217 \\
\hline IINPUT & 218 \\
\hline IINPUT & 219 \\
\hline IINPUT & 220 \\
\hline IINPUT & . 221 \\
\hline IINPUT & . $\quad 222$ \\
\hline IINPUT & . 223 \\
\hline IINPUT & 224 \\
\hline IINPUT & . 225 \\
\hline IINPUT & 228 \\
\hline IINPUT & 227 \\
\hline IINPUT & 228 \\
\hline IINPUT & . 229 \\
\hline IINFUT & . 230 \\
\hline IINPUT & 231 \\
\hline IINPUT & 232 \\
\hline IINPUT & 233 \\
\hline IINPUT & 234 \\
\hline IINPUT & 235 \\
\hline IINPUT & 238 \\
\hline IINPUT & 237 \\
\hline IINPUT & 238 \\
\hline IINPUT & 239 \\
\hline IINPUT & . 240 \\
\hline IINPUT & . 241 \\
\hline IINPUT & 242 \\
\hline IINPUT & . 243 \\
\hline IINPUT & . 244 \\
\hline
\end{tabular}




\begin{tabular}{|c|c|c|c|}
\hline & IUPDAT $=1$ & IINPUT & 245 \\
\hline c & & IINPUT & 248 \\
\hline c & LDOP UPDATES SYSTEU CONSTANTS & IINPUT & . 247 \\
\hline \multirow[t]{3}{*}{$\mathrm{C}$} & & IINPUT & . 248 \\
\hline & LPNT $=2$ & IINPUT & . 249 \\
\hline & $I=0$ & IINPUT & . 258 \\
\hline \multirow[t]{2}{*}{296} & CONTINUE & IINPUT & . 251 \\
\hline & $\mathrm{I}=\mathrm{I}+1$ & IINPUT & . 252 \\
\hline \multirow[t]{5}{*}{308} & CONTINUE & IINPUT & . 253 \\
\hline & WRITE (IOUT, 746) CLABE (I), CDIMEN(I) & IINPUT & . 254 \\
\hline & IF (ICNT.GT.1) WRITE (IOUT,75\%) CONST(I) & IINPUT & . 255 \\
\hline & READ (IN, * ERR $=416) \operatorname{CONST}(I)$ & IINPUT & . 256 \\
\hline & IF (I.LT.NCONST) GO TO 296 & IINPUT & . 257 \\
\hline \multicolumn{2}{|c|}{310 CONTINUE } & IINPUT & . 258 \\
\hline c & & IINPU & . 259 \\
\hline$c$ & \multirow{2}{*}{ ENTER NUMBER OF SAMPLES TO TAKE } & IINPUT & . 280 \\
\hline c & & IINPUT & . 281 \\
\hline & $L P N T=3$ & IINPUT & . 262 \\
\hline \multirow[t]{8}{*}{326} & CONTIMUE & IINPUT & . 263 \\
\hline & WRITE (IOUT,760) WXSN & IINPUT & . 264 \\
\hline & READ (IN, $*$ ERR=416) NSN & IINPUT & . 265 \\
\hline & IF (NSAH.LT.1) GD TD 320 & IINPUT & . 266 \\
\hline & IF (NSAH.GT.MXSAN) THEN & IINPUT & . 267 \\
\hline & WRITE (IOUT, 770) WXXSA & IINPUT & . 288 \\
\hline & GO TD 320 & IINPUT & . 269 \\
\hline & ENDIF & IINPUT & . 278 \\
\hline C & & IINPUT & . 271 \\
\hline c & ENTER PRINT INTERVAL & IINPUT & . $\quad 272$ \\
\hline \multirow[t]{2}{*}{$c$} & & IINPUT & . 273 \\
\hline & $L P N T=4$ & IINPUT & . 274 \\
\hline \multirow[t]{4}{*}{330} & CONTINUE & IINPUT & . 275 \\
\hline & WRITE (IOUT, 789) & IINPUT & . 278 \\
\hline & $R E A D(I N, *, E R R=410)$ NPRT & IINPUT & . 277 \\
\hline & IF ((NPRT.LT.1).OR. (NPRT.GT.NSAM)) GO TO 410 & IINPUT & 278 \\
\hline C & & IINPUT & . 279 \\
\hline C & ENTER THE NUMBER OF INTERVALS FOR STRATIFIED SNPLING AND & IINPUT & 280 \\
\hline C & INITIALIZE SAMPLING ARRAYS. ONIT IF NO INPUT VARIABLES & IINPUT & . 281 \\
\hline c & ARE DISTRIBUTED. & IINPUT & . 282 \\
\hline \multirow[t]{6}{*}{ c } & & IINPUT & . 283 \\
\hline & LPNT $=6$ & IINPUT & . 284 \\
\hline & NLEVE $=1$ & IINPUT & . 285 \\
\hline & IF (NSTOC.NE. $)$ ) OO TO 370 & IINPUT & . 286 \\
\hline & $I=W A \times L \sqrt{S}$ & IINPUT & . 287 \\
\hline & IF (I.GT. MAXLEV) I= MAXLEV & IINPUT & . 288 \\
\hline \multirow[t]{10}{*}{346} & CONTINUE & IINPUT & . 289 \\
\hline & WRITE (IOUT,799) I & IINPUT & . 290 \\
\hline & READ (IN, * ERR=416) NLEVE & IINPUT & . 291 \\
\hline & IF ((NLEVE.LT.1).DR. (NLEVE. GT.I)) GO TO 340 & IINPUT & - 292 \\
\hline & IF (NLEVE.GT.NSAN) THEN & IINPUT & . 293 \\
\hline & WRITE (IOUT, B60) NLEVE, NSN & IINPUT & 294 \\
\hline & CO TO 340 & IINPUT & . 295 \\
\hline & ENDIF & IINPUT & - 296 \\
\hline & DO $360 \mathrm{~N}=1$, NYAR & IINPUT & . 297 \\
\hline & DD $350 \mathrm{I}=1$, NLEY日 & I INPUT & . 298 \\
\hline
\end{tabular}




\begin{tabular}{|c|c|c|c|}
\hline & $\operatorname{MPTSP}(I, N)=0$ & IINPUT & 299 \\
\hline 358 & CONTINUE & IINPUT & 360 \\
\hline 368 & CONTINUE & IINPUT & 301 \\
\hline \multicolumn{2}{|c|}{370 CONTINUE } & IINPUT & 302 \\
\hline c & & IINPUT & 383 \\
\hline c & \multirow[t]{2}{*}{ DАTA ECHO } & IINPUT & 304 \\
\hline \multirow{13}{*}{ I } & & IINPUT & 395 \\
\hline \multicolumn{2}{|r|}{ IF (IDAT.GT.,) THEM } & IINPUT & . 366 \\
\hline & IF (ICNT.GT.1) WRITE (IOAT,510) & IINPUT & 387 \\
\hline & WRITE (IDAT, B10) & IINPUT & 368 \\
\hline & TRITE (IDAT, 826) TILE, JRUNID, STTLE, USRNAN, ICNT & IINPUT & 309 \\
\hline & DD $399 \mathrm{~N}=1$, NYAR & IINPUT & 310 \\
\hline & $I T=I T Y P E(N)$ & IINPUT & . 311 \\
\hline & WRITE (IDAT,838) VLABE (N), VDIMEN(N), LABE(IT) & IINPUT & . 312 \\
\hline & IF (IT.EQ.1) URITE (IDAT, 840) PAR(N,1), PAR(N,2) & IINPUT & . 313 \\
\hline & IF ((IT.EQ.2), OR. (IT.ER.3)) URITE (IDAT,850) PAR(N,1), PAR(N,2) & IINPUT & . 314 \\
\hline & IF ((IT.EQ.4).OR. (IT.EQ.5)) WRITE (IDAT,860) PAR(N,1),PAR(N,2) & IINPUT & . 315 \\
\hline & IF (IT. ME. B) GO To 380 & IINPUT & . 318 \\
\hline & $C=1 . / \operatorname{PAR}(N, 1)$ & IINPUT & . 317 \\
\hline & WRITE (IDAT, 870) C & IINPUT & . 318 \\
\hline \multirow[t]{7}{*}{380} & CONTINUE & IINPUT & 319 \\
\hline & IF (IT. EQ.7) WRITE (IDAT, BBG) PAR $(N, 1), \operatorname{PAR}(N, 2)$ & IINPUT & . 328 \\
\hline & IF (IT. EQ.8) TRITE (IDAT, 898) PAR(N,1), PAR(N, 2), PAR(N,3) & IINPUT & 321 \\
\hline & IF (IT.EQ.9) WRITE (IDAT,908) (JJNF(I,N), I=1, NLLEN) & IINPUT & . 322 \\
\hline & IF (IT.EQ. 18) WRITE (IDAT, 916) (JJNF $(I, N), I=1$, NLLEN) & IINPUT & . 323 \\
\hline & IF (IT.ER, NTYPE) VRITE (IDAT,926) PAR(N,1) & IINPUT & 324 \\
\hline & IF (ILIU(N).GT.ø) WRITE (IDAT, 936) VIU(N,1), $\operatorname{VIM}(N, 2)$ & IINPUT & 325 \\
\hline \multirow[t]{4}{*}{390} & CONTINUE & IINPUT & . 326 \\
\hline & URITE (IDAT, 940) & IINPUT & . 327 \\
\hline & DO $400 \mathrm{I}=1$, KCONST & IINPUT & . 328 \\
\hline & TRITE (IDAT, 950) CLABE (I), CDIMEN(I),CONST(I) & IINPUT & . 329 \\
\hline \multirow[t]{8}{*}{400} & CONTINUE & IINPUT & . 330 \\
\hline & IRITE (IDAT,960) NSN, NPRT & IINPUT & . 331 \\
\hline & IF (NLEVE.GT.1) THEN & IINPUT & . 332 \\
\hline & WRITE (IDAT,979) NLEYE & IINPUT & . 333 \\
\hline & ESE & IINPUT & . 334 \\
\hline & VRITE (IDAT,980) & IINPUT & . 335 \\
\hline & ENDIF & IINPUT & . 338 \\
\hline & ENDIF & IINPUT & . 337 \\
\hline \multicolumn{2}{|l|}{ c } & IINPUT & . 338 \\
\hline \multicolumn{2}{|r|}{ RETURN } & IINPUT & . 339 \\
\hline c & & IINPUT & . 346 \\
\hline \multirow{2}{*}{\multicolumn{2}{|c|}{ WRITE WARNS OF ERROR ON INPUT }} & IINPUT & . 341 \\
\hline c & & IINPUT & . 342 \\
\hline \multicolumn{2}{|c|}{410 CONTINUE } & IINPUT & . 343 \\
\hline \multicolumn{2}{|r|}{ WRITE (IOUT, 999) } & IINPUT & . 344 \\
\hline \multicolumn{2}{|r|}{ GO TO $(138,398,328,339,34 \theta)$, LPNT } & IINPUT & . 345 \\
\hline \multicolumn{2}{|l|}{ c } & IINPUT & . 346 \\
\hline \multicolumn{2}{|r|}{ 580 FORWAT (/1X, 'WOUL YOU LIKE TO UPDATE THE SYSTEN VARIABLES (Y/N)?' } & IINPUT & . 347 \\
\hline \multicolumn{2}{|c|}{$+, 1 X, \wedge 1)$} & IINPUT & . 348 \\
\hline \multicolumn{2}{|c|}{510 FORNAT (A1) } & IINPUT & . 349 \\
\hline \multicolumn{2}{|r|}{526 FORWAT (/1X, 'INPUT OF DISTRIBUTION TYPES AND PARAMETERS') } & IINPUT & . 350 \\
\hline \multicolumn{2}{|r|}{536 FORUAT $(/ 1 X, 14, ') ', A 12,{ }^{\prime}(', A 12, ')$ OISTRIQUTION') } & IINPUT & 351 \\
\hline 540 & FORUAT $\left.\left(8 X, I_{2},{ }^{\prime}\right) ',, A 28\right)$ & IINPUT & . 352 \\
\hline
\end{tabular}


550 FORUAT $\left(8 X, I 2,{ }^{1}\right)$ PREVIOLSLY DEFINED DISTRIBUTION. ')

IINPUT $\quad 353$

560 FOPUAT ( $1 X,{ }^{\prime}$ ETTER THE MMIBER FOR THE DESIRED DISTRIBUTION, ', $\left.A 1\right)$

576 FORUAT $\left(1 X, X^{\prime}\right.$ 'DISTRIQUTION TYPE IS ', A2g)

580 FORMAT ( $1 x^{\prime}$ ' $E$ ETTER THE MEAN OF THE DISTRIBUTION, ', $\left.A 1\right)$

590 FOPUAT (1X,' 'ECTER THE STANDARD DEVIATION. ', T38, A1)

896 FORMAT (/1X, '** ENTERED VNLUE OF ',1PE12.4,' IS OUT GF RANGE.')

610 FOPAMT (1X, 'ENTER THE MEDIAN VNLUE FOR THE DISTRIBUTIDN. ',A1)

626 FOPUAT (1X,' 'THE WEAN OF THE UNDERLYING NORUA DISTRIBUTION IS', IP

+E12.4/1X, 'ENTER THE STANDARD DEVIATION OF THIS NORLAL DISTRIBUTION $\rightarrow$ ' ', (1)

630 FORUT (1X, 'ENTER THE MINIMU AND UAXIMN EXPONENTS FOR THE OISTRI - BUTION. ')

649 FORUAT (1X, 'ENTER THE LOIER AND UPPER ENDPOINTS FOR THE UNIFORM DI +STRIBUTIOK. ')

658 FORUAT (IX, 'ENTER THE LONER AND UPPER VALUES FOR THE BERNOULLI DIS +TRIQUTION. ')

686 FORUAT (IX, 'ENTER THE PROBABILITY 9,1 OF THE LOWER VALUE OCCURIN + Q. ')

870 FORUAT (/1X, 'THE CONFIDENCE FUNCTION WILL BE READ FROU AN EXTERNAL

- FILE. '/1X, 'ENTER the NANE OF THE FILE CONTAINING tHE CUMLLATIVE'

$+/ 1 X$, 'CONFIDENCE FUNCTION DATA FOR THIS VARIABLE.')

680 FORMT ( $/ 1 X_{1}$ ' THE DATA POINTS IILL BE READ FROM AN EXTERMAL FILE.' $/$

$+1 X_{1}$ 'ENTER THE RANE OF THE FILE CONTAINING'/1X, 'THE DATA POINTS FOR

- THIS INPUT VARIARLE.')

699 FORMT (28A4)

760 FORMAT (1X, 'ENTER THE YNLUE FOR THE YARIAQLE. ',A1)

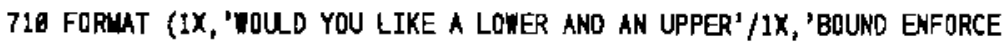
+D ON THIS YARIABLE $(Y / N)$ ?',, 1$)$

720 FORUAT ( $1 x_{1}$ 'ENTER THE YNUES FOR THE LOWER AND UPPER BDUNDS. ')

730 FORMA (/1X, 'VOU O YDU LIKE TO UPOATE THE SYSTEN CONSTANTS (Y/N)?' $+, 1 \times, A I)$

740 FORUAT (/1X,' 'ENTER THE YNLUE FOR ',A12,2X, '(',A12,')')

756 FORAAT (EX,' (CURRETT YNLUE IS ',1PE12.5, $\left.{ }^{\prime}\right)^{\prime}$ )

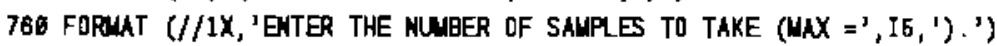

776 FORUAT (/1X, '** ERROR ** ENTERED YNUE EXCEEDS PRDGRAM LIMIT DF', + I5)

780 FORUAT (/1X, 'ENTER THE PRINT INTERVAL FOR DUTPUT DATA. ',A1)

790 FORMT ( $/ 1 X_{1}$ ' 'ENTER THE RWBER OF INTERYNS FOR STRATIFIED SNIPLING $+(M A X=', 15, ')$. ')

806 FORMAT (1X, I5,' INTERYALS IS TOD WANY, ONLY ',I5,' SAMPLES CHOSEN.

')

B10 FORMT (/1X,

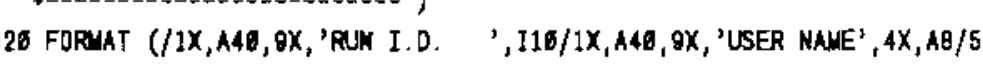

+EX, 'DATA SET MUBER ', I5///1X, '*--- ECHO OF INPUT OATA')

836 FORUAT $\left(/ 12, A 12,{ }^{\prime}\left({ }^{\prime}, A 12,{ }^{\prime}\right) ' / 15 X_{1}\right.$ 'OISTRIBOTION IS ', A26)

846 FORUAT (17X, 'MEAN VNLUE $='$, T35, 1PE12.4/17X, 'STND DEVIATION $='$, T3 $+5, E 12.4)$

850 FORMT (17X, 'CONSTANTS FDR THE UNDERLYING NORUAL OISTRIBUTION ARE'

$+/ 17 X$, 'WEAN YNLUE $='$, T36,1PE12.4/17X, 'STND DEVIATION =', T35, E12.4)

869 FORMT (17X, 'MIN, WAX EXPONENTS FOR THE UNDER YING'/17X, 'UNIFORN D

+ISTRIBUITON ARE' $/ 17 X_{1}{ }^{\prime}$ MIN = ',1PE12.4', UAX = ', E12.4)

878 FORAAT (17X, 'NEAN VNLUE IS', T35,1PEI2, 4)

B8B FORMAT (17X, 'LOWER ENDPOINT =', T35, 1PE12,4/17X, 'UPPER ENDPOINT =',

IINPUT . 354

IINPUT . 355

IINPUT . 356

IINPUT . 357

IINPUT - 358

IINPUT $\quad 359$

IINPUT , 368

IINPUT . 361

IINPUT , 362

IINPUT . 363

IINPUT . 364

IINPUT . 365

IINPUT - 386

IINPUT . 367

IINPUT - 388

IINPUT - 369

IINPUT . 370

IINPUT . 371

IINPUT - $\quad 372$

IINPUT - 373

IINPUT - 374

IINPUT , 375

IINPUT - 376

IINPUT . 377

IINPUT . 378

IINPUT . 379

IINPUT . 388

IINPUT - 381

IINPUT . 382

IINPUT . 383

IINPUT . 384

IINPUT . 385

IINPUT . 386

IINPUT - 387

IINPUT - 386

IINPUT $\quad 389$

IINPUT - 398

IINPUT . 391

IINPUT . 392

IINPUT . 393

IINPUT . 394

IINPUT - 395

IINPUT - 398

IINPUT . 397

IINPUT . 398

IINPUT . 399

IINPUT . 460

IINPUT $\quad 461$

IINPUT . 462

IINPUT . 403

IINPUT . 404

IINPUT . 405

IINPUT . 466 
$\rightarrow T 35, E 12.4)$

IINPUT $\quad .497$

896 FORMT (17X, 'LOWER YNUE $=$ ', T35, 1PE12.4/17X, 'UPPER YALUE $={ }^{\prime}$, T35, E1

$+2.4 / 17 X$, 'LOW YNLUE PRCB $=', T 35, E 12.4)$

909 FORMT (17X, 'THE CONFIDEICE FUNCTION WIL BE READ AS A PIECE WISE'

$\rightarrow / 17 X$, 'CONTINUOUS DATA FUACTION FROU THE EXTERNAL FILE: '/18X, 20A4)

910 FORMT (17X,'THE RANDOU DATA POINTS WILL BE READ'/17X, 'FRON THE EX

$\rightarrow$ TERNAL FILE: '/1BX, 20A4)

920 FORUT (17X, 'CONSTANT VALUE $=', T 35,1$ PE12.4)

938 FORMT (17X, 'LOTER BQUND $\approx ', T 35,1 P E 12.4 / 17 X$, 'UPPER BOUND $={ }^{\prime}, T 35$, E1

$+2.4)$

946 FORUAT (/)

950 FORUAT (1X, 'YALUE FOR ', A12, 2X, '(',A12, $\left.{ }^{1}\right)$ IS ', IPE12.4)

980 FORUAT ( $/ / 1 X$, 'NUMBER OF SAMPLES IS ',IB/1X, 'PRINT INTERVAL IS',4X,

$+\mathrm{IB})$

970 FORMT (1X, 'STRATIFIED SAMPLING CHOSEN, USING ', I5, ' LEVELS. ')

980 FORMT (1 $X_{1}$ 'STRATIFIED SAMPLING NOT CHOSEN.')

990 FORUAT (/1X, '***** ERROR ON INPUT, TRY AGIN *****'/)

END

EAT PACSTAT.LOAD

COUO PACSTAT.LOAD

DATE

SEG

LOAD

PPACSTAT

LOAD * $>B .>8$ PACSTAT

LOAD $>$ B. $>$ Q DATOUT

LOAD *>B.>Q DATSET

LOAD *>B.>B EXPECT

LOAD *>B.>B IINPUT

LOAD $*>$ B.>B PPLT

LOAD *>B.>Q RIOAT

LOAD *>B.>B_R2OAT

LOAD *>B.>B REORDR

LOAD *>B.>Q RNCS

LOAD *>B.>B STATOT

LOAD $*>B .>B$ VARSET

LOAD *)B. $>B$ WRSTOC

LOAD SYSTEUS>UTIL>RANDQW>B. >B_ALNORW

LOAD SYSTES>UTIL>RANDOU>B. >B BERNLI

LOAD SYSTES>UTIL)RANDEM)B.) B_DEXPF

LDAD SYSTESS

LDAD SYSTEMS $>U T I L>R A N D O M>B .>B$ DNORM

LOAO SYSTEAS)UTIL>RANDOY $>B .>B$ LNNORM

LOAD SYSTESS>UTIL>RANDOM>B.>B LNNORL

LOAD SYSTES $>U T I L>R A N D O W>B .>B$ LNUNIF

LOAD SYSTESSYUTIL>RANDOM>B. SB LOGNORN

LOAD SYSTEUS $>U T I L>R A N D O U>B .>B$ LOGNORLL

LOAD SYSTEUS>UTIL>RANDOU>B. >E_LDQUNIF

LOAD SYSTEAS>UTIL>RANDOU $>8 .>B$ NORWL

LOAD SYSTEUS>UTIL)RANDOU)B. >B NORULL

LOAD SYSTESSUTIL>RANDOU $>B$. \B STATS

LOAD SYSTES) UTIL) PANDOU>B. >B_UA1

LOAD SYSTESSUTIL) RANDOW)B. >B_UVIS

LOAD SYSTEUS>UTIL>RANDOW>B.>B_XUDIST

\begin{tabular}{|c|c|}
\hline NPUT & 408 \\
\hline NPUT & 48 \\
\hline NPUT & 410 \\
\hline NPUT & 41 \\
\hline VPUT & 418 \\
\hline YPUT & 413 \\
\hline पPUT & . 41 \\
\hline PUT & 41 \\
\hline NPUT & 416 \\
\hline NPUT & 417 \\
\hline NPUT & 418 \\
\hline NPUT & 41 \\
\hline NPUT & 428 \\
\hline NPUT & . 421 \\
\hline NPUT & $.42 z$ \\
\hline INPUT & 42 \\
\hline IINPUT & 42 \\
\hline JCLPRIM & \\
\hline JCLPRIM & \\
\hline JCLPRIM & - \\
\hline JCLPRIM & \\
\hline JCLPRIM & \\
\hline JCLPRIM & \\
\hline CLPRIM & \\
\hline JCLPRIM & \\
\hline JCLPRIM & 1 \\
\hline JCLPRIM & . \\
\hline JCLPRIM & 1 \\
\hline JCLPRIM & 13 \\
\hline JCLPRIM & 14 \\
\hline JCLPRIM & 15 \\
\hline JCLPRIM & 18 \\
\hline JCLPRIM & 17 \\
\hline JCLPRIM & 18 \\
\hline JCLPRIM & 19 \\
\hline JCLPRIM & 20 \\
\hline JCLPRIM & . \\
\hline JCLPRIM & 22 \\
\hline JCLPRIM & 23 \\
\hline JCLPRIM & 2 \\
\hline JCLPRIM & 25 \\
\hline JCLPRIM & 28 \\
\hline JCLPRIM & 27 \\
\hline JCLPRIM & $2 E$ \\
\hline JCLPRIM & 28 \\
\hline JCLPRIM & 30 \\
\hline JCLPRIM & 31 \\
\hline JCLPRIM & 32 \\
\hline JCLPRIM & 35 \\
\hline JCLPRIY & 34 \\
\hline JCLPRIM & 35 \\
\hline & 38 \\
\hline & 37 \\
\hline
\end{tabular}




\begin{tabular}{|c|c|c|c|}
\hline & SYSTEXS>UTIL>FELIB>B.>B_WHEADR & JCLPRIM & 38 \\
\hline & SYSTESS>UTIL>USRLIB>B.>B_ASG & JCLPRIM & 39 \\
\hline & SYSTEUS>UTIL>USRLIB>B.>B_IDNUW & JCLPRIM & 46 \\
\hline & SYSTES)(UTIL>USRLIB>B. >B_IDPLT & JCLPRIM & 41 \\
\hline & SYSTES>UTIL>USRLIB>B.>B_PLINIT & JCLPRIM & 42 \\
\hline & SYSTESS>UTIL>USRLIB>B.>B_QSORT & JCLPRIM & 43 \\
\hline & STSTESS)UTIL>USPLIB>B.>B_R日EEAS & JCLPRIM & 44 \\
\hline & SYSTEUS>UTIL>USRL_IB>B.>B_SORT & JCLPRIM & 45 \\
\hline & 4) B. >B_SOLN & JCLPRIM & 46 \\
\hline & AD * $>$ B.>B_BLKDAT & JCLPRIM & 47 \\
\hline & DISSPLA_9.0 & JCLPRIM & 48 \\
\hline & APPLB & JCLPRIL & 49 \\
\hline LI & & JCLPRIM & 50 \\
\hline & 77774 & JCLPRIM & 51 \\
\hline SA & & JCLPRIH & 52 \\
\hline & & JCLPRIM & 53 \\
\hline QU & & JCLPRIM & 54 \\
\hline & $-E$ & JCLPRIM & 55 \\
\hline & SUBRDUTINE LNNORU (N, RNDVNL,RP1, RP2) & LNNORM & 2 \\
\hline C & & LNNORH & 3 \\
\hline C & ROUTINE FOR TRANSFOFING A UNIFORALY DISTRIBUTED DATA SET ON THE & LNNORW & 4 \\
\hline c & INTERVAL 11 TO A NATURAL LOGNORWALY DISTRIQUTED DATA SET. & LNNOPM & 5 \\
\hline C & & LNNDRI & 6 \\
\hline C & $=$ THE NUMBER OF ITESS IN THE DATA SET & LNNORN & 7 \\
\hline c & RNDVAL = YECTOR OF UNIFOFY Y DISTRIBUTED YALUES ON INPUT. EACH & LNNOFW & 8 \\
\hline C & VALUE WST BE BETIEEN ZERO AND ONE. & LNNDR & . \\
\hline C & $=$ YECTOR OF LOGNORYALLY DISTRIBUTED YALUES ON DUTPUT. & LNNORM & 10 \\
\hline c & = MEAN YNLUE FDR THE UNDERYYING NORUAL DISTRIBUTION. & LNNORM & 11 \\
\hline C & $=$ STAKDARD DEYIATION FOR THE UNDERYYING NDRUAL DISTRIBUTION. & LNNORM & 12 \\
\hline C & & LNNORM & 13 \\
\hline & DIUENSIDN & LNNQRI & 14 \\
\hline & * $\quad$ RNDVAL $(N)$ & LNNORM & 15 \\
\hline C & & LNNORN & 16 \\
\hline C & NATURN LOGNORUAL DISTRIBUTION & LNNORM & 17 \\
\hline C & & LNNORM & 18 \\
\hline & Do $100 \mathrm{I}=1, \mathrm{~N}$ & LNNORN & 19 \\
\hline & RV=RNDVAL(I) & LNNDFN & 20 \\
\hline & RV=DNORU (RV, RP1, RP2) & LNNDRM & 21 \\
\hline & RNDYAL(I) $=\operatorname{EXP}(R V)$ & LNNORM & 22 \\
\hline & CONTINUE & LNNORM & 23 \\
\hline & RETURN & LNNORM & 24 \\
\hline & END & LNNORN & 25 \\
\hline & SUBROUTINE LNNOAL (N, RNDVAL, RP1, RP2, AMIN, AWAX) & LNNOA4 & 2 \\
\hline C & & LNNQRML & 3 \\
\hline C & ROUTINE FOR TRANSFORUING $A$ UNIFOFAY DISTRIBUTED DATA SET ON THE & LNNOFY & 4 \\
\hline C & INTERVAL 0,1 TO A NATURAL LDGNDRWALY DISTRIEUTED DATA SET, WITH & LNNARAL & 5 \\
\hline C & THE CONSTRAINT THAT ALL OUTPUT VALUES LIE BETHEEN SPECIFIED LIUITS. & LNNDFAL & 6 \\
\hline C & & LNNDRM & 7 \\
\hline C & $=$ THE NUMBER OF DATA VALUES IN VECTOR RNDYAL. & LNNORLL & 8 \\
\hline C & RNDVAL = ON INPUT, THE VECTOR OF UNIFORILY OISTRIBUTED VALUES. & LNNORIL & 9 \\
\hline C & = ON DUTPUT, THE VECTOR OF LOGNORUALLY DISTRIBUTED VALUES. & LNNORIL & 10 \\
\hline$c$ & $=$ WEAN YALUE FOR THE DESIRED UNDERLYING NORUAL DISTBUTION. & LNNORIL & 11 \\
\hline C & $=$ STAKD. DEY FOR THE DESIRED UNDERLYING NORMAL DISTRIBUTION. & LNNORIL & 12 \\
\hline C & AMIN = MININA LIUIT VALUE FOR THE LOGNORMALLY DISTRIBUTEO DATA. & LNNORML & 13 \\
\hline
\end{tabular}




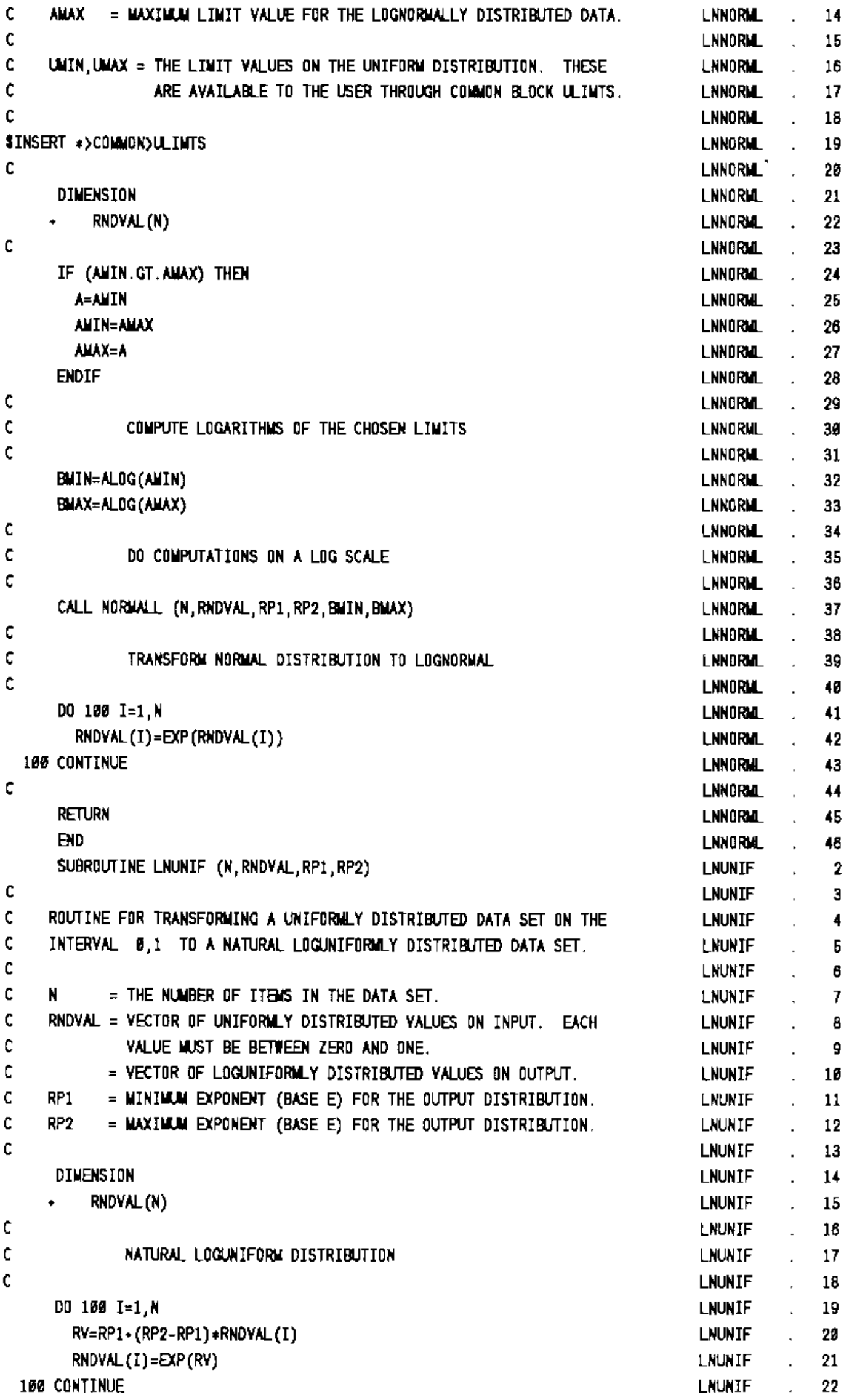




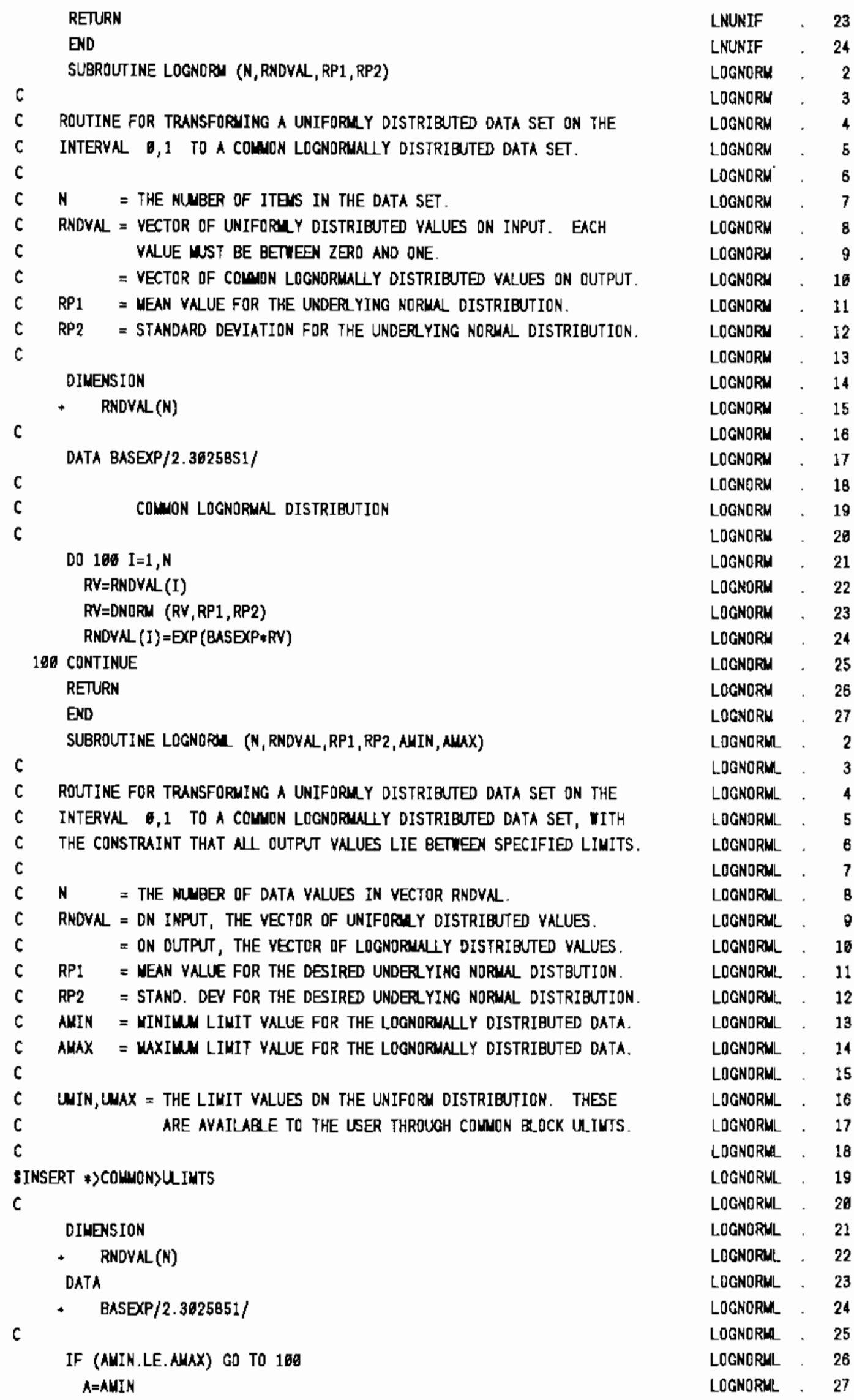




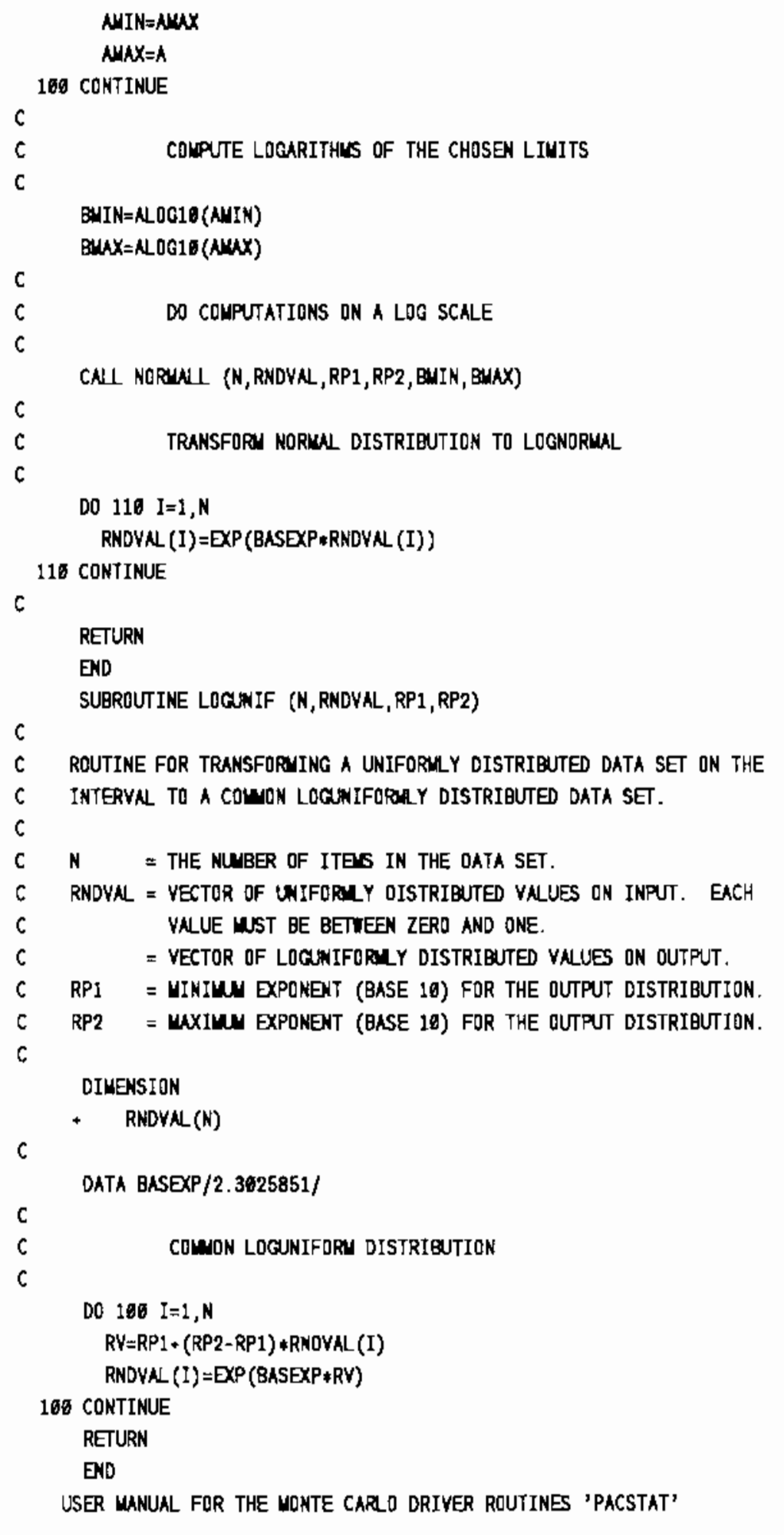

THIS IS A BRIE DOCUNENT DESCRIBING THE MONTE CARLA DRIVER ROUTINES WHICH ARE REERED TO AS PACSTAT. THESE ROUTINES ARE DESIGNED TO NLLOW A PROGRAMER TO SET UP A MONTE CARLO SILALATION OF A PHYSICAL PROCESS IN A NINIMU AUOUNT DF TIME.

\begin{tabular}{|c|c|}
\hline IORLL & \\
\hline OGNORLL & \\
\hline IGNORWL & \\
\hline JGNORLL & \\
\hline JGNORLL & \\
\hline JGNORLL & \\
\hline JGNORLE" & \\
\hline JGNORML & \\
\hline JGNORH & \\
\hline JGNORWL & \\
\hline GNORWL & \\
\hline JGNORLL & 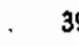 \\
\hline DGNORLL & 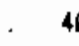 \\
\hline JGNORML & \\
\hline JGNORLL & \\
\hline IGHORN & - \\
\hline JGNORHL & 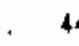 \\
\hline OGNORLL & \\
\hline DGNORLL & \\
\hline GNORL & \\
\hline OGNORLL & . \\
\hline GUNIF & \\
\hline OGUNIF & \\
\hline JCUNIF & 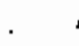 \\
\hline DQUNIF & 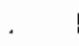 \\
\hline 00 & 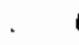 \\
\hline LOCUNIF & . \\
\hline LOCUNIF & . \\
\hline LOCUNIF & . \\
\hline Bat & . \\
\hline OCUNIF & . \\
\hline LOCUNIF & . \\
\hline JCUNIF & . \\
\hline لالمD & . \\
\hline OCUNIF & . \\
\hline OCUNIF & . \\
\hline DCUNIF & . \\
\hline 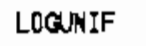 & - \\
\hline DGUNIF & . \\
\hline OGUNIF & . \\
\hline DGUNIF & . \\
\hline LDGUNIF & . \\
\hline LOGUNIF & . \\
\hline LOGUNIF & . \\
\hline LDGUNIF & . \\
\hline LOGUNIF & . \\
\hline WANUAL & 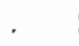 \\
\hline WANUAL & \\
\hline WANUAL & \\
\hline WANUAL & \\
\hline WANUAL & \\
\hline WANUAL & \\
\hline$A N \cup A$ & \\
\hline NUA & \\
\hline
\end{tabular}


THE USER MST DEINE A SET OF INPUT AND OUTPUT VARIABLES, AND SUPPLY A ROUTINE (OR SET OF ROUTINES) IHICH DEFINE THE PKYSICAL PROCESS GEING UDDELLED. THE MONTE CARLO DRIVER ROUTINES PROMPT THE USER FOR A STOCASTIC OEFINITION DF EACH INPUT VARIABLE, AND THEN RUN THE PHYSICAL MODEL A NUMER OF TIUES.

FDR EACH RUN, THE INPUT YARIABLES WILL BE SAMPLED FROM THEIR DEFINED DISTRIBUTIONS. THE DUTPUT YARIABLES FOR EACH SAMPLE ARE STORED, AND $\lambda$ CRUDE STATISTICAL ANALYSIS IS PERFORUED ON THESE OUTPUT DISTRIBUTIONS. TABLES OF CONFIDENCE VALUES VERSLS DATA VALUES ARE ALSO SET UP FOR CHOSEN DUTPUT VARIABLES. THIS DATA WAY BE PLOTTED, AND/OR WRITTEN TO AN OUTPUT BINARY FILE FOR LATER PROCESSING.

\section{PROGRANING INFDRUATION}

PACSTAT IS WRITTEN USING THE FORTRAN 77 STANDARD. AN EFFORT WAS MADE TO GENERATE COOINO THICH WOUD BE AS EASY TO CONVERT TO OTHER MACHINES AS POSSIBLE. THE ORIGINAL CODE IAS DEVEOPED ON A PRINE 750

THREE ROUTINES TIL REQUIRE SONE MACHINE/INSTALLATION DEPENDENT CODING. THESE ARE (1) ASG, WHICH OPENS DATA FILES DURING PROGRAM EXECUTION, (2) REEEAS, WHICH CLOSES DATA FILES DURING PROGRAN EXECUTION, AND (3) IONU, WHICH BUILOS A UNIQUE, TEN DIGIT RUN IDENTIFIER FOR IDENTIFYING EACH RUN OF THE PROGRAL. THIS RUN IDENTIFIER IS IMPORTANT, AS IT IS TRITTEN TO THE FILE HEADER DF ALL PACSTAT OUTPUT FILES, AND CAN BE USED FOR IDENTIFYING DATA FILES.

THE PROGRAN IAS DESIGNED AROUND THE DISSPLA GRAPHICS SOFTTARE PACKAGE, FROM INTEGRATED SOFTIARE SYSTEMS CORPORATION, SAN OIEGO, CALIFURNIA, 92121. IF THE GRAPHICS CALLS CANNOT BE SATISFIED BY THE SYSTEM, THE DISSPLA SUBROUTINE DONEPL, AND THE PACSTAT ROUTINES PPLT AND PLINIT, MST BE CONENTED OUT OR REPLACED BY DUNYY ROUTINES. ALL OF THE CALLS TO DISSPLA, AND TO PACSTAT ROUTINE IDPLT, ILL THEN BE RENOVED FROM THE PROGRAM. THE PLOTTING CAPABILITY ILL ALSO BE GQNE.

PACSTAT IS INTENDED TO RUN INTERACTIVEYY, ITH THE USER DEFINING THE RERUIRED VARIABLE DISTRIBUTIONS DURING THE PROGRAH EXECUTION. THE INPUT AND OUTPUT LOGICAL UNIT NUMBER FOR THIS USER INTERACTION ARE STORED IN VARIABLES 'IN' AND 'IOUT,' THESE ARE SET AT THE TOP OF THE MAIN ROUTINE. ALSO SET HERE ARE THE LOGICA UNIT NLABERS FOR THE TWO OUTPUT FILES, PRINTED AND BINARY (IDAT AND IDATB), AND A UNIT NLMBER FOR ANY INPUT FILES WHICH WAY BE NEEDED (INFIL).

\section{INPUT/OUTPUT VARIAQLE DEINIIION}

THE INPUT AND QUTPUT VARIABLES LUST BE DEINED BY THE USER AS THE FIRST STEP IN SETTING UP PACSTAT TD RUN. THIS IS DONE GY DATA STATENENTS IN A BLOCK DATA ROUTINE. THIS BLDCK DATA IAS ORIOINALLY NALED BKDAT, BUT AS IT IS NEVER 'CALLED', THE NAME IS NOT IMPORTANT.

A SAMPLE COPY OF THE BLOCK DATA ROUTINE IS ENCLDSED WITH THIS DOCUMENT. THE YARIABLES WHICH MST BE DEFINED BY THIS ROUTINE ARE LISTED BEDU. THESE VARIABLES ARE CONTAINED IN COMMON BLOCKS IDCOM, PBLK1, PQKK2, AND PBLK3.

$$
\begin{array}{ll}
\text { PRG } & =\text { A CHARACTER*B PROGRAW NAME IDENTIFIER. } \\
\text { VERNUN } & =\text { THE PROGRAN VERSION NUMBER (FLOATINO POINT) }
\end{array}
$$

THESE ARE BOTH PRINTED TO THE PACSTAT OUTPUT FILES. THEY ARE 
USEU THEX SEVERAL DIFFERETT MODES ARE BEING EUHINED YITH THE WONTE CARLO TECHNIQUE (EACH MODE REQUIRING A OIFFERENT PROCRAY NANE), AND VHEN A SINGE WOEL IS BEIMG REINED (REQUIRING NE VESSION NUAEES). THESE LABES ARE IMPORTANT TOOLS IN TRACING A DATK SET TO ITS SOURCE PROGRAU.

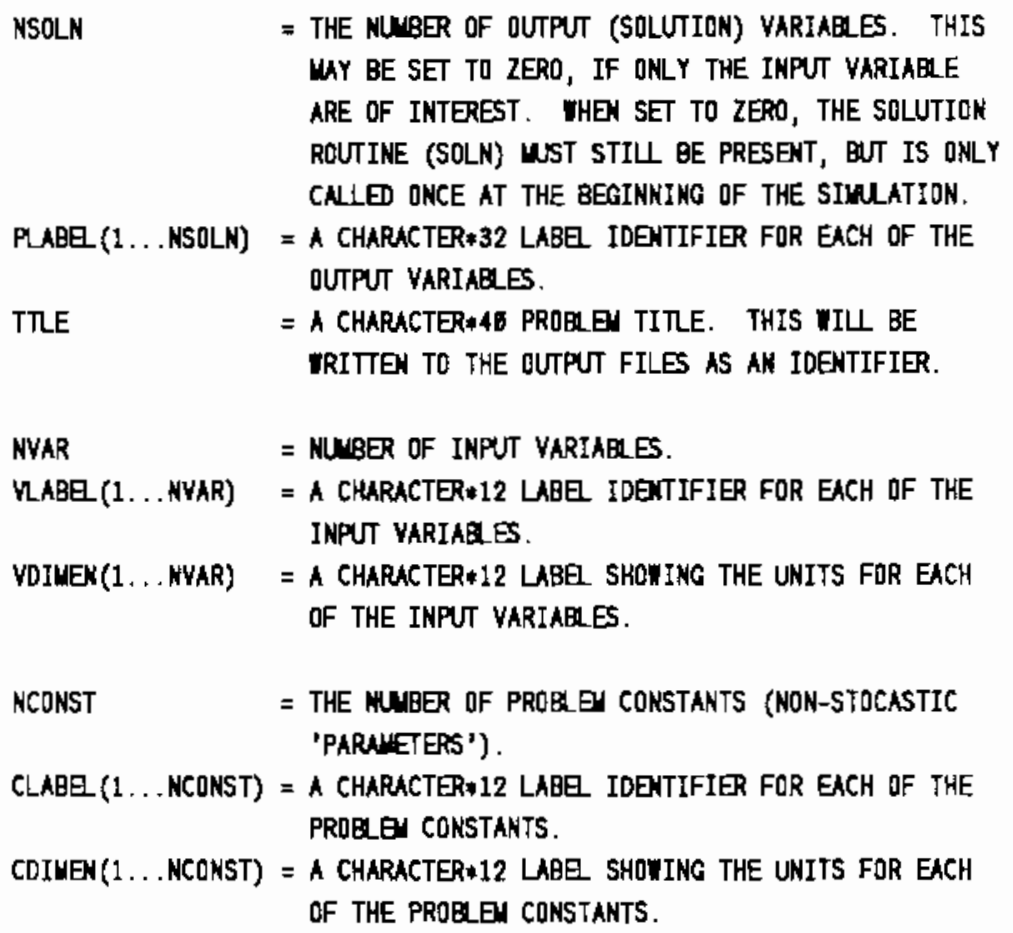

THE CHARACTER VARIARES ARE NOT IMPORTANT TO MUMERC SUCCESS OF THE PROGRN. HONEVER, THEY ARE USED IN THE INPUT AND OUTPUT PROMPTS, AKD IN THE PRINTED OUTPUT. THIS YAKES IT EASIER FDR THE USER TO INSURE CORRECT DATA ON INPUT. IT NLSO INSURES BETTER QUALITY CONTROL ON THE DATA, AS $\Perp$ OF THE DATA YALUES OK THE PRINTOUT ARE LABELED VITH BOTH A NAME ANO OIMENSIONS.

THE PROBLE CONSTANTS ARE DESIGKED TO NLON PARNETERS WHICH ARE

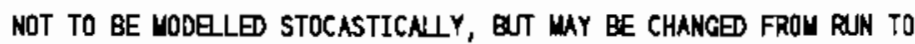
RUN. THIS LETS THE LSER CHANGE THESE PARANETERS DURING THE EXECUTION, RATHER THAN BY RE-COMPILING THE SOURCE CODE. ALSO, THE CONSTANTS ARE WRITTES TO THE DUTPUT PRINT FILE, ANO BECOME A PART OF THE WRITTEN DQCLAENTATION FOR THE RUN.

\section{PROQEI SOLUTION ROUTINES}

THE DRIVER ROUTINES IL LODP ON THE NUABER OF SNMPLES TO BE RUN. FOR EACH SAMPLE, EACH OF THE INPUT VARIABLES IS COMPUTED FROM A USER

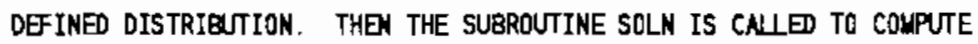
THE OUTPUT VARIABES ('SOLUTIONS') FRON THE INPUT VARIABES.

THE ROUTINE SOLN UST BE CODED FOR THE PROREN AT HAND. IT IS USED TO INTERFACE THE MONTE CARLO DRIVER ROUTINES TO THE MODELING CODE. THE INPUT DATA IS TAKEX FROY ARRAY YINPT, AND PLACED INTO THE INPUT VARIARES FOR THE NUWERIC MODE BEING SIUUATED. THE NODE IS EXECUTED, AND THE OUTPUT DATA IS PLACED INTO ARRAY VALU, TO BE RETURNED TO THE MONTE CARLD DRIVER ROUTINES FOR STORAGE AND SUBSEQUENT ANALYSIS.

\begin{tabular}{|c|c|}
\hline WAKUNL & \\
\hline WANUAL & \\
\hline WANUAL & 86 \\
\hline WANUAL & 67 \\
\hline WANUAL & 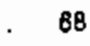 \\
\hline MANUAL & 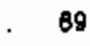 \\
\hline WANUAL & \\
\hline MANUAL & 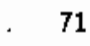 \\
\hline WANUAL & 32 \\
\hline WANUNL & 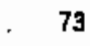 \\
\hline MANUAL & \\
\hline WANUAL & 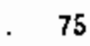 \\
\hline WANUAL & 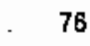 \\
\hline MANUAL & . \\
\hline WANUAL & 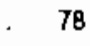 \\
\hline MANUAL & . \\
\hline WANUAL & \\
\hline WANUAL & . \\
\hline WAHUAL & . \\
\hline MANUAL & 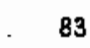 \\
\hline WANUAL & . \\
\hline MANUAL & 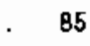 \\
\hline MANUAL & 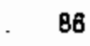 \\
\hline MANUAL & 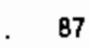 \\
\hline MANUAL & 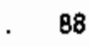 \\
\hline MANUAL & 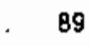 \\
\hline MANUAL & 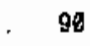 \\
\hline WANUAL & . \\
\hline MANUAL & . \\
\hline MANUNL & . \\
\hline MANUN & . \\
\hline WANUAL & 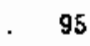 \\
\hline MANUAL & 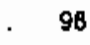 \\
\hline MANUAL & 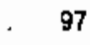 \\
\hline WAMUAL & . \\
\hline WANUAL & . \\
\hline MANUAL & . 100 \\
\hline WANUAL & . 101 \\
\hline WANUKL & . 162 \\
\hline YANUAL & . $\quad 103$ \\
\hline MANUAL & , 104 \\
\hline WANUAL & . 105 \\
\hline WANUAL & . 106 \\
\hline WANUAL & . $\quad 167$ \\
\hline WANUAL & . 168 \\
\hline HANUML & . 108 \\
\hline MANUAL & . 110 \\
\hline WANUAL & . 111 \\
\hline WANUAL & 112 \\
\hline MANUAL & . 113 \\
\hline MANUAL & . 114 \\
\hline WANUAL & 11 \\
\hline MANUAL & . 116 \\
\hline WANUAL & . 117 \\
\hline
\end{tabular}


IT IS IUPORTANT TO CDRRECTLY IDENTIFY THE INPUT AND DUTPUT VARIABLES (VINPT(1), VINPT(2), ETC...) UITH THE PHYSICAL QUANTITIES FOR WHICH EACH VARIABLE STANDS.

THE ROUTINE SOLN WUST CONCERN ITSEF ITH THE VARIABQES LISTED BE DW. THESE VARIABLES ARE CONTAINED IN COMON BLDCKS PBLK4, PB.K5 AND INOUT.

\begin{tabular}{|c|c|}
\hline IDAT & $\begin{aligned}= & \text { FILE UNIT NUNBER FOR OUTPUT PRINT FILE. CUTPUT } \\
& \text { FILE HAS NOT BEEN OPENED IF IDAT IS ZERO. }\end{aligned}$ \\
\hline IQUT & $\begin{aligned}= & \text { FILE UNIT NLMBER FOR THE USERS TERMINAL (FOR } \\
& \text { PRINTING ANY ERROR/DIAGNOSTIC MESSAGES). }\end{aligned}$ \\
\hline ICNT & $\begin{aligned} \text { = DATA SET NUMBER FOR THIS RUN. NFTER A PROCESS HAS } \\
\text { BEEN SIMUAED, THE ORIVER CODE NLLONS THE USER } \\
\text { THE OPTION OF RE-RUNING THE SIMUATION, WITHDUT } \\
\text { TERUINATING THE PROGRAM EXECUTION. BOTH RUNS ARE } \\
\text { IDENTIFIED BY THE SAME RUN ID. ONLY THE DATA } \\
\text { DATA SET NUMBER UNIQUEY INDENTIFIES THE DATA. }\end{aligned}$ \\
\hline INDEX & $\begin{aligned}= & \text { THE SAMPLE NLMEER WHICH IS CURRENTLY BEING } \\
& \text { SIMUATED. NDTE THAT, IF THE SOLUTION ROUTINES } \\
& \text { NEED INITIALIZATION ONLY ONCE FOR NLL THE RUNS } \\
& \text { BEING WAOE, THIS MUST BE DONE ONLY WHEN INDEX }=1 .\end{aligned}$ \\
\hline \multicolumn{2}{|c|}{ CONST $(1 \ldots$ KCONST $)=$ THE CONSTAKTS WHICH HAVE BEEN DEFINED. } \\
\hline VNLU $(1 \ldots$ NSOLN $)$ & $\begin{aligned}= & \text { THE DUTPUT RESUTS FOR THE PROCESS MDDE. THE } \\
& \text { ROUTINE SOLN WUST SET EACH OUTPUT VARIABLE OF THE } \\
& \text { UODE INTO THE APPROPIATE ITEU IN THIS ARRAY. }\end{aligned}$ \\
\hline VINPT (1 . . NVAR) & $\begin{aligned}= & \text { THESE ARE THE INPUT RANODH VARIABLES FOR THE } \\
& \text { CURRENT SANPLE. THE INPUT VARIABLS TO THE } \\
& \text { PROCESS BEING WODELLED LUST BE SET TO THE } \\
& \text { CORRESPONOING VALUES IN ARRAY VINPT. }\end{aligned}$ \\
\hline
\end{tabular}

EXTERNAL INPUT VARIABLE DEINITION

PACSTAT ALLOWS TWO OPTIONS FOR DEFINING RANDOM INPUT VARIABLES EXTERNAШY TO THE PROGRA. THESE WAY BE USEFU IF A DESIRED DISTRIBUTION IS NOT PART OF THE SOFTUARE, OR IF 'CORRELATED' DATA SETS ARE REQUIRED.

THE FIRST OPTION ALLOTS THE USER TO DEFINE A CLMUUATIVE CONFIDENCE FUNCTION TO BE USED IN COMPUTING INPUT VARIABES. THIS FUNCTION UUST GE APPRDXIUATED AS A PIECE WISE LINEAR FUNCTION, DEINED BY DATA POINTS IN AN EXTERNA DATA FILE. EACH LINE OF THE FILE CONTAINS ONE DRTA FOINT (TWO NLMBERS) OF THE FUNCIION. THE FIRST NUMBER ON EACH LINE MUST BE THE DESIRED VARIABLE VNLUE, AND THE SECOND NLABER LUST BE THE CONFIDENCE LEVE (ZERO TO DNE) CORRESPONDING TO THAT VARIABLE VALUE. THESE WUST BE IN ASCENDING ORDER IN THE INPUT FILE.

WHEN THIS OPTION IS USED, PACSTAT FIRST COMPUTES A SET OF RANDOM NUMBERS ('CONFIDENCE LEVES') ON THE INTERVAL 6,1. THESE ARE USED IN CONJUNCTION ITH THE DEFINED CUMUATIVE CONFIDENCE FUNCTION TO COMPUTE YARIABLE YNLUES CORRESPONDING TO EACH CONFIDENCE LEVB. THESE NUMBERS WILL BE COLPUTED IN A RANDON OROER. CONFIDENCE LEYES LESS THAN ONE OR GREATER THAN ZERO ARE IGNORED. IF THE ENTIRE CONFIDENCE INTERVAL 0,1 IS NDT COYERED BY THE FUNCTION, RANCOLYY GENERATED CONFIDENCE WLUES OUTSIDE OF THE INTERVAL IILL RETURN ITH THE APPROPRIATE END POINT

\begin{tabular}{|c|c|}
\hline WANUAL & 110 \\
\hline WANUAL & 119 \\
\hline MANLAL & 128 \\
\hline WANUAL & 12 \\
\hline WANUAL & $12 r$ \\
\hline WANUN & 123 \\
\hline MNUN & 12 \\
\hline WANUNL & $12:$ \\
\hline WANUAL & . 12 \\
\hline WANUAL & . 12 \\
\hline WANUAL & . 12 \\
\hline WANUNL & . 12 \\
\hline WANUNL & . 13 \\
\hline MANUAL & . 13 \\
\hline WANUAL & . 13 \\
\hline WANUAL & . 133 \\
\hline WANUA & . 134 \\
\hline WANUNL & . 13 \\
\hline MANUAL & . 13 \\
\hline MANUAL & . 137 \\
\hline WANURL & . 13 \\
\hline MANUNL & 139 \\
\hline WANUAL & 14 \\
\hline MANUAL & . 14 \\
\hline MANUKL & 14 \\
\hline WANUNL & . 14 \\
\hline MANUAL & . 14 \\
\hline MANUAL & . 14 \\
\hline MANUAL & . 148 \\
\hline WANUAL & . 147 \\
\hline MANUNL &.$\quad 14 \varepsilon$ \\
\hline MANUNL & . 14 \\
\hline MANUNL & . 15 \\
\hline MANUNL & . 15 \\
\hline MANUNL & . 15 \\
\hline MANUAL & 15 \\
\hline MANUNL & . 15 \\
\hline WANUAL & . 15 \\
\hline WANUAL & . 15 \\
\hline MANUAL & . 15 \\
\hline WANUAL & . 15 \\
\hline MANUAL & 15 \\
\hline MANUNL & . 16 \\
\hline WANUA & . 16 \\
\hline MANUNL & . 16 \\
\hline MANUNL & 16 \\
\hline MANUAL & . 16 \\
\hline MANUAL & . 18 \\
\hline WANUAL & .26 \\
\hline MANUAL & .16 \\
\hline WANUAL & 16 \\
\hline WANUAL & . 16 \\
\hline MANUAL & 1 \\
\hline WANUAL & $x$ \\
\hline
\end{tabular}


YALUE.

THE SECOND OPIION NUOTS THE USER TO ENTER RANDON DATA POINTS DIRECTLY. THESE DATA POINTS MST BE IRITTEN IN AN EXTERNAL FILE, DNE POINT PER LINE. PACSTAT NIL READ THESE DATA YALUES, AND USE THEN DIRECTLY, IN ORDER, IN THE MONTE CARLO RUNS TO BE MADE. IF THERE ARE FETER DATA POINTS IN THIS FILE THAN SNPLES DESIRED, THE DATA WHICH IS PRESENT IS REPEATED, IN ORDER, A SLFFICIENT MUHER OF TIUES TO CREATE ENOUGH DATA.

BOTH OPTIONS RERUIRE THE USER TO ENTER THE FILE NAME THERE THE DATA IS STORED. THESE NAMES ARE STORED URTIL THE DATA IS NEEDED. EACH FILE IS OPENED, READ, AND CLOSED INDEPENDENTLY. THIS NLOWS THE USER TO USE THE SAUE FILE FOR SEVERAL DIFFERENT INPUT VARIABLES. $\amalg$ OF THE EXTERNAL FILES, USED FQR INPUT VARIABLE DEINITION, ARE READ FREE FORM.

PROGRAU EXECUTION

THIS IS A BRIE LIST DF THE PROGRAN PRDMPTS AND APPROPRIATE USER RESPONSES.

SEG PACSTAT

THIS COWHA EXECUTES THE CODE ' PPACSTAT' ON THE PRIMOS OPERATING SYSTENS

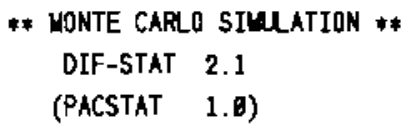

SIMPLE DIFFUSION RELEASE NODE

THESE PRINTOUTS IDENTIFY THE SOURCE CODE NALE, YERSION NUMEER, AND THE TITLE OF THE PRORE BEING SIMUATED. THE CODE NAME AND YERSION NUMBER (VARIABLES PRG AND VERNU, SET IN BLOCK DATA) ARE ECHOED HERE, ALONG ITH THE DRIYER CODE NAYE (PACSTAT) AND YERSIDN NUABER.

ENTER DUTPUT DATA FILE NANE.

(ENTER 'NONE' TO OUIT)

ENTER THE NAME OF THE OUTPUT FILE FOR THE PRINTED OUTPUT. THIS FILE WILL CONTAIN ALL OF THE INPUT DATA ECHO AND OUTPUT DIAGNOSTICS. BY ENTERING 'NONE', THIS DUTPUT WIL BE DIVERTED TO THE USER'S TERMINAL, RATHER THAN TO AN OUTPUT FILE.

ENTER BINARY DATA OUTPUT FILE NALE

(ENTER 'NONE' TO OMIT)

ENTER THE NANE DF THE OUTPUT GINARY FILE. THIS FILE WIL CONTAIN BOTH THE INPUT AND OUTPUT YARIABLE YALUES FOR EACH SAMPLE TAKEN, AS wEL AS THE PRDBLE TITLES AND LABES, AND THE VALUES OF THE PROBLE CONSTANTS. IT CAN BE READ LATER FOR POST PROCESSING THE DATA (E.G. PLOTTING), GY ENTERING 'NONE', THIS FILE WILL NOT BE CREATED.

THIS FILE IS IRITTEN PRIOR TO THE SORTING OF THE DATA. (THE

\begin{tabular}{|c|c|}
\hline UANUAL & . 172 \\
\hline WANUAL & 173 \\
\hline WANUAL & 174 \\
\hline WANUAL & 175 \\
\hline WAKUALE. & 176 \\
\hline WANUAL & . $\quad 177$ \\
\hline WANUAL & 178 \\
\hline WANUAL & 179 \\
\hline WANUAL & . 180 \\
\hline WANUAL & . 181 \\
\hline WANUAL & . 182 \\
\hline WANUAL & . 183 \\
\hline WANUAL & . 184 \\
\hline MANUAL & . 185 \\
\hline MANUAL & . 186 \\
\hline WANUAL & . 187 \\
\hline MANUAL & . 188 \\
\hline MANUAL & . 189 \\
\hline MANUAL & . 196 \\
\hline MANUAL & . 191 \\
\hline MANUAL & . 192 \\
\hline MANUAL & . 193 \\
\hline MANUAL & . 194 \\
\hline MANUAL & . 195 \\
\hline MANLAL & . 196 \\
\hline MANUAL & . 197 \\
\hline WANUAL & . 198 \\
\hline MANUAL & . 199 \\
\hline MANUAL & . 200 \\
\hline MANUAL & . 201 \\
\hline MANUAL. & . 292 \\
\hline MANUAL & . 203 \\
\hline NANUAL & . 204 \\
\hline MANUAL & . 205 \\
\hline MANUAL & . 206 \\
\hline MANUAL & . 207 \\
\hline MANUAL & . 268 \\
\hline MANUAL & . 289 \\
\hline WANUAL & . 210 \\
\hline MANUAL & . 211 \\
\hline MANUAL & . 212 \\
\hline WANUAL & . 213 \\
\hline WANUAL & 214 \\
\hline WANUAL & . 215 \\
\hline WANUAL & . 216 \\
\hline WANUAL & , 217 \\
\hline WANUAL & 218 \\
\hline WANUAL & 219 \\
\hline MANUAL & 228 \\
\hline WANUAL & 221 \\
\hline WANUAL & 222 \\
\hline WANUAL & 223 \\
\hline WANUAL & . 224 \\
\hline VANU & 225 \\
\hline
\end{tabular}


SORTING IS A NESESSARY PART OF A VONTE CARLO SILULATION.) THIS N $\amalg$ ONS THE USER TO DIRECTLY CORREATE THE INPUT AND OUTPUT VALUES FOR EACH SAMPLE. ANY SAMPLES YHICH FAIL DURRING THE RUM IILL BE NOYED TO THE END OF THE OUTPUT ARRAYS, HHICH EASES THE HANDLING AND EXNINIATION OF BOTH THE SUCCESSFU AND UNSUCCESSFUL SAMPLE DRTA VALUES.

ENTER LABE FDR THIS RUN (WXX 40 CHARACTERS)

IHIS IS A CHARACTER LABE FOR THE CURRENT RUN. IT HEPS TO IOENTIFY THE INPUT DATA SET. TOGETHER, THE PROBLE TITLE AND THIS LABE REPRESENT THE DATA SET TITLE. THE PROBLEN TITLE IS USED AS THE PLOT TITLE, WHILE THIS LABE IS USED AS A SUB-TITLE ON THE PLOT.

WOUD YOU LIKE TO CONTINUE OF QUIT IF AN ERROR IS ENCQUNTERED IN THE SOLUTION ROUTINE (C/Q)?

ON OCCASSION, THE HODE BEING ANALYSED MAY FAIL FOR A RANGE OF INPUT VARIARE VMLUES, AND IT WAY NOT BE EYIOENT BEOREHAND WHAT VALUES OF THOSE VARIABRES ILL CAUSE MODE FAILURE. HOWEVER, IT IS A SIMPLE WATTER TO HAVE THE SOLUTION ROUTINE RETURN IITH AN ERROR POINTER SET WHEN THIS OCCURS. THIS IS POINTER IERR IN THE CNLL LIST OF ROUTINE SOLN.

FOR SOME PROBQES, IT IS DESIREARE TO CONTINUE WDDE $\perp$ ING WHEN AN ERROR OCCURS IN THE SOLUTION ROUTINE. ENTER A ' $C$ ' HERE TO ACTIYATE THIS OPTION. AT THE END OF THE RUN, THE SAMPLES IN ERROR WIL BE SDRTED TO THE ENO OF THE DATA SETS, AND IGNDRED IN ALL SUESEqUENT ANALYSIS (PRINTING AND PLOTIING). NOTE THAT THE ENTIRE INPUT AND OUTPUT DATA ARRAYS ARE STIL PRINTED TO THE BINARY OUTPUT FILE, SO THAT ANALYSIS DF THE DATA IN ERROR WAY BE WADE.

GNTER A 'Q' HERE IF YOU WOUD PREER HAVING THE STOCASTIC WOOELING STOPPED ON AN ERROR FROH THE SOLUTION ROUTINE.

INPUT OF DISTRIBUIION TYPES AND PARAMETERS

1) WOLEC DIFF (U*2/YR ) DITRIBUTION

1) NORAAL

2) NATURAL LDGNORNAL

3) CONON LOGNORALL

4) NATURAL LOQUNIFCRM

5) CONON LOGUNIFORN

6) EXPONENTIAL

7) UNIFBRI

8) BERNOULI

8) EXTERNAL CONF FUNCTN

16) EXTERNAL DATA POINTS

11) CONSTANT

ENTER THE NUMEER FOR THE DESIRED DISTRIEUTION.

THIS PROUPT ASKS THE USER TO DEINE THE OISTRIBUTION TYPE FOR THE INPUT YARIABLE NAMED 'MOLEC DIFF,' ENTER THE NUMBER CORRESPONDING TD THE DESIRED DISTRIBAIION. THE FOLLOWING

\begin{tabular}{|c|c|}
\hline WANUAL & . 226 \\
\hline WANUAL & . 227 \\
\hline WANUAL & . 228 \\
\hline WANUAL & . 229 \\
\hline WANUNL & . 230 \\
\hline WANUNL & . 231 \\
\hline WNUNL & . 232 \\
\hline WNUNL & . 233 \\
\hline WANUNL & . 234 \\
\hline WANUAL & . 235 \\
\hline MANUAL & . 236 \\
\hline MANUNL & . $\quad 237$ \\
\hline WANUAL & . 238 \\
\hline MANUAL & . 239 \\
\hline WANUAL & . 246 \\
\hline MANUAL & . 241 \\
\hline MANUAL & . 242 \\
\hline MANUNL & . 243 \\
\hline MANUAL & . 244 \\
\hline MANUAL & . 245 \\
\hline MANUAL & . 246 \\
\hline MANUAL & . 247 \\
\hline MANUAL & . 246 \\
\hline WANUAL & . 249 \\
\hline WANUAL & . 256 \\
\hline WANUAL & . 251 \\
\hline MANUAL & . 252 \\
\hline WANUAL & . 253 \\
\hline WANUAL & . 254 \\
\hline MANUN & . 255 \\
\hline MANUAL & . 258 \\
\hline MANUAL & . 257 \\
\hline WANUNL & . 258 \\
\hline WANUAL & . 259 \\
\hline MANURL & . 250 \\
\hline HANUAL & . 261 \\
\hline WANUAL & . $\quad 262$ \\
\hline MANUAL & . 263 \\
\hline MANUAL & . 264 \\
\hline MANUAL & . 265 \\
\hline MANUAL & . 268 \\
\hline MANUAL & . 267 \\
\hline HANUNL & . 268 \\
\hline MANUAL & . 269 \\
\hline WANUAL & . 276 \\
\hline MANUAL & . 271 \\
\hline MANUAL & . 272 \\
\hline MANUAL & . 273 \\
\hline MANUAL & . 274 \\
\hline WANUAL & . 275 \\
\hline WANUAL & . 278 \\
\hline MANUAL & . 277 \\
\hline MANUAL & . 278 \\
\hline MANUAL & . $\quad 279$ \\
\hline
\end{tabular}


PROMPTS WIU ASK FOR THE DEINING PARUNEIERS OF THE DISTRIBUTION WHICH IS CHOSEN, AND WILL YARY ACCDRDINCEY.

DISTRIBUTION TYPE IS NORUN

INPUT UNITS ARE $\quad M * \star 2 / Y R$

ENTER THE MEAN OF THE DISTRIBUTION.

ENTER THE MEAN OF THE DESIRED NORUAL DISTRIBUTION.

ENTER THE STANDARD DEVIATION.

ENTER THE STANDARD DEVIATION OF THE DESIRED NORWAL DISTRIBUTION.

WDLLD YDU LIKE A LDTER AND AN UPPER

gOUND ENFORCED ON THIS VARIAQE (Y/N)?

PACSTAT AШDWS THE USER TO SET UPPER AND LDWER LIMITS ON THE DISTRIBUTION FOR THIS INPUT VARIAQE. THIS IS USEEU IHEN A DISTRIBUTION TAIL EXTEUDS TO AN UNREALISTIC VNLUE (E.G., BELON ZERO). IF 'Y' IS ENTERED HERE, THE LSER WILL BE PROMPTED FOR THE UPPER AND LOTER LIUITS FOR USE ON THE INPUT YARIABLE. NOTE THAT DISTRIBUTIONS WHICH HAVE INHERENT ENDPOINTS (I.E. THE UNIFORN, LOCUNIFORN, AND CONSTANT DISTRIBUTIONS) ILL NOT GENERATE THIS PROMPT.

2) POROSITY (OIMENSIONLES) DISTRIBUTION

1) NORMAL

2) MATURAL LOGNORUAL

3) COWON LOGNORMA

4) NATURAL LOCUNIFORN

5) COMON LOGUNIFORY

8) EXPONENTIAL

7) UNIFOR

8) BERNOULI

9) EXTERNAL CONF FUNCTN

10) EXTERNAL DATA POINTS

11) CONSTANT

ENTER THE NMWER FOR THE DESIRED DISTRIQNTION.

THIS PROUPT IS REPEATED ONCE FOR EACH INPUT VARIAQLE WHICH THE USER HAS DEFINED IN THE QLOCK DATA ROUTINE.

ENTER THE VALUE FOR SCALE FACTOR (OIMENSIONLES),

THIS IS A PROMPT FOR THE VALUE OF THE SYSTE CONSTANT NAMED 'SCALE FACTOR'. THIS PROMPT ILL BE WRITTEN ONCE FOR EACH OF THE CONSTANTS WHICH THE USER HAS DEFINED IN THE BLOCK DATA RQUTINE.

ENTER THE NWBER OF SAMPLES TO TAKE (WAX = 16900)

ENTER THE NUMBER OF STOCASTIC SAMPLES TO TAKE. THE MAXIMU NUMBER OF SAMPLES MAY YARY, DEPENDING ON THE PROBLEN. THE

\begin{tabular}{|c|c|}
\hline WANUAL & . 28 \\
\hline WANUAL & 281 \\
\hline MANUAL & 282 \\
\hline MANUNL & 283 \\
\hline WANUAL & 284 \\
\hline WANUAL & 285 \\
\hline WANUAL & 286 \\
\hline WANUAL & 287 \\
\hline MANUN & 288 \\
\hline WANUAL & 289 \\
\hline WANUAL & 298 \\
\hline WANUAL & 291 \\
\hline WANUAL & . 292 \\
\hline WANUAL & 293 \\
\hline WANUNL & 294 \\
\hline WANUAL & 295 \\
\hline WANUNL & 298 \\
\hline MANUAL & 297 \\
\hline WANUAL & 298 \\
\hline MANUAL & 295 \\
\hline MANUAL & 360 \\
\hline MANUAL & 30 \\
\hline MANUAL & 30 \\
\hline MANUAL & 36 \\
\hline MANUAL & 30 \\
\hline MANUAL & 30 \\
\hline MANUAL & $30 t$ \\
\hline WANUAL & 307 \\
\hline WANUAL & 308 \\
\hline MANUAL & 380 \\
\hline WANUAL & 318 \\
\hline WANUAL & 31 \\
\hline WANUAL & 312 \\
\hline HANUAL & 315 \\
\hline WANUAL & 314 \\
\hline WANUAL & 315 \\
\hline WANUAL & 316 \\
\hline WANUNL & 317 \\
\hline MANUAL & 318 \\
\hline WANUAL & 315 \\
\hline WANUAL & 328 \\
\hline MANUAL & 32 \\
\hline WANUAL & 322 \\
\hline WANUAL & 323 \\
\hline WANUAL & 00 \\
\hline MANUAL & 325 \\
\hline WANUAL & 326 \\
\hline WANUAL & 327 \\
\hline WANUAL & 0 \\
\hline WANUAL & $=0$ \\
\hline WANUAL & 338 \\
\hline WANUAL & 331 \\
\hline WANUAL & 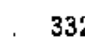 \\
\hline WANUNL & \\
\hline
\end{tabular}

B. 39 
MENORY AUOCATION TECHNIQUE ATTGMPTS TO PERUIT AS WANY SAMPLES AS POSSIBLE, WITHIN THE AVAILABLE SPACE. THE MXIMN VALUE PRINTED IITH IHIS PROUPT VIU ALWAYS BE ENFORED.

ENTER THE PRINT INTERYAL FOR OUTPUT DATA.

THE PRINT INTERYAL SETS THE NUMBER OF SAMPLES, FROM THE OUTPUT DATA SET, IHICH IL BE PRINTED IN THE CONFIDENCE TABLES. THIS DUTPUT DATA IS USUNYY A LARGE DATA SET (E.G. 1060 VALUES). THE CONFIDENCE TARES WIL CONTAIN ONLY EVERY 'N-TH' DATA VALUE, WHEN N IS THE PRINT INTERVAL. THE SWNLEST AND LARGEST DATA YALUES IILL ALMAYS BE PRINTED, REGARDLES OF THE PRINT INTEYAL.

ENTER THE NUMBER OF INTERNALS FOR STRATIFIED SAMPLING (MAX= 196).

STRATIFICATION DIVIDES THE VARIABLE DISTRIBUTIONS INTO A NUMER OF INTERYALS OF EQUAL AREA. IT THEN FORCES THE SAME NUMBER OF SALPLES TO BE CHOSEN FRON EACH INTERNAL. THIS CREATES A MORE UNIFORAL DISTRIQUTED SET OF RANDOU YARIARES FOR A GIVEN NUMBER OF SNARES.

THE USER HERE MST ENTER THE NUMBER OF INTERYALS DESIRED FOR STRATIFICATION. EATERING A VALUE OF ONE WI DEEAT THE STRATIFICATION PROCEDURES. THIS PROUPT, AND STRATIFICATION, WILL BE OMITTED IF NONE OF THE INPUT VARIABLES ARE DISTRIBUTED.

ENTER AN INITIAL SEED NUMBER FOR THE RANDOM NUMBER GENERATDR. (A RENL, DOUBLE PRECISION MUBER BETEEFN 1.0 AND 2.0E+9)

THE PRINT RANDOM NUMEER GENERATOR REQUIRES AN SEED VALUE, WHICH WST BE A DOURE PRECISION NUABER BETWEEN 1. AND 2.6E+9. THIS ALLONS THE USER TO CHANGE THE RANDON SEQUENCE FROU RUN TO RUN.

THE MONTE CARLO SIMLATION IS RUN AT THIS POINT. THE INPUT VARIABLES ARE COLPUTED FROU THE DESIRED DISTRIBUTIONS, AND THE OUTPUT VARIARES ARE COMPUTED IN THE ROUTINE SOLN. THIS SOLUTION ROUTINE IS CALLED ONCE FOR EACH SAMPLE DESIRED.

CERTAIN RUN TIME DIAGNDSTICS MAY BE PRINTED TO THE USERS TERAINAL AT THIS POINT, DEPENDING ON THE NATURE DF THE PROBLEI.

AVAILABLE SOLUTIONS ARE

פ) EXIT LIST

1) TOTAL RELEASE (NG)

2) TRAVEL TIHE (YRS)

3) - ENTER A NEI PRINT INTERYAL -

WHICH WOUD YOU LIKE (ENTER NUABER)?

THIS PRDUPT ASKS WHICH OUTPUT DATA VNLUE FHAT DATA SHOUD BE PRINTED AND/OR PLOTTED. ENTER THE NUABER CORRESPONDING TO THE DESIRED OUTPT VARIABLE. ENTER \& TO EXIT THIS PART OF THE CDOE.

\begin{tabular}{|c|c|}
\hline IANUAL & 334 \\
\hline WANUAL & 335 \\
\hline UANUAL & 338 \\
\hline WANUAL & 337 \\
\hline UANUAL & 338 \\
\hline WANUAL & 339 \\
\hline WANUAL & 346 \\
\hline HANUAL. & 341 \\
\hline MANUAL & 342 \\
\hline MANUAL & . 343 \\
\hline WANUAL & . $\quad 344$ \\
\hline WANUAL & 345 \\
\hline WANUAL & . 346 \\
\hline WANUAL & . $\quad 347$ \\
\hline WANUAL & . 348 \\
\hline WANUAL & . 349 \\
\hline WANUAL & . 358 \\
\hline WANUAL & . $\quad 351$ \\
\hline WAKUAL & . $\quad 352$ \\
\hline MANUAL & 353 \\
\hline WAKUAL & . 354 \\
\hline MANUAL & . $\quad 355$ \\
\hline WANUAL & . 356 \\
\hline WANUAL & 367 \\
\hline WANUAL & .358 \\
\hline WANUAL & 359 \\
\hline WANUAL & 360 \\
\hline WANUAL & 361 \\
\hline WANUAL & 362 \\
\hline WANUAL & 363 \\
\hline WANUAL & 364 \\
\hline WANUAL & . 365 \\
\hline WANUAL & 386 \\
\hline MANUAL & 387 \\
\hline WAKUAL & 368 \\
\hline WANUAL & 369 \\
\hline WANUAL & 370 \\
\hline WANUAL & 371 \\
\hline WANUAL & 372 \\
\hline WANUAL & 373 \\
\hline MANUAL & 374 \\
\hline WANUAL & 375 \\
\hline WANUAL & 378 \\
\hline MANUAL & 377 \\
\hline MANUN & 378 \\
\hline MANUAL & 379 \\
\hline MANUAL & 380 \\
\hline WANUAL & 381 \\
\hline WANUAL & 382 \\
\hline WANUAL & 383 \\
\hline MANUNL & 384 \\
\hline MANUAL & 385 \\
\hline WANUNL & 386 \\
\hline MANUNL & 387 \\
\hline
\end{tabular}


THE LAST OPTION, A NE PRINT INTERVAL, WIL N NAYS FOLON THE

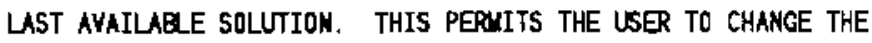
PRINT INTERVA VHICH WAS ENTERED EARLIER.

WHICH WOUD YQU LIKE

1) CUMUATIVE PROBABILITY

2) CDUPLgientARY CUMUATIVE PROBABILITY

ENTER 1 DR 2

THE DATA MAY BE PRINTED AND/GR PLOTTED AS A FUNCTION OF EITHER THE CUMLATIVE PROBABILITY, OR THE COMPLENENTARY CUMULATIVE PROBABILITY, ENTER THE VALUE CORRESPONDING TO THE DESIRED DATA SET TYPE. A SAMPLE OUTPUT TABLE IS PRINTED BEIOU.

**--- OUTPUT CUMULATIVE PROBABILITIES

FOR STOCASTIC VARIABLE, TRAYE TIME (YRS)

\begin{tabular}{|c|c|c|}
\hline SAMPLE & VARIAQLE & CGMULATIVE \\
\hline NLABER & VALUE & CONFIDEACE \\
\hline 1 & $6.1716 \mathrm{E}-61$ & 0.18006 \\
\hline 2 & 0.7712E-01 & 0.20080 \\
\hline 3 & 0.1895 & 0.36000 \\
\hline 4 & .2822 & 6. 46050 \\
\hline 5 & 0.3484 & 6.50000 \\
\hline 6 & 0.4288 & 0.80000 \\
\hline 7 & 6.7398 & 6.79000 \\
\hline 8 & 1.882 & 0.88096 \\
\hline 9 & 1.883 & 0.98080 \\
\hline 16 & 2.016 & 1.00900 \\
\hline
\end{tabular}

WOUD YOU LIKE THE DATA PLDTIED $(Y / N)$ ?

THE PLOTIING OPTION ALIOWS ONE TO CREATE A PLOT OF THE DESIRED OUTPUT VARIABLE (ABCISSA) VERSLS THE CONFIDEKCE LEYE (ORDINATE). IF CHOSEA, THE USER UST DEINE THE PLOTTING DEVICE DEVICE (CURREAT TERIINAL) AND PAGE SIZE, PRIDR TO ONLY THE FIRST PLAT.

AVAILABLE SOLUTIONS ARE

•) EXIT LIST

1) TOTAL REEEASE (UM)

2) TRAYE TIUE (YR)

3) - ENTER A NEI PRINT INTERYAL -

WHCH TOUD YDU LIKE (ENTER NLMBER)?

THIS PROUPT IS REPEATED UNTIL A ZERD IS EATERED.

CREATE ANOTHER DATA SET $(Y / N)$ ?

BY ENTERING A 'Y' HERE, THE USER WAY RESTART THE NONTE CARLO SIMUATION. THE PROGRAL ILL PROMPT FOR UPDATED PARAMETERS FOR THE INPUT VARIAEEE AND CONSTANTS. IF AN ' $N$ ' IS ENTERED, THE PROGRAN EXECUTION IS ERDED.

\begin{tabular}{|c|c|}
\hline & .00 \\
\hline MANUAL & 38 \\
\hline MANUAL & 39 \\
\hline UANUAL & 391 \\
\hline WANUAL & . $\quad 392$ \\
\hline MANUAL & . 393 \\
\hline WANUAL & . 394 \\
\hline WANUAL & . 395 \\
\hline MANUNL & . 398 \\
\hline MANUNL & . 397 \\
\hline MANUAL & . 398 \\
\hline WANUAL & . 399 \\
\hline MANUAL & . 460 \\
\hline MANUAL & .401 \\
\hline MANUAL & . 402 \\
\hline MANUAL & . 463 \\
\hline WANUAL & . 464 \\
\hline MANUNL & .495 \\
\hline WANUAL & . 406 \\
\hline MANUAL & . 497 \\
\hline WANUAL & . 488 \\
\hline WANUAL & . 469 \\
\hline MANUAL & . 410 \\
\hline MANUAL & . 411 \\
\hline WANUN & . 412 \\
\hline WANUAL & . 413 \\
\hline WANUAL & 414 \\
\hline WANUAL & 415 \\
\hline WANUAL & 416 \\
\hline WANUAL & . 417 \\
\hline WANUA & 418 \\
\hline WANUNL & 418 \\
\hline WANUAL & 428 \\
\hline WANUAL & 421 \\
\hline MANUNL & 422 \\
\hline WANUAL & 423 \\
\hline MANUAL & . 424 \\
\hline WANUAL & 425 \\
\hline MANUAL & . 426 \\
\hline MANUAL & 427 \\
\hline MANUAL & 428 \\
\hline MANUAL & 429 \\
\hline WANUAL & 436 \\
\hline MANUAL & 431 \\
\hline MANUAL & 432 \\
\hline MANUN & 433 \\
\hline MANUAL & 434 \\
\hline WANUAL & 435 \\
\hline MANUAL & 436 \\
\hline MANUAL & 437 \\
\hline MANUAL & 438 \\
\hline MANUAL & 439 \\
\hline MANUAL & . 440 \\
\hline MANUAL & 44 \\
\hline
\end{tabular}


WHEN A NEI DATA SET IS CREATED, THE PRINTED AND BIMARY DUTPUT DATA ARE ADDED ON TO THE EXISTING DATA FILES. NET FILES ARE NOT OPENED.

SUBRDUTINE NORULL (N, RNDYN, RPI, RP2)

C ROUTINE FOR COUPUTING A NORULLY DISTRIBUTED DATA SET FROW A

C UNIFORLY DISTRIBUTED DATA SET DN THE INTERYAL $\$, 1$.

C

\begin{tabular}{|c|c|}
\hline ANUNL & 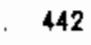 \\
\hline IANUAL & 443 \\
\hline IANUAL & 444 \\
\hline IANUAL & 445 \\
\hline WANUAL & 446 \\
\hline UANUNL & 447 \\
\hline NORUAL & 2 \\
\hline NORNAL & 3 \\
\hline NORMAL & 4 \\
\hline NORWAL & 5 \\
\hline NORNAL & 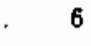 \\
\hline NDRNAL & 7 \\
\hline NORNAL & 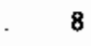 \\
\hline NORMAL & . \\
\hline NORMAL & 10 \\
\hline NORWAL & 11 \\
\hline NORMAL & 12 \\
\hline NORIAL & 13 \\
\hline NORUAL & 14 \\
\hline NORMAL & 15 \\
\hline NORMAL & 16 \\
\hline NORMAL & 17 \\
\hline NORUNL & 18 \\
\hline NDRWAL & 19 \\
\hline IRINL & 28 \\
\hline NQRMAL & 21 \\
\hline NORWAL & 22 \\
\hline NORUAL & 23 \\
\hline NORWAL & 24 \\
\hline NORUAL & 25 \\
\hline NORMALL & 0 \\
\hline NORNALL & 3 \\
\hline NORMALL & 4 \\
\hline NDRNNL & 5 \\
\hline NORNALL & 8 \\
\hline NORWN & 7 \\
\hline NORMALL & a \\
\hline NORMALL & 9 \\
\hline NORMALL & 10 \\
\hline NORMALL & 11 \\
\hline NORLALL & 12 \\
\hline NORHALL & 13 \\
\hline NORHALL & 14 \\
\hline NORMALL & 15 \\
\hline NORNALL & 16 \\
\hline NDRNALL & 17 \\
\hline NORWALL & 18 \\
\hline NORWALL & 19 \\
\hline NORWALL & 20 \\
\hline NORIALL & 21 \\
\hline NORYALL & 22 \\
\hline NORNALL & 23 \\
\hline NORMALL & 2 \\
\hline NORUALL & 25 \\
\hline
\end{tabular}




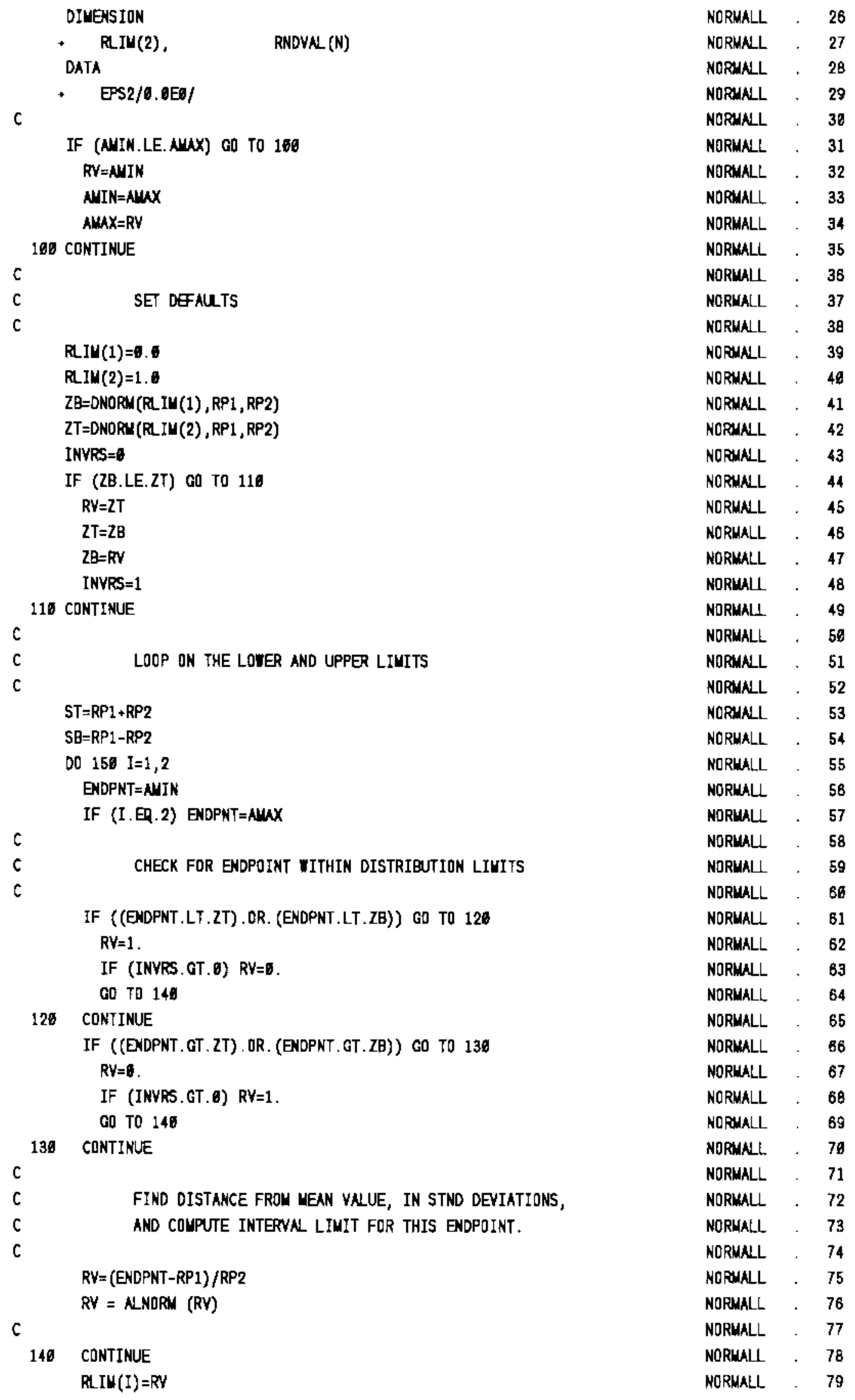




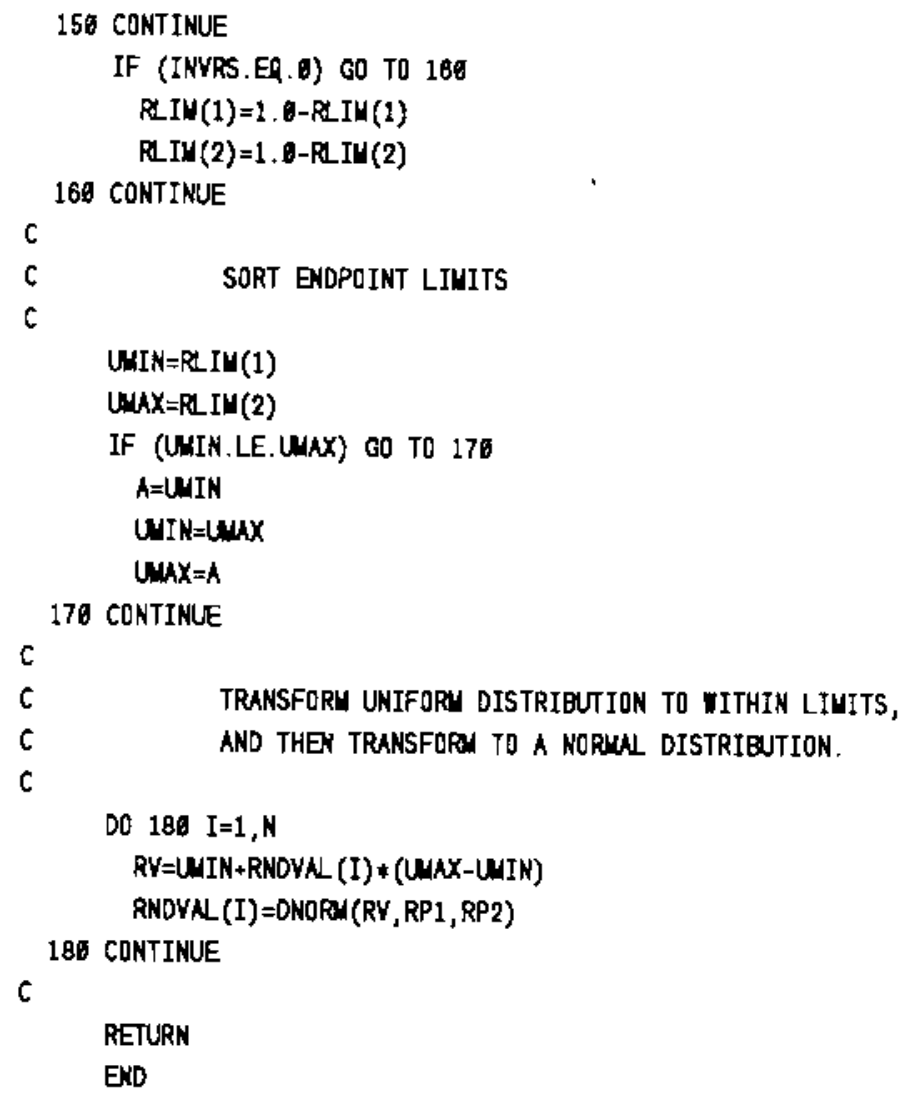

THIS IS A SAMPLE DUTPUT PRINT FILE. THIS FILE WAS CREATED BY THE. MONTE CARLO TEST CASE DEINED BY THE BLOCK DATA AND SOLUTION ROUTINES LISTED ABOYE.

MASS STDRAGE AND REEEASE RATE INTO LAYER CARBON-14

**--- ECHD DF INPUT DATA

MOLEC DIFFSV (MET**2/YEAR) DISTRIBUTION IS COLNDN LDCUNIFORN MIN, WAX EXPONENTS FOR THE UNDERLYING UNIFOR DISTRIBUITON ARE

MIN $=-3.0080 E+69, \quad W A X=-2.0906 E+96$

RETARDATION (UNITLESS ) DISTRIBUTION IS CONSTANT CONSTANT YALUE $=1.6969 \mathrm{E}+69$

RUN I.O. 312861320 USER NAME LANGFORD DATA SET NUMBER 1

\begin{tabular}{|c|c|}
\hline RWALL & 85 \\
\hline NORWLLL & 81 \\
\hline NORWALL & 82 \\
\hline NORHALL & 83 \\
\hline NORMALL & 84 \\
\hline NORWALL & 85 \\
\hline NORNALL & 86 \\
\hline NORNALL & 87 \\
\hline NORUNL & 88 \\
\hline NDRUALL & 89 \\
\hline NORUNLL & 90 \\
\hline NORWNL & 91 \\
\hline NORUALI & 92 \\
\hline NORLALL & 93 \\
\hline NORMALL & 94 \\
\hline NORMALL & 95 \\
\hline NORMALL & 98 \\
\hline NGRWALL & 97 \\
\hline NORWALL & 98 \\
\hline NORNALL & 99 \\
\hline NORANAL & . 169 \\
\hline NOFANLL & . 101 \\
\hline NOFANLL & . 162 \\
\hline NDFUALL & . 103 \\
\hline NORUALL & . 164 \\
\hline NORNALL & . 105 \\
\hline DUTPUT & 2 \\
\hline DUTPUT & 3 \\
\hline DUTPUT & 4 \\
\hline QUTPUT & 5 \\
\hline DUTPUT & 6 \\
\hline QUTPUT & 7 \\
\hline QUTPUT & 8 \\
\hline QUTPUT & 9 \\
\hline QUTPUT & 16 \\
\hline DUTPUT & 11 \\
\hline QUTPUT & 12 \\
\hline OUTPUT & 13 \\
\hline OUTPUT & 14 \\
\hline OUTPUT & 15 \\
\hline OUTPUT & 16 \\
\hline OUTPUT & 17 \\
\hline QUTPUT & 18 \\
\hline OUTPUT & 19 \\
\hline QUTPUT & 20 \\
\hline DUTPUT & 21 \\
\hline OUTPUT & 22 \\
\hline DUTPUT & 23 \\
\hline DUTPUT & 24 \\
\hline OUTPUT & 25 \\
\hline OUTPUT & 26 \\
\hline DUTPUT & 27 \\
\hline OUTPUT & 28 \\
\hline DUTPUT & 29 \\
\hline
\end{tabular}




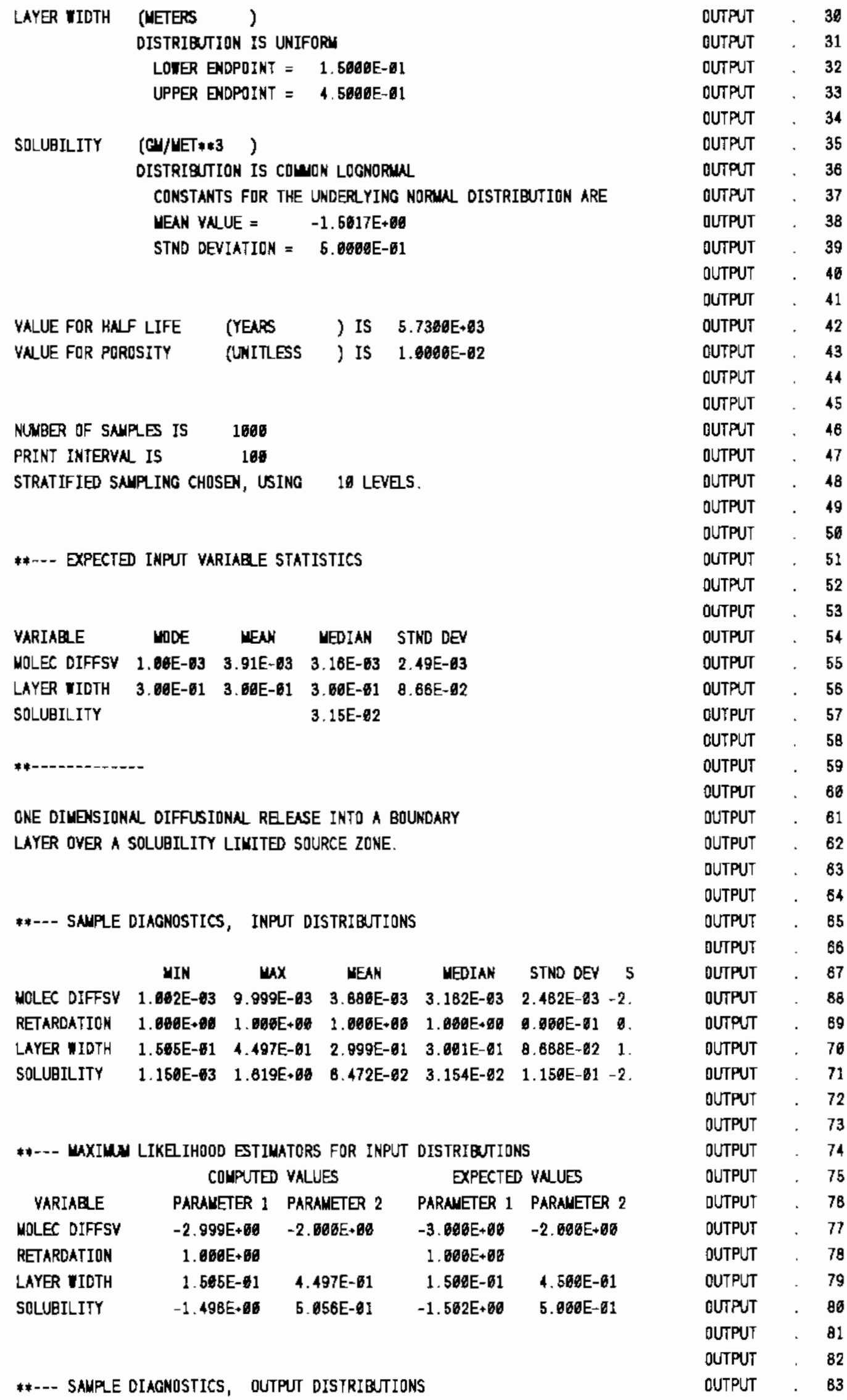




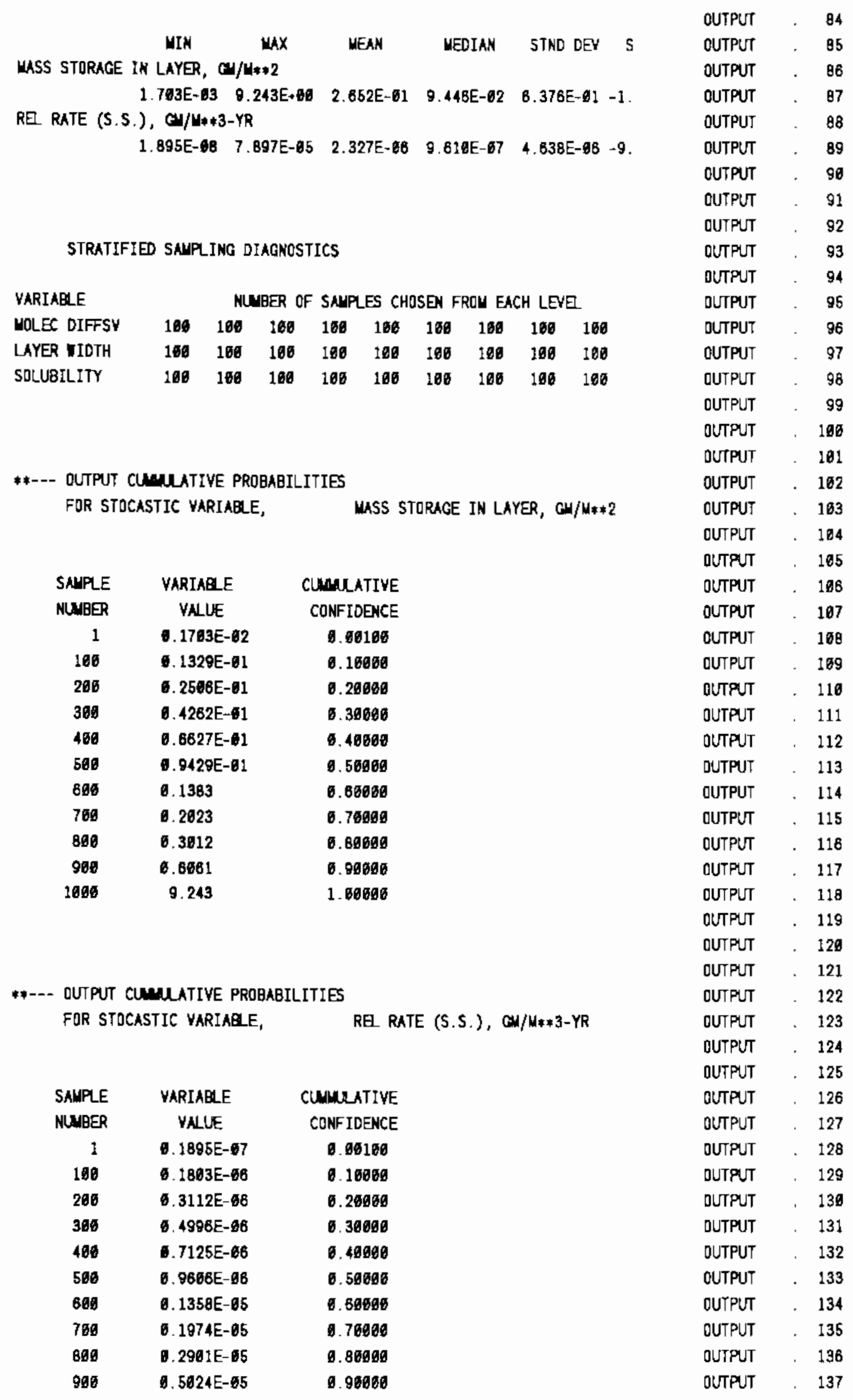


THE FDLOWING IS A LIST OF YARIABLES FOR TKE PACSTAT MONTE CARLO DRIVER ROUTINES. NOTE THAT ONLY COMAON BLOCKS PQLK1 THROLCH PQLK6 ARE NEEDED BY THE LSER DEINARLE ROUTIMES SOLN (SOLUTION CDLPUTATION) AND BLKDAT (BLOCK DATA DEINING THE PROBLEN).

THIS LIST IS CURRENT AS OF MARCH 5, 1988.

\section{PARALETER BLOCK PPARA}

ISIZE = WXIWN DATA SET SIZE. THIS SETS THE DIMENSIDNS OF THE LARGE ARRAYS FOR STORING INPUT AND OUTPUT YARIAQRES.

WXXCNT = WXXILU NLMBER OF CONSTANTS A PROBLEN WAY CONTAIN

MAXDST = WAXIMN NLMBER DF DATA POINTS FOR A USER DEINED CUMULATIVE DISTRIQUTION FUNCTION.

MAXLEV = MAXIMN NUMBER OF LEVES FOR STRATIFICATION.

MAXNS = THE WAXIMU NUMBER OF MONTE CARLO SAMPLES WHICH ARE ALLDWED PER RIN OF THE DRIVER CDOE.

MAXSOL = MAXIMW NUMBER OF 'SQLUTIONS' (OUTPUT VARIABLES) NШOWED.

MAXYAR = MAXIMN NUMBER OF INPUT YARIABLES A PROQLEU WAY CONTAIN.

NIMEN = MXXINM FILE NAME LENGTH (NUMBR OF CHAR*4 WORDS).

NTYPE = MAXINM NUMBER OF DISTRIBUTION TYPES PRESENT. IF THIS PROGRAM LIMIT IS CHANGED, THE ACCOMPANYING LIST OF DISTRIBUTIONS UST ALSO BE UPGRADED. THIS MILL OCCUR IN ROUTINES IINPY, EXPECT, STATDT, AND RNGS.

\section{COMMON BLOCK PQLK1}

NCONST = MUMER OF CONSTANTS FOR THE CURRENTLY DEINED PROQEE. (BLKDT)

NSOLN = NUUGER OF SOLUTIONS FOR THE CURRENTLY DEINED PRORLEI. (BLKDAT)

NYAR = NLMBER OF INPUT YARIABLES FOR THE CURRENTLY DEFINED PROBLEU. (BLKDAT)

COLNON BLOCK PQLK2

NSTTL = NUMBER OF CHARACTERS IN THE PLDTTING SUB-TITLE. (PACSTAT)

NTTL = NUMBER OF CHARACTERS IN THE PLOTING TIFLE. (PACSTAT)

STTLE = PLOTTING SUB-TITLE, UP TO 40 CHARACTERS. (PACSTAT)

TTLE = PLOTTING TITLE, UP TO 40 CHARACTERS. (BLXDAT)

COMNON BLOCK PQLK3

\footnotetext{
CLABE = LABEES FOR THE SYSTEM CONSTANTS FOR THE CURRENTLY DEINED PROBLE, UP TO 12 CHARACTERS. (BLKDAT)

CDINEN = LABESS FOR THE UNITS DF THE SYSTEN CONSTANTS, UP TO 12 CHARACTERS. (BLKDAT)

PLABE = LABES FOR THE SOLUTIONS OF THE CURRENTLY DEINED PROBLEN, UP TO 32 CHARACTERS. (BLXDAT)

ИABE = LABELS FOR THE INPUT YARIAQLES FOR THE CURRENTLY DEINED
}

\begin{tabular}{|c|c|}
\hline TPUT & $13 \mathrm{E}$ \\
\hline OUTPUT & 139 \\
\hline OUTPUT & 140 \\
\hline OUTPUT & 141 \\
\hline OUTPUT & 142 \\
\hline OLTPUT & 143 \\
\hline OLTPUT & 144 \\
\hline OUTPUT & . 145 \\
\hline OUTPUT & . 146 \\
\hline OUTPUT & . 147 \\
\hline OUTPUT & . 148 \\
\hline OLTPUT & . 149 \\
\hline DUTPUT & 150 \\
\hline QUTPUT & 151 \\
\hline DUTPUT & . 152 \\
\hline DUTPUT & 153 \\
\hline OUTPUT & 154 \\
\hline DUTPUT & . 155 \\
\hline DUTPUT & . 156 \\
\hline OUTPUT & 157 \\
\hline OUTPUT & . 158 \\
\hline DUTPUT & . 159 \\
\hline OUTPUT & . 160 \\
\hline OUTPUT & 161 \\
\hline DUTPUT & . 162 \\
\hline DUTPUT & . 163 \\
\hline DLTPUT & . 184 \\
\hline DUTPUT & . 165 \\
\hline OLTPUT & . 166 \\
\hline OUTPUT & . $\quad 167$ \\
\hline OLTPUT & . 168 \\
\hline घUTPUT & . $\quad 169$ \\
\hline OUTPUT & . $\quad 179$ \\
\hline DUTPUT & . 171 \\
\hline DUTPUT & . 172 \\
\hline वUTPण & . 173 \\
\hline DUTPUT & . 174 \\
\hline OUTPUT & . 175 \\
\hline OUTPUT & . 178 \\
\hline OUTPUT & . 177 \\
\hline OUTPUT & . 178 \\
\hline DUTPUT &.$\quad 179$ \\
\hline DUTPUT & . 180 \\
\hline OUTPUT & . 181 \\
\hline DUTPUT & . $\quad 182$ \\
\hline DUTPUT & . 183 \\
\hline OUTPUT & . 184 \\
\hline OUTPUT & 185 \\
\hline OUTPUT & 186 \\
\hline OUTPUT & 187 \\
\hline OUTPUT & . $\quad 188$ \\
\hline QUTPUT & 189 \\
\hline DUTPUT & 196 \\
\hline OUTPUT & . 191 \\
\hline
\end{tabular}


PRQQRE, UP TO 12 CHARACTERS. (BCKDAT) OUTPUT . 192

VDIUEN = LABES FOR THE UNITS OF THE INPUT VARIABLES FOR THE CURRENTLY OUTPUT . 193

DGINED PROBEE, UP TO 12 CHARACTERS. (BLKDAT) OUTPUT . 194

\section{COMEN BOCK PBLKA}

OUTPUT . 195

$\begin{aligned} \text { CONST = } & \text { VALUES FOR THE SYSTEU CONSTANTS. (IINPUT) } \\ \text { VALU = } & \text { ARRAY FOR TEUPORARY STORAGE OF THE SOLUTION VALUES, FOR } \\ & \text { TRANSFER FROU SUGROUTINE SOLN TO THE YAIN ROUTINE. (SOLN) } \\ \text { YINPT = } & \text { ARRAY FOR TEUPORARY STORAGE OF THE INPUT VARIABLE YALUES, } \\ & \text { FOR TRURSFER TO SUBRDUTINE SOLN. (DATSET) }\end{aligned}$

\section{COMON BLOCK PBLS}

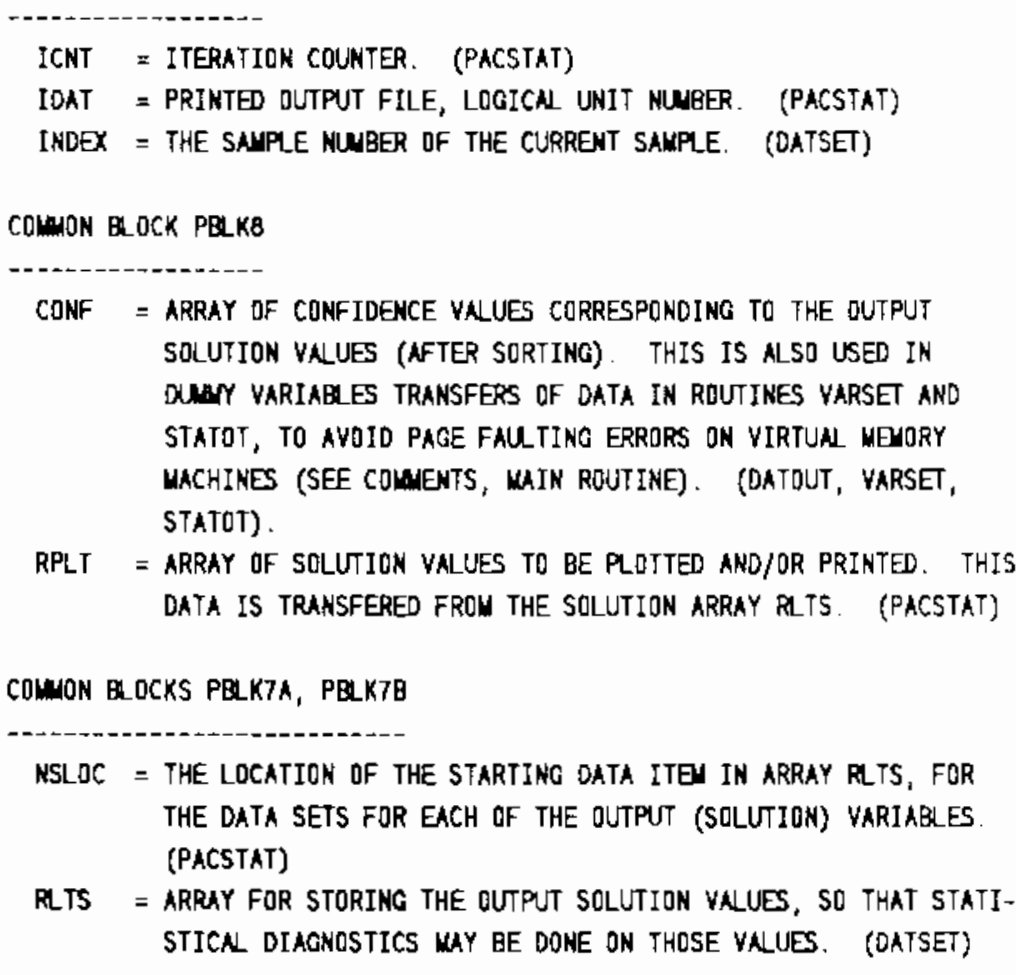

COIMON BLOCKS PBLKAA, PBLK8B

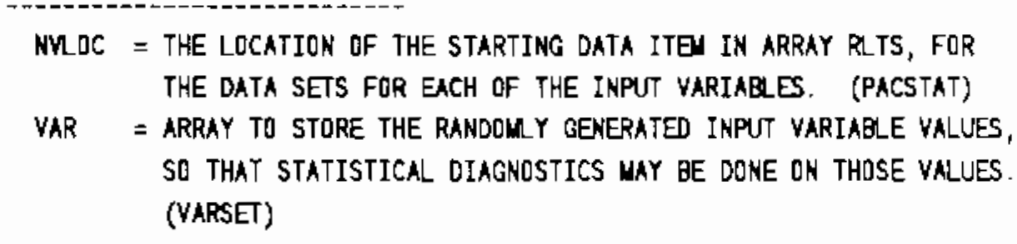

\section{COMNON BLOCK PBLKG}

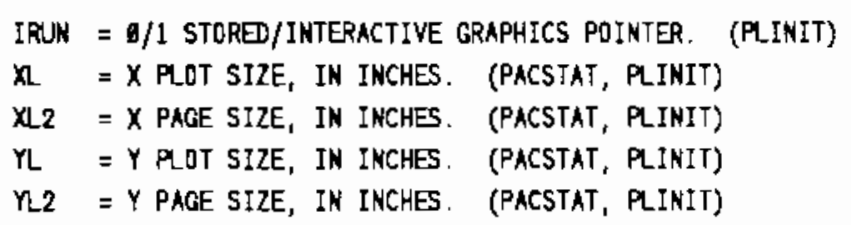




\begin{tabular}{|c|c|c|c|}
\hline \multirow{5}{*}{$\begin{array}{l}\text { FAVG } \\
\text { FluXX } \\
\text { FUED }\end{array}$} & \multirow{2}{*}{ = AVERAGES FOR THE COMPUTE SOLUTION DISTRIBUTIONS. (STATS) } & \multirow{2}{*}{$\begin{array}{l}\text { OUTPUT } \\
\text { OUTPUT }\end{array}$} & \multirow{2}{*}{$\begin{array}{l}248 \\
247\end{array}$} \\
\hline & & & \\
\hline & $=$ = WXILUS FOR THE CONPUTE SOLUTION DISTRIBTUIONS. (STATS) & OUTPUT & 248 \\
\hline & $=$ MEDIAN VNLUES FOR THE COUPUTED SOLUTIONS DISTRIBUTION. & DUTPUT & 249 \\
\hline & (STATQT) & DUTPUT & 250 \\
\hline FUIN & $=$ = UINILUS FOR THE COUPUTED SOLUTION DISTRIBUTIONS. (STATS) & OUTPUT & 251 \\
\hline \multirow[t]{2}{*}{ FSK厂 } & = SKENESS YNLUES FOR THE COUPUTED SOLUTION DISTRIBUTIONS. & DUTPUT & 152 \\
\hline & (STATS) & OUTPUT & 253 \\
\hline \multirow[t]{2}{*}{ FSTD } & $=$ STANDARD DEVIATION VNLUES FOR THE CDMPUTED SCLUTION & DUTPUT & 254 \\
\hline & DISTRIBUTIONS. (STATS) & OUTPUT & $25 \mathrm{~S}$ \\
\hline \multirow[t]{2}{*}{ YAVG } & $=$ AVERAGES FOR THE INPUT RANDOM VARIAQLE DISTRIBUTIONS. & OUTPUT & 256 \\
\hline & (STATS) & DUTPUT & 257 \\
\hline \multirow[t]{2}{*}{ nux } & $=$ MAXIUUSS FOR THE INPUT RANDOU VARIABLE DISTRIBUTIONS. & OUTPUT & 258 \\
\hline & (STATS) & DUTPUT & 259 \\
\hline \multirow[t]{2}{*}{ WED } & $=$ MEDIAN VNLUES FOR THE INPUT RANDOM VARIABLE DISTRIBUTIONS. & OUTPUT & 289 \\
\hline & (STATOT) & OUTPUT & 261 \\
\hline \multirow[t]{2}{*}{ WIN } & $=$ UINILUS FOR THE INPUT RANDON VARIABLE DISTRIBUTIONS. & QUTPUT & 262 \\
\hline & (STATS) & DUTPUT & 263 \\
\hline \multirow[t]{2}{*}{ VSKW } & = SKEMESS VNLUES FOR THE INPUT RANDOU VARIABLE DISTRIBUTIONS. & OUTPUT & 264 \\
\hline & (STATS) & OUTPUT & 265 \\
\hline \multirow[t]{3}{*}{ VSTD } & $=$ STANDARD DEVIATION VRLUES FOR THE YNPUT RANDOW VARIABLE & OUTPUT & 268 \\
\hline & DISTRIQUTIONS. (STATS) & QUTPUT & 267 \\
\hline & & QUTPUT & 268 \\
\hline \multicolumn{2}{|c|}{ CQMON BLOCK PBLK11 } & QUTPUT & 269 \\
\hline \multicolumn{2}{|c|}{ 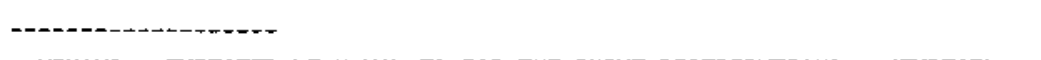 } & OUTPUT & 270 \\
\hline \multirow{2}{*}{\multicolumn{2}{|c|}{$\begin{array}{l}\text { VINAVG = EXPECTED WEAN VNUES FOR THE INPUT DISTRIBUTIONS. (EXPECT) } \\
\text { VINMED = EXPECTED WEDIAN YAUES FOR THE INPUT DISTRIBUTIONS. (EXPECT) }\end{array}$}} & OUTPUT & 271 \\
\hline & & QUTPUT & 272 \\
\hline \multicolumn{2}{|r|}{ VINMOD = EXPECTED MODE FOR THE INPUT DISTRIBUTION. (EXPECT) } & OUTPUT & 273 \\
\hline \multicolumn{2}{|r|}{ VINSTD = EXPECTED STANDARD DEVIATIONS FOR THE INPUT DISTRIBITIONS. } & OUTPUT & 274 \\
\hline & (EXPECT) & DUTPUT & 275 \\
\hline & & OUTPUT & 276 \\
\hline \multicolumn{2}{|c|}{ CDMAN BQDCK PBLK12 } & DUTPUT & 277 \\
\hline \multicolumn{2}{|c|}{ - } & DUTPUT & 278 \\
\hline \multirow[t]{2}{*}{ NERR } & $=$ AN ERROR POINTER, INDICATING WHICH SAMPLES REUURNED WITH AN & OUTPUT & 279 \\
\hline & ERROR POINTER. (DATSET) & OUTPUT & 286 \\
\hline NLMERR & $=$ THE NUMBER OF SAMPLES WHICH ERROR TERUINATE. (DATSET) & DUTPUT & 281 \\
\hline & & OUTPUT & 282 \\
\hline COLMON B & LOCK PBLK13 & OUTPUT & 283 \\
\hline ----- & $-\cdots$ & OUTPUT & 284 \\
\hline NLEYE & $=$ THE NUMBER OF LEYESS OF STRATIFICATION TO USE. MAY BE & OUTPUT & 285 \\
\hline & REDUCED BY ROUTINE UQ1S. (IINPUT) & OUTPUT & 288 \\
\hline NPTSPL & $=$ THIS COUNTS THE NLMBER OF SAUPLES, FOR EACH INPUT VARIABLE, & OUTPUT & 287 \\
\hline & WHICH HAVE BEEN TAKEN FROM EACH STRATIFICATION LEVE. & OUTPUT & 288 \\
\hline & (RNCS, IINPUT) & DUTPUT & 289 \\
\hline NSTOC & $=\varnothing / 1$ POINTER INDICATES IF ALL DF THE VARIABLES ARE CONSTANT, & OUTPUT & 299 \\
\hline & OR IF AT LEAST ONE IS STOCATICALLY MODELED. THIS IMPACTS & OUTPUT & 291 \\
\hline & STRATIFICATION AND DIAGNOSTICS. (IINPU) & OUTPUT & 292 \\
\hline & & DUTPUT & 293 \\
\hline COMON B & SLDCK PQLK14 & OUTPUT & 294 \\
\hline & - & OUTPUT & 295 \\
\hline LABEL & $=A$ CHARACTER\#26 ARRAY CONTAINING THE DISTRIBUTION TYPE & OUTPUT & 286 \\
\hline & MAMES. THIS IS SET VIA A DATA STATEMENT. (IINPUT) & OUTPUT & 297 \\
\hline & & DUTPUT & 298 \\
\hline $\operatorname{NON}$ & $X X P B$ & OUTPUT & 299 \\
\hline
\end{tabular}




\begin{tabular}{|c|c|c|c|}
\hline \multirow{4}{*}{ ILIN } & & DUTPUT & 300 \\
\hline & $=0 / 1$ POINTER INDICATES IF THE CORRESPONDING VARIABLE HAS & DUTPUT & 381 \\
\hline & ENFORCED UPPER AND LOFER LIMITS DN ITS DISTRIBUTION. & OUTPUT & 382 \\
\hline & (IINPUT) & OUTPUT & 393 \\
\hline \multirow[t]{2}{*}{ ITYPE } & $=$ INIDICATES THE DISTRIQUIOUN TYPE FOR THE CORRESPONDING & OUTPUT & \\
\hline & INPUT VARIABLE. (IINPUT) & OUTPUT & 165 \\
\hline \multirow[t]{3}{*}{ NLIM } & $=$ DIAGNDSTIC WHICH INDICATES HOW MANY OF THE RANDOM SAMPLE & OUTPUT & 366 \\
\hline & VALUES FOR THE INPUT VARIABLES WERE ABDVE OR BEOW THE USER & DUTPUT & 307 \\
\hline & SEECTED DISTRIBETION LIMITS. (RNGS) & OUTPUT & 368 \\
\hline \multirow[t]{2}{*}{ PAR } & $=$ ARRAY WHICH HOLOS THE DFINING PARNMETERS FOR THE DISTRI- & OUTPUT & \\
\hline & BUTIONS FOR EACH VARIABLE. (IINPUT) & OUTPUT & 020 \\
\hline \multirow[t]{3}{*}{ RLIN } & $=$ CONTAINS YALUES DF THE RANDCM VARIABLE ON THE INTERVAL 9,1 & DUTPUT & 311 \\
\hline & WHICH TRANSFORN TO THE LOHER AND UPPER LIMITS FOR EACH & DUTPUT & 312 \\
\hline & LINITED DISTRIBETION. (RNCS) & DUTPUT & 313 \\
\hline \multirow[t]{3}{*}{ VIM } & $=$ DESIRED LIMITS ON THE DISTRIBUTION FOR EACH INPUT VARIABLE. & DUTPUT & 314 \\
\hline & THESE ARE ONLY ACTIVATED UPON USER REQUEST, FOR THE NORUAL, & OUTPUT & 315 \\
\hline & LOGNOPUNL, AND EXPCNENTIAL DISTRIBUTIONS. (IINPUT) & OUTPUT & 316 \\
\hline \multirow[t]{3}{*}{ nIMT } & = EFECTIVE ENDPOINT LIMITS ON THE DISTRIQUTION FOR EACH INPUT & OUTPUT & 317 \\
\hline & VARIABLE. (RNGS) & OUTPUT & \\
\hline & & OUTPUT & 319 \\
\hline \multicolumn{2}{|c|}{ COLAION BLDCK PBLK16 } & DUTPUT & 326 \\
\hline------ & 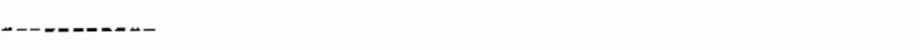 & OUTPUT & 021 \\
\hline \multirow{2}{*}{ DSEED } & $=$ THE RANDOU NUMBER SEED VALUE. REAL*8. (PACSTAT, Uø1) & OUTPUT & 322 \\
\hline & & OUTPUT & 3 \\
\hline \multicolumn{2}{|c|}{ COMON BLOCK PBLK17 } & DUTPUT & 324 \\
\hline \multirow{4}{*}{ ICCD } & --------- & OUTPUT & 325 \\
\hline & $=\theta / 1$ POINTER TD INDICATE IF CUMULATIVE OR COMPLEMENTARY & OUTPUT & 328 \\
\hline & CLMULATIVE PROBAGILITIES ARE TO BE PLOTTED/PRINTED. & DUTPUT & 327 \\
\hline & (DATOUT) & OUTPUT & \\
\hline \multirow[t]{2}{*}{ ISOLN } & = OUTPUT DATA VARIABLE ('SCLUTION') NUMBER FOR PLOTIING & OUTPUT & 329 \\
\hline & AND/OR PRINTING. (DATOUT) & OUTPUT & \\
\hline \multirow[t]{3}{*}{ IQUIT } & $=8 / 1$ POINTER TO INDICATE IF THE SAMPLING SHOUD STOP OR & OUTPUT & \\
\hline & CONTINUE NHEN AN ERRQR IS FLAGGED IN THE USER SUPPLIED & DUTPUT & \\
\hline & SOLUTION RDUTINE, SOLN. (PACSTAT) & QUTPUT & \\
\hline \multirow[t]{4}{*}{ IUPDAT } & $=6 / 1$ POINTER INDICATES IF THE INPUT STOCASTIC PARAMETERS & OUTPUT & \\
\hline & (VARIABLES OR CONSTANTS) HAVE BEEN UPDATED SINCE THE LAST & OUTPUT & \\
\hline & ITERATION OF RUNS. ONLY USED NHEN MUTIPLE 'RUNS' ARE MADE & OUTPUT & 33 \\
\hline & IN A SINCE EXECUTION OF THE PRDGRAM. (IINPUT) & DUTPUT & \\
\hline \multirow[t]{3}{*}{ MAXSAN } & I = THE MAXIMM NLNBER OF STOCHASTIC SAMFLES YHICH MAY BE TAKEN, & OUTPUT & 33 \\
\hline & GIVEN THE NLMBER OF INPUT AND OUTPUT VARIABLES, AND THE ARRAY & DUTPUT & 33 \\
\hline & SIZES (ISIZE). (PACSTAT) & OUTPUT & 34 \\
\hline \multirow[t]{2}{*}{ NPRT } & $=$ PRINT INTERYAL FOR THE OUTPUT DATA. (OATOUT, IINPUT, & QUTPUT & 34 \\
\hline & PACSTAT) & OUTPUT & 34 \\
\hline NSAM & $=$ THE NUMBER OF SAUPLES FDR THE CURRENT RUN. (IINPUT, PACSTAI) & DUTPUT & 343 \\
\hline \multirow[t]{3}{*}{ NSNUC } & $=$ THE NUMEER OF SAMPLES WHICH RAN WITHOUT ERROR FAILURE. & DUTPUT & 34 \\
\hline & (PACSTAT) & DUTPUT & 34 \\
\hline & & DUTPUT & 34 \\
\hline \multicolumn{2}{|c|}{ COMNON PQLK1B } & OLTPUT & 34 \\
\hline & 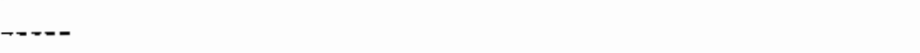 & DUTPUT & 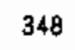 \\
\hline \multirow{2}{*}{ JJNF } & $=A$ LIST OF FILE NAMES FUR DATA WHICH IS TO BE READ FROM & OUTPUT & 3 \\
\hline & EXTERNAL FILES. (IINPUT) & OUTPUT & 35 \\
\hline \multirow[t]{2}{*}{ INFIL } & = FILE UNIT NLNBER FOR DATA TO BE READ FROU EXTERNAL & OUTPUT & 35 \\
\hline & FILES. (PACSTAT) & OUTPUT & 352 \\
\hline INDIST & $=$ THE NUMBER OF DISCRETE POINTS DFINING A CUMLULATIVE & OUTPUT & 353 \\
\hline
\end{tabular}


CONFIOENCE FUNCTIDN, THEN THAT FUNCIIDN IS READ FRDN

AN EXTERNAL FILE. (R2DAT)

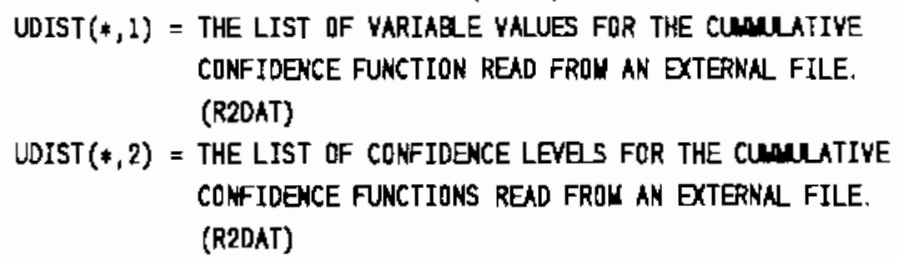

COWON IDCOH

\begin{tabular}{|c|c|}
\hline JPUNID & = RUN IDENTIFIER OF THE CURRENT RUN, (IONUN) \\
\hline $\mathrm{FL}$ & $\begin{aligned}= & \text { FILE TYPE IDENTIFIER FOR THE OUTPUT BINARY FILES. } \\
& \text { (PACSTAT). }\end{aligned}$ \\
\hline FLT (MAXSRC) & $\begin{aligned}= & \text { FILE TRACEBACK ON BINARY I/O FILES. (RHEADR, NOT } \\
& \text { USED BY PACSTAT) }\end{aligned}$ \\
\hline NRUNID ( $M N X S R C)$ & $\begin{aligned}= & \text { FILE TRACEBACK IDENTIFIERS. (RHEADR, NDT USED BY } \\
& \text { PACSTAT) }\end{aligned}$ \\
\hline NSRC & $\begin{aligned}= & \text { NLMBER OF TRACEBACK FILES ON BINARY I/D FILES, } \\
& \text { (RHEAOR, NOT USED BY PACSTAT) }\end{aligned}$ \\
\hline PRO & = MONTE CARLO SIMLATION PROGRAU NAHE. (BLKDAT) \\
\hline PRGT (MAXSRC) & $\begin{aligned}= & \text { SOURCE CODE NAMES OF TRACEBACK FILES. (RHEADR, NOT } \\
& \text { USED EY PACSTAT) }\end{aligned}$ \\
\hline TITLE(2ס) & $=$ TITLE FOR BINARY I/O FILE HEAOER. (PACSTAT) \\
\hline USRNAM & = NAME OF CURRENT PROGRAN USER. \\
\hline USRNLT (NAXSRC) & $\begin{aligned}= & \text { USER NAME ON TRACEEACK FILES. (RHEAOR, NOT USED BY } \\
& \text { PACSTAT) }\end{aligned}$ \\
\hline VERNUN & $\begin{aligned}= & \text { VERSION MUMBER OF MDNTE CARLO SIULATTION PROGRAM. } \\
& \text { (BLKDAT) }\end{aligned}$ \\
\hline
\end{tabular}

COWON BLOCX INOUT

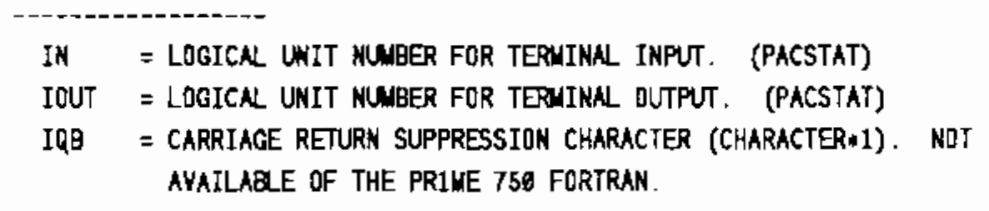

\section{COHUON QLOCK STRATI}
MAXLEV = THE MAXIMN NUMBER DF STARTIFICATION LEYES WHICH MAY BE HANDLE BY THE UNIFORN DISTRIBUTION ROUTINE UDIS.
NSPL = A COUNTER IHICH SHOWS THE NLABER OF POINTS TAKEN FRON EACH LEVE DF STRATIFICATION. IT IS UPDATED EACH TIME A NET, STARTIFIED UNIFOFW DISTRIBUTION IS CREATED. (UQ1S)

COMON BDCX ULIMTS

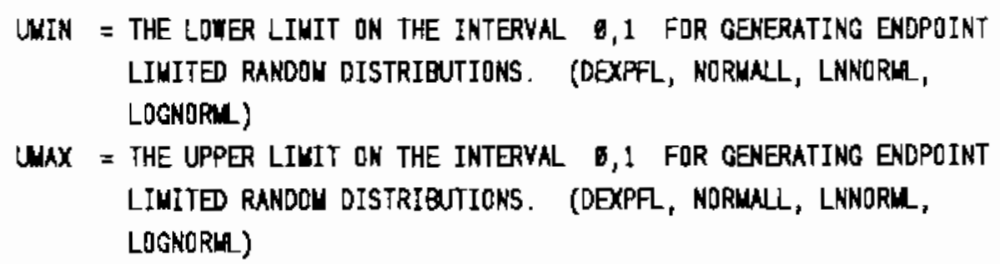



SIMUATION. AN OUTPUT YARIAQLE (OR SEVERAL OUTPUT YALRIABCES) IS IS COMPUTED FRON SEYERN INPUT YARIABLES, THE INPUT YARIABCES CAN TAKE ON ANY OF EIGHT DIFFERENT DISTRIBUTION TYPES, DHICH THE USER CHOOSES (RDUTINE IINPUT). THE USER WUST ALSO SPECIFY THE DEINING PARALETERS OF THE DISTRIQUTION FOR EACH INPUT VARIABLE.

A PRINTOUT OF A CHOSEN DUTPUT YARIABEE IS GIVEN IITH DIAGNGSTICS ON BOTH THE OUTPUT ANO INPUT DISTRIQNIIONS. THIS IS DIRECTED TO AN OPTIDNAL, FORUTTED OUTPUT FILE, OUTPUT MAY ALSO BE URITTEN TO A BIMARY OUTPUT FILE, FOR POST PROCESSING.

THE ROUTINE BLKDAT AKD SOLN WST BE PROVIDED BY THE USER. INPUT AND OUTPUT YARIAQLE NAMES AND DIMENSIONS (UNITS) ARE SET IN BLOCK DATA (ROUTINE BLKDAT). THE YALUE OF NYAR SETS THE NUMBER OF INPUT VARIABLES. THE VALUE OF NSOLN SETS THE NUMBER OF AVAILABLE SOLUTIONS. IF THESE EXCEDD PROGRAM LIMITS, AN ERROR VIU OE PRINTED AND THE RUN TERINATED.

SEYERAL USER DEFINABLE CONSTANTS ARE PEPITTED. THE NUMBER OF CONSTANTS IS SET IN BLOCK DATA AS VARIABLE NCONST. THE NAMES AND DINENSIONS OF EACH CONSTANT WUST ALSO BE SET IN BLOCK DATA. DNE WUST NOT CHOSE WORE CONSTANTS THAN THE PROGRN IS DIUENSIONED TO ACCOUODATE. WCONST = ZERO IS PERMITTED.

WTIPLE SOLUTIONS FOR ANY GIVEX PRABLE ARE NLOWED. NLL OF THE SOLUTIONS UST BE COMPUTED IN ROUTINE SOLN. ROUTINE SOLN IS CALLED ONCE FOR EACH RANDOU SNPLE. IT TAKES THE INPUT VALUES FRDM ARRAY VINPT(1... NYAR) (COMON RLOCX PBLKA). OUTPUT VALUES WUST BE STORED IN ARRAY VALU(1... NSOLN) (COLEON BLOCK PQLK4) FOR RETURN TO THE CA $\perp I N G$ ROUTINES. THESE DUTPUT VALUES ARE WOVED TO ARRAY RLTS FOR LOWG TERU STORAGE, AND THE TO ARRAY RPLT (ROUTINE DATOUT) FOR PLOTTING AND DIAGNOSTICS. ONLY DNE SOLUTION WAY BE PLOTTED/ PRINTED AT A TIME.

WACHINE OEPENDENT CODING FOR THIS PROGRAN IS RESTRICTED TO RDUTINES ASG, RELEAS (OPENING AND CLOSING FILES, AND IDNUN (FILE IDENTIFIERS).

THE RANDOW MUABER GENERATOR, UB1(DSEFD), IS A FORTRAN CODED ROUTINE. IT IS ONLY CALLED FROU THE MAIN ROUTINE AND IN ROUTINE UפIS. THE SEED, DSEED, IS A DOURLE PRECISION REAL NUMAER, AND IS THE OHLY ITEN IN COWON BLOCK PBLK16.

THE PARAMETER ISIZE SETS THE WAXILUM NUMBER OF RANDOM SAMPLES WHICH WAY BE USED. THE PARAMETER WAXNS SETS THE UAXIHUM MUMBER OF SNAPLS PER INPUT/OUTPUT VARIABLE WHICH WAY BE COMPUTED. THIS REQUIRES THAT WAXNS MUST BE LESS THAN OR EQUAL TO ISIZE. NDTE THAT SOME TRANSFERING OF RANDOW ARRAYS IS DONE IN ROUTINES VARSET AND STATOT, WHICH IS ONLY NECESSARY ON VIRTUAL MENORY COMPUTERS, THERE THE PARAMETER ISIZE EXCEEDS THE PAGE SIZE. ALSO, IF MAXNS EXCEFDS HALF THE PAGE SIZE, THE COLONON BLOCK PBLK6 WAY NEED TO BE CHANGED. 


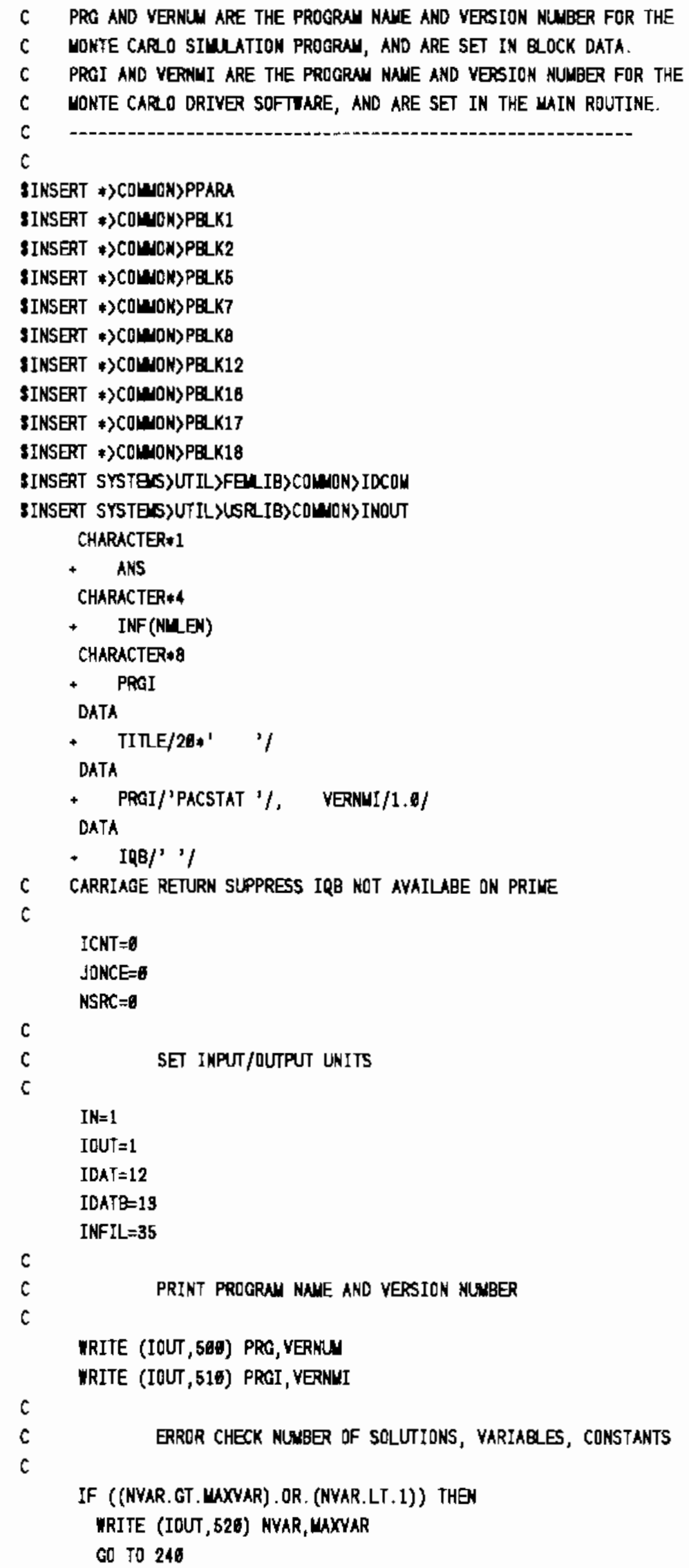




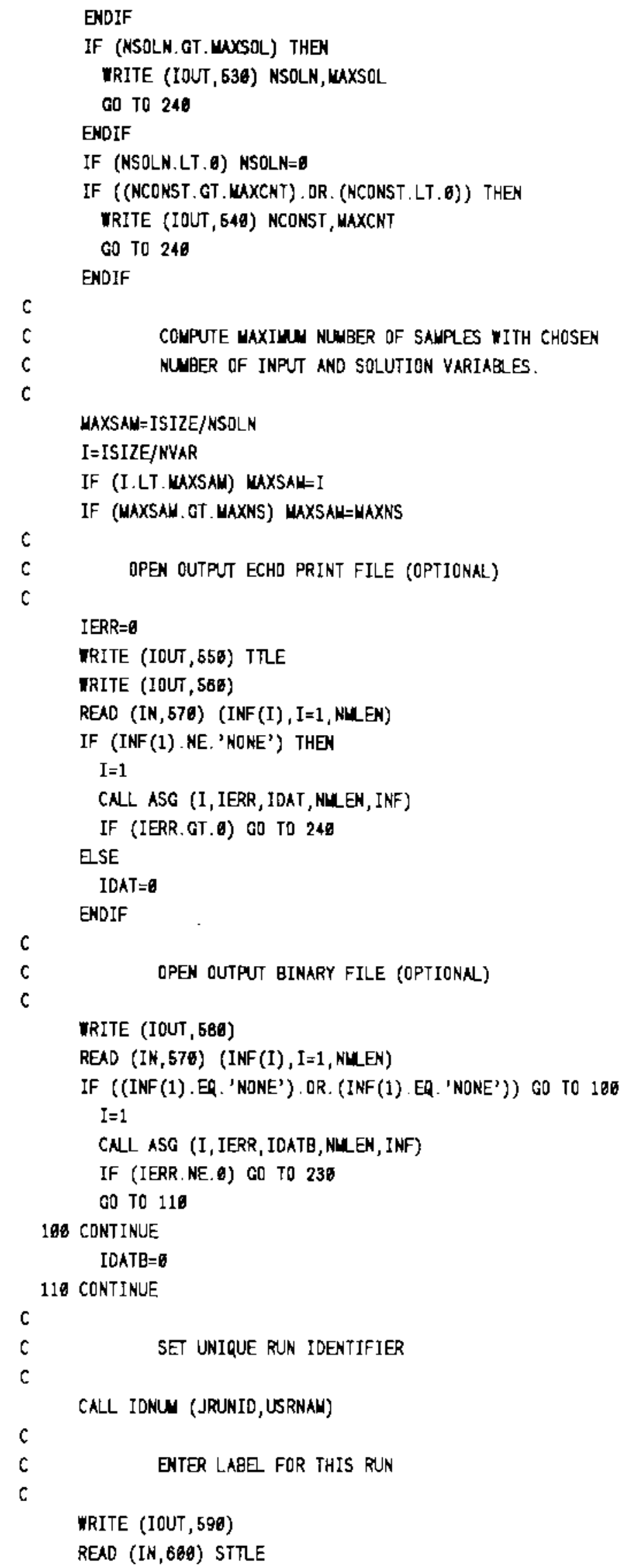

\begin{tabular}{|c|c|}
\hline ACSTAT & 110 \\
\hline CSTAT & , \\
\hline CSTAT & 11 \\
\hline CSTAT & 113 \\
\hline ACSTAT & 114 \\
\hline ACSTAT & 115 \\
\hline ACSTAT & 116 \\
\hline ACSTAT & 117 \\
\hline ACSTAT & 118 \\
\hline ACSTAT & 119 \\
\hline ACSTAT & 120 \\
\hline ACSTAT & 121 \\
\hline ACSTAT & 122 \\
\hline PACSTAT & 123 \\
\hline PACSTAT & 124 \\
\hline PACSTAT & 125 \\
\hline PACSTAT & 126 \\
\hline PACSTAT & 127 \\
\hline PACSTAT & 128 \\
\hline PACSTAT & 129 \\
\hline PACSTAT & 136 \\
\hline PACSTAT & 131 \\
\hline PACSTAT & 132 \\
\hline PACSTAT & 133 \\
\hline PACSTAT & 134 \\
\hline PACSTAT & 135 \\
\hline PACSTAT & 136 \\
\hline ACSTAT & 137 \\
\hline ACSTAT & 138 \\
\hline PACSTAT & 139 \\
\hline PACSTAT & 148 \\
\hline ACSTAT & 141 \\
\hline PACSTAT & 142 \\
\hline PACSTAT & 43 \\
\hline PACSTAT & 144 \\
\hline PACSTAT & 145 \\
\hline PACSTAT & 148 \\
\hline PACSTAT & . 147 \\
\hline PACSTAT & 148 \\
\hline PACSTAT & 149 \\
\hline PACSTAT & 150 \\
\hline PACSTAT & 151 \\
\hline PACSTAT & 152 \\
\hline PACSTAT & 153 \\
\hline PACSTAT & 154 \\
\hline PACSTAT & 155 \\
\hline PACSTAT & 158 \\
\hline PACSTAT & 157 \\
\hline PACSTAT & 158 \\
\hline PACSTAT & 159 \\
\hline SACSTAT & 160 \\
\hline ACSTAT & $1 \in 1$ \\
\hline PACSTAT & 62 \\
\hline ACSTAT & 163 \\
\hline
\end{tabular}


$c$

$c$

c

$K=41$

129 CONTINUE

$\mathrm{K}=\mathrm{K}-1$

IF ((TTLE(K:K).EQ.' ') .AND. (K.GT.1)) GO TO 120

IF ((TTLE(1:1).EQ.' '),AND. (K.EQ.1)) K=1

NTTL $=K$

$\mathrm{K}=41$

138 CONTINUE

$x=k-1$

IF ((STTLE(K:K).ER.' ').AND.(K.GT.1)) GO TO 136

IF ((STTLE(1:1).EQ. ' ') . AND. (K.EQ.1)) $K=0$

NSTTL $=x$

$c$

c CREATE TITLE FOR THIS RUN

C

DO $140 I=1,10$

$\mathrm{J}=\mathrm{I}+10$

$\mathrm{I} 2=4 * \mathrm{I}$

$\mathrm{I} 1=\mathrm{I} 2-3$

$\operatorname{TITLE}(\mathrm{I})=\operatorname{TTL}(\mathrm{I} 1: 12)$

IITLE(J)=STTLE $\{$ I $1:$ I 2$)$

146 CONTINUE

c

c OPTION TO TGRNATE OR CONTINUE ON SOLUTION ERROR

$c$

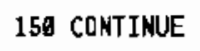

IQUIT $=0$

IF (NSOLN.GT.6) THEM

WRITE (IOUT, B10)

READ (IN, 626) ANS

IF ((ANS. NE. 'C'). AND. (ANS. NE. 'Q')) THEN

IF ((ANS. NE. ' $c$ ') . AND. (ANS.NE. ' $q$ ')) GO TO 158

ENDIF

IF ((ANS. EQ. ' 'Q') .OR. (ANS. ER. ' 'q')) IQUIT=1

ENDIF

c

$c$

c

180 CONTINUE

ICNT=ICNT + 1

$c$

c

CNLL IINPUT

c

C SET DATA ARRAY LDCATION POINTERS

$c$

DO $170 \mathrm{I}=1, \mathrm{~N}$ YAR

$\operatorname{NMLCC}(I)=(I-1) * N S A M+1$

179 CONTINUE

IF (NSOLN.GT.6) THEN

\begin{tabular}{|c|c|}
\hline CSTAT & 164 \\
\hline ACSTAT & 185 \\
\hline PACSTAT & 166 \\
\hline PACSTAT & 187 \\
\hline PACSTAT & 188 \\
\hline PACSTAT & 169 \\
\hline PACSTAT & 178 \\
\hline PACSTAT & 171 \\
\hline PACSTAT & 172 \\
\hline PACSTAT & 173 \\
\hline PACSTAT & 174 \\
\hline PACSTAT & 175 \\
\hline PACSTAT & 176 \\
\hline PACSTAT & 177 \\
\hline PACSTAT & 178 \\
\hline PACSTAT & 179 \\
\hline PACSTAT & 180 \\
\hline PACSTAT & 181 \\
\hline PACSTAT & 182 \\
\hline PACSTAT & 183 \\
\hline PACSTAT & 184 \\
\hline ACSTAT & 185 \\
\hline PACSTAT & 188 \\
\hline PACSTAT & 187 \\
\hline PACSTAT & 188 \\
\hline ACSTAT & I89 \\
\hline ACSTAT & 190 \\
\hline ICSTAT & 191 \\
\hline ACSTAT & 192 \\
\hline ACSTAT & 193 \\
\hline ACSTAT & 194 \\
\hline ACSTAT & 195 \\
\hline ACSTAT & 196 \\
\hline ICSTAT & 197 \\
\hline ACSTAT & 198 \\
\hline ACSTAT & 199 \\
\hline ACSTAT & 260 \\
\hline ACSTAT & 281 \\
\hline PACSTAT & 282 \\
\hline PACSTAT & 203 \\
\hline PACSTAT & 204 \\
\hline 'ACSTAT & 285 \\
\hline 'ACSTAT & 296 \\
\hline PACSTAT & 297 \\
\hline PACSTAT & 208 \\
\hline PACSTAT & 269 \\
\hline PACSTAT & 210 \\
\hline PACSTAT & 211 \\
\hline PACSTAT & 212 \\
\hline ACSTAT & 213 \\
\hline CSTAT & 214 \\
\hline ESTAT & 215 \\
\hline STAT & 216 \\
\hline STRAT & 217 \\
\hline
\end{tabular}




\begin{tabular}{|c|c|c|c|}
\hline & DO $188 I=1$, NSDLN & PACSTAT & 218 \\
\hline & $\operatorname{NSLDC}(I)=(I-1) * N S N+1$ & PACSTAT & 219 \\
\hline 188 & CONTINUE & PACSTAT & .220 \\
\hline & ENDIF & PACSTAT & . 221 \\
\hline c & & PACSTAT & . 222 \\
\hline c & USER ENTERS SEED FOR RANDOM NLMBER GEKERATOR & PACSTAT & 223 \\
\hline c & & PACSTAT & . 224 \\
\hline 1960 & CONTINUE & PACSTAT & 225 \\
\hline & IF (ICNT.LT.2) THEN & PACSTAT & . 226 \\
\hline & WRITE (IOUT,630) & PACSTAT & 227 \\
\hline & READ (IN, *,ERR=196) 10 & PACSTAT & . 228 \\
\hline & IF ((WO.LT.2.).OR. (WD.GT.2.E+9)) GO TO 190 & PACSTAT & . 229 \\
\hline & DSEFB $=$ OBLE $(T 0)$ & PACSTAT & . 230 \\
\hline & $A=U Q 1$ (DSEFD) & PACSTAT & . 231 \\
\hline & EMDIF & PACSTAT & . 232 \\
\hline C & & PACSTAT & . 233 \\
\hline C-- & 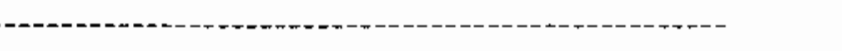 & PACSTAT & 234 \\
\hline c & & PACSTAT & . 235 \\
\hline c & SET INPUT VARIAQQE ARRAYS & PACSTAT & . 236 \\
\hline$c$ & & PACSTAT & . 237 \\
\hline & CALL VARSET (IERR) & PACSTAT & . 238 \\
\hline & IF (IERR.NE. $\sigma$ ) THEN & PACSTAT & . 239 \\
\hline & WRITE (IOUT,640) & PACSTAT & 246 \\
\hline & IF (IDAT.GT. E) VRITE (IDAT, B46) & PACSTAT & . 241 \\
\hline & GO TO 220 & PACSTAT & . 242 \\
\hline & ENDIF & PACSTAT & . 243 \\
\hline c & & PACSTAT & . 244 \\
\hline C-.-... & 列 & PACSTAT & . 245 \\
\hline $\mathrm{C}$ & & PACSTAT & . 246 \\
\hline c & COUPUTE AND PRINT THEDRETICAL DISTRIBUTION SPECS & PACSTAT & . 247 \\
\hline C & & PACSTAT & . 248 \\
\hline & $C A L \perp$ EXPECT & PACSTAT & . 249 \\
\hline$c$ & & PACSTAT & . 250 \\
\hline C------ & - & PACSTAT & . 251 \\
\hline$c$ & & PACSTAT & . 252 \\
\hline C & COMPUTE OUTPUT YARIABLE VALUES & PACSTAT & 253 \\
\hline C & & PACSTAT & . 254 \\
\hline & CALL DATSET (IERR) & PACSTAT & . 255 \\
\hline & IF (IERR.NE. 6) CO TO 208 & PACSTAT & 256 \\
\hline & NSAMC=NSAM-NLMERR & PACSTAT & . 257 \\
\hline C & & PACSTAT & . 258 \\
\hline c & CHECK FOR GOOD SOLUTIONS & PACSTAT & . 259 \\
\hline c & & PACSTAT & . 289 \\
\hline & IF (NUMERR.EQ.NSAN) THEN & PACSTAT & . 261 \\
\hline & WRITE (IOUT,65б) & PACSTAT & . 262 \\
\hline & $I=N S O L N$ & PACSTAT & $\quad 263$ \\
\hline & $N S O L N=6$ & PACSTAT & . 264 \\
\hline & CALL STATOT & PACSTAT & . 265 \\
\hline & NSOLN=I & PACSTAT & . 286 \\
\hline & GO TO 220 & PACSTAT & . 267 \\
\hline & ENDIF & PACSTAT & . 268 \\
\hline C & & PACSTAT & . 269 \\
\hline$c$ & RE-ORDER AROUND ANY BAD SOLUTIONS WHICH WERE FOUND & PACSTAT & 270 \\
\hline$c$ & & PACSTAT & . 271 \\
\hline
\end{tabular}




\begin{tabular}{|c|c|c|c|}
\hline & NSAK =NSA & PACSTAT & . 272 \\
\hline & IF (NUERR.GT.0) CN丩 REOROR & PACSTAT & . 273 \\
\hline & GO TO 210 & PACSTAT & . 274 \\
\hline C & & PACSTAT & . 275 \\
\hline C-n-- & 足- & PACSTAT & . 276 \\
\hline C & & PACSTAT & . 277 \\
\hline C & SAL YAGE GOQD DATA ON ERROR STOP & PACSTAT & . 278 \\
\hline C & & PACSTAT & . 279 \\
\hline 200 & CENT INUE & PACSTAT & . 280 \\
\hline & $A=$ FLOAT (NPRT) /FLOAT (NSAN) & PACSTAT & . 281 \\
\hline & NSAC=NSAH & PACSTAT & . 282 \\
\hline & WRITE (IOUT, 689) INDEX & PACSTAT & . 283 \\
\hline & IF (IDAT.GT. O) WRITE (IOAT, B66) INOEX & PACSTAT & . 284 \\
\hline & IF (NSA.LT.1) GO TO 228 & PACSTAT & . 285 \\
\hline & WRITE (IOUT, 870) & PACSTAT & . 286 \\
\hline & IF (IDAT.GT. ) WRITE (IDAT, B7б) & PACSTAT & . 287 \\
\hline & NPRT $=I N T(F L A T(N S N) * A)$ & PACSTAT & . 288 \\
\hline & IF (NPRT.LE. 1 ) NPRT=1 & PACSTAT & . 289 \\
\hline 210 & CONTINUE & PACSTAT & . 298 \\
\hline c & & PACSTAT & . 291 \\
\hline$c$ & IRITE BINARY OUTPUT FILE HEADER, ON FIRST ITERATIDN ONLY & PACSTAT & . 292 \\
\hline c & & PACSTAT & . 283 \\
\hline & IF (ICNT.EQ.1) THEN & PACSTAT & . 294 \\
\hline & IHENDR $=1$ & PACSTAT & . 295 \\
\hline & $\mathrm{FL}=$ 'STDCAST' & PACSTAT & . 298 \\
\hline & 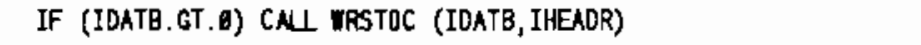 & PACSTAT & . 297 \\
\hline & ENOIF & PACSTAT & . 298 \\
\hline C & & PACSTAT & . 299 \\
\hline c & WRITE DATA TO BINARY OUTPUT FILE & PACSTAT & . 360 \\
\hline C & & PACSTAT & . 361 \\
\hline & IHEADR=0 & PACSTAT & . 362 \\
\hline & IF (IDATB.GT. C) CAL WRSTOC (IDATB, IHEADR) & PACSTAT & . 383 \\
\hline C & & PACSTAT & . 304 \\
\hline C & CHECK OUTPUT STATISTICS & PACSTAT & . 305 \\
\hline C & ** THIS ROUTINE SORTS INPUT AND DUTPUT VARIABLES & PACSTAT & . 396 \\
\hline C & * IN ASCENDING OROER (TO COMPUTE MEDIAN) & PACSTAT & . 307 \\
\hline C & & PACSTAT & . 368 \\
\hline & CALL STATOT & PACSTAT & . 369 \\
\hline C & & PACSTAT & 310 \\
\hline$c$ & PRINT/PLDT QUTPUT DATA & PACSTAT & . 311 \\
\hline C & & PACSTAT & 312 \\
\hline & IF (NSOLN.GT. $)$ ) CALL DATDUT (JONCE) & PACSTAT & 313 \\
\hline c & & PACSTAT & 314 \\
\hline$c$ & ANDTHER RUN OR STOP & PACSTAT & 315 \\
\hline C & & PACSTAT & 318 \\
\hline 220 & CONTINUE & PACSTAT & . 317 \\
\hline & WRITE (IOUT, 689) IR日 & PACSTAT & . 318 \\
\hline & READ $(I N, 620)$ ANS & PACSTAT & 319 \\
\hline & IF ((ANS. NE. 'Y') . AND. (ANS .NE. 'N')) THEY & PACSTAT & 320 \\
\hline & IF ((ANS.NE. 'y') AND. (ANS.NE. 'n')) GO TO 229 & PACSTAT & 321 \\
\hline & ENDIF & PACSTAY & 322 \\
\hline & IF ((ANS.EQ. 'Y'). OR. (ANS.EQ. 'y')) GO TO 180 & PACSTAT & . 323 \\
\hline$c$ & & PACSTAT & . 324 \\
\hline C & END OF PROGRH & PACSTAT & 325 \\
\hline
\end{tabular}


IF (JONCE, GT. .) CN丩 DONEPL

IF (IDATB.GT.B) CN丩 REEAS (IDATB)

238 CONTINUE

IF (IDAT.GT. .) CAL RECEAS (IDAT)

240 CONTINUE

STOP

c

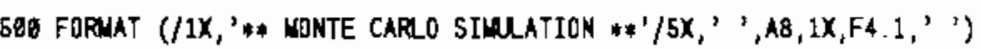

516 FORUAT $\left(5 X,{ }^{\prime}\left(', A B, 1 X, F 4.1,{ }^{\prime}\right) '\right)$

520 FORMAT (/1X,' 'ERROR, THE NUMEER OF INPUT VARIARLES $=', I 6, '$ EXCEEDS

+ THE'/1 $1 x_{1}$ 'PROGRAN LIMIT OF ',13,' , DR IS LESS THAN ONE. '/1X, 'PROGR - AH STOP.')

539 FORMAT (/1X, 'ERROR, THE NUMBER OF AVAILABLE SOLUTIONS =', I5, ' EXC +EDS PROGRA LIUIT DF ', I3, '.'/1X, 'PROGRAM STDP.')

540 FORMAT (/1X, 'ERROR, MUMER OF CONSTANTS $=', 15, '$ EXCEEDS PRDGRAM $L$ +IMIT OF ',13,' ' $11 \mathrm{X}$, 'PROGRAM STOP.')

550 FQRNAT $(/ 1 X, A 46)$

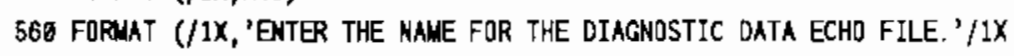
•, '(' "NDNE'' TO ONIT)')

576 FORAMT (2ER4)

589 FORUAT (/1X, 'ENTER THE NAME FOR THE BINARY OUTPUT DATA FILE. '/1X,' +('MONE' TO OMIT)')

599 FORMT (/1X, 'ENTER LABE FOR THIS RUN (MAX 40 CHARACTERS)')

6EO FORMAT (A4B)

610 FORMAT (/1X, 'TOUD YOU LIKE TO CONTINUE DR QUIT IF AN ERROR'/1X, 'I +S ENCQUNTERED IN THE SOLUTION ROUTINE (C/Q)? ')

B26 FORMAT (A1)

636 FORUAT (/1X, 'ENTER AN INITIAL SEED NLMBER FOR THE RANDDM NUMBER GE +NERATOR. ' $/ 1 X^{\prime}$ ' '(A RENL NUMBER BETHEEN 1.6 AND 2.8E+9)')

640 FORMAT (/1X, ${ }^{\prime} * \#$ ERROR COMPUTING INPUT DISTRIBUTION DATA. THIS ITE + RATION WAS'/4X, 'ABORTED DUE TO AN INABILITY TO COMPUTE AND/OR READ

+ THE DESIRED'/4X, 'RANDON SAMPLES.')

858 FDRMAT (/1X, '*** ERROR, NO SUCCESSFU SAMPLES WERE COUPUTED. '/5X,'

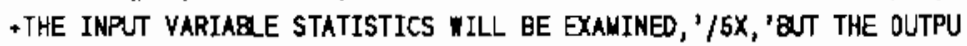
+T DAIA TILL BE IGNORE, AND THE BINARY'/5X, 'OUTPUT FILE RECORD WIL +L NOT BE TRITTEN.')

860 FORMAT (///5X,'--- ERROR ABORT, SAMPLE NUMBER ',I5)

676 FDRMAT (5X,"--- PROCEDURE NILL CDNTINUE WITH REDUCED SAMPLE SPACE +')

B8E FDRMAT (/1X,' 'CREATE ANOTHER DATA SET $(Y / N)$ ? ',Al)

BND

SUBROUTINE PLINIT (XL2, n2, IRUN)

$c$

ROUTINE FOR CHOSING INTERACTIVE/NON-INTERACTIVE PLOTIING, SETIING PAGE SIZE, AND INITIALIZING PLOTTING PACKAGE.

XL2, YL2 = PAGE SIZE (INCHES, INITIALIZED BY CALING ROUTINE)

IRUN $=1 / 0$ INTERACTIVE/NON-INTERACTIVE PLDTTING

C

SINSERT \#)COMMON)INOUT

$c$

CHARACTER*1

$+\quad$ ANS

c 


\begin{tabular}{|c|c|c|c|}
\hline$c$ & INTER/NON-INTERACTIVE PLOTIING & PLINIT & 14 \\
\hline C & & PLINIT & 15 \\
\hline & IRUN=1 & PLINIT & 16 \\
\hline 108 & CONTINUE & PLINIT & 17 \\
\hline & WRITE (IOUT, 560) & PLINIT & 18 \\
\hline & READ $(I N, *$, ERR $=1$ 108) KDEY & PLINIT & 19 \\
\hline & IF ((KDEY.LT.6).OR. (KDEY.GT.5)) GO TO 100 & PLINIT & 20 \\
\hline c & & PLINIT & 21 \\
\hline c & INIT PAGE SIZE (XL2, YL2). NLOW USER MODIFICATION. & PLINIT & 22 \\
\hline c & & PLINIT & 23 \\
\hline & IF (XL2.LE. .) XL2=14.2 & PLINIT & 24 \\
\hline & IF (YL2.LE. .) YL2=16.1 & PLINIT & 25 \\
\hline 110 & CONTINUE & PLINIT & 26 \\
\hline & TRITE (IOUT, 510) XL2, YL2, IQB & PLINIT & 27 \\
\hline & READ (IN, 526) ANS & PLINIT & 28 \\
\hline & IF ((ANS.NE. 'Y') AND. (ANS.NE. 'N')) TKEN & PLINIT & 29 \\
\hline & IF ((ANS. NE. 'y'), AND. (ANS.NE. 'n')) GO TO 110 & PLINIT & 30 \\
\hline & ENDIF & PLINIT & 31 \\
\hline & IF ((ANS.ER.'Y'), OR. (ANS.EQ. 'y')) THEN & PLIKIT & 32 \\
\hline 120 & CONTIME & PLINIT & 33 \\
\hline & TRITE (IOUT, 536) & PLINIT & 34 \\
\hline & READ $(I N, *, E R R=120) X 2, Y L 2$ & PLINIT & 35 \\
\hline & IF (XL2.LF.8.) XL2=8. & PLINIT & 36 \\
\hline & IF (YL2.LT.6.) YL2=6. & PLINIT & 37 \\
\hline & ENDIF & PLINIT & 38 \\
\hline c & & PLINIT & 39 \\
\hline c & GRAPHICS INITIALIZATIONS & PLINIT & 40 \\
\hline c & & PLINIT & 41 \\
\hline & $\mathrm{KDEV}=\mathrm{KDEV}+1$ & PLINIT & 42 \\
\hline & GD TO $(130,140,150,160,170,180)$, KDEV & PLINIT & 43 \\
\hline $\mathrm{C}$ & & PLINIT & 44 \\
\hline 136 & CONTINUE & PLINIT & 45 \\
\hline & CNL COMPRS & PLINIT & 46 \\
\hline & IRUN=ø & PLINIT & 47 \\
\hline & GO TO 490 & PLINIT & 48 \\
\hline C & & PLINIT & 49 \\
\hline 140 & CONTINUE & PLINIT & 50 \\
\hline & CA TK TO14 $(126,0)$ & PLINIT & 51 \\
\hline & G0 TO 490 & PLINIT & 52 \\
\hline c & & PLINIT & 53 \\
\hline 158 & CONTINUE & PLINIT & 54 \\
\hline & 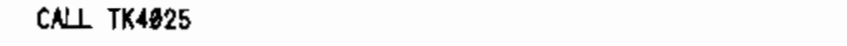 & PLINIT & 55 \\
\hline & GD TO 499 & PLINIT & 56 \\
\hline C & & PLINIT & 57 \\
\hline 186 & CONTINUE & PLINIT & 58 \\
\hline & CNL TK4054 $(120, \theta)$ & PLINIT & 59 \\
\hline & GO to 490 & PLINIT & 60 \\
\hline C & & PLINIT & 61 \\
\hline 176 & CONTINUE & PLINIT & 62 \\
\hline & CAL REGIS $(1, \emptyset)$ & PLINIT & 63 \\
\hline & CO TO 490 & PLINIT & 64 \\
\hline c & & PLINIT & 85 \\
\hline 189 & CONTINUE & PLINIT & 66 \\
\hline & CALL SLNRYT (120) & PLINIT & 67 \\
\hline
\end{tabular}




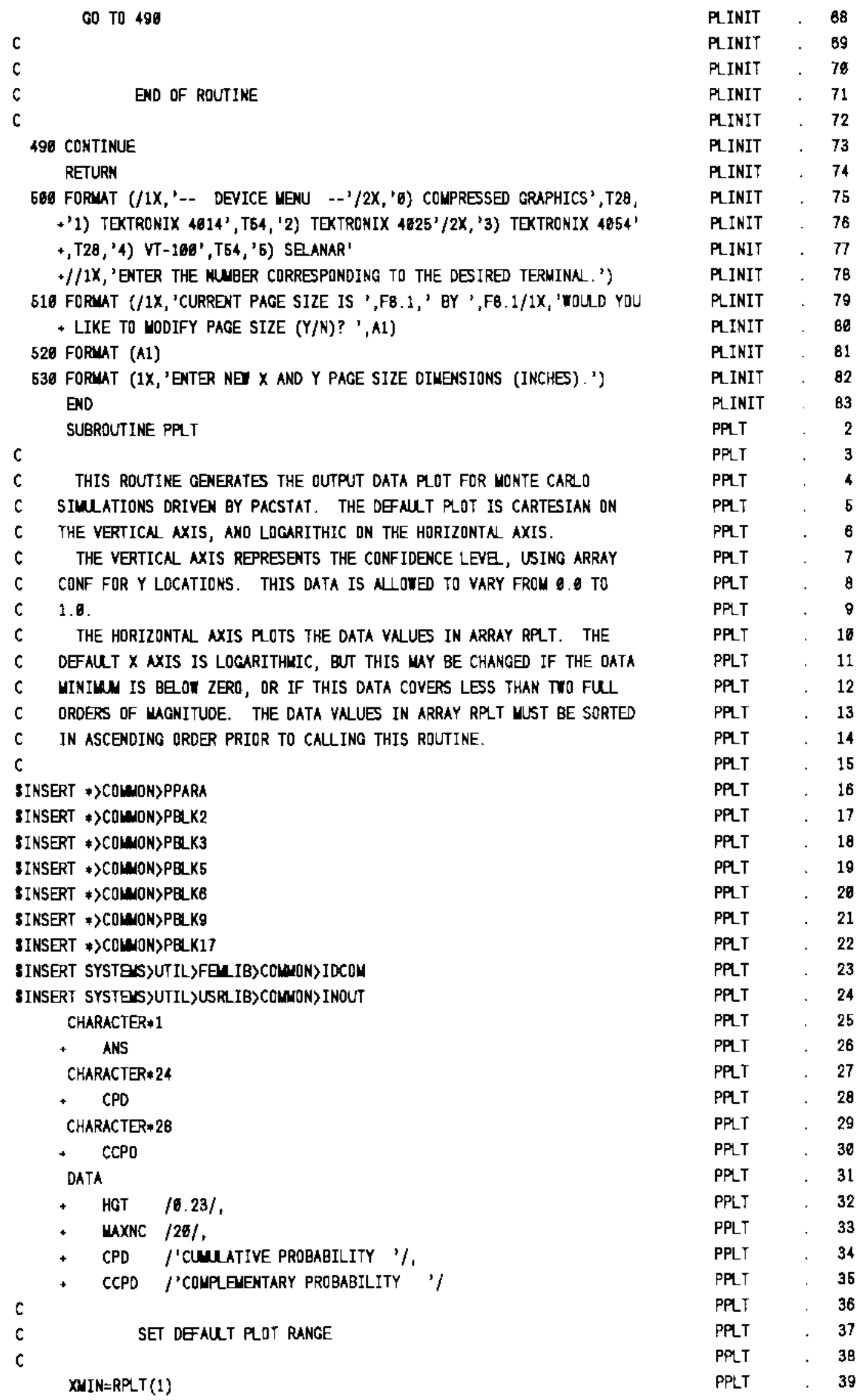




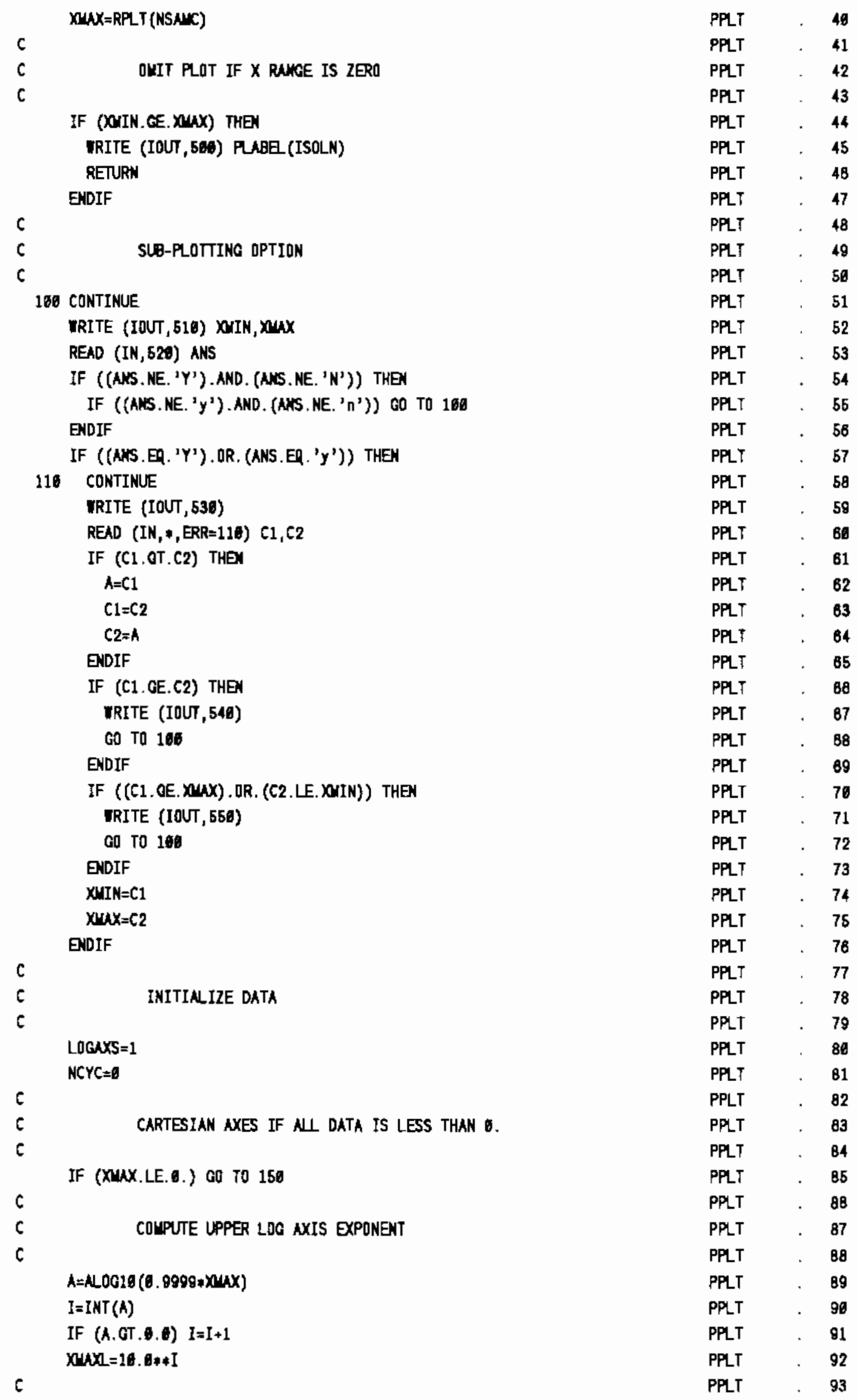




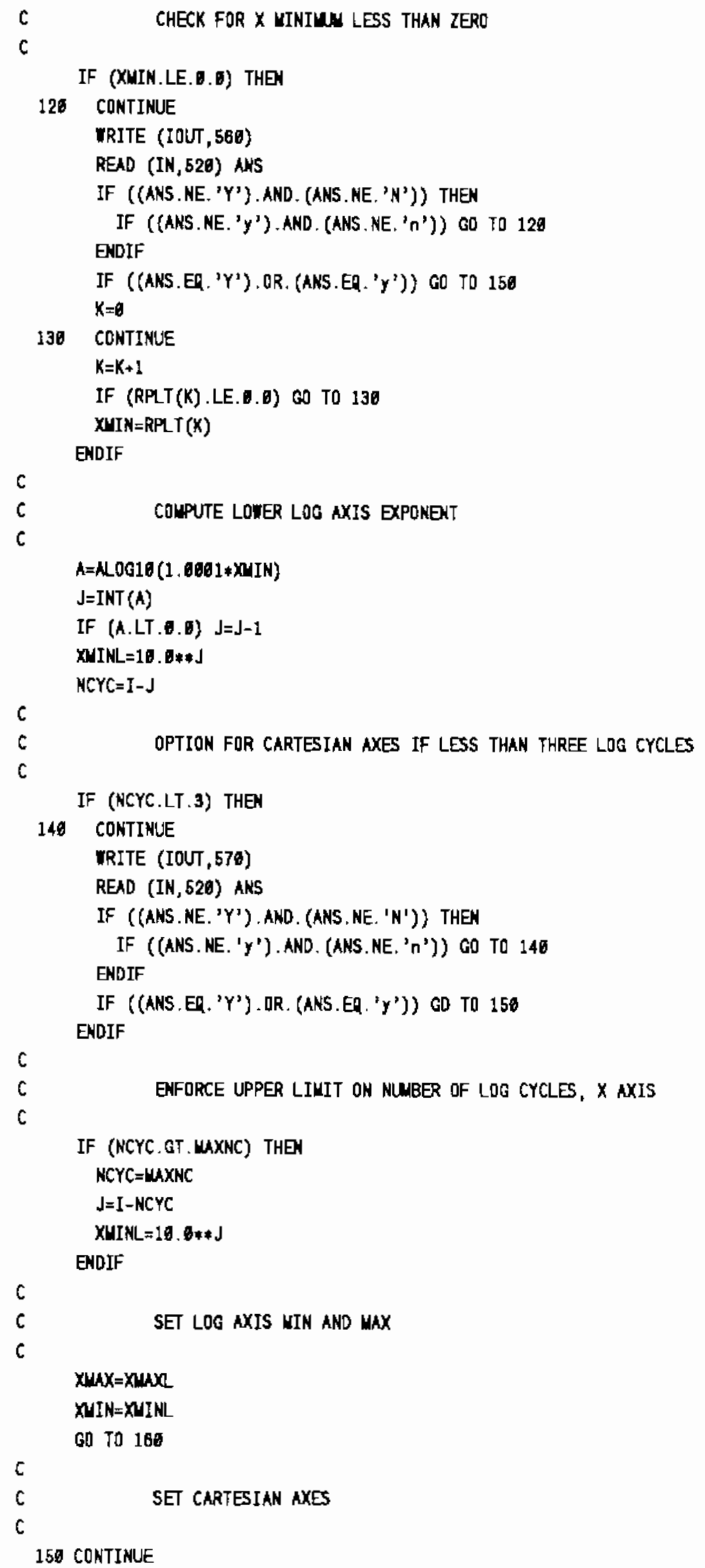




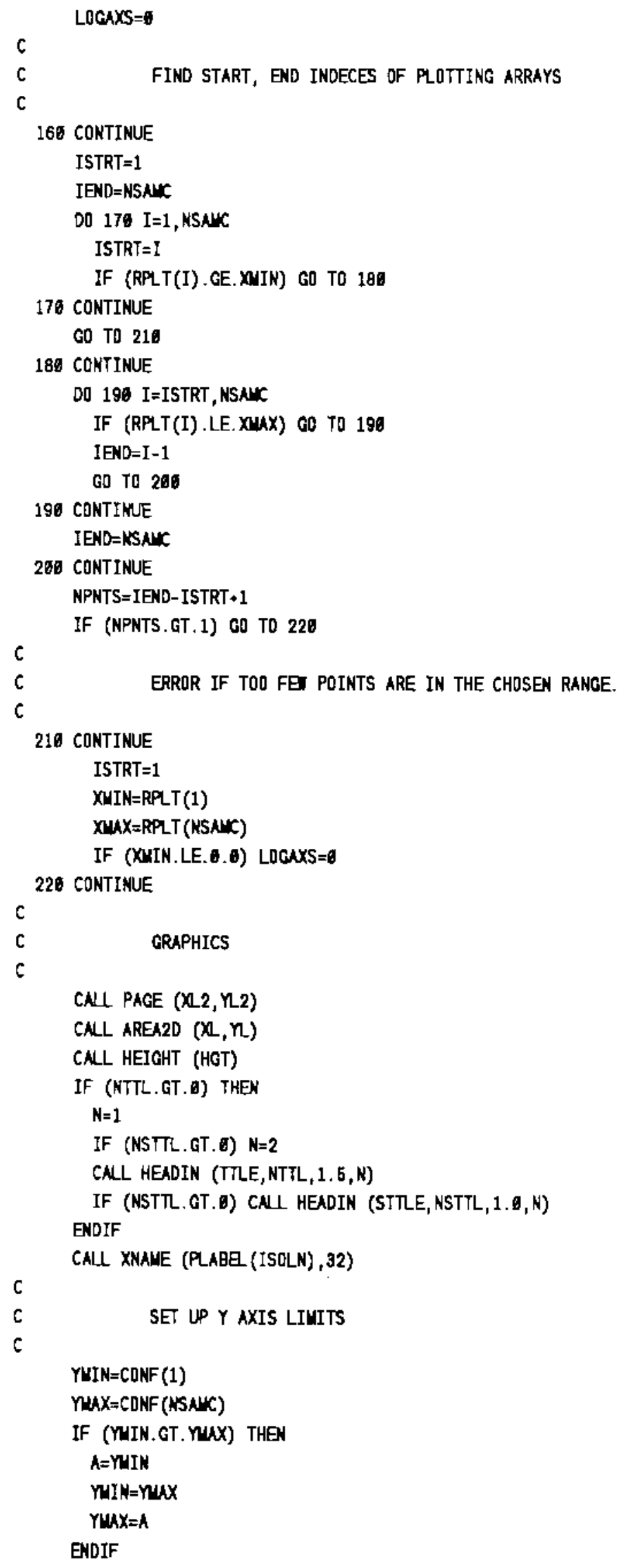




\begin{tabular}{|c|c|c|c|}
\hline & WIN=FLOAT(INT (YIN)) & PPLT & 202 \\
\hline & 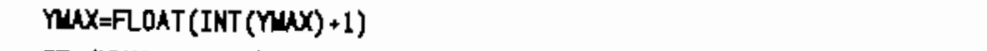 & PPLT & . 203 \\
\hline & IF $(n u x, G T .1,0) \quad n u x=1.0$ & PPLT & . 204 \\
\hline$c$ & & PPLT & 205 \\
\hline C & SEMI-LOG AXES & PPLT & . 206 \\
\hline c & & PPLT & . 207 \\
\hline & IF (LOCNXS.EQ.1) GO TO 230 & PPLT & 208 \\
\hline & $X N=X L / F D A T(N C Y C)$ & PPLT & 289 \\
\hline & $Y K=($ YAX $-Y I N) / Y L$ & PPLT & . 210 \\
\hline & CAL YKNE $(1 \mathrm{H}, \theta)$ & PPLT & . 211 \\
\hline & CALL XLOG (XNIN,XN, MIN,YN) & PPLT & 212 \\
\hline & IF (ICCD. EQ.1) THEN & PPLT & . 213 \\
\hline & CALL YGRXXS (YUIN, 'SCALE', YWAX, ML,CPD, 22,0.,0.) & PPLT & . 214 \\
\hline & 且SE & PPLT & . 215 \\
\hline & CAL YGRAXS (NIN, 'SCALE', MLAX, YL, CCPD, 25,0.,0.) & PPLT & . 216 \\
\hline & ERIF & PPLT & . 217 \\
\hline & GO TO 240 & PALT & . 218 \\
\hline C & & PPLT & . 219 \\
\hline C & CARTESIAN AXES & PPLT & . 220 \\
\hline C & & PPLT & . 221 \\
\hline & 38 CONTIMUE & PPLT & - 222 \\
\hline & IF (ICCD.EQ.1) THEN & PPLT & . 223 \\
\hline & CNLL YNNE (CPD, 22) & PPLT & 224 \\
\hline & ESE & PPLT & . 225 \\
\hline & CNШ YNANE (CCPD, 25) & PPLT & . 226 \\
\hline & ENDIF & PPLT & . 227 \\
\hline & CAШ GRAF (XVIN, 'SCALE', XWAX, YMIN, 'SCALE', $n N X$ ) & PPLT & - 228 \\
\hline & 40 CONTINUE & PPLT & . 229 \\
\hline C & & PPLT & . 230 \\
\hline c & PLOT DATA & PPLT & - 231 \\
\hline C & & PPLT & . $\quad 232$ \\
\hline & $I=1$ & PALT & . 233 \\
\hline & CAШ CURYE (RPLT(ISTRT), CDNF(ISTRT), NPNTS, I) & PPLT & . 234 \\
\hline C & & PPLT & . 235 \\
\hline C & LABE PLOT & PPLT & . 238 \\
\hline$c$ & & PPLT & . 237 \\
\hline & $X I D=-8.78$ & PPLT & , 238 \\
\hline & $Y I D=Y 2+8.80$ & PPLT & . 239 \\
\hline & CALL INTND (ICNF, XID, YID) & PPLT & . 240 \\
\hline & CN HEIGHT (0.13) & PPLT & . 241 \\
\hline & $X I D=X-6.40$ & PPLT & . $\quad 242$ \\
\hline & $Y I D=Y+1.45$ & PPLT & . 243 \\
\hline & CALI IDPLT (JRUNID, XID, YID) & PPLT & . 244 \\
\hline c & & PPLT & . 245 \\
\hline C & END PLOT & PPLT & . 246 \\
\hline c & & PPLT & . 247 \\
\hline - & $N=0$ & PPLT & . 248 \\
\hline & CAШ ENDPL (N) & PPLT & . 249 \\
\hline & IF (IRUN.EQ.1) CNU ERASE & PPLT & . 258 \\
\hline C & & PPLT & . 251 \\
\hline & RETURN & PPLT & . 252 \\
\hline c & & PPLT & . 253 \\
\hline & FOREAT $\left(/ / 1 X_{1}\right.$ '*** ERROR *** WIN AND WAX VALUES FROH SOLUTION ' $/, 15$ & PPLT & 254 \\
\hline & $+X_{1}$ A32,' ARE EQUAL. '/5X,'NO PLOT VILL BE VADE OF THIS DATA.') & PPLT & . 255 \\
\hline
\end{tabular}




\begin{tabular}{|c|c|c|}
\hline 510 FORUAT (/1X, 'DATA MINIMU $=$ ',1PE11.3/1X, 'OATA WAXIUN $=$ ', E11.3/ & PPLT & 256 \\
\hline -1X, 'rOUO YOU LIKE A SUB-PLOT ON THE OATA NXIS (Y/N)?') & PPLT & 257 \\
\hline 520 FORLAT (A1) & PPLT & 258 \\
\hline 530 FORUAT (/1X, 'ENTER THE MIN ARD WAX OATA VALUES FOR PLOTTING. ') & PPLT & 259 \\
\hline 546 FORMAT (/1X,'* ERROR, ENTERED XWIN IS LESS THAN ENTERED XNAX.') & PPLT & 260 \\
\hline 550 FORUAT $\left(/ 2 X,{ }^{\prime} *\right.$ ERROR, ENTERED XNIN AND XUAX ARE OUT OF THE RANGE 0 & PPLT & 261 \\
\hline +F THE DATA.') & PPLT & 262 \\
\hline 560 FORUAT (//1X,'THE MINIMN DATA YALUE IS LESS THAN ZERO. '/1X, 'MOUD & PPLT & 263 \\
\hline + YOU PREER A CARTESIAN NXIS ON THE DATA PLOT ABCISSA $(Y / N)$ ?') & PPLT & 264 \\
\hline 576 FORUAT (//1X,'THERE ARE LESS THAN TWO FUL LOGARITHU CYCLES DN THE & PPLT & 265 \\
\hline + OUTPUT DATA. '/1X, 'WOUL YOU PREFER A CARTESIAN AXIS ON THE DATA P & PPLT & 286 \\
\hline +LOT ABCISSA $(Y / N) ? ')$ & PPLT & 267 \\
\hline END & PPLT & 268 \\
\hline SUBROUTIKE PQSORT (N,IFIN, $11, A$ ) & PQSORT & 2 \\
\hline 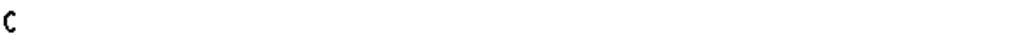 & PQSORT & 3 \\
\hline THIS SUBROUTINE IMPLEVENS THE RUICK SORT (PARTITIONING) & PQSORT & 4 \\
\hline ALGORITHU ON THE CURRENT PARTITION OF THE ARRAY A. AN EXTENSIVE & PQSORT & 5 \\
\hline EFORT IS MAE TO FIND A GOOD MEDIAN VALUE, AND TO CHECK FDR ALL & PRSORT & B \\
\hline INPUT VALUES BEING EQUAL, OR BEING OF DNLY TYO VALUES. THIS & PQSORT & 7 \\
\hline SPEEDS EXECUTION IN CERTAIN CASES WHICH ARE LIKEY YO ARISE IN & PQSORT & 8 \\
\hline SOME SPECIFIC USES OF THIS QUICK SORT ROUTINE. & PQSORT & 8 \\
\hline C & PQSORT & 10 \\
\hline$A=$ INPUT ARRAY TO SORT. & PQSORT & 11 \\
\hline AMED = MEDIAK VALUE USED FOR PARTITIDNING. & PQSORT & 12 \\
\hline II = LDTER INOEX OF QUUICK SORTER SEARCH. & PQSORT & 13 \\
\hline ON RETURN, THIS INDICATES THE INDEX IN ARRAY A OF THE & PQSORT & 14 \\
\hline NE PARTITION LOCATION. & PQSORT & 15 \\
\hline I2 = UPPER INOEX OF QUICX SORTER SEARCH. & PQSORT & 16 \\
\hline IFIN = PDINTER INOICATES THIS PARTITION IS COMPLETEY SORTED. & PQSORT & 17 \\
\hline IM = INDECES OF THREE WEDIAN VALUES (LESS THAN MAXINUN AND & PASDRT & 18 \\
\hline GREATER THAN NINIMW) IN ARRAY A, FOR SEEECTING A 'COOD' & PQSDRT & 19 \\
\hline UEDIAN FOR ARRAY PARTITIONING. & PQSORT & 20 \\
\hline INED = INDEX OF THE WEDIAN VALUE IN ARRAY A. & PQSORT & 21 \\
\hline$N=$ NUNBER OF ITES IN ARRAY $A$. & PQSORT & 22 \\
\hline C & PQSORT & 23 \\
\hline DIMENSIOK & PQSORT & 24 \\
\hline$+\quad A(N)$ & PQSORT & 25 \\
\hline ( & PQSORT & 26 \\
\hline CHECX ARRAY SIZE FOR BEING SWALL & PQSORT & 27 \\
\hline$C$ & PQSORT & 28 \\
\hline IF (N.GT.10) 60 TO 160 & PQSORT & 29 \\
\hline CALL SORT $(N, A)$ & PQSORT & 30 \\
\hline GO TO $2 B \theta$ & PASORT & 31 \\
\hline 160 CONTINUE & PASORT & 32 \\
\hline IFIN $=8$ & PQSORT & 33 \\
\hline C & PQSORT & 34 \\
\hline SEARCH FOR TMREE MEDIAN VALUES & PQSORT & 35 \\
\hline$c$ & PQSORT & 36 \\
\hline$K=N / 5$ & PQSORT & 37 \\
\hline 116 CONTINUE & PQSORT & 38 \\
\hline IMIN $=1$ & PQSORT & 39 \\
\hline$I M A X=1$ & PQSDRT & 40 \\
\hline$M I N=A(1)$ & PQSORT & 41 \\
\hline AMAX $=$ AUIN & PQSORT & 42 \\
\hline
\end{tabular}




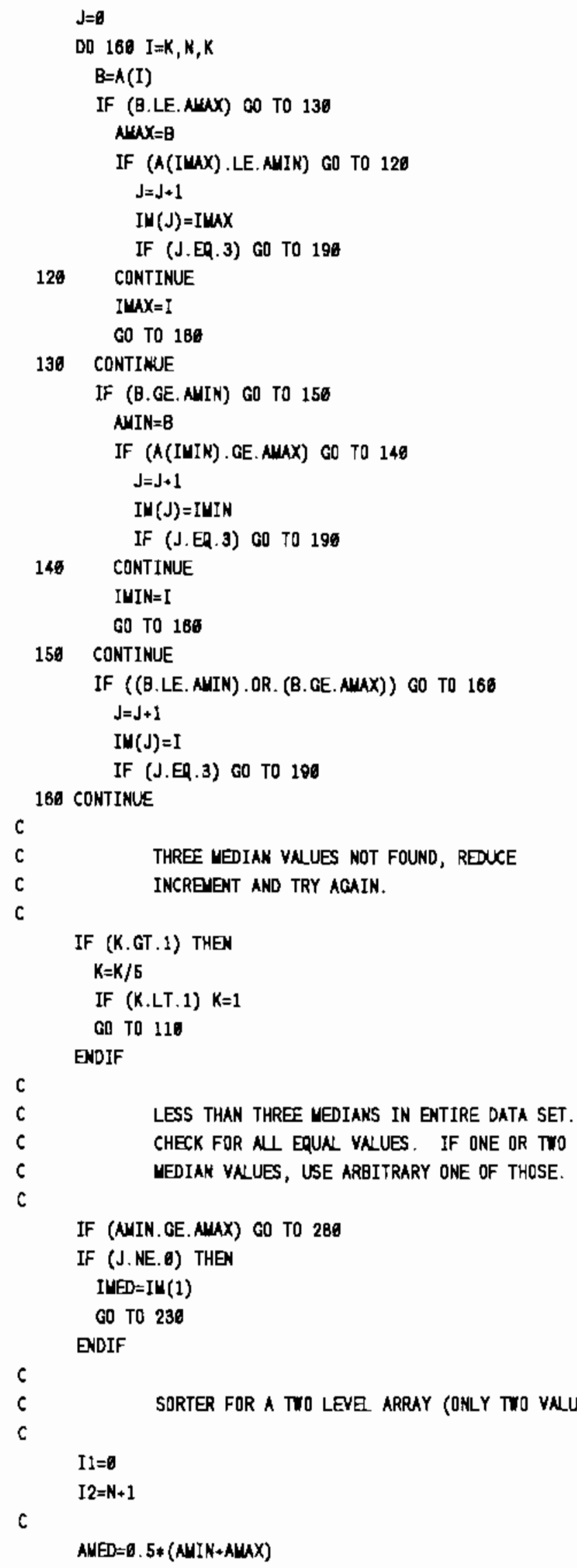




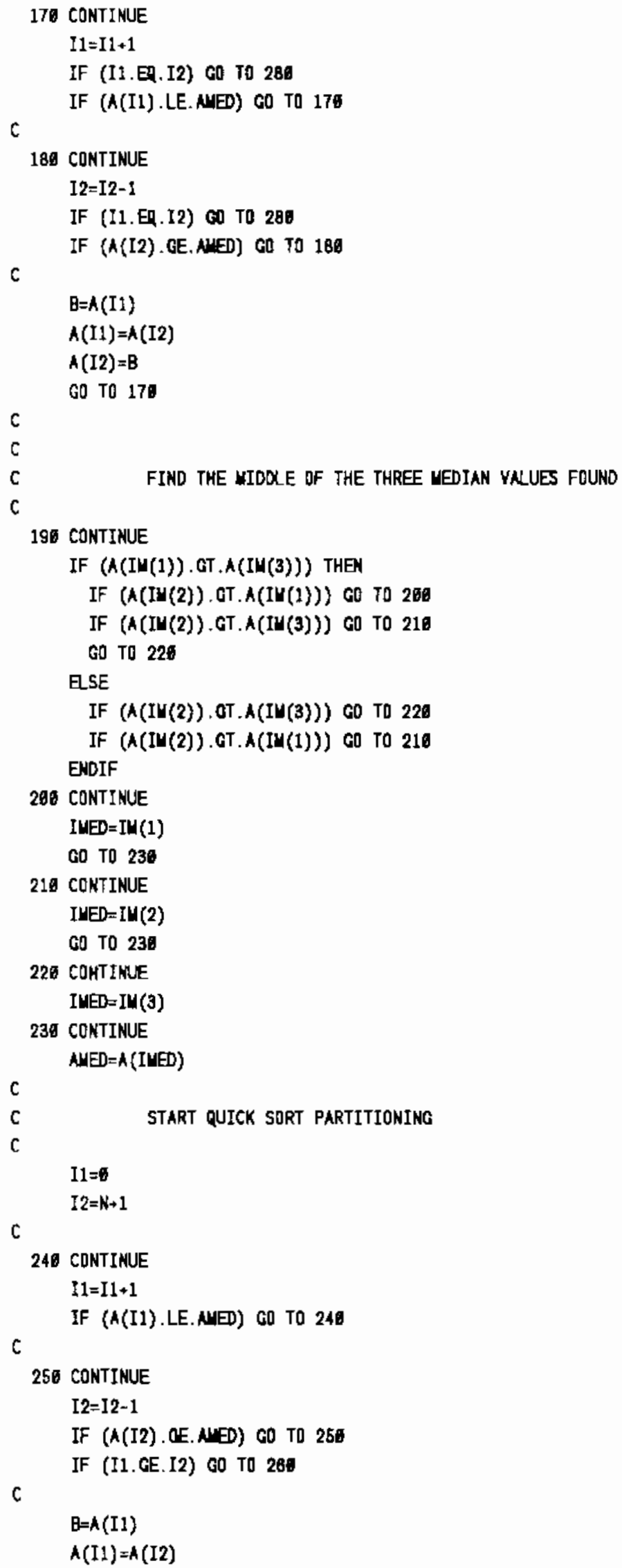




\begin{tabular}{|c|c|c|c|}
\hline & $A(12)=B$ & PQSORT & . 151 \\
\hline & GD TO 240 & PQSORT & . 152 \\
\hline$c$ & & PQSORT & . 153 \\
\hline C & BOTTOM OF PARTITIONING, INSERT THE MEDIAN VALUE & PQSORT & . 154 \\
\hline c & INTO THE CORRECT PDSITION IN THE ARRAY A. & PQSDRT & . 155 \\
\hline c & $\cdot$ & PQSORT & . 156 \\
\hline 260 & CONTINUE & PQSORT & . 157 \\
\hline & IF (I1, OT. IUED) I1=I1-1 & PQSORT & . 158 \\
\hline & IF (I1.EQ. INED) CD TO 270 & PQSORT & . 159 \\
\hline & $B=A(I 1)$ & PQSORT & 160 \\
\hline & $A(I 1)=A(I M E D)$ & PQSQRT & . 161 \\
\hline & $A(I N E D)=\theta$ & PQSORT & . 182 \\
\hline 270 & CONTINUE & PQSORT & . 163 \\
\hline & RETURN & PQSERT & . 164 \\
\hline C & & PQSORT & . 165 \\
\hline C & SET FINISH WARKER AND RETURN & PQSORT & . 166 \\
\hline$c$ & & PQSORT & . 167 \\
\hline 280 & CONTINUE & PQSQRT & . 168 \\
\hline & IFIN $=1$ & PQSORT & . 189 \\
\hline & RETURN & PQSORT & .170 \\
\hline & END & PQSORT & . 171 \\
\hline & SUBROUTINE QSORT $(N, h)$ & QSORT & 2 \\
\hline c & & QSORT & 3 \\
\hline $\mathrm{c}$ & THIS IS A QUICK SORTING NLORITHM. THIS PORTION OF THE ROUTINE & QSORT & 4 \\
\hline c & CONTROLS THE PARTITIONING OF THE ARRAY A. THE PARTIAL QUICK & QSORT & 5 \\
\hline C & SORTING ROUTINE PQSORT IS APPLIED RECURSIVELY TO EACH SECTION OF & QSORT & 6 \\
\hline$c$ & THE INPUT ARRAY A. & QSORT & 7 \\
\hline C & THIS SORTING ROUTINE REQUIRES THE PRESENCE OF A SIMPLE SORIING & QSORT & 8 \\
\hline C & ROUTINE WHICH IS EFICIENT FOR ARRAYS OF LESS THAN TEN ITEMS. & QSORT & 9 \\
\hline$c$ & THIS SORTING ROUTINE IS CALLED BY THE NAHE 'SORT', IN BOTH THIS & QSORT & 16 \\
\hline c & ROUTINE AND THE ROUTINE PQSORT. & QSORT & 11 \\
\hline C & NOTE THAT THE NGORITHU WAY FAIL IF WAXSTK IS LESS THAN TWO. & QSORT & 12 \\
\hline$c$ & & QSQRT & 13 \\
\hline C & $A$ = ARRAY TO BE SORTED. & QSORT & 14 \\
\hline C & $N=$ NLNBER OF ITES IN ARRAY A. & QSORT & 15 \\
\hline$c$ & IC = INDEX OF DIVIDER OF THE NE PARTITIONS OF ARRAY $A, A S$ & QSORT & 16 \\
\hline C & FOUND BY ROUTIYE PQSORT. & QSORT & 17 \\
\hline$c$ & IFIN = POINTER, INDICATES ROUTINE PQSDRT HAS FINISHED SORTING THE & QSORT & 18 \\
\hline$c$ & CURREAT PARTITION OF ARRAY A (RATHER THAN CREATING A NEW & QSORT & 19 \\
\hline$c$ & PARTITIONING). & QSORT & 20 \\
\hline$c$ & IL = STACK ARRAY, CONTAINS THE LOWER INDEX OF EACH PARTITION OF & QSORT & 21 \\
\hline$c$ & THE INPUT ARRAY A. & QSORT & 22 \\
\hline C & IU = STACK ARRAY, CONTAINS THE UPPER INDEX OF EACH PARTITIDN OF & QSORT & 23 \\
\hline$c$ & THE INPUT ARRAY $A$. & QSORT & 24 \\
\hline c & NS = STACK POINTER, LOCATES ACTIVE PARTITION OF ARRAY A. & QSDRT & 25 \\
\hline c & & QSORT & 26 \\
\hline & PARAMETER MXXSTK=20 & QSORT & 27 \\
\hline & DIMENSION & QSORT & 28 \\
\hline & IL (WAXSTK), & QSORT & 29 \\
\hline$c$ & & QSORT & 30 \\
\hline & $N S=1$ & QSORT & 31 \\
\hline & IL $(1)=1$ & QSORT & 32 \\
\hline & $I U(1)=N$ & QSQRT & 33 \\
\hline c & & QSORT & 34 \\
\hline
\end{tabular}




\begin{tabular}{|c|c|c|c|}
\hline 106 & CONTINUE & QSDRT & 35 \\
\hline & $L=I U(N S)-I L(N S)+1$ & QSDRT & 36 \\
\hline & $C N \amalg$ PQSORT (U,IFIN, IC, $A(I L(N S)))$ & QSORT & 37 \\
\hline c & & QSDRT & 38 \\
\hline & IF (IFIN.EQ.日) GO TO 110 & QSORT & 38 \\
\hline & $N S=N S-1$ & QSORT & 40 \\
\hline & IF (NS.LE. O) RETURN & QSORT & 41 \\
\hline & co TO 190 & QSORT & 42 \\
\hline 110 & CONTINUE & QSORT & 43 \\
\hline C & & QSORT & 44 \\
\hline & $I C=I C+I L(N S)-1$ & QSORT & 45 \\
\hline & $k=N S+1$ & QSORT & 46 \\
\hline c & & QSDRT & . 47 \\
\hline & IF (K.LE. MNXSTK) GO TO 120 & QSDRT & 48 \\
\hline & $\mathbf{H}=\mathrm{IC}-\mathrm{IL}(\mathrm{NS})$ & QSORT & 49 \\
\hline & CALL SORT $(U, A(I L(N S)))$ & QSORT & 58 \\
\hline & $I C=I C+1$ & QSORT & . \\
\hline & $\mathbf{U}=\mathrm{IU}(\mathrm{NS})-\mathrm{IC}+1$ & QSORT & . \\
\hline & CND SORT $(M, A(I C))$ & QSORT & 53 \\
\hline & $N S=N S-1$ & QSORT & 54 \\
\hline & GO TO 186 & QSORT & . \\
\hline 128 & CONTINUE & QSORT & . \\
\hline C & & QSORT & 57 \\
\hline & $\operatorname{IL}(K)=I L(N S)$ & QSDRT & 58 \\
\hline & $\mathrm{IU}(\mathrm{K})=\mathrm{IC}-1$ & QSORT & 59 \\
\hline & $I L(N S)=I C+1$ & QSORT & . \\
\hline & $N S=K$ & QSORT & 61 \\
\hline & GO TO 108 & QSORT & 82 \\
\hline & END & QSORT & 63 \\
\hline C & & QSORT & 64 \\
\hline$c$ & - & QSORT & 85 \\
\hline$c$ & & QSORT & 66 \\
\hline & SUBROUTINE R1DAT (IERR,N, KSN, RNDYAL) & RIDAT & . \\
\hline C & & RIDAT & . \\
\hline$c$ & ROUTINE TO READ RANDON INFUT VARIABLE DATA FROM AN EXTERNAL FILE. & RIDAT & . \\
\hline C & & R1DAT & . \\
\hline C & IERR = ERROR FLAO. & RIDAT & . \\
\hline C & IEXT = EXTERNAL FILE UNIT NUMAER. & RIDAT & . \\
\hline$c$ & N = VARIABLE MUBER BEING READ. & RIDAT & . \\
\hline$C$ & NSAM = NLUBER OF SAMPLES TO READ FROM THE FILE. & RIDAT & . \\
\hline c & RNDYN = ARRAY TO FILL ITTH RANDOM VNLUES & RIDAT & 10 \\
\hline c & & RIDAT & 11 \\
\hline IINSER & $R T *>C O W O N>P P A R A$ & RIDAT & 12 \\
\hline IINSER & RT *COWONYPBLK3 & RIDAT & 13 \\
\hline INSER & RT $\#>C O W O N>P B R K 1 B$ & RIDAT & 14 \\
\hline INSER & RT SYSTEXS>UTIL>USRLIB)(OUNON)INOUT & RIDAT & 15 \\
\hline & DIMENSION & RIDAT & 16 \\
\hline & $+\quad$ RNDVN(NSN) & RIDAT & 17 \\
\hline$c$ & & R1DAT & 18 \\
\hline$c$ & & RIDAT & 18 \\
\hline & $I=1$ & R1DAT & 20 \\
\hline 169 & CONTINUE & R1DAT & 21 \\
\hline & READ (INFIL, *,END=110,ERR=150) RNDVAL(I) & R1DAT & 22 \\
\hline & $I=I+1$ & R1DAT & 23 \\
\hline
\end{tabular}




\begin{tabular}{|c|c|c|}
\hline & IF (I.LE.NSN) GO 70100 & RIDAT \\
\hline & RETURN & RIDAT \\
\hline$c$ & & RIDAT \\
\hline$c$ & END OF FILE & RIDAT \\
\hline$c$ & & RIDAT \\
\hline 110 & CONTINUE & RIDAT \\
\hline & IF (I.GT.1) GD TO 120 & R1DAT \\
\hline & WRITE (IOUT, 596) & R1DAT \\
\hline & GO TO 180 & RIDAT \\
\hline 120 & CONTINUE & R1DAT \\
\hline & $I=I-1$ & R1DAT \\
\hline & TRITE (IDUT, 510) I & RIDAT \\
\hline & IRITE (IOUT, 520) VABE (N), (JJMF $(J, N), J=1, N$ LEN) & RIDAT \\
\hline & WRITE (IOUT,536) NSN & R1DAT \\
\hline & $K=I$ & RIDAT \\
\hline 130 & CONTINUE & R1DAT \\
\hline & $\mathrm{J}=\mathrm{K}+1$ & R1DAT \\
\hline & $K=J+I-1$ & R1DAT \\
\hline & IF (K.GT.NSN) K=NSN & R1DAT \\
\hline & $D 0140 \mathrm{~L}=\mathrm{J}, \mathrm{K}$ & R1DAT \\
\hline & $t=L-J+1$ & R1DAT \\
\hline & RNDVAL(L)=RNDVAL(N) & RIDAT \\
\hline 140 & CDNTINUE & R1DAT \\
\hline & IF (K.LT.NSN) OO TO 130 & RIDAT \\
\hline & RETURN & RIDAT \\
\hline$c$ & & RIDAT \\
\hline$c$ & ERROR READING FILE & R1DAT \\
\hline $\mathrm{C}$ & & RIDAT \\
\hline 150 & CONTINUE & RIDAT \\
\hline & WRITE (IOUT, 548) I & RIDAT \\
\hline 180 & CONTINUE & RIDAT \\
\hline & WRITE (IOUT, 550) & R1DAT \\
\hline & TRITE (IOUT, 520) VABE (N), (JJNF $(J, N), N=1, N$ LEN) & RIDAT \\
\hline & IERR=1 & RIDAT \\
\hline & RETURK & RIDAT \\
\hline C & & R1DAT \\
\hline $590 \mathrm{~F}$ & FORMAT (/1X,'** ERROR, END OF FILE ENCOUNTERED WHILE READING RANDO & RIDAT \\
\hline & + D DATA POINTS'/4X, 'FROL AN EXTERNAL FILE, NO USABLE DATA WAS FOUN & RIDAT \\
\hline & $+D$ ON THIS FILE.') & RIDAT \\
\hline $510 F$ & FORUAT $\left(/ 1 X,{ }^{\prime} *\right.$ WARING, END OF FILE ENCOUNTERED AFTER READING ',I & R1DAT \\
\hline & +5,' DATA VALUES'/4X, 'FROY THE EXTERNAL FILE (DATA VALUES READ FROM & RIDAT \\
\hline & + AN EXTERNAL FILE). ') & RIDAT \\
\hline $526 \mathrm{~F}$ & FORUAT ( $4 X_{1}$ 'DATA WAS BEING READ FOR INFUT VARIAQLE',A12,' '/4X,'A & R1DAT \\
\hline & -ND WAS BEING READ FROU EXTERNAL FILE:'/4X,20A4) & R1DAT \\
\hline $530 \mathrm{~F}$ & FORWAT (4X,'THE ENERED DATA WL BE DUPLICATED A SUFFICIENT NUMBE & R1DAT \\
\hline & 4R OF '/4X, 'TIUES TO CREATE THE RERUIRED ', I5,' SAMPLE VALUES, ') & RIDAT \\
\hline $540 F$ & FDRUAT (/1X, '* ERROR EICDUNTERED WHILE REAOIMG RANDOM DATA POINTS & R1DAT \\
\hline & - FROU AN'/4X,'EXPERKAL DATA FILE. THIS ERROR OCCURRED ON THE ',I5 & RIDAT \\
\hline & $+,{ }^{\prime}-\mathrm{TH}^{\prime} / 4 \mathrm{X},{ }^{\prime}$ LINE OF THE FILE. $\left.{ }^{\prime}\right)$ & R1DAT \\
\hline $550 \mathrm{~F}$ & FDRUAT (4X,'RUN TERUINATED FROM ROUTINE RDDAT.' ) & R1DAT \\
\hline & EKD & R1DAT \\
\hline & SUBROUTINE R2DAT (IERR,N) & R2DAT \\
\hline C & & R2DAT \\
\hline TH & THIS ROUTINE READS THE USER DEFINABLE CONFIDENCE FUNCTION FOR THE & R2DAT \\
\hline
\end{tabular}




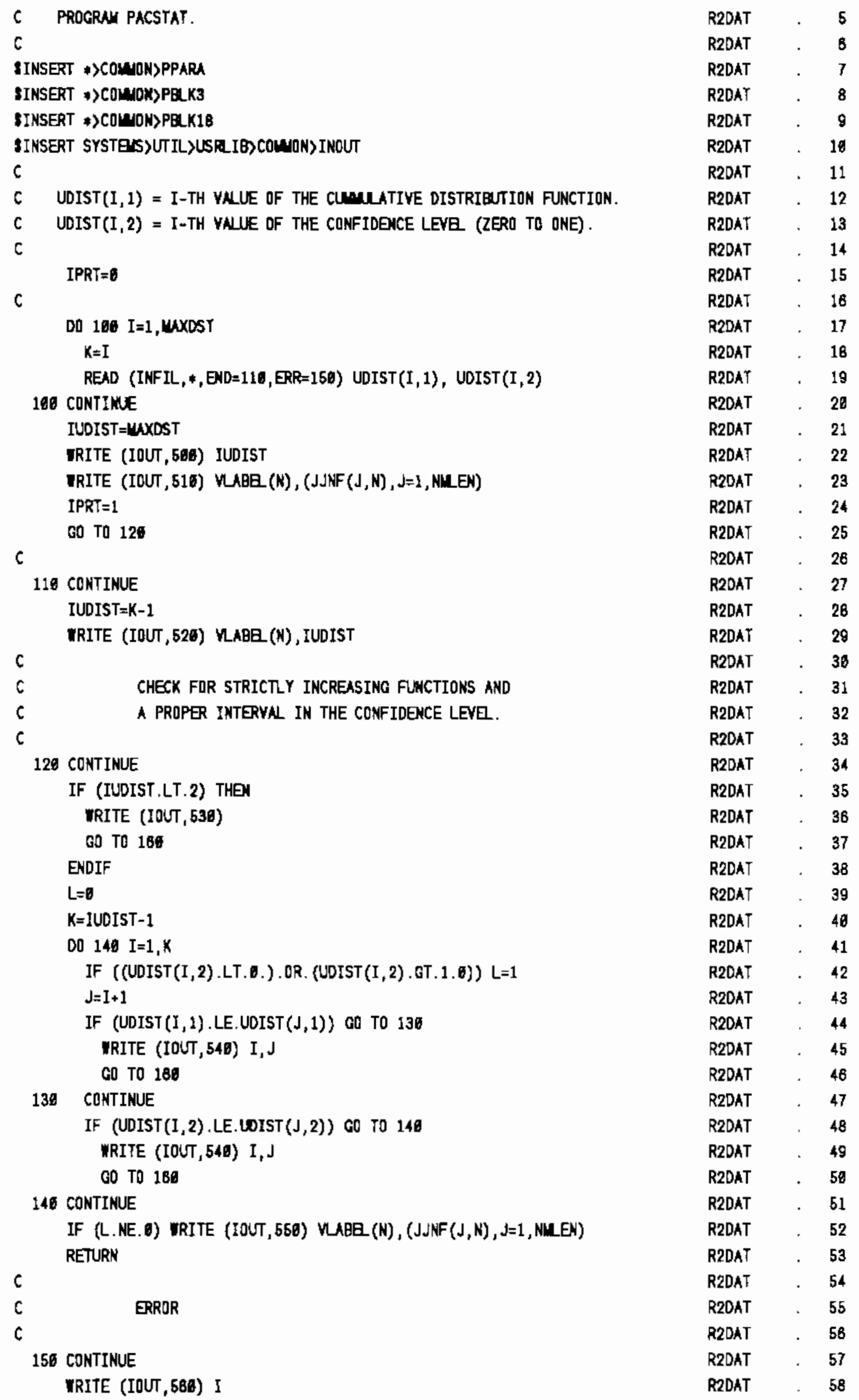

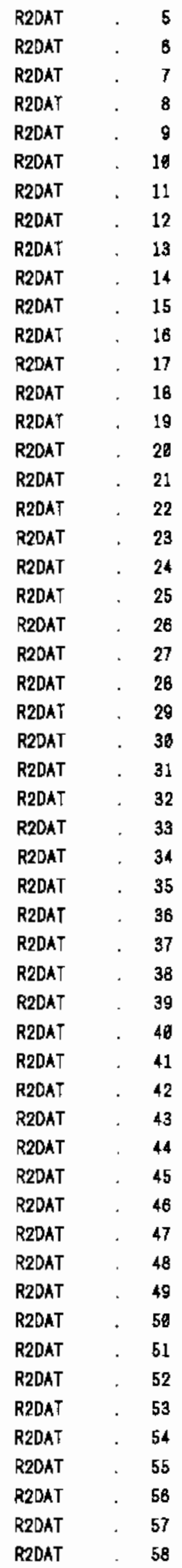




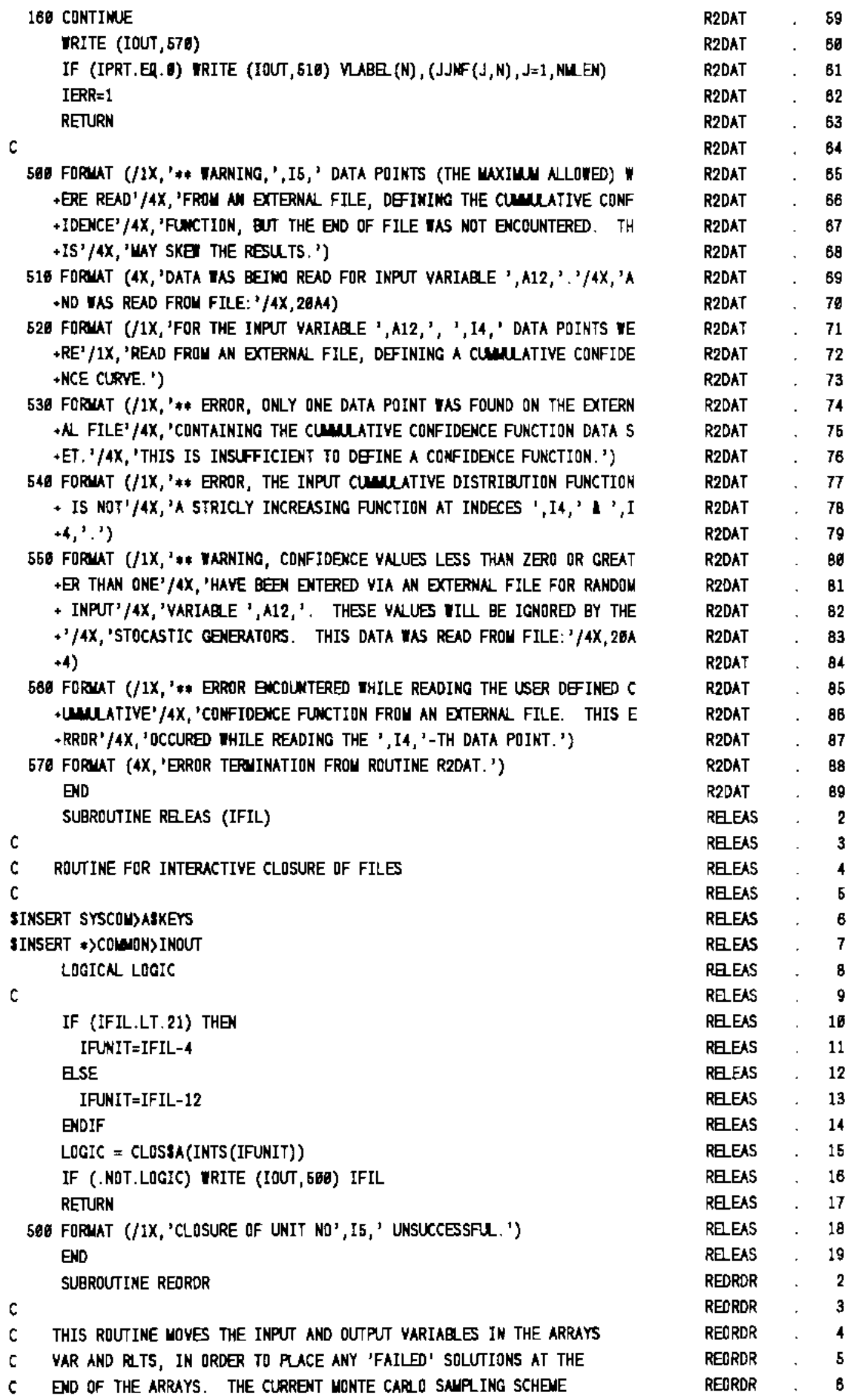


C REQUIRES THAT N SUCCESSFU SAMPLE BE LISTED CONTICUDUSLY IN THE

REORDR . 7

$c$ OUTPUT ARRAYS. HONEVER, IT WAY OFTEN BE DESIRED TO SAVE THE FAILED

REORDR - 8

C SAMPLES FOR LATER ANALYSIS.

C ARRAY CONF IS USED HERE AS TEMPORARY STORAGE OF THE FAILED

C SAPLE YNLUES.

C

IINSERT *)COWON)PPARA

IINSERT *>COUNDPPQK1

SINSERT *)CQUNON>PQK5

SINSERT * COUNON>PQKB

SINSERT *COMON>PQX7

IINSERT *>CONDN>PQLXB

SINSERT •)COWON>PEX 12

SINSERT $\Rightarrow$ CONONSPQLK17

SINSERT SYSTE'S>UTIL)USRLIB>COWON)INOUT

$C$

c

c

LOOP YOVES SAMPLES WHICH FAILED TD THE END OF EACK LIST

NSAC $=$ MSAM- MUERR

IF (NSAX. Eq.0) THEN

TRITE (IOUT, 580) NSNM

IF (IDAT.GT. ) IRITE (IOUT, 560) NSAM

GO TO 260

ENDIF

IF (NUNERR.EQ.6) GO TO 200

c

c

c

ADJUST INPUT YARIABLE LIST

IF (NYAR.LT.1) OO TO 140

DO $130 I=1$, NYAR

$\mathrm{NF}=0$

$N B=N$ NOC (I) -1

DO $110 \mathrm{~J}=1$, NSN

$\mathrm{NI}=\mathrm{Ne}+\mathrm{J}$

IF (NERR(J).EM.6) 00 TO 100

$\mathrm{NF}=\mathrm{NF}+1$

$\operatorname{CONF}(\mathrm{NF})=\mathrm{YAR}(\mathrm{N} 1)$

GO TO 110

100 CONTINUE

N2=N1-NF

VAR(N2) $=$ VAR(N1)

110 CONTINUE

$N G=N Y L O C(I)+N S N C-1$

DC $120 \mathrm{~J}=1$, NLUERR

$K=N 8+J$

$\operatorname{VAR}(X)=\operatorname{CONF}(J)$

120 CONTINUE

136 CONTINUE

\$46 CONTINUE

c

C ADJUST OUTPUT VARIABLE LIST

$c$

IF (NSOLN.LT.1) GO TO 190

DO 1 BO I=1, NSOLN

REOROR · 9

REORDR - 16

REORDR - 11

REDRDR - 12

REORDR - 13

REORDR . 14

REORDR . 15

REORDR - 16

REORDR - 17

REDRDR - 18

REDRDR . 19

REDRDR - 20

REDRDR - 21

REDRDR - 22

REORDR . 23

REORDR 、 24

REORDR 、 25

REDRDR . 26

REORDR - 27

REOROR - 28

REORDR . 29

REORDR . 36

REDRDR - 31

REORDR - 32

REORDR - 33

REDRDR - 34

REORDR - 35

REORDR . 36

REORDR - 37

REORDR - 38

REDRDR - 39

REORDR - 40

REORDR - 41

REORDR . 42

REDRDR . 43

REDRDR - 44

REORDR - 45

REDRDR - 46

RESRDR ～ 47

REDRDR । 48

REORDR . 49

REORDR . 50

REOROR - 51

REOROR . 52

REORDR . 53

REDROR . 54

REDRDR - 55

REORDR - 56

REDRDR - 57

REORDR . 58

REDRDR . 59

REORDR - 60 


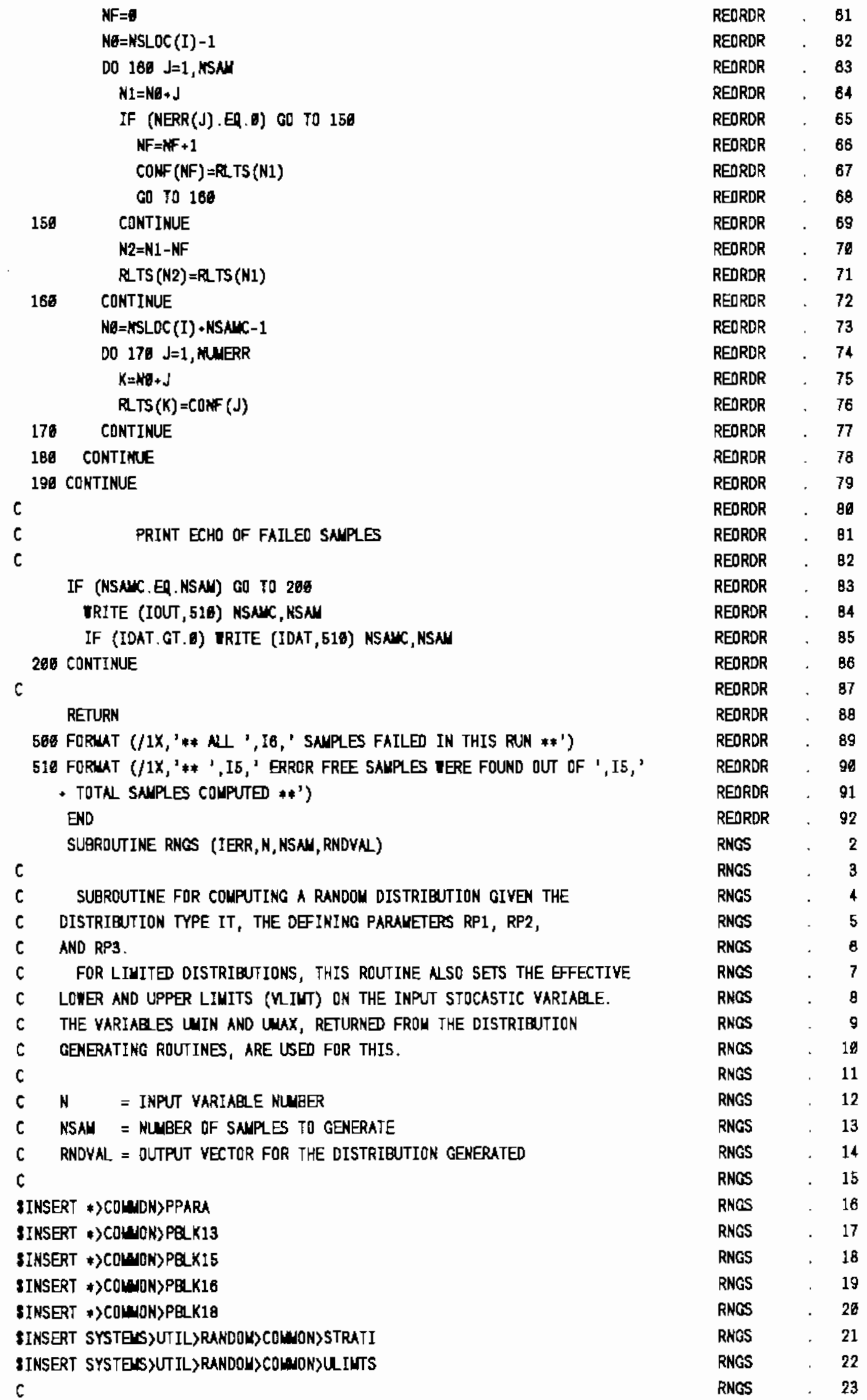




\begin{tabular}{|c|c|c|c|}
\hline & DIUENSIOH & RNGS & 24 \\
\hline - & RNDYAL (NSN) & RNGS & 25 \\
\hline & DATA & RNGS & 26 \\
\hline+ & IONE/1/ & RNGS & 27 \\
\hline C & & RNCS & 28 \\
\hline & $R P 1=\operatorname{PAR}(N, 1)$ & RNGS & 29 \\
\hline & RP2=PAR $(N, 2)$ & RNAS & 30 \\
\hline & $R P 3=P A R(N, 3)$ & RNGS & 31 \\
\hline c & & RNCS & 32 \\
\hline & $I T=I T Y P E(N)$ & RNAS & 33 \\
\hline & $N I N=\operatorname{VIIM}(N, 1)$ & RNCS & 34 \\
\hline & $N M A X=\operatorname{VIU}(N, 2)$ & RNGS & 35 \\
\hline$c$ & & RNGS & . 36 \\
\hline 6 & OPEN EXTERNAL FILE FDR EXTERNALY DEINED DISTRIBUTIONS. & RNCS & 37 \\
\hline C & & RNGS & . 38 \\
\hline & IF ((IT.ME.9). AND. (IT.NE.19)) GO TO 160 & RNCS & 39 \\
\hline & $I=0$ & RNCS & 48 \\
\hline & CN丩 ASG (I, IERR, INFIL, MLEN, JJNF $(1, N))$ & RNCS & 41 \\
\hline & IF (IERR. NE. D) RETURN & RNGS & 42 \\
\hline C & & RNGS & . 43 \\
\hline$c$ & PEPFORN DATA POINT READ AT THIS POINT & RNGS & 44 \\
\hline C & & RNGS & 45 \\
\hline & IF (IT.ER.9) GD TO 260 & RNAS & 46 \\
\hline & CAU RIDAT (IERR,N,NSN, RNDVN) & RNOS & 47 \\
\hline & CA REEAS (INFIL) & RNCS & 48 \\
\hline & RETURN & RNGS & 49 \\
\hline $100 \mathrm{C}$ & CDNTINUE & RNCS & 50 \\
\hline C & & RNCS & 51 \\
\hline C & COUPUTE BASE UNIFORN DISTRIBUTIDN & RNGS & 52 \\
\hline c & & RNOS & 53 \\
\hline & CNLL UOIS (NSN, RNDYN, DSEED, MLEYE) & RNGS & 54 \\
\hline C & & RNAS & 55 \\
\hline$c$ & SAYE STRATIFICATION DIAGNDSTICS & RNCS & 56 \\
\hline C & & RNGS & 57 \\
\hline & DO $110 \mathrm{I}=1$, NLEY日 & RNGS & 58 \\
\hline & NPTSPL $(I, N)=N S P(I)$ & RNGS & 59 \\
\hline $110 \mathrm{C}$ & CONTINUE & RNCS & 80 \\
\hline c & & RNGS & B1 \\
\hline C & CHOSE DISTRIBUTION & RNOS & 62 \\
\hline C & & RNOS & 63 \\
\hline & CO TO $(126,130,140,150,160,170,180,200,220,230,246)$, IT & RNGS & 84 \\
\hline C & & RNAS & 85 \\
\hline C & NORUAL DISTRIBUTION & RNCS & B6 \\
\hline C & & RNGS & 67 \\
\hline $120 \mathrm{C}$ & CONTINUE & RNGS & 68 \\
\hline & IF (ILIU(N).EQ.E) TKEN & RNGS & 69 \\
\hline & CNШ NORUN (NSN, RNDYAL, RP1, RP2) & RNGS & 70 \\
\hline & RETURN & RNCS & 71 \\
\hline & 日SE & RNGS & 72 \\
\hline & CAШ NORWШ (NSN, RNDVA, RP1,RP2, NMIN, NWXX) & RNCS & 73 \\
\hline & RLI $I N(N ; 1)=\operatorname{ININ}$ & RNCS & 74 \\
\hline & $\operatorname{ReI} I(N, 2)=\omega M x$ & RNGS & 75 \\
\hline & CNШ NORUN (IONE, UIN, RP1, RP2) & RNCS & 76 \\
\hline & CN NORUN (IDNE, LWXX,RP1,RP2) & RNGS & 7 \\
\hline
\end{tabular}




\begin{tabular}{|c|c|c|c|}
\hline & IF (UMIN.gT.LWX) THEN & RNGS & 78 \\
\hline & D=WIN & RNGS & 79 \\
\hline & $W I N=w N x$ & RNGS & 88 \\
\hline & WHX $=$ ro & RNGS & 61 \\
\hline & ENDIF & RNGS & 82 \\
\hline & $\operatorname{VIIT}(N, 1)=\operatorname{UIN}$ & RNGS & 83 \\
\hline & $\operatorname{VIIT}(N, 2)=U \mathrm{UHX}$ & RNGS & 84 \\
\hline & 6070288 & RNGS & 85 \\
\hline & ENDIF & RNCS & 86 \\
\hline $\mathrm{c}$ & & RNGS & 87 \\
\hline c & NATURAL LOGNORUAL DISTRIBUTIDN & RNGS & 88 \\
\hline$c$ & & RNGS & 89 \\
\hline 130 & CONTINUE & RNGS & 96 \\
\hline & IF (ILIU $(\boldsymbol{N})$, EQ. $\boldsymbol{\theta})$ THEN & RNGS & 91 \\
\hline & CALL LNNORU (NSAU, RNDYAL, RP1, RP2) & RNGS & 92 \\
\hline & RETURN & RNGS & 93 \\
\hline & ESE & RNGS & 94 \\
\hline & CALL LNNOR1 (NSAU, RNDYAL, RP1, RP2, NMIN, AMAX) & RNGS & 95 \\
\hline & $\operatorname{RLIN}(N, 1)=\operatorname{MIN}$ & RNGS & 96 \\
\hline & $\operatorname{RLIU}(N, 2)=\operatorname{LNAX}$ & RNGS & 97 \\
\hline & CALL LNNORM (IONE, UMIN, RP1, RP2) & RNGS & 98 \\
\hline & CALL LNNORA (IONE, LUX, RPI, RP2) & RNGS & . 99 \\
\hline & IF (UMIN.GT.UMX) THEM & RNGS & . 100 \\
\hline & $W=U$ MIN & RNGS & . 161 \\
\hline & UIIN=Unax & RNES & . 162 \\
\hline & WAX $=$ WD & RNGS & . 103 \\
\hline & ENDIF & RNGS & . 184 \\
\hline ; ; & $V \mathrm{~L} I \mathrm{~T}(\mathrm{~N}, 1)=U \mathrm{I} N$ & & \\
\hline & $\operatorname{VIIT}(N, 2)=\operatorname{Lux}$ & RNCS & . 186 \\
\hline & GO TO 260 & RNCS & . 107 \\
\hline & ENDIF & RNCS & . 108 \\
\hline C & & RNGS & . 109 \\
\hline $\mathrm{C}$ & COMON LOGKORUAL OISTRIBUTIDN & RNGS & 110 \\
\hline$c$ & & RNCS & . 111 \\
\hline 140 & CONTINUE & RNCS & . 112 \\
\hline & IF (ILIM(N).EQ.ø) THEN & RNGS & 113 \\
\hline & CALL LIGNDAN (NSAM, RNOVAL, RP1, RP2) & RNCS & . 114 \\
\hline & RETURN & RNGS & . 115 \\
\hline & ESE & RNGS & . 116 \\
\hline & CALL LOGNDR1 (NSNM, RNOVNL, RP1, RP2, AMIN, AMAX) & RNGS & . 117 \\
\hline & $\operatorname{RIIU}(N, 1)=U M I N$ & RNGS & . 118 \\
\hline & $\operatorname{RII}(N, 2)=\operatorname{LN} \times x$ & RNGS & . 119 \\
\hline & $C N \perp$ LOGNOR\# (IONE, UNIN,RP1,RP2) & RNGS & . 120 \\
\hline & CALL LOGNDR (IONE, UMX, RP1, RP2) & RNGS & . 121 \\
\hline & IF (WIN.GT.UWAX) THEA & RNGS & . 122 \\
\hline & TD=UNIN & RNGS & . 123 \\
\hline & $W I N=L U X$ & RNGS & . 124 \\
\hline & $\ln 4 x=110$ & RNGS & . 125 \\
\hline & ENDIF & RNGS & . 126 \\
\hline & $\operatorname{VIIT}(N, 1)=\operatorname{UIN}$ & RNGS & . 127 \\
\hline & $\operatorname{VIIT}(N, 2)=\operatorname{Lux}$ & RNGS & . 128 \\
\hline & CO IO 269 & RNGS & . 129 \\
\hline & ENOIF & RNGS & . 138 \\
\hline$c$ & & RNGS & . 131 \\
\hline
\end{tabular}


$c$

c

150 CONTINUE

CAШ LNUNIF（NSN, RNDYN, RP1, RP2)

RETURN

c COWON LOCUNIFOR DISTRIBUTION

c

160 CDNTINUE

CNL LOCUNIF (NSAY, RNDVAL, RP1, RP2)

RETURK

c

EXPONENTIAL DISTRIQUTION

$c$

179 CONTINUE

IF (ILIU(N) , ER, 6) THEN

CN山 DEXPF (NSAL, RNDVAL, RP1)

RETURN

ESE

CND DEXPFL (WSNM, RNDYAL, RP1, NIN, NAX)

$\operatorname{RLIN}(N, 1)=\omega$ IN

$\operatorname{RLIM}(N, 2)=\operatorname{SunX}$

CAU DEXPF (IDNE, UIN, RP1)

CNU DEXPF (IONE, WNX, RP1)

IF (UIN.GT. LWXX) THEN

$M D=($ NIN

UIN $=$ UwXX

Wux $=$ mo

ENDIF

$\operatorname{VIIMT}(N, 1)=\operatorname{LIN}$

$\operatorname{VIITT}(k, 2)=\operatorname{WN} x$

GO TO 286

ENDIF

C

C UNIFOR DISTRIBUTION

C

180 CDNTINUE

DO $199 I=1$, NSN

RNDYAL (I) $=R P 1+R N D Y A L(I) *(R P 2-R P 1)$

190 CONTIKUE

RETURN

C

C BERNDULI DISTRIQUTION

C

209 CONTINUE

CNU BERNLI (NSAL, POIDYN, RP3)

DO $210 \mathrm{I}=1$, NSAL

$R Y=R P 1$

IF (RNDYAL(I).GT. .) RV=RP2

RNDVAL(I) $=$ RV

210 CONTIMUE

RETURN

RNCS

133

RNGS . 134

RNGS $\quad 135$

RNCS $\quad 136$

RNCS $\quad 137$

RNCS $\quad 138$

RNGS . 139

RNGS . 146

RNCS , $14 !$

RNCS 142

RNGS 143

RNES 144

RNGS 145

RNGS . 146

RNGS . 147

RNGS . 148

RNGS . 149

RNGS - 168

RNGS . 151

RNCS . 152

RNGS . 153

RNGS $\quad 154$

RNGS . 155

RNGS . 158

RNGS . 157

RNGS . 158

RNGS . 159

RNCS 180

RNCS 161

RNCS . 162

RNCS . 163

RNGS 164

RNGS . 185

RNGS - 166

RNCS 、 167

RNES - 188

RNCS $\quad 160$

RNGS . 170

RNGS $\quad 171$

RNCS - 172

RNCS , 173

RNGS 174

RNGS $\quad 175$

RNGS - 176

RNOS 177

RKCS . 178

RNCS . 179

RNCS $\quad 180$

RNCS . 181

RNCS . 182

RNCS . 183

RNGS + 184

C

C CONFIDENE FUNCTION DN EXTERNAL FILE 
c

220 CONTINUE

CN R2OAT (IERR,N)

CAU XUDIST (NSNM, RKDVN, IUDIST, UDIST $(1,1), \operatorname{UDIST}(1,2)$ )

CNI RELEN (INFIL)

RETURH

$c$

C

236 CONTINUE

RETURN

c

c DISCREFE DISTRIBTUION

c

240 CORTINLE

DO $250 \mathrm{I}=1$, NSAN

RNDYN $(I)=$ RP1

250 CONTINUE

RETURN

$c$

c EFOREE UPPER/LOWER LIMITS FOR END POINT LIMITING

$c$

260 CONTINUE

$\operatorname{NLIU}(N, 1)=0$

$\operatorname{NLIU}(N, 2)=0$

DD $280 I=1, N S N$

$A=$ RNDVAL(I)

IF (A.GE. ANIN) CO TO 270

RNOYAL $(I)=$ ANIN

$\operatorname{NLI}(N, 1)=\operatorname{NLIM}(N, 1)+1$

GO TO 288

270 CONTIMUE

IF (A.LE. N $N A$ ) OO TO 280

RNDVAL(I) $=$ NWXX

$\operatorname{MLIN}(N, 2)=\operatorname{NLIV}(N, 2)+1$

280 CONTINUE

RETURN

END

SUBROUTINE SOLN (IERR)

6

C THIS SAMPLE PRORLE WODES THE UASS STORAGE AND RELASE RATE

c (STGADY STATE) OF A CDNTAMINANT, IHICH IS DIFFUSING INTO A BOUNDARY

C LAYER AWAY FROY A SOLUBILITY LIMITED SOURCE. DUTSIDE THE BOUNDARY

C LAYER, CONVECTION IS ASSUED TO RENOVE THE CONTAMINANT AT A FAST

C RATE. THE MODE IS ONE DIUENSIONAL, CARTESIAN DIFFUSION, AND

C OUTPUT VALUES ARE 'PER SQUARE UETER' OF SOURCE ZONE.

C

C INPUT VARIARLES/DATA

C ICNT = DATA SET NUMBER ( OF INPUT VARIABLE UPDATES)

C IERR = ERROR POINTER

c INDEX = SALPLE NUWEER BF CURRENT RUM

c

c VINPT (1) = WDLECUAR DIFFUSIVITY, WET **2/YEAR

C VINPT (2) = RETARDATION FACTOR

\begin{tabular}{|c|c|}
\hline RNGS & . 188 \\
\hline RNGS & . 187 \\
\hline RNCS & + $\quad 188$ \\
\hline RNOS & . $\quad 189$ \\
\hline RNES &.$\quad 190$ \\
\hline RNES & . 191 \\
\hline RHCS & . 182 \\
\hline RNCS & . 193 \\
\hline RNCS & . 194 \\
\hline RNCS & . 195 \\
\hline RNCS & . 198 \\
\hline RNGS & . $\quad 197$ \\
\hline RNCS & . 198 \\
\hline RNCS & . 199 \\
\hline RNCS & . 260 \\
\hline RNCS & . 261 \\
\hline RNCS & . $\quad 282$ \\
\hline RNGS & . 203 \\
\hline RNCS & . 204 \\
\hline RNCS & . $\quad 205$ \\
\hline RNCS & . 208 \\
\hline RNCS & . 207 \\
\hline RHCS & . 268 \\
\hline RNOS & . 289 \\
\hline RNCS & . 210 \\
\hline RNGS & . 211 \\
\hline RNGS & . 212 \\
\hline RNCS & . 213 \\
\hline RNGS & . 214 \\
\hline RNCS & . 215 \\
\hline RNCS & . 216 \\
\hline RNCS & . $\quad 217$ \\
\hline RNGS & . 218 \\
\hline RNCS & . 219 \\
\hline RNGS & . 226 \\
\hline RNCS & . 221 \\
\hline RNGS & . 222 \\
\hline RNCS & . $\quad 223$ \\
\hline SOLN & . \\
\hline SOLN & 3 \\
\hline SOLLN & 4 \\
\hline SOLN & . \\
\hline SOLN & . \\
\hline SOLN & . \\
\hline SOLN & . \\
\hline SOLN & . \\
\hline SDLN & 10 \\
\hline SOLN & 11 \\
\hline SOLN & 12 \\
\hline SOLN & 13 \\
\hline SOLN & 14 \\
\hline SDLN & 15 \\
\hline SOLN & 16 \\
\hline SDLN & 17 \\
\hline
\end{tabular}




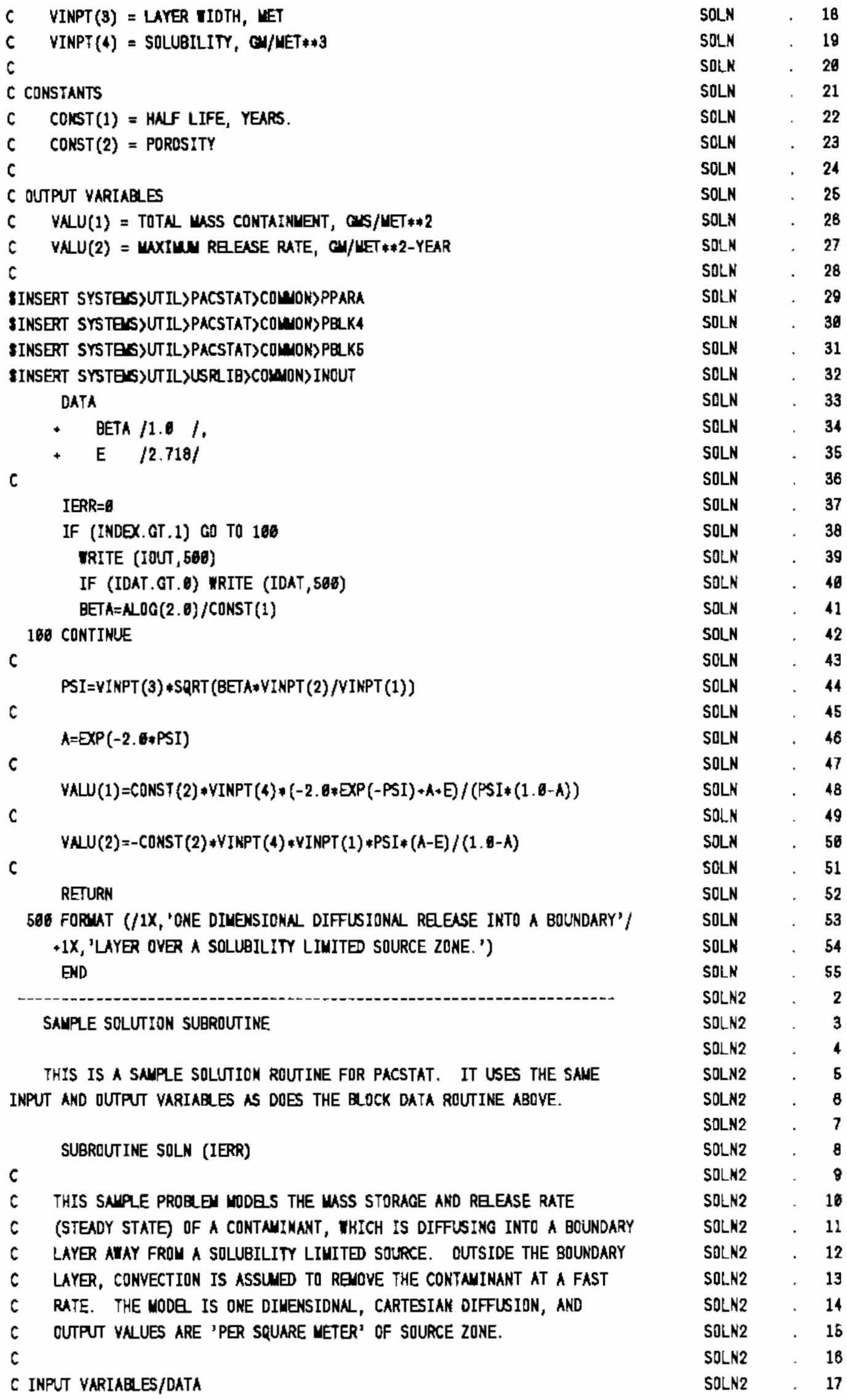




\begin{tabular}{|c|c|c|c|}
\hline c & = DATA SET MUBER (I DF INPUT VARIAQE UPDATES) & SOLN2 & 18 \\
\hline$c$ & = ERROR PLINTER & SOLN2 & 19 \\
\hline$c$ & $=$ SAMPLE NUMER OF CURREXT RUN & SOLN2 & 20 \\
\hline$c$ & & SOLN2 & 21 \\
\hline 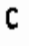 & VINPT $(1)=$ MOLECULAR DIFFUSIVITY, MET**2/YEAR & SOLN2 & 22 \\
\hline C & VINPT (2) = RETARDATION FACTOR & SOLN2 & 23 \\
\hline C & VINPT (3) = LAYER TIDTH, UET & SOLN2 & 24 \\
\hline C & YINPT $(4)=$ SDLLOILITY, 4 M/UET**3 & SOLN2 & 25 \\
\hline$C$ & & SOLN2 & 26 \\
\hline \multicolumn{2}{|c|}{ C CONSTANTS } & SOLN2 & 27 \\
\hline C & $\operatorname{CONST}(1)=$ HNF LIFE, YEARS. & SOLN2 & 28 \\
\hline C & CONST $(2)=$ PORASITY & SDLN2 & 29 \\
\hline$c$ & & SOLN2 & 30 \\
\hline \multicolumn{2}{|c|}{ C OUTPUT VARIARES } & SOLN2 & 31 \\
\hline C & VALU $(1)=$ TOTAL WASS CONTAINUEXT, GS/MET:*2 & SOLN2 & 32 \\
\hline C & VALU(2) = MAIMN REEESE RATE, G/NET**2-YEAR & SOLN2 & 33 \\
\hline$c$ & & SOLN2 & . 34 \\
\hline \multicolumn{2}{|r|}{ 3INSERT SYSTES\UTIL>PACSTAT>COMON>PPARA } & SOLN2 & 35 \\
\hline \multicolumn{2}{|r|}{ SINSERT SYSTES>UTIL>PACSTAT>COWON>PQK4 } & SOLN2 & 36 \\
\hline \multicolumn{2}{|r|}{ SINSERT SYSTES>UTIL>PACSTATDCOYON>PQK5 } & SOLN2 & 37 \\
\hline \multicolumn{2}{|r|}{ SINSERT SYSTEXS>UTIL>USRLIB>COLMON)INOUT } & SOLN2 & 38 \\
\hline & & SOLN2 & 39 \\
\hline \multicolumn{2}{|r|}{$+\quad$ 㫙A $/ 1.0 \mathrm{l}$} & SOLN2 & 46 \\
\hline \multicolumn{2}{|r|}{$+\quad E \quad / 2.718 /$} & SOLN2 & 41 \\
\hline \multicolumn{2}{|r|}{ 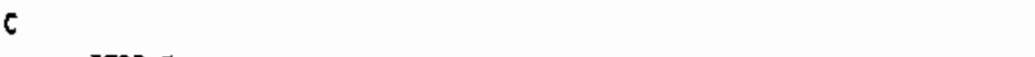 } & SOLN2 & 42 \\
\hline \multicolumn{2}{|r|}{ IERR $=0$} & SOLN2 & 43 \\
\hline \multicolumn{2}{|r|}{ IF (INDEX.GT.1) 00 TO 160} & SOLN2 & 44 \\
\hline \multicolumn{2}{|r|}{ IRITE (IOUT, EAS) } & SOLN2 & 45 \\
\hline \multicolumn{2}{|r|}{ IF (IDAT.GT. I) WRITE (IDAT, 506) } & SOLN2 & 46 \\
\hline \multicolumn{2}{|r|}{ BETA=ALDG $(2,0) / C D N S T(1)$} & SOLN2 & . 47 \\
\hline \multicolumn{2}{|c|}{100 CONTIME } & SOLN2 & . 48 \\
\hline \multicolumn{2}{|l|}{$c$} & SOLN2 & 49 \\
\hline & PSI $=V I N P T(3) * S Q R T($ BETA*VINPT (2)/VINPT(1)) & SOLN2 & 50 \\
\hline \multirow[t]{2}{*}{$c$} & & SOLN2 & $\$ 1$ \\
\hline & $A=E \times P(-2.6 * P S I)$ & SOLN2 & . 52 \\
\hline \multirow[t]{2}{*}{$c$} & & SOLN2 & . 53 \\
\hline & $\operatorname{VALU}(1)=\operatorname{CONST}(2) * \operatorname{VINPT}(4) *(-2.0 * \operatorname{EXP}(-\mathrm{PSI})+A+E) /(\operatorname{PSI} *(1.0-A))$ & SOLN2 & 54 \\
\hline c & & SOLN2 & 55 \\
\hline & $\operatorname{VALU}(2)=-\operatorname{CQNST}(2) * \operatorname{VINPT}(4) * \operatorname{VINPT}(1) * \operatorname{PSI} *(A-\mathrm{E}) /(1,0-A)$ & SDLN2 & 56 \\
\hline C & & SOLN2 & 57 \\
\hline & RETURN & SOLN2 & 58 \\
\hline & FOFUAT (/1X, 'ONE DIMEXSIONAL DIFFUSIDNAL RELEASE INTO A BDUNDARY'/ & SOLN2 & 59 \\
\hline & +1X, 'LAYER OYER A SOLUBILITY LIUITED SOURCE ZONE. ') & SOLN2 & 68 \\
\hline & END & SOLN2 & . 61 \\
\hline & & SOLN2 & 62 \\
\hline & SUBRQUTINE SORT $(\mathrm{N}, \mathrm{A})$ & SORT & . \\
\hline$c$ & & SDRT & . \\
\hline C & SORT IS A SIMPLE SHEL SORT. & SQRT & . \\
\hline C & ITES ARE SORTED IN ASCENDING ORDER. & SORT & . \\
\hline c & & SORT & . \\
\hline$c$ & A IS THE ARRAY OF ITES TO SORT. & SORT & . \\
\hline$c$ & N IS THE NUNBER OF ITES IN ARRAY A. & SORT & . \\
\hline c & & SDRT & . \\
\hline & DIMENSION & SORT & 16 \\
\hline
\end{tabular}




\begin{tabular}{|c|c|c|c|}
\hline & $\rightarrow \quad A(1)$ & SORT & 11 \\
\hline & IF (N.LT.2) RETURN & SORT & 12 \\
\hline & $I D=N$ & SORT & 13 \\
\hline & CONTINUE & SORT & 14 \\
\hline & $\mathrm{ID}=\mathrm{ID} / 2$ & SORT & 15 \\
\hline & $\mathrm{IB}=1$ & SORT & 16 \\
\hline & GO TO 120 & SORT & 17 \\
\hline & CONTINUE & SORT & 18 \\
\hline & $I B=I B+1$ & SORT & 19 \\
\hline & IA (IB.LE.ID) GO TO 120 & SORT & 20 \\
\hline & $\mathrm{IF}^{2}($ ID.GT.1) 60 TO 108 & SORT & 21 \\
\hline & RETURN & SQRT & 22 \\
\hline & 8 CONTINUE & SORT & 23 \\
\hline & $I=I B$ & SORT & 24 \\
\hline 130 & CONTINUE & SORT & 25 \\
\hline & $K=I+I D$ & SORT & 28 \\
\hline & IF $(A(I) . L E . A(K))$ OO TO 160 & SORT & 27 \\
\hline & $T=A(K)$ & SORT & 28 \\
\hline & $h(K)=A(I)$ & SORT & 28 \\
\hline & $J=I$ & SORT & . $\quad 38$ \\
\hline 140 & CONTINUE & SORT & 31 \\
\hline & $K=J-I D$ & SORT & 32 \\
\hline & IF (K.LT.1) OO IO 150 & SORT & 33 \\
\hline & IF $(T . G T . A(K))$ GO TO 150 & SORT & 34 \\
\hline & $A(J)=A(K)$ & SORT & 35 \\
\hline & $J=k$ & SQRT & 36 \\
\hline & CO TO 140 & SORT & 37 \\
\hline 150 & CDNTINUE & SORT & 38 \\
\hline & $A(J)=T$ & SORT & 39 \\
\hline 160 & CONTINUE & SORT & 40 \\
\hline & $I=I+I 0$ & SORT & 41 \\
\hline & IF $(I+I D . L E, N)$ OD TO 139 & SORT & 42 \\
\hline & GO TO 116 & SORT & 43 \\
\hline & END & SORT & 44 \\
\hline & SUBROUTINE STATDT & STATOT & . \\
\hline C & & STATOT & . \\
\hline C & THIS ROUTINE CNLLS LIBRARY ROUTINE STATS TD COUPUTE THE WEAN, & STATOT & . \\
\hline C & MEDIAN, STANDARD DEVIATIDN, AND SKENESSS FOR THE INPUT AND OUTPUT & STATOT & . \\
\hline C & VARIAQLE DISTRIBUTIDNS WHICH HAVE BEEN GENERATED. DIAGNOSTICS FOR & STATOT & . \\
\hline$c$ & INFUT VARIABEE STRATIFICATION, AND WAXIMN LIKE YHDDO ESTIMATORS & STATOT & . \\
\hline C & ARE ALSO GENERATED. & STATOT & 8 \\
\hline c & ALL OF THESE DIATNASTICS ARE PRINTED TO THE DLTTPT PRINT FILE & STATDT & . \\
\hline C & OR USERS TERINAL BY TKIS ROUTINE. & STATDT & 10 \\
\hline C & DATA FOR STRATIFIED SNIPLING IS PRINTED ONLY THEN MORE THAN & STATOT & 11 \\
\hline $\mathrm{C}$ & DNE LEYE OF SAMPLING HAS BEEN CHOSEN. & STATOT & 12 \\
\hline $\mathrm{C}$ & THE DATA IN ARRAYS VAR AND RLTS ARE TRANSFERED TD ARRAY CONF & STATOT & 13 \\
\hline C & PRIOR TO CNL ROUTINE STATS. THIS AVOIDS A PRINE PAGING ERROR. & STATOT & 14 \\
\hline C & & STATOT & 15 \\
\hline C & **** THIS ROUTINE SORTS THIS INPUT AND OUTPUT YARIABLE ARRAYS & STATOT & 16 \\
\hline C & **** IN ASCENDING ORDER, VIA A CALL TO SUBROUTINE QSORT. & STATOT & 17 \\
\hline C & & STATOT & 18 \\
\hline \$INSE & ERT $*>C O L N O N>P P A R A$ & STATOT & 19 \\
\hline 3 INSE & ERT $\Rightarrow$ COLON>PBCK1 & STATOT & 26 \\
\hline IINSER & ERT *>COWON>PELK3 & STATDT & 21 \\
\hline
\end{tabular}


SINSERT \# COMONDPEL

SINSERT ^>COLON>PQCK

SINSERT *>CONON>PELT

IINSERT *>COLONDPELB

SINSERT \#CONON)PELK1.

IINSERT *)COUDN>PELK13

IINSERT *>CONOK>PEL15

IINSERT \#)COWOW>PELK17

IINSERT SYSTES) YTIL)(USRLIB)COLAON)INOUT

REN $* 8$

- A, B, EASEXP

OIUENSION

- RD(2),

CHARACTER*1

Fo(2)

$+\quad$ AKS

DATA

+ BASEXP/2.363DE/

$c$

C SET OUTPUT MIT MUBER

c

III $=$ IDAT

IF (IOAT.EA.6) III=IOUT

c

C ***** INPUT DISTRYQUTIONS *****

$c$

IF (NYAR.LT.1) OO TO 140

DO $130 \mathrm{~N}=1$, NYAR

$N 1=N$ NOC $(N)-1$

DO $160 \mathrm{~J}=1$, NSNIC

$K=J+N 1$

$\operatorname{CONF}(J)=Y A R(K)$

100 CONTINUE

CNL STATS (NSNX, CONF, WIN(N), $\operatorname{WNX(N),~VAVG(N),~VSTO(N),VSKW(N))}$

INPUT VARIABLE SDRT AND MEDIAN COUPUTATION

$c$

WED $(N)=0$.

CALL QSORT (NSAX, CONF)

IF (NSALC.LT.10) GD TO 110

$I=N S A N C / 2$

$\mathrm{J}=\mathrm{I}$

IF (MOD (NSN'C, 2), Eq.

$\operatorname{WED}(N)=(\operatorname{CONF}(\mathrm{I})+\operatorname{CONF}(\mathrm{J})) / 2$

116 CONTINUE

DO $120 \mathrm{~J}=1$, NSAIC

$\mathrm{K}=\mathrm{N} 1 \bullet \mathrm{J}$

$\operatorname{VAR}(K)=\operatorname{CONF}(J)$

120 CONTIME

130 CONTINUE

146 CONTINUE

c

$c$

***** OUTPUT DISTRIBUTIONS *****

IF (NSOLK.LT.1) CO TO 190

\begin{tabular}{|c|c|c|}
\hline STATDT & & \\
\hline STATOT & & \\
\hline STATOT & & \\
\hline STATOT & & \\
\hline STATOT & & \\
\hline STATOT & & \\
\hline STATOT & & \\
\hline STATOT & & \\
\hline STATOT & & \\
\hline STATOT & & \\
\hline STATOT & & \\
\hline STATOT & & \\
\hline STATOT & & \\
\hline STATOT & & \\
\hline STATOT & & \\
\hline STATOT & & \\
\hline STATOT & & \\
\hline STATOT & & \\
\hline STATOT & & \\
\hline STATOT & & \\
\hline STATOT & & \\
\hline STATOT & & \\
\hline STATOT & & \\
\hline STATOT & & \\
\hline STATOT & & \\
\hline STATOT & & \\
\hline STATDT & & \\
\hline STATOT & & \\
\hline STATOT & & \\
\hline STATQT & & \\
\hline STATOT & & \\
\hline STATOT & & \\
\hline STATOT & & \\
\hline STATOT & & \\
\hline STATOT & & \\
\hline STATOT & & \\
\hline STATOT & & \\
\hline STATOT & & \\
\hline STATOT & & \\
\hline STATOT & & \\
\hline STATOT & & \\
\hline STATOT & & \\
\hline STATOT & & \\
\hline STATOT & & \\
\hline STATOT & & \\
\hline STATOT & & \\
\hline STATOT & & \\
\hline STATOT & & \\
\hline STATOT & & \\
\hline STATDT & & \\
\hline STATOT & & \\
\hline STATOT & & \\
\hline STATOT & & \\
\hline STATDT & & \\
\hline
\end{tabular}




\begin{tabular}{|c|c|c|c|}
\hline & DO $186 \mathrm{~N}=1$, NSOLN & STATOT & 78 \\
\hline & $\operatorname{FAYG}(N)=\mathbb{R L T S}(\operatorname{NSLOC}(N))$ & STATOT & 77 \\
\hline & FNED $(N)=0.0$ & STATOT & 78 \\
\hline & $\operatorname{FSTD}(N)=0$ & STATOT & 79 \\
\hline & $F S K(N)=0$. & STATOT & 80 \\
\hline & IF (NSAXC.LE.1) CO TO 180 & STATOT & 81 \\
\hline$c$ & & STATOT & 82 \\
\hline & $N 1=N S L O C(N)-1$ & STATOT & 83 \\
\hline & DO $150 \mathrm{~J}=1$, NSAXC & STATOT & 84 \\
\hline & $K=J+K \mathbf{L}$ & STATOT & 85 \\
\hline & $\operatorname{CONF}(J)=\mathbb{R} \operatorname{TS}(K)$ & STATOT & 88 \\
\hline 150 & CONTIME & STATOT & B7 \\
\hline & CNL STATS (NSAX, CONF, FUIN $(N)$, FUNX(N), FAYG $(N), F S T D(N), F S K M(N))$ & STATOT & 88 \\
\hline $\mathrm{C}$ & & STATOT & 89 \\
\hline c & OUTPUT YARIABLE SORT ANO MEDIAN COMPUTATIOK & STATOT & 96 \\
\hline C & & STATDT & 91 \\
\hline & CNL QSORT (NSNC,CONF) & STATOT & 92 \\
\hline & If (NSNKC.LT.18) GO TO 180 & STATOT & 93 \\
\hline & $I=K S N L C / 2$ & STATOT & 94 \\
\hline & $J=I$ & STATOT & 95 \\
\hline & IF (UDO(NSNK, 2).EQ.6) J=J+1 & STATOT & 96 \\
\hline & FMED $(N)=\{\operatorname{CONF}(I)+\operatorname{CDNF}(J)) / 2$. & STATOT & 97 \\
\hline 169 & CONTIMUE & STATOT & 98 \\
\hline & DO $17 \% \mathrm{~J}=1$, NSNLC & STATOT & 99 \\
\hline & $K=\mathbf{N} 1+J$ & STATOT & . 180 \\
\hline & $\operatorname{RLTS}(K)=\operatorname{CONF}(J)$ & STATDT & . 181 \\
\hline 170 & CONTIMUE & STATOT & . 192 \\
\hline 188 & CONTINUE & STATOT & 183 \\
\hline 190 & CONTINUE & STATQT & . 104 \\
\hline$c$ & & STATOT & . 105 \\
\hline c & PRINT YNUES TO CUTPUT FILE DR USER'S TERHINAL & STATOT & . 186 \\
\hline C & & STATOT & . 167 \\
\hline$c$ & IMPUT VARIAELE STATISYICS & STATOT & . $10 \mathrm{~B}$ \\
\hline$c$ & & STATOT & . 169 \\
\hline & IF (NYAR.LT.1) GO TO 336 & STATOT & . 118 \\
\hline & IEXT $=1$ & STATOT & $\therefore 111$ \\
\hline & WRITE (III, 560) & STATOT & . 112 \\
\hline & TRITE (III, 510) & STATOT & .113 \\
\hline & DO $290 \mathrm{~N}=1$, NYAR & STATOT & . 114 \\
\hline & VRITE (III, 528) $\operatorname{VABE}(N), \operatorname{VIN}(N), \operatorname{Whx}(N), \operatorname{VAVG}(N), \operatorname{WED}(N)$, & STATOT & . 115 \\
\hline & + VSTD(N), YSKW (N) & STATOT & . 116 \\
\hline & 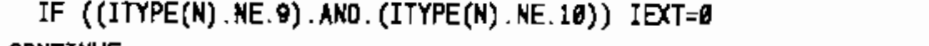 & STATOT & . 117 \\
\hline 2000 & CDNTINUE & STATOT & . 118 \\
\hline c & & STATOT & . 119 \\
\hline$c$ & COMPUTE MAXIMN LIKEIHDCD ESTIMKTORS FDR INPUT & STATOT & 120 \\
\hline C & VARIAELES (ONEY FOR INTERNALY DEINED VARIABLES). & STATQT & . 121 \\
\hline $\mathrm{C}$ & & STATOT & - 122 \\
\hline & IF (IEXT.EQ.1) GO TO 330 & STATOT & . 123 \\
\hline & WRITE (III, 538) & STATOT & . 124 \\
\hline & ISTAR $=0$ & STATOT & . 125 \\
\hline & DO $328 \mathrm{~N}=1$, NVAR & STATOT & . 126 \\
\hline & ANS $=1$ & STATOT & 127 \\
\hline & IF (ILIU(N).NE. G) THEN & STATOT & . 128 \\
\hline & ANS=' & STATOT & . 129 \\
\hline
\end{tabular}




\begin{tabular}{|c|c|c|c|}
\hline \multicolumn{2}{|c|}{ ISTAR $=1$} & \multirow{2}{*}{$\begin{array}{l}\text { STATOT } \\
\text { STATOT }\end{array}$} & \multirow{2}{*}{$\begin{array}{l}130 \\
\text {. } 131\end{array}$} \\
\hline & ENDIF & & \\
\hline & $N 1=N$ NOC $(N)$ & STATOT & . 132 \\
\hline & $N 2=N 1+N S N C_{-1}$ & STATOT & . 133 \\
\hline & $N 3=N 1-1$ & STATOT & . 134 \\
\hline & $\operatorname{RN}(1)=\operatorname{PAR}(N, 1)$ & STATOT & 135 \\
\hline & $\operatorname{RN}(2)=\operatorname{PAR}(N, 2)$ & STATOT & . 136 \\
\hline & CO TO $(210,220,220,250,250,280,270,280,320,320,300)$, ITYPE(N) & STATOT & . 137 \\
\hline c & & STATOT & . 138 \\
\hline C & NORUN & STATOT & . 139 \\
\hline C & & STATOT & . 148 \\
\hline \multirow[t]{5}{*}{210} & CDNTIRE & STATOT & . 141 \\
\hline & $R D(1)=Y A Y G(N)$ & STATOT & . 142 \\
\hline & $R O(2)=Y S T D(K)$ & STATOF & . 143 \\
\hline & $I=2$ & STATOT & . 144 \\
\hline & GO TO 310 & SFATOT & . 145 \\
\hline$c$ & & STATQT & . 146 \\
\hline$c$ & LDGKORMLL & STATOT & . 147 \\
\hline c & & STATOT & . 148 \\
\hline \multirow[t]{7}{*}{220} & CONTINUE & STATOT & . 149 \\
\hline & $A=1.6 D \theta$ & STATOT & . 150 \\
\hline & IF (ITYPE(N).EQ.3) A=BASEXP & STATOT & . 151 \\
\hline & $B=6 . \pm D E$ & STATOT & . 152 \\
\hline & DO $236 \mathrm{~K}=1$, NSAX & STATOT & . $\quad 153$ \\
\hline & $J=k+1 / 3$ & STATOT & . 154 \\
\hline & $B=B+D R E(A L O G(\operatorname{VAR}(J))) / A$ & STATOT & . 155 \\
\hline \multirow[t]{6}{*}{236} & CONTINUE & STATOT & . 158 \\
\hline & $\operatorname{RD}(1)=S N C L(B) / F L O A T($ KSAC) & STATOT & . 157 \\
\hline & $B=0.000$ & STATOT & . 158 \\
\hline & DO $240 \mathrm{~K}=1$, NSAC & STATOT & . 159 \\
\hline & $J=K+N 3$ & STATOT & . 180 \\
\hline & $B=B+\operatorname{DBLE}((\operatorname{ALOG}(\operatorname{YAR}(J)) / A-R D(1)) * * 2)$ & STATOT & . 161 \\
\hline \multirow[t]{4}{*}{240} & CONTINUE & STATOT & . 162 \\
\hline & $\operatorname{RD}(2)=S Q R T(S N C L(B) / F L A T(N S N K C))$ & STATOT & . 163 \\
\hline & $I=2$ & STATOT & . 184 \\
\hline & GO TO 310 & STATOT & . 185 \\
\hline$C$ & & STAFOT & . 186 \\
\hline $\mathrm{C}$ & LOCUNIFORU & STATOT & . 167 \\
\hline $\mathrm{c}$ & & STATOT & . 168 \\
\hline \multirow[t]{7}{*}{250} & CONTINUE & STATOT & . 169 \\
\hline & $A=1.000$ & STATOT & . 170 \\
\hline & IF (ITYPE(N).EQ.5) A=BASEXP & STATOT & . 171 \\
\hline & $\operatorname{RD}(1)=A L O G(\operatorname{VAR}(N 1)) / S N C$ (A) & STATOT & .172 \\
\hline & $\operatorname{RD}(2)=\operatorname{LOOG}($ YAR $(\mathrm{K} 2)) /$ SNCL $(A)$ & STATOT & . 173 \\
\hline & $I=2$ & STATOT & . 174 \\
\hline & GO TO 310 & STATOT & . 175 \\
\hline C & & STATOT & . 176 \\
\hline$c$ & EXPONENTIAL. & STATOT & . $\quad 177$ \\
\hline$c$ & & STATOT & . $\quad 178$ \\
\hline \multirow[t]{5}{*}{260} & CONTINUE & STATOT & . 179 \\
\hline & $\operatorname{RD}(1)=\operatorname{VAVG}(\boldsymbol{N})$ & STATQT & . 180 \\
\hline & $\operatorname{RN}(1)=1.6 / \operatorname{PAR}(N, 1)$ & STATOT & . 181 \\
\hline & $I=1$ & STATOT & . $\quad 182$ \\
\hline & GO TO 310 & STATOT & . 183 \\
\hline
\end{tabular}




\begin{tabular}{|c|c|c|c|}
\hline c & & STATOT & . 184 \\
\hline$c$ & UNIFORM & STATOT & . 185 \\
\hline C & & STATOT & . 188 \\
\hline 276 & CONTINUE & STATQT & . 187 \\
\hline & $J=N \operatorname{MoC}(N)$ & STATOT & . 188 \\
\hline & $R D(1)=\operatorname{VAR}(K 1)$ & STATQT & . 189 \\
\hline & $R D(2)=V A R(N 2)$ & STATOT & . 190 \\
\hline & $I=2$ & STATOT & . 191 \\
\hline & GO TO 316 & STATOT & . 192 \\
\hline c & & STATOT & . 193 \\
\hline C & BERNOU $\perp I$ & STATOT & . 194 \\
\hline 6 & & STATQT & . 195 \\
\hline 289 & CONTINUE & STATOT & . 298 \\
\hline & $\operatorname{RN}(1)=\operatorname{PAR}(N, 3)$ & STATOT & . 197 \\
\hline & IF $((\operatorname{VAR}(N 1)$. EQ. VAR(N2)) .OR. (NSALC.LT.2)) THEN & STATQT & . 198 \\
\hline & $\operatorname{RD}(1)=0.6$ & STATOT & . 199 \\
\hline & $I=1$ & STATQT & . 200 \\
\hline & GO TO 319 & STATOT & . 281 \\
\hline & ENDIF & STATOT & . 282 \\
\hline & $A=\operatorname{VAR}(\mathrm{N} 1)$ & STATOT & 203 \\
\hline & $K=\varnothing$ & STATOT & 264 \\
\hline 299 & CONTIME & STATDT & . 265 \\
\hline & $k=k+1$ & STATOT & . 208 \\
\hline & $J=N 3+K$ & STATOT & . 297 \\
\hline & IF (A.GE.VAR(J)) GO TO 290 & STATOT & . 268 \\
\hline & RD(1) $=($ FLAT $(K)-6.5) / F L O A T(N S A K C)$ & STATOT & - 269 \\
\hline & $I=1$ & STATQT & . 210 \\
\hline & GO TO 310 & STATQT & . 211 \\
\hline C & & STATOT & 212 \\
\hline C & CONSTANT & STATOT & . 213 \\
\hline C & & STATOT & . 214 \\
\hline 360 & CONTIMUE & STATOT & . 215 \\
\hline & $R D\{1)=\operatorname{VAR}(N 1)$ & STATOT & 218 \\
\hline & $I=1$ & STATOT & . 217 \\
\hline C & & STATOT & 218 \\
\hline 310 & CONTINUE & STATOT & 219 \\
\hline & IF (I.OT.1) THEN & STATOT & 220 \\
\hline & URITE (III, 540) VABE (N), ANS, RD (1), RD(2), RU(1), RM(2) & STATOT & . 221 \\
\hline & ESE & STATOT & - 222 \\
\hline & WRITE (III, 559) VABE (N), ANS, RD(1), RM(1) & STATOT & . 223 \\
\hline & ENDIF & STATUT & . 224 \\
\hline 326 & CONTINUE & STATOT & 225 \\
\hline & IF (ISTAR.NE. D) URITE $(1 \mathrm{II}, 566)$ & STATOT & - 228 \\
\hline 336 & CDNTINUE & STATOT & . 227 \\
\hline C & & STATOT & . 228 \\
\hline C & OUTPUT VARIABLE STATISTICS & STATOT & . 229 \\
\hline c & & STATOT & . 230 \\
\hline & IF (NSOLN.LT.1) G0 TO 350 & STATOT & . 231 \\
\hline & WRITE (III, 570) & STATOT & . 232 \\
\hline & WRITE $(I I I, 510)$ & STATOT & . 233 \\
\hline & DO $348 \quad N=1, N S O L N$ & STATOT & . 234 \\
\hline & WITE (III, 586) PLABE (N) & STATOT & . 235 \\
\hline & VRITE $(I I I, 598)$ FNIN(N),FMX(N), FAYG(N), FMED $(N), F S T D(N), F S K W(N)$ & STATOT & . 238 \\
\hline 340 & CONTINUE & STATOT & . 237 \\
\hline
\end{tabular}




\begin{tabular}{|c|c|c|c|}
\hline \multicolumn{2}{|c|}{356 CONTINUE } & \multirow{2}{*}{$\begin{array}{l}\text { STATDT } \\
\text { STATDT }\end{array}$} & \multirow{2}{*}{$\begin{array}{r}238 \\
-\quad 239\end{array}$} \\
\hline c & & & \\
\hline c & \multirow{2}{*}{ PRINT RUBER OF RAHDDH SALPLES REJECTED DUE TD LIUTS } & STATOT & . 248 \\
\hline$c$ & & STATOT & 241 \\
\hline \multicolumn{2}{|r|}{ IF (NYAR.LT.1) GO TO 420} & STATOT & 242 \\
\hline \multicolumn{2}{|r|}{ DD $3 B O N=1$, NYAR } & STATOT & 243 \\
\hline \multicolumn{2}{|r|}{ IF (ILIN(N).ER.0) O0 TO 380} & STATOT & 244 \\
\hline \multicolumn{2}{|r|}{ IF ((MIU(N,1),EQ.0),AND. (NLIM(N,2).EQ.0)) GQ T0 390} & STATOT & 245 \\
\hline \multicolumn{2}{|r|}{ WRITE (IOUT,606) VABE (N) } & STATOT & 246 \\
\hline \multicolumn{2}{|r|}{ IF (IDAT.NE.6) WRITE (IDAT, 6E6) VABEL(N) } & STATOT & 247 \\
\hline \multicolumn{2}{|r|}{ IF (NLIU(N,1).ER. E) GO TO 360} & STATOT & 248 \\
\hline \multicolumn{2}{|r|}{ IRITE (IOUT, 610) NLIM(N,1) } & STATOT & 249 \\
\hline \multicolumn{2}{|r|}{ IF (IDAT, NE. } & STATOT & 250 \\
\hline \multirow[t]{4}{*}{360} & CONTINUE & STATOT & . 251 \\
\hline & IF (NLIN(N,2).Eg.ø) CO TO 370 & STATOT & . 252 \\
\hline & IRITE (IDUT, 629) NLIM $(N, 2)$ & STATOT & . 253 \\
\hline & IF (IDAT.NE. б) WRITE (IOAT,620) NLIM(N,2) & STATOT & . 254 \\
\hline \multicolumn{2}{|c|}{376 CONTIMUE } & STATOT & 255 \\
\hline \multicolumn{2}{|c|}{380 COXTINUE } & STATOT & 258 \\
\hline \multicolumn{2}{|l|}{$c$} & STATOT & . 257 \\
\hline C & \multirow[t]{2}{*}{ STRATIFIED SAMPING DIAGNOSTICS } & STATOT & . 258 \\
\hline C & & STATOT & . 259 \\
\hline \multicolumn{2}{|r|}{ IF ((NLEVE.LT.2).OR. (IDAT.EQ.9)) GO TO 410} & STATOT & . 280 \\
\hline & IRITE (IDAT, 636) & STATOT & . 281 \\
\hline & DD $400 \mathrm{~N}=1$, INAR & STATOT & . 282 \\
\hline & IF (ITYPE(N).ER.NTYPE) GO TO 400 & STATOY & . 283 \\
\hline & IF (ITYPE(N).ER.10) G0 TO 460 & STATOT & 264 \\
\hline & K=NLEYB & STATOT & . 285 \\
\hline & IF $(K . G T .10) \quad K=10$ & STATOT & . 266 \\
\hline & NRITE (IDAT, 640) VAABE (N), (NPTSPL $(I, N), I=1, K)$ & STATOT & . 267 \\
\hline & IF (K.E.NLEVE) CD TO 460 & STATOT & 268 \\
\hline 390 & CONTINUE & STATOT & . 269 \\
\hline & $J=k+1$ & STATOT & . 270 \\
\hline & $K=K \cdot 10$ & STATOT & . 271 \\
\hline & IF (K.GT. MEYB) K=NLEVE & STATOT & . 272 \\
\hline & WRITE (IDAT, B5A) (NPTSPL $(I, N), I=J, K)$ & STATOT & 273 \\
\hline & IF (K.LT. MEVEZ) GO TO 390 & STATOF & . 274 \\
\hline 460 & CONTINUE & STATAT & 276 \\
\hline 410 & CONTINUE & STATOT & 276 \\
\hline c & & STATOT & . 277 \\
\hline 420 & CONTIMUE & STATOT & . 278 \\
\hline & RETURN & STATQT & . 279 \\
\hline 500 & FORUT (//1X,'**--- SAMPLE DIAGNOSTICS, INPUT DISTRIEUTIONS') & STATOT & . 280 \\
\hline 516 & FORUT (/T19, 'UIN', T39, 'WAX', T46, 'UEAN', T51, 'UEDIAN', TB1, 'STND DEV & STATOT & . 281 \\
\hline & $+^{\prime}$, T72, 'SKENESS'\} & STATOT & . 282 \\
\hline 528 & FORUT $(1 X, A 12,1 X, 1$ PE10.3, $5(1 X$, E10.3) $)$ & STATOT & . 283 \\
\hline 539 & FORUT (//1X,'**--- WXXIUM LIKEIHOOD ESTIMATORS FOR INPUT DISTRI & sTATOT & . 284 \\
\hline & +ВUTIONS'/T24, 'CDLPUTED VALUES', T52, 'EXPECTED VNLUES'/3X, 'VARIABLE' & STATOT & . 285 \\
\hline & $\rightarrow$, T26, 'PARANETER 1', T33, 'PARANETER 2', T48, 'PARALETER 1',T61, 'PARAME & STATOT & . 288 \\
\hline & +TER 2') & STATOT & . 287 \\
\hline $540 \mathrm{P}$ & FORUAT $(1 X, A 12,3 X, A 1, T 20,1 P E 16.3,3 X, E 10.3, T 48, E 16.3, T 61$, E16.3) & STATOT & . 288 \\
\hline 550 & FORUAT $(1 X, A 12,3 X, A 1, T 2 \theta, 1 P E 10.3, T 48, E 10.3)$ & STATOT & . 289 \\
\hline 566 & FORMT (T33,'* INDICATES ENDPOINT LIMITING WAY WOVE THE '/T35, 'DIS & STATOT & 290 \\
\hline & +TRIQUTION AWAY FRON THE EXPECTED YALUES. ') & STATOT & . $\quad 291$ \\
\hline
\end{tabular}




\begin{tabular}{|c|c|c|}
\hline 570 FORUAT (//1X, ${ }^{\prime} * *---$ SNAPLE DIAGNOSTICS, OUTPUT DISTRIBUTIONS') & STATOT & $\begin{array}{r}292 \\
293\end{array}$ \\
\hline 580 FORUT $(1 \times, 13 \times, 1 P E 16.3,5(1 X, E 10.3))$ & STATOT & 294 \\
\hline 86E FORUAT $\left(/ / 3 X_{1}{ }^{\prime}\right.$ FOR THE DISTRIBUTION IN $\left.', A 12\right)$ & STATOT & 295 \\
\hline 816 FORUT (3X,I6,' RANDOY SAHFLE TERE BEOW THE LDWER LIMIT, AND SET & STATDT & 298 \\
\hline + TO THAT LIMIT.') & STATOT & 297 \\
\hline 629 FORUAT (3X, I6,' RANDOU SAMPLES WERE ABOVE THE UPPER LIMIT, AND SET & STATOT & . 298 \\
\hline • TO THAT LIUIT.') & STATOT & 299 \\
\hline 830 FORUT (//7X,'STRATIFIED SAIPLING DIAGNDSTICS'//1X, 'YARIABLE',17X & STATOT & 386 \\
\hline,+ 'NUMBER DF SAMPLES CHOSEN FRDM EACH LEYE') & STATOT & 381 \\
\hline 640 FORUT $(1 X, A 12,2 X, 10(1 X, I 5))$ & STATDT & 302 \\
\hline 656 FORMT $(15 X, 10(1 X, I 5))$ & STATOT & . 363 \\
\hline END & STATOT & 304 \\
\hline SUBROUTINE STATS (N, RNDVA, NIN, AMAX, AMEAN, STDEY, SKEY) & STATS & 2 \\
\hline & STATS & 3 \\
\hline THIS ROUTINE COUPUTES THE MINIMM, MAXIMN, MEAN, STANDARD & STATS & 4 \\
\hline DEVIATION, AND SKEINESS FOR THE DATA SET STORED IN VECTOR RNDVAL. & STATS & 5 \\
\hline & STATS & 6 \\
\hline REN $* 8$ & STATS & 7 \\
\hline$+\quad A, A V G, B, B S A N, C, C S A N, D$, BASEXP & STATS & 8 \\
\hline DIMENSION & STATS & 9 \\
\hline$+\quad$ RNDVAL(N) & STATS & 19 \\
\hline DATA & STATS & 11 \\
\hline$\rightarrow \quad$ BASEXP/2.3E3DO/ & STATS & 12 \\
\hline & STATS & 13 \\
\hline & STATS & 14 \\
\hline BSAM=OBLE(FLOAT $(N))$ & STATS & 15 \\
\hline$C S N=B S A M+1.6 D \theta$ & STATS & 16 \\
\hline & STATS & 17 \\
\hline AMEAN=RNDVAL(1) & STATS & 18 \\
\hline STDEY $=0$. & STATS & 19 \\
\hline$S K E Y=0.0$ & STATS & 26 \\
\hline IF (N.LE. 1) OB TO 498 & STATS & 21 \\
\hline & STATS & 22 \\
\hline COLPUTE AYERAGE, WIN AND WAX VALUE & STATS & 23 \\
\hline & STATS & 24 \\
\hline AWAX $=R$ NOVAL(1) & STATS & 25 \\
\hline NMIN=ANAX & STATS & 26 \\
\hline$A V G=O B L E(A N A X)$ & STATS & 27 \\
\hline$D 0100 \mathrm{I}=2, \mathrm{~N}$ & STATS & $2 B$ \\
\hline RV=RNDVAL (I) & STATS & 29 \\
\hline$A V G=A V G+D Q E E(R V)$ & STATS & 30 \\
\hline IF (RV.LT. NIN) AMIN=RV & STATS & 31 \\
\hline IF (RY.GT. $A M A X) \quad A H N X=R Y$ & STATS & 32 \\
\hline 108 CONTINUE & STATS & 33 \\
\hline$A Y G=A Y G / B S N$ & STATS & 34 \\
\hline NMEAN=SNCL (AVG) & STATS & 36 \\
\hline & STATS & 36 \\
\hline COUPUTE STAND DEY AND SKEINESS & STATS & 37 \\
\hline ; & STATS & 38 \\
\hline$\theta=0.00$ & STATS & 39 \\
\hline$C=6.6 \mathrm{DO}$ & STATS & 40 \\
\hline $00110 I=1, N$ & STATS & 41 \\
\hline$A=A Y G-D Q L E(R N D V A L(I))$ & STATS & 42 \\
\hline
\end{tabular}




\begin{tabular}{|c|c|c|c|}
\hline & $B=B+A * A$ & STATS & 43 \\
\hline & $C=C+A \neq A \neq A$ & STATS & 44 \\
\hline & 10 CONTIMUE & STATS & 45 \\
\hline & STDEY=SIME (DSQRT (B/CSAN) & STATS & 46 \\
\hline & SKEI $=$ SNCL $((D A B S(C / C S A N)) * * 0.3333333 D 8)$ & STATS & 47 \\
\hline & IF (C.LT. . 6DO) SKET=-SKE & STATS & 40 \\
\hline 6 & & STATS & 49 \\
\hline & 80 CONTINUE & STATS & 58 \\
\hline & RETURN & STATS & 51 \\
\hline & END & STATS & 52 \\
\hline & REN FUNCTION UA1( DSEED ) & UE1 & 2 \\
\hline$C *$ & & UE1 & 3 \\
\hline$C *$ & & UE1 & 4 \\
\hline C* & PURPOSE: & 101 & 5 \\
\hline$C *$ & & var & . \\
\hline$C *$ & THIS FUNCTION GENERATES A REN*4 RAKDOU UNIFORA $(0,1)$ VARIATE & W1 & 7 \\
\hline C* & GIVEN AN INITIN YALUE OF THE SEED, DSEED. THE INPUT SEED & Ua1 & 8 \\
\hline$C_{*}$ & IS UPDATED TO A NE SEED USING A LINEAR CONGRUENTN METHOD. & Ua1 & . \\
\hline C* & & Uo1 & 10 \\
\hline$C_{*}$ & CURREUT USAGE IS FOR THE PRIME 760, 32 BIT WORD LEYGTH. & U01 & 11 \\
\hline C* & & Uo1 & . 12 \\
\hline C* & DSEED = REN $\approx 8$ SEED FOR RANDOU NUMBER GENERATOR. WUST BE BETWEEN & U1 & . 13 \\
\hline C* & ONE AND TIO TO THE THIRTY FIRST POWER. & 101 & . 14 \\
\hline$C_{*}$ & & Us1 & . 15 \\
\hline$C *$ & REGRENCE: & Ua: & . 16 \\
\hline C* & & Uo1 & . 17 \\
\hline$C_{*}$ & LEIIS, COODMN, ANO MILER (1969). 'A PSEUDO-RANDOW NUMBR & บo1 & . $\quad 18$ \\
\hline C* & GENERATOR FQR THE SYSTEI/366" IEU SYSTEIS JOURNAL, YOL. B, & Uo1 & 19 \\
\hline & No. $2, P P, 138-145$ & Uo1 & . 20 \\
\hline C* & & UO1 & . 21 \\
\hline$C *$ & & UO1 & . $\quad 22$ \\
\hline & REAL*8 B, M, DSEED & U01 & . 23 \\
\hline C & & U01 & . 24 \\
\hline & DATA В / $16807.60+60 /$ & Ua1 & 25 \\
\hline & DATA 山 / 2147483647.60+06/ & Uo1 & . 28 \\
\hline C & & Wo1 & . $\quad 27$ \\
\hline & DSEED $=\operatorname{DINT}(\operatorname{OMD}(B * D S E E D, M))$ & Uo1 & - 28 \\
\hline C & & Lo1 & . $\quad 29$ \\
\hline & UO1 = SNEL ( DSEED/U ) & Ua1 & . 30 \\
\hline $\mathrm{C}$ & & U日1 & . $\quad 31$ \\
\hline & RETURN & Uo1 & 32 \\
\hline & END & Uat & 33 \\
\hline & SUBROUIINE UBIS (N, RNDYAL, DSEED, NLEVE) & UO1S & . \\
\hline c & & Ua1s & 3 \\
\hline c & THIS ROUTINE COMPUTES A VECTOR OF RANDOLY GENERATED NUMBERS ON & Uo1s & 4 \\
\hline $\mathrm{C}$ & THE INTERVN 0,1 , IFH STRATIFICATION. STRATIFICATION IS IGNORED & Uo1s & 5 \\
\hline$c$ & IF ONLY ONE LEYE. IS REQUESTED. & UO1S & . \\
\hline c & STRATIFICATION IS 'CHECKED', BUT NOT ACTED UPON UNTIL AT LEAST & Uo1S & . \\
\hline $\mathrm{C}$ & HALF OF IHE CHOSEN LEVES ARE FU工. THIS WAKES THE ROUTINE RUN & Lo1s & 8 \\
\hline C & WODERATEY FAST FOR THE WHJURITY OF THE TIUE, SLOWING TO ENSURE & U日1S & . \\
\hline C & STRATIFICATION ONLY ON THE LAST FE DATA POINTS COLLETED. & Uo1s & . 10 \\
\hline$c$ & & Uo1S & . 11 \\
\hline ᄃ & DSEED = RELL $* 8$ SEED FOR RANDQW NUNBER GENERATOR. WST BE BETWEEN & Uo1s & . 12 \\
\hline C & ONE AND TTO TO THE THIRTY FIRST POWER. & Uo15 & 13 \\
\hline
\end{tabular}




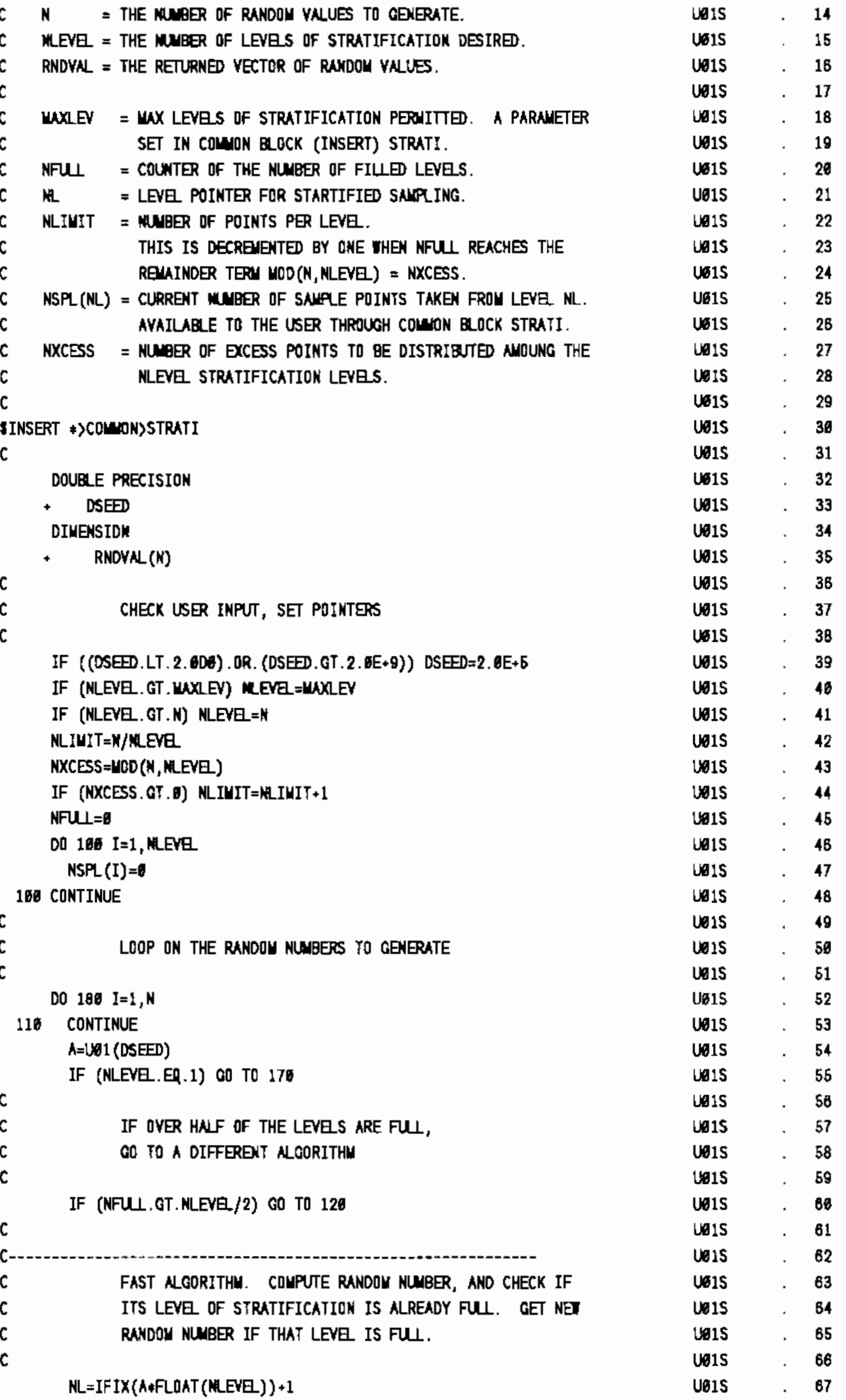


IF (NSPL(NL).CE. NLINIT) G0 T0 110

Ua1S . 68

c IMCREMENT COUNTER AND SAVE YNUE

vo1S . 69

c

$c$

GO TO 150

vais . 76

Uo1s . 71

Uo1s . 72

$c$

Ua1S . 73

C

C

C SLOW NGORITH, RANDOLY LOCATES AN ENPTY STRATIFICATION

UV15 . 74

UB1S . 76

Uo1S . 75

va1s $\quad 77$

LEYH AND FORCE NE RANDOU NUMBER TO BE IN THAT LEYE. JOIS . 78

C

120 CDNTINUE

$c$

c

c

FIND $A$ LEVE AT RANDOU WHICH IS NOT FILLED

Ua1S $\quad 79$

Wis . 80

Ua1S . B1

Uo1s . 82

Wa1s . 83

LIFIX(A*FLDAT(NLEVE-NFUL) )+1 U61S . 84

$\mathrm{KL}=0$ Ua1S. 85

DO $130 \mathrm{~J}=1$, NLEYH $\quad$ UO1S . 86

$\mathrm{K}=\mathrm{J}$

IF (NSPL(J) .LT, NLIUIT) NL=NL+1

UeIS . B7

130 CONTINLE

STOP 'UOIS'

$\begin{array}{ll}\text { Uo1S } & 88 \\ \text { Uo15 } & 89\end{array}$

U915 . 86

va1s . 91

148 CONTINUE Ua1S $\quad 92$

$M=K \quad$ UB1S . 93

C C Ue1S . 94

C COUPUTE EIDPOINTS FOR THAT LEYE AND UNIS 95

6 FIND A RANDOH VALUE WITHIN THAT LEVE. va1S . 96

C $\quad$ Uais $\quad .97$

B=FLOAT(NL-1)/FLOAT(HLEYH) $\quad$ UOS1S . 98

T=FLOAT(ML)/FLOAT(NLEVE) $\quad$ UD1S . 99

$A=B+\angle B 1(D S E D) *(T-B) \quad$ Ua1S * 106

C-

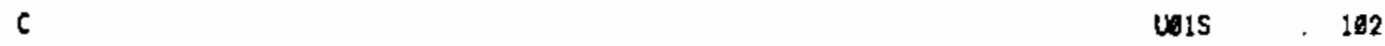

6 INCRENENT \$ ITES IN CURRENT LEVE, CURRENT VARIABLE LOIS . IB3

C $\quad$ UO1S , 104

1SE CONTINUE UDIS , 165

NSPL(NL)=NSPL(KL)+1 U91S . 196

\begin{tabular}{|c|c|c|c|}
\hline C & & ueis & 107 \\
\hline C & CHECK TO SEE IF ITES THIS LEVE HAS REACHED LIMIT & weis & . 188 \\
\hline$c$ & & Uo1S & . 169 \\
\hline & IF (NSPL(ML).LT. NLINIT) GO TO 170 & Uo1S & 110 \\
\hline C & & ve1s & 111 \\
\hline$c$ & INCREATT FUL LEYHS COUNTER & Uo1s & 112 \\
\hline $\mathrm{C}$ & & Uo1S & 113 \\
\hline & NFU $=N F U \sqcup+1$ & We1s & 114 \\
\hline$c$ & & Uo1s & 115 \\
\hline C & IF FUL LEYES = NUNBER DF EXCESS LEVES, WST & ve1s & 116 \\
\hline C & DECREEEIT PERUISSAQRE LEVE SIZE, AND RE-COUNT & veis & $1 \sqrt{11}$ \\
\hline$c$ & THE NUYER OF FULL LEVES. & Lo1S & 118 \\
\hline$c$ & & U61S & 119 \\
\hline & IF (NFU,.NE. NXCESS) GO TO 17B & Ue1S & 128 \\
\hline & RIMIT=NILIT-1 & Uo1s & 121 \\
\hline
\end{tabular}




\begin{tabular}{|c|c|c|c|}
\hline & DO $180 \mathrm{~J}=1$, NLEVE & Wais & 122 \\
\hline & IF (NSPL(J).ER.NLIMIT) FUL=NFUL+1 & UB1S & 123 \\
\hline 180 & CONTIIXE & Uø1S & 124 \\
\hline$c$ & & ue1s & . 125 \\
\hline C & SET THE RANDOU VARIABLE INTO THE DUTPUT ARRAY & Uais & . 126 \\
\hline c & & Wois & 127 \\
\hline 179 & CONTIME & UBis & . 128 \\
\hline & RNDVN $(I)=h$ & Leis & . 129 \\
\hline 180 & CONTINUE & uB1s & . 139 \\
\hline & RETJRN & Uøis & 131 \\
\hline & END & uals & . 132 \\
\hline & SUBROUTINE VARSET (IERR) & VARSET & 2 \\
\hline c & & VARSET & 3 \\
\hline C & THIS ROUTINE COMPUTES THE RANDOU INPUT VARIARLES VALUES FRON & VARSET & 4 \\
\hline c & THE SPECIFIED DISTRIBUTIONS. & VARSET & 5 \\
\hline$c$ & & VARSET & 6 \\
\hline $\mathrm{C}$ & NOTE THE RANDOU OATA IS FIRST PLACED INTO ARRAY CONF BY THE & VARSET & 7 \\
\hline c & RANDON MUBER GENERATOR ROUTINES. THE DATA IS THEN NOVED INTD & VARSET & 8 \\
\hline c & THE STORAGE ARRAY VAR. THIS TECHNIQUE IS REQUIRED BY VIRTUAL & VARSET & 9 \\
\hline$c$ & MENORY CDMPUTERS, TO ENSURE PRDPER PAGINO OF THE DATA, WHEN THE & VARSET & 10 \\
\hline$c$ & PARAMETER ISIZE EXCEEDS THE PAGE SIZE. & VARSET & 11 \\
\hline c & & VARSET & 12 \\
\hline SINSER & RT $\nRightarrow$ COUNN>PPARA & VARSET & 13 \\
\hline SINSER & $R T *>C O M D N>P B L K I$ & VARSET & 14 \\
\hline SINSER & RT $>$ CQMON)PBLE & VARSET & 15 \\
\hline SINSER & RT $\gg$ CONON)PBLKa & VARSET & 16 \\
\hline SINSER & RT *CONOK>PBLK17 & VARSET & 17 \\
\hline$c$ & & VARSET & 18 \\
\hline$c$ & LOOP ON THE NUMBER OF VARIABLES & VARSET & 19 \\
\hline$c$ & COUPUTE A RANOOU SAMPLE SET FDR EACH VARIABLE & VARSET & 20 \\
\hline C & & VARSET & 21 \\
\hline & IERR $=0$ & VARSET & 22 \\
\hline & DO $110 \mathrm{~N}=1$, NYAR & VARSET & 23 \\
\hline & CND RAOS (IERR, N, NSNN, CONF) & VARSET & 24 \\
\hline & IF (IERR.NE. 6) RETURN & VARSET & 25 \\
\hline & $J=N$ NOC $(n)-1$ & VARSET & 26 \\
\hline & DO $180 I=1$, NSAN & VARSET & 27 \\
\hline & $K=I+J$ & VARSET & 28 \\
\hline & $\operatorname{VAR}(K)=\operatorname{CONF}(I)$ & VARSET & 29 \\
\hline 100 & CONTINUE & VARSET & 30 \\
\hline 110 & CONTINUE & VARSET & 31 \\
\hline & RETURN & VARSET & 32 \\
\hline & 510 & VARSET & 33 \\
\hline & SUBROUTINE WHEADR (IFLO, IERR) & WHEADR & 2 \\
\hline C & & THEADR & 3 \\
\hline C & THIS ROUTINE WRITES FILE HEADER RECORD(S) TD THE BINARY DATA & WHEADR & 4 \\
\hline C & FILES ASSOCIATED WITH THE WGNUN ANO CHAINT PROGRAUS. THE FIRST & WHEADR & 5 \\
\hline C & RECGRD CONTAINS IMFORMTION CONCERNING THE IDENTITY OF THE CURRENT & WHEADR & 6 \\
\hline C & FILE. THE SECOND RECORD, IF NEEDED, CONTAINS A BRIE SUMARY OF & WHEADR & 7 \\
\hline C & THE FILES WHICH TERE LSED AS SOURCE DATA FOR THE RUN WHICH CREATED & WHEADR & a \\
\hline C & THE CURRENT FILE. & WHEADR & 9 \\
\hline C & & WHEADR & 16 \\
\hline $\mathrm{C}--\mathrm{CON}$ & NTROL DATA & THEADR & 11 \\
\hline C & IFLO = OUTPUT FILE UNIT NLWER & WHEADR & 12 \\
\hline
\end{tabular}


C IERR = ERROR POINTER, = FOR SUCCESSFU HEADER WRITE

C--HEADER RECORD IHFORUATION

C NSRC = MUBER OF FILE TRACEAACK RECORDS

C JRUNID = IDEUTIFIER (DATE/TINE) OF SOURCE RUN

C USRNA = NANE OF USER WHO INITIATED SOURCE RUN (CHAR*8)

C PRG = NAME OF SOURCE PROGRAM (CHAR*B)

C FL = FILE TYPE NALE (CHAR*8)

C VERNUN = VERSION MUBER OF SOURCE PROGRN

C TITLE = TITLE FROU SQURCE RUN (CHAR*BO)

$\mathrm{C}$

C--TRACERACK RECQRD IFORATION (ONIT IF NSRC=0)

C NRUNID(I) = IDEITIFIER OF INPUT FILE I

C FLT(I) = FILE TYPE OF INPUT FILE I (CHAR $* 8)$

C PRGT(I) = SQURCE PROGRAN OF INPUT FILE I (CHAR*8)

C USRNUT(I) = SQURCE PROGRA USER (CHAR*B)

C

SINSERT SYSTESS>UTIL>FELIB>COMON>IDCOU

3INSERT SYSTEAS>UTIL) USRLIB>CONON>INOUT

IERR $=0$

IF (NSRC.GT. WNSSR) THEX

WRITE (IDUT, 569) NSR, MXSRC

IERR $=1$

RETURN

ENDIF

C INITIN FILE HEADER

c

TRITE (IFLO) NSRC, JRUNID, USRNAN, FL, PRG, YERMU, (TITLE(I) , I=1, 26)

6

c

C

TRACERACK HEADER

IF (NSRC.GT.ø) WRITE (IFLO) (NRUNID(I),FLT(I), PRGT(I), USRNUT(I),

- $I=1$, NSRC)

RETURN

500 FORMT (/1X, ${ }^{1} * * *$ ERRIR, ',I5,' SOURCE FILES EXCEDS PROGRAW WXXIM

+4 OF ',I3,' $1 / 5 X$, 'ABORT IN ROUTINE THEADR.' $)$

END

SUBROUTINE WRSTOC (TOATB, IHEAOR)

$c$

THIS ROUTINE WRITES THE GINARY FILE FOR PROGRAN PACSTAT. BOTH

THE HEADER AND PACSTAT OUTPUT DATA ARE TRITTEN BY THIS CODE.

IDATB = BINARY FILE UNIT NUUEER

IHEADR = FOR DATA WRITE

$=1$ FOR HENOER TRITE

IINSERT $\Rightarrow$ CQWON>PPARA

IINSERT \#)COWON>PQEKI

SINSERT *) COMON)PQLK2

IINSERT $*>$ COINON $>$ PQLX3

SINSERT *)COMUN)PQLK4

SINSERT *)COMUN)PELKK

SINSERT *)COMUN PEQLK7
THEADR . 13

WHEADR , 14

THEADR , 15

THEADR , 16

THEADR . 17

WHEADR - 18

WEADR - 19

WHEAOR . 20

THEADR . 21

MHEADR . 22

WHEADR . 23

THEAOR . 24

WHEAOR . 25

THEADR . 26

WHEADR . 27

WHEAOR . 28

WHEADR . 29

WHEAOR . 30

THEAOR . 31

WHEADR . 32

MHEAOR . 33

VHEADR . 34

WHEADR . 35

VHEADR . 36

WHEADR , 37

WHEADR - 38

WHEADR , 39

THEADR + 46

WHEADR , 41

WHEADR . 42

MHEADR \& 43

THEAOR . 44

THEADR - 45

THEADR 、 48

WHEROR . 47

WHEADR . 48

WHEADR . 49

WHEKOR . 50

WRSTOC . 2

WRSTOC $\quad 3$

IRSTOC , 4

IRSTOC . 5

IRSTOC . 6

IRSTOC . 7

NRSTOC . 8

WRSTOC , 9

WRSTDC . 10

WRSTOC . 11

WRSTOC , 12

WRSTOC . 13

WRSTOC . 14

IRSTOC - 15

VRSTOC . 18

IRSTOC . 17 


\begin{tabular}{|c|c|c|}
\hline IINSERT $*>C O M A N>P E L X$ & MRSTOC & 18 \\
\hline SINSERT \#)COMON>PELK16 & WRSTOC & 18 \\
\hline SINSERT *XCOMON>PELK17 & URSTOC & 28 \\
\hline SINSERT SYSTESSUTIL>FELIB>COMON>IDCOM & TRSTOC & 21 \\
\hline 3INSERT SYSTES) UTIL>ULRLIB)COMON>INOUT & YRSTOC & 22 \\
\hline C & MRSTOC & 23 \\
\hline ERROR CHECK CAH PARANETERS & TRSTOC & 24 \\
\hline $\mathrm{C}$ & WRSTOC & 25 \\
\hline IF ((IHEADR.LT.G).OR. (IHEADR.GT.1)) THEN & WRSTOC & 26 \\
\hline VRITE (IDUT, 5ø0) IHEADR & MRSTOC & 27 \\
\hline GD TO 110 & WRSTOC & 28 \\
\hline ENDIF & IRSTOC & 29 \\
\hline IF (IDATB.LE. $\theta$ ) THEM & MRSTOC & 30 \\
\hline WRITE (IOUT,516) IOATB & DRSTOC & 31 \\
\hline GD TO 116 & TRSTOC & 32 \\
\hline ENDIF & WRSTOC & 33 \\
\hline c & URSTOC & 34 \\
\hline VRITE HEADER & MRSTOC & 35 \\
\hline C & MRSTDC & 36 \\
\hline IF (IHEADR. Eq. & WRSTOC & 37 \\
\hline REIIND (IDATB) & WRSTOC & $3 B$ \\
\hline NSRC $=$ & VRSTOC & 39 \\
\hline CAD WHEADR (IDATB, IERR) & WRSTOC & 46 \\
\hline IF (IERR, NE. A) STBP & WRSTOC & 41 \\
\hline$I=6$ & WRSTLC & 42 \\
\hline WRITE (IDATB) NSOLN, NYAR, NCCNST, NTTL, NSTTL, I, I, I & WRSTOC & 43 \\
\hline IF (NSOLN.GT.9) WRITE (IDATB) (PLABE (I), I=1, NSOLN) & IRSTOC & 44 \\
\hline IF (NYAR, GT, 6) URITE (IDATB) (VABE (I), VDIUEN(I), I=I, NYAR) & TRSTOC & 45 \\
\hline IF (NCDNST.GT. U) URITE (IDATB) (CLABEI (I),CDINEN(I), I=1, NCONST) & WRSTOC & 46 \\
\hline RETURN & WRSTOC & 47 \\
\hline C & VRSTOC & 48 \\
\hline TRITE FILE DATA & WRSTOC & 49 \\
\hline c & WRSTOC & 50 \\
\hline 160 CONTINUE & WRSTOC & 51 \\
\hline N1=NYAR*NSAN & IRSTDC & 52 \\
\hline N2=NSOLN*NSA & VRSTDC & 53 \\
\hline$I=0$ & WRSTOC & 54 \\
\hline WRITE (IDATB) ICNT, NSN, NSALC, I, I, I & WRSTOC & 55 \\
\hline IF (NCONST.GT. W) WRITE (IDATB) (CONST(I), I=1, NCONST) & MRTOC & 56 \\
\hline IF (NVAR.GT.E) THEV & WRSTOC & 57 \\
\hline WRITE (IDATB) (ITYPE(I), PAR(I, 1), PAR(I, 2), PAR(I, 3), I=1, NYAR) & MRSTOC & 58 \\
\hline WRITE (IDATB) (VAR(I), I=1,N1) & WRSTOC & 59 \\
\hline ENDIF & WRSTOC & 80 \\
\hline IF (NSOLN.GT.9) IRITE (IDATE) (RLTS(I), I=1,N2) & WRSTDC & 81 \\
\hline$c$ & WRSTDC & 62 \\
\hline RETURN & IRSTOC & 63 \\
\hline C & WRSTOC & 64 \\
\hline 110 CONTINUE & IRSTQC & 65 \\
\hline WRITE (IOUT, 520) & MRSTOC & 86 \\
\hline RETURN & WRSTOC & 67 \\
\hline c & WRSTOC & 68 \\
\hline 509 FORUA (/1X, 'ERROR, BINARY FILE YRITE. IHERDR UNDEFINED, $=$ ',I5) & MRSTOC & 89 \\
\hline 510 FORUAT (/1X,'ERROR, BINARY FILE VRITE. IDATB =', I5) & WRSTOC & 79 \\
\hline 528 FOFOMT (IX, 'ROUTIKE WRSTOC.') & WRSTOC & 71 \\
\hline
\end{tabular}


END

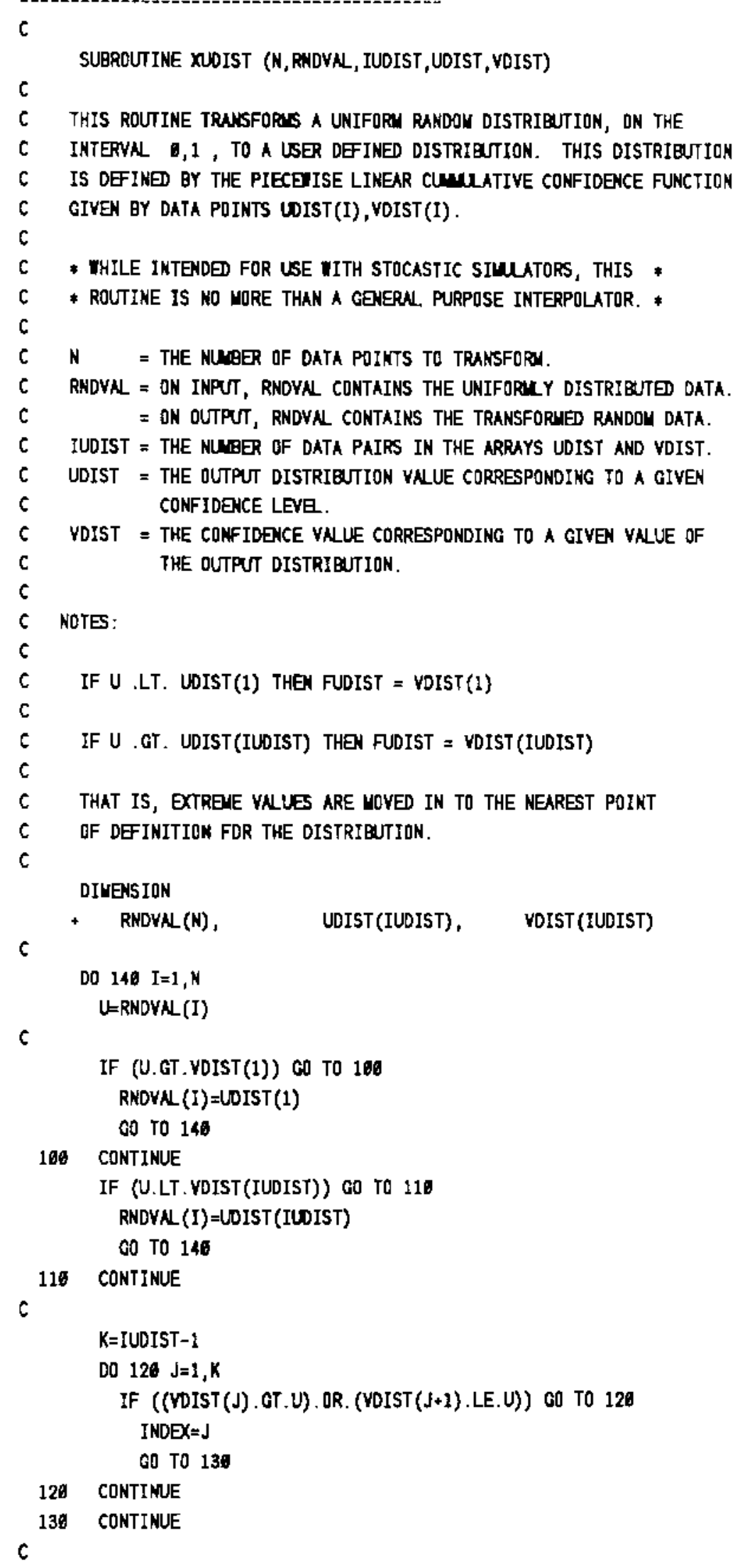

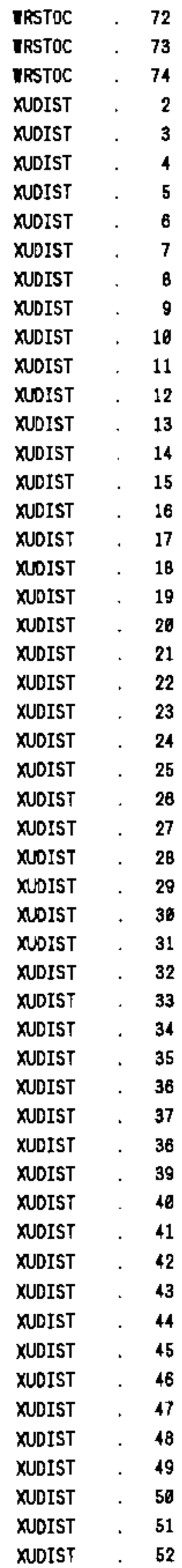




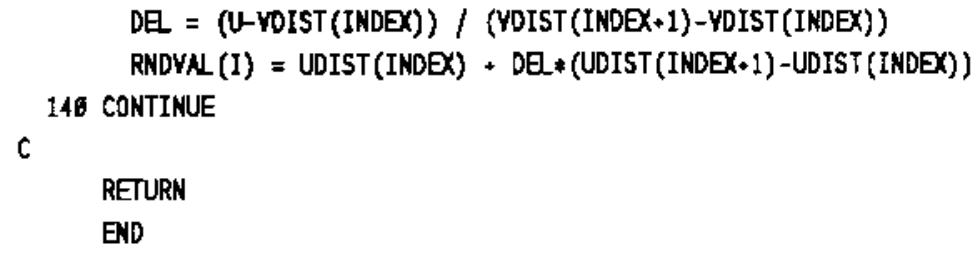

$\begin{array}{lll}\text { XUDIST } & \cdot 53 \\ \text { XUDIST } & \cdot & 54 \\ \text { XUDIST } & \cdot & 65 \\ \text { XUDIST } & \cdot & 56 \\ \text { XUDIST } & \cdot & 57 \\ \text { XUDIST } & 58\end{array}$



APPENDIX C

PACSTAT_B4.FOR (FINAL BATCH VERSION, FORTRAN SOURCE FILE) 


\begin{tabular}{|c|c|c|c|}
\hline & FUNCTION ALNORN $(x)$ & ALNORI & 2 \\
\hline C & & NLNDRM & 3 \\
\hline$c$ & ALGQRITHW FROU APPL. STATIST. (1973) VOL. 22, HO. 3, PP 424-427. & NNORM & 4 \\
\hline C & & ALNORI & . \\
\hline C & EVNUATES THE AREA UNDER A STANDARD NORWN CURVE (MEAN $=0.0$, & ALNDRM & . \\
\hline C & STAKDARD DEVIATION $=1.0$ ), INTEGRATING FROM UINUS INFINITY TO $x$. & NNORM & 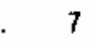 \\
\hline C & A POLYNOMIN UETHDD IS USED. & NLNOA & 8 \\
\hline C & & ALNORH & 8 \\
\hline & REN $* 8$ & ALNORH & 16 \\
\hline & $+\quad A$, & NLNDRM & 11 \\
\hline & LOGICAL & NLNOW & 12 \\
\hline & - POSTIV & ALMORM & 13 \\
\hline & DATA & NLNORU & 14 \\
\hline & $+\operatorname{CON} / 1.28 /$ & ALNORN & 15 \\
\hline & $+\quad$ UTZERO / $9.50 /$ & ALNORM & 16 \\
\hline$c$ & & ALNORM & 17 \\
\hline & POSTIV=. TRUE. & NLNORM & 18 \\
\hline & IF $(X . L T .8 . \emptyset)$ PDSTIV=.FNLSE. & ALNORM & 19 \\
\hline & $T=\operatorname{ABS}(X)$ & ALNORN & 28 \\
\hline & $Z=\operatorname{DQLE}(T)$ & ALNORN & 21 \\
\hline$c$ & & ALNDRH & 22 \\
\hline & IF (T.LT.UTZERO) GO TO 100 & ALNORU & 23 \\
\hline & $A \perp N O R=8.8$ & ALNDRH & 24 \\
\hline & GO TO 139 & ALNOPU & 25 \\
\hline 208 & CONTINUE & ALNOW & 28 \\
\hline & $Y=0.500+2.2$ & ALNORH & 27 \\
\hline & IF (T.GT.CON) GO TO 118 & ALNORM & 28 \\
\hline c & & ALNDRM & 29 \\
\hline & $A=0.500-Z *(0.398942280444 D 9-0.39998343850400 * Y /$ & ALNORN & 30 \\
\hline & - $(Y+5.76885486458 D 9-29.821355788800 /$ & ALNORN & 31 \\
\hline & $+(Y+2.6243312187906+48.695993669200 /$ & ALNORN & 32 \\
\hline & $+(Y+5.92885724438 D G))))$ & ALNORH & 33 \\
\hline & GO TO 120 & ALNORN & 34 \\
\hline 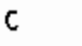 & & ALNORI & 35 \\
\hline 118 & CONTINUE & ALNORM & 38 \\
\hline & $A=0.39894228 \mathrm{~B} 385 * \operatorname{DEXP}(-Y) /$ & ALNORM & 37 \\
\hline & $+1.0000961538208 /$ & ALNORU & 38 \\
\hline & $+(Z+3.988647840-4 \cdot 1.9881538138408 /$ & HEIDI3 & 9 \\
\hline & $+(Z+4.838591280800-15.156897245106 /$ & HEIOI3 & 16 \\
\hline & - $(Z+4.838591280800+15.158897245108 /$ & ALNORU & 41 \\
\hline & - $\quad(Z+0.742388924627 D 8+30.78993383400 /$ & ALNORH & 42 \\
\hline & $+(Z+3.9981941781108))))))$ & ALNORL & 43 \\
\hline$c$ & & ALNORH & 44 \\
\hline 126 & CONTINUE & ALNORU & 45 \\
\hline & ALNORM = SNGL $(A)$ & ALNORM & 48 \\
\hline 130 & CONTINUE & ALNORM & 47 \\
\hline & IF (POSTIV) ALNDRM = $3.6-A L N D R M$ & ALNDRM & 48 \\
\hline & RETURN & ALNORM & 49 \\
\hline & END & AINORM & 58 \\
\hline & SUBROUTINE ASG (ITYP, IERR, IFIL, NLEN, INF) & ASG & 2 \\
\hline c & & ASO & . \\
\hline c & ROUTINE FOR INTERACTIVE ASSIGNMENT OF FILES ON PRIME & ASG & . \\
\hline$c$ & INPUT IS ITYP = 1 FOR WRITE FILE, $\approx$ FOR READ ONLY & ASG & . \\
\hline C & = OTHER FOR READ/WRITE FILE (WUST BE EXISTING FILE) & ASG & . \\
\hline
\end{tabular}




\begin{tabular}{|c|c|c|c|}
\hline$c$ & IERR = ERRDR RETURN FLAG & ASG & 7 \\
\hline c & IFIL = UNIT NUMBER TO USE & ASG & 8 \\
\hline$c$ & NLEN = DIUENSION OF INF & NSG & 8 \\
\hline c & INF = NANE OF INPUT FILE (CHAR $* 4$, DTUENSION = NLEN $)$ & ASG & 16 \\
\hline$c$ & & NSG & 11 \\
\hline$c$ & DESIGNED FOR PRIME 250 AND 750 . FUNITS 1-4, 40-42, 51, & NSG & 12 \\
\hline$c$ & 55 AND UNITS 21-2B ARE COMITTED ON THESE WACHINES. & NSG & 13 \\
\hline c & & ASG & 14 \\
\hline & COMNON /INDUT/ & INOUT & . \\
\hline & + IN, IOUT, IQB, IECHO & ECHO & 86 \\
\hline & CHARACTER $\approx 1$ & INOUT & 4 \\
\hline & $+\quad \mathrm{IQB}$ & INOUT & 5 \\
\hline$c$ & & INOUT & . \\
\hline c & & INOUT & 7 \\
\hline C & & NSG & 16 \\
\hline C - & PRIME - SINSERT SYSCOMDASKEYYS & ASG & 17 \\
\hline & LOGICAL LDGIC & ASG & 18 \\
\hline & CHARACTER $* 1$ & ASG & 19 \\
\hline & $+\quad$ ANS & ASG & 20 \\
\hline & CHARACTER $* 4$ & ASG & 21 \\
\hline & - INF(NLEN) & ASG & 22 \\
\hline & IERR=0 & ASO & 23 \\
\hline $\mathrm{C}$ & & NSG & . 24 \\
\hline C & CHECK FOR FILE NLREADY OPEN ON THIS UNIT NUMBER & ASG & 25 \\
\hline$c$ & & ASG & 26 \\
\hline & REIIND (IFIL, ERR=106) & ASG & 27 \\
\hline & WRITE (IOUT, 500) IFIL, (INF(I), I=1,NLEN) & ASO & . $\quad 28$ \\
\hline & GO TO 180 & ASG & 29 \\
\hline & 00 CONTINUE & ASG & 30 \\
\hline & NLL4=4*NLEN & ASG & 31 \\
\hline C & & ASG & . 32 \\
\hline C & CHECX FOR AШOWABLE UNIT NUMBER. SET PRIME OFFSET. & ASG & . 33 \\
\hline C & & ASG & . 34 \\
\hline & IF (IFIL.LE.4) G0 TO 110 & $A S G$ & 35 \\
\hline & IF ((IFIL.GE. 21). AND. (IFIL.LE. 28)) GO TO 110 & ASG & 36 \\
\hline & IF ((IFIL.GE. 40). AND. (IFIL.LE. 42)) GO TO 110 & ASG & 37 \\
\hline & IF ((IFIL.EQ.51). OR. (IFIL.EQ.55)) GO TO 110 & ASG & 38 \\
\hline & IF (IFIL.GT.127) GO TO 116 & ASG & 39 \\
\hline & GD TO 120 & ASG & 40 \\
\hline & 10 CONTINUE & ASG & 41 \\
\hline & WRITE (IOUT,516) IFIL & ASG & 42 \\
\hline & 20 CONTINUE & ASG & 43 \\
\hline & IF (IFIL.LT.21) THEN & ASG & 44 \\
\hline & IFUNIT $=I F I L-4$ & ASG & . 45 \\
\hline & 日SE & ASG & 48 \\
\hline & IFUNIT $=$ IFIL -12 & ASG & . 47 \\
\hline & ENDIF & ASG & 48 \\
\hline C & & ASG & . 49 \\
\hline $\mathrm{C}$ & CHECK TO SEE IF FILE NREADY EXISTS & ASG & 56 \\
\hline C & & ASG & 51 \\
\hline & 39 CONTINUE & NSG & . 52 \\
\hline & LDGIC = EXSTSA (INF, INTS (NNL4)) & NSG & . 53 \\
\hline c & & ASG & . 54 \\
\hline C & READ ONLY OR READ/WRITE FILE & ASG & . 55 \\
\hline
\end{tabular}




\begin{tabular}{|c|c|c|c|}
\hline C & FILE WUST ALREADY EXIST & ASG & 56 \\
\hline C & & ASG & 57 \\
\hline & IF (ITYP.NE.1) THEN & ASG & 58 \\
\hline & IF (. HOT.LDGIC) THEN & ASG & 59 \\
\hline 14 & CONTINUE & ASG & 60 \\
\hline & WRITE (IQUT, 528) (INF(I), I=1, NLEN) & ASG & 81 \\
\hline & WRITE (IOUT, 530) & ASG & 62 \\
\hline & READ $(I N, 540)$ ANS & NSG & 83 \\
\hline & WRITE (IECHO, 540) ANS & ЕСHO & 26 \\
\hline & IF ((ANS.NE. 'Y') AND. (ANS. NE. 'N' $\left.\left.N^{\prime}\right)\right)$ THEN & ASG & B4 \\
\hline & IF ((ANS. NE. 'y') AND. (ANS. NE. 'n')) GO TO 140 & ASG & 65 \\
\hline & ENDIF & 150 & 68 \\
\hline & IF ((ANS.ER. 'Y') OR. (ANS.EQ.' 'y')) THEN & ASG & 67 \\
\hline & WRITE (IDUT,550) IQB & ASG & 68 \\
\hline & READ (IN, 560) (INF (I), I=1, NLEN) & ASG & 69 \\
\hline & TRITE (IECHO, 560) (INF (I), I=1, NLEN) & ECHO & 27 \\
\hline & GO TO 136 & 150 & 70 \\
\hline & gSSE & ASG & 71 \\
\hline & GO TO 180 & ASG & 72 \\
\hline & ENDIF & ASG & 73 \\
\hline & 日SE & ASG & 74 \\
\hline & IF (ITYP.EQ.8) THEN & ASG & 75 \\
\hline C $\mathrm{L}$ & OSIC $=$ DPENSA (ASREAD+ABSAUF, INF, INTS (NLL4),INTS(IFUNIT) $)$ & ASO & 78 \\
\hline & a & ASG & 77 \\
\hline$c \mathrm{~L}$ & OGIC = OPEN\$ (ASRDDR+AASAN, INF, INTS (NML4),INTS(IFUNIT)) & ASG & 78 \\
\hline & ENDIF & ASG & 79 \\
\hline & ENDIF & ASG & 80 \\
\hline & 日SE & ASG & 81 \\
\hline C & & 150 & 82 \\
\hline C & WRITE ONLY FILE. CHECK WITH USER BEORE & ASG & 83 \\
\hline$c$ & DEEETING AN EXISTING FILE. & ASG & 84 \\
\hline c & & ASG & 85 \\
\hline & IF (LOGIC) THEN & ASG & 86 \\
\hline 15 & CONTINUE & ASG & 87 \\
\hline & WRITE (IOUT, 570) (INF(I), I=1, NLEN) & ASG & 88 \\
\hline & WRITE (IOLT, 580) IQB & ASG & 89 \\
\hline & REAO (IN, 546) ANS & ASG & 90 \\
\hline & WRITE (IECHO, 546) ANS & ECHO & 28 \\
\hline & IF ((ANS.NE. 'Y') . AND. (ANS.NE. 'N')) TKEN & 150 & 91 \\
\hline & IF ((ANS.NE. ' $y$ ') . AND. (ANS. NE. ' $n '))$ GO TO 150 & 150 & 92 \\
\hline & ENDIF & ASG & 93 \\
\hline & IF ((ANS.EQ. 'N') OR. (ANS.EQ. 'n')) THEN & ASG & 94 \\
\hline 16 & CONTINUE & ASG & 95 \\
\hline & WRITE (IOLT, 530) IQ日 & ASO & 96 \\
\hline & READ (IN, 546) ANS & ASG & 97 \\
\hline & WRITE (IECHO, 540) ANS & ECHO & 29 \\
\hline & IF ((ANS.NE. 'Y') . AND. (ANS.NE. 'N')) THEN & ASG & 98 \\
\hline & IF ((ANS.NE. 'y'), AND. (ANS. NE. 'n')) GO TO 168 & ASG & 99 \\
\hline & ENDIF & ASO & 100 \\
\hline & IF ((ANS.EQ.'N'), OR. (ANS.EQ. 'n')) GO TO 180 & NSG & . 191 \\
\hline & ANS $=$ 'N' & NSO & 102 \\
\hline & ENDIF & NSG & . 193 \\
\hline & IF ((ANS.EQ. 'Y') , OR. (ANS. EQ.' 'y')) THEN & ASG & . 184 \\
\hline C L L & DGIC = DELEA(INF, INTS $($ NLL4) $)$ & ASG & . 185 \\
\hline
\end{tabular}




\begin{tabular}{|c|c|c|c|}
\hline & BSE & ASG & 108 \\
\hline & WRITE (IOUT, 556) IQB & ASC & . 197 \\
\hline & READ (IN, 560) (INF(I), I=1, NLEN) & ASG & . 108 \\
\hline & WRITE (IECHO , 566) (INF (I),I=1, NLEN) & ECHI & 30 \\
\hline & GO TO 138 & ASO & . 169 \\
\hline & EMOIF & ASO & . 116 \\
\hline & ENDIF & ASG & . 111 \\
\hline C & LDGIC = OPEMSA (ASHRIT+ASAMF, INF, INTS(NM4), INTS(IFUNIT)) & ASG & 112 \\
\hline & ENDIF & ASG & . 113 \\
\hline C & & ASO & . 114 \\
\hline & IF (LOGIC) RETURN & ASG & . 115 \\
\hline$c$ & & ASG & . 116 \\
\hline & 176 CONTINUE & ASO & . 117 \\
\hline & WRITE (ICUT, 590) & ASG & . 118 \\
\hline & WRITE (IOUT, 530) IQB & ASO & . 119 \\
\hline & READ (IN, 546) ANS & ASG & . 120 \\
\hline & VRITE (IECHO, 546) & ECHO & 31 \\
\hline & IF ((ANS.NE. 'Y') . AND. (ANS.NE. 'N')) THEN & ASG & . 121 \\
\hline & IF ((ANS.NE. ' $\left.y^{\prime}\right)$. ANO. (ANS.NE. ' $\left.n^{\prime}\right)$ ) CO TO 170 & ASG & . 122 \\
\hline & ENDIF & ASG & 123 \\
\hline & IF ((ANS.EQ. 'N').OR. (ANS.EQ. 'n')) GO TO 180 & ASG & .124 \\
\hline & WRITE (IOUT, 550) IQB & ASO & . 125 \\
\hline & RERO (IN, 566) (INF (I), I=1, NLEN) & ASG & . 126 \\
\hline & WRITE (IECHO, 560) (INF $(I), I=1$, NLEN) & ECHO & 32 \\
\hline & G0 TO 130 & ASG & 127 \\
\hline c & & ASG & . 128 \\
\hline & 180 CONTINUE & ASG & . 128 \\
\hline & WRITE (IOUT, 680) & ASG & . 130 \\
\hline & IERR $=1$ & ASG & . 131 \\
\hline & RETURN & ASG & . 132 \\
\hline$c$ & & ASG & . 133 \\
\hline & 586 FORHAT (/1X, 'FILE UNIT ',I5,' IS ALREAOY ASSIGNED TO AN OPEN FILE. & ASO & . 134 \\
\hline & ${ }^{\prime} ' / 1 X_{1}{ }^{\prime}$ ATTEMPT TO ASSIGN FILE',2044) & ASG & . 135 \\
\hline & 510 FORUT (/1X, 'FILE UNIT', I5," IS IN POSSIBLE CONFLICT IITH PRIME SY & ASG & - 136 \\
\hline & $\bullet$ TEI UNITS' $D$ & ASO & . 137 \\
\hline & 520 FORUAT (/IX, 'UNAELE TO LOCATE FILE',20A4) & ASO & . 138 \\
\hline & 539 FORWAT $\left(1 X,{ }^{1} 00\right.$ YOU ISH TD TRY AGIN $(Y / N)$ ? $\left.{ }^{\prime}, A 1\right)$ & ASG & . 139 \\
\hline & 546 FORWAT (A1) & ASG & . 140 \\
\hline & 558 FORMAT (1X,'ENTER FILE NAME ',A1) & ASG & - 141 \\
\hline & 560 FORUAT (28A4) & ASO & . 142 \\
\hline & 570 FORMT (1X, 'FILE', 20A4) & ASG & . 143 \\
\hline & 580 FORMAT (1X,' 'ALREADY EXISTS. N RIGHT TO DEETE (Y/N)? ',A1) & ASG & . 144 \\
\hline & 598 FORUAT (/1X, 'FILE ASSIGNMEHT UNSUCCESSFL. ') & ASG & . 145 \\
\hline & BOB FORUAT (1X, 'PROGRAM ABORTED IN RQUTINE ASG. ') & ASG & . 148 \\
\hline & END & ASG & . 147 \\
\hline & SUBROLTINE ASGVAX (NLEN, INF, IFLAG) & ECHO & 33 \\
\hline$c$ & & ASGVAX & 3 \\
\hline C & THIS IS A SUBROUTINE TKAT TAKES THE PLACE OF SUBROUTINE ASO & ASGVAX & 4 \\
\hline c & IN THE PRINE VERSION. IT BASICN & ASGVAX & 5 \\
\hline c & TO READ AND WRITE AND SETS VARIABIE MOOETYP WHICH OENOTES & ASGVAX & 6 \\
\hline$c$ & WHETHER YOU WANT TO RUN IN BATCH OR INTERACTIVE MODE. & ASGVAX & 7 \\
\hline$c$ & II SHOLLD TO BE NOTED THAT THIS ROUTINE IS ONLY TO BE USED ON & ASGVAX & 8 \\
\hline$c$ & VAX SYSTEUS. AUTHOR: C.L. BARTLEY & ASGYAX & 9 \\
\hline C & & ASGVAX & 10 \\
\hline
\end{tabular}




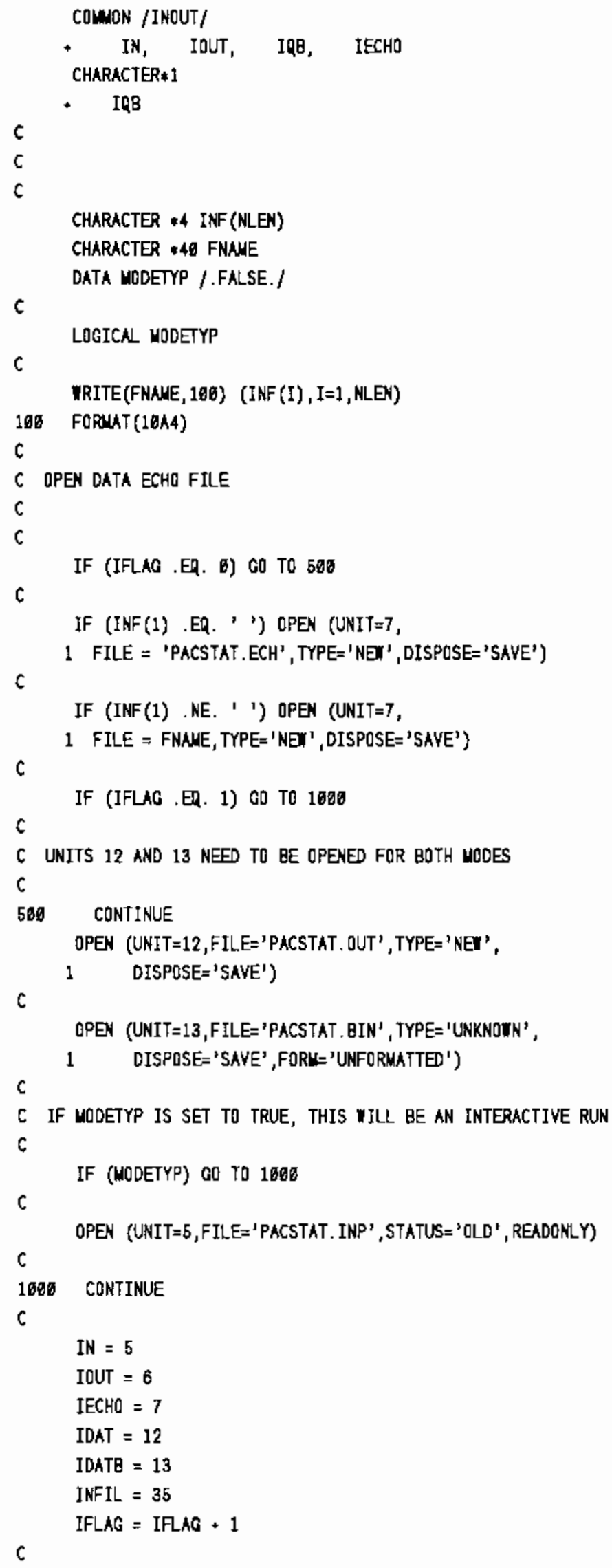

\begin{tabular}{|c|c|}
\hline INOUT & . \\
\hline ECHO & 86 \\
\hline INOUT & . \\
\hline INOUT & 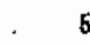 \\
\hline INOUT & . \\
\hline INOUT & . \\
\hline ASGVAX & 12 \\
\hline ECHO & 34 \\
\hline ECHO & 35 \\
\hline $\mathrm{BAYCH}$ & 14 \\
\hline ASGVAX & 14 \\
\hline ASGVAX & 15 \\
\hline ASGVAX & 16 \\
\hline ECHO & 36 \\
\hline ECHO & 37 \\
\hline ECHD & 38 \\
\hline ECHO & 39 \\
\hline ECHO & 48 \\
\hline ECHO & 41 \\
\hline ECHO & 42 \\
\hline $\mathrm{ECHO}$ & 43 \\
\hline ECHO & 44 \\
\hline ECHO & 45 \\
\hline ECHD & 48 \\
\hline ECHD & . \\
\hline ECHO & 48 \\
\hline ECHO & 49 \\
\hline ECHO & 58 \\
\hline ASGVAX & 17 \\
\hline ASGVAX & 18 \\
\hline ASGVAX & 19 \\
\hline ECHO & 51 \\
\hline ASGVAX & 26 \\
\hline ASGVAX & 21 \\
\hline ASGVAX & 22 \\
\hline ASGVAX & 23 \\
\hline ASGVAX & 24 \\
\hline ASGYAX & . \\
\hline ASGVAX & 26 \\
\hline ASGVAX & 27 \\
\hline ASGVAX & 28 \\
\hline ASGVAX & 29 \\
\hline ASGVAX & 38 \\
\hline ASGVAX & 31 \\
\hline NSGVAX & 32 \\
\hline ASGVAX & 33 \\
\hline ASGVAX & 34 \\
\hline ASGVAX & 35 \\
\hline ECHO & 52 \\
\hline AsGVAX & 36 \\
\hline ASGVAX & 37 \\
\hline ASQVAX & 38 \\
\hline ECHO & 53 \\
\hline ASGVAX & 39 \\
\hline
\end{tabular}




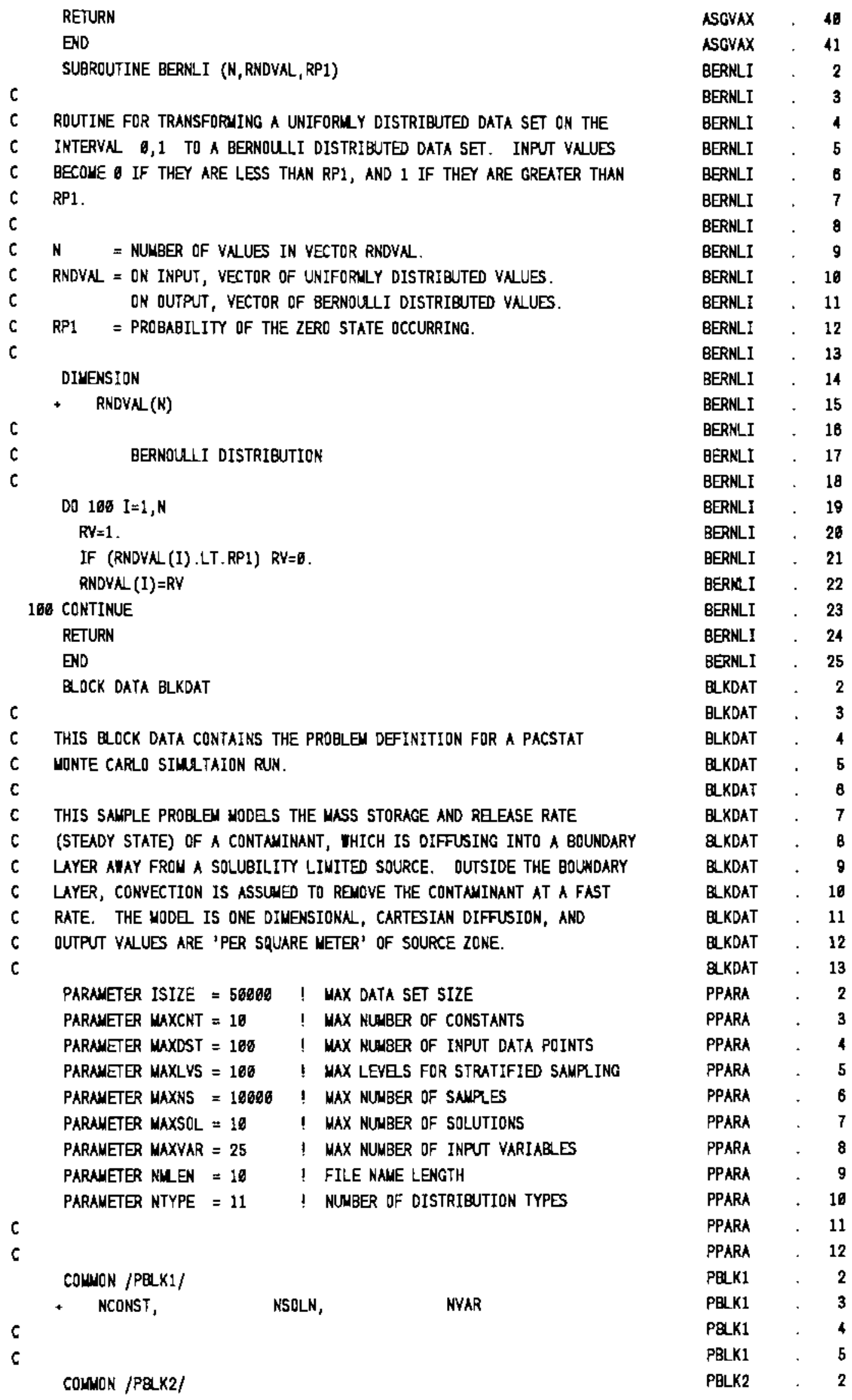

C. 6 


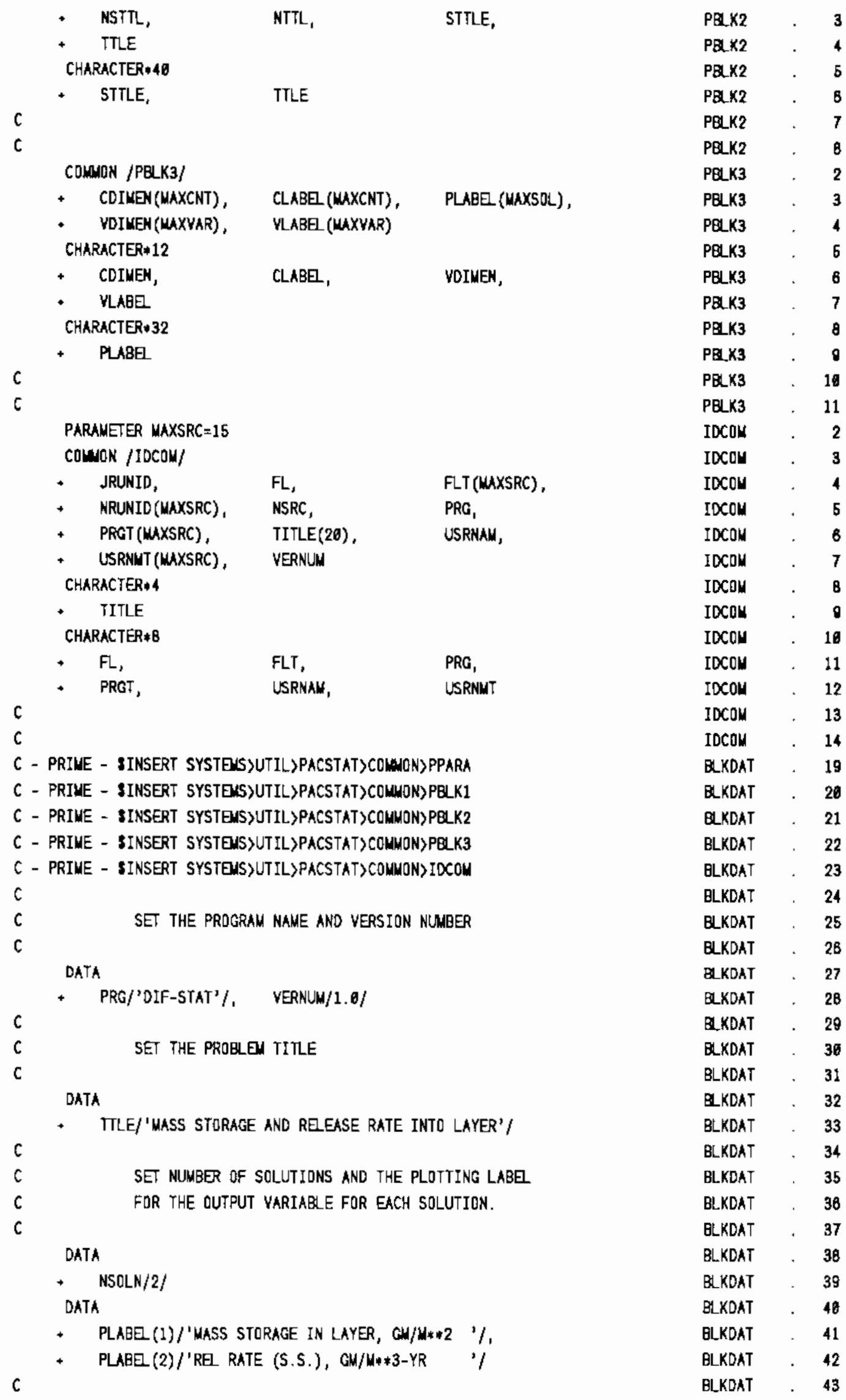




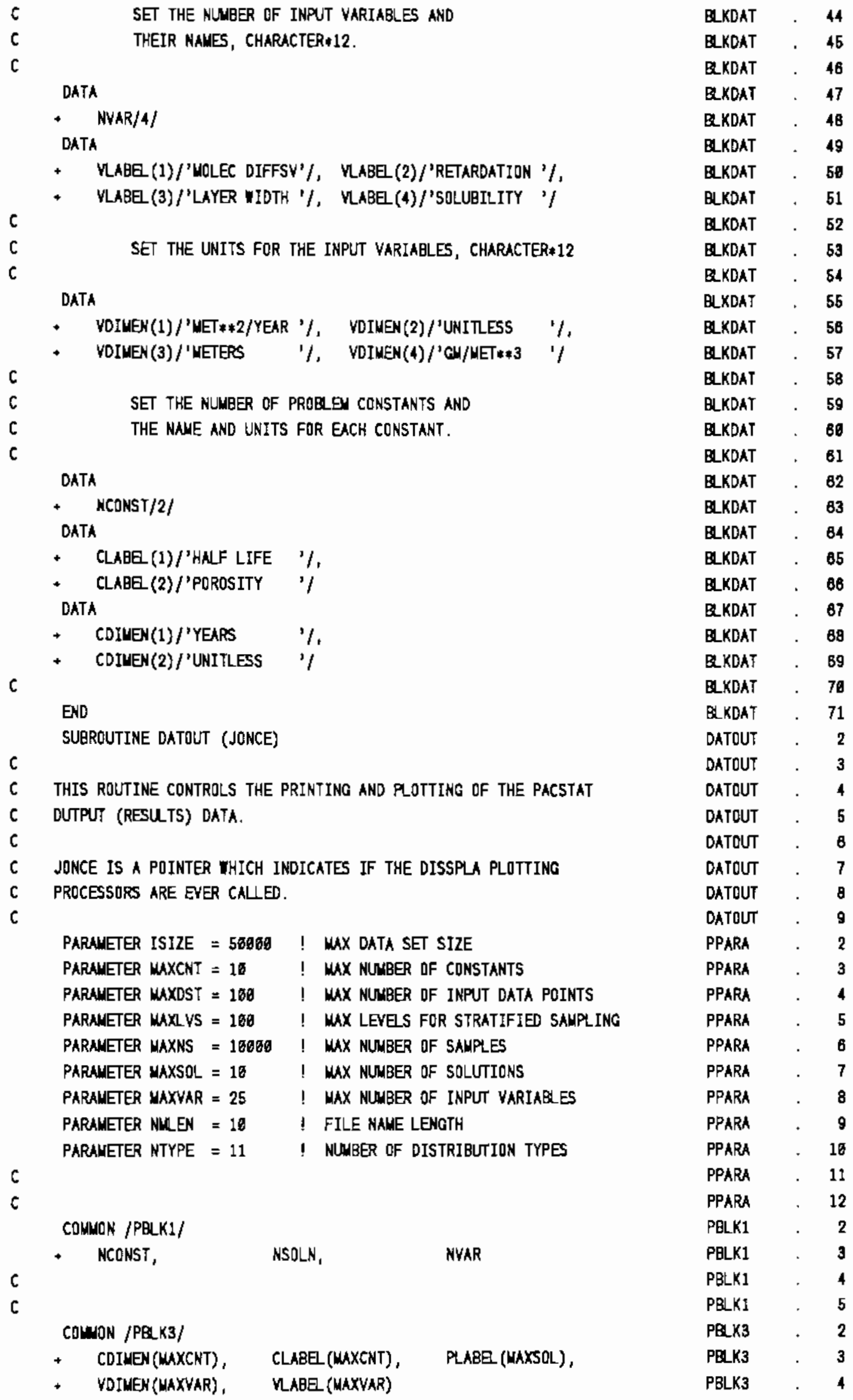

C. 8 


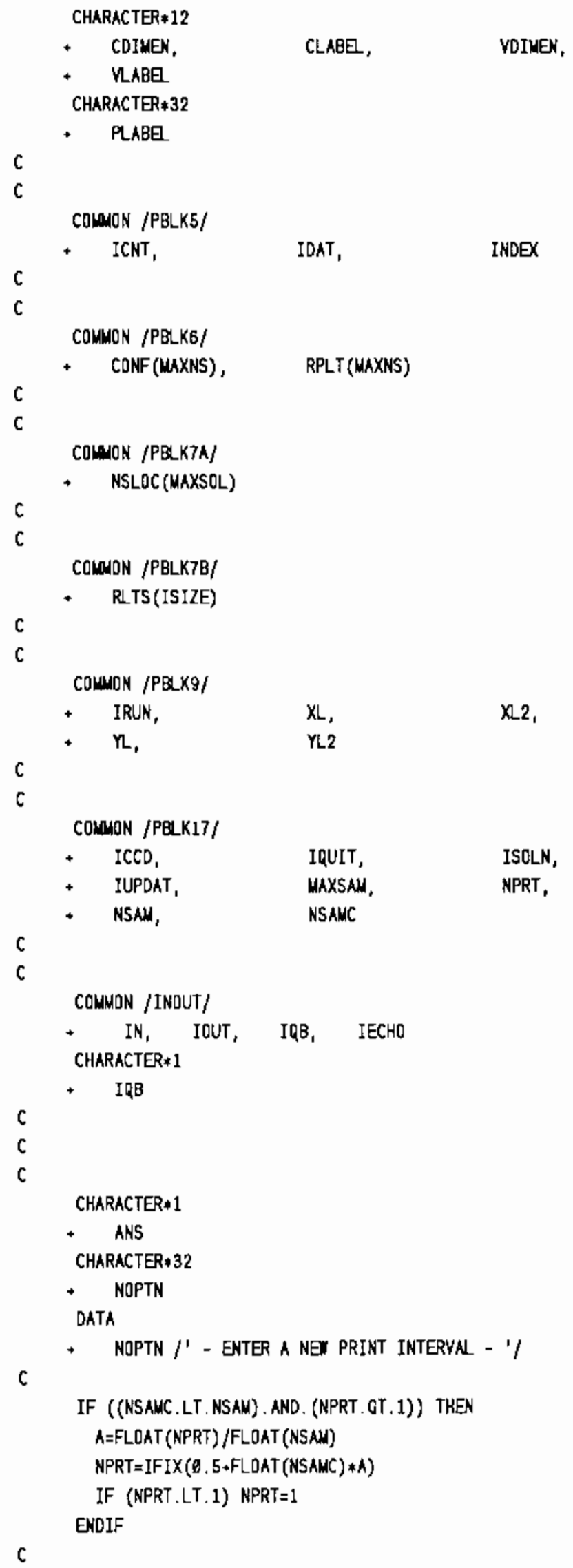

VOIMEN,

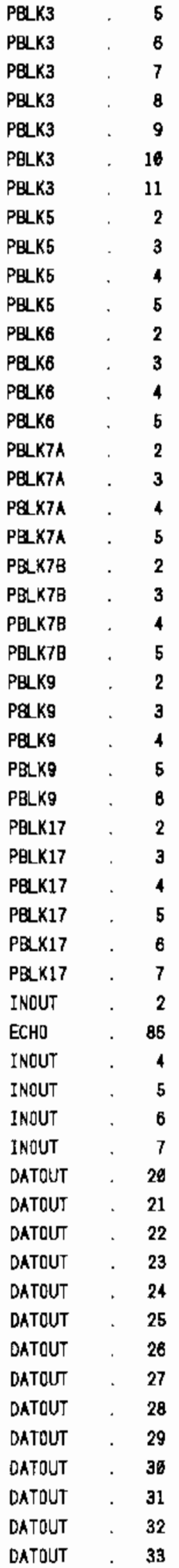




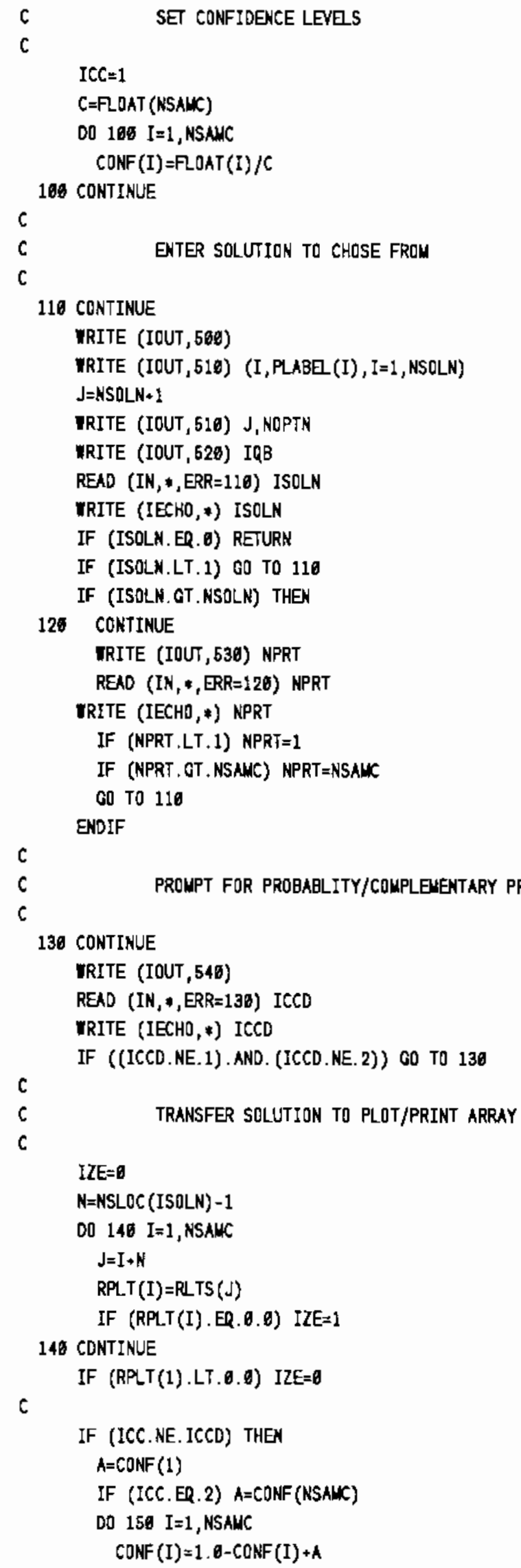




\begin{tabular}{|c|c|c|c|}
\hline 150 & CONTINUE & DАTOUT & 85 \\
\hline & $I C C=I C C D$ & DATOUT & 88 \\
\hline & ENDIF & DATOUT & 87 \\
\hline $\mathrm{C}$ & & DATOUT & 88 \\
\hline$c$ & PRINT RESULTS & DATOUT & 89 \\
\hline C & & DATDUT & 90 \\
\hline$c$ & OUTPUT DATA TABLE KEADERS & DATDLT & 91 \\
\hline c & & DATOUT & 92 \\
\hline & WRITE (IOUT, 550) PLABE (ISOLN) & DАTOUT & 93 \\
\hline & IF (IDAT.GT.6) WRITE (IDAT, 550) PLABE(ISOLN) & DATDUT & 94 \\
\hline & IF (IZE. EQ.1) THEK & DATDUT & 95 \\
\hline & WRITE (IOUT, 586) & OATOUT & 98 \\
\hline & IF (IDAT.CT. ) WRITE (IDAT, 560) & OATOUT & 97 \\
\hline & ENDIF & DATOU: & 98 \\
\hline & IF (ICCD.EA. 2) THEN & DATOUT & 98 \\
\hline & WRITE (IOUT, 57ø) & DATOUT & . 100 \\
\hline & IF (IDAT,GT. D) WRITE (IDAT,57ठ) & DATOUT & . 161 \\
\hline & ESE & DATOUT & . 102 \\
\hline & NRITE (IOUT, 586) & DATOUT & . 103 \\
\hline & IF (IDAT.GT.g) WRITE (IDAT, 580) & DATOUT & .104 \\
\hline & ENDIF & DATOUT & . 165 \\
\hline c & & DATOUT & . 166 \\
\hline c & FIRST DATA POINT, IF NOT PRINTED BY DEFALT & OATOUT & . 107 \\
\hline$c$ & & DATOUT & . $\quad 308$ \\
\hline & NSTRT $=$ NPRT & DATOUT & . 169 \\
\hline & IF (NPRT.GT.1) THEN & DATOUT & . 110 \\
\hline & WRITE (IOUT, 596) 1, RPLT(1), CONF(1) & OATOUT & . 111 \\
\hline & IF (IDAT.GT.6) WRITE (IDAT, 596) 1, RPLT(1), CONF (1) & DATOUT & . $\quad 112$ \\
\hline & IF (ICCD.EQ.2) NSTRT=NSTRT+1 & DATOUT & . 113 \\
\hline & ENDIF & DATDUT & . 114 \\
\hline$c$ & & DATOUT & 115 \\
\hline$c$ & PRINTING LOQP & DATDUT & . 116 \\
\hline c & & DATOUT & . $\quad \$ 17$ \\
\hline & $J=6$ & DATOUT & . 118 \\
\hline & $K=\varnothing$ & DATOUT & . 119 \\
\hline & DO 160 I=NSTRT, NSAMC, NPRT & DATOUT & . 126 \\
\hline & $J=I$ & DATOUT & . 121 \\
\hline & 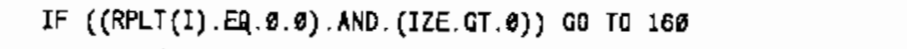 & DATOUT & . 122 \\
\hline & VRITE (IOUT, 590) I, RPLT(I), CONF(I) & DATOUT & . 123 \\
\hline & IF (IDAT.GT.6) WRITE (IDAT,590) I, RPLT(I), CONF (I) & DATQUT & . 124 \\
\hline & $k=k+1$ & DATOUT & . 125 \\
\hline 160 & CONTINUE & DATOUT & 128 \\
\hline$c$ & & DATDUT & . 127 \\
\hline C & LAST DATA POINT, IF NOT PRINTED BY DEFALLT & DATQUT & . 128 \\
\hline C & & DATOUT & . 129 \\
\hline & IF (J.NE. NSAMC) THEN & DATOU: & . 130 \\
\hline & WRITE (IOUT, 596) NSAMC, RPLT (NSANC), CONF (NSAMC) & DATQUT & . 131 \\
\hline & IF (IDAT.GT.6) WRITE (IOAT, 596) NSAMC, RPLT(NSNC), CONF (NSANC) & DATOUT & . 132 \\
\hline & ENDIF & DATOUT & . 133 \\
\hline C & & DATOUT & . 134 \\
\hline C & WARNING IF ALL DATA POINTS ARE ZERO & DATOUT & . 135 \\
\hline C & & DATOUT & 138 \\
\hline & IF (K.EQ.ø) WRITE (IOUT,606) PLABE (ISOLN) & DATOUT & . 137 \\
\hline C & & DATOUT & . 138 \\
\hline
\end{tabular}




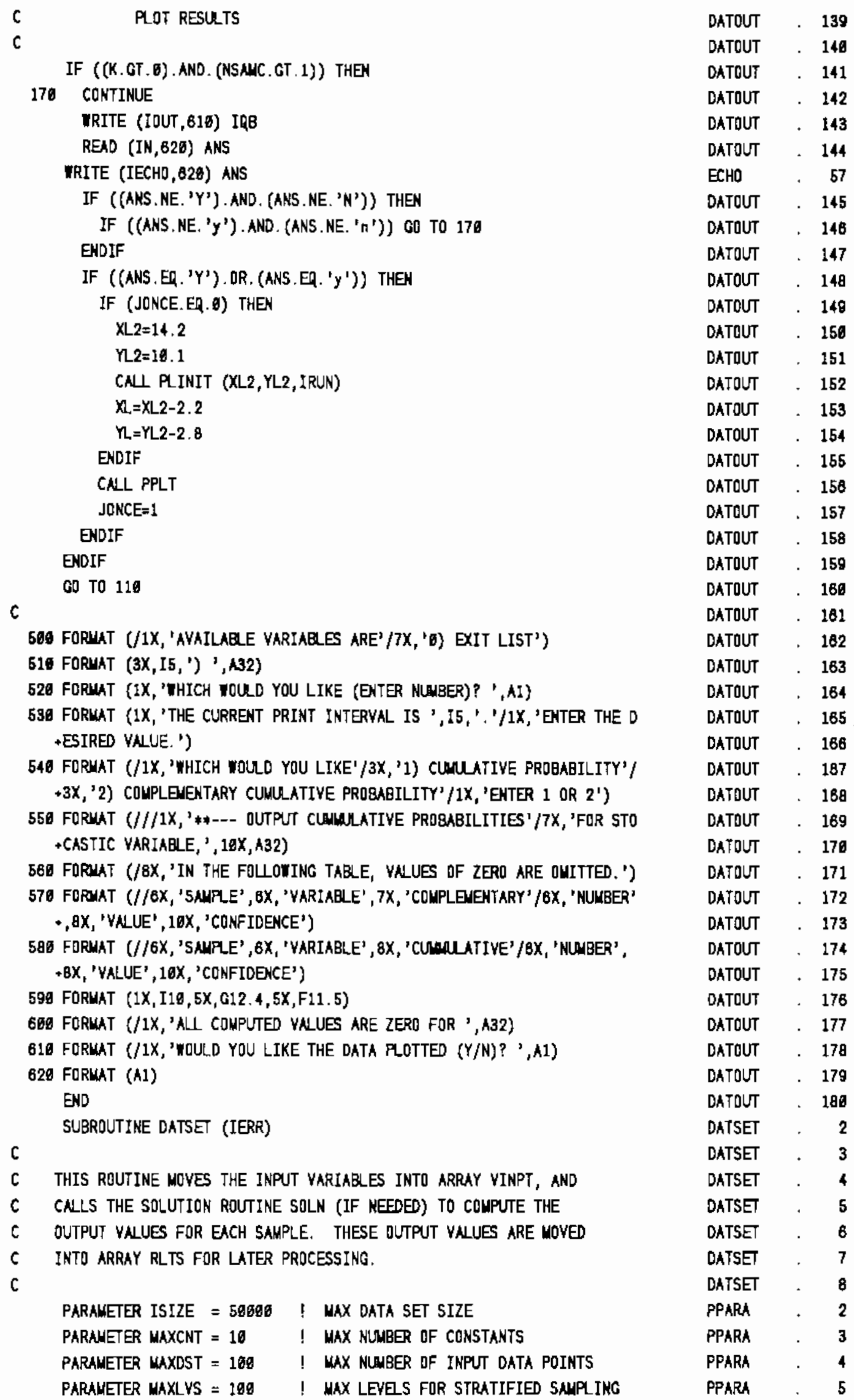




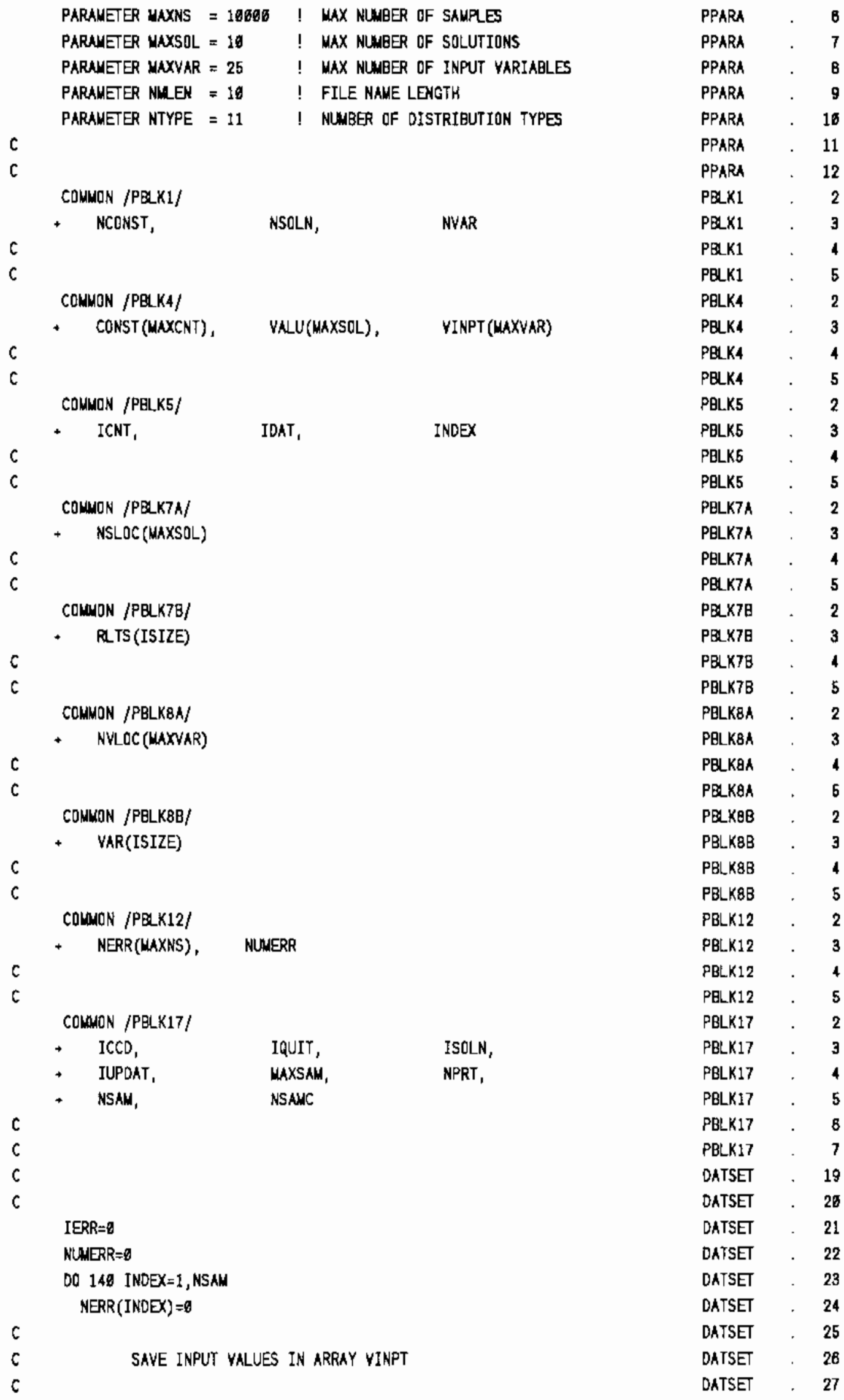

C. 13 


\begin{tabular}{|c|c|c|c|}
\hline & DD $100 I=1$, NYAR & DATSET & 28 \\
\hline & $J=N Y L O C(I)+I N D E X-1$ & DATSET & . $\quad 29$ \\
\hline & $\operatorname{VINPT}(I)=\operatorname{VAR}(J)$ & DATSET & . 30 \\
\hline 160 & CONTINUE & DATSET & 31 \\
\hline c & & DATSET & 32 \\
\hline c & SOLUTION ROUTINE CALLED ONCE WHEN NSOLN=0 & DATSET & 33 \\
\hline C & & DATSET & . 34 \\
\hline & IF (NSOLN.GT. G) GO TO 110 & DATSET & . 35 \\
\hline & IF (INDEX.EQ.1) CNLL SOLN (IERR) & DATSET & 36 \\
\hline & CO TO 140 & DATSET & 37 \\
\hline 119 & CONTINUE & DATSET & 38 \\
\hline C & & DATSET & 39 \\
\hline C & COUPUTE OUTPUT YARIABLES & DATSET & . 48 \\
\hline C & & DATSET & . 41 \\
\hline & CALL SOLN (IERR) & DATSET & .42 \\
\hline & IF (IERR. EQ.ब) GD TO 120 & OATSET & . 43 \\
\hline & IF (IQUIT.GT. B) GO TO 158 & OATSET & . 44 \\
\hline & NUNERR $=N U$ NERR +1 & DATSET & . 45 \\
\hline & NERR (INDEX) =INDEX & OATSET & . 46 \\
\hline & IERR $=6$ & OATSET & . 47 \\
\hline 120 & CONTINUE & DATSET & . 48 \\
\hline C & & DATSET &.$\quad 49$ \\
\hline C & SAYE YNLUES IN OUTPUT ARRAY & DATSET & 56 \\
\hline$c$ & & DATSET & . 51 \\
\hline & DO $130 \mathrm{I}=1, \mathrm{NSOLN}$ & DATSET & . 62 \\
\hline & $J=N S L O C(I)+I N D E X-I$ & DATSET & . 53 \\
\hline & $\operatorname{RLTS}(J)=Y N L U(I)$ & DATSET & . 54 \\
\hline 139 & CONTINUE & DATSET & 55 \\
\hline 140 & CONTINUE & DATSET & So \\
\hline & RETURN & DATSET & 57 \\
\hline c & & DATSET & 58 \\
\hline 158 & CONTINUE & DATSET & 59 \\
\hline & IERR $=1$ & DATSET & 60 \\
\hline & NSAL $=I N D E X-1$ & DATSET & $\theta 1$ \\
\hline & RETURN & DATSET & 62 \\
\hline & EX:D & DATSET & 63 \\
\hline & SUBROUTIAE DCLAIM (IOUT) & DCLAIU & 2 \\
\hline c & & DCLAIN & . \\
\hline C & THIS ROUTINE PRINTS A VERIFICAIION/BENCHMARKING DISCLAIMER TO & DCLAIN & 4 \\
\hline c & LOGICAL UNIT IOUT. & DCLAIM & 5 \\
\hline C & & DCLAIN & 6 \\
\hline & WRITE (IOUT, 500) & DCLAIM & 7 \\
\hline C & & DCLAIH & 8 \\
\hline & RETURN & DCLAIM & 9 \\
\hline 580 & FORUAT (/IX, 'Results froin this code are based on the use of unveri & DCLAIM & . 10 \\
\hline & +fied'/1x, 'softurare and are not for use in ticense related applicat & DCLAIM & . 11 \\
\hline & + ions. "/) & DCLAIM & . 12 \\
\hline & END & DCLAIM & 13 \\
\hline & SUBROUTINE DEXPF (N,RNDVN, RPI) & DEXPF & . \\
\hline C & & DEXPF & . \\
\hline c & ROUTINE FOR TRANSFORNING A UNIFORLEY DISTRIBUTED DATA SET ON THE & DEXPF & . \\
\hline$c$ & INTERYAL 6,1 TO AN EXPONENTINLLY DISTRIBUTED DATA SET. & DEXPF & . \\
\hline$c$ & & DEXPF & 8 \\
\hline C & $=$ THE NUMBER OF ITEUS IN THE DATA SET. & DEXPF & . \\
\hline
\end{tabular}




\begin{tabular}{|c|c|c|c|}
\hline c & RNDVN = VECTOR OF UNIFORILY DISTRIBUTED VNLUES ON INPUT. EACH & DEXPF & B \\
\hline$c$ & YALUE WST BE BETWEEN ZERO AND DNE. & DEXPF & 9 \\
\hline c & = VECTOR OF EXPONENTINLLY DISTRIEUTED VNLUES ON OUTPUT. & DEXPF & 10 \\
\hline C & $=$ MEAN VNLUE FOR THE EXPONENTINL DISTRIQNTION. & DEXPF & 11 \\
\hline C & & DEXPF & 12 \\
\hline & DIMENSION & DEXPF & 13 \\
\hline & $+\quad$ RNDVNL(N) & DEXPF & 14 \\
\hline C & & DEXPF & 15 \\
\hline C & EXPONENTIAL DISTRIBUTION & DEXPF & 16 \\
\hline C & & DEXPF & 17 \\
\hline & DO $196 I=1, N$ & DEXPF & 18 \\
\hline & $R V=1, \nabla-R N D Y A L(I)$ & DEXPF & 19 \\
\hline & IF (RV.LT.1.6E-21) RV=1. gE-21 & DEXPF & 28 \\
\hline & RNOVNL(I) $=-A L O G(R V) / R P 1$ & DEXPF & 21 \\
\hline 108 & CONTINUE & DEXPF & 22 \\
\hline & RETURN & DEXPF & 23 \\
\hline & END & DEXPF & 24 \\
\hline & SUBROUTINE DEXPFL (N, RNDVAL, RP1, ANIN, ANAX) & DEXPFL & 2 \\
\hline C & & DEXPFL & 3 \\
\hline c & ROUTINE FOR TRANSFORMING A UNIFORLLY DISTRIBUTED DATA SET ON THE & DEXPFL & 4 \\
\hline $\mathrm{C}$ & INTERVAL 9,1 TO AN EXPONENTIALLY DISTRIBUTED DATA SET, WITH THE & DEXPFL & 5 \\
\hline C & CDNSTRAINT THAT ALL DUTPUT VALUES LIE BETWEEN SPECIFIED LIMITS. & DEXPFL & 6 \\
\hline C & & DEXPFL & 7 \\
\hline C & $=$ THE NUMBER DF ITENS IN THE RANDON VARIAGLE VECTOR RNDVN. & DEXPF & 8 \\
\hline C & RNDVAL $=$ ON INPUT, $A$ VECTOR OF N RANDOW VNLUES, UNIFORULY & DEXPFL & 9 \\
\hline C & OISTRIUTED, ON THE INTERVAL ZERD TO ONE. & DEXPFL & 10 \\
\hline c & $=$ ON OUTPUT, A VECTOR OF N RANDOW VNLUES, EXPONENTINLLY & DEXPF & 11 \\
\hline C & DISTRIBUTED WITH 'MEAN' RP1, AND WITH VNLUES BEL OW ANIN & DEXPFL & 12 \\
\hline $\mathrm{C}$ & AND AEDVE NMAX DELETED FRDM THE DISTRIEUTION. & DEXPFL & 13 \\
\hline c & RP1 = MENN VALUE FDR THE DESIRED EXPONENTIN DISTRIEUTION. & DEXPFL & 14 \\
\hline C & ANIN = MINIMUN LIMIT VALUE FOR THE OUTPUT DISTRIBUTION. & DEXPFL & 15 \\
\hline c & AWAX = MAXIMUN LIMIT VALUE FOR THE OUTPUT DISTRIBUTION. & DEXPFL & 18 \\
\hline c & & DEXPFL & 17 \\
\hline C & UMIN, UNAX = LIMITS DN THE UNIFORM DISIRIBUTION. THESE ARE & DEXPFL & 18 \\
\hline c & AVALIABLE TO THE USER THROUGH COWMAN BLOCK ULIMTS. & DEXPFL & 19 \\
\hline c & & DEXPFL & 20 \\
\hline & COKMON /ULINTS/ & UIMTS & 2 \\
\hline & $+\quad$ UwAX, & ULIMTS & 3 \\
\hline C & & UIMTS & 4 \\
\hline c & & DEXPFL & 22 \\
\hline & DIMENSION & OEXPFL & 23 \\
\hline & $+\quad$ RNDVAL $(N)$ & DEXPFL & 24 \\
\hline & OATA & OEXPFL & 25 \\
\hline & $+\quad$ IONE/1/ & DEXPFL & 26 \\
\hline C & & DEXPFL & 27 \\
\hline & IF (RP1.LJ.1.) RP1=ABS (RP1) & DEXPFL & $2 B$ \\
\hline & IF (AMIN.LE. AMAX) GO TO 100 & DEXPFL & 29 \\
\hline & $M D=A N I N$ & DEXPFL & 30 \\
\hline & AMIN=AWAX & DEXPFL & 31 \\
\hline & $A M A X=W D$ & DEXPFL & 32 \\
\hline 160 & CONTINUE & OEXPFL & 33 \\
\hline & IF (AMIN.LF.AMAX) GD TO 110 & DEXPFL & 34 \\
\hline & $A M I N=\sigma$. & DEXPFL & 35 \\
\hline & $A M A X=138, * R P 1$ & DEXPFL & 38 \\
\hline
\end{tabular}




\begin{tabular}{|c|c|c|}
\hline \multirow{2}{*}{119 CONTINUE } & DEXPF & 37 \\
\hline & DEXPFL & 38 \\
\hline \multirow[t]{2}{*}{ UNIFORU DISTRIBUTIDN LINITS } & DEXPFL & 39 \\
\hline & DEXPF & 40 \\
\hline UMIN=1.0-EXP(-RP1*AUIN) & DEXPF & 41 \\
\hline WAX $=1,9-\operatorname{ExP}(-\mathrm{RP} 1 * \lambda \mathrm{MAX})$ & DEXPFL & 42 \\
\hline RY $=U \mathbf{M I N}$ & DEXPFL & 43 \\
\hline CNLL DEXPF (IONE, RV, RP1) & DEXPF & 44 \\
\hline $\mathrm{B}=\mathrm{RV}$ & DEXPFL & 45 \\
\hline$R V=L$ LNAX & DEXPF & 46 \\
\hline CNLL DEXPF (IONE,RV,RP1) & DEXPF & 47 \\
\hline$T=R Y$ & DEXPF & 48 \\
\hline IF (B.LE.T) CO TO 120 & DEXPFL & 49 \\
\hline LNIN=1,0-UMIN & DEXPF & 50 \\
\hline UWAX $=1,0-U$ WAX & DEXPF & 51 \\
\hline \multirow[t]{2}{*}{120 CONTINUE } & DEXPFL & 52 \\
\hline & DEXPFL & . 53 \\
\hline \multirow[t]{2}{*}{ ADJUST UNIFORN DISTRIBUTION TO NEI LIUITS } & DEXPF & . 54 \\
\hline & DEXPFL & . 55 \\
\hline NO=LNAX-UMIN & DEXPFL & 58 \\
\hline$D 0130 I=1, N$ & DEXPFL & 57 \\
\hline $\operatorname{RNDYAL}(I)=U M I N+\operatorname{RNOVAL}(I) * W D$ & DEXPFL & . 58 \\
\hline \multirow[t]{2}{*}{139 CONTINUE } & DEXPF & 59 \\
\hline & DEXPF & 80 \\
\hline \multirow[t]{2}{*}{ CONPUTE EXPONENTINL DISTRIBUTIDN } & DEXPF & . 81 \\
\hline & DEXPF & . 82 \\
\hline CNL DEXPF（N,RNDVN, RP1) & DEXPF & 83 \\
\hline RETURN & DEXPF & 64 \\
\hline END & DEXPF & 85 \\
\hline FUNCTION DNORM (RNDYAL,RP1,RP2) & DNORN & 2 \\
\hline & DNORN & 3 \\
\hline ROUTINE FOR TRANFORMING A SINGLE YNLUE FROU $A$ UNIFORUY & DNORM & 4 \\
\hline DISTRIBUTED DATA SET ON THE INTERVNL 6,1 TO A SINGLE YNLUE IN A & DNORH & 5 \\
\hline NORULLY OISTRIBUTED DATA SET. & DNORM & 6 \\
\hline & DNORU & 7 \\
\hline A POLYNDNIAL METHDD IS USED. & DNORN & 8 \\
\hline REEERENCE ABRNDUTITZ \& STEGUN, PAGE 933. & DNORM & 9 \\
\hline & DNQRH & 10 \\
\hline RNDYN = INPUT RANDOW YNLUE BETMEEN O AND 1. & DNORH & 11 \\
\hline RP1 = WEAN VALUE FOR THE NORUAL DISTRIBUTION. & DNORM & 12 \\
\hline RP2 = STANDARO DEYIATION FOR THE NORLAL DISTRIBUTIDN. & DNORM & 13 \\
\hline & DNORM & 14 \\
\hline LOGICAL & DNDRM & 15 \\
\hline - NEGTIV & DNORM & 16 \\
\hline DATA & ONORW & 17 \\
\hline - $\quad C \theta, C 1, C 2 / 2.515517,0.882853,0.616328 /$ & DNDRM & 18 \\
\hline DATA & DNORM & 19 \\
\hline$+\quad D 1, D 2, D 3 / 1.432788,6.189269,0.061398 /$ & DNORM & 28 \\
\hline & DNORM & 21 \\
\hline NORWAL DISTRIBUTION & DNORM & 22 \\
\hline & DNORW & 23 \\
\hline$P=R N D Y N$ & DNCRM & 24 \\
\hline NEGTIV=. TRUE. & DNORW & 25 \\
\hline IF (P.LT.0.5) GO TO 160 & DNORN & 26 \\
\hline
\end{tabular}




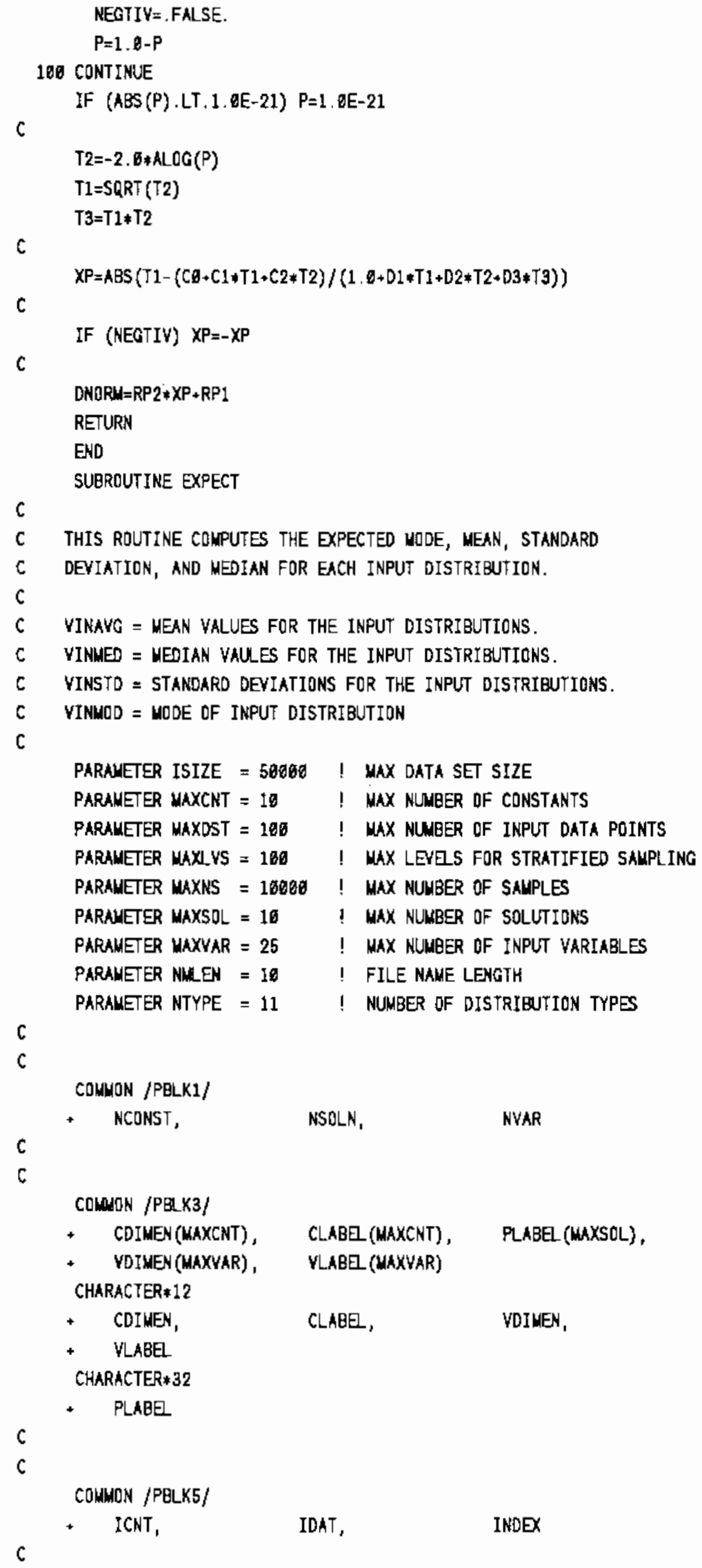


CONON /PBLK11/

+ VINAYG(MAXYAR), VINMED (MAXYAR), VINMOD(WAXVAR),

- VINSTD(MAXYAR)

c

c

CONON /PBLK13/

+ nleva,

NPTSPL (WAXLYS, WAXYAR),

- NSTOC

C

c

COMMON /PBLK15/

- ILIM(MAXYAR)

- par(maXVAR, 3),

- VIMT (MAXVAR, 2)

c

c

COLON /INOUT/

- IN, IDUT, IQB, IECHO CHARACTER*1

+ IQB

$c$

c

REN $* 8$

$\rightarrow \quad A, B, C, D, F, G$

DATA

- BASEXP/2.3625/, ENAX/5.E+1/, EMIN/2.E-2/

c

C

c

OUTPUT FILE UNIT

III $=$ IDAT

IF (IDNT.EQ.8) III=IOUT

$c$

$c$

c

c

c

c

c

LIHITS=0

OUIT TABLE OF EXPECTED INPUT DISTRIBUTION PARAMETERS

IF AL OF THE VARIABLES ARE CONSTANTS (NSTOC=1), ARE ENDPOINT LIMITED, OR ARE READ FROU AN EXTERNAL FILE. SET CQUNTER 'LIKITS' TO INDICATE HOW MANY VARIABLES ARE ENDPOINT LIMITED.

$J=0$

DO $100 N=1$, NYAR

IF (ILIM(N) . NE. D) LIMITS=LIMITS + I

IF ((ILIM(N) , NE, \&) , OR. (ITYPE(N) $, G T, 8)$ ) J=J+1

196 CONTINUE

IF (LIMITS.EQ. NYAR) GO TO 240

IF (J.EQ.NYAR) GO TO 260

IF (NSTOC.ER.1) GO TO 280

$c$

c

\section{TABLE HEADER}

IF (III.GT.6) THEN

WRITE (III, 500)

WRITE (III, 510)

\begin{tabular}{|c|c|}
\hline PBLK6 & \\
\hline PBLK1I & \\
\hline PBLK1: & \\
\hline PBLK1I & \\
\hline PBLK1I & \\
\hline PBLK11 & \\
\hline PBLK13 & \\
\hline PBLK13 & \\
\hline PBLK13 & \\
\hline PBLK13 & \\
\hline PBLK13 & \\
\hline PBLK15 & \\
\hline PBLK15 & \\
\hline PBLK15 & \\
\hline PBLK15 & \\
\hline PBLK15 & \\
\hline PBLK15 & \\
\hline INOUT & \\
\hline $\mathrm{ECHD}$ & \\
\hline INOUT & \\
\hline INOUT & \\
\hline INOUT & \\
\hline INOUT & \\
\hline EXPECT & \\
\hline EXPECT & \\
\hline EXPECT & \\
\hline EXPECT & \\
\hline EXPECT & \\
\hline EXPECT & \\
\hline EXPECT & \\
\hline EXPECT & \\
\hline EXPECT & \\
\hline EXPECT & \\
\hline EXPECT & \\
\hline ВXPECT & + \\
\hline EXPECT & • \\
\hline EXPECT & . \\
\hline EXPECT & \\
\hline EXPECT & \\
\hline EXPECT & \\
\hline EXPECT & \\
\hline EXPECT & \\
\hline EXPECT & \\
\hline EXPECT & . \\
\hline EXPECT & \\
\hline EXPECT & \\
\hline EXPECT & \\
\hline EXPECT & \\
\hline EXPECT & \\
\hline EXPECT & \\
\hline EXPECT & \\
\hline EXPECT & \\
\hline EXPECT & \\
\hline EXPECT & \\
\hline
\end{tabular}

C. 18 


\begin{tabular}{|c|c|c|c|}
\hline & ENDIF & EXPECT & 51 \\
\hline c & & EXPECT & 52 \\
\hline c & INPUT VARIABLE EXPECTED STATISTICS. OMIT ANY VARIABLE & EXPECT & 53 \\
\hline C & WHICH IS EKDPOINT LIWITED. INDICATED EY ILIM(N) $>\varnothing$. & EXPECT & 54 \\
\hline C & & EXPECT & 55 \\
\hline & DO $230 \mathrm{~N}=1$, NYAR & EXPECT & 56 \\
\hline & IF (ILIM(N).GT.0) GO TO 230 & EXPECT & 57 \\
\hline & $A=0$. & EXPECT & 58 \\
\hline & $E=6$. & EXPECT & 59 \\
\hline & $C=6$. & EXPECT & 68 \\
\hline & $D=0$. & EXPECT & 61 \\
\hline & $I O M I T=0$ & EXPECT & 82 \\
\hline & $R P 1=P A R(N, 1)$ & EXPECT & 63 \\
\hline & $\operatorname{RP2}=P A R(N, 2)$ & EXPECT & 64 \\
\hline c & & EXPECT & 85 \\
\hline$c$ & DISTRIQUTION TYPE & EXPECT & 86 \\
\hline $\mathrm{c}$ & & EXPECT & 87 \\
\hline & IF (ITYPE(N).GE.NTYPE) GO TO 238 & EXPECT & 68 \\
\hline & GO TO $(110,120,130,140,160,180,190,260,230,230)$ ITYPE(N) & EXPECT & 69 \\
\hline c & & EXPECT & 76 \\
\hline$c$ & NORMAL DISTRIBUTION & EXPECT & 71 \\
\hline c & & EXPECT & 72 \\
\hline 116 & CDNTINUE & EXPECT & 73 \\
\hline & $A=D B L E(R P 1)$ & EXPECT & 74 \\
\hline & $B=D E L E(R P 2 * R P 2)$ & EXPECT & 75 \\
\hline & $C=D Q E(R P 1)$ & EXPECT & 78 \\
\hline & $D=D B L E(R P 1)$ & EXPECT & 77 \\
\hline & GO TO 218 & EXPECT & 78 \\
\hline c & & EXPECT & 79 \\
\hline$c$ & NATURAL LOGNOPUAL DISTRIEUTION & EXPECT & 80 \\
\hline c & & EXPECT & 81 \\
\hline 129 & CONTINUE & EXPECT & 82 \\
\hline & $A=8.000$ & EXPECT & 83 \\
\hline & $B=0.000$ & EXPECT & 84 \\
\hline & $G=D E L E(R P 1+0,5 * R P 2 * R P 2)$ & EXPECT & 85 \\
\hline & IF ((G.GE. ENIN). AND. (G.LE. EMAX)) THEN & EXPECT & 88 \\
\hline & $k=\operatorname{DEXP}(0)$ & EXPECT & 87 \\
\hline & $B=D E X P(D Q L E(2, \sigma *(R P 1+R P 2 * R P 2)))-D B L E(A * \lambda)$ & EXPECT & 88 \\
\hline & ESSE & EXPECT & 89 \\
\hline & IOWIT $=1$ & EXPECT & 90 \\
\hline & ENDIF & EXPECT & 91 \\
\hline & $\mathrm{C}=\mathrm{DEXP}(\mathrm{OBLE}(\mathrm{RP} 1))$ & EXPECT & 92 \\
\hline & $0=0.600$ & EXPECT & 93 \\
\hline & $G=D B L E(R P 1-R P 2 * R P 2)$ & EXPECT & 94 \\
\hline & IF ((G.GE. ENIN), AND. (G.LE. ENAX)) THEN & EXPECT & 95 \\
\hline & $D=D E X P(C)$ & EXPECT & 96 \\
\hline & 日SE & EXPECT & 97 \\
\hline & IONIT $=I O M I T+2$ & EXPECT & 98 \\
\hline & ENDIF & EXPECT & 99 \\
\hline & GO FD 210 & EXPECT & . 100 \\
\hline $\mathrm{C}$ & & EXPECT & . 181 \\
\hline$c$ & COMMON LOGNORMAL DISTRIBUTION & EXPECT & . 102 \\
\hline $\mathrm{c}$ & & EXPECT & . 183 \\
\hline 130 & CONTINUE & EXPECT & . 104 \\
\hline
\end{tabular}

C. 19 


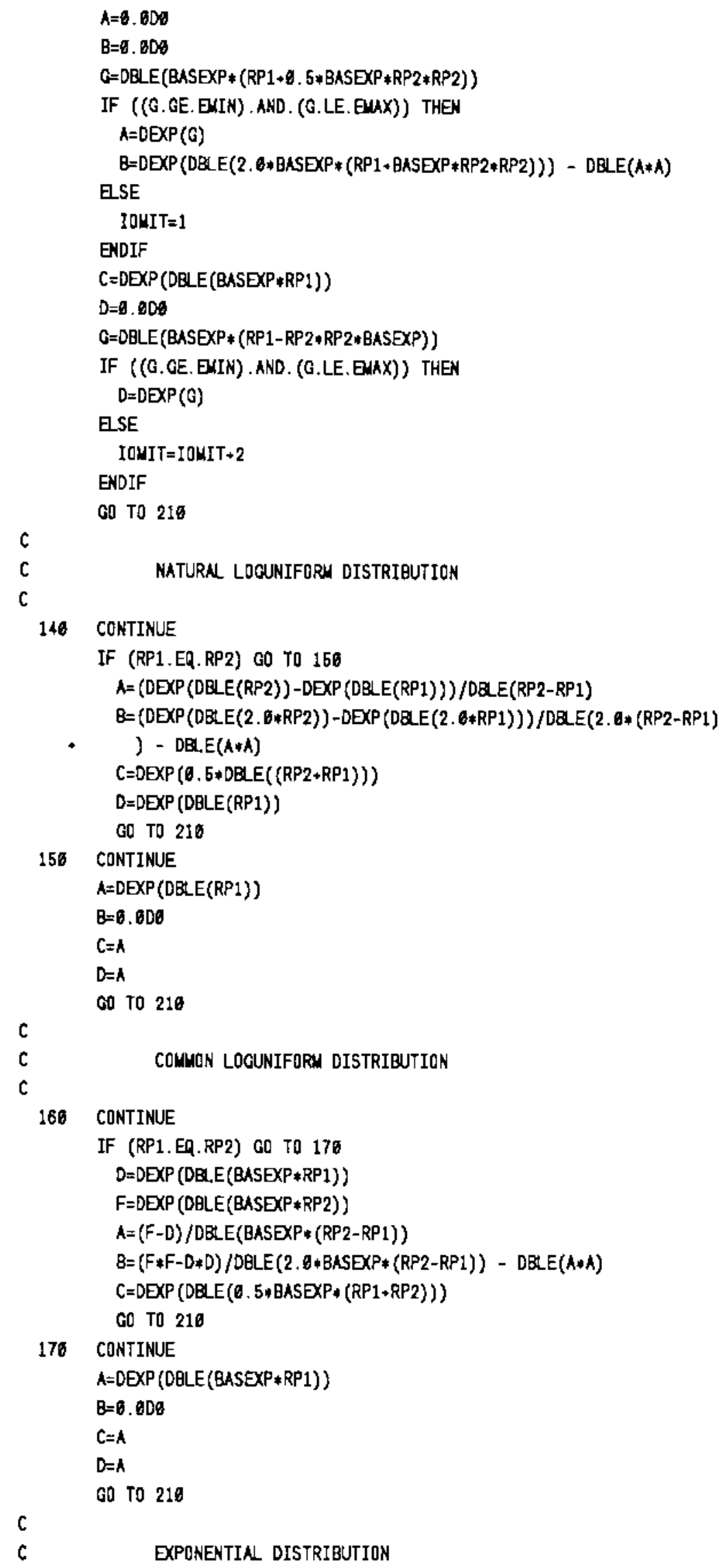


C

188 CONTINUE

$A=D Q E(1.0 / R P 1)$

$B=A \neq A$

$C=A \neq \operatorname{DBLE}(A \operatorname{LOG}(2.6))$

$D=9.60 \%$

GO TO 219

C

c

$c$

198

$A=\operatorname{DBLE}(6.5 *(R P 1+R P 2))$

$B=D Q E((A B S(R P 2-R P 1) / 3.464117)) * * 2$

$C=A$

$D=A$

GO TO 210

C

$c$

c

200

CONTINUE

$A=\operatorname{RP} 1 * \operatorname{PAR}(N, 3)+\operatorname{RP} 2 *(1 . \sigma-\operatorname{PAR}(N, 3))$

IONIT $=4$

c

C

c

210 CONTINUE

VINAVG $(N)=$ SNGL $(A)$

$B=$ DSRRT $(B)$

VINSTD $(N)=$ SNCL $(\theta)$

VINUED $(N)=$ SNGL $(C)$

$\operatorname{VINHOO}(N)=S N G L(0)$

IF (IDMIT, NE. G) GO TO 226

WRITE (III, 526) VLAGQ $(N), 0, A, C, B$

GO TO 230

226

CONTINUE

IF (IOMIT. EQ . 1) WRITE (III, 530) VABEl (N) , O,C

IF (IONIT. EQ.2) MRITE (III, 540) VABE (N), $A, C, B$

IF (IOMIT .EQ .3) WRITE (III, 550) VABE (N), C

IF (IOMIT.EA.4) WRITE (III, 540) VLABE (N), A

230 CONTINUE

IF (IDAT.GT.6) VRITE (III, 560)

$c$

c

C

240 CONTINUE

IF (LIMITS.EQ.ø) GO TO 260

YRITE (III, 570)

DO $258 \mathrm{~N}=1$, NVAR

IF (ILIM(N).EQ.0) 00 TO 250

WRITE (III, 588) VLABE (N), VLIMT $(N, 1), \operatorname{VINT}(N, 2), R \operatorname{IN}(N, 1)$,

$\operatorname{RLIM}(N, 2)$

250 CONTINUE

IF (IDAT.GT.0) WRITE (III, 566)

260 CONTINUE

\begin{tabular}{|c|c|}
\hline EXPECT & . 159 \\
\hline EXPECT & 160 \\
\hline EXPECT & 181 \\
\hline EXPECT & 162 \\
\hline EXPECT & 163 \\
\hline EXPECT & 184 \\
\hline EXPECT & 185 \\
\hline EXPECT & 168 \\
\hline EXPECT & 187 \\
\hline EXPECT & 168 \\
\hline EXPECT & . 169 \\
\hline EXPECT & .170 \\
\hline EXPECT & . 171 \\
\hline EXPECT & . $\quad 172$ \\
\hline EXPECT & 173 \\
\hline EXPECT & . 174 \\
\hline EXPECT & . 175 \\
\hline EXPECT & . 178 \\
\hline EXPECT & . $\quad 177$ \\
\hline EXPECT & . 178 \\
\hline EXPECT & . 179 \\
\hline EXPECT & 180 \\
\hline EXPECT & . 181 \\
\hline EXPECT & 182 \\
\hline EXPECT & . 183 \\
\hline EXPECT & 184 \\
\hline EXPECT & 185 \\
\hline EXPECT & . 186 \\
\hline EXPECT & 187 \\
\hline EXPECT & . 188 \\
\hline EXРECT & 189 \\
\hline EXPECT & . 190 \\
\hline EXPECT & . 191 \\
\hline EXPECT & . $\quad 192$ \\
\hline EXPECT & . 193 \\
\hline EXPECT & . 194 \\
\hline EXPECT & . 195 \\
\hline EXPECT & 198 \\
\hline EXPECT & 197 \\
\hline EXPECT & . 198 \\
\hline EXPECT & 199 \\
\hline EXPECT & 260 \\
\hline EXPECT & . 201 \\
\hline EXPECT & . 282 \\
\hline EXPECT & 283 \\
\hline EXPECT & 284 \\
\hline EXPECT & . 265 \\
\hline EXPECT & . 206 \\
\hline EXPECT & 287 \\
\hline EXPECT & . 268 \\
\hline EXPECT & 269 \\
\hline EXPECT & 218 \\
\hline EXPECT & 211 \\
\hline EXPEC & 212 \\
\hline
\end{tabular}

C.21 
666 FORWT (//1X,'**--- EXPECTED INPUT VARIABLE STATISTICS')

EXPECT $\quad 213$

510 FORWT (//1X, 'VARIABZE', T18, 'WODE', T28, 'WEAN', T37, 'WEDIAN', T46, 'St $\rightarrow$ NO DEV')

520 FORUA (1X,A12,1X,1PEg. 2,3(1X,E9.2))

530 FORWAT (1X,A12,1X,1PE9.2,11X,E9.2)

B49 FORUAT (1X, A12, 18X, 3(1X,1PE9.2))

550 FORUAT (1X,A12,21X, IPES . 2)

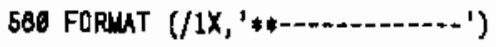

576 FORMAT ( $/ 1 X_{2}$ 'EFECTIVE LIMITS ON THE INPUT OISTRIBUTION VARIABLES'

+/4X, 'VARIABLE', 16X, 'VARIABLE LIMITS', 13X, 'INTERYAL LIMITS'/22X, 'LO

+NER', 7X, 'UPPER', 10X, 'LOWER', 7X, 'UPPER')

580 FORUAT (1X, A12,5X,1PE10.3,2X,E10.3,5X,6PF10,4,2X,F10.4)

END

SUBROUTINE IDNUN (IRUNID, USRNAM)

THIS ROUTINE CREATES $\&$ UNIQUE RUN IDENTIFIER NUMBER, UTILIZING

THE DATE AND TIME AT WHICH THIS ROUTINE IS CNLED. THE SYSTEN

USER NAME IS ALSO PLACED INTO A CHARACTER VARIABLE. THIS

INFORWATIDN IS USED TO INSURE TRACEABILITY AND ACCDUNTABILITY

FOR DATA FILES FRON SIMLATION PROGRAUS.

IRUNID = RUN IDENTIFIER, AS FOLLONS:

DIGITS $9-10$ = MONTH DF CURREAT DATE.

DIGITS 7-8 = DAY OF THE MONTK ( 1 TO 31).

DIGITS $5-6=$ CURRENT YEAR.

DIGITS 3-4 = CURRENT HOUR (6 TO 23).

DIGITS 1 2 = CURRENT UINUTE (6 TO 59).

USRNAL = 8 CHARACTER NAME OF THE CURREAT SYSTEN USER.

c

INTESER $* 4$

+ IRUNID, JW, JDD, JYY,

- JHR, JUIN

C

LOCAL ARRAY FOR READING DATE AND TIME

INTEGER.2

- LOCN (15)

CHARACTER $* 8$

$+\quad$ ASDATE

CHARACTER $* 8$

- TIME,

USRN,

USRNAM

CHARACTER*16

+ DATE

EQRIVALEMCE

- (ASDRTE, LOCAL(1)),

- (USRN, LOCAL(13))

$c$

C GET CURRENT TIME AND DATE

c TIMDAT RETURNING:

c

ASCII: LOCAL(1) $=M$ (MONTH)

LOCAL(2) $=D O$ (DAY)

$\operatorname{LOCAL}(3)=Y Y$ (YEAR)

BINARY: LOCN (4)=WINUTES

LOCN $(5)=$ SECONDS

\begin{tabular}{|c|c|}
\hline XXPECT & . 213 \\
\hline EXPECT & 214 \\
\hline EXPECT & 215 \\
\hline EXPECT & . 216 \\
\hline EXPECT & . 217 \\
\hline EXPECT & . 218 \\
\hline EXPECT & . 218 \\
\hline EXPECT & . 220 \\
\hline EXPECT & . 221 \\
\hline EXPECT & . 222 \\
\hline ЕХРECT & . 223 \\
\hline EXPECT & . 224 \\
\hline ЕХPECT & + 225 \\
\hline ЕХPECT & - 226 \\
\hline ЕХРЕCT & . 227 \\
\hline IDNUN & 2 \\
\hline IDNUI & 3 \\
\hline IDNUM & 4 \\
\hline IDNU & 5 \\
\hline IDNUM & B \\
\hline IDNUN & . \\
\hline IDNUM & 6 \\
\hline IDNT & 9 \\
\hline IDNUM & 10 \\
\hline IDHUN & 11 \\
\hline IDNUY & 12 \\
\hline IONUM & 13 \\
\hline IDNUA & 14 \\
\hline IDNLA & 15 \\
\hline IDNCN & 16 \\
\hline IDNLM & 17 \\
\hline IDNUM & 18 \\
\hline IDNUN & 18 \\
\hline IDNUY & 20 \\
\hline IDNW & 21 \\
\hline IONUY & 22 \\
\hline IDNUM & 23 \\
\hline IDNUN & 24 \\
\hline IDNUM & 25 \\
\hline IDNUM & 25 \\
\hline IDNU & 27 \\
\hline IDNUM & 28 \\
\hline IDNUM & 29 \\
\hline IDNUM & 30 \\
\hline IDNUN & 31 \\
\hline IDNUM & 32 \\
\hline IDNUY & 33 \\
\hline IONUX & 34 \\
\hline IDNUN & 35 \\
\hline YDNUY & 36 \\
\hline IONUM & 37 \\
\hline IDNU & 38 \\
\hline IDNUH & 39 \\
\hline IDNUN & 46 \\
\hline
\end{tabular}


c

C CNL TIMDAT (LOCNL(1), INTS(18))

C CNL OATESA (DATE)

C CNL TIMEHA (TIME)

$c$

READ (ASDATE, EDE) JUM, JDD, JYY

$J H R=\operatorname{LOCNL}(4) / 60$

$J M I N=$ LOCAL(4)-JHR*6D

$J y=J 4 M * 100080000$

$J D D=J D D * 1960606$

$J Y Y=J Y Y * 160 B 0$

$J H R=J H R * 160$

IRUNID $=J U W+J D O+J Y Y+J K R+J M I N$

USRNAM=USRN

c

RETURN

500 FORUA (3I2)

END

SUBROUTINE IDPLT (IRUNID,XID,YID)

c

c SURROUTINE IDPLT TAOS A PLOT WITH AN INTEGER RUN ID IN A

C LSER-SPECIFIED LOCATION. THIS IS DONE IN TWO PARTS, AS

c DISSPLA CANNOT HANDLE FEN DIGIT INTEGERS.

c

DATA

- IDATE/16060/

C

C

$c$

IUDY=IRUNID/IDATE

IF (IMDY.GT. D) THEN

CALL INTND (IMDY, XID, YID)

EISE

CNLL MESSAG ('000B', $4, X I D, Y I D)$

ENDIF

c

$c$

$c$

ITME=IRUNID-IMDY *IDATE

DO $160 \mathrm{I}=1,4$

$J=4-I$

IF (J.GT. ) THEN

$J=16 * J$

ELSE

$J=1$

ENDIF

IF (ITUE.GE. J) GO TJ 110

CNLL MESSAG (' $\theta$ ', 1, 'ABUT', 'ABUT')

100 CONTINUE

CD TO 120

110 CONTINUE

CALL INTNO (ITHE, 'AEUT', 'ABUT')

C

120 CONTINUE

\begin{tabular}{|c|c|}
\hline IDNUN & \\
\hline IDNUN & \\
\hline IDNLM & \\
\hline IONLW & \\
\hline IDNUM & . \\
\hline IDNUM & \\
\hline IDNUN & \\
\hline IDNUM & \\
\hline IDNUI & . \\
\hline IONU & \\
\hline IDNUM & \\
\hline IDNUM & . \\
\hline IDNUA & \\
\hline IDNUM & \\
\hline IDNUW & \\
\hline IDNUA & \\
\hline IONLN & \\
\hline IONUN & \\
\hline IDPLT & \\
\hline IDPLT & \\
\hline IOPLT & \\
\hline IDPLT & \\
\hline IOPLT & \\
\hline IDPLT & \\
\hline IDPLT & \\
\hline IDPLT & \\
\hline IDPLT & \\
\hline IDPLT & \\
\hline IDPLT & \\
\hline IDPLT & \\
\hline IDPLT & \\
\hline IDPLT & \\
\hline IDPLT & \\
\hline IDPLT & \\
\hline IOPLT & \\
\hline IDPLT & \\
\hline IDPT & \\
\hline IOPLT & . \\
\hline IDPLT & \\
\hline IDPLT & \\
\hline IDPLT & . \\
\hline IDPLT & \\
\hline IDPLT & \\
\hline IDPLT & \\
\hline IDPLT & \\
\hline IDPLT & \\
\hline IDPLT & \\
\hline IDPLT & \\
\hline IDPLT & \\
\hline IDPLT & \\
\hline IDPLT & \\
\hline IDPLT & \\
\hline IDPLT & \\
\hline IDPLT & \\
\hline
\end{tabular}




\begin{tabular}{|c|c|c|c|}
\hline & RETURN & IOPLT & 38 \\
\hline & END & IDPLT & 39 \\
\hline & SUBROUTINE IDVAX (IRUNID, USRNAN) & IDVAX & . \\
\hline C & & IDVAX & . \\
\hline C & THIS ROUTINE IS THE VAX 11/780 ERUIVALENT OF THE PRIME 750 & IDVAX & . \\
\hline c & PACSTAT IDNUN SUBROUTINE. IT'S PURPOSE IS THE SAME AS IDNUA, & IOVAX & . \\
\hline C & TO CREATE A UNIQUE RUN IDENTIFIER, UTILIZING THE DATE AND TIUE & IOVAX & 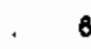 \\
\hline C & SYSTEY'S LIBRARIES AT THE TIME THIS ROUTINE IS CALLED. THE & IDVAX & . \\
\hline 6 & SYSTEI USER NAME IS ALSO PLACED INTO A CHARACTER VARIABE. TKIS & IDVAX & . \\
\hline$c$ & IFORUTION IS USED TO INSURE TRACEABILITY AND ACCOUNTABILITY FOR & IDVhX & . \\
\hline C & DATA FILES FRON SIUUAATION RUNS. AUTHOR: C.L. BARTLEY & IDVAX & 10 \\
\hline C & & IOVAX & . \\
\hline C & & IDVAX & 12 \\
\hline C & & IOVAX & 13 \\
\hline c & IRUNID = RUN IDENTIFIER, AS FDLLONS: & IOVAX & . \\
\hline C & DIGITS $\$-10=$ MONTH OF CURRENT DATE. & IDVAX & 15 \\
\hline c & DIGITS $7-8=$ DAY OF THE MONTH $\left(\begin{array}{lll}1 & \text { TO } 31\end{array}\right)$. & IOVAX & 18 \\
\hline C & DIGITS 6-6 = CURRENT YEAR. & IOVAX & 17 \\
\hline C & DIGITS 3-4 = CURRENT HOUR ( 6 TO 23). & IDVAX & 18 \\
\hline c & DIGITS $1-2=$ CURRENT WINUTE ( 0 TO 59 ). & IOVAX & 19 \\
\hline C & USRNAM = 8 CHARACTER NAME OF THE CURRENT SYSTEY USER. & IOVhx & 20 \\
\hline c & & IDVAX & 21 \\
\hline & INTEGER*4 & IDVAX & 22 \\
\hline & + IRUNID, $\quad J M M_{1} \quad J D D_{1} \quad J Y Y_{1}$ & IDVAX & \\
\hline & $+\quad J H R, \quad$ JWIN & IDVAX & 24 \\
\hline$c$ & LOCAL ARRAY FOR READING DATE AND TIME & IDVAX & 25 \\
\hline & INTEGER*2 & IOVhX & 26 \\
\hline & - $\quad$ LOCAL(15) & IDVAX & 27 \\
\hline & CHARACTER $* 8$ & IDVAX & 28 \\
\hline & $+\quad$ TIH, & IDYAX & 28 \\
\hline & CHARACTER $* 18$ & IOVAX & 38 \\
\hline & - DATE & IDVAX & 31 \\
\hline & ERUIVALENCE & IOVAX & 32 \\
\hline & $+\quad$ (ASOATE, & IDVAX & . \\
\hline & - $\quad$ (USRN, & IDVMX & 34 \\
\hline$c$ & & IDVhX & 35 \\
\hline c & GET CURRENT TIME AND DATE & IDVAX & 36 \\
\hline c & IDATE RETURNING: & IOVAX & 37 \\
\hline$c$ & $\operatorname{LOCAL}(1)=N U$ (WONTH) & IDVhX & 38 \\
\hline C & $\operatorname{LOCAL}(2)=D D(D A Y)$ & IDVAX & 39 \\
\hline$c$ & LOCAL (3) $=$ YY (YEAR) & IOVAX & 48 \\
\hline C & TIUE RETURNING: & IDVAX & 41 \\
\hline C & JHR $\quad(1)=$ HOURS & IOVAX & 42 \\
\hline C & JMIN (5)=NINUTES & IDVAX & 43 \\
\hline$c$ & & IDVAX & 44 \\
\hline 6 & & IOVAX & 45 \\
\hline & $C N \perp \operatorname{IDNTE}(\operatorname{LOCA}(1), \operatorname{LOCNL}(2), \operatorname{LOCAL}(3))$ & IDVAX & 48 \\
\hline C & & IDVAX & 47 \\
\hline & $J W=L O C A L(1)$ & IDVAX & 48 \\
\hline & $J D D=\operatorname{LDCA}(2)$ & IDVAX & 49 \\
\hline & $J Y Y=\operatorname{LOCAL}(3)$ & IDVAX & . \\
\hline & CALL TIME (TIM) & IOVAX & 51 \\
\hline c & & IDVAX & 52 \\
\hline & READ (TIM, 500) JHR, JMIN & IDVhX & 63 \\
\hline
\end{tabular}




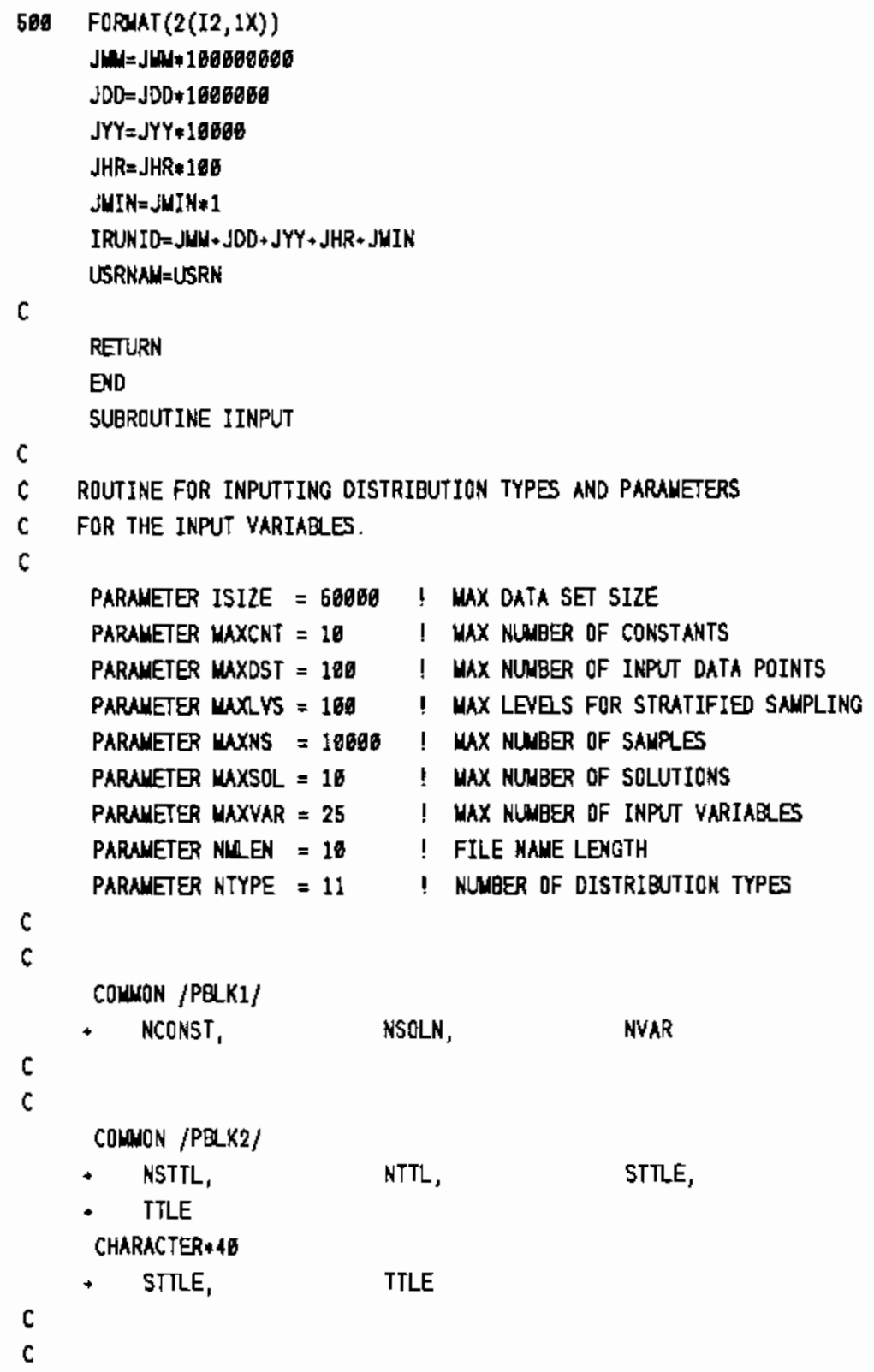


$c$

COLMON /PBLK13/

- NLEVE, NPTSPL (WAXLVS, WAXVAR),

$c$

c

COMAN /PBLK14/

- LABE (NTYPE)

CHARACTER $* 29$

- LABE

$c$

$c$

COMON /PBLK15/

- ILIN(MNVAR)

- PAR(MNYVAR,3),

- VIITt (WKYYAR, 2)

$c$

c

COMAON /PBLK17/

- ICCO,

- IUPDAT,

- NSAY,

$c$

$c$

COLNON /PELK18/

- JJNF (NUEN, MHXVAR), INFIL,

- UDIST(MAXDST, 2)

CHARACTER*4

+ JJNF

$c$

c

PARAMETER WAXLEY $=208$ ! WAX LEVELS OF STRATIFICATION

COMON /STRATI/

- NSPL(HAXLEV)

C

c

PARAMETER MAXSRC $=15$

COWON/IDCOM/

+ JRUNID,

$\rightarrow \quad$ NRUNID (MXSRC),

- PRGT (WAXSRC),

- USRNMT (MAXSRC), CHARACTER $* 4$

- TITLE

CHARACTER*B

+ Fl,

* PROT,

c

$c$

CONMON /INOUT/

- IN, IOUT, IQB, IECHO CHARACTER*1

+ IQB
PBLK5 - 4

PBLK5 . 5

PBLK13 . 2

PQXX13 + 3

PBLK13 . 4

PBLK13 . 6

PBLK13 - 6

PBLK14 - 2

PBLX14 . 3

PBLK14 - 4

PBLK14.5

PBLK14 . 6

PBLK14 . 7

PQK15 . 2

PQXXI5 , 3

$P Q \times 15$. 4

PBLK15 . 5

PBLK15 - 8

PBLK15 + 7

PBLK17 , 2

PBLK17 . 3

PBLK17 . 4

PBLK17 . 5

PBLK17 . 8

PBLK17 . 7

PBLK1B . 2

PBLK1B . 3

PBLK18 . 4

PBLK1B - 5

PBLK1B , 6

PBLK18 . 7

PBLK18 . 8

STRATI . 2

STRATI + 3

STRATI - 4

STRATI . 5

STRATI . 8

IDCON . 2

IDCON . 3

IOCOM . 4

IDCOM 5

IDCOM . 6

IDCON . 7

IDCON . $B$

IDCOM $\quad \theta$

IDCON . 18

IDCOM $\quad 11$

IDCON - 12

IDCON . 13

IDCON $\quad 14$

INOUT . 2

ECHO . 88

INOUT . 4

INOUT . 5 


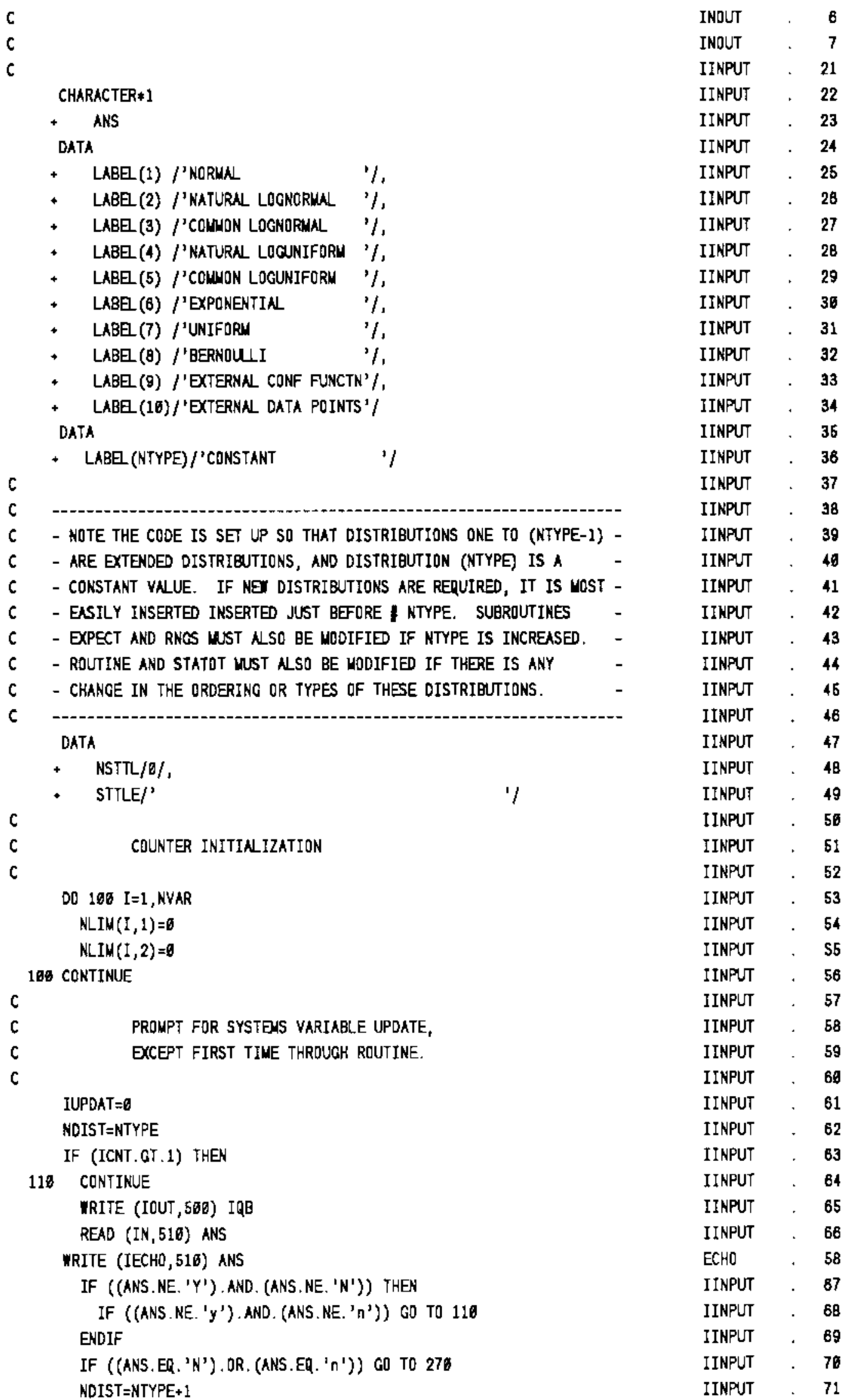




\begin{tabular}{|c|c|c|c|}
\hline & ENDIF & IINPUT & 72 \\
\hline & IUPDAT=1 & IINPUT & 73 \\
\hline & \multirow{4}{*}{ LOOP UPDATES SYSTEN VARIABLES } & IINPUT & 74 \\
\hline c & & IINPUT & 75 \\
\hline c & & IINPUT & 76 \\
\hline \multirow[t]{4}{*}{ c } & & IINPUT & 77 \\
\hline & WRITE (IOUT, 520) & IINPUT & 78 \\
\hline & LPNT $=1$ & IINPUT & 79 \\
\hline & $\mathrm{N}=6$ & IINPUT & 88 \\
\hline \multirow[t]{3}{*}{129} & CONTINUE & IINPUT & 81 \\
\hline & $N=N+1$ & IINPUT & 82 \\
\hline & $\operatorname{ILIM}(N)=0$ & IINPUT & 83 \\
\hline \multicolumn{2}{|l|}{ c } & IINPUT & 84 \\
\hline c & \multirow{2}{*}{ INPUTS DISTRIBUTIONS/PARAMETERS FOR SYSTENS VARIARLES } & IINPUT & 85 \\
\hline$c$ & & IINPUT & 88 \\
\hline \multirow[t]{11}{*}{138} & CONTINUE & IINPUT & 87 \\
\hline & VRITE (IDUT, 536) N, VLABE (N), VDIMEN(N) & IINPUT & 88 \\
\hline & WRITE (IOUT, 546) (I, LAEE (I), I=1, NTYPE) & IINPUT & 89 \\
\hline & IF (ICKT.GT.1) WRITE (IOUT,550) NDIST & IINPUT & 98 \\
\hline & URITE (IOUT, 560) IQB & IINPUT & 91 \\
\hline & READ (IN, $*$, ERR=416) IT & IINPUT & 82 \\
\hline & VRITE (IECHO, *) IT & ECHO & 59 \\
\hline & IF ((IT.LT.1).DR. (IT.GT.NDIST)) GO TO 410 & IINPUT & 93 \\
\hline & IF (IT.GT.NTYPE) OO TO 260 & IINPUT & 94 \\
\hline & WRITE (IOUT, 570) LABE(IT) & IINPUT & 95 \\
\hline & $\operatorname{ITYPE}(N)=I T$ & IINPUT & 86 \\
\hline c & & IINPUT & 97 \\
\hline c & NORMAL DISTRIBUTION & IINPUT & 88 \\
\hline \multirow[t]{5}{*}{ c } & & IINPUT & . 99 \\
\hline & IF (IT. NE.1) GO TO 150 & IINPUT & . 180 \\
\hline & VRITE (IOUT, 580) IQB & IINPUT & .101 \\
\hline & READ (IN,*, ERR=416) PAR $(N, 1)$ & IINPUT & . $\quad 192$ \\
\hline & WRITE (IECHO,*) PAR(N,I) & ECHO & 60 \\
\hline \multirow[t]{9}{*}{140} & CONTINUE & IINPUT & . 103 \\
\hline & WRITE (IOUT, 596) IQB & IINPUT & . 104 \\
\hline & $\operatorname{READ}(\mathrm{IN}, *, E R R=410) \operatorname{PAR}(\mathrm{N}, 2)$ & IINPUT & . 105 \\
\hline & WRITE (IECHO, *) PAR(N,2) & ECHO & 61 \\
\hline & IF (PAR(N, 2).LT.Q.) THEN & IINPUT & . 106 \\
\hline & WRITE (IOUT, 6EQ) PAR $(N, 2)$ & IINPUT & . 167 \\
\hline & GO TO 146 & IINPUT & . 108 \\
\hline & ENDIF & IINPUT & .109 \\
\hline & GO TO 258 & IINPUT & .110 \\
\hline c & & IINPUT & . 111 \\
\hline C & LOGNORMAL DISTRIBUTIONS & IINPUT & . $\quad 112$ \\
\hline C & & IINPUT & . 113 \\
\hline \multirow[t]{2}{*}{150} & CONTINUE & IINPUT & . 114 \\
\hline & IF ((IT.NE. 2).AND. (IT.NE.3)) GO TO $18 \theta$ & IINPUT & . 115 \\
\hline \multirow[t]{7}{*}{180} & CONTINUE & IINPUT & . 116 \\
\hline & WRITE (IOUT,61日) IQB & IINPUT & . 117 \\
\hline & $\operatorname{READ}(I N, *, E R R=410) \operatorname{PAR}(N, 1)$ & IINPUT & . 118 \\
\hline & WRITE (IECHD, *) PAR(N,1) & ECHO & 82 \\
\hline & IF $(\operatorname{PAR}(N, 1) \cdot$ LE. $\theta . \theta)$ THEN & IINPUT & . 119 \\
\hline & WRITE (IOUT,600) PAR(N,1) & IINPUT & . 120 \\
\hline & GO TO 166 & IINPUT & . 121 \\
\hline
\end{tabular}




\begin{tabular}{|c|c|c|c|}
\hline \multicolumn{2}{|c|}{ ENDIF } & \multirow{2}{*}{$\begin{array}{l}\text { IINPUT } \\
\text { IINPUT }\end{array}$} & \multirow{2}{*}{$\begin{array}{r}122 \\
. \quad 123\end{array}$} \\
\hline & $\operatorname{PAR}(N, 1)=A \perp \operatorname{OG}(\operatorname{PAR}(N, 1))$ & & \\
\hline & IF (IT.EQ.3) PAR(N,1)=PAR $(N, 1) / 2.362585$ & IINPUT & . 124 \\
\hline \multirow[t]{9}{*}{170} & CONTINUE & IINPUT & 125 \\
\hline & WRITE (IOUT, 620) PAR(N,1),IQB & IINPUT & . 128 \\
\hline & READ $(I N, *, E R R=410) \operatorname{PAR}(N, 2)$ & IINPUT & . 127 \\
\hline & URITE (IECHO, *) PAR(N,2) & ECHO & 83 \\
\hline & IF (PAR(N, 2),LT, O.) THEN & IINPUT & . 128 \\
\hline & WRITE (IOUT, 660) PAR(N,2) & IINPUT & . 129 \\
\hline & $G 0$ T0 178 & IINPUT & . 138 \\
\hline & ENDIF & IINPUT & . 131 \\
\hline & GO TO 258 & IINPUT & . 132 \\
\hline c & & IINPUT & . 133 \\
\hline $\mathrm{C}$ & UNIFORM LOGNORWN DISTRIBUTIONS & IINPUT & . 134 \\
\hline$c$ & & IINPUT & . 135 \\
\hline \multirow[t]{11}{*}{188} & CONTINUE & IINPUT & . 136 \\
\hline & IF ((IT.NE.4).AND. (IT.NE.5)) GO TO 196 & IINPUT & 137 \\
\hline & WRITE (IOUT,638) & IINPUT & . 138 \\
\hline & $\operatorname{READ}(I N, *, E R R=416) \operatorname{PAR}(N, 1), \operatorname{PAR}(N, 2)$ & IINPUT & . 138 \\
\hline & WRITE (IECHO, *) PAR $(N, 1), \operatorname{PAR}(N, 2)$ & ECHO & 64 \\
\hline & IF (PAR(N,1).GT.PAR(N,2)) THEN & IINPUT & 140 \\
\hline & $C=\operatorname{PAR}(N, 1)$ & IINPUT & . 141 \\
\hline & $\operatorname{PAR}(N, 1)=\operatorname{PAR}(N, 2)$ & IINPUT & . 142 \\
\hline & $\operatorname{PAR}(N, 2)=C$ & IINPUT & .143 \\
\hline & ENOIF & IINPUT & 144 \\
\hline & 60 To 280 & IINPUT & . 145 \\
\hline \multirow{2}{*}{$c^{196}$} & CONTINUE & IINPUT & 148 \\
\hline & & IINPUT & . 147 \\
\hline c & EXFONENTIAL DISTRIBUTION & IINPUT & . 148 \\
\hline \multirow[t]{2}{*}{ c } & & IINPUT & 149 \\
\hline & IF (IT.NE.8) GO TO 216 & IINPUT & . 156 \\
\hline \multirow[t]{10}{*}{286} & CONTINLE & IINPUT & . 151 \\
\hline & IRITE (IOUT, 580) & IINPUT & 152 \\
\hline & READ $(I N, *, E R R=110)$ PAR $(N, 1)$ & IINPUT & . 153 \\
\hline & WRITE (IECHO, *) PAR $(N, 1)$ & ECHO & 85 \\
\hline & IF (PAR(N,1).LE.0.) THEN & IINPUT & 154 \\
\hline & WRITE (IOUT,6B0) PAR(N,1) & IINPUT & 155 \\
\hline & OO TO 208 & IINPUT & . 158 \\
\hline & ENOIF & IINPUT & 157 \\
\hline & $\operatorname{PAR}(N, 1)=1,0 / \operatorname{PAR}(N, 1)$ & IINPUT & .158 \\
\hline & GO TO 250 & IINPUT & . 159 \\
\hline \multicolumn{2}{|l|}{ c } & IINPUT & . 160 \\
\hline C & UNIFDRM DISTRIBUTION & IINPUT & . 181 \\
\hline \multicolumn{2}{|r|}{ (2) - } & IINFUT & . 162 \\
\hline \multirow[t]{10}{*}{218} & CONTINUE & IINPUT & . 163 \\
\hline & IF (IT.NE.7) GO TO 220 & IINPUT & . 184 \\
\hline & WRITE (IOUT, 646) & IINPUT & . 165 \\
\hline & $\operatorname{READ}(\operatorname{IN}, *, E R R=410) \operatorname{PAR}(N, 1), \operatorname{PAR}(N, 2)$ & IINPUT & . $18 B$ \\
\hline & URITE (IECHO, *) PAR $(N, 1), \operatorname{PAR}(N, 2)$ & ECHO & 68 \\
\hline & IF (PAR(N,1) GT PAR $(\mathrm{N}, 2))$ THEN & IINPUT & 187 \\
\hline & $\mathrm{C}=\operatorname{PAR}(\mathrm{N}, 1)$ & IINPUT & . 168 \\
\hline & $\operatorname{PAR}(N, I)=\operatorname{PAR}(N, 2)$ & IINPUT & .169 \\
\hline & $\operatorname{PAR}(N, 2)=C$ & IINPUT & .176 \\
\hline & ENDIF & IINPUT & . 171 \\
\hline
\end{tabular}




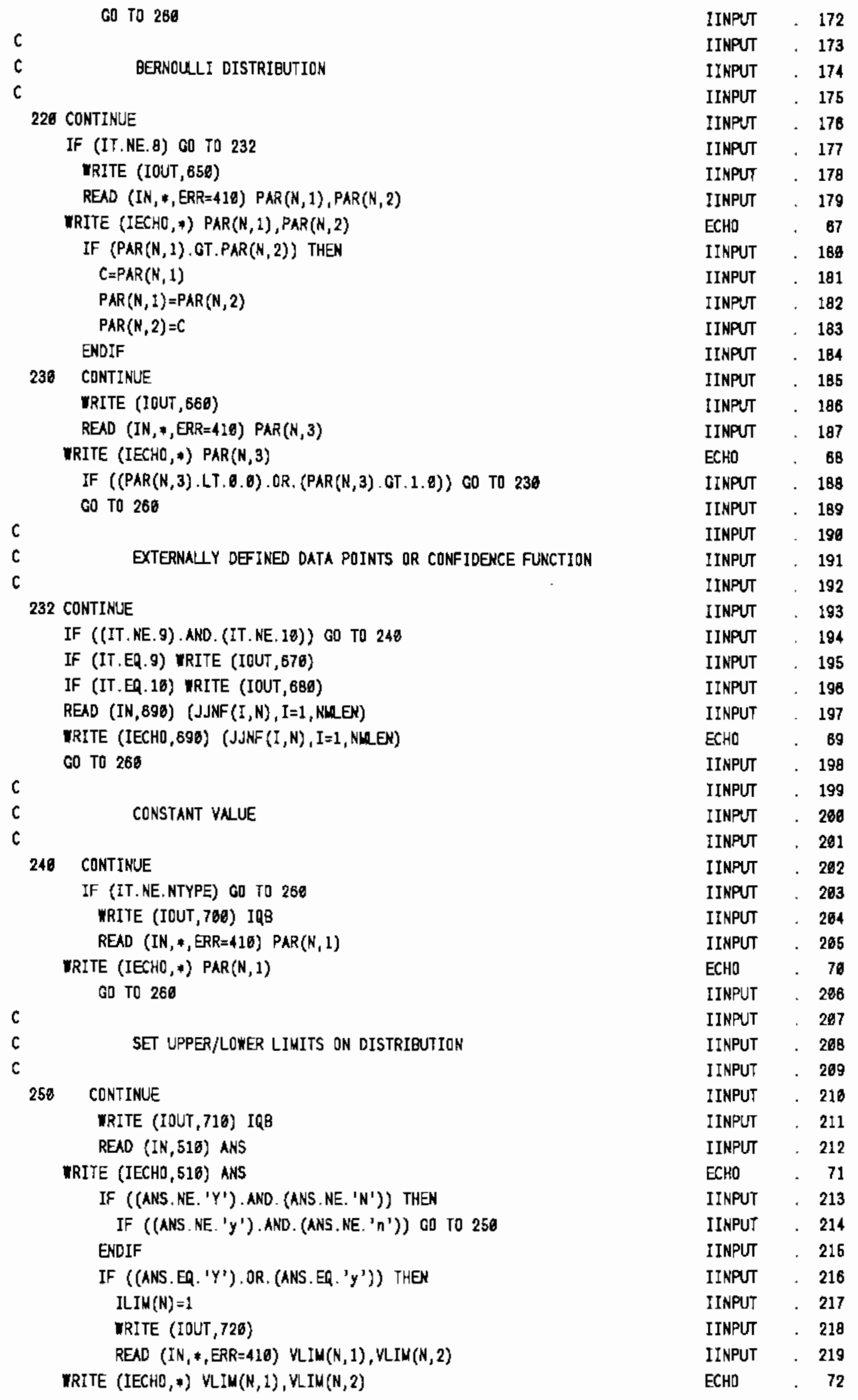




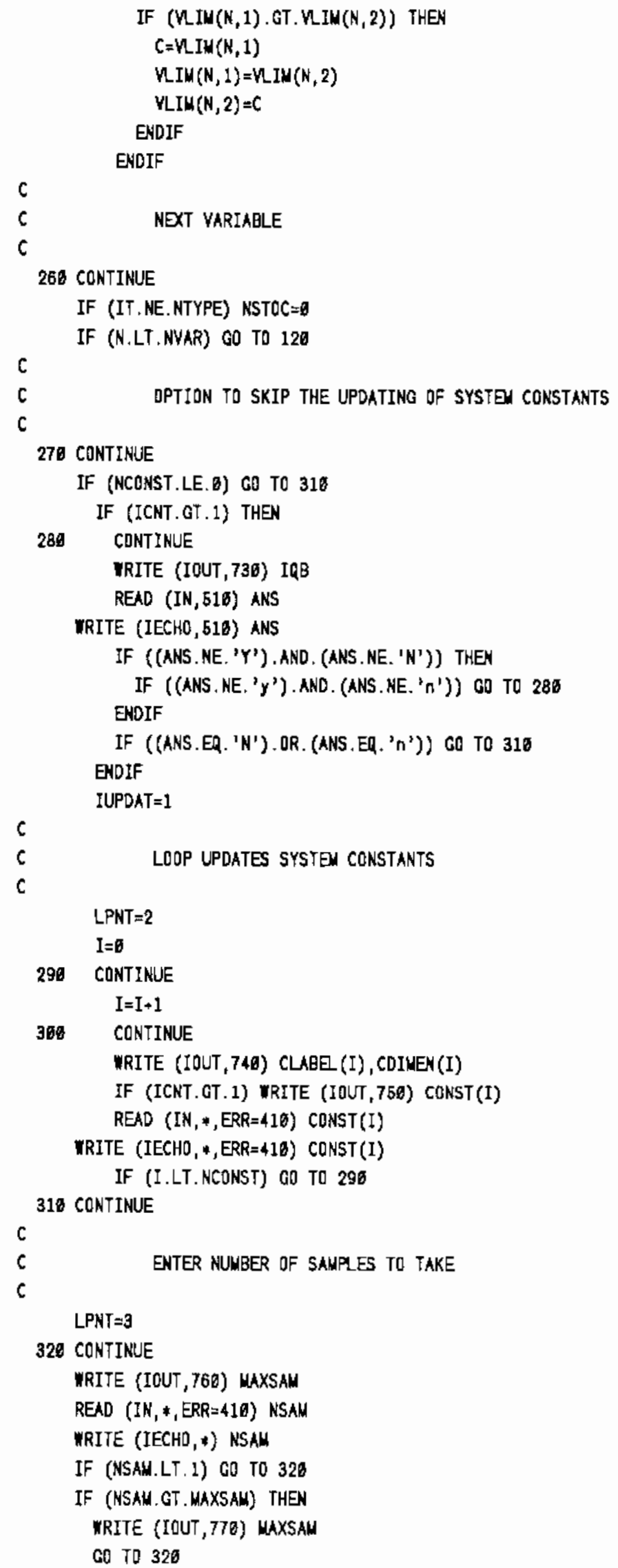




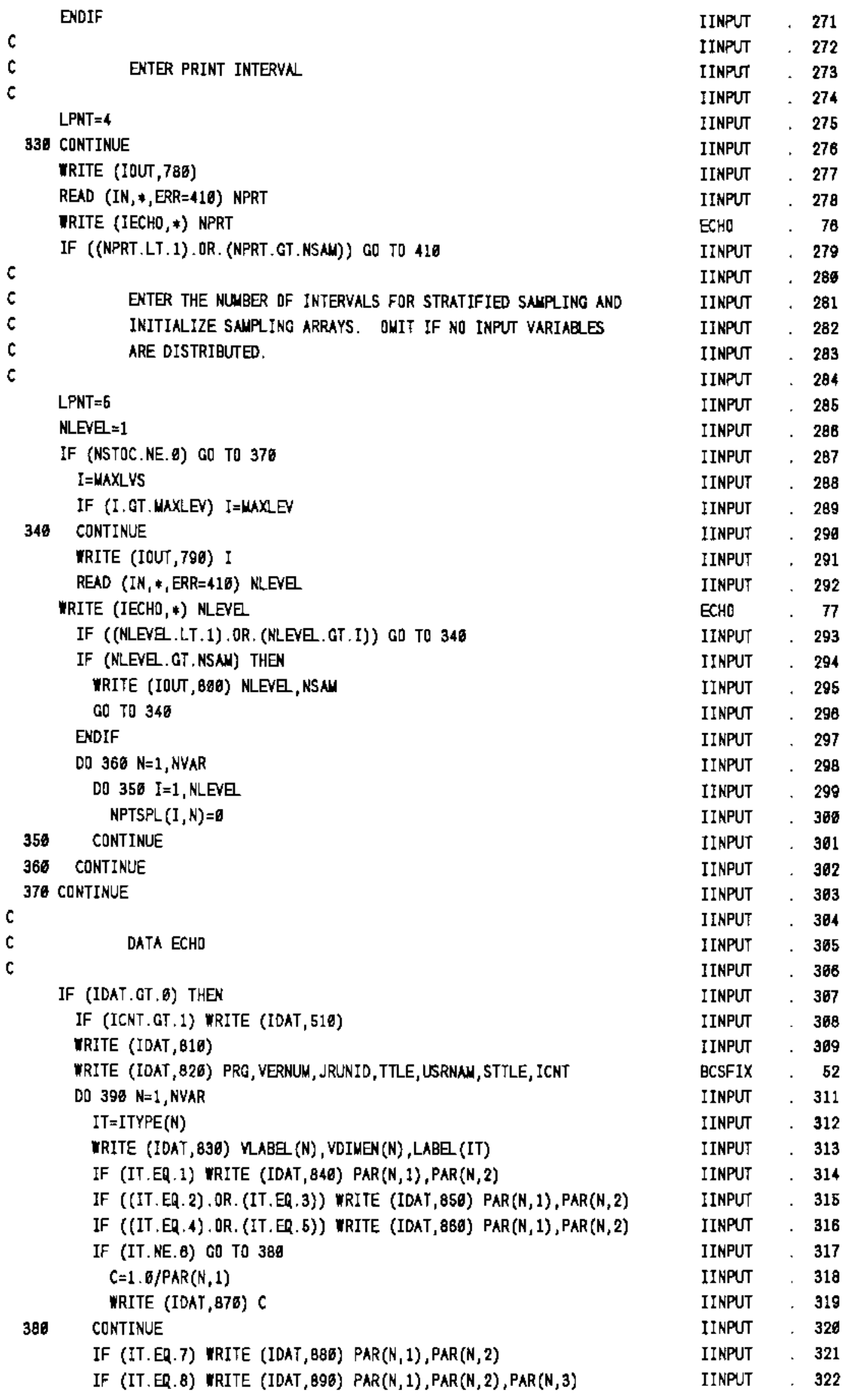


IF (IT.ER, 9) WRITE (IDAT, 9ø8) (JJNF(I,N), I=1, NLLEN)

IF (IT.EQ. 16) WRITE (IDAT, 916) (JJNF( $I, N), I=I$, NLLEN)

IF (IT. EQ.NTYPE) WRITE (IDAT, S29) PAR(N,1)

IF (ILIM(N) . GT. 9) WRITE (IDAT, 936) $\operatorname{VIM}(N, 1), \operatorname{VIM}(N, 2)$

396

CONTINUE

WRITE (IDAT, 940)

DO $406 \mathrm{I}=1$, NCONST

WRITE (IDAT, 959) CLABEL (I), CDIMEN(I), CONST(I)

480

CONTINUE

MRITE (IDAT, 960) NSAM, NPRT

IF (NLEVE.GT.1) THEN

WRITE (IDAT, 978) NLEVE

보

IRITE (IDAT, 986)

ENDIF

ENDIF

$c$

RETURN

$c$

$c$

c

410 CONTINUE

WRITE (IOUT, 996)

GO TO $(130,360,320,330,340)$, LPNT

C

596 FORUAT (/1X, 'WOLLD YOU LIKE TD UPDATE THE SYSTEM VARIABLES (Y/N)?' $\rightarrow, 1 X, A 1)$

E10 FORHAT (A1)

526 FORMAT ( $/ 1 X_{1}{ }^{\prime}$ INPUT OF DISTRIBUTION TYPES AND PARANETERS')

536 FORMAT $(/ 1 X, I 4, ')$ ',A12,' (',A12,') DISTRIBUTION')

540 FORMAT $(B X, 12, ')$ ',A26)

650 FORUA (BX, $12, ')$ PREVIOUSLY DEFINED DISTRIBUTION.' $)$

568 FORMAT (1X, 'ENTER THE NLMEER FOR THE DESIRED DISTRIBUTION. ', A1)

570 FORMAT (1X,'DISTRIQUTION TYPE IS ', A28)

586 FORMAT (1X,' 'ENTER THE MEN OF THE DISTRIQUTION. ',A1)

590 FORUAT (1X,'ENTER THE STANDARD DEVIATION. ',T38, $A 1$ )

660 FORMAT ( $/ 1 X,{ }^{\prime} * *$ ENTERED VALUE OF ',1PE12.4,' IS OUT OF RANGE. ')

610 FORMAT (1 $1 X_{1}$ 'ENTER THE MEDIAN YNLUE FOR THE DISTRIBUTION. ', 'AI)

626 FORMAT (IX, 'THE MEAN OF THE UNDERLYING NORMAL DISTRIBUTION IS ',1P

+E12.4/1X, 'ENTER THE STANDARD DEVIATION OF THIS NORWAL DISTRIBUTION $\left.+{ }^{2}, A 1\right)$

630 FORMAT (1X,' 'ENTER THE MININUM AND MAXIMUM EXPONENTS FOR THE DISTRI +BUTION. ')

846 FORUAT (1X, 'ENTER THE LOWER AND UPPER ENDPOINTS FOR THE UNIFORM DI - STRIBUTION. ')

856 FORMAT (IX, 'ENTER THE LOWER AND UPPER VNLUES FOR THE BERNOLLLI DIS +TRIBUTION. ')

680 FDRMAT (IX, 'ENTER THE PROBABILITY Q,1 OF THE LOWER VALUE OCCURIN $+Q$. ')

676 FORMAT (/1X, 'THE CONFIDENCE FUNCTION WIL BE READ FROM AN EXTERNAL - FILE. ' $/ 1 X$, 'ENTER THE NAME OF THE FILE CONTAINING THE CUMALATIVE' $-/ 1 x_{1}$ 'CONFIDENCE FUNCTION DATA FOR THIS VARIABLE.')

6B@ FORMAT (/1X, 'THE DATA POINTS WILL BE READ FROM AN EXTERNAL FILE. '/ +1X, 'ENTER THE NAME OF THE FILE CONTAINING'/1X, 'THE DATA POINTS FOR

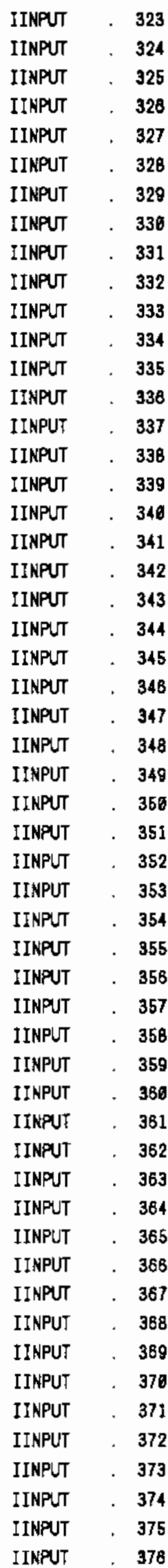

C. 33 
- THIS INPUT VARIAQLE. ')

690 FORMAT (20K4)

700 FOROAT (1X, 'ENTER THE YALUE FOR THE YARIABLE. ',$A 1$ )

710 FOFUAT (1X, 'WOULD YOU LIKE A LOWER AND AN UPPER'/1X, 'BCUND ENFORCE -D ON BHIS VARIABLE (Y/N)? ',A1)

720 FORMAT (1X, 'ENTER THE VALUES FOR THE LONER AND UPPER BOUNDS.')

736 FORAMT (/IX, 'YOUD YOU LIKE TO UPDATE THE SYSTEM CONSTANTS (Y/N)?' $+, 1 \mathrm{X}, \mathrm{A1})$

740 FORUAT (/1X, 'ENTER THE VALUE FOR ',A12,2X,' $\left(', A 12,{ }^{\prime}\right) '$ ')

750 FORMAT (5X,'(CURRENT YALUE IS ',1PE12.5,')')

768 FORMAT (//1X, 'ENTER THE NLMAER OF SAMPLES TO TAKE (MAX =',15, ').')

770 FORMAT (/1X, '** ERROR ** ENTERED VALUE EXCEEDS PROGRAN LIMIT DF', $+I 5)$

780 FORWAT (/1X, 'ENTER THE PRINT INTERVAL FOR OUTPUT DATA. ',A1)

790 FORMAT (/1X, 'ENTER THE NUMBER OF INTERVALS FOR STRATIFIED SAMPLING + (MAX=',I5, '). ')

880 FORHAT (1X, I5,' INTERVALS IS TDO MANY, ONLY', I5,' SAMPLES CHOSEN. $+1)$

810 FDRUAT (/1X,'

820 FORMAT $\left(/ 1 X, A 8,2 X,{ }^{\prime}\left[{ }^{\prime}, F 5.2,{ }^{\prime}\right] ', T 51\right.$, 'RUN I.D. ',I10, $/ 1 X, A 40,9 X, ' U$ -SER NAME', $4 X, A B / 1 X, A 40,9 X$, 'DATA SET NUMBER ', I5////1X, 'TABLE $1.1,3$ $+X$, 'ECHO OF THE INPUT DATA')

836 FORMAT $(/ 5 X, A 12, '(', A 12, ') ' / 35 X$, 'DISTRIBUTION IS ',A20)

846 FORMAT $\left(22 X_{1}\right.$ 'WEAN VALUE $='$, T35, 1PE12,4/17X, 'STND DEVIATION $='$, T3 $+5, E 12,4)$

850 FORMAT (22X, 'CONSTANTS FOR THE UNDERLYING NORML DISTRIBUTION ARE' $+/ 17 X$, 'WEAN VALUE $=1$, T35,1PE12,4/17X, 'STND DEVIATION $={ }^{\prime}$, T35,E12.4)

860 FORMAT (22X, 'MIN, MAX EXPONENTS FOR THE UNDERLYING' $/ 17 X$, 'UNIFORM D +ISTRIBUITDN ARE' $/ 17 X$, 'MIN = ',1PE12.4,', MAX = ',E12.4)

B7ø FORMAT (22X, 'WEAN VALUE IS', T35,1PE12.4)

886 FORHAT (22X, 'LQWER ENDPOINT =', T35, 2PE12,4/17X, 'UPPER ENDPDINT =', $+T 35, E 12.4)$

899 FORMAT (22X, 'LOWER VALUE $=$ ', T35, 1PE12,4/17X, 'UPPER VALUE $=1, T 35$, E1 $+2.4 / 17 X$, 'LOT YALUE PRDB $=', T 35, E 12.4$ )

960 FORMAT (22X, 'THE CONFIDENCE FUNCTION WIL BE READ AS A PIECE WISE' - /17X, 'CONTINUDUS DATA FUNCTION FROH THE EXTERNAL FILE:'/18X, 20A4)

910 FORUAT (22X, 'THE RANDOW DATA POINTS ILL BE READ'/17X, 'FROW THE EX -TERNAL FILE:'/18X, 20A4)

920 FORNAT (22X, 'CONSTANT VALUE $=', T 35,1 P E 12.4$ )

930 FORMAT (22X, 'LOWER BOUND $=', T 35,1$ PE12,4/17X, 'UPPER BOUND =', T35, E1 $+2.4)$

940 FDRMAT (/)

8 S6 FORMAT (5X, 'VALUE FOR' ', $12,2 X,{ }^{\prime}\left({ }^{\prime}, A 12,{ }^{\prime}\right)$ IS ', 1PE12.4)

960 FORMAT (//5X, 'NUMBER OF SAMPLES IS ', IB/1X, 'PRINT INTERVNL IS', 4X, -IB)

970 FORMAT (5X, 'STRATIFIED SAMPLING CHOSEN, USING ', I5,' LEVES. ')

980 FORUAT (5X, 'STRATIFIED SANPLING NDT CHOSEN. ')

990 FQRWAT (/1X, '***** ERROR ON INPUT, TRY AGAIN *****'/)

END

SUBROUTINE LNNORM (N, RNDYAL, RP1, RP2)

C

ROUTINE FOR TRANSFORMING A UNIFORLLY DISTRIBUTED DATA SET ON TKE

C INTERVAL 9,1 TO A NATURAL LOGNORMALEY DISTRIBUTED DATA SET.

\begin{tabular}{|c|c|}
\hline IPUT & 377 \\
\hline IINPUT & 378 \\
\hline INPUT & 379 \\
\hline INPUT & 386 \\
\hline INPUT & 381 \\
\hline INPUT & 382 \\
\hline IINPUT & 383 \\
\hline IINPUT & 384 \\
\hline IINPUT & 385 \\
\hline IINPUT & 388 \\
\hline IINPUT & 387 \\
\hline IINPUT & 388 \\
\hline IINPUT & . 389 \\
\hline IINPUT & 390 \\
\hline IINPUT & . 391 \\
\hline IINPUT & . 392 \\
\hline IINPUT & 393 \\
\hline IINPUT & . 394 \\
\hline IINPUT & . 395 \\
\hline IINPUT & . 396 \\
\hline BCSFIX & 53 \\
\hline BCSFIX & 5 \\
\hline BCSFIX & 5 \\
\hline BCSFIX & 56 \\
\hline BCSFIX & 5 \\
\hline BCSFIX & 58 \\
\hline BCSFIX & 5 \\
\hline BCSFIX & 6 \\
\hline BCSFIX & B: \\
\hline BCSFIX & 62 \\
\hline BCSFIX & 63 \\
\hline BCSFIX & $B$ \\
\hline BCSFIX & $8:$ \\
\hline BCSFIX & 86 \\
\hline BCSFIX & 87 \\
\hline BCSFIX & 8 \\
\hline BCSFIX & 69 \\
\hline BCSFIX & . \\
\hline BCSFIX & 7 \\
\hline BCSFIX & 72 \\
\hline BCSFIX & 7 \\
\hline BCSFIX & 7 \\
\hline BCSFIX & 7 \\
\hline BCSFIX & 7 \\
\hline BCSFIX & 7 \\
\hline BCSFIX & 7 \\
\hline BCSFIX & 7 \\
\hline BCSFIX & 8 \\
\hline BCSFIX & 8 \\
\hline IINPUT & . 42 \\
\hline LNNORM & \\
\hline LNNORM & \\
\hline LNNORM & \\
\hline LNNORM & \\
\hline
\end{tabular}




\begin{tabular}{|c|c|c|c|}
\hline C & & LNNORU & 6 \\
\hline C & $=$ THE NUNBER OF ITEUS IN THE DATA SET & LNNOFU & 7 \\
\hline$C$ & RNDVN = VECTOR OF UNIFORMLY DISTRIBUTED VALUES ON INPUT. EACH & LNNORL & B \\
\hline$C$ & YALUE WST BE BETWEEN ZERO AND ONE. & LNNORH & 9 \\
\hline C & $=$ VECTOR OF LOGNORMALLY DISTRIBUTED VALUES ON OUTPUT. & LNNORM & 10 \\
\hline C & $=$ MEAN YALUE FDR THE UNDERLYING NORMN DISTRIQUTION. & LNNORM & 11 \\
\hline C & $=$ STANDARD DEVIATION FDR THE UNDERLYING NORMN DISTRIBUTION. & LNNORH & 12 \\
\hline$C$ & & LNNORM & 13 \\
\hline & DIMENSION & LNNORY & 14 \\
\hline & - $\quad$ RNOVAL $(N)$ & LNNORM & 15 \\
\hline$c$ & & LNNDRM & 16 \\
\hline C & NATURAL LOGNORLAL DISTRIBUTION & LNNDRM & 17 \\
\hline C & & LNNORN & 18 \\
\hline & DO $190 \mathrm{I}=1, \mathrm{~N}$ & LNNORM & 19 \\
\hline & RV=RNDVAL(I) & LRNORM & 29 \\
\hline & $R V=D N O R H(R V, R P 1, R P 2)$ & LNNORM & 21 \\
\hline & RNDVNL(I) $=\operatorname{EXP}(R V)$ & LNNDRM & 22 \\
\hline 190 & CONTINUE & LNNORM & 23 \\
\hline & RETURN & LNNORM & 24 \\
\hline & END & LNMORM & 25 \\
\hline & SUBRCUTINE LNNORUL (N, RNDVN, RP1, RP2, NMIN, AMAX) & LNNORUL & 2 \\
\hline$C$ & & LNNARML & 3 \\
\hline C & ROUTINE FDR TRANSFORNING A UNIFORILY DISTRIBUTED DATA SET ON THE & LNNORML & 4 \\
\hline C & INTERVAL 9,1 TO A NATURAL LOGNORUNLLY DISTRIBUTED DATA SET, ITTH & LNNORLL & 5 \\
\hline C & THE CONSTRAINT THAT ALL OUTPUT VNLUES LIE BETWEEN SPECIFIED LIMITS. & LNNQRUL & 6 \\
\hline$c$ & & LNNORLI. & 7 \\
\hline C & $=$ THE NUMBER OF OATA VALUES IN VECTOR RNDVAL. & L.NNORUL & B \\
\hline$c$ & RNOVNL = BN INPUT, THE VECTOR OF UNIFORLLY DISTRIBUTED VALUES. & LNNORL, & 9 \\
\hline C & = ON DUTPUT, THE VECTOR OF LOGNDRMAUY DISTRIBUTED VALUES. & LNNBRNL & 10 \\
\hline$c$ & $=$ WEAN YNUE FOR THE DESIRED UNOERLYING NORWAL DISTBUTION. & LNNORAL & 11 \\
\hline$c$ & $=$ STAND. DEY FOR THE DESIRED UNDERLYING NORUAL OISTRIBUTIDN. & LNNORUL & 12 \\
\hline C & AMIN = MINIMUH LIMIT YNUUE FOR THE LOGNORMALLY DISTRIBUTED DATA. & LNNORUL & 13 \\
\hline C & AMAX = WAXIWHM LIMIT VALUE FOR THE LOGNORWALLY DISTRIBUTED DATA. & LNNORXL & 14 \\
\hline$c$ & & LNNORLL & 15 \\
\hline C & UMIN, UMAX = THE LIMIT VALUES ON THE UNIFORW DISTRIBUTION. THESE & LNNORM & 16 \\
\hline c & ARE AVRILABLE TO THE USER THROUGH COWON BLOCK ULIMTS. & LNNQRML & 17 \\
\hline C & & LNNORML & 18 \\
\hline & COMMON /ULIMTS/ & ULIMTS & 2 \\
\hline & + UNAX, & ULINTS & 3 \\
\hline C & & ULINTS & 4 \\
\hline C & & LNNORML & 20 \\
\hline & DIMENSION & LNNORML & 21 \\
\hline & - $\quad$ RNDVAL $(\mathrm{N})$ & LNNORIML & 22 \\
\hline c & & LNWORML & 23 \\
\hline & IF (AMIN.GT.AMAX) THEN & LNNORLL & 24 \\
\hline & $A=A M I N$ & LNNORM & 25 \\
\hline & AMIN $=A M A X$ & LNNORIL & $2 B$ \\
\hline & AMAX $=A$ & LNNORIML & 27 \\
\hline & ENDIF & LNNORML & 28 \\
\hline$c$ & & LNNDRML & 29 \\
\hline$c$ & COMPUTE LOGARITHMS OF THE CHOSEN LIMITS & LNNORML & 30 \\
\hline C & & LNNDRM & 31 \\
\hline & $B M I N=N L O G(A M I N)$ & LNNORML & 32 \\
\hline & $\operatorname{BNAX}=A \operatorname{LOG}(A \cup A X)$ & LNNORNL & 33 \\
\hline
\end{tabular}




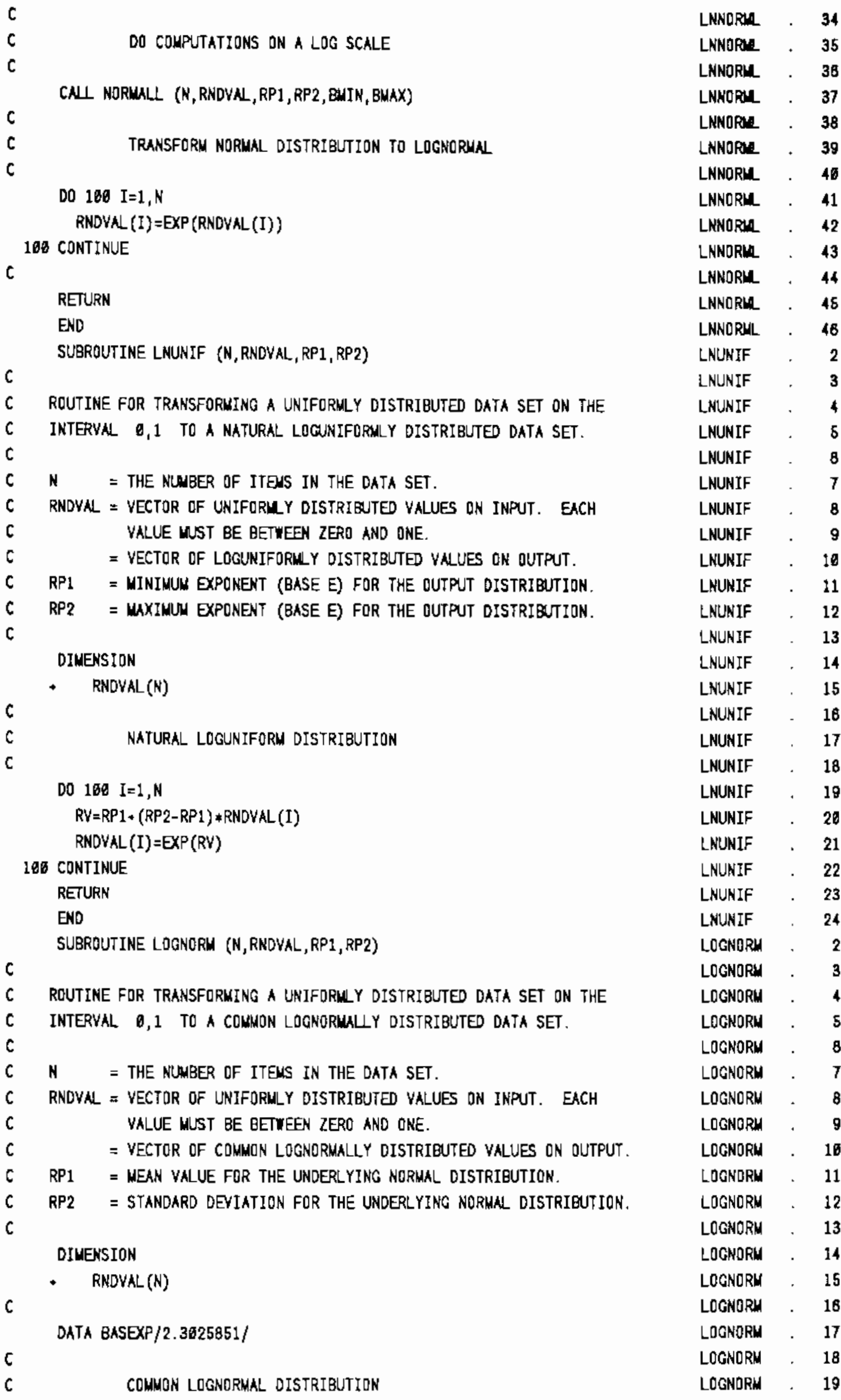


DO $196 \mathrm{I}=1, \mathrm{~N}$

RV=RNDVAL(I)

$R V=O N Q R N(R V, R P 1, R P 2)$

206 CONTINUE

RNDVAL(I) $=$ EXP (BASEXP*RV)

RETURN

END

SUEROUTINE LOGNORNL (N, RNDVAL, RP1, RP2, ANIN, AMAX)

c

RDUTINE FOR TRANSFORUING A UNIFORMYY DISTRIBUTED DATA SET ON THE

INTERVAL 0,1 TO A COMMON LDGNORMALLY DISTRIBUTED OATA SET, MITH

THE CONSTRAINT THAT ALL OUTPUT VALUES LIE BETWEEN SPECIFIED LIMITS.

N = THE NUMBER OF DATA VRLUES IN VECTOR RNDVAL.

RNDVAL = ON INPUT, THE VECTOR DF UNIFORULY DISTRIBUTED VALUES.

= ON OUTPUT, THE VECTOR OF LOGNORMALLY DISTRIBUTED VNLUES.

RP1 = MEAN VNLUE FOR THE DESIRED UNDERLYING NORMAL DISTBUTIDN.

RP2 = STAND. DEV FOR THE DESIRED UNDERLYING NORMAL DISTRIBUTION.

ANIN = MINIMUM LIMIT VALUE FOR THE LOGNORUNLLY DISTRIBUTED DATA.

AMAX = MAXIMUM LIMIT VALUE FOR THE LOGNORMALLY DISTRIBUTED DATA.

UMIN, UMAX = THE LIMIT VALUES ON THE UNIFORM DISTRIBUTION. THESE ARE AVAILABLE TO THE USER THROUGH COMMON BLOCK ULIMTS.

$c$

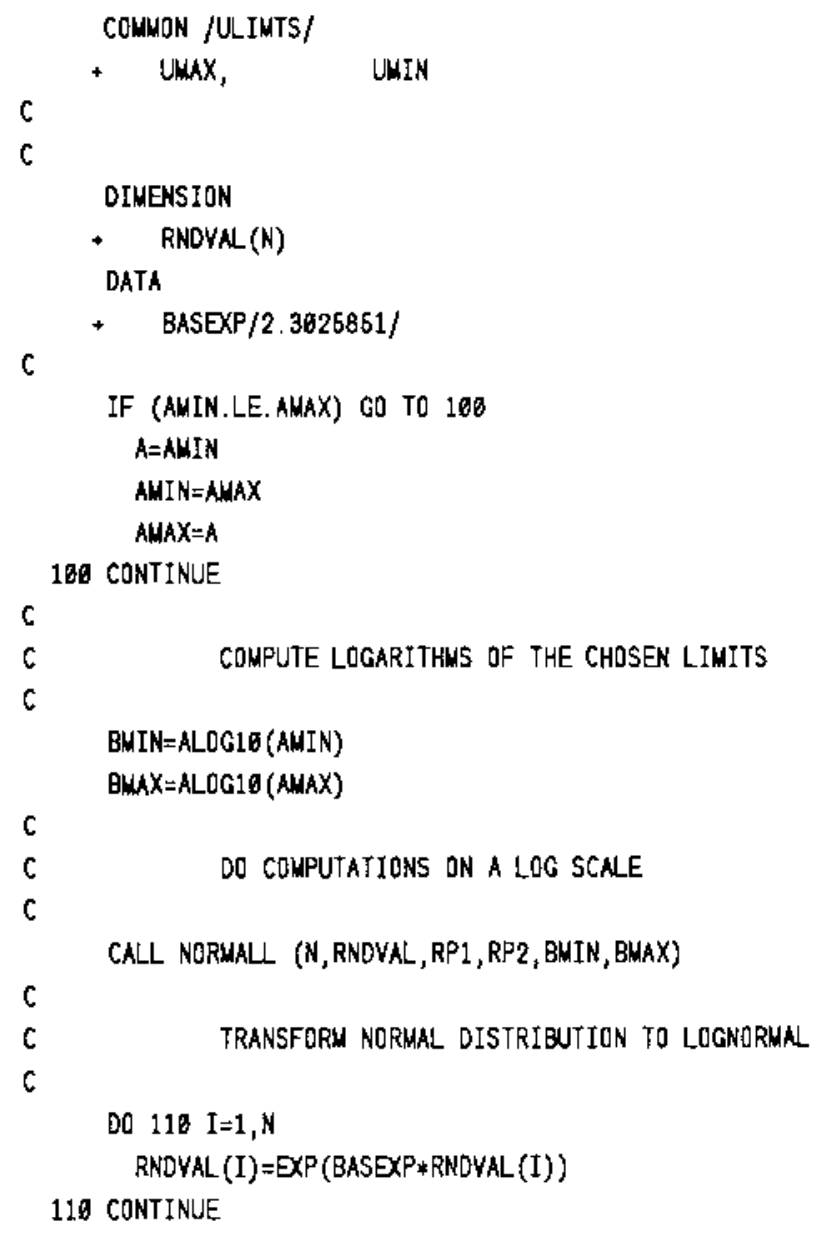


RETURN

END

SUBROUTINE LOGUNIF (N, RNDYAL, RP1, RP2)

$c$

ROUTINE FOR TRANSFORNING A UNIFORLLY DISTRIBUTED DATA SET ON THE

INTERVAL TO A COMMON LOCUNIFORMLY DISTRIDUTED DATA SET.

N $\approx$ THE NUMBER DF ITENS IN THE DATA SET.

RNDYAL = VECTOR OF UNIFORULY DISTRIBUTED VALUES ON INPUT. EACH VALUE WUST BE BETWEEN ZERO AND ONE.

= VECTOR OF LOGUNIFORMLY DISTRIBUTED VALUES ON OUTPUT.

RP1 = MINIWUN EXPONENT (BASE 10) FOR THE DUTPUT DISTRIBUTION.

RP2 = MAXINUN EXPONENT (BASE 10) FOR THE DUTPUT DISTRIBUTION.

C

DIMENSION

+ RNDVAL(N)

c

DATA BASEXP/2.3825851/

c

$c$

c

DO $100 \mathrm{I}=1, \mathrm{~N}$

$R V=R P 1 *(R P 2 * R P 1) * R N D V A L(I)$

RNDVNL(I) $=$ EXP (BASEXP*RV)

160 CONTINUE

RETURN

END

SUBROUTINE NDRMAL（N, RNDVAL, RP1, RP2)

RQUTINE FOR COMPUTING A NORMALLY DISTRIBUTED DATA SET FROM A

UNIFOPALY DISTRIBUTED DATA SET ON THE INTERVAL 0,1 .

N = THE NUMBER OF ITENS IN THE DATA SET

RNDVAL = VECTOR DF UNIFORMLY DISTRIBUTED VNLUES ON INPUT. EACH VALUE WUST BE BETWEEN ZERO AND ONE.

= VECTOR OF NORWALLY DISTRIQUTED VALUES ON OUTPUT.

RP1 = MEAN VALUE FOR THE NOPOAL DISTRIBUTION.

RP2 = STANDARD DEYIATION FOR THE NORMAL DISTRIBUTION.

c

DIMENSION

- RNDVAL(N)

c

$\mathrm{c}$

$c$

NORMAL DISTRIBUTION

DO $116 \mathrm{I}=1, \mathrm{~N}$

$R Y=R N O V A L(I)$

RNDYAL(I) $=\operatorname{DNDRM}(R V, R P 1, R P 2)$

110 CONTINUE

c

RETURN

END

$c$

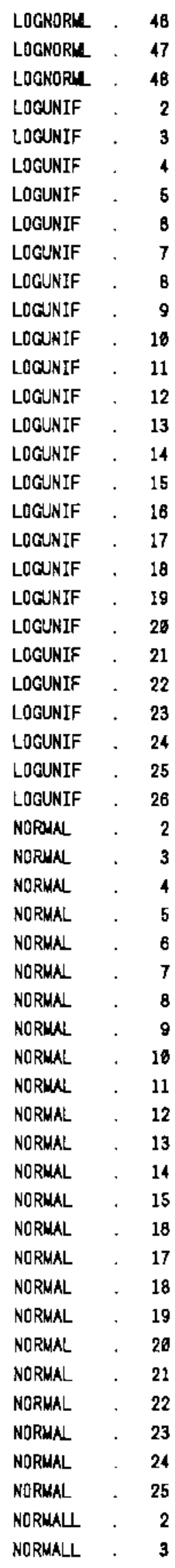


ROUTINE FOR TRANSFORNING A UNIFORULY OISTRIQUTED DATA SET ON THE

INTERVAL 0,1 TO A NORWALLY DISTRIBUTED DATA SET, WITH THE

C CONSTRAINT THAT ALL OUTPUT VALUES LIE BETWEEN SPECIFIED LIMITS.

$c$

C RPI = WEAN VALUE FOR THE DESIRED NORMAL DISTRIBUTION.

C RP2 = STANOARO DEV FOR THE DESIRED NORHAL DISTRIEUTION.

C AMIN = WINIMUM LIMIT VALUE FOR THE NORMALLY DISTRIBUTED DATA.

C AMAX = WAXIMUM LIMIT VALUE FOR THE NORWALLY DISTRIBUTED DATA.

C N = THE NUMBER OF DATA VALUES IN VECTOR RNDVAL.

C RNDYAL $=$ ON INPUT, THE VECTOR OF UNIFORNLY DISTRIBUTED VALUES.

C $=$ ON DUTPUT, THE VECTOR OF NORNALLY DISTRIBUTED VALUES.

c

C NOTE THAT THE NORMAL DISTRIBUTION GENERATOR, DNORM, WST BE A ONE

C TO ONE TRANFORWATION BETWEEN THE UNIFORM AND NORMAL DISTRIBUTIONS.

C EACH WEAN OF THE UNIFORM DISTRIGUTION (0.5) WUST TRANSFORM TO THE

c WEAN OF THE NORIAL DISTRIBUTION (RP1).

$c$

C WMIN, UWAX = THE LIMIT VALUES ON THE UNIFIRN DISTRIBUTION. THESE

c ARE AVAILAQLE TO THE USER THROUGH COWWON BLOCK UIMTS.

$c$

COUMON /ULIUTS/

+ UNAX, UMIN

$c$

$c$

DIHENSION

+ RLIM(2), $\quad$ RNDVAL(N)

DATA

c

- EPS2/0.0EQ/

IF (AMIN.LE. AWAX) GO TO 100

RV=AMIN

AMIN $=$ ALAX

A $A X X=R V$

108 CONTINUE

c SET DEAULTS

$c$

$\operatorname{RLIM(1)}=6.6$

$\operatorname{RLIM(2)=1.6}$

$Z B=D N O R H(R L I M(1), R P 1, R P 2)$

$Z T=\operatorname{DNORM}(R L I M(2), R P 1, R P 2)$

INYRS $=0$

IF (ZB.LE.ZT) GO TO 110

$R Y=Z T$

$Z T=Z B$

$\mathrm{ZB}=\mathrm{R} \mathrm{V}$

INVRS $=1$

116 CONTINUE

c

$c$

$c$

$S T=R P 1+R P 2$

$S B=R P 1-R P 2$

DO $150 \mathrm{I}=1,2$

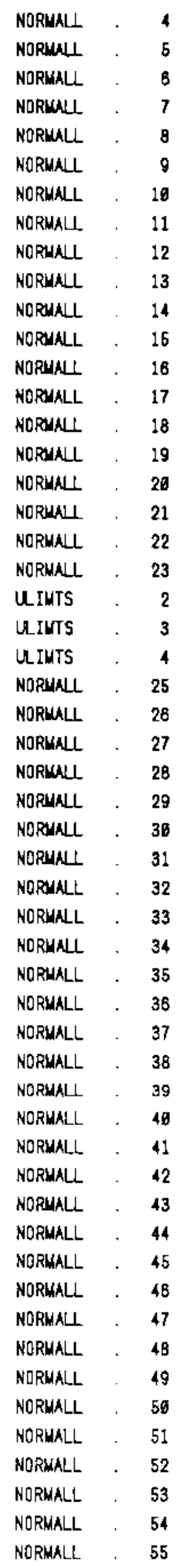




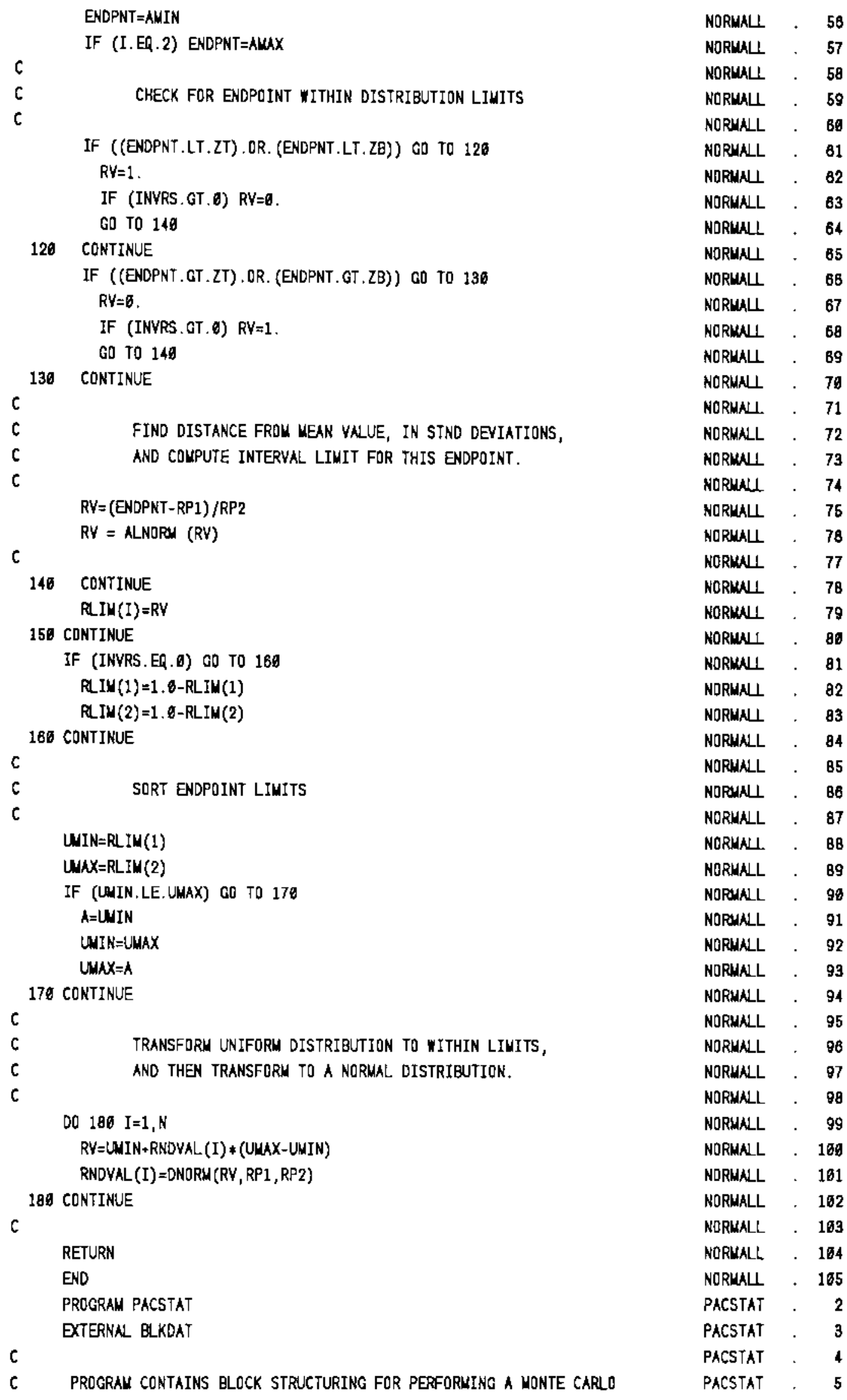


C SIMULATION, AN OUTPUT VARIABLE (OR SEVERAL OUTPUT VALRIABLES) IS

PACSTAT + 6

c IS COMPUTED FROM SEVERAL INPUT VARIABLES. THE INPUT VARIABLES CAN

C TAKE ON ANY OF EIGHT DIFFERENT DISTRIBUTION TYPES, WHTCH THE USER

C CHODSES (ROUTINE IINPUT). THE USER MUST ALSO SPECIFY THE DEINING

PARAMETERS OF THE DISTRIBUTION FOR EACH INPUT VARIABLE.

$A$ PRINTOUT OF $A$ CHDSEN OUTPUT VARIABLE IS GIVEN WITH DIAGNOSTICS ON BOTH THE OUTPUT AND INPUT DISTRIBUTIONS. THIS IS DIRECTED TO AN OPIIONAL, FORMATTED OUTPUT FILE. OUTPUT WAY ALSO BE WRITTEN TO A BINARY OUTPUT FILE, FOR POST PROCESSING.

THE ROUTINE BLKDAT AND SOLN WUST BE PROVIDED BY THE USER.

INPUT AND OUTPUT VARIABLE NAMES AND DIMENSIONS (UNITS) ARE SET IN BLOCK DATA (ROUTINE ELKOAT). THE VALUE OF NYAR SETS THE NLMBER OF INPUT VARIABLES. THE VALUE OF NSOLN SETS THE NUMBER GF AVAILABLE SQLUTIONS. IF THESE EXCEED PRDGRAM LIMITS, AN ERROR WILL BE PRINTED AND THE RUN TERWINATED.

SEVERN USER DEINABLE CONSTANTS ARE PERMITTED. THE NUMBER OF CONSTANTS IS SET IN BLOCK DATA AS VARIABLE NCONST. THE NAMES ANO DIMENSIONS OF EACH CONSTANT MUST ALSO BE SET IN BLOCK DATA. ONE WUST NOT CHOSE MORE CONSTANTS THAN THE PROGRAM IS DINENSIONED TO ACCOMDDATE. NCONST = ZERO IS PERUITTED.

MUTIPLE SDLUTIONS FOR ANY GIVEN PROBLEM ARE NLLOWED. NLL OF THE SOLUTIONS MUST BE COMPUTED IN ROUTINE SOLN. ROUTINE SOLN IS CALLED ONCE FOR EACH RANDOM SALPLE. IT TAKES THE INPUT VALUES FROM ARRAY VINPT (1... NVAR) (COUMON BLOCK PQLX4). OUTPUT VRLUES MUST BE STORED IN ARRAY VRLU(1... NSOLN) (COMHON ELOCK PELK4) FOR RETURN TO THE CALLING ROUTINES. THESE OUTPUT VALUES ARE MOVED TO ARRAY RLTS FOR LONG TERM STDRAGE, AND THE TO ARRAY RPLT (RQUTINE DATDUT) FDR PLOTTING AND DIAGNOSIICS. DNLY ONE SOLUTION LAY BE PLOTTED/ PRINTED AT A TIME.

MACHINE DEPENDENT CODING FOR THIS PROGRAN IS RESTRICTED TO ROUTINES ASG, RELEAS (OPENING AND CLOSING FILES, AND IDNUM (FILE IDENTIFIERS).

THE RANOOM NUMBER GENERATOR, UV1 (DSEED), IS A FORTRAN CODED ROUTINE. IT IS ONLY CALLED FRON THE MAIN ROUTINE AND IN ROUTINE U $1 S$. THE SEED, DSEED, IS A DOUBLE PRECISION REAL NUMBER, AND IS THE ONLY ITEN IN CONMON BLOCX PBLK16.

THE PARAMETER ISIZE SETS THE MAXIMUM NUMBER OF RANDOM SAMPLES WhICH WAY BE USED. THE PARAMETER MAXNS SETS THE MAXIMUN NUMBER DF SAMPLES PER INPUT/DUTPUT VARIABLE WHICH MAY BE COMPUTED. THIS REQUIRES THAT MAXNS WUST BE LESS THAN OR EqUAL TO ISIZE. NDTE THAT SOME TRANSFERING OF RANDOW ARRAYS IS DONE IN ROUTINES VARSET AND STATOT, WICH IS ONLY NECESSARY ON VIRTUAL MENORY COMPUTERS, WERE THE PARAMETER ISIZE EXCEEDS THE PAGE SIZE. ALSO, IF WAXNS EXCEEDS HALF THE PAGE SIZE, THE COMMON BLOCK PBLKG MAY NEED TO BE CHANGED.

PRG AND VERNUM ARE THE PROGRAM NAME AND VERSION NUMBER FOR THE HONTE CARLO SIMULATION PROGRAM, AND ARE SET IN BLOCK DATA. PRGI ANO VERNMI ARE THE PROGRAM NAME AND VERSION NUMBER FOR THE 


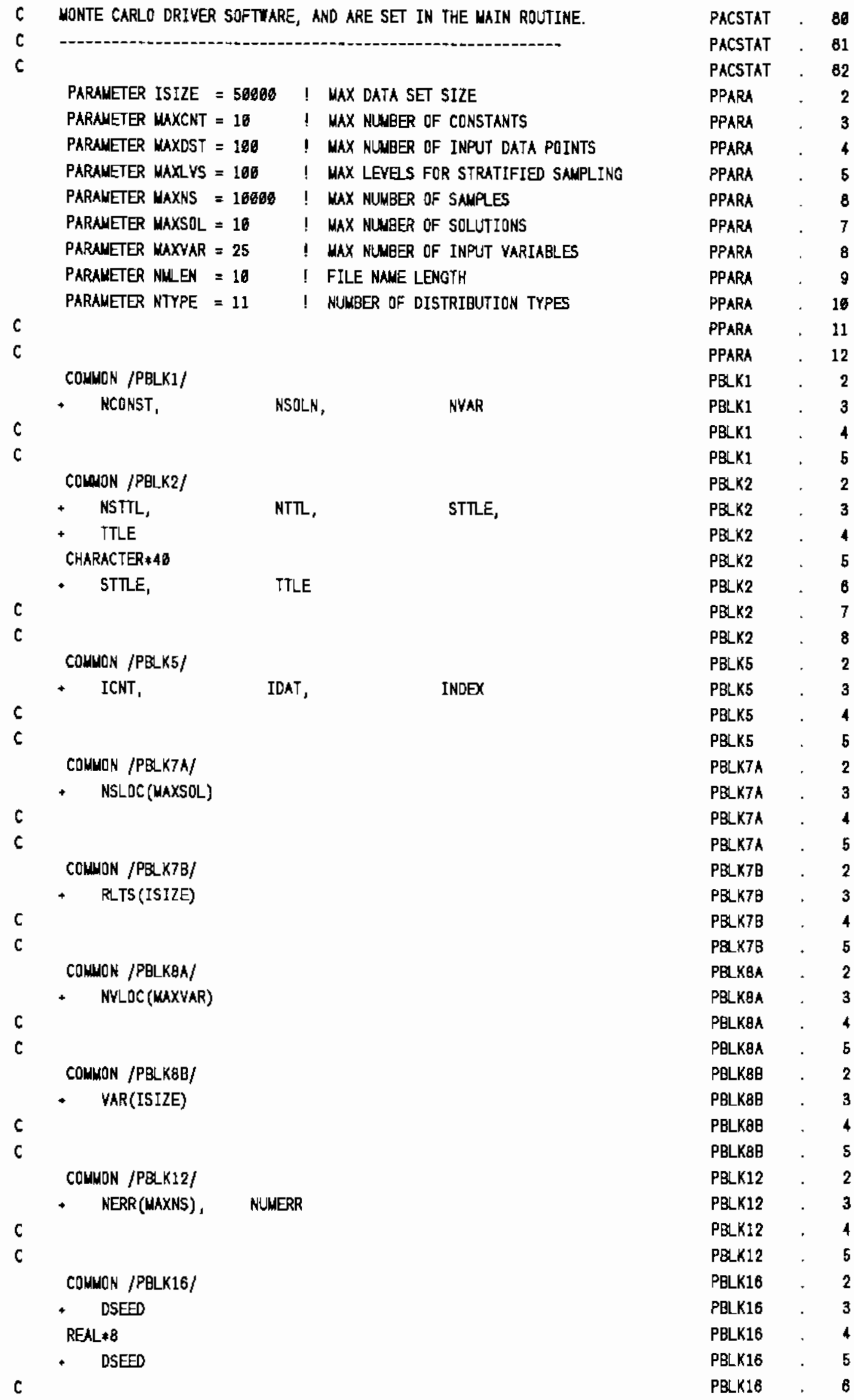


c

COWHON /PELK17/

$+\quad$ ICCD,

- IUPDAT,

- NSN,

$c$

c

COMNON /PBLKIB/

- JJNF (NMLEN, MAXVAR), INFIL,

- UDIST (MAXDST, 2)

CHARACTER*4

- JJNF

c

c

PARAMETER MAXSRC $=15$

COMNON / IDCOM/

+ JRUNID,

- NRUNID(MAXSRC),

- PRGT(MAXSRC),

+ USRNITT (MAXSRC), CHARACTER*4

- title

CHARACTER $* 8$

+ $\mathrm{FL}$,

c

+ PRGT,

c

COMMON / INOUT/

- IN, IDUT, IQB, IECHO CHARACTER" 1

- IQB

$c$

c

CHARACTERN 1

- ANS

CHARACTER:4

- INF(NMLEN)

CHARACTER*B

- PRGI

C DQUBLE PRECISION WXSEED

DATA

- TITLE/26*' '/

DATA

- PRGI/'PACSTAT $\%$ VERNMI/1.6/ DATA

- IQB/' '/

REAL *4 MNSEED

REAL $* B$ WXSEED

$c$

C SET YARIAELE WODEYAX TO TRUE FOR RUNWING ON THE VAX

$c$

OATA MODEVAX /.FALSE./

LOGICAL MODEVAX

CHARACTER INREC $* 86$
IQUIT,

MAXSN,

NSAMC

ISOLN,

NPRT,

IUDIST,

FL,

TITLE(20),

VERNUN

FLT,

USRNAM,

PRG,

USRNUT

PRG,

USRNAN,
FLT (MAXSRC),

PQXK16 . 7

PBLK17 , 2

PBLK17 . 3

PQLK17 . 4

PELK17 . 5

PQLK17 - 6

PQLK17 $\quad 7$

PQLX18 . 2

PQK18 . 3

PQLK18 . 4

PBLK18 - 5

PQLK18 . 6

PELK1B . 7

PBLK1B , 8

IDCOM 2

IDCON , 3

IOCOM . 4

IDCOU . 5

IDCOW - 6

IDCOM $\quad 7$

IDCON , 8

IDCON . 9

IDCOM 10

IDCOM, 11

IDCOY $\quad 12$

IDCOM $\quad 13$

IDCOM, 14

INOUT $\quad 2$

ECHO . 88

INDUT . 4

INOUT . 5

INEUT - 6

INOUT . 7

PACSTAT . 77

PACSTAT . 78

PACSTAT $\quad 78$

PACSTAT . 86

PACSTAT . 81

PACSTAT . 82

HEIDI3 \& 1

PACSTAT - 83

PACSTAT . 84

PACSTAT - 85

PACSIAT . 86

PACSTAT . 87

PACSTAT . BB

HEIDI3 . 2

HEIDI3 . 3

ECHO . 1

ECHO . 2

$\mathrm{ECHD}$. 3

ECHO . 4

ECHO . 5

BATCH . 1 
ICNT $=6$

JONCE $=0$

NSRC $=9$

UNSEED $=1.8$

WXSEFD $=(2,6 * * 31)-2$.

c

C

C

$c$

c

c

c

c

IN $=5$

IOUT $=6$

IECHO $=7$

IDAT $=12$

IDATB $=13$

IFLAG $=\varnothing$

CNI NSOVAX (NLLEN, INF, IFLAG)

c PRINT EANNER PAGE

C

CN VERSION

KARD $=0$

50 CONTINUE

READ (IK, 10600) INREC

$K A R D=K A R D+1$

IRITE(IOUT, 11E06) INREC, KARD

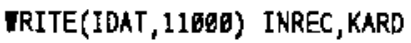

READ(INREC, 18061) KIND

WRITE (IECHO, 11968) INREC, KARO

c

IF (KIND.EQ.4H****) GO TO 58

BACKSPACE IN

c

C

$\mathrm{C}$

WRITE (IOUT, 506) PRG, VERNLM

WRITE (IDUT, 5IB) PRGI, VERNHI

CNLL DCLAIH (IOUT)

ERROR CHECX NUMEER OF SOLUTIONS, VARIABLES, CONSTANTS

IF ((NVAR.GT. WAXYAR).OR. (NVAR.LT.1)) THEN

IRITE (IOUT, 526) NVAR, WAXVAR

CO TD 240

ENDIF

IF (NSDLN.GT. MAXSOL) THEN

WRITE (IOUT, 536) NSOLN, MAXSOL

GO TO 240

ENDIF

IF (NSOLN, LT, B) NSOLN= $\theta$

\begin{tabular}{|c|c|}
\hline PACSTAT & 89 \\
\hline PACSTAT & 96 \\
\hline PACSTAT & 91 \\
\hline PACSTAT & 92 \\
\hline PACSTAT & 93 \\
\hline HEIDI3 & 4 \\
\hline HEIDI3 & 5 \\
\hline PACSTAT & 94 \\
\hline BCSFIX & 1 \\
\hline BCSFIX & 2 \\
\hline BCSFIX & 3 \\
\hline BCSFIX & 4 \\
\hline BCSFIX & $s$ \\
\hline PACSTAT & 96 \\
\hline PACSTAT & 97 \\
\hline PACSTAT & 98 \\
\hline $\mathrm{ECHO}$ & 6 \\
\hline PACSTAT & 99 \\
\hline PACSTAT &.$\quad 100$ \\
\hline ECHO & 7 \\
\hline ECHO & 8 \\
\hline PACSTAT & . $\quad 103$ \\
\hline PACSTAT & . 184 \\
\hline PACSTAT & . 185 \\
\hline PACSTAT & . 186 \\
\hline BATCH & 2 \\
\hline BATCH & 3 \\
\hline BATCH & 4 \\
\hline ВАТСН & 5 \\
\hline BATCH & 6 \\
\hline BATCH & 7 \\
\hline BATCH & 8 \\
\hline ECHO & 9 \\
\hline ECHO & 10 \\
\hline BATCH & 9 \\
\hline BATCH & 16 \\
\hline PACSTAT & . $\quad 107$ \\
\hline PACSTAT & $10 B$ \\
\hline PACSTAT & . 109 \\
\hline PACSTAI & . 110 \\
\hline PACS $¥ A T$ & , $\quad 111$ \\
\hline BCSFIX & 6 \\
\hline PACSTAT & . $\quad 112$ \\
\hline PACSTAT & . 113 \\
\hline PACSTAT & . 114 \\
\hline PACSTAT & . 115 \\
\hline PACSTAT & . 116 \\
\hline PACSTAT & . 117 \\
\hline PACSTAT & . $\quad 118$ \\
\hline PACSTAT & . 119 \\
\hline PACSTAT & . 120 \\
\hline PACSTAT & . 121 \\
\hline PACSTAT & . 122 \\
\hline PACSTAT & . 123 \\
\hline
\end{tabular}




\begin{tabular}{|c|c|c|}
\hline IF ((NCONST.GT. MAXCNT).OR. (NCONST.LT. & PACSTAT & 124 \\
\hline IRITE (IOUT, 540) NCONST, MAXCNT & PACSTAT & . 125 \\
\hline CD TO 246 & PACSTAT & . 128 \\
\hline ENDIF & PACSTAT & . 127 \\
\hline c & PACSTAT & . 128 \\
\hline COWPUTE WAXIMU NUMBER OF SAMPLES WITH CHOSEN & PACSTAT & . 129 \\
\hline NUMBER OF INPUT AND SOLUTION VARIABLES. & PACSTAT & . 130 \\
\hline c & PACSTAT & . 131 \\
\hline WAXSAM = ISIZE/NYAR & PACSTAT & . 132 \\
\hline$I=$ ISIZE & PACSTAT & 133 \\
\hline IF (NSDLN , GT, B) I=ISIZE/NSOLN & PACSTAT & . 134 \\
\hline IF (I .LT. MAXSAM) WAXSAM=I & PACSTAT & 136 \\
\hline IF (MAXSAM,GT, WAXNS) MAXSAM=WAXNS & PACSTAT & . 136 \\
\hline c & PACSTAT & 137 \\
\hline DPEN DUTPUT ECHD PRINT FILE (OPTIONAL) & PACSTAT & . 138 \\
\hline C & PACSTAT & . 139 \\
\hline IERR $=0$ & PACSTAT & .140 \\
\hline WRITE (IOUT, 55B) TTLE & PACSTAT & . 141 \\
\hline IF (MDOEVAX) GO TO 65 & ECHD & . 11 \\
\hline WRITE (IOUT, 560) & PACSTAT & . 142 \\
\hline 65 CONTINUE & ECHO & 12 \\
\hline WRITE (IOUT, 585) & ECHO & 13 \\
\hline READ $(I N, 570)(I N F(I), I=1, N M L E N)$ & PACSTAT & 143 \\
\hline CAШ ASGVAX (NLLEN, INF, IFLAG) & ECHO & 14 \\
\hline MRITE (IECHD, 57g) (INF (I), I=1,NMLEN) & ECHD & 15 \\
\hline IF (INF(1), NE. 'NONE') THEN & PACSTAT & . 144 \\
\hline$I=1$ & PACSTAT & . 145 \\
\hline C - PRIME - CALL ASG (I, IERR, IOAT, NLLEN, INF) & PACSTAT & . 148 \\
\hline IF (IERR.OT.D) GO TO 248 & PACSTAT & . 147 \\
\hline WRITE (IOAT, 586) PRGI, VERNMI & BCSFIX & 7 \\
\hline CALL DCLAIN (IDAT) & BCSFIX & 8 \\
\hline 日SE & PACSTAT & 148 \\
\hline$I D A T=\sigma$ & PACSTAT & 149 \\
\hline ENDIF & PACSTAT & . 150 \\
\hline c & PACSTAT & 151 \\
\hline OPEN OUTPUT BINARY FILE (OPTIONAL) & PACSTAT & .162 \\
\hline c & PACSTAT & . 153 \\
\hline IF (WODEVAX) G0 TO 75 & ECHO & . 16 \\
\hline WRITE(IOUT, 590) & BCSFIX & . \\
\hline REKD (IN, 570) (INF (I), I=1, NMLEN) & PACSTAT & .155 \\
\hline 75 CONTINUE & ECHO & 17 \\
\hline WRITE (IDUT, 595) & ECHO & 18 \\
\hline WRITE (IECHO ,570) (INF (I), I=1, NMLEN) & ECHO & 19 \\
\hline IF ((INF (1). EQ. 'NONE'). DR. (INF (1). EQ. 'NONE')) CO TO 100 & PACSTAT & 156 \\
\hline$I=1$ & PACSTAT & 157 \\
\hline C - PRIME - CALL ASG (I, IERR, IDATB, NMLEN, INF) & PACSTAT & 158 \\
\hline IF (IERR.NE. O) GO TO 230 & PACSTAT & 159 \\
\hline GO TO 110 & PACSTAT & 166 \\
\hline 160 CONTINUE & PACSTAT & . 161 \\
\hline IDATB $=\varnothing$ & PACSTAT & 162 \\
\hline 110 CONIINUE & PACSTAT & . 163 \\
\hline C & PACSTAT & . 184 \\
\hline SET UNIQUE RUN IDENTIFIER & PACSTAT & . 185 \\
\hline $\mathrm{C}$ & PACSTAT & . 186 \\
\hline
\end{tabular}




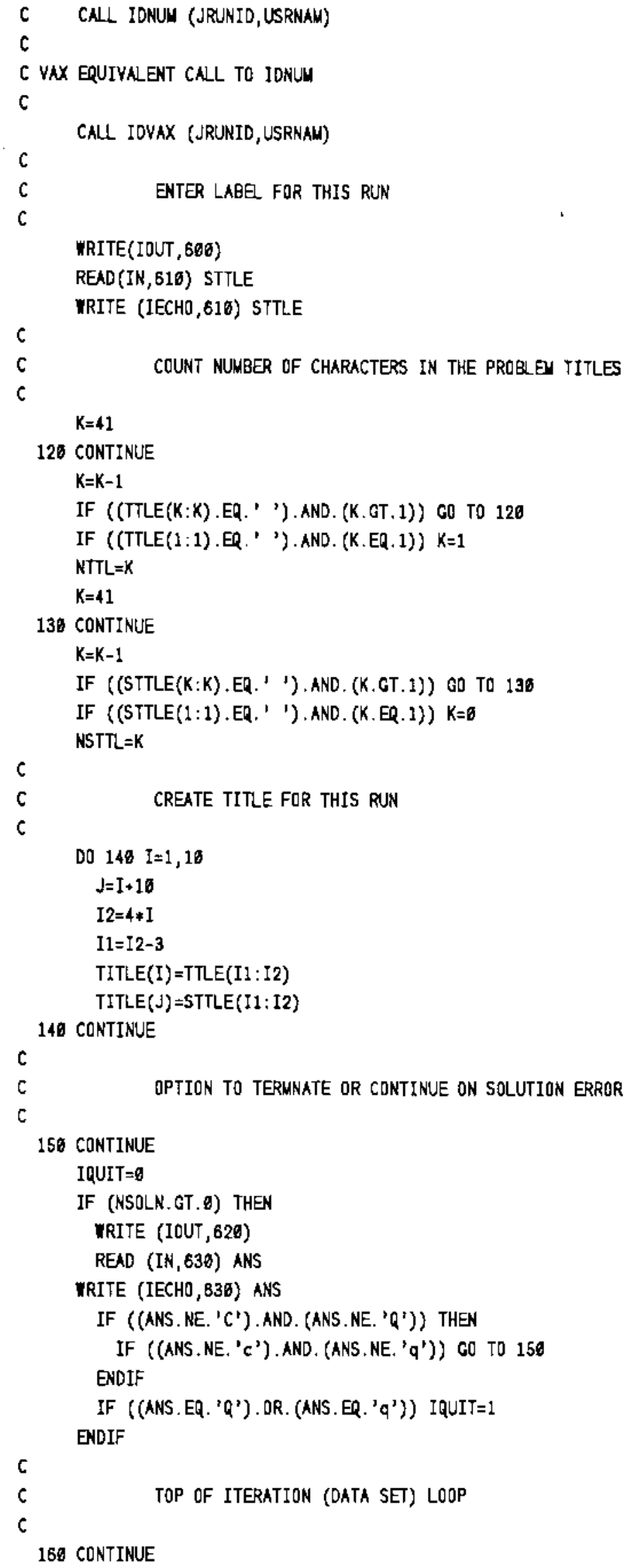




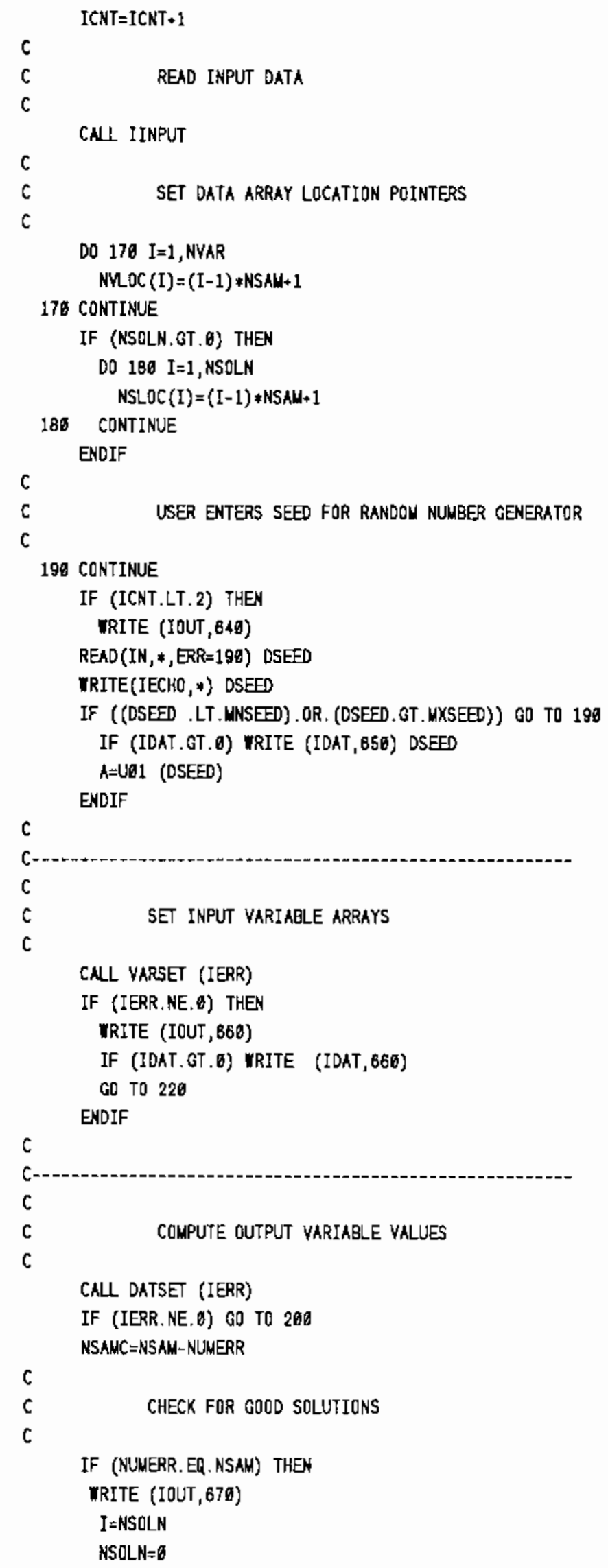


CNL STATOT

PACSTAT . 278

NSOLN=I

CO TO 228

ENDIF

PACSTAT . 279

PACSTAT . 286

PACSTAT . 281

PACSTAT , 282

RE-ORDER AROUND ANY BAD SOLUTIONS WHICH MERE FOUND

NSAMC=NSAM

IF (NUWERR.GT.6) CNLL REDRDR

GO TO 210

C

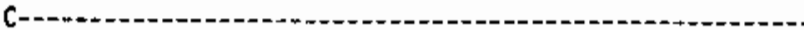

c

$c$

SALVAGE GOOD DATA ON ERROR STOP

260 CONTINUE

$A=F L O A T$ (NPRT) $/ F L O A T$ (NSAM)

NSAMC=NSAM

WRITE (IOUT, 680) INDEX

IF (IDAT,GT.0) WRITE (IDAT, 68ס) INDEX

IF (NSAM.LT.1) GO TO 220

WRITE (IUUT, 898)

IF (IDAT, GT,, ) WRITE (IDAT, 698)

NPRT $=I N T(F L O A T(N S A M) * A)$

IF (NPRT.LE.b) NPRT=1

218 CONTINUE

C

C

C

IF (ICNT.EQ.1) THEN

IHEADR $=1$

$\mathrm{FL}=$ 'STOCAST '

IF (IDATE.GT. $)$ ) CNL WRSTOC (IOATB, IHEADR)

ENDIF

C

C

c

IHEADR $=\varnothing$

IF (IDATB.GT.6) CALL WRSTOC (IDATB, IHEADR)

c

c

C

c

CNע STATOT

C

C

c

c

c

220 CONTINUE

WRITE (IOUT, 790) IQQB
PACSTAT . 283

PACSTAT - 284

PACSTAT , 285

PACSTAT . 288

PACSTAT - 287

PACSTAT . 288

PACSTAT . 289

PACSTAT . 290

PACSTAT , 291

PACSTAT . 292

PACSTAT - 293

PACSTAT . 294

PACSTAT . 295

BCSFIX . 19

BCSFIX . 20

PACSTAT . 298

BCSFIX . 21

SCSFIX . 22

PACSTAT , 301

PACSTAT . 302

PACSTAT . 393

PACSTAT . 304

PACSTAT - 385

PACSTAT . 306

PACSTAT . 367

PACSTAT - 368

PACSTAT , 309

PACSTAT . 310

PACSTAT . 311

PACSTAT , 312

PACSTAT - 313

PACSTAT , 314

PACSTAT , 315

PACSTAT - 316

PACSTAT 317

PACSTAT - 318

PACSTAT . 319

PACSTAT - 328

PACSTAT - 321

PACSTAT . 322

PACSTAT . 323

PACSTAT . 324

PACSTAT . 325

PACSTAT . 326

PACSTAT . 327

PACSTAT . 328

PACSTAT - 329

PACSTAT . 330

BCSFIX , 23 


\begin{tabular}{|c|c|c|}
\hline READ (IN, 636) ANS & BCSFIX & 24 \\
\hline TRITE (IECHD, 630) ANS & ECHO & 23 \\
\hline IF ((ANS. NE. 'Y') . AND. (ANS. NE. 'N')) THEN & PACSTAT & . 333 \\
\hline IF ((ANS. NE. 'y'). AND. (ANS. NE. ' $n '))$ GO TO 228 & PACSTAT & . 334 \\
\hline ENDIF & PACSTAT & . 335 \\
\hline IF ((ANS.EQ. 'Y') ,OR. (ANS.EQ. 'Y')) GO TO 160 & PACSTAT & . 336 \\
\hline 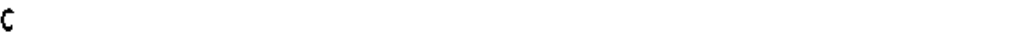 & PACSTAT & .337 \\
\hline EKD OF PROGRAM & PACSTAT & . 338 \\
\hline C & PACSTAT & . 339 \\
\hline IF (JONCE.GT. O) CALL DONEPL & PACSTAT & . $\mathbf{3 4 0}$ \\
\hline IF (IDATB.GT. & PACSTAT & . 341 \\
\hline 230 CONTINUE & PACSTAT & . 342 \\
\hline IF (IDAT.GT.g) CNLL RELEAS (IDAT) & PACSTAT & . 343 \\
\hline 240 CONTINUE & PACSTAT & . 344 \\
\hline STOP & PACSTAT & . 345 \\
\hline $\mathrm{C}$ & PACSTAT & . 346 \\
\hline 500 FORUAT $\left(/ 1 X_{1}{ }^{\prime} * *\right.$ MONTE CARLO SIMUATION $\left.* *^{\prime} / 5 X, ', A 8,1 X, F 4.1,{ }^{\prime} '\right)$ & PACSTAT & . 347 \\
\hline 510 FORMAT $\left(5 X,{ }^{\prime}(', A 8,1 X, F 4,1, ') '\right)$ & PACSTAT & . $\mathbf{3 4 8}$ \\
\hline 520 FORMAT (/1X, 'ERROR, THE NLWBER OF INPUT VARIABLES $='$ 'I5,' EXCEEDS & PACSTAT & . 349 \\
\hline + THE'/1X, 'PROGRAN LIMIT DF ',I3,', DR IS LESS THAN ONE.'/1X, 'PROGR & PACSTAT & . 350 \\
\hline$\rightarrow$ AN STQP. ' & PACSTAT & . 351 \\
\hline 530 FORWAT (/1X, 'ERROR, THE NUMBER DF AVAILABLE SDLUTIONS =',I5,' EXC & PACSTAT & . 352 \\
\hline +EEDS PROGRAM LIMIT OF ',I3,'.'/1X, 'PROGRAM STOP.' ') & PACSTAT & $\cdot 353$ \\
\hline 546 FORWUT (/1X, 'ERROR, NUMEER OF CONSTANTS =',I5,' EXCEEDS PROGRAM L & PACSTAT & . 354 \\
\hline +IHIT OF ',I3,'.'/1X, 'PROGRAM STOP.') & PACSTAT & . 355 \\
\hline 659 FORNAT $(/ 1 X, 14 \sigma)$ & PACSTAT & . 356 \\
\hline 566 FORMAT (/1X, 'ENTER THE NAME FOR THE DIAGNLSTIC DATA ECHO FILE. ' $/ 1 X$ & PACSTAT & . 357 \\
\hline$+_{1}^{\prime}($ 'NONE'" TO ONIT)') & PACSTAT & . 358 \\
\hline 565 FORMAT (/1X, 'ENTER THE NAME FOR THE DATA ECHD FILE, ' $/ 1 \mathrm{X}$ & ECHD & 24 \\
\hline${ }^{+}, '($ OR A CARRIAGE RETURN TO DEFAULT TO DATA FILE PACSTAT.ECH)') & ECHD & 25 \\
\hline 570 FDRNAT (29A4) & PACSTAT & . 359 \\
\hline 680 FORMAT $\left(1 H 1,1 X,{ }^{\prime}\left({ }^{\prime}, A 8,1 X, F 5,2,{ }^{\prime}\right)^{\prime}\right)$ & BCSFIX & 25 \\
\hline 596 FORMAT (/1X, 'ENTER THE NAME FOR THE BINARY OUTPUT DATA FILE. '/1X,' & BCSFIX & 26 \\
\hline -("NONE' 'TO OUIT)') & BCSFIX & 27 \\
\hline 595 FORMAT(/1X, 'ERTER THE NAME FOR THE BINARY OUTPUT DATA FILE,' $/ 1 X_{1}$ ' & BCSFIX & 28 \\
\hline -(DR A CARRIAGE RETURN TD DEFAUT TD DATA FILE PACSTAT. GIN)') & ECSFIX & 29 \\
\hline 860 FORHAT (/1X,'ENTER LABEL FOR THIS RUN (MAX 46 CHARACTERS)') & ECSFIX & 30 \\
\hline 619 FQRUAT (A4E) & BCSFIX & 31 \\
\hline 620 FORMAT (/1X, 'WOUD YOU LIKE TO CONTINUE OR QUIT IF AN ERROR'/1X,'I & BCSFIX & 32 \\
\hline +S ENCOUNTERED IN THE SOLUTION ROUTINE (C/Q)? ') & BCSFIX & 33 \\
\hline 830 FORMAT (A1) & BCSFIX & 34 \\
\hline 640 FORMAT (/1X,' ENTER AN INITIAL SEED NUMBER FOR THE RANDOM NUMBER GE & BCSFIX & 35 \\
\hline -NERATOR.' $\left./ 1 X,{ }^{\prime}(A \text { REN NUMEER EETWEEN } 1.6 \text { ANO }(2 * 31)-2 .)^{\prime}\right)$ & BCSFIX & 36 \\
\hline 65ઇ FORMAT (/5X,'INITIAL RANDOM SEED NUMBER IS ',1PDI4.7) & BCSFIX & 37 \\
\hline $68 \sigma$ FORMAT $\left(/ 1 \mathrm{X},{ }^{\prime} * *\right.$ ERROR COMPUTING INPUT DISTRIBUTION DATA. THIS ITE & BCSFIX & 38 \\
\hline •RATION WAS'/4X, 'ABORTED DUE TO AN INABILITY TO CONPUTE AND/OR READ & BCSFIX & . \\
\hline + THE DESIRED'/4X,'RANDOM SAUPLES. ') & BCSFIX & 49 \\
\hline 670 FORNAT $\left(/ 1 X_{1}{ }^{\prime} * * *\right.$ ERROR, ND SUCCESSFUL SAMPLES WERE COMPUTED.' $/ 5 X_{1}^{\prime}$ & BCSFIX & 41 \\
\hline •THE INPUT VARIABLE STATISTICS WILL BE EXAMINED, '/5X, 'BUT THE DUTPU & BCSFIX & $\cdot$ \\
\hline +T DATA WILL BE IGNORED, AND THE BINARY'/5X, 'OUTPUT FILE RECORD WIL & BCSFIX & 43 \\
\hline +L NDT BE WRITTEN. ') & BCSFIX & 44 \\
\hline 689 FORMAT $(/ / / 5 X, '---$ ERROR ABORT, SALPLE NUNBER ',I5) & BCSFIX & 45 \\
\hline 696 FORMT (5X,'--- PROCEDURE WILL CONTINUE WITH REDUCED SAMPLE SPACE. & BCSFIX & 46 \\
\hline$+')$ & BCSFIX & 47 \\
\hline
\end{tabular}




\begin{tabular}{|c|c|c|c|}
\hline 700 & FORWAT $(/ 1 X$, 'CREATE ANOTHER DATA SET $(Y / N)$ ? ',A1) & BCSFIX & 48 \\
\hline 10606 & FDRMAT (ABG) & BCSFIX & 48 \\
\hline 10601 & FORUAT (2BA4) & BCSFIX & 50 \\
\hline 11096 & FORNAT $(5 X, A 80,10 X, I 5)$ & BCSFIX & 5. \\
\hline & EKD & PACSTAT & 380 \\
\hline & SUEROUTINE PLINIT (XL2, YL2, IRUN) & PLINIT & 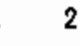 \\
\hline $\mathbf{C}$ & & PLINIT & \\
\hline$c$ & ROUTINE FOR CHOSING INTERACTIVE/NON-INTERACTIVE PLOTTING, & PLINIT & \\
\hline c & SETTING PAGE SIZE, AND INITIALIZING PLOTTING PACKAGE. & PLINIT & \\
\hline c & X2, YL2 = PAGE SIZE (INCHES, INITIALIZED BY CAШING ROLTINE) & PLINIT & 6 \\
\hline c & IRUN $=1 / \sigma$ INTERACTIVE/NON-INTERACIIVE PLDTTING & PLINIT & 7 \\
\hline C & & PLINIT & 5 \\
\hline & COLMON /INOUT/ & INOUT & 2 \\
\hline & - IN, IOUT, IQB, IECHO & ECHO & 86 \\
\hline & CHARACTER $\# 1$ & INOUT & \\
\hline & $+\quad I Q B$ & INOUT & 5 \\
\hline c & & INDUT & 6 \\
\hline$c$ & & INDUT & 7 \\
\hline C & & PLINIT & 10 \\
\hline & CHARACTER $\# 1$ & PLINIT & 11 \\
\hline & $\rightarrow \quad$ ANS & PLINIT & 12 \\
\hline$c$ & & PLINIT & 13 \\
\hline c & INTER/NON-INTERACTIVE PLOTIING & PLINIT & 14 \\
\hline C & & PLINIT & 15 \\
\hline & IRUN=1 & PLINIT & 16 \\
\hline 100 & CONTINUE & PLINIT & 17 \\
\hline & URITE (IDUT, 600) & PLINIT & IB \\
\hline & READ (IN, $*$,ERR=100) KDEV & PLINIT & 19 \\
\hline & IRITE (IECHD, *) XDEY & ECHO & 78 \\
\hline & IF ((KDEY.LT.6).OR. (KDEY.GT.5)) GO TO 106 & PLINIT & 28 \\
\hline$c$ & & PLINIT & 21 \\
\hline c & INIT PAGE SIZE (XL2,YL2). ALLON USER MDDIFICATION. & PLINIT & 22 \\
\hline C & & PLINIT & 23 \\
\hline & IF (XL2.LE.6.) $\times 2.2=14.2$ & PLINIT & 24 \\
\hline & IF (YL2.LE. 0.) YL2=10.1 & PLINIT & 25 \\
\hline 110 & CONTINUE & PLINIT & 26 \\
\hline & IRITE (IOUT, 510) XL2, Y22, IGB & PLINIT & 27 \\
\hline & READ (IN, 526) ANS & PLINIT & 28 \\
\hline & VRITE (IECHO, 520) ANS & ECHO & 74 \\
\hline & IF ((ANS.NE. 'Y'), AND. (ANS. NE. 'N')) THEN & PLINIT & 29 \\
\hline & IF ((ANS. NE, 'y') AND. (ANS.NE, ' $n '))$ GD TO 118 & PLINIT & 3 \\
\hline & ENDIF & PLINIT & 31 \\
\hline & IF ((ANS.EQ, 'Y'), OR. (ANS.EQ. ' $\left.y^{\prime}\right)$ ) THEN & PLINIT & 32 \\
\hline 126 & CONTINUE & PLINIT & 33 \\
\hline & WRITE (IOUT, 530) & PLINIT & 34 \\
\hline & $\operatorname{READ}(\mathrm{IN}, *, E R R=12 \theta) \times L 2, Y L 2$ & PLINIT & 35 \\
\hline & WRITE (IECHO,$*) \times \mathrm{L} 2, \mathrm{YL2}$ & ECHO & 8 \\
\hline & IF (XL2.LT.B.) XL2=8. & PLINIT & 36 \\
\hline & IF (YL2.LT.6.) YL2=8. & PLINIT & 37 \\
\hline & ENDIF & PLINIT & 38 \\
\hline$c$ & & PLINIT & 39 \\
\hline$c$ & GRAPHICS INITIALIZATIONS & PLINIT & 46 \\
\hline C & & PLINIT & 41 \\
\hline & $K D E V=K D E V+1$ & PLINIT & \\
\hline
\end{tabular}


C

130 CONTINUE

CALL COMPRS

IRUN $=0$

GD TO 496

C

146 CONTINUE

CALL TK4ס14 $(120,6)$

GO TO 496

C

156 CONTINUE

CALL TK4625

GO TO 498

C

180 CDNTINUE

CNLL TK4654 $(126,6)$

GO TO 496

c

179 CONTINUE

CALL REGIS $(1,6)$

GO TO 490

C

180 CONTINUE

CNLL SLNRVT (120)

GO TO 496

c

c

$c$

$c$

490 CONTINUE RETURN

560 FORMAT (/1X, '-- DEVICE WENU --'/2X, ' $\theta$ ) COMPRESSED GRAPHICS', T28, -'1) TEXTRONIX 4014',T54, '2) TEXTRONIX 4625'/2X, '3) TEXTRONIX 4854' $\bullet, T 28$, '4) VT-106' 'T54, 'S) SELANAR'

$+/ / 1 X$, 'ENTER THE NUMBER CORRESPONDING TO THE DESIRED TERMINAL. ')

516 FORMAT (/1X, 'CURRENT PAGE SIZE IS ',F8.1,' BY ',FB.1/1X, 'WOLLD YOU

+ LIKE TO MaOIFY PAGE SIZE $(Y / N)$ ? ', A $)$

520 FORWAT (A1)

530 FORMAT (1X, 'ENTER NEN $X$ AND $Y$ PAGE SIZE DIMENSIONS (INCHES). ') END

SUBROUTINE PPLT

c

C

$\mathrm{C}$

$$
c
$$

\section{c}

$$
c
$$

c

\section{cons}

$$
c
$$

THIS ROUTINE GENERATES THE OUTPUT DATA PLOT FOR MONTE CARLO SILLATIONS ORIVEN BY PACSTAT. THE DEFAULT PLDT IS CARTESIAN ON THE VERTICAL AXIS, AND LOGARITHIC ON THE HORIZONTAL AXIS.

THE VERTICAL AXIS REPRESENTS THE CONFIDENCE LEVE, USING ARRAY CONF FOR Y LOCATIONS. THIS DATA IS NLLOWED TO VARY FROM $0 . \emptyset$ TD 1. D.

THE HORIZONTAL AXIS PLOTS THE DATA VNLUES IN ARRAY RPLT. THE DEFAULT $X$ AXIS IS LOGARITHMIC, BUT THIS MAY BE CHANGED IF THE DATA MINIMUM IS BE OW ZERI, DR IF THIS DATA COVERS LESS THAN TWO FUL

\begin{tabular}{|c|c|}
\hline DINIT & 43 \\
\hline PLINIT & 44 \\
\hline PLINIT & 48 \\
\hline PLINIT & 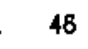 \\
\hline PLINIT & 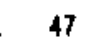 \\
\hline PLINIT & 48 \\
\hline PLINIT & 48 \\
\hline PLINIT & 50 \\
\hline PLINIT & \\
\hline PLINIT & \\
\hline PLINIT & \\
\hline PLINIT & \\
\hline PLINIT & \\
\hline PLINIT & \\
\hline PLINIT & 57 \\
\hline PLINIT & 58 \\
\hline PLINIT & 69 \\
\hline PLINIT & 80 \\
\hline PLINIT & 81 \\
\hline PLINIT & 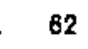 \\
\hline PLINIT & 83 \\
\hline PLINIT & 84 \\
\hline PLINIT & 66 \\
\hline PLINIT & 68 \\
\hline PLINIT & 67 \\
\hline PLINIT & 68 \\
\hline PLINIT & 69 \\
\hline PLINIT & 76 \\
\hline PLINIT & 71 \\
\hline PLINIT & 72 \\
\hline PLINIT & 73 \\
\hline PLINIT & 14 \\
\hline PLINIT & 10 \\
\hline PLINIT & 76 \\
\hline PLINIT & 77 \\
\hline PLINIT & 78 \\
\hline PLINIT & 78 \\
\hline PLINIT & 80 \\
\hline PLINIT & 81 \\
\hline PLINIT & 82 \\
\hline PLINIT & 83 \\
\hline PPLT & \\
\hline PPLT & \\
\hline PPLT & \\
\hline PPLT & \\
\hline PPLT & \\
\hline PPLT & \\
\hline PPLT & \\
\hline PPLT & \\
\hline PPLT & 1 \\
\hline PPLT & 1 \\
\hline PPLT & 12 \\
\hline PPLT & 1 \\
\hline PPLT & 14 \\
\hline
\end{tabular}
DRDERS DF WAGNITUDE. THE DATA VALUES IN ARRAY RPLT NUST BE SORTED IN ASCENDING ORDER PRIOR TO CALLING THIS ROUTINE. 
PARAKETER ISIZE $=50806$ ! MAX DATA SET SIZE

PARUMETER MAXCNT $=10 \quad !$ MAX NUMBER OF CONSTANTS

PARAMETER MAXDST $=108 \quad !$ MAX NUMBER OF INPUT DATA PDINTS

PARAMETER MAXLVS $=168 \quad !$ MAX LEVES FOR STRATIFIED SAMPLING

PARRMETER MAXNS $=16060 !$ MAX NUMBER OF SAMPLES

PARANETER MAXSOL $=16$ ! MAX NUMBER OF SOLUTIONS

PARANETER MAXYAR $=25$ ! MAX NUMBER OF INPUT VARIABLES

PARNAETER NMLEY $=16$ ! FILE NAME LENGTH

PARAHETER NTYPE $=11$ ! NUMGER OF OISTRIBUTION TYPES

$c$

c

COWWN /PQLK2/

$+\quad$ NSTTL,

+ TTLE

CHARACTER *46

c

- STTLE,

c

CONMON /PBEK3/

- CDIMEN(MAXCNT),

- VDimen (MAXYAR), CHARACTER $* 12$

- CDIMEN.

+ vabe日

CHARACTER $* 32$

- Pla昍

C

c

COMMON /PELK5/

- ICNT,

c

c

COMNON /PBLXB/

+ CONF (MAXNS),

c

c

CONKON /PBLKG/

- IRUN,

- $\mathrm{YL}_{1}$

c

c

COMNDN /PBLK17/

- ICCD,

- IUPDAT,

+ NSAM,

c

c

PARAMETER MAXSRC $=15$

COMMON / IOCOM/

- JRUNID,

- NRUNid (MAXSRC),

- PRGt(MAXSRC),

- USRNLT (MAXSRC).
NTTL,

STRE,

TTLE

CLABE (MAXCNT),

VLABE (MAXVAR)

CLABE,

CLABE,

IDAT,

RPLT (MAXNS)

$\mathrm{XL}$,

YL2

$X\llcorner 2$,

VDINEN,

PLABE (MAXSDL),

INDEX

IQUIT,

MAXSAM,

NSAMC

$\mathrm{FL}$,

NSRC,

TITLE(20), VERNUM

\begin{tabular}{|c|c|}
\hline PPLT & 15 \\
\hline PPARA & 2 \\
\hline PPARA & 3 \\
\hline PPARA & 4 \\
\hline PPARA & 5 \\
\hline PPARA & 0 \\
\hline PPARA & 1 \\
\hline PPARA & 0 \\
\hline PPARA & $\sigma$ \\
\hline PPARA & 10 \\
\hline PPARA & 11 \\
\hline PPARA & 12 \\
\hline PBLK2 & 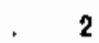 \\
\hline PBLK2 & . \\
\hline PBLK2 & 4 \\
\hline PBLK2 & 5 \\
\hline PBLK2 & B \\
\hline PBLK2 & 7 \\
\hline PBLK2 & 8 \\
\hline PBLKK & 2 \\
\hline PBLK3 & 3 \\
\hline PBLK3 & 4 \\
\hline PBLK3 & 5 \\
\hline PBLK3 & 8 \\
\hline PBLK3 & 7 \\
\hline PBLKK3 & B \\
\hline PBLKK3 & 9 \\
\hline PBLK3 & 16 \\
\hline PBLK3 & 11 \\
\hline PBLK5 & 2 \\
\hline PBLKS & 3 \\
\hline PBLK5 & 4 \\
\hline PQLKS & $\delta$ \\
\hline PQLKKB & 2 \\
\hline PQKB & 3 \\
\hline PBALKB & 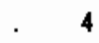 \\
\hline PBLKKB & 5 \\
\hline PQLKg & 2 \\
\hline PBLLK9 & 3 \\
\hline PBLKG & 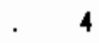 \\
\hline PBLKg & 5 \\
\hline PBLKG & 6 \\
\hline PBLLK17 & 2 \\
\hline PBLK17 & 3 \\
\hline PBLK17 & 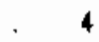 \\
\hline PBLK17 & - \\
\hline PBLK17 & 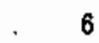 \\
\hline PBLK17 & 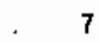 \\
\hline IOCOM & 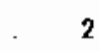 \\
\hline IOCOW & 3 \\
\hline IDCOM & 4 \\
\hline $\mathrm{OCOM}$ & 5 \\
\hline COM & 6 \\
\hline OM & \\
\hline
\end{tabular}




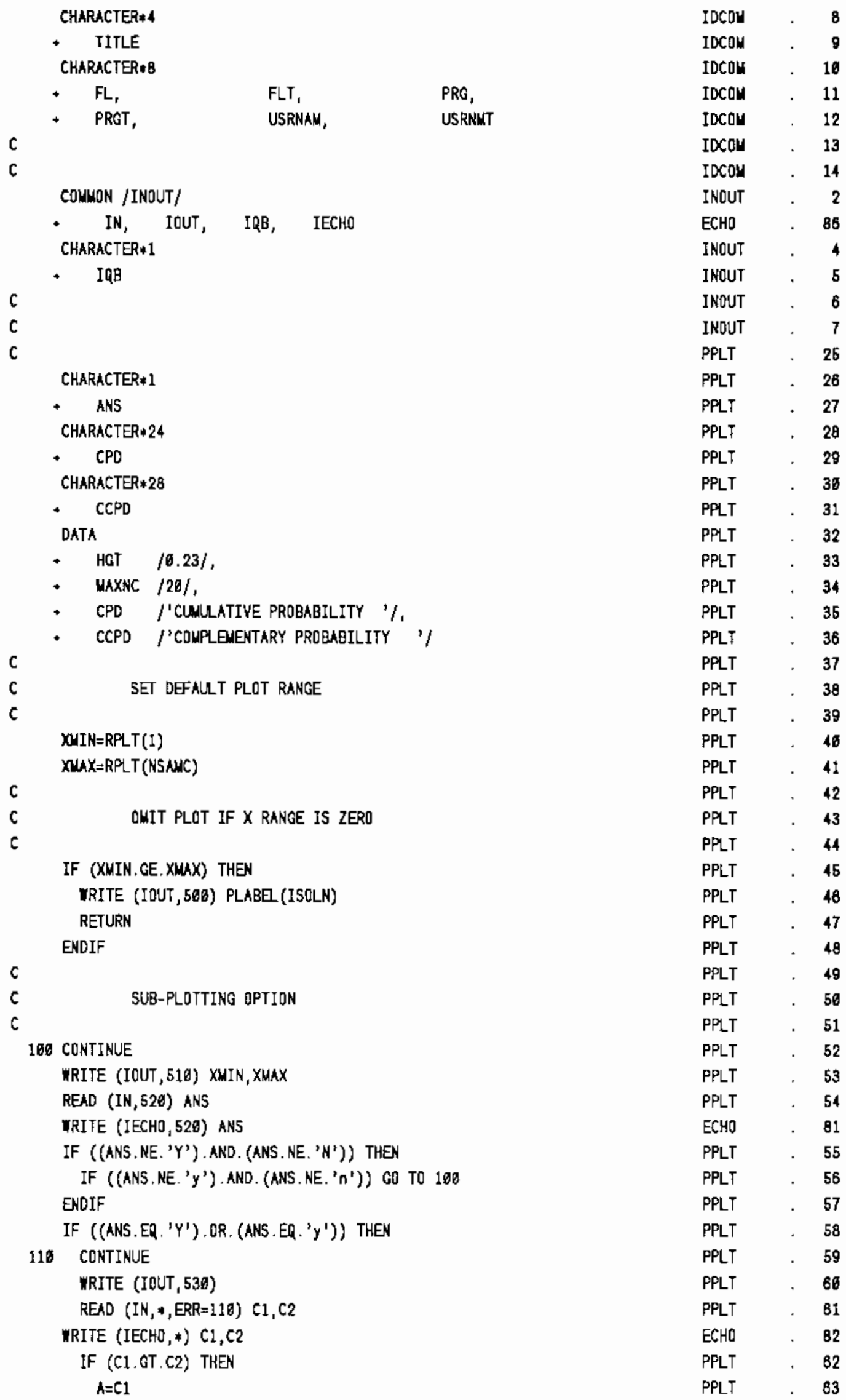




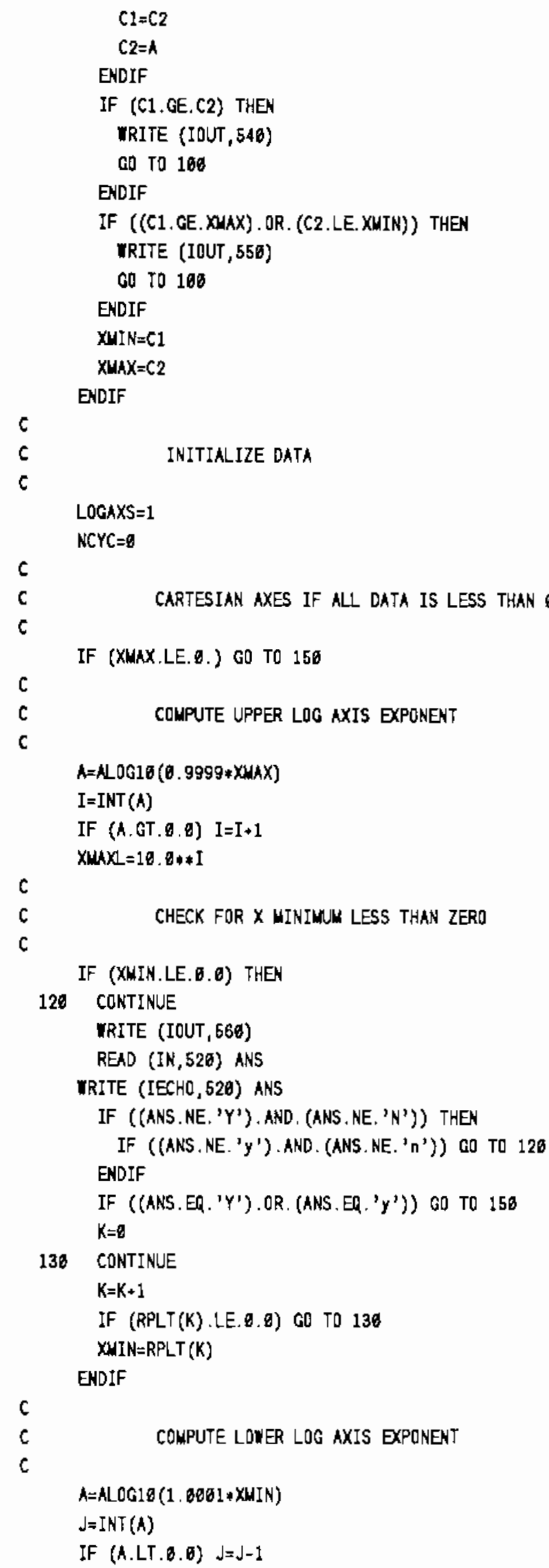




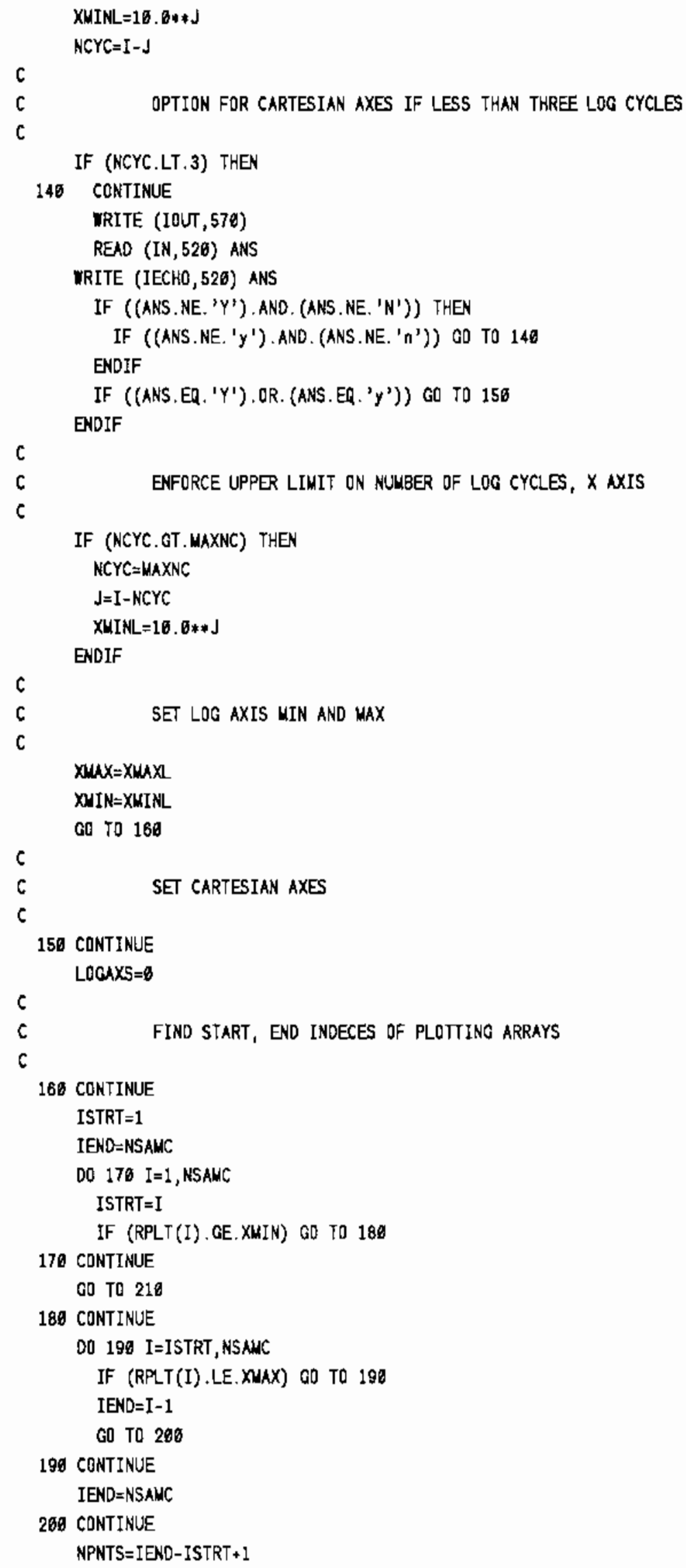




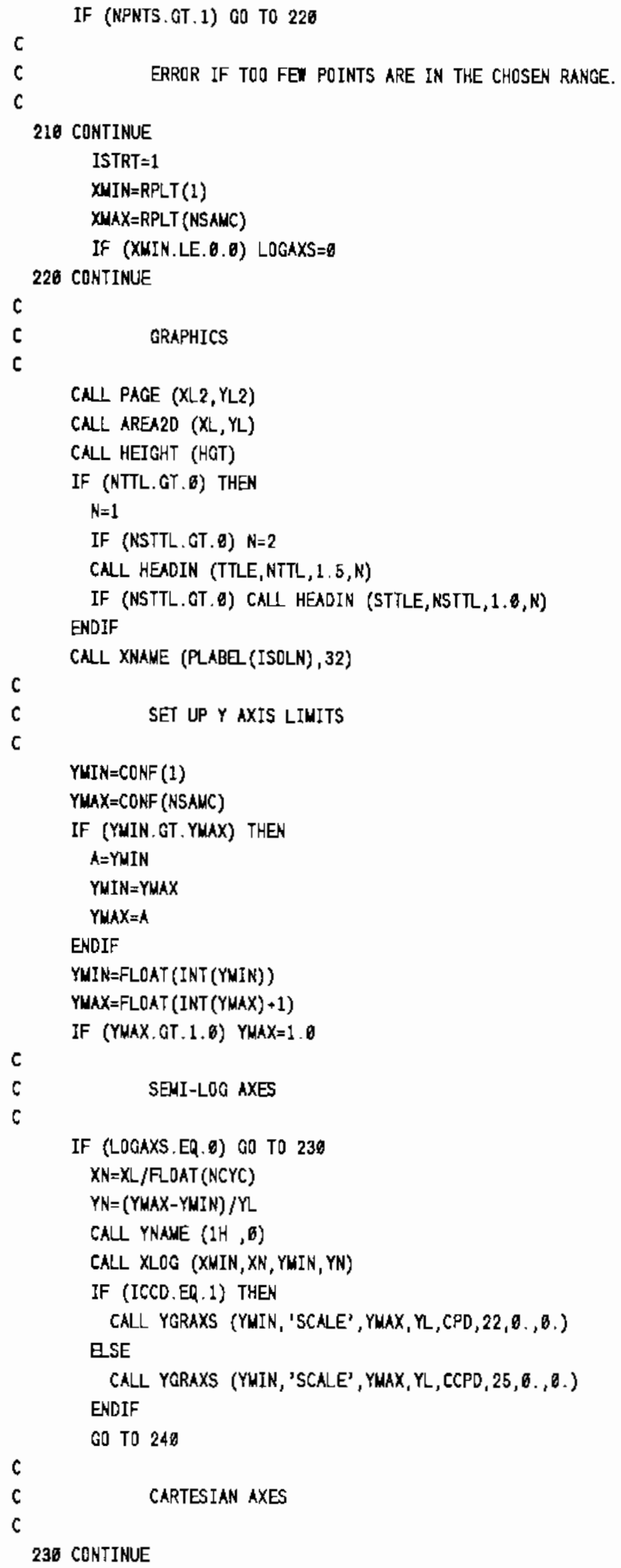

PPLT . 170

PPLT , 171

PPLT . 172

PPLT $\quad .173$

PPLT . 174

PPLT $\quad 178$

PPLT $\quad 176$

PPLT . 177

PPLT . 178

PPLT 179

PPLT . 180

PPLT - 181

PPLT . 182

PPLT . 183

PPLT 184

PPLT 185

PPLT . 188

PPLT 187

PPLT 188

PPLT - 189

PPLT . 190

PPLT $\quad$. 191

PPLT - 192

PPLT $\quad 193$

PPLT . 194

PFLT . 195

PPLT . 198

PPLT - 197

PPLT . 198

PPLT $\quad 199$

PPLT . 206

PPLT . 201

PPLT . 262

PPLT . 263

PPLT . 204

PPLT . 295

PPLT . 206

PPLT $\quad . \quad 267$

PPLT . 208

\begin{tabular}{ll} 
PPLT $\quad 209$ \\
\hline
\end{tabular}

PPLT . 210

PPLT . 211

PPLT . 212

PPLT . 213

PPLT . 214

PPLT - 215

PPLT , 218

PPLT $\quad 217$

PPLT - 218

PPLT $\quad 219$

PPLT ， 220

PPLT $\quad 221$

PPLT . 222

PPLT . 223 


\begin{tabular}{|c|c|c|}
\hline IF (ICCD.EQ.1) THEN & PPLT & 224 \\
\hline CALL YNANE (CPD, 22) & PPLT & . 225 \\
\hline ESE & PPLT & . 228 \\
\hline CALL YNAHE $(C C P D, 25)$ & PPLT & . 227 \\
\hline ENDIF & PPLT & . 228 \\
\hline CNI GRAF (XUIN, 'SCALE', XWAX, YMIN, 'SCALE', YMAX) & PPLT & . 229 \\
\hline 246 CONTINUE & PPLT & . 230 \\
\hline C & PPLT & . 231 \\
\hline PLOT DATA & PPLT & . 232 \\
\hline c & PPLT & . 233 \\
\hline$I=1$ & PPLT & . 234 \\
\hline CAШ CURVE (RPLT(ISTRT), CONF (ISTRT), NPNTS, I) & PPLT & . 235 \\
\hline (25) & PPLT & 236 \\
\hline LABE PLOT & PPLT & . 237 \\
\hline C & PPLT & . 238 \\
\hline$X I D=-9.78$ & PPLT & . 239 \\
\hline$Y I D=Y L+0.80$ & PPLT & . 246 \\
\hline CALL INTNO (ICNT,XID, YID) & PPLT & . 241 \\
\hline CALL HEIGHT (6.13) & PPLT & . 242 \\
\hline$X I D=X L-0.40$ & PPLT & . 243 \\
\hline$Y I D=Y L+1.45$ & PPL $T$ & . 244 \\
\hline CAШ IDPLT (JRUNID,XID, YIO) & PPLT & . 245 \\
\hline C & PPLT & 248 \\
\hline END PLOT & PPLT & 247 \\
\hline c & PPLT & . 248 \\
\hline$N=0$ & PPLT & . 249 \\
\hline CAL ENDPL (N) & PPLT & . 250 \\
\hline IF (IRUN.EQ.1) CALL EPUSE & PPLT & . 251 \\
\hline C & PPLT & . 252 \\
\hline RETURN & PPLT & . 253 \\
\hline$c$ & PPLT & . 254 \\
\hline SOS FORUAT $\left(/ / 1 X, x^{\prime} * * *\right.$ ERROR *** MIN ANO WAX VALUES FRDN SOLLTION $/ /, 15$ & PPLT & . 265 \\
\hline$+X, A 32, '$ ARE EQUAL. '15X, 'NO PLOT WIL BE WADE OF THIS OATA.') & PPLT & . 258 \\
\hline 516 FORHAT (/1X, 'DATA MINIMUN = ',1PE11.3/1X, 'DATA MAXIMUM $={ }^{\prime}$, E11.3/ & PPLT & . 257 \\
\hline +1X, 'WOULD YOU LIXE A SUB-PLOT ON THE DATA AXIS (Y/N)?') & PPLT & . 258 \\
\hline 526 FORUAT (AI) & PPLT & . 269 \\
\hline 53§ FORMAT (/1X, 'ENTER THE MIN AND WAX DATA YALUES FOR PLOTTING.') & PPLT & . 280 \\
\hline 546 FORMAT ( $/ 1 X_{1}{ }^{\prime} *$ ERROR, ENTERED XWIN IS LESS THAN ENTERED XMAX.') & PPLT & . 281 \\
\hline 558 FORMAT $\left(/ 1 X,{ }^{\prime} *\right.$ ERROR, ENTERED XNIN AND XMAX ARE OUT OF THE RANGE $O$ & PPLT & . 282 \\
\hline ^F THE DRTA.') & PPLT & . 263 \\
\hline 568 FORMAT (//1X,'THE WINIMUM DATA VALUE IS LESS THAN ZERO.' $/ 1 X_{1}$, 'WOULD & PPLT & 284 \\
\hline + YDU PREFER A CARTESIAN AXIS ON THE DATA PLOT ABCISSA $\left.(Y / N) ?^{\prime}\right)$ & PPLT & . 265 \\
\hline $57 \%$ FORMAT (//1X, 'THERE ARE LESS THAN TYO FULL LOGARITHM CYCLES ON THE & PPLT & . 268 \\
\hline + OUTPUT DATA.' $/ 1 X_{1}$ 'WOULO YQU PREFER A CARTESIAN AXIS ON THE OATA P & PPLT & . 267 \\
\hline +LOT ABCISSA $\left.(Y / N) ?^{\prime}\right)$ & PPLT & . 268 \\
\hline END & PPLT & 269 \\
\hline SUBROUTINE PQSORT ( $N$, IFIN, II, $A$ ) & PQSORT & 2 \\
\hline c & PQSORT & 3 \\
\hline THIS SUBROUTINE IUPLEMENTS THE QUICK SORT (PARTITIONING) & PQSERT & 4 \\
\hline ALGORITHW ON THE CURRENT PARTITION OF THE ARRAY A. AN EXTENSIVE & PQSORT & 5 \\
\hline EFFORT IS MADE TO FIND A GOOD MEDIAN VALUE, AND TO CHECK FOR AШ & PQSORT & 6 \\
\hline INPUT VALUES BEING EQUAL, OR BEING OF ONLY THO YNLUES. THIS & PQSORT & 7 \\
\hline SPEEDS EXECUTION IN CERTAIN CASES WHICH ARE LIKEY TO ARISE IN & PQSORT & 8 \\
\hline SOME SPECIFIC USES OF THIS QUICK SORT ROUTINE. & PQSORT & 9 \\
\hline
\end{tabular}




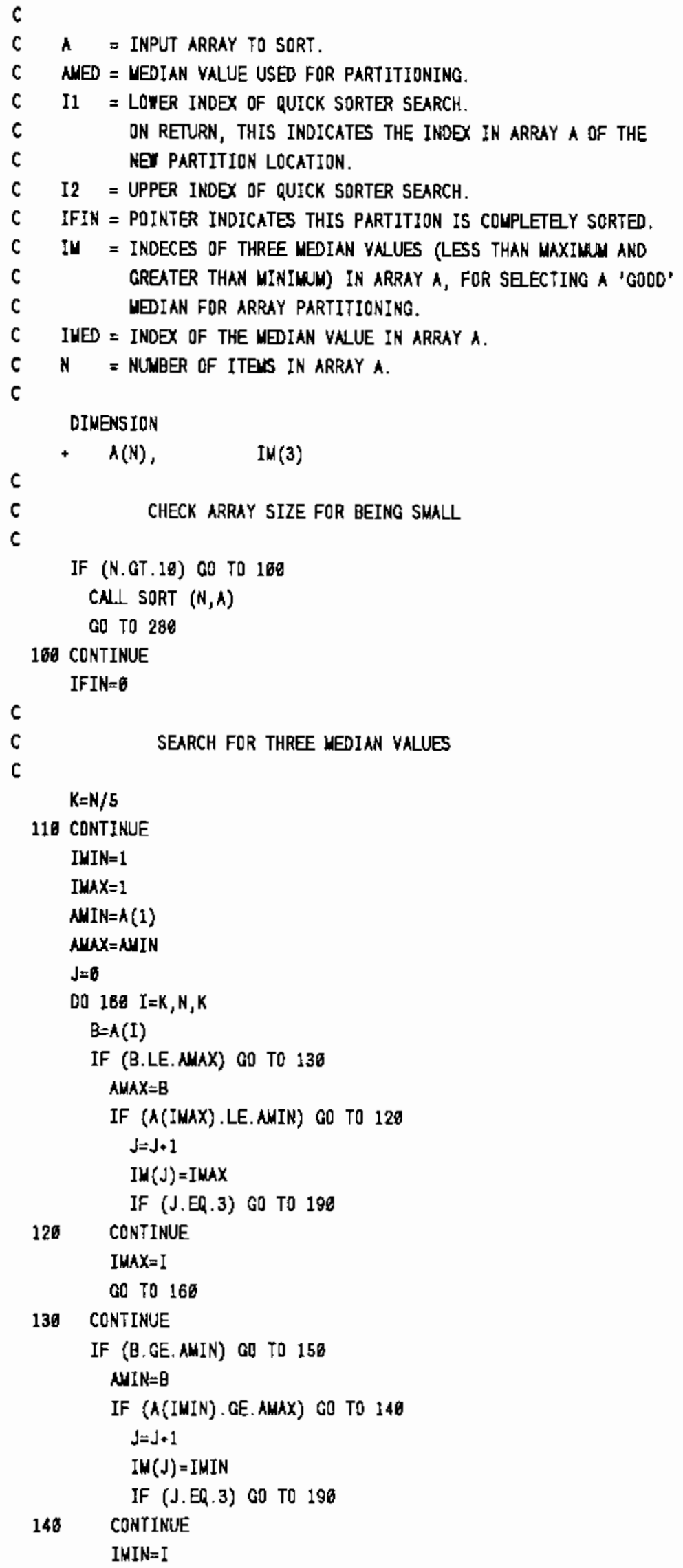

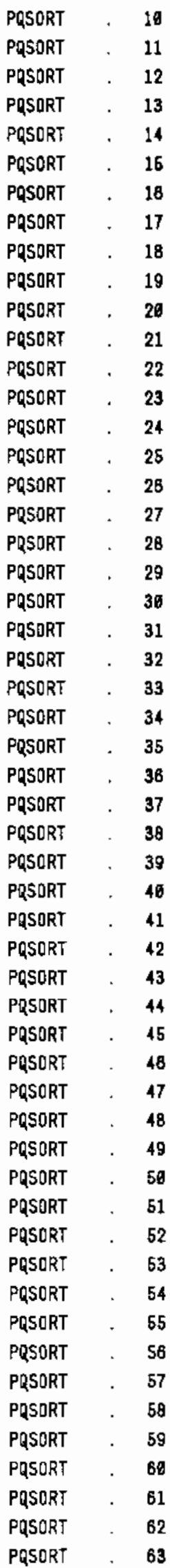




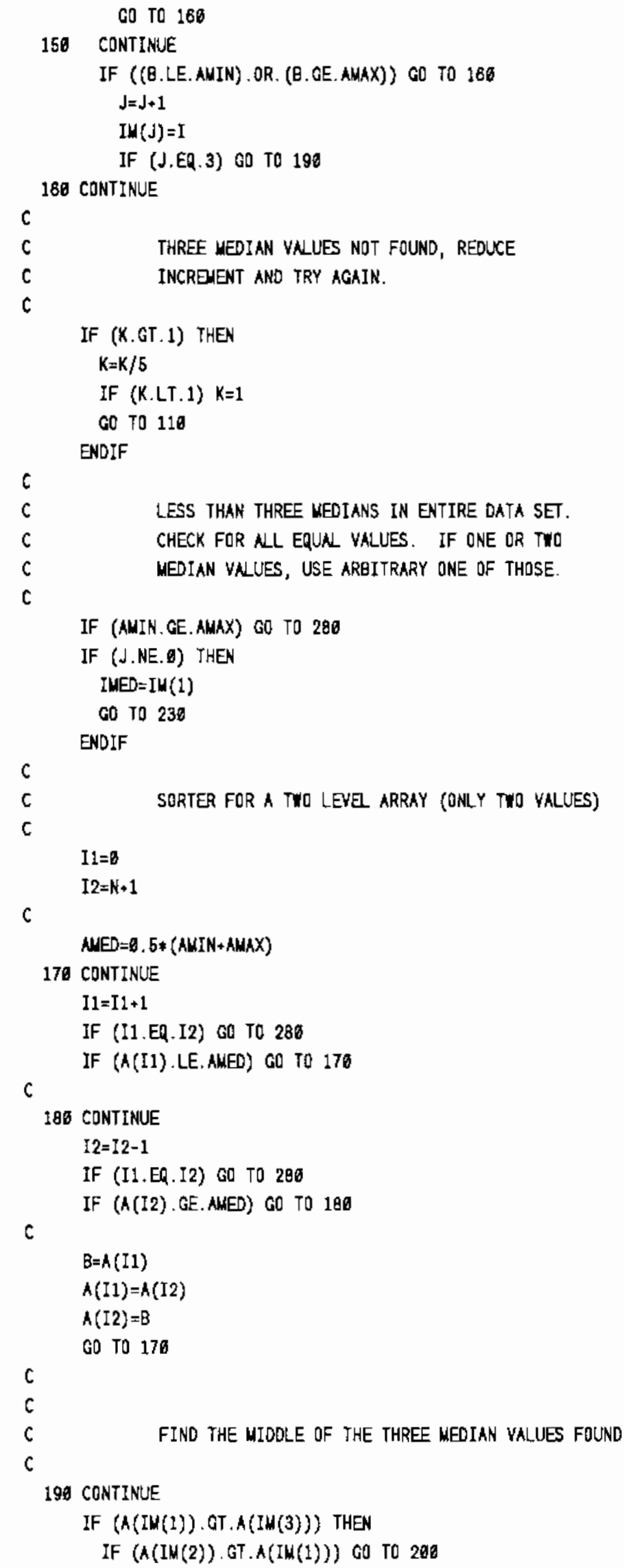

\begin{tabular}{|c|c|}
\hline PQSQRT & 84 \\
\hline PQSORT & 65 \\
\hline PQSORT & 68 \\
\hline PQSORT & 67 \\
\hline PQSORT & 88 \\
\hline PQSORT & 69 \\
\hline PQSORT & 70 \\
\hline PQSDRT & 71 \\
\hline PQSORT & 72 \\
\hline PQSORT & 73 \\
\hline PQSORT & 74 \\
\hline PQSORT & 75 \\
\hline PASORT & 76 \\
\hline PQSORT & $n$ \\
\hline PQSORT & 78 \\
\hline PQSQRT & 79 \\
\hline PQSORT & 86 \\
\hline PQSORT & 81 \\
\hline PQSORT & 82 \\
\hline PQSORT & B3 \\
\hline PQSORT & 84 \\
\hline PQSORT & 85 \\
\hline PQSORT & 88 \\
\hline PQSORT & 87 \\
\hline PQSORT & 88 \\
\hline PQSQRT & 69 \\
\hline PQSORT & 96 \\
\hline PQSDRT & 91 \\
\hline PQSDRT & 92 \\
\hline PQSORT & 93 \\
\hline PQSORT & 94 \\
\hline PQSORT & 95 \\
\hline PQSORT & 98 \\
\hline PQSORT & 97 \\
\hline PQSORT & 98 \\
\hline PQSORT & 99 \\
\hline PQSORT & . 100 \\
\hline PQSORT & . 101 \\
\hline PQSORT & 102 \\
\hline PQSORT & . 103 \\
\hline PQSORT & . 104 \\
\hline PQSURT & . 165 \\
\hline PQSORT & 166 \\
\hline PQSORT & 187 \\
\hline PQSORT & 108 \\
\hline PQSDRT & 189 \\
\hline PQSORT & 110 \\
\hline PQSORT & 111 \\
\hline PQSORT & . 112 \\
\hline PQSORT & . $\quad 113$ \\
\hline PQSORT & 114 \\
\hline PQSORT & 115 \\
\hline PQSORT & 116 \\
\hline PQSORT & 117 \\
\hline
\end{tabular}




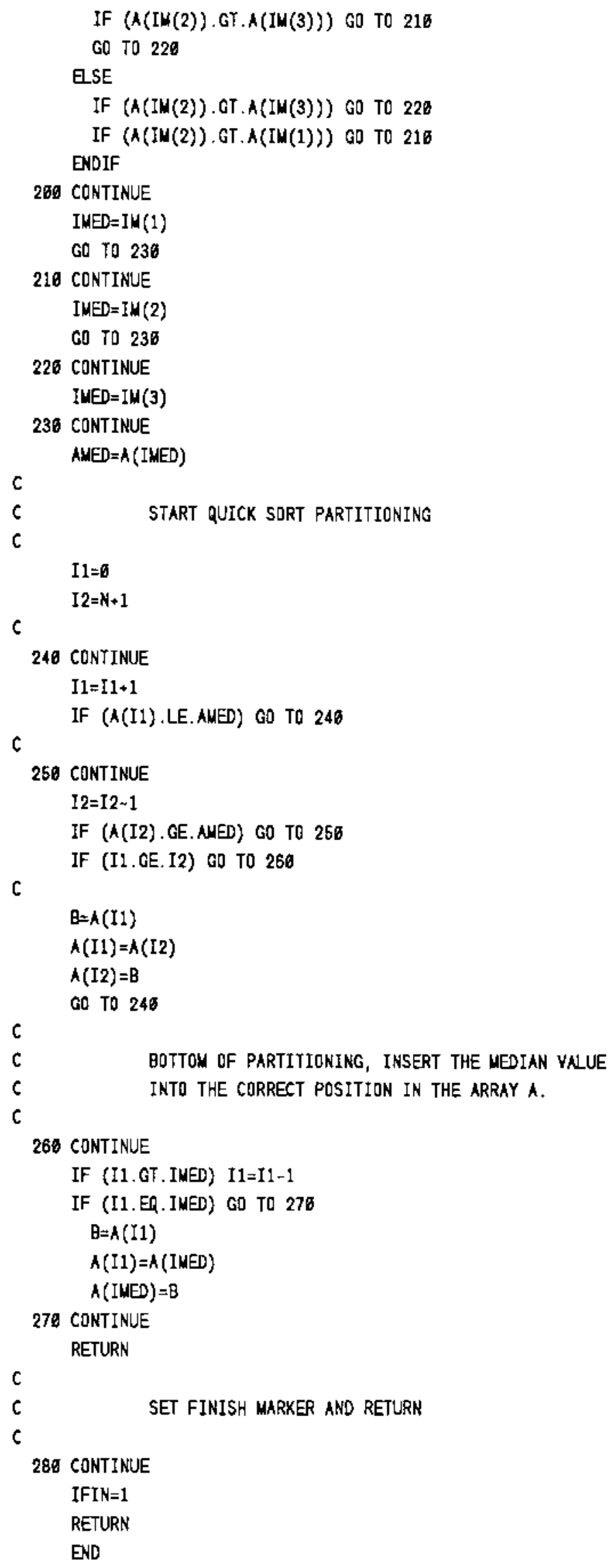


SUBROUT INE QSORT $(N, A)$

THIS IS $A$ RUICK SORTING NGORITHM. THIS PORTION DF THE ROUTINE CONTROLS THE PARTITIDNING OF THE ARRAY A. THE PARTIAL QUICK SORTING ROUTINE PQSORT IS APPLIED RECURSIVEY TO EACH SECTION DF THE INPUT ARRAY A.

THIS SORTING ROUTINE REQUIRES THE PRESENCE OF A SIUPLE SORTING ROUTINE WHICH IS EFICIERT FOR ARRAYS OF LESS THAN TEK ITEUS.

THIS SORTING ROUTINE IS CNLED BY THE NAME 'SORT', IN BOTH THIS ROUTINE AND THE ROUTINE PQSORT.

WOTE THAT THE NGORITH YAY FAIL IF WAXSTK IS LESS THAN TWO.

$A$ = ARRAY TO BE SORTED.

$*$ = NUMBER OF ITENS IN ARRAY A.

IC = INDEX OF DIVIDER OF THE NEW PARTITIONS OF ARRAY A, AS FDUND BY ROUTINE PQSORT.

IFIN = PDINTER, INDICATES ROUTINE PQSORT HAS FINISHED SORTING THE CURRENT PARTITION OF ARRAY A (RATHER THAN CREATING A NaI PARTITIONING).

IL = STACK ARRAY, CONTAINS THE LOWER INDEX OF EACH PARTITION OF THE INPUT ARRAY A.

IU = STACK ARRAY, CONTAINS THE UPPER INDEX OF EACH PARTITION OF THE INPUT ARRAY A.

NS = STACK POINTER, LOCATES ACTIVE PARTITION OF ARRAY A.

c

PARAMETER MAXSTK $=2 \sigma$

DINEKSION

- $\quad A(N), \quad$ IL(MXSTK), IU(WXXSTK)

c

NS $=1$

IL $(1)=1$

$\operatorname{IU}(1)=N$

c

100 CONTINUE

$U=I U(N S)-I L(N S)+1$

CALL PQSORT ( $M, I F I N, I C, A(I L(N S)))$

C

IF (IFIN.EQ. E) GO TO 116

NS $=$ NS -1

IF (NS.LE. 0) RETURN

GO TO 160

110 CONTINUE

$c$

$I C=I C+I L(N S)-1$

$K=N S+1$

$c$

IF (K.LE. MAXSTK) G0 TO 128

$M=I C-I L$ (NS)

CALL SORT $(M, A(I L(N S)))$

$I C=I C+1$

$\mathrm{W}=\mathrm{I} \cup(N S)-I C+1$

CALL SORT $(M, \wedge(I C))$

NS=NS-1

GO TO 186

QSORT

QSORT

QSORT

QSORT

QSORT

QSDRT

QSORT

QSORT

QSORT

QSORT

QSORT

QSORT

QSORT

QSORT

QSORT

QSORT

QSORT - 19

QSORT . 20

QSORT - 21

QSORT . 22

QSORT . 23

QSDRT - 24

QSDRT . 25

QSDRT - 26

QSORT - 27

QSORT - 2B

QSORT . 29

QSORT . 30

QSORT - 31

QSDRT - 32

QSORT . 33

QSORT . 34

QSORT - 35

QSORT - 36

QSORT , 37

QSDRT . 38

QSDRT . 39

QSORT . 40

QSORT . 41

QSORT . 42

QSORT . 43

QSORT . 44

QSORT . 45

QSORT . 46

QSORT . 47

QSORT . 48

QSORT . 49

QSORT $\quad 50$

QSORT . \$1

QSDRT . 52

QSORT . 53

QSDRT . 54

QSORT - 55 


\begin{tabular}{|c|c|}
\hline QSERT & \\
\hline QSORT & \\
\hline QSORT & \\
\hline QSORT & \\
\hline QSORT & \\
\hline QSORT & \\
\hline QSORT & \\
\hline QSORT & \\
\hline QSDRT & \\
\hline QSORT & \\
\hline QSORT & \\
\hline R1DAT & \\
\hline R1DAT & \\
\hline R1DAT & \\
\hline R1DAT & \\
\hline R1DAT & \\
\hline R1DAT & \\
\hline R1DAT & \\
\hline R1DAT & \\
\hline R1DAT & \\
\hline R1DAT & \\
\hline PPARA & \\
\hline PPARA & \\
\hline PPARA & \\
\hline PPARA & \\
\hline PPARA & \\
\hline PPARA & \\
\hline PPARA & \\
\hline PPARA & ${ }^{\circ}$ \\
\hline PPARA & 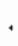 \\
\hline PPARA & . \\
\hline PPARA & \\
\hline PQLK3 & \\
\hline PELKJ & ${ }^{\circ}$ \\
\hline$P Q \times 3$ & . \\
\hline PBXX3 & \\
\hline PBLK3 & 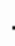 \\
\hline PBLK3 & 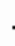 \\
\hline PBLK3 & \\
\hline PBLK3 & \\
\hline PELK3 & \\
\hline PELK3 & \\
\hline PBLK18 & 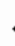 \\
\hline PBLK18 & \\
\hline PBLK18 & \\
\hline PBLK18 & 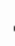 \\
\hline PELK1B & \\
\hline PBLK18 & \\
\hline PBLK1B & \\
\hline INOUT & \\
\hline ECHO & \\
\hline INDUT & \\
\hline INOUT & \\
\hline INOUT & \\
\hline
\end{tabular}

COMON /PBLK3/

+ CDIMEN(MAXCNT), CLABEL (MAXCNT), PLABE (MAXSOL),

- VDIMEN(MAXYAR), VLABE (MAXYAR) CHARACTER*12

- COINEN, CLABE, VDIMEN,

- vlabel

CHARACTER*32

- PLABE

c

C

COMMON /PBLK18/

+ JJNF (NLEEN, MAXVAR), INFIL, IUDIST,

- UDIST (MAXDST, 2)

CHARACTER*4

+ JJNF

$\mathrm{C}$

$c$

COLNON /INOUT/

- in, ioUt, IQB, iecho

CHARACTER*1

c

+ IQE 
+R OF ' $/ 4 X$, 'TIMES tO CREATE THE RERUIRED ', I5, ' SAMPLE VALUES.')

540 FQRNAT (/1X,'* ERROR EKCOUNTERED WHILE READING RANDOM DATA POINTS

- FROH AN'/4X, 'EXPERNAL DATA FILE. THIS ERROR OCCLRRED ON THE ', I5

* '-TH'/4X, 'LINE OF THE FILE. ')

550 FORMAT (4X, 'RUN TERMINATED FROM ROUTINE RDDAT.')

END

SUBRLUTINE R2OAT (IERR, N)

C

C THIS ROUTINE READS THE USER DEFINABLE CONFIDENCE FUNCTION FQR THE

C PRDGRAH PACSTAT.

c

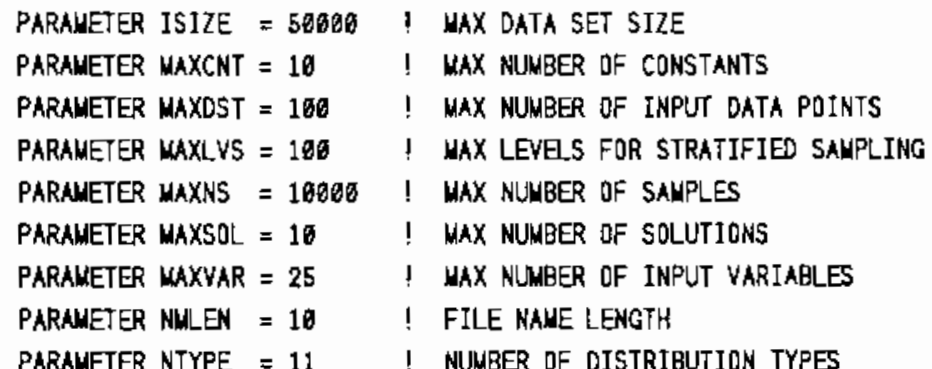

C

$c$

COMNON /PGLK3/

+ COIMEN (MAXCNT), CLABE (MAXCNT), PLABE (MAXSOL),

- VOINEN (WAXVAR), VLABE (WAXVAR)

CHARACTER: 12

+ COIMEN,

$+\quad$ VLABB

CHARACTER $* 32$

- Plake

c

c

CQLDNON/FRK18/

+ JJNF (NLLEN, WAXVAR), INFIL,

+ UDIST (MAXDST, 2)

CHARACTER*4

$+\quad$ J.JNF

$c$

c

COMNON /INOUT/

+ in, IOUT, IQB, IECHO

CHARACTER*1

$+\quad$ IQB

$c$

c

c

c

C UDIST $(I, 1)=$ I-TH VALUE OF THE CUNHULATIVE DISTRIBUTION FUNCTIDN.

c $\operatorname{UDIST}(I, 2)=I-T H$ VALUE OF THE CONFIDENCE LEYE (ZERO TO ONE).

$c$

IPRT $=\varnothing$

$c$

DO $200 \quad I=1$, MAXDST

$k=I$

\begin{tabular}{|c|c|}
\hline R1DAT & $\begin{array}{l}+\quad 69 \\
\end{array}$ \\
\hline R1DAT & 70 \\
\hline RIDAT & . 71 \\
\hline RIDAT & . $\quad 72$ \\
\hline R1DAT & . 73 \\
\hline R1DAT & . 74 \\
\hline R2DAT & 2 \\
\hline R2DAT & 3 \\
\hline R2DAT & 4 \\
\hline R2DAT & 5 \\
\hline R2DAT & 6 \\
\hline PPARA & 2 \\
\hline PPARA & 3 \\
\hline PPARA & 4 \\
\hline PPARA & 5 \\
\hline PPARA & 6 \\
\hline PPARA & 7 \\
\hline PPARA & 8 \\
\hline PPARA & 9 \\
\hline PPARA & . 10 \\
\hline PQARA & . $\quad 11$ \\
\hline PPARA & . $\quad 12$ \\
\hline PBK3 & 2 \\
\hline PELK3 & 3 \\
\hline PQLK3 & 4 \\
\hline PBLK3 & 5 \\
\hline PBLKK3 & 6 \\
\hline PBLK3 & 7 \\
\hline PQK3 & $B$ \\
\hline PELK3 & 8 \\
\hline PBLK3 & . $\quad 10$ \\
\hline PBLK3 & 11 \\
\hline 9BLK18 & 2 \\
\hline PBLK18 & 3 \\
\hline PBLK1B & 4 \\
\hline PBLK18 & 5 \\
\hline PBLK18 & . \\
\hline PBLK18 & 7 \\
\hline PELK18 & B \\
\hline INOUT & $\cdot$ \\
\hline ECHO & 86 \\
\hline INOUT & . \\
\hline INOUT & . \\
\hline INDUT & . \\
\hline INOUT & . \\
\hline R2DAT & . $\quad 11$ \\
\hline R2DAT & . $\quad 12$ \\
\hline R2DAT & . $\quad 13$ \\
\hline R2DAT & 14 \\
\hline$R 2 D A T$ & . $\quad 15$ \\
\hline R2DAT & . $\quad 16$ \\
\hline R2DAT & 17 \\
\hline R2OAT & . 18 \\
\hline R2OAT & 19 \\
\hline
\end{tabular}


READ (INFIL, * END $=110$, ERR $=156) \operatorname{UDIST}(I, 1), \operatorname{UDIST}(I, 2)$

\begin{tabular}{|c|c|}
\hline R2OAT & $2 \theta$ \\
\hline R2DAT & 21 \\
\hline R2DAT & 22 \\
\hline R2DAT & 23 \\
\hline R2DAT & 24 \\
\hline R2OAT & 25 \\
\hline R2OAT & 26 \\
\hline R2DAT & 27 \\
\hline R2DAT & 28 \\
\hline R2DAT & 29 \\
\hline $\mathrm{R} 2 \mathrm{OAT}$ & 38 \\
\hline R2DAT & 31 \\
\hline R2DAT & 32 \\
\hline R2DAT & 33 \\
\hline R2OAT & 34 \\
\hline R2DAT & 35 \\
\hline R2OAT & 36 \\
\hline R2OAT & 37 \\
\hline R2OAT & 38 \\
\hline R2DAT & 39 \\
\hline R2DAT & 48 \\
\hline R2DAT & 41 \\
\hline R2DAT & 42 \\
\hline R2OAT & 43 \\
\hline R2OAT & 44 \\
\hline R2OAT & 45 \\
\hline R2OAT & 46 \\
\hline R2OAT & 47 \\
\hline R2DAT & 48 \\
\hline R2OAT & 49 \\
\hline R2DAT & 58 \\
\hline R2DAT & 51 \\
\hline R2OAT & S2 \\
\hline R2OAT & 53 \\
\hline R2DAT & 54 \\
\hline R2DAT & 55 \\
\hline R2OAT & 56 \\
\hline R2OAT & 57 \\
\hline R2OAT & 58 \\
\hline R2OAT & 59 \\
\hline R2OAT & 60 \\
\hline R2OAT & 61 \\
\hline R2DAT & 62 \\
\hline R2DAT & 63 \\
\hline $\mathrm{R} 2 \mathrm{OAT}$ & 64 \\
\hline R2DAT & 65 \\
\hline R2OAT & 66 \\
\hline R2OAT & 67 \\
\hline R2DAT & 68 \\
\hline R2OAT & 69 \\
\hline R2OAT & 70 \\
\hline R2DAT & 71 \\
\hline R2DAT & . 72 \\
\hline R2DAT & . 73 \\
\hline
\end{tabular}

110 CONTINUE

IUDIST $=\mathrm{K}-1$

WRITE (IOUT, 52\%) VLABE (N), IUDIST

C

CHECX FOR STRICTLY INCREASING FUNCTIDNS AND

A PROPER INTERVN IN THE CONFIOENCE LEVEL.

129 CONTINUE

IF (IUDIST.LT.2) THEN

IRITE (IOUT, 536)

GO TO 160

ENDIF

$L=8$

$K=I U D I S T-1$

DO $146 \quad I=1, K$

IF ((UDIST $(I, 2)$, LT. U.). OR. (UDIST $(I, 2) . G T .1 .6)) L=1$

$J=I+1$

IF (UDIST $(I, 1) . \operatorname{LE} . \operatorname{UDIST}(J, 1))$ GO TO 138

MRITE (IOUT, 546) I, J

GO TO 160

136

CONIINUE

IF (UDIST $(I, 2)$.LE. UDIST $(J, 2))$ GO TO 140

MRITE (IOUT, 540) $I, J$

GO TO 180

148 CONTINUE

IF (L.NE. .) WRITE (IDUT, 550) VLABEL (N), (JJNF $(J, N), J=1, N M L E N)$

RETURN

C

C

C

150 CONTINUE

NRITE (IOUT, 566) I

186 CONTINUE

URITE (IOUT, 570)

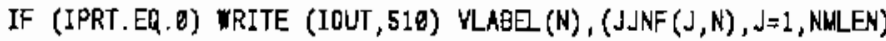

IERR $=1$

RETURN

$c$

56g FORMAT (/1X, '** MARNING,', I5,' OATA POINTS (THE MAXIUUM ALOWED) * -ERE READ'/4X, 'FRJM AN EXTERNAL FILE, DEFINING THE CUMNUATIVE CONF +IOENCE'/4X, 'FUNCTION, BUT THE END OF FILE NAS NDT ENCOUNTERED. TH $\rightarrow$ IS'/4X, 'WAY SKEX THE RESUTS. ')

516 FORMAT (4X,'DATA YAS BEING READ FOR INPUT VARIABLE', $A 12, ', 1 / 4 X, ' A$ +ND WAS READ FROW FILE: '/4X,20A4)

526 FORWAT ( $/ 1 X$, 'FOR THE INPUT VARIABle ',A12,', ',I4,' DATA POINTS "E $+R E ' / 1 X$, 'REAO FROM AN EXTERNAL FILE, OEFININO A CUMULATIVE CONFIDE

$$
\text { C. } 65
$$


+NCE CURVE. ')

R2DAT . 74

530 FORMAT (/1X,'** ERROR, ONLY ONE DATA POINT WAS FDUND DN THE EXTERN

R2DAT . 75

+NL FILE'/4X,' CONTAINING THE CULULATIVE CONFIDENCE FUNCTION DATA S +ET. '/4X, 'THIS IS INSUFFICIENY TO DEFINE A CONFIDENCE FUNCTION.')

R2DAT , 76

R2DAT $\quad 77$

549 FORMAT (/1X,'** ERROR, THE INPUT CUNMUATIVE DISTRIBUTION FUNCTION

R2DAT $\quad 78$

+ IS NOT'/4X, 'A STRICLY INCREASING FUNCTION AT INDECES ',I4,' 2 ', I

R2DAT . 79 $.4, .^{\prime \prime)}$

550 FDRUAT (/1X,'** "ARNING, CONFIDENCE VALUES LESS THAN ZERO OR GREAT

R2OAT . BO

R2DAT . $B 1$

-ER THAN ONE'/4X, 'HAVE BEEN ENTERED VIA AN EXTERNAL FILE FOR RANDON

R2DAT $\quad 82$

- INPUT'/4X, 'VARIABLE ',A12,'. THESE VALUES YLL BE IGNORED BY THE

R2DAT . 83

-1/4X,'STOCASTIC GENERATQRS. THIS DATA WAS REAO FRON FILE: '/4X, $26 \mathrm{~A}$ 4)

560 FORUAT (/1X,'** ERROR ENCOUNTERED WHILE READING THE USER DEINED $C$ - UMUULATIVE' $/ 4 X$, 'CONFIDENCE FUNCTION FRON AN EXTERNAL FILE. THIS E +RROR'/4X, 'DCCURED WRILE READING THE ', I4,' '-TH DATA POINT. ')

570 FORMAT (4X,'ERROR TERMINATION FROU ROUTINE R2DAT.')

END

SUBROUTINE RELEAS (IFIL)

c

ROUTINE FOR INTERACTIVE CLOSURE OF FILES

c

COMUON /INOUT/

+ IN, IDUT, IQE, IECho

CHARACTER 1

+ IQB

c

c

C - PRIME - SINSERT SYSCOW>A\$KEYS

LOGICAL LOGIC

c

IF (IFIL.LT.21) THEN

IFUNIT=IFIL -4

ESE

IFUNIT $=$ IFIL- 12

ENDIF

C LOGTC $=$ CLOSSA (INTS (IFUNIT))

$c$

C CLOSING VAX OPEA STATENENTS

c

CLOSE (UNIT=7)

CLOSE (UNIT=12)

CLOSE (UNIT $=13$ )

LOGIC = .TRUE.

IF (.NOT.LOGTC) WRITE (IOUT, 506) IFIL

RETURN

560 FORMAT (/1X, 'CLOSURE OF UNIT NO', 15, ' UNSLCCESSFUL. ')

ERD

SUBROUTINE REORDR

6

THIS ROUTINE MOVES THE INPUT AND IUTPUT YARIABLES IN THE ARRAYS

VAR AND RLTS, IN ORDER TO PLACE ANY 'FAILED' SOLUTIONS AT THE

END OF TRE ARRAYS. THE CURREAT WONTE CARLO SAMPLING SCHENE

REQUIRES THAT ALL SUCCESSFU SAMPLES BE LISTED CONTIGUOUSLY IN THE

C OUTPUT ARRAYS. HOMEVER, IT MAY OFTEN BE OESIRED TO SAVE THE FATLED

R2DAT . 84

R2OAT . B5

R2DAT $\quad 86$

R2DAT - 87

R2DAT . 88

R2DAT - 89

R2DAT . 90

REEAS - 2

REEAS - 3

RELEAS . 4

RELEAS : 5

INOUT - 2

ECHO $\quad 68$

INLUT . 4

INDUT . 5

INOUT . $\mathrm{B}$

INOUT - 7

RELEAS - 7

RELEAS - 8

REEEAS 9

RELEAS - 10

REEEAS , 11

RELEAS - 12

RELEAS - 13

RELEAS - 14

RELAS - 15

REIEAS - 15

REIEAS - 17

REIEAS - 18

ECHO $\quad 85$

RELEAS - 19

RELEAS - 20

REIEAS . 21

RELEA - 22

REEES - 23

RELES . 24

REIEAS - 25

REORDR . 2

REORDR . 3

REORDR . 4

REORDR - 5

REORDR . 6

REORDR * 7

REORDR . B

C. 66 


\begin{tabular}{|c|c|c|c|c|c|}
\hline C & \multicolumn{3}{|l|}{ SAMPLES FOR LATER ANALYSIS. } & REORDR & 9 \\
\hline C & \multicolumn{3}{|c|}{ ARRAY CONF IS USED HERE AS TEMPORARY STORAGE OF THE FAILED } & REOROR & 10 \\
\hline C & \multicolumn{3}{|l|}{ SAMPLE VALUES. } & REORDR & 11 \\
\hline C & & REORDR & 12 \\
\hline & & $! W$ & WAX DATA SET SIZE & PPARA & 2 \\
\hline & PARAMETER WAXCNT $=10$ & 14 & WAX NUNBER OF CONSTANTS & PPARA & 3 \\
\hline & PARANETER WAXDST $=160$ & $!$ & WAX NUWBER OF INPUT DATA POINTS & PPARA & 4 \\
\hline & PARAMETER MAXLVS = 106 & ! & WAX LEVES FLR STRATIFIED SAMPLING & PPARA & 5 \\
\hline & PARAMETER MAXNS = 10000 & $!$ & WAX NUMBER OF SAMPLES & PPARA & 6 \\
\hline & PARAMETER MAXSOL = 10 & 14 & WAX NUWBER DF SOLUTIONS & PPARA & 7 \\
\hline & PARNUETER WAXVAR $=2 S$ & $! W$ & WAX NLMEER OF INPUT VARIABLES & PPARA & 8 \\
\hline & PARAMETER NMEN $=10$ & $! F$ & FILE NAME LENGTH & PPARA & g \\
\hline & \multirow[t]{3}{*}{ PARAMETER NTYPE $=11$} & $! N$ & NUMBER OF DISTRIBUTION TYPES & PPARA & 16 \\
\hline$c$ & & & & PPARA & 11 \\
\hline C & & & & PPARA & 12 \\
\hline & \multicolumn{3}{|l|}{ COUNDN /PBLK1/ } & PELK1 & 2 \\
\hline & \multirow[t]{3}{*}{ - NCONST, } & \multicolumn{2}{|c|}{ NSOLN, } & PQLK1 & 3 \\
\hline \multicolumn{3}{|l|}{ C } & & PBLKI & . \\
\hline \multirow[t]{3}{*}{ C } & & & & PBLK1 & 5 \\
\hline & \multicolumn{3}{|l|}{ COWMON /PBLK5/ } & PQLK5 & 2 \\
\hline & $\rightarrow \quad$ ICNT, & & INDEX & PELK5 & 3 \\
\hline C & & & & PQLK5 & 4 \\
\hline C & & & & PBLK5 & 5 \\
\hline & CONAN /PBLK6/ & & & $\mathrm{PB}, \mathrm{KB}$ & 2 \\
\hline & - CONF (WAXNS), & $L T(M A$ & AXNS) & PBLK6 & 3 \\
\hline$c$ & & & & PQLKB & 4 \\
\hline C & & & & PRLKB & 8 \\
\hline & COMON /PBLK7N/ & & & PBLK7A & 2 \\
\hline & $+\quad$ NSLOC (wAXSOL) & & & PBLK7A & 3 \\
\hline$c$ & & & & PQLX7A & 4 \\
\hline $\mathrm{C}$ & & & & PRLK7A & 5 \\
\hline & COMHON /PBL.K7B/ & & & PBLK7B & 2 \\
\hline & + $\quad$ RLTS(ISIZE) & & & PBLK>B & 3 \\
\hline$c$ & & & & PBLK7B & 4 \\
\hline$c$ & & & & PELK7B & 5 \\
\hline & COMWON /PELKBN/ & & & PBLXBA & 2 \\
\hline & - $\quad$ NVLOC (MAXVAR) & & & PBLK8A & 3 \\
\hline$c$ & & & & PELKBA & 4 \\
\hline C & & & & PQLKBA & 5 \\
\hline & CONMON /PELKBB/ & & & PBLK8B & 2 \\
\hline & $+\quad$ VAR(ISIZE) & & & PELK8B & 3 \\
\hline$c$ & & & & PQL KBB & 4 \\
\hline C & & & & PBLKBB & 5 \\
\hline & COHMON /PBLK12/ & & & PBLK12 & 2 \\
\hline & - NERR (MAXNS), & & & PBLK12 & 3 \\
\hline$c$ & & & & PALL12 & 4 \\
\hline$c$ & & & & PBLK12 & 5 \\
\hline & COWHON /PBLK17/ & & & PBLK17 & 2 \\
\hline & $+\quad$ ICCD & UIT, & ISOLN, & PELK17 & 3 \\
\hline & $+\quad$ IUPDAT, & XSAM, & NPRT, & PBLK17 & 4 \\
\hline & $+\quad$ NSAM, & $A M C$ & & PBLK17 & 5 \\
\hline$C$ & & & & PBLK17 & 6 \\
\hline C & & & & PBLK17 & 7 \\
\hline & COWHON /INOUT/ & & & INOUT & . \\
\hline
\end{tabular}




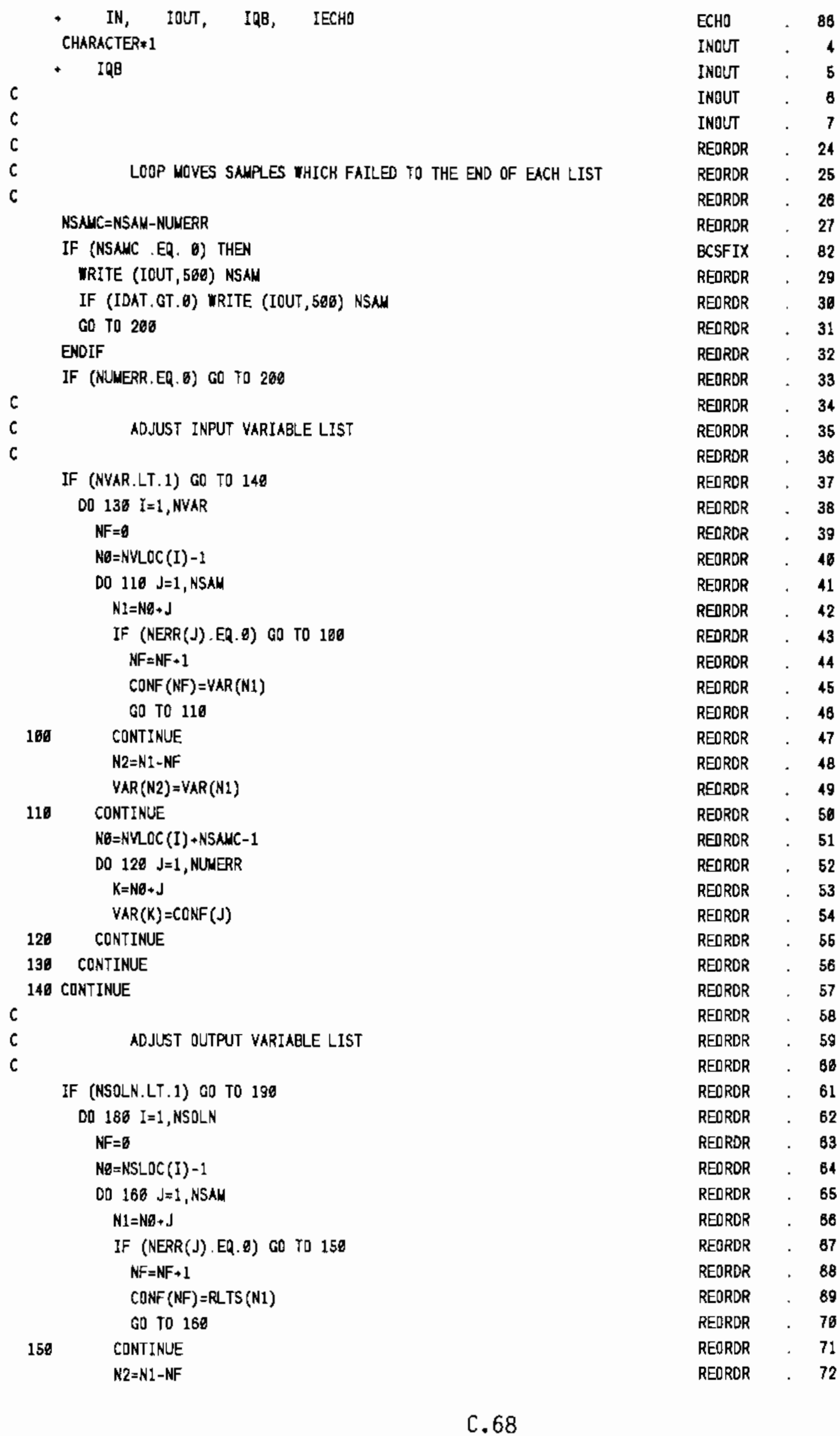




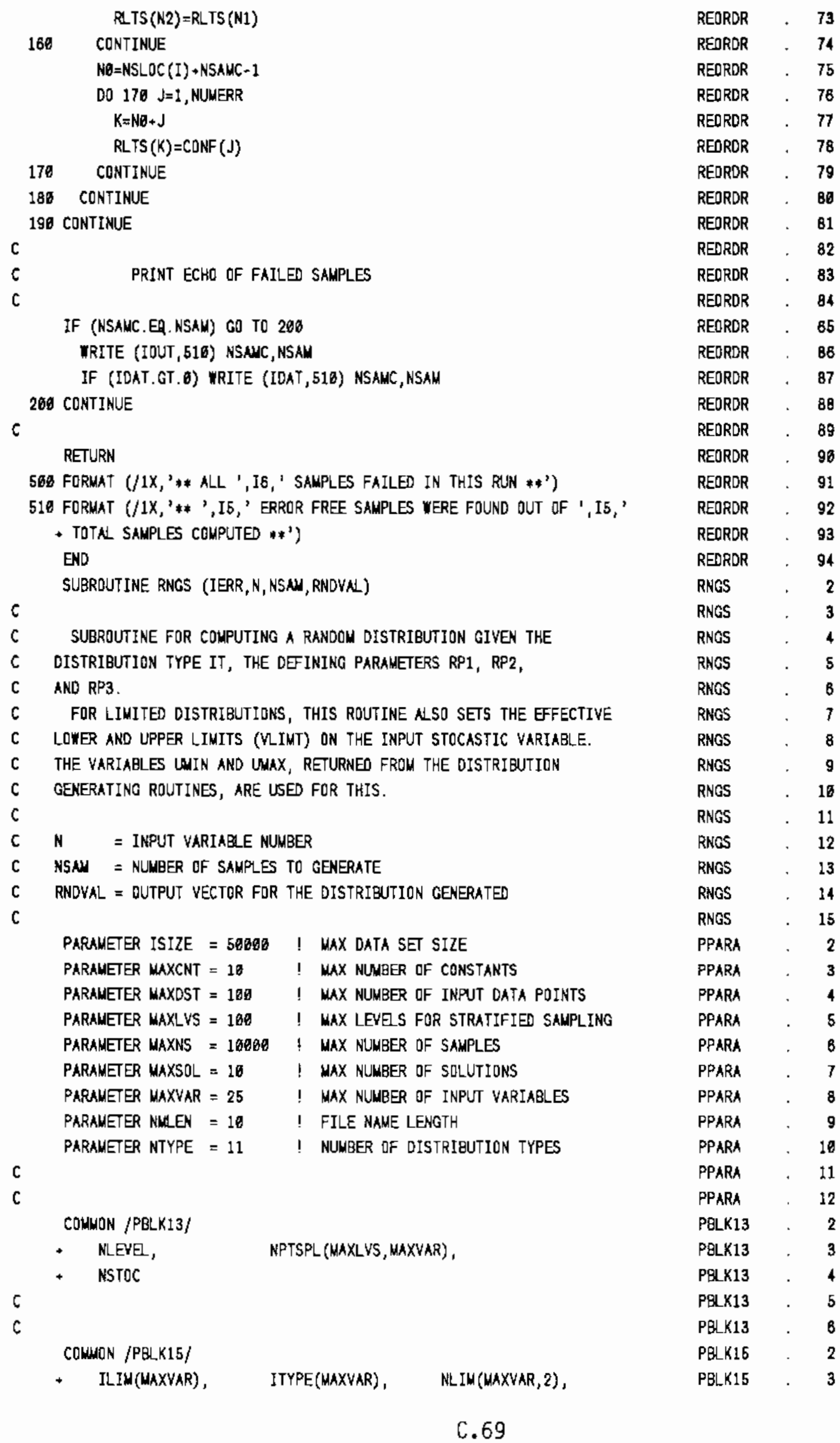




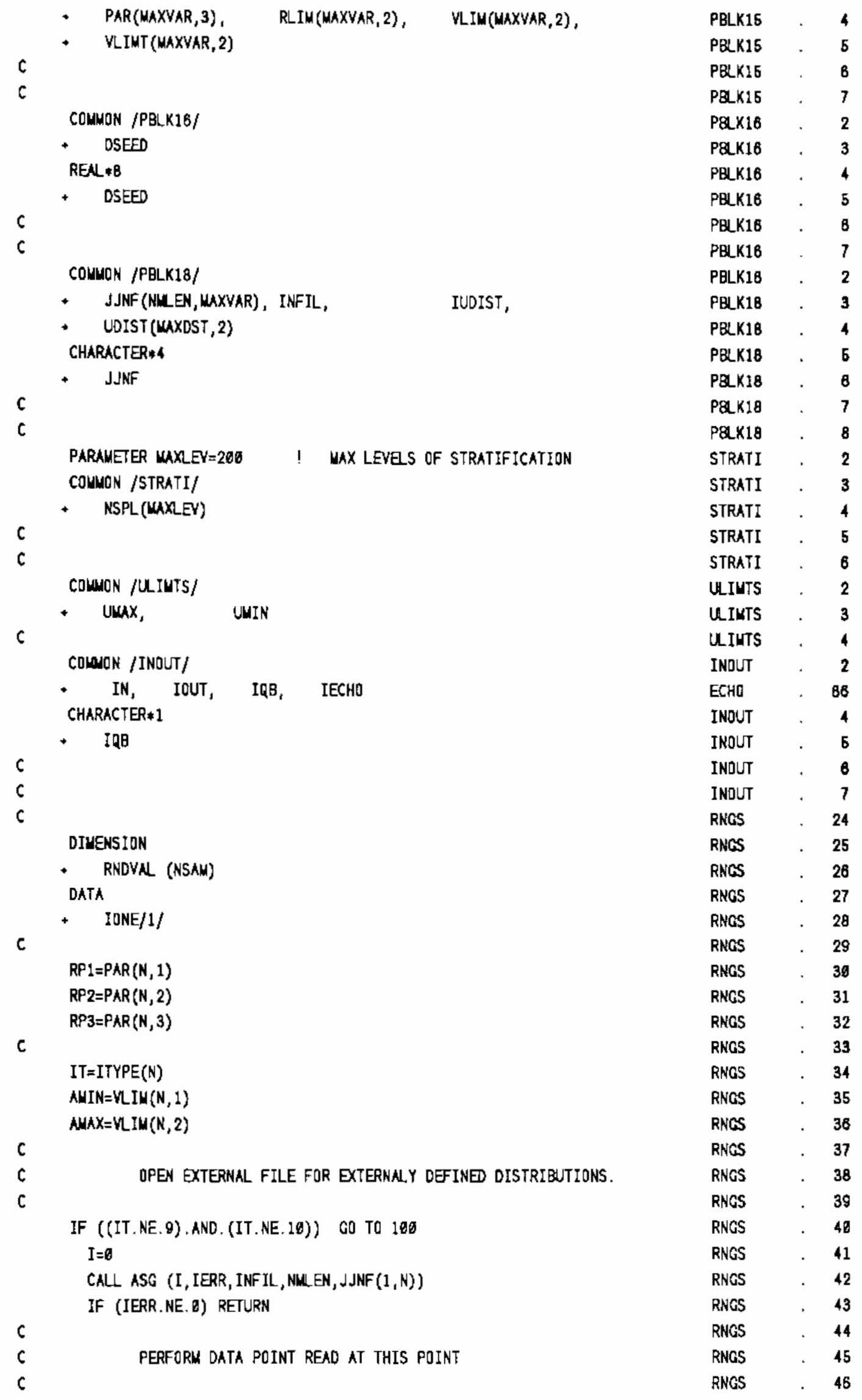

\section{70}




\begin{tabular}{|c|c|c|c|}
\hline & IF (IT.EQ.9) GO TO 190 & RNGS & 47 \\
\hline & CALL R1DAT (IERR,N,NSAM, RNDVAL) & RNGS & 48 \\
\hline & CAШ REEAS (INFIL) & RNGS & 49 \\
\hline & RETURN & RNGS & 50 \\
\hline 160 & CONTINUE & RNGS & 51 \\
\hline C & & RNGS & 52 \\
\hline C & COUPUTE BASE UNIFORU DISTRIBUTION & RNGS & 53 \\
\hline c & & RNGS & 54 \\
\hline & CALL Uø1S (NSAH, RNDYAL, DSEFD, NLEYE) & RNGS & 55 \\
\hline C & & RNGS & 56 \\
\hline C & SAVE STRATIFICATION DIAGNOSTICS & RNGS & 57 \\
\hline C & & RNGS & 58 \\
\hline & DO $110 \mathrm{I}=1, \mathrm{NLEYE}$ & RNGS & 59 \\
\hline & $\operatorname{NPTSPL}(I, N)=N S P L(I)$ & RNCS & 60 \\
\hline 110 & CONTINUE & RNGS & 61 \\
\hline C & & RNGS & 62 \\
\hline C & CHOSE DISTRIBUTION & RNGS & 63 \\
\hline C & & RNGS & 64 \\
\hline & GD TO $(120,130,14 \theta, 150,160,176,180,260,220,230,240)$, IT & RNGS & 65 \\
\hline C & & RNGS & 68 \\
\hline C & NORMAL DISTRIBUTION & RNGS & 67 \\
\hline C & & RNGS & 68 \\
\hline 129 & CONTINUE & RNGS & 69 \\
\hline & IF (ILIM(N) .EQ.ø) THEN & RNGS & 76 \\
\hline & CALL NORMAL (NSAN, RNDVAL, RP1, RP2) & RNGS & 71 \\
\hline & RETURN & RNGS & 72 \\
\hline & ELSE & RNGS & 73 \\
\hline & CAL NORMALL (NSAH, RNDVAL, RP1, RP2, AKIN, NMAX) & RNGS & 74 \\
\hline & $\operatorname{RLIM}(N, 1)=\operatorname{LIIN}$ & RNGS & 76 \\
\hline & $\operatorname{RLIM}(N, 2)=U M A X$ & RNGS & 78 \\
\hline & CALL NORWAL (IONE, UMIN, RP1, RP2) & RNGS & 77 \\
\hline & CALL NORMAL (IONE, UMAX, RP1, RP2) & RNGS & 78 \\
\hline & IF (UWIN.GT.UMAX) THEN & RNGS & 79 \\
\hline & WD=UNIN & RNGS & 86 \\
\hline & UWIN=UNAX & RNGS & 81 \\
\hline & UWAX=WD & RNCS & 82 \\
\hline & ENOIF & RNGS & B3 \\
\hline & $\operatorname{VINT}(N, 1)=$ UNIN & RNGS & 84 \\
\hline & $\operatorname{VINT}(N, 2)=\operatorname{UMAX}$ & RNGS & 85 \\
\hline & 60 TO 260 & RNGS & 86 \\
\hline & ENDIF & RNGS & 87 \\
\hline c & & RNGS & 88 \\
\hline C & NATURAL LOGNORMAL DISTRIBUTION & RNGS & 89 \\
\hline C & & RNGS & 90 \\
\hline 130 & CONTINUE & RNGS & 91 \\
\hline & IF (ILIM(N).EQ.8) THEN & RNGS & 92 \\
\hline & CALL LNNORM (NSAM, RNDVAL, RP1, RP2) & RNGS & 93 \\
\hline & RETURN & RNGS & 94 \\
\hline & 日SE & RNGS & 95 \\
\hline & CALL LNNORML (NSAN, RNDVAL, RP1, RP2, AMIN, AMAX) & RNGS & 96 \\
\hline & $\operatorname{RLI}(N, 1)=$ UNIN & RNGS & 97 \\
\hline & $\operatorname{RLIM}(N, 2)=U M A X$ & RNGS & 98 \\
\hline & CALL LNNORH (IONE, UNIN,RP1, RP2) & RNGS & 99 \\
\hline & CALL LNNORW (IONE, UWAX,RP1, RP2) & RNGS & . 100 \\
\hline
\end{tabular}




\begin{tabular}{|c|c|c|c|}
\hline & IF (UMIN.GT. UAAX) THEN & RNGS & 101 \\
\hline & WD=LMIN & RNGS & 102 \\
\hline & UMIN=LMAX & RNGS & . 193 \\
\hline & $U N A X=m$ & RNGS & . 104 \\
\hline & ENDIF & RNGS & .105 \\
\hline & $\operatorname{VIUT}(N, 1)=\operatorname{UNIN}$ & RNGS & . 186 \\
\hline & $\operatorname{VLIMT}(N, 2)=U W A X$ & RNGS & 187 \\
\hline & GO TO $26 \theta$ & RNGS & . 108 \\
\hline & ENDIF & RNGS & 169 \\
\hline $\mathrm{c}$ & & RNGS & . 110 \\
\hline c & COUMON LOGNORWAL DISTRIBUTION & RNGS & . 111 \\
\hline$c$ & & RNGS & . 112 \\
\hline 1400 & CONTINUE & RNGS & . 113 \\
\hline & IF (ILIM(N).EQ.ø) THEN & RNGS & . 114 \\
\hline & CALL LOGNORM (NSAM, RNDVAL, RP1, RP2) & RNGS & . 115 \\
\hline & RETURN & RNGS & . 118 \\
\hline & 日SE & RNGS & 117 \\
\hline & CALL LOGNORL (NSAM, RNDVAL, RP1, RP2, AMIN, AMAX) & RNGS & . 118 \\
\hline & $\operatorname{RLIM}(N, 1)=U M I N$ & RNGS & 119 \\
\hline & $\operatorname{RLIM}(N, 2)=U$ MAX & RNGS & . 126 \\
\hline & CALL LOGNORN (IONE, UNIN, RP1, RP2) & RNGS & . 121 \\
\hline & CALL LOGNORM (IONE, UNAX, RP1, RP2) & RNGS & . 122 \\
\hline & IF (UWIN.GT.LANX) THEN & RNGS & . 123 \\
\hline & WD=UMIN & RNCS & . 124 \\
\hline & UNIN= UNAX & RNCS & . 125 \\
\hline & WWAX $=10$ & RNGS & . 128 \\
\hline & ENDIF & RNGS & . 127 \\
\hline & $\operatorname{VIMT}(N, 1)=\operatorname{LMIN}$ & RNGS & . 128 \\
\hline & $\operatorname{VLIMT}(N, 2)=\operatorname{LNAX}$ & RNGS & . 129 \\
\hline & GO TO 260 & RNGS & 130 \\
\hline & ENDIF & RNGS & . 131 \\
\hline c & & RNCS & . 132 \\
\hline c & NATURAL LOGUNIFORU DISTRIEUTION & RNGS & 133 \\
\hline c & & RNGS & 134 \\
\hline $150 \mathrm{C}$ & CONTINUE & RNGS & . 135 \\
\hline & CALL LNUNIF (NSAM, RNDYAL, RP1, RP2) & RNGS & . 136 \\
\hline & RETURN & RNCS & . 137 \\
\hline c & & RNGS & . 138 \\
\hline C & COLMON LOGUNIFORM DISTRIBUTION & RNGS & 138 \\
\hline c & & RNGS & . 140 \\
\hline $168 \mathrm{C}$ & CONTINUE & RNGS & . 141 \\
\hline & CALL LDQUNIF (NSAM, RNDYAL, RP1, RP2) & RNGS & . 142 \\
\hline & RETURN & RNGS & 143 \\
\hline C & & RNGS & 144 \\
\hline$c$ & EXPONENTIAL DISTRIBUTION & RNGS & 145 \\
\hline$c$ & & RNGS & . 148 \\
\hline $178 \mathrm{C}$ & CONTINUE & RNGS & . 147 \\
\hline & IF (ILIM(N) .EQ.ø) THEN & RNGS & . 148 \\
\hline & CAL DEXPF (NSAM, RNDVAL, RP1) & RNGS & 149 \\
\hline & RETURN & RNGS & . 150 \\
\hline & ESE & RNGS & 151 \\
\hline & CALL DEXPFL (NSAM, RNDVAL, RP1, ANIN, AMAX) & RNGS & 152 \\
\hline & $\operatorname{RLIM}(N, 1)=\operatorname{UMIN}$ & RNGS & . 153 \\
\hline & $\operatorname{RLIM}(N, 2)=U M A X$ & RNGS & 154 \\
\hline
\end{tabular}




\begin{tabular}{|c|c|c|c|}
\hline & CALI DEXPF (IONE, UNIN, RP1) & RNGS & . 155 \\
\hline & CNL DEXPF (IONE, UWAX,RP1) & RNGS & . 156 \\
\hline & IF (UMIN.GT.UWX) THEN & RNGS & . 157 \\
\hline & WD=UMIN & RNGS & . 158 \\
\hline & UNIN=UWAX & RNGS & 159 \\
\hline & UWAX $=\mathbb{W}$ & RNGS & . 160 \\
\hline & ENDIF & RNGS & . 161 \\
\hline & $\operatorname{VLIMT}(N, 1)=U N I N$ & RNGS & . 182 \\
\hline & $\operatorname{nINT}(N, 2)=U N k X$ & RNGS & 183 \\
\hline & GO TO 260 & RNGS & 164 \\
\hline & ENDIF & RNGS & .165 \\
\hline C & & RNGS & . 168 \\
\hline C & UNIFORN DISTRIBUTION & RNGS & . 167 \\
\hline c & & RNGS & . 168 \\
\hline 180 & CONTINUE & RNGS & . 169 \\
\hline & $00198 \mathrm{I}=1, \mathrm{NSNH}$ & RNGS & . 170 \\
\hline & $\operatorname{RNDVAL}(I)=R P 1+\operatorname{RNDVN} L(I) *(R P 2-R P 1)$ & RNGS & . 171 \\
\hline 190 & CONTINUE & RNGS & 172 \\
\hline & RETURN & RNGS & 173 \\
\hline C & & RNGS & 174 \\
\hline $\mathrm{C}$ & BERNAULI DISTRIBUTION & RNGS & . 175 \\
\hline c & & RNGS & . 176 \\
\hline 200 & CONTINUE & RNGS & . 177 \\
\hline & CALL BERNLI (NSAM, RNDVAL, RP3) & RNGS & . 178 \\
\hline & DO $210 \mathrm{I}=1$, NSAN & RNGS & . 179 \\
\hline & $R V=R P 1$ & RNGS & 180 \\
\hline & IF (RNDVN (I).GT, 6.$) R V=R P 2$ & RNGS & 181 \\
\hline & $\operatorname{RNDVAL}(I)=R V$ & RNGS & . 182 \\
\hline 218 & CONTINUE & RNGS & . 183 \\
\hline & RETURN & RNGS & 184 \\
\hline C & & RNGS & 185 \\
\hline C & CONFIDENCE FUNCTION ON EXTERNAL FILE & RNGS & 186 \\
\hline C & & RNGS & . 187 \\
\hline 226 & CDNTINUE & RNGS & 188 \\
\hline & CAL R2DAT (IERR,N) & RNGS & . 189 \\
\hline & CAL XUDIST (NSAM, RNDVN, IUDIST, UDIST $(1,1), \operatorname{UDIST}(1,2))$ & RNCS & 190 \\
\hline & CAD REEAS (INFIL) & RNGS & . 191 \\
\hline & RETURN & RNGS & . 192 \\
\hline C & & RNGS & . 193 \\
\hline C & DATA POINTS DN AN EXTERNAL FILE (DUMMY STATENENT) & RNGS & 194 \\
\hline $\mathrm{C}$ & & RNGS & 195 \\
\hline 230 & CONTINUE & RNGS & . 196 \\
\hline & RETURN & RNGS & . 197 \\
\hline C & & RNGS & 198 \\
\hline c & DISCRETE DISTRIETUION & RNGS & 199 \\
\hline C & & RNGS & .200 \\
\hline 240 & CONTINUE & RNGS & . 201 \\
\hline & DO $258 \mathrm{I}=1$, NSAM & RNGS & 282 \\
\hline & RNDVAL (I) $=R P 1$ & RNGS & 203 \\
\hline 256 & CONTINUE & RNGS & 204 \\
\hline & RETURN & RNGS & 265 \\
\hline$c$ & & RNGS & 266 \\
\hline C & ENFORCE UPPER/LDWER LIMITS FOR END POINT LIMITING & RNGS & . 207 \\
\hline C & & RNGS & . 298 \\
\hline
\end{tabular}




\begin{tabular}{|c|c|c|c|c|}
\hline \multirow[t]{2}{*}{280} & \multicolumn{2}{|l|}{ CONTINUE } & RNGS & . 269 \\
\hline & \multicolumn{2}{|l|}{$\operatorname{NLIU}(N, 1)=0$} & RNCS & . 210 \\
\hline \multicolumn{3}{|c|}{$\operatorname{NLIN}(N, 2)=0$} & RNGS & . 211 \\
\hline \multicolumn{3}{|c|}{ DO $286 I=1$, NSAM } & RNCS & . 212 \\
\hline \multicolumn{3}{|c|}{$A=$ RNDVAL (I) } & RNGS & . 213 \\
\hline \multicolumn{3}{|c|}{ IF (A.GE.ANIN) GO TO 270} & RNGS & . 214 \\
\hline \multicolumn{3}{|c|}{ RNOVAL (I) = AMIN } & RNGS & 215 \\
\hline \multicolumn{3}{|c|}{$N \operatorname{LI}(N, 1)=N \operatorname{IM}(N, 1)+1$} & RNGS & . 216 \\
\hline \multicolumn{3}{|c|}{ GO TO 280} & RNGS & 217 \\
\hline \multirow[t]{4}{*}{270} & \multicolumn{2}{|l|}{ CONTINUE } & RNGS & . 218 \\
\hline & \multicolumn{2}{|l|}{ IF (A.LE. AWAX) GO TO 280} & RNCS & . 218 \\
\hline & \multicolumn{2}{|l|}{ RNDVAL $(I)=N A X$} & RNCS & . 220 \\
\hline & \multicolumn{2}{|l|}{$\operatorname{MLIM}(N, 2)=\operatorname{NLIM}(N, 2)+1$} & RNGS & . 221 \\
\hline \multirow[t]{4}{*}{280} & \multicolumn{2}{|l|}{ CONTINUE } & RNGS & . 222 \\
\hline & \multicolumn{2}{|l|}{ RETURN } & RNGS & . 223 \\
\hline & \multicolumn{2}{|l|}{ END } & RNGS & . 224 \\
\hline & \multicolumn{2}{|l|}{ SUBROUTINE SOLN (IERR) } & SOLN & . \\
\hline C & & & SOLN & . \\
\hline C & THIS SNMPLE PRORLEA MDOEES & THE WASS STORACE AND REZEASE RATE & SOLN & . \\
\hline C & (STEADY STATE) OF A CONTAM. & NANT, WICH IS DIFFUSING INTO $A$ BOUNDARY & SOLN & . \\
\hline C & LAYER AWAY FRON A SOLUBILI & Y LINITED SDURCE. OUTSIDE THE QOUNDARY & SOLN & . \\
\hline c & LAYER, CONYECTION IS ASSLNE & D TO RENDVE THE CONTANINANT AT A FAST & SOLN & . \\
\hline C & RATE. THE NDOE IS ONE DI & ENSIONAL, CARTESIAN DIFFUSION, AND & SOLN & . \\
\hline C & OUTPUT VALUES ARE 'PER SQUI & RE METER' OF SOURCE ZONE. & SDLN & . \\
\hline C & & & SOLN & 10 \\
\hline C INPU & DUT VARIABLES/DATA & & SOLN & 11 \\
\hline c & $=$ DATA SET NUME & ER ( & SOLN & . \\
\hline$c$ & = ERROR POINTE & & SQLN & . \\
\hline C & $=$ SAMPLE NUMBE & OF CURRENT RUN & SALN & . \\
\hline C & & & SOLN & 15 \\
\hline C & VINPT (1) = MOLECUAR DIFFUS & IVITY, WET**2/YEAR & SDLN & 18 \\
\hline C & VINPT(2) = RETARDATION FAC & & SOLN & . \\
\hline C & VINPT(3) = LAYER WIDTH, WE & & SOLN & . \\
\hline C & VINPT (4) = SOLUBILITY, GM/ & $E T * * 3$ & SILN & . \\
\hline C & & & SOLN & . \\
\hline C CONS & NSTANTS & & SOLN & . \\
\hline$c$ & $\operatorname{CONST}(1)=$ HALF LIFE, YEAR & & SOLN & . \\
\hline C & CONST (2) = POROSITY & & SOLN & . \\
\hline C & & & SOLN & . \\
\hline C OUTP & TPUT VARIABLES & & SOLN & . \\
\hline$c$ & VALU $(1)=$ TOTAL MASS CONTA & NMENT, GMS/MET**2 & SOLN & . \\
\hline C & $V A L U(2)=$ MAXIMUM REIEASE & ATE，GM/NET**2-YEAR & SOLN & . \\
\hline C & & & SOLN & . \\
\hline & PARAMETER ISIZE $=58000$ & ! MAX DATA SET SIZE & PPARA & . \\
\hline & PARANETER MAXCNT $=10$ & I WMX NUWBER OF CONSTANTS & PPARA & . \\
\hline & PARAMETER MAXDST = $10 B$ & ! MAX NLABER OF INPUT DATA POINTS & PPARA & . \\
\hline & PARAMETER MAXLYS = 106 & ! MAX LEVES FDR STRATIFIED SNMPLING & PPARA & . \\
\hline & PARAMETER MAXNS $=10600$ & ! MAX NUMEER OF SAMPLES & PPARA & . \\
\hline & PARAMETER WAXSOL $=10$ & ! WAX NUNBER OF SOLUTIONS & PPARA & . \\
\hline & PARAMETER MAXVAR $=25$ & ! WAX NUMBER OF INPUT VARIABLES & PPARA & . \\
\hline & PARAMETER NMLEN $=10$ & ! FILE NXME LENGTH & PPARA & . \\
\hline & PARAMETER NTYPE = 11 & I NUMBER OF DISTRIBUTION TYPES & PPARA & 10 \\
\hline$c$ & & & PPARA & . \\
\hline$c$ & & & PPARA & . \\
\hline
\end{tabular}




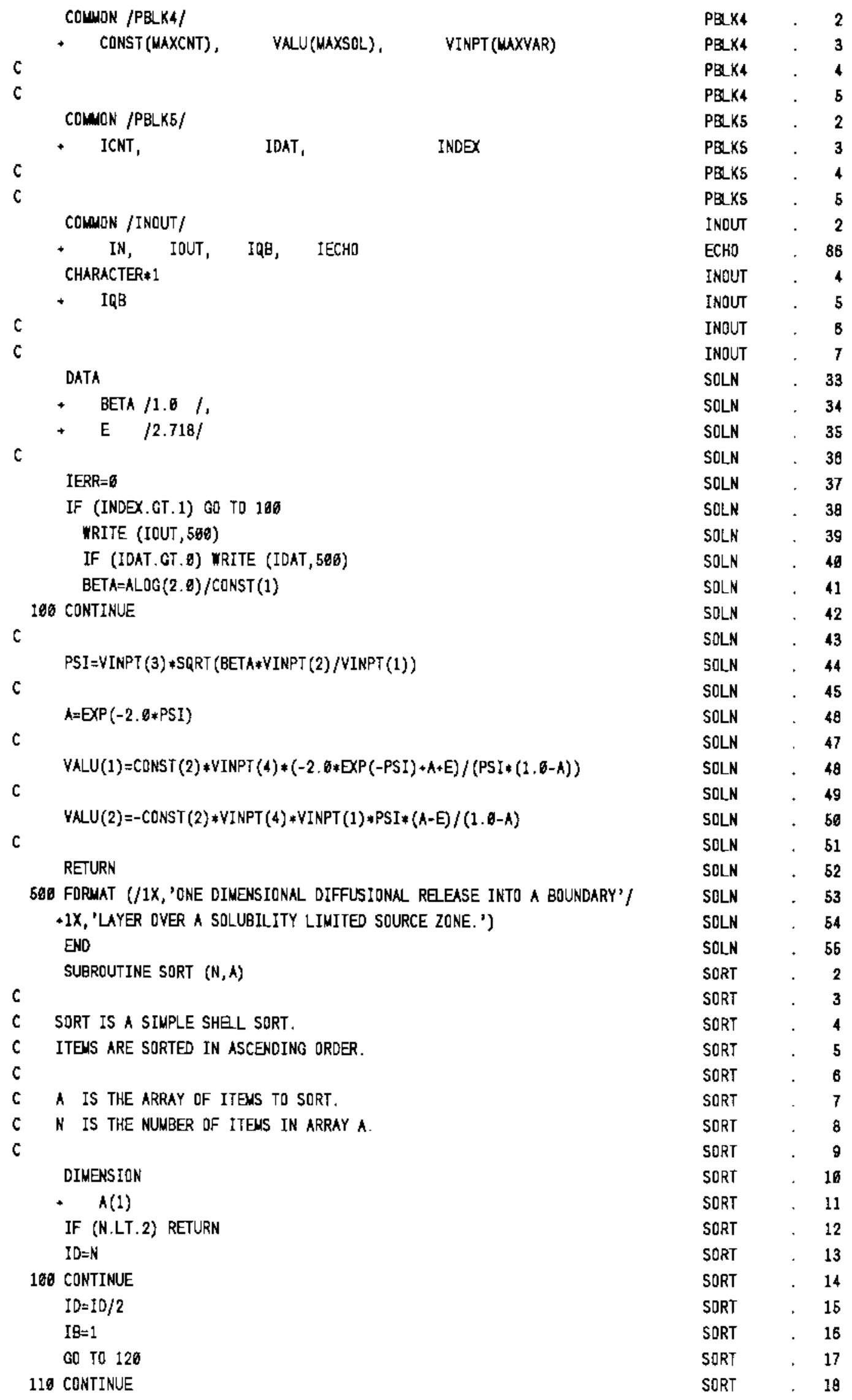


$I \mathrm{I}=\mathrm{IB}+1$

IF (IB.LE. ID) GO TO 126

SORT . 19

IF (ID.GT.1) GO TO 160

SORT $\quad 28$

RETURN

SORT $\quad 21$

120 CONTINUE

SORT . 22

$I=I B$

136 CONTINUE

$\mathrm{X}=\mathrm{I}+\mathrm{ID}$

IF $(A(I) . L E . A(K))$ GO TO $18 \theta$

$T=A(K)$

$A(K)=A(I)$

$\mathrm{J}=\mathrm{I}$

140 CONTINUE

$K=J-I D$

IF (K.LT.1) GO TO 150

IF (T.GT.A(K)) GO TO 150

$A(J)=A(K)$

$J=K$

GO TO 140

SQRT . 23

SORT . 24

SORT . 25

SORT . 26

SORT . 27

SORT $\quad 28$

SORT . 29

SQRT + 30

SORT . 31

SORT . 32

SORT - 33

SORT . 34

SORT - 35

SDRT . 38

156 CONTINUE

SORT . 37

$A(J)=T$

169 CONTINUE

$\mathrm{I}=\mathrm{I} \bullet \mathrm{IO}$

IF (I•ID.LE.N) GO TO 130

GO TO 110

ENO

SUBROUTINE STATOT

C

c

$c$

$c$

c

C

THIS ROUTINE CALLS LIBRARY ROUTINE STATS TO COUPUTE THE UEAN, MEDIAN, STANDARD DEVIATION, AND SKEINESS FDR THE INPUT AND OUTPUT

VARIAELE DISTRIBUTIONS WHICH HAVE BEEN GENERATED. DIAGNDSTICS FOR INPUT VARIABLE STRATIFICATYON, AND MAXIMLN LIKE YHOOD ESTIMATORS ARE ALSO GEAERATED.

ALL OF THESE DIATNOSTICS ARE PRINTED TO THE DUTPUT PRINT FILE OR USERS TERMINAL BY THIS ROUTINE.

DATA FOR STRATIFIED SALPLING IS PRINTED ONLY WHEN WDRE THAN ONE LEYE OF SAMPLING HAS BEEN CHLSEN.

THE DATA IN ARRAYS VAR AND RLTS ARE TRANSFERED TO ARRAY CONF

PRIOR TO CALL ROUTINE STATS. THIS AVOIDS A PRINE PAGING ERROR.

*** THIS ROUTINE SDRTS THIS INPUT ANO OUTPUT VARIAELE ARRAYS

*** IN ASCENDING ORDER, VIA A CALL TO SUBROUTINE QSORT.

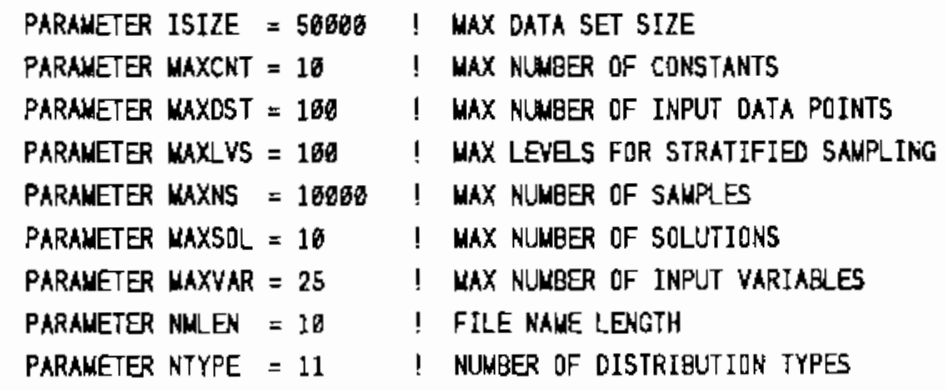

SORT - 38

SORT . 39

SORT . 48

SORT . 41

SORT + 42

SORT . 43

SORT . 44

STATOT , 2

STATDT - 3

STATOT . 4

STATOT - 5

STATOT . 6

STATOT . 7

STATOT - 8

STATOT . 9

STATOT - 10

STRTOT - 11

STATDT - 12

STATOT + 13

SIATET - 14

STATRT - 15

STATOT + 16

STATOT . 17

STATOT - 18

PPARA . 2

PPARA . 3

PPARA . 4

PPARA . 5

PPARA . 8

PPARA . 7

PPARA . 8

PPARA . 9

PPARA - 16

PPARA . 11

PPARA 12 


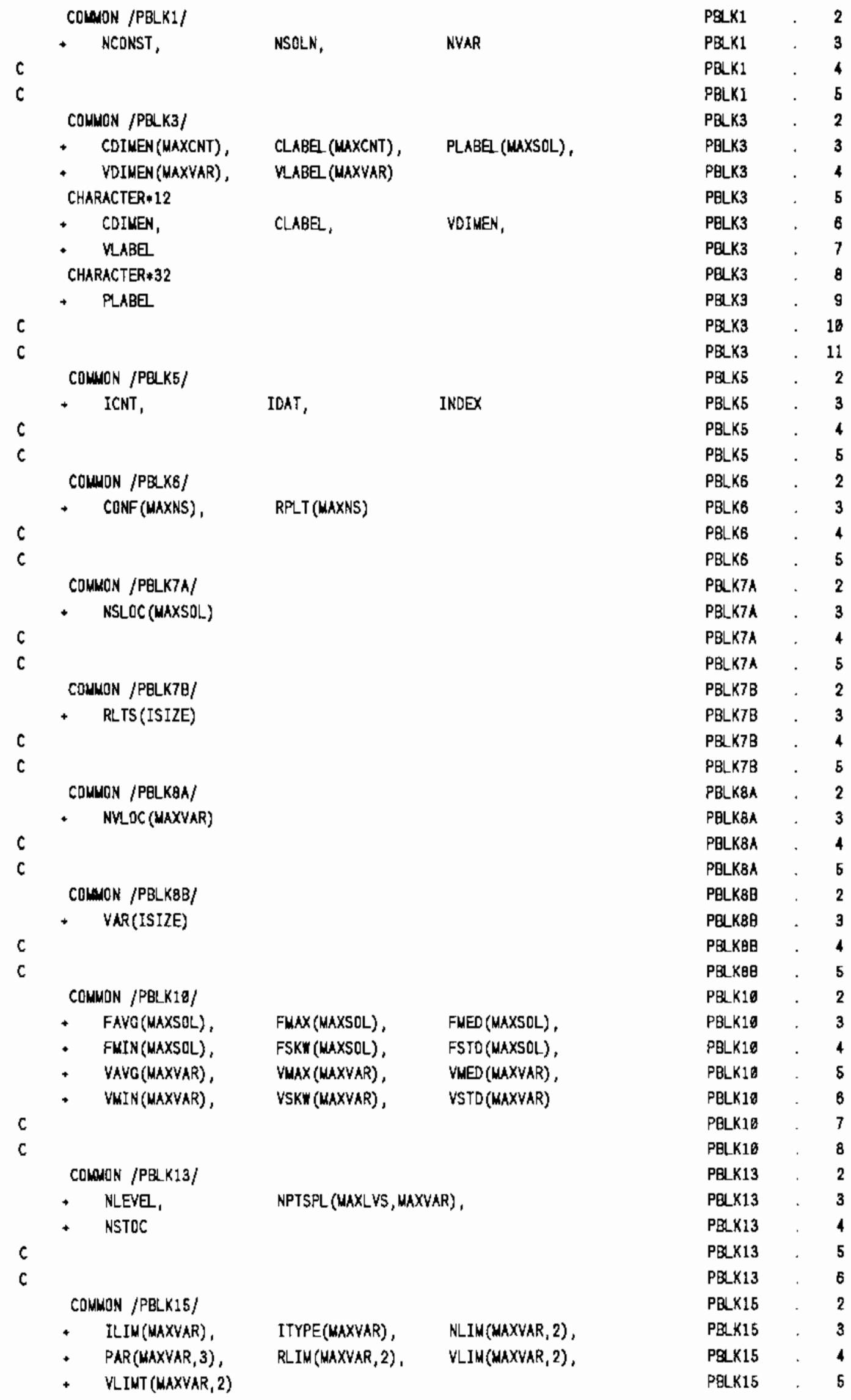


c

c

COLMON /PBLK17/

- ICCD,

+ IUPDAT,

- NSAM,

c

C

COMNON /INOUT/

- IN, IDUT, IQB, IECHO

CHARACTER*1

- IQB

$c$

c

REAL $* 8$

- A, B, BASEXP

DINENSION

- RD(2),

CHARACTER*1

- ANS

DATA

$+\quad$ BASEXP/2.38300/

C

c

c

III $=I D A T$

IF (IDAT.EQ. B) III=IOUT

c

c

c

WRITE (III, 506)

CALL EXPECT

c

c

$c$

IF (NVAR.LT.1) CO TO 148

DO $130 \mathrm{~N}=1$, NVAR

$\mathrm{N} 1=\mathrm{N} \operatorname{NLC}(\mathrm{N})-1$

DO $100 \mathrm{~J}=1$, NSAMC

$\mathrm{K}=\mathrm{J}+\mathrm{N} 1$

$\operatorname{CONF}(J)=\operatorname{VAR}(K)$

190 CONTINUE

CALL STATS (NSAMC, CONF, VMIN(N), $\operatorname{WMAX}(N), \operatorname{VAVG}(N), \operatorname{VSTD}(N), \operatorname{VSKM}(N))$

c

$c$

c

ISOLN,

NPRT,

NSAMC

RN(2)

UT UNT NUMBER

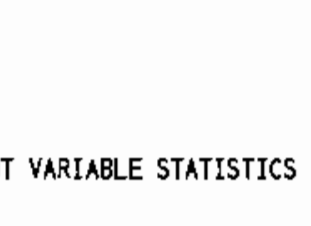




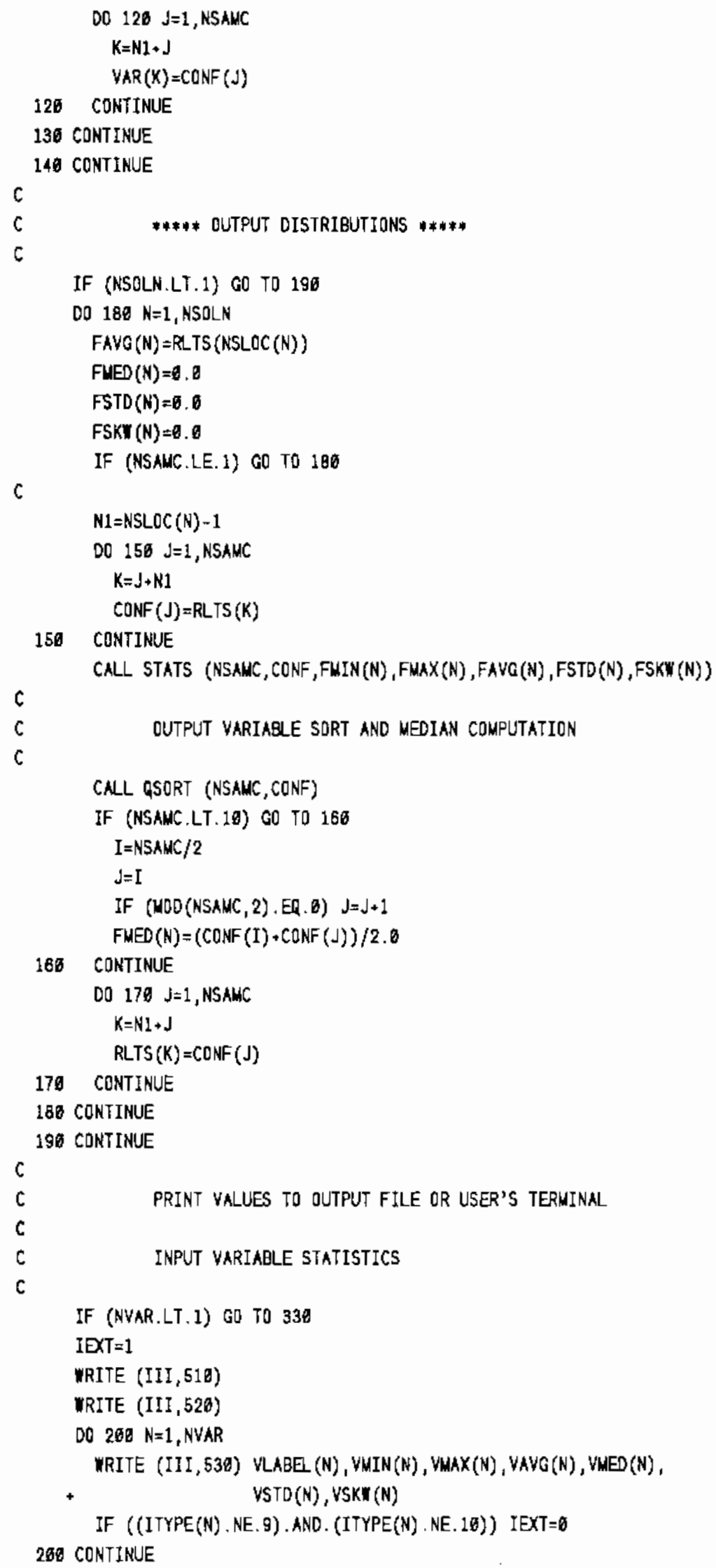




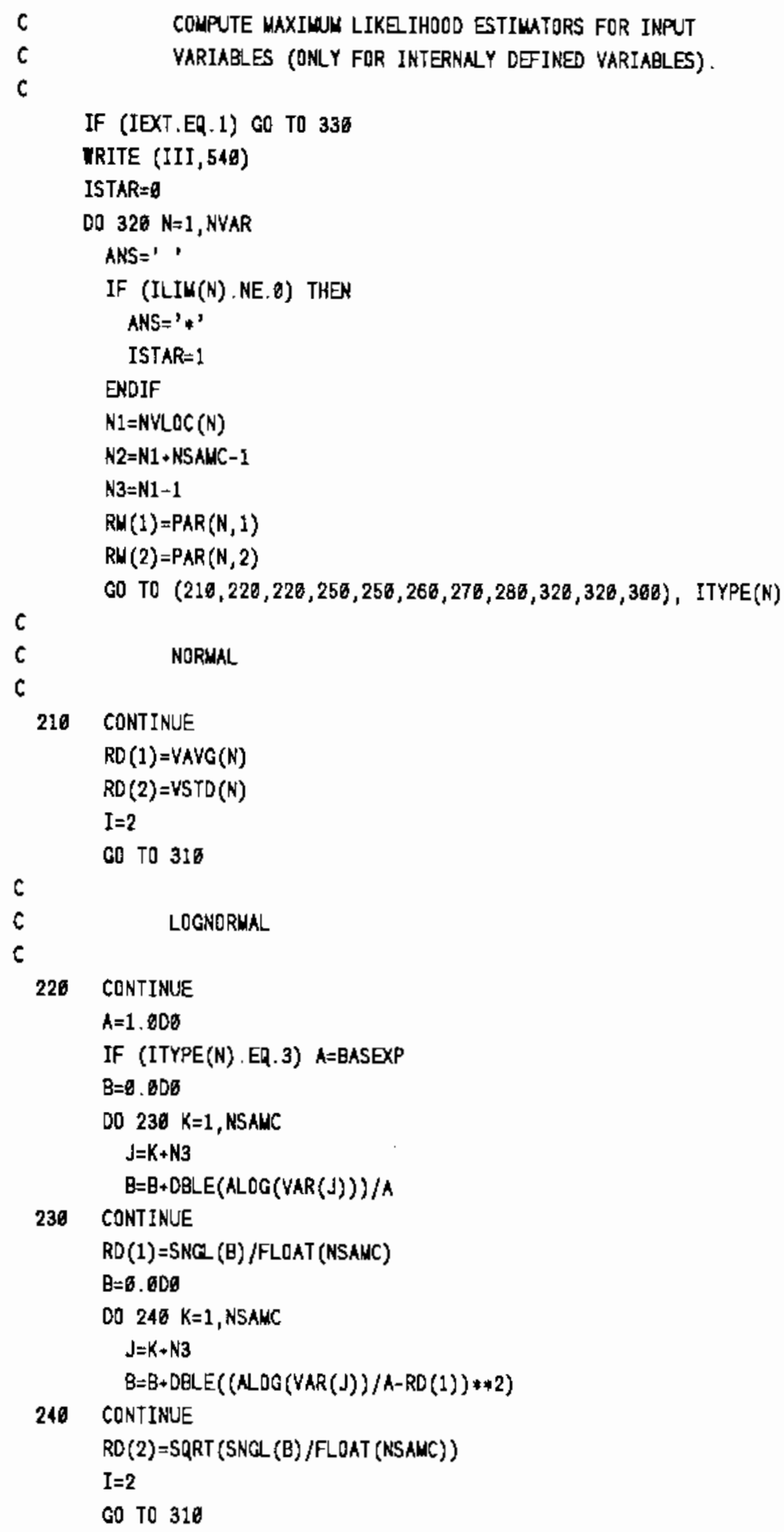




\begin{tabular}{|c|c|c|c|}
\hline & $I=2$ & STATOT & . 178 \\
\hline & GO TO 310 & STATOT & . 177 \\
\hline c & & STATOT & . 178 \\
\hline c & EXPONENTIRL & STATOT & . 178 \\
\hline c & & STATOT & . 180 \\
\hline 260 & CONTINUE & STATOT & . 181 \\
\hline & $R D(1)=\operatorname{VAVG}(N)$ & STATOT & . 182 \\
\hline & $R M(1)=1.0 / \operatorname{PAR}(N, 1)$ & STATQT & . 183 \\
\hline & $I=1$ & STATOT & . 184 \\
\hline & GD TO 310 & STATOT & . 185 \\
\hline C & & STATQT & . 186 \\
\hline C & UNIFORM & STAYOT & 187 \\
\hline C & & STATOT & . 188 \\
\hline 278 & CONTINUE & STATOT & . 189 \\
\hline & $J=N Y L O C(N)$ & STATQT & . 190 \\
\hline & $R D(1)=\operatorname{VAR}(N 1)$ & STATOT & . 191 \\
\hline & $\mathrm{RD}(2)=\operatorname{VAR}(\mathrm{N} 2)$ & SFATOT & . 192 \\
\hline & $I=2$ & STATOT & . 193 \\
\hline & GO TO 310 & STATDT & . 194 \\
\hline c & & STATOT & . 195 \\
\hline C & BERNOULLI & STATOT & . 198 \\
\hline c & & STATOT & . 197 \\
\hline 286 & CONTINUE & STATOT & . 198 \\
\hline & $\operatorname{RM}(1)=\operatorname{PAR}(N, 3)$ & STATOT & . 199 \\
\hline & IF ((VAR(N1).ER. $\operatorname{VAR}(N 2))$, OR. (NSANC.LT.2)) THEN & STATOT & . 200 \\
\hline & $\operatorname{RD}(1)=0.0$ & STATOT & 201 \\
\hline & $I=1$ & STRTOT & . 292 \\
\hline & GO TO 310 & STATOT & 203 \\
\hline & ENOIF & STATOT & . 264 \\
\hline & $A=\operatorname{VAR}(\mathrm{N} 1)$ & STATOT & . 285 \\
\hline & $K=0$ & STATOT & . 208 \\
\hline 298 & CONTINUE & STATOT & . 207 \\
\hline & $K=K+1$ & STATOT & . 208 \\
\hline & $J=N 3+K$ & STATOT & . 209 \\
\hline & IF $(A, G E . Y A R(J))$ GO TO 298 & STATOT & . 210 \\
\hline & $R D(1)=(F L O A T(K)-6.5) / F L O A T(N S A M C)$ & STATOT & 211 \\
\hline & $I=1$ & STATOT & . 212 \\
\hline & GO TO 318 & STATOT & . 213 \\
\hline C & & STATOT & . 214 \\
\hline C & CONSTANT & STATOT & . 215 \\
\hline C & & STATOT & . 216 \\
\hline 360 & CONTINUE & STATOT & . 217 \\
\hline & $R D(1)=Y A R(N 1)$ & STATOT & . 218 \\
\hline & $I=1$ & STATOT & . 219 \\
\hline c & & STATOT & . 228 \\
\hline 310 & CONTINUE & STATOT & . 221 \\
\hline & IF (I.GT.1) THEN & STATOT & . 222 \\
\hline & WRITE (III, 550) VLABEL (N), ANS, RD(1), RD(2), RM(1), RM(2) & BCSFIX & 92 \\
\hline & ESE & STATOT & . 224 \\
\hline & MRITE (III, 560) VLABEL (N), ANS, RD(1), RM(1) & BCSFIX & 93 \\
\hline & ENOIF & STATOT & . 226 \\
\hline 320 & CONTINUE & STATOT & . 227 \\
\hline & IF (ISTAR.NE. & BCSFIX & 94 \\
\hline 330 & CONTINUE & STATOT & . 229 \\
\hline
\end{tabular}


c

506 FORMAT (1H1, IX, 'TABLE 2. INPUT VARIABLE STATISTICS')

\begin{tabular}{|c|c|}
\hline CSFIX & 95 \\
\hline SFIX & 96 \\
\hline SFIX & 97 \\
\hline SFIX & 98 \\
\hline SFIX & 99 \\
\hline SFIX & 166 \\
\hline SFIX & 161 \\
\hline SFIX & 102 \\
\hline CSFIX & 103 \\
\hline CSFIX & 104 \\
\hline CSFIX & 105 \\
\hline CSFIX & 108 \\
\hline CSFIX & 107 \\
\hline CSFIX & 108 \\
\hline BCSFIX & 109 \\
\hline BCSF IX & . 110 \\
\hline CSFIX & . $\quad 111$ \\
\hline SCSFIX & . $\quad 112$ \\
\hline CSFIX & . 113 \\
\hline BCSFIX & . 114 \\
\hline BCSFIX & . 115 \\
\hline BCSFIX & . 116 \\
\hline BCSFIX & . $\quad 117$ \\
\hline BCSFIX & . 118 \\
\hline BCSFIX & . $\quad 119$ \\
\hline BCSFIX & 126 \\
\hline BCSFIX & 121 \\
\hline BCSFIX & . $\quad 122$ \\
\hline BCSFIX & 123 \\
\hline BCSFIX & . 124 \\
\hline BCSFIX & 125 \\
\hline BCSFIX & 128 \\
\hline BCSFIX & 127 \\
\hline BCSFIX & 128 \\
\hline BCSFIX & 129 \\
\hline BCSFIX & 130 \\
\hline BCSFIX & 131 \\
\hline BCSFIX & 132 \\
\hline BCSFIX & 193 \\
\hline BCSFIX & . 134 \\
\hline BCSFIX & . 135 \\
\hline BCSFIX & . 136 \\
\hline BCSFIX & . 137 \\
\hline BCSFIX & . 138 \\
\hline 8CSFIX & . $\quad 139$ \\
\hline BCSFIX & . 140 \\
\hline BCSFIX & . 141 \\
\hline BCSFIX & . 142 \\
\hline BCSFIX & . 143 \\
\hline BCSFIX & . 144 \\
\hline BCSFIX & . 145 \\
\hline BCSFIX & . 148 \\
\hline BCSFIX & . 147 \\
\hline 3CSFIX & 148 \\
\hline
\end{tabular}




\begin{tabular}{|c|c|c|}
\hline 516 FORMAT (//1X,'*\#-.. SAMPLE DIAGNDSTICS, INPUT DISTRIBUTIONS') & BCSFIX & . 149 \\
\hline 520 FORWAT (/T19, 'WIN', T36, 'MAX',T40, 'WEAN', T51, 'WEDIAN', T61, 'STND DEV & BCSFIX & .150 \\
\hline$+{ }^{\prime}$, T72, 'SKEWNESS') & BCSFIX & . 151 \\
\hline 530 FORAT $(1 X, A 12,1 X, 1 P E 18,3,5(1 X, E 10.3))$ & BCSFIX & . 152 \\
\hline 549 FORNAT (//1X,'**--- MAXIMUM LIKEIHOOD ESTIMATORS FOR INPUT DISTRI & BCSFIX & . 153 \\
\hline -BUTIDNS'/T24, 'COMPUTED VALUES', T52, 'EXPECTED VALUES'/3X, 'VARIABLE' & ECSFIX & . 154 \\
\hline,+ T26, 'PARAMETER 1',T33, 'PARAMETER 2',T48, 'PARAMETER 1',T61, 'PARAME & BCSFIX & . 155 \\
\hline -TER 2') & BCSFIX & . 158 \\
\hline 556 FORMAT $(1 X, A 12,3 X, A 1$, T20, IPE19.3, 3X,E10.3, T48, E16. 3, T61, E16.3) & BCSFIX & . 157 \\
\hline 566 FORMAT $(1 X, \wedge 12,3 X, A 1, T 26,1$ PE10.3, T48, E16.3) & BCSFIX & 158 \\
\hline 570 FORMAT (T33, '" INDICATES ENDPOINT LIMITING MAY WOVE THE '/T35, 'DIS & BCSFIX & . 159 \\
\hline$\rightarrow$ TRIBUTION AMAY FROM THE EXPECTED VALUES. ') & BCSFIX & . 168 \\
\hline 580 FORNAT (//3X,'FOR THE DISTRIBUTION IN', A12) & BCSFIX & . 161 \\
\hline 598 FORWAT $(3 X, 16$, ' RANDOW SAMPLES WERE BEOW THE LOMER LIMIT, AND SET & BCSFIX & . 162 \\
\hline + TO THAT LIMIT. ') & ECSFIX & . 163 \\
\hline BgG FORMAT (3X,I8,' RANDON SAMPLES WERE ABOVE THE UPPER LIMIT, AND SET & BCSFIX & 184 \\
\hline + TO THAT LIMIT.') & BCSFIX & 165 \\
\hline 810 FORMAT $(/ / 7 X$, 'STRATIFIED SAMPLING DIAGNOSTICS' $/ / 1 X$, 'VARIABLE', $17 X$ & BCSFIX & 168 \\
\hline •, 'NUMBER DF SAMPLES CHOSEN FROM EACH LEVE') & BCSFIX & 187 \\
\hline 620 FORMAT $(1 X, A 12,2 X, 10(1 X, I 5))$ & BCSFIX & 168 \\
\hline 630 FORMAT $(15 X, 10(1 X, I 5))$ & BCSFIX & . 169 \\
\hline 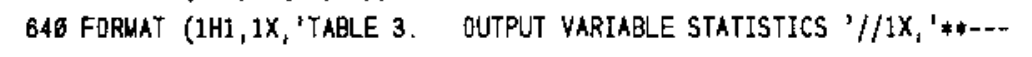 & BCSFIX & . 178 \\
\hline + SAMPLE DIAGNOSTICS, OUTPUT DISTRIBUTIONS') & BCSFIX & . 171 \\
\hline 656 FORMT $(1 X, A 32)$ & BCSFIX & . 172 \\
\hline 660 FORUAT $(1 X, 13 X, 1 P E 10,3,5(1 X, E 10.3))$ & BCSFIX & . 173 \\
\hline END & BCSFIX & . 174 \\
\hline SUBROUTINE STATS (N, RNDVAL, AMIN, AMAX, AMEAN, STDEY, SKEW) & STATS & 2 \\
\hline & STATS & 3 \\
\hline THIS RQUTINE COMPUTES THE MINIMUM, MAXIMUM, WEAN, STANDARD & STATS & 4 \\
\hline DEVIATION, AND SKEWNESS FOR THE DATA SET STORED IN VECTOR RNDVAL. & STATS & 5 \\
\hline & STATS & B \\
\hline$R E N=8$ & STATS & 7 \\
\hline$+\quad A, A V G, G, B S A M, C, C S A M, D, B A S E X P$ & STATS & 8 \\
\hline DIMENSION & STATS & 9 \\
\hline$+\quad$ RNDVAL $(N)$ & STATS & 10 \\
\hline DATA & STATS & 11 \\
\hline$+\quad B A S E X P / 2.303 D Q /$ & STATS & 12 \\
\hline C & STATS & 13 \\
\hline$C$ & STATS & 14 \\
\hline BSAN=DELE(FLOAT $(N))$ & STATS & 15 \\
\hline$C S A M=B S A M+1 . B D B$ & STATS & 16 \\
\hline$C$ & STATS & 17 \\
\hline AMEAN=RNDVAL(1) & STATS & 18 \\
\hline $\mathrm{STDEY}=\emptyset . \emptyset$ & STATS & 19 \\
\hline SKEW $=0 . \varnothing$ & STATS & 20 \\
\hline IF (N.LE.1) GO TO 490 & STATS & 21 \\
\hline$C$ & STATS & 22 \\
\hline CGMPUTE AVERAGE, MIN AND WAX VALUE & STATS & 23 \\
\hline 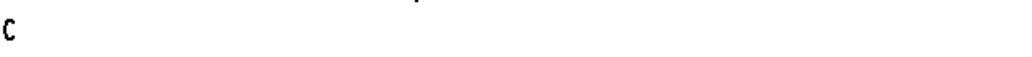 & STATS & 24 \\
\hline AMAX $=R$ NOVAL (1) & STATS & 25 \\
\hline AMIN $=A M A X$ & STATS & 26 \\
\hline$A Y G=D B L E(A M A X)$ & STATS & 27 \\
\hline DD $106 \quad I=2, N$ & STATS & 28 \\
\hline$R V=R N D V A L(I)$ & STATS & 29 \\
\hline
\end{tabular}




\begin{tabular}{|c|c|c|}
\hline$A V G=A V G+D E L E(R V)$ & STATS & 30 \\
\hline IF (RV.LT. ANIN) ANIN=RV & STATS & 31 \\
\hline IF (RV.GT, AMAX) AHAX=RV & STATS & 32 \\
\hline 106 CONTINUE & STATS & 33 \\
\hline AVG $=A V G / E S A N$ & STATS & +34 \\
\hline ALEAN $=S N C$ C (AVG) & STATS & . $\quad 35$ \\
\hline c & STATS & 36 \\
\hline COMPUTE STAND DEY AND SKEYNESS & STATS & . $\quad 37$ \\
\hline 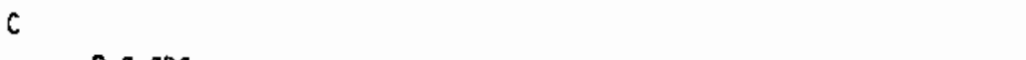 & STATS & . $\quad 38$ \\
\hline$B=0.800$ & STATS & . 39 \\
\hline$C=8.808$ & STATS & 40 \\
\hline $00118 I=1, N$ & STATS & 41 \\
\hline$A=A V G-D B L E(R N D V A L(I))$ & STATS & . $\quad 42$ \\
\hline$B=B+A * A$ & STATS & . 43 \\
\hline$C=C+A+A * A$ & STATS & 44 \\
\hline 110 CONTINUE & STATS & 45 \\
\hline STDEY $=$ SNGL (DSQRT (B/CSAN)) & STATS & . 46 \\
\hline SKE $=S N G L((D A B S(C / C S A M)) * * 6.333333306)$ & STATS & . $\quad 47$ \\
\hline IF (C.LT.0.60D) SKEW=-SKEH & STATS & 48 \\
\hline c & STATS & . $\quad 49$ \\
\hline 490 CONTINUE & STATS & . $\quad 56$ \\
\hline RETURN & STATS & . 51 \\
\hline END & STATS & . $\quad 52$ \\
\hline REAL FUNCTION U木1 ( DSEFD) & U81 & 2 \\
\hline C* & U81 & 3 \\
\hline C* & Uฮ1 & 4 \\
\hline C* PURPQSE: & UB1 & 5 \\
\hline C* & Uæ1 & 6 \\
\hline THIS FUNCTION GENERATES $A$ REAL 44 RANDOW UNIFQRM $(0,1)$ VARIATE & บด1 & 7 \\
\hline GIVEN AN INITIAL VALUE OF THE SEED, DSEED. THE INPUT SEED & Ue1 & 8 \\
\hline IS UPDATED TO A NEW SEFD USING A LINEAR CONGRUENTAL WETHOD. & U81 & 9 \\
\hline C* & UB1 & . 16 \\
\hline CURRENT USAGE IS FOR THE PRIME 75a, 32 BIT WORD LENGTH. & U日1 & 11 \\
\hline$C_{*}$ & U日1 & . 12 \\
\hline DSEED = REAL B SEED FOR RANDOW NUMBER GENERATOR. LUST BE BETTEEN & บ81 1 & . 13 \\
\hline GNE AND TWO TO THE THIRTY FIRST POKER. & Uø1 & 14 \\
\hline C* & Uol & 15 \\
\hline C* REFERENCE: & Ue1 & 16 \\
\hline C* & U⿺𠃊1 & . 17 \\
\hline LENIS, GOODWAN, AND MILLER (1969). 'A PSEUDO-RANDOM NUMEER & UE1 & 18 \\
\hline GEKERATOR FOR THE SYSTEN/368"' IBM SYSTEUS JDURNAL, VOL. 8 , & บ 1 & 19 \\
\hline ND. 2 , PP. 136-145. & U01 & . 20 \\
\hline$C *$ & Uळ1 & 21 \\
\hline$C_{*}$ & บด1 1 & . $\quad 22$ \\
\hline RENL*B B, $M$, DSEED & Uø1 & . $\quad 23$ \\
\hline C & Uø1 1 & . 24 \\
\hline DATAB B $16807,00+00 /$ & บอ1 & . $\quad 25$ \\
\hline DATA / $/ 2147483647.00+00 /$ & U81 & . 26 \\
\hline C & Uø1 & . 27 \\
\hline DSEED $=$ DINT( DMOD $(\mathrm{B} *$ DSEED, M) $)$ & บข1 & . $\quad 28$ \\
\hline c & U⿻1 & . $\quad 29$ \\
\hline Uø1 = SNGL $(D S E E D / N)$ & U日1 1 & . 30 \\
\hline c & U81 & . 31 \\
\hline RETURN & U81 & . $\quad 32$ \\
\hline
\end{tabular}




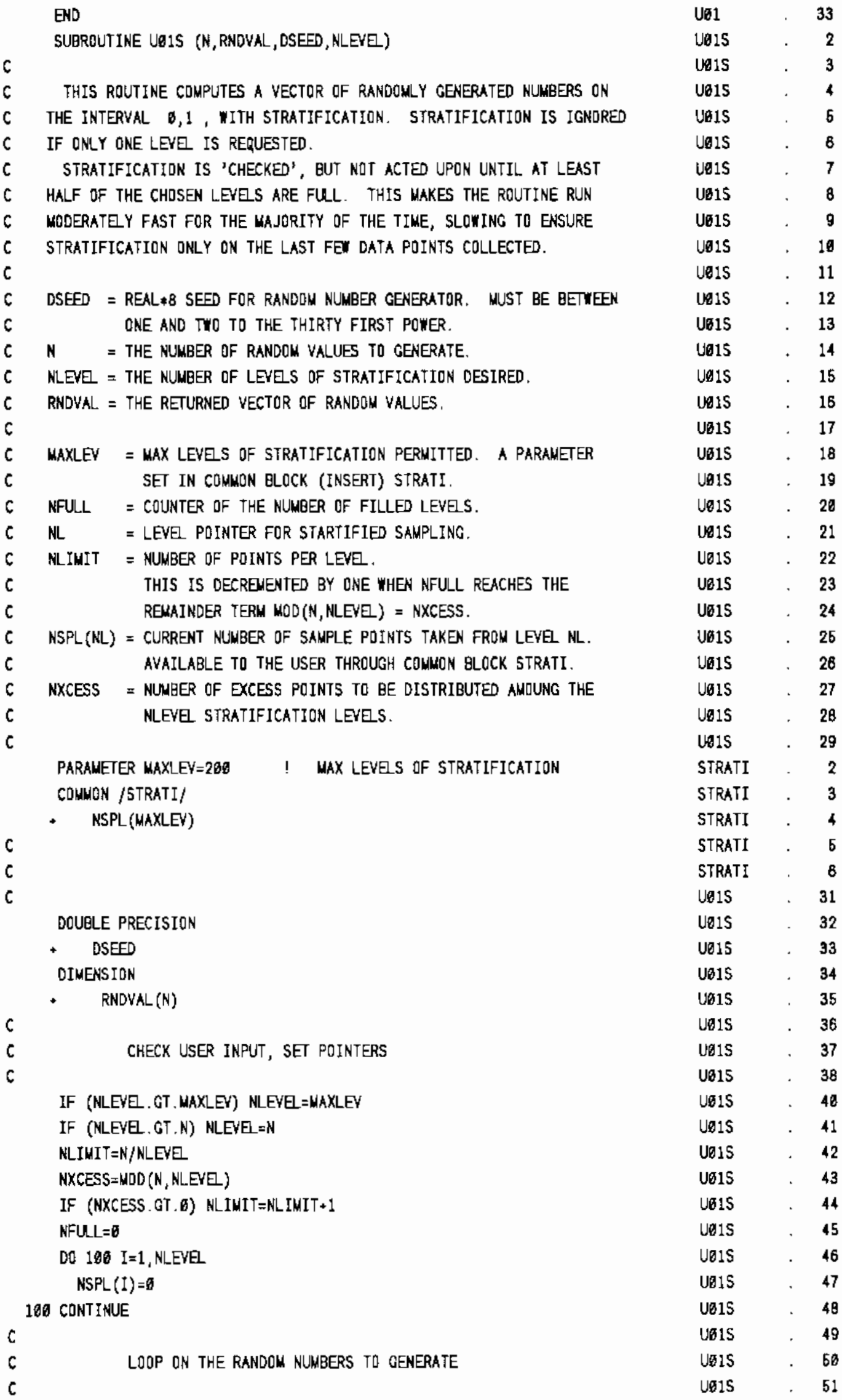




\begin{tabular}{|c|c|c|c|}
\hline \multicolumn{2}{|r|}{ DO $180 I=1, N$} & Ua:S & 52 \\
\hline \multirow[t]{3}{*}{118} & CONTINUE & UB1S & 53 \\
\hline & $A=\cup Q 1$ (DSEFD) & U61S & 54 \\
\hline & IF (NLEVE. EQ.1) GO TO $17 \theta$ & U日1s & 55 \\
\hline C & & Ua1S & 86 \\
\hline C & IF OVER HALF OF THE LEVES ARE FULL, & Uø1S & 57 \\
\hline c & GO TO A DIFFERENT ALGORITHM & Ue1s & 58 \\
\hline C & & U日1S & 59 \\
\hline & IF (NFULL.GT.NLEVE/2) GO TO 120 & vais & 60 \\
\hline C & & UB1S & 61 \\
\hline C---- & 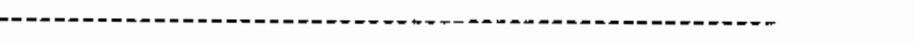 & Uø1S & 62 \\
\hline c & FAST ALGORITHM. COMPUTE RANDOM NUMBER, ANO CHECK IF & U日1S & 63 \\
\hline C & ITS LEVE DF STRATIFICATION IS NLREAOY FULL, GET NEX & UE1S & 64 \\
\hline C & RANDON NUMBER IF THAT LEVE IS FULL. & UB1S & 65 \\
\hline c & & Ua1S & 68 \\
\hline & NL=IFIX $(A * F L O A T(N L E Y B)) \cdot 1$ & Ue1S & . 67 \\
\hline & IF (NSPL(NL).GE. NLINIT) GO TO 118 & Ua1S & . $\quad 68$ \\
\hline C & & Uø1S & . $\quad 69$ \\
\hline C & INCREMENT COUNTER AND SAVE VALUE & Uø1S & 76 \\
\hline C & & Ue1S & . 71 \\
\hline & CO TO 158 & Ua1S & . 72 \\
\hline C & & UB1S & . 73 \\
\hline C---- & 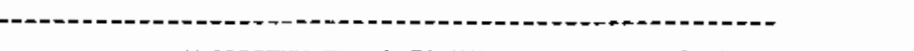 & Uø1S & 74 \\
\hline c & **** ALGORITHM FDR OVER HALF THE LEVES FULL **** & Vo1s & 75 \\
\hline C & & UQ1S & . 76 \\
\hline C & SLOW ALGORITHM. RANDOLLY LOCATES AN EAPTY STRATIFICATION & VA15 & 77 \\
\hline C & LEVE AND FORCES NEN RANDOH NUMBER TO BE IN THAT LEYE. & Uß1S & . 76 \\
\hline$c$ & & U61S & 79 \\
\hline 120 & CONTINUE & U日1S & 86 \\
\hline$c$ & & UQ1S & 81 \\
\hline$c$ & FIND A LEYE AT RANDOW WHICH IS NOT FILLED & Ue1S & . 82 \\
\hline C & & Uø1S & 83 \\
\hline & $W=\operatorname{IFIX}(A * F L O A T(N L E Y E L, N F U L L))+1$ & U615 & 84 \\
\hline & $N L=0$ & UQ1S & 85 \\
\hline & DD $130 \mathrm{~J}=1, \mathrm{NLEVE}$ & Ue1S & 86 \\
\hline & $K=J$ & Uø1S & 87 \\
\hline & IF (NSPL(J).LT, NLIMIT) NL=NL+1 & UQ1S & 88 \\
\hline & IF (NL.EA.W) GO TO 140 & vais & B9 \\
\hline 130 & CONTINUE & Uø1S & 96 \\
\hline & STOP 'UB1S' & U日1S & 91 \\
\hline 140 & CONTINUE & Ua15 & 92 \\
\hline & $\mathrm{NL}=\mathrm{K}$ & U61S & 93 \\
\hline C & & UQ1S & . 84 \\
\hline C & COMPUTE ENOPOINTS FOR THAT LEYEI AND & Ue1S & 95 \\
\hline$c$ & FIND A RANDOW VALUE WITHIN THAT LEYEL. & Ua1s & 96 \\
\hline$c$ & & veis & . 97 \\
\hline & $B=F L D A T(N L-1) / F L O A T$ (NLEYE) & UB1S & 98 \\
\hline & $T=F L O A T(N L) / F L O A T$ (NLEVEL) & U61S & 99 \\
\hline & $A=B+U B 1(D S E E D) *(T-B)$ & Ue1S & . 168 \\
\hline C---n & 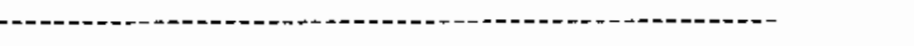 & UB1S & . 101 \\
\hline C & & Ua1S & . 102 \\
\hline C & INCREMENT \# ITEMS IN CURRENT LEVE,, CURRENT VARIABLE & U日1S & . 103 \\
\hline C & & Uo1s & . 164 \\
\hline 156 & CONTINUE & U日is & . 105 \\
\hline
\end{tabular}




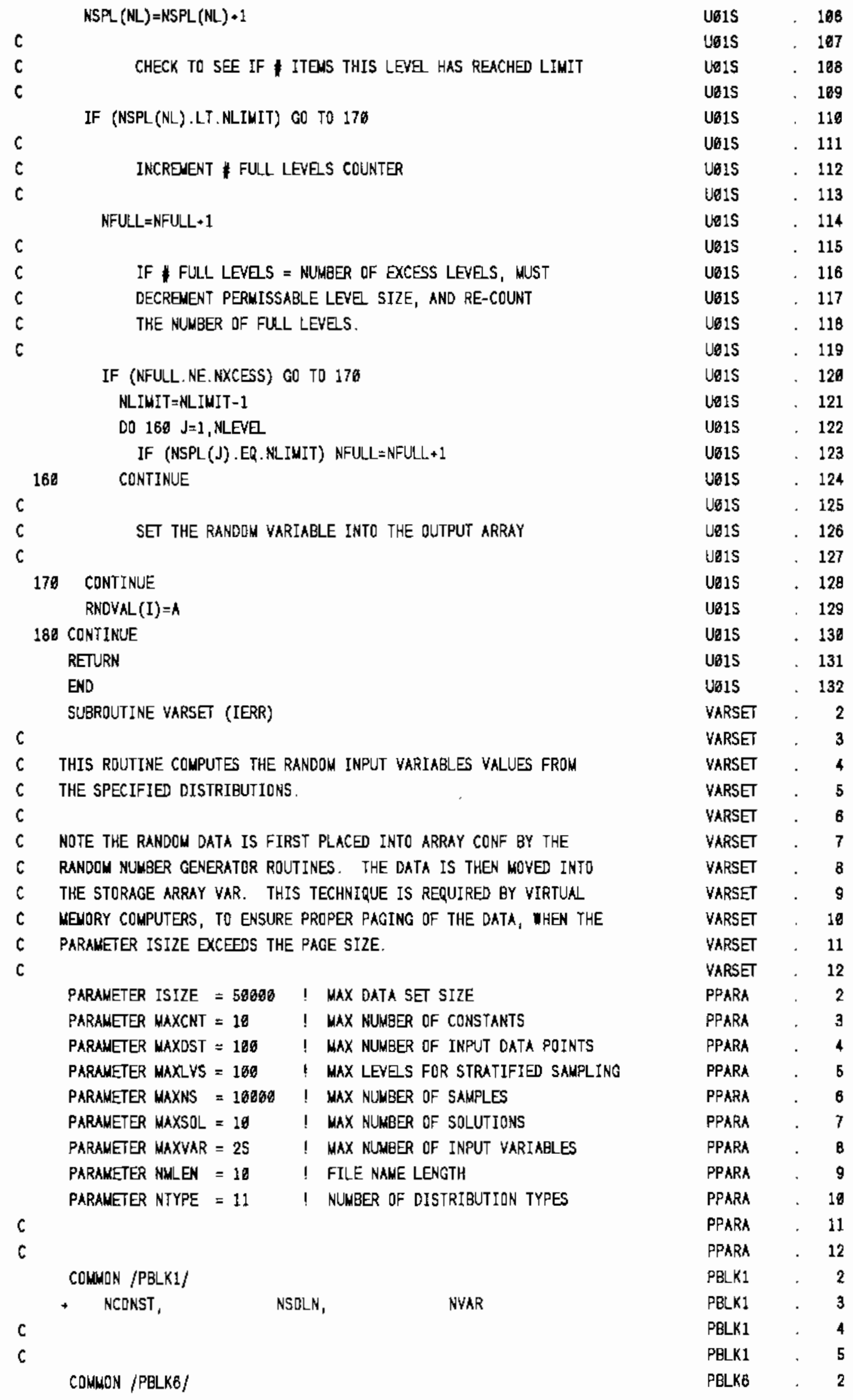




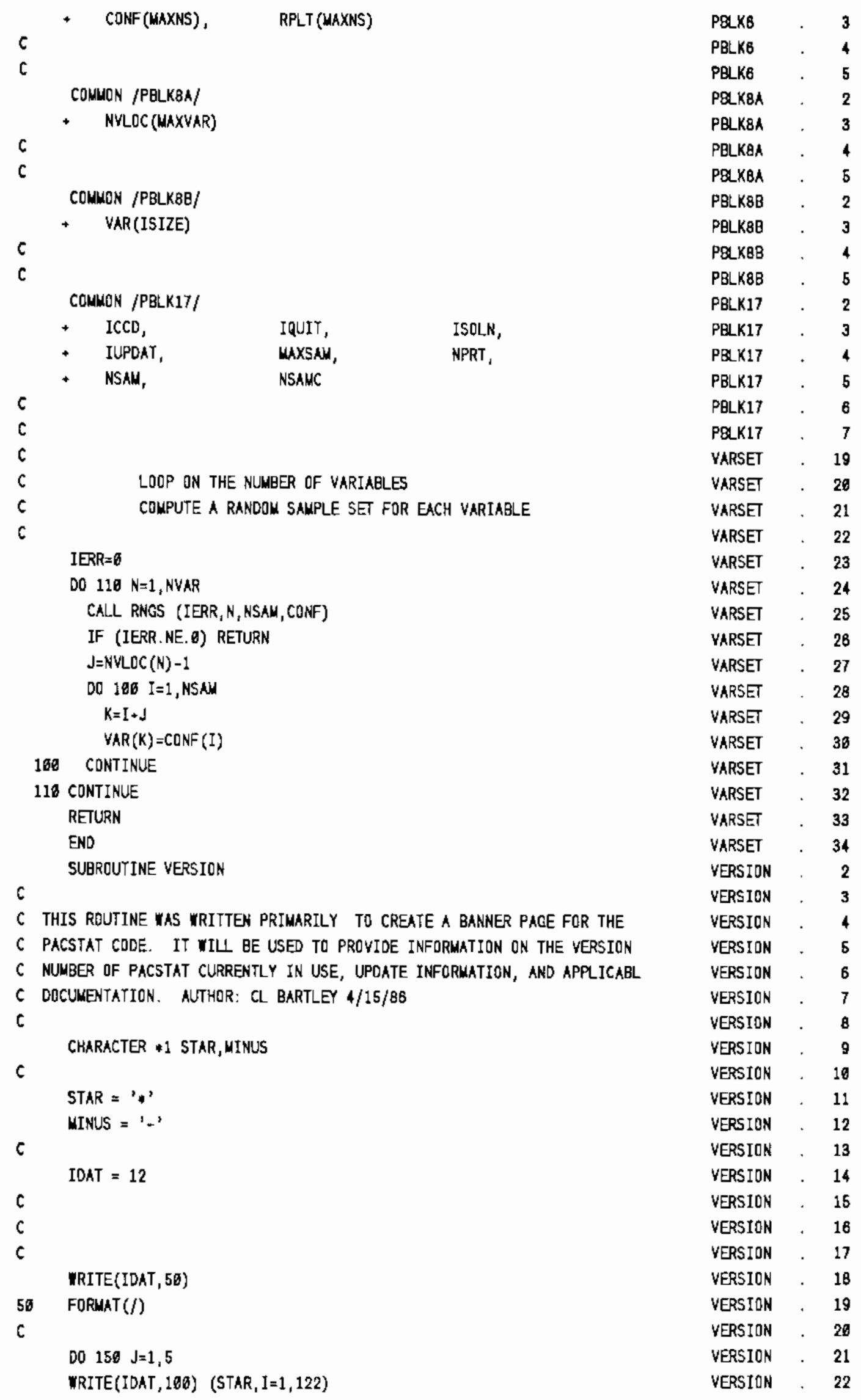




\begin{tabular}{|c|c|c|c|c|}
\hline 180 & \multicolumn{2}{|l|}{ FORUAT $(3(5) \times 122 A 1 /))$} & VERSION & 23 \\
\hline 150 & \multicolumn{2}{|l|}{ CONTINUE } & VERSION & 24 \\
\hline \multirow[t]{3}{*}{ c } & & & VERSION & 25 \\
\hline & \multicolumn{2}{|l|}{ DO $25 \delta \mathrm{J}=1,2$} & VERSION & 28 \\
\hline & \multicolumn{2}{|l|}{ WRITE(IDAT, 206) (STAR, I=1,46) } & VERSION & 27 \\
\hline 260 & \multicolumn{2}{|l|}{ FORMAT $(5 X, 20 A 1,82 X, 20 A 1 /)$} & VERSION & 28 \\
\hline 250 & \multicolumn{2}{|l|}{ CONTINUE } & VERSION & 29 \\
\hline \multirow[t]{2}{*}{ c } & & & VERSION & 30 \\
\hline & \multicolumn{2}{|l|}{ WRITE(IOAT, 386) (STAR, I=1,48) } & VERSIDN & 31 \\
\hline 360 & \multicolumn{2}{|c|}{ FORMAT (5X, 29A1,34X,'P A C S T A T', 35X,29A1) } & VERSION & 32 \\
\hline \multirow[t]{2}{*}{ C } & & & VERSION & 33 \\
\hline & \multicolumn{2}{|l|}{ MRITE $($ IDAT, 400) (STAR, I=1,40) } & VERSION & 34 \\
\hline \multirow[t]{2}{*}{408} & \multirow{2}{*}{\multicolumn{2}{|c|}{ FORMAT $(/ / 5 X, 20 A 1,23 X$, 'CODE VERSIDN AND UPDATE INFORMATION', 24X, }} & VERSION & 35 \\
\hline & & & VERSION & 36 \\
\hline \multirow[t]{2}{*}{$c$} & & & VERSION & 37 \\
\hline & WRITE(IDAT, 506) (STAR, I=1,46) & & VERSION & 38 \\
\hline \multirow[t]{2}{*}{590} & FORMAT $\left(/ 5 X, 20 A 1,22 X,{ }^{2}\right.$ 'WITTEN FOR PRIN & 750 BY D. LANGFORD', & VERSION & 39 \\
\hline & $124 \times, 20 A 1)$ & & VERSION & 48 \\
\hline \multirow[t]{2}{*}{ C } & & & VERSION & 41 \\
\hline & WRITE(IDAT, 600) $(S T A R, I=1,40)$ & & VERSION & 42 \\
\hline \multirow[t]{2}{*}{600} & FORMAT $(/ 5 X, 20 A 1,11 X$, 'CONVERTED TO VA) & $1 / 78 \sigma$ BY C. BARTLEY AND " & VERSION & 43 \\
\hline & $1^{\prime} M$. BUDDEN $\left.-4 / 86^{\prime}, 13 \times, 26 \wedge 1\right)$ & & VERSION & 44 \\
\hline \multirow[t]{2}{*}{ C } & & & VERSION & 45 \\
\hline & RITE $(I D A T, 700) \quad(S T A R, I=1,40)$ & & VERSION & 48 \\
\hline 700 & FORMAT $(/ 5 X, 20 A 1,26 X, ' A P P L I C A B L E$ DOCUN & ITS TO FULLOW', 26X, 26A1) & VERSIDN & 47 \\
\hline \multirow[t]{2}{*}{ c } & & & VERSION & 48 \\
\hline & WRITE(IDAT, 860) (STAR, I=1,40) & & VERSION & 49 \\
\hline 880 & FDRMAT $(/ 5 X, 20 A 1,28 X$, 'VAX VERSION NUME & $\left.(B 4)^{\prime}, 28 \times, 28 \times 1\right)$ & VERSION & 60 \\
\hline C & & & VERSION & $\$ 1$ \\
\hline & MRITE $(I D A T, 816) \quad(S T A R, I=1,4 \theta)$ & & VERSION & 52 \\
\hline 810 & FORAAT $\left(/ 5 X, 26 A 1,5 X,{ }^{\prime} D I R=[B W I P 86, P A C\right.$ & AT. B4 $\left.\}^{\prime}, 52 X, 20 A 1\right)$ & VERSION & 59 \\
\hline & WRITE(IDAT, 828) (STAR, I=1,48) & & VERSION & 54 \\
\hline 828 & FORMAT $\left(/ 5 X, 20 A 1,10 X,{ }^{\prime}\right.$ PACSTAT_B4.SRC & WITNESS SQURCE FILE', $34 X_{1}$ & VERSION & 55 \\
\hline & $\$ 20 A 1)$ & & VERSION & 56 \\
\hline & WITE(IDAT, 896) (STAR, I=1, 46) & & VERSION & 57 \\
\hline 830 & FORWAT $\left(/ 5 X, 20 A 1,10 X X_{1}{ }^{\prime}\right.$ PACSTAT_B4.OPL & WITNESS OLD PROGRAN LIBRAR & VERSION & 58 \\
\hline & $\left.3 Y^{\prime}, 26 x, 26 \wedge 1\right)$ & & VERSION & 59 \\
\hline & WRITE(IDAT, 840) (STAR, I=1,40) & & VERSIDN & 68 \\
\hline 840 & FORHAT $(/ 5 X, 20 A 1,18 X$, 'PACSTAT_B4.0L8 & WITNESS OBJECT LIBRARY', & VERSIDN & 81 \\
\hline & $\$ 31 x, 20 A 1)$ & & VERSION & 62 \\
\hline & WITE(IDAT, 85 $) \quad(S T A R, I=1,4 \theta)$ & & VERSION & 63 \\
\hline 850 & FORMAT (/5X,20A1,16X, 'PACSTAT_B4,FOR & MITNESS FORTRAN COWPILE & VERSION & 84 \\
\hline & SFILE', 23X,28A1) & & VERSION & 65 \\
\hline & MRITE(IDAT, 666) (STAR, I=1,46) & & VERSION & 66 \\
\hline 868 & FORMAT (/5X,20A1, 10X, 'PACSTAT_B4.LIS & FORTRAN LIST FILE', 36X, & VERSION & 67 \\
\hline & $\$ 20 \wedge 1)$ & & VERSIDN & 68 \\
\hline & WRITE $($ IDAT, 816) $\quad(S T A R, I=1,40)$ & & VERSION & 69 \\
\hline & DO $950 \mathrm{~J}=1,5$ & & VERSION & 70 \\
\hline & MRITE(IDAT, 200) $($ STAR, I=1,40) & & VERSION & 71 \\
\hline 950 & CONTINUE & & VERSION & 72 \\
\hline $\mathrm{C}$ & & & VERSIDN & 73 \\
\hline & Do $1050 \mathrm{~J}=1,5$ & & VERSION & 74 \\
\hline & WRITE(IDAT, 108) (STAR, I=1, 122) & & VERSION & 75 \\
\hline 1058 & CONTINUE & & VERSION & 76 \\
\hline
\end{tabular}




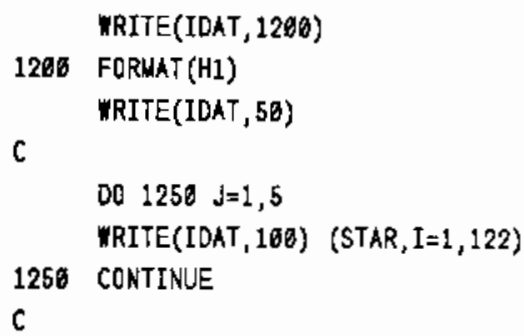

\begin{tabular}{|c|c|}
\hline & \\
\hline RSION & \\
\hline RSION & \\
\hline ERSION & \\
\hline RSIDN & \\
\hline ON & \\
\hline ERSION & \\
\hline ERSION & 8 \\
\hline ESION & \\
\hline RSION & \\
\hline CRSION & \\
\hline ERSION & 88 \\
\hline ERSION & 89 \\
\hline ERSION & \\
\hline ERSION & \\
\hline ERSION & 9 \\
\hline ERSION & 93 \\
\hline ERSION & \\
\hline ERSION & \\
\hline ERSION & . \\
\hline VERSION & 97 \\
\hline ERSION & 98 \\
\hline ERSION & 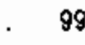 \\
\hline ERSION & 160 \\
\hline ERSION & 101 \\
\hline IERSION & 102 \\
\hline VERSION & 103 \\
\hline VERSION & 104 \\
\hline VERSION & 165 \\
\hline ON & 106 \\
\hline ERSION & 107 \\
\hline VERSION & 168 \\
\hline $2 N$ & 189 \\
\hline ERSIDN & 110 \\
\hline RSION & 111 \\
\hline VERSION & 112 \\
\hline ESION & 113 \\
\hline ERSION & 114 \\
\hline VERSION & 115 \\
\hline RSION & 116 \\
\hline YERSION & 117 \\
\hline VERSION & 118 \\
\hline ERSION & 119 \\
\hline VERSIDN & 120 \\
\hline ERSION & 121 \\
\hline VERSION & 122 \\
\hline VERSION & 123 \\
\hline VERSION & 124 \\
\hline YERSION & 125 \\
\hline VERSION & 100 \\
\hline VERSIDN & 127 \\
\hline VERSION & 128 \\
\hline ERSIDN & $12 s$ \\
\hline ESION & 138 \\
\hline
\end{tabular}




\begin{tabular}{|c|c|c|c|c|}
\hline & \multicolumn{2}{|c|}{ VRITE(IDAT, 18B) (STAR, I=1,122) } & VERSION & 131 \\
\hline 2608 & \multicolumn{2}{|l|}{ CONTINUE } & VERSION & 132 \\
\hline \multicolumn{3}{|l|}{ c } & VERSION & 133 \\
\hline \multirow[t]{6}{*}{1380} & \multicolumn{2}{|c|}{ FORMAT $(5 X, 20 A 1,10 X$, 'THESE CARDS ARE IDENTIFIED BY THE PATTERN' } & VERSION & 134 \\
\hline & \multicolumn{2}{|c|}{ s' '****' IN COLUMNS 1 - 4 OF EACH OATA CARD.') } & VERSION & 135 \\
\hline & \multicolumn{2}{|l|}{ WITE(IDAT, 1200) } & VERSION & 138 \\
\hline & \multicolumn{2}{|l|}{ RETURN } & VERSION & 197 \\
\hline & \multicolumn{2}{|l|}{ END } & VERSION & 138 \\
\hline & \multicolumn{2}{|c|}{ SUBROUTINE WHEADR (IFLL, IERR) } & WHEADR & 2 \\
\hline \multicolumn{3}{|l|}{ C } & WHEADR & $\mathbf{3}$ \\
\hline$c$ & \multicolumn{2}{|c|}{ THIS RDUTINE WRITES FILE HEADER RECORD(S) TO THE BINARY DATA } & WHEADR & 4 \\
\hline C & \multicolumn{2}{|c|}{ FILES ASSOCIATED WITH THE MAGNUM AND CHAINT PROGRAMS. THE FIRST } & WHEADR & 5 \\
\hline C & \multicolumn{2}{|c|}{ RECORD CONTAINS INFORMATION CONCERNING THE IOENTITY OF THE CURRENT } & WHEAOR & 6 \\
\hline C & \multicolumn{2}{|c|}{ FILE. THE SECOND RECORD, IF NEFDED, CONTAINS A BRIEF SUMMARY OF } & WHEADR & 7 \\
\hline C & \multicolumn{2}{|c|}{ THE FILES WHICH WERE USED AS SOURCE DATA FOR THE RUN WHICH CREATED } & WHEADR & 8 \\
\hline C & \multicolumn{2}{|l|}{ THE CURRENT FILE. } & WHEAOR & 9 \\
\hline \multicolumn{3}{|l|}{ C } & WHEADR & 10 \\
\hline \multicolumn{3}{|c|}{ C--CONTROL DATA } & WHEAOR & 11 \\
\hline c & \multicolumn{2}{|c|}{ IFLO = OUTPUT FILE UNIT NUWBER } & WHEADR & 12 \\
\hline C & IERR $=$ ERROR POINTER, $=\theta$ & FOR SUCCESSFUL HEADER WRITE & WEADR & 13 \\
\hline C & & & WHEADR & 14 \\
\hline C--HE & EADER RECORD INFORWATION & & WHEADR & 16 \\
\hline C & NSRC = NUNBER OF FILE T & ACEBACK RECORDS & WHEADR & 16 \\
\hline C & JRUNID = IDENTIFIER (DATE & TIME) OF SOURCE RUN & WHEADR & 17 \\
\hline$c$ & USRNAH = NAME OF USER $* H O$ & INITIATED SOURCE RUN (CHAR*8) & WEADR & 18 \\
\hline C & $=$ NAME OF SOURCE $P$ & OGRAM (CHAR*8) & WHEADR & 19 \\
\hline$c$ & $=$ FILE TYPE NAHE (C & $\mathrm{HAR} * \mathrm{~B})$ & MHEADR & 20 \\
\hline c & VERNUM = VERSION RUMBER OF & SOURCE PROGRAM & WHEADR & 21 \\
\hline C & TITLE = TITLE FROW SOURCE & RUN (CHAR*86) & WHEADR & 22 \\
\hline $\mathrm{C}$ & & & WHEADR & 23 \\
\hline C--TR & RACEBACK RECORD INFORWATIOI & (ONIT IF NSRC $=6$ ) & WHEADR & 24 \\
\hline C & NRUNIO $(\mathrm{I})=$ IDENTIFIER OF & INPUT FILE I & WEADR & 25 \\
\hline C & $=$ FILE TYPE OF & NPUT FILE I (CHAR*B) & WHEADR & 26 \\
\hline C & PRGT(I) = SOURCE PROGRA & OF INPUT FILE I (CHAR*8) & MHEADR & 27 \\
\hline C & $\operatorname{USRNAT}(I)=$ SOURCE PROGRA & USER (CHAR*8) & WHEADR & $2 B$ \\
\hline c & & & WHEADR & 29 \\
\hline & PARAMETER ISIZE $=50000$ & ! WAX DATA SET SIZE & PPARA & 2 \\
\hline & PARAMETER MAXCNT $=16$ & ! MAX NUMBER OF CONSTANTS & PPARA & 3 \\
\hline & PARANETER MAXDST $=100$ & ! MAX NUMBER OF INPUT DATA POINTS & PPARA & 4 \\
\hline & PARANETER HAXLVS $=100$ & ! MAX LEVESS FOR STRATIFIED SAMPLING & PPARA & 5 \\
\hline & PARAMETER MAXNS $=10000$ & ! MAX NUWHER OF SAMPLES & PPARA & 8 \\
\hline & PARAMETER WAXSOL $=10$ & ! WAX NUMBER OF SOLUTIONS & PPARA & 7 \\
\hline & PARAMETER MAXVAR $=25$ & I WAX NUWBER OF INPUT VARIABRES & PPARA & 8 \\
\hline & PARAMETER NMLEN $=16$ & ! FILE NAME LENGTH & PPARA & g \\
\hline & PARAMETER NTYPE $=11$ & ! NUWEER OF DISTRIBUTION TYPES & PPARA & 16 \\
\hline C & & & PPARA & 11 \\
\hline C & & & PPARA & 12 \\
\hline & PARANETER MAXSRC $=15$ & & IDCOH & 2 \\
\hline & COWMON /IDCOM/ & & IDCON & 3 \\
\hline & $+\quad$ JRUNID, & FLT (MAXSRC), & IOCOM & 4 \\
\hline & $+\quad$ NRUNID (MAXSRC) & PRG, & IDCOM & \\
\hline & $+\quad$ PRGT (WAXSRC) & USRNAM, & IOCOM & \\
\hline & $+\quad$ USRNAT (MAXSRC) & ERNUM & IDCON & \\
\hline & CHARACTER*4 & & IDCON & \\
\hline
\end{tabular}




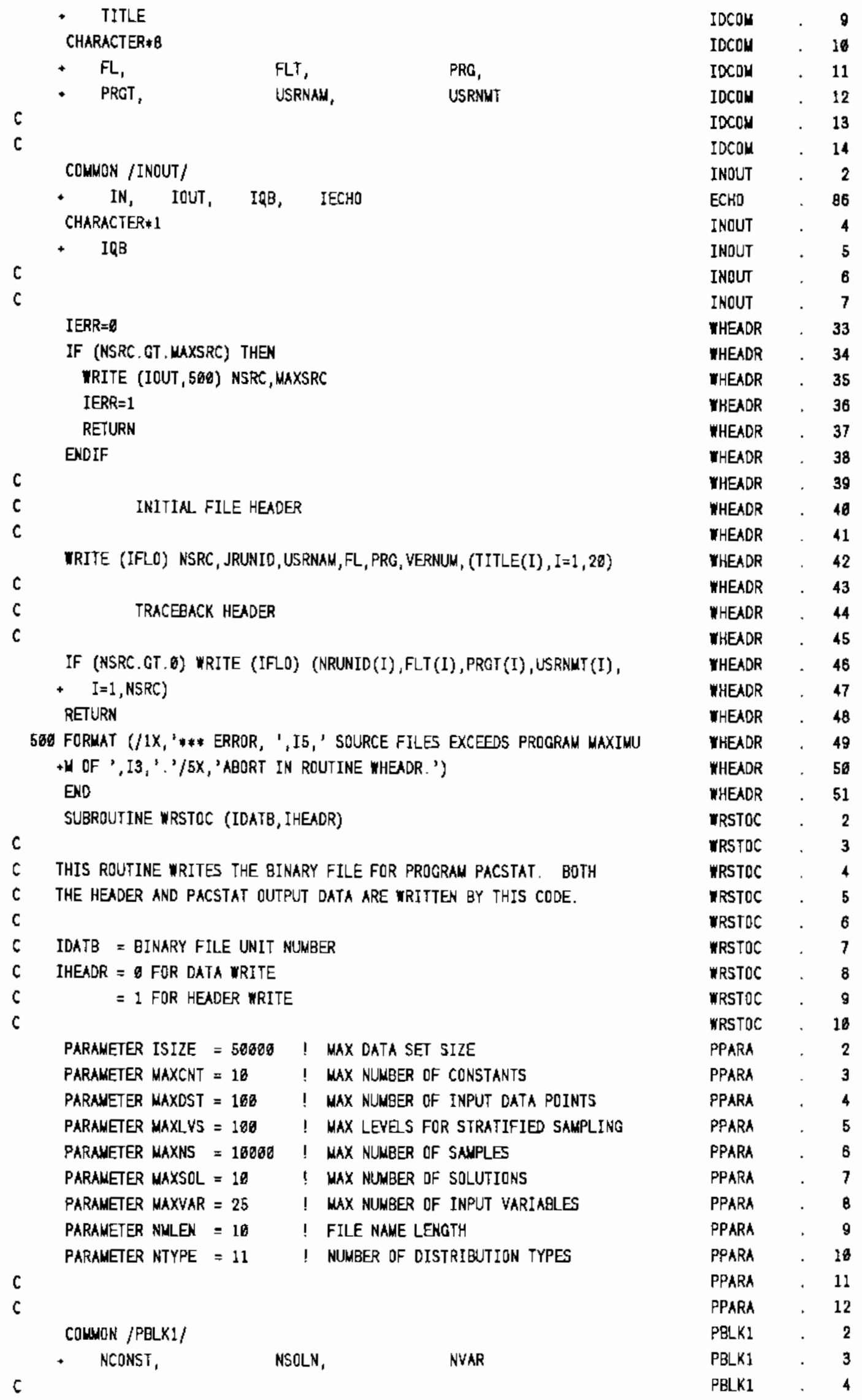


c

CONHON /PBLK2/

+ NSTTL,

- tre

CHARACTER:46

c

- STtLe,

c

COMNON /PBLK3/

+ CoImen( (naXCNT),

- VDIMEN(MAXVAR), CHARACTER*12

+ CDINEN,

+ Vlabel

CHARACTER $* 32$

- plabe

$c$

c

CDMMON /PELK4]

- CONST (MAXCNT)

6

c

COWNON /PBLK5/

- ICNT,

c

c

CDMMON /PELX7A/

- NSLOC (MAXSOL)

c

c

COMMON /PBLK78/

$+\quad$ RLTS(ISIZE)

c

c

COMNON /PBLKEA/

- NYLOC (MAXYRR)

$c$

c

COMNON /PBLKBB/

$c$

- var(ISIZE)

c

COMMON /PBLK15/

- ILIM(MAXYAR),

- par(MAXYar,3),

- VLIMT (MAXVAR, 2)

$c$

c

COMNDN /PBLX17/

$+\quad \mathrm{ICCD}_{1}$

+ IUPOAT,

+ NSAM,

$c$

C
NTTL, STTLE,

TTLE

CLABE (MAXCNT), PLABE (MAXSOL),

VLABE (MAXYAR)

CLABE,

VDIMEN,

VALU(MAXSOL),

VINPT (MAXVAR)

IDAT,

INDEX

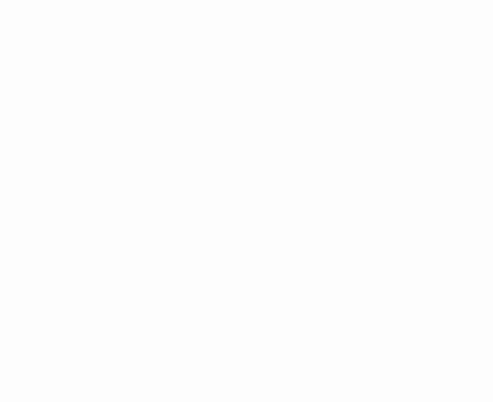

ISOLN, NPRT,

\begin{tabular}{|c|c|}
\hline PBLKI & \\
\hline PELK2 & \\
\hline PBL.K2 & \\
\hline PBLK2 & \\
\hline PBLK2 & \\
\hline PQLK2 & \\
\hline PELK2 & \\
\hline PBLK2 & \\
\hline PBLK3 & \\
\hline PBLK3 & \\
\hline PQLK3 & \\
\hline PBLK3 & \\
\hline PBLK3 & \\
\hline PELK3 & \\
\hline PEL X3 & \\
\hline PBLK3 & \\
\hline PBLK3 & \\
\hline PBLK3 & \\
\hline PELK4 & \\
\hline PBLKK4 & \\
\hline PBLK4 & \\
\hline PBLK4 & \\
\hline PELK5 & \\
\hline PELK5 & \\
\hline PBLX5 & \\
\hline PBLK5 & \\
\hline PBLK7A & \\
\hline PBLK7A & \\
\hline PBLK7A & \\
\hline PELKTA & \\
\hline PBLK7B & \\
\hline PBLK7B & \\
\hline PBLK7B & \\
\hline PBLK7B & \\
\hline PBLKBA & \\
\hline PBLKBA & \\
\hline PBLK8A & \\
\hline PQLKBA & \\
\hline PBLKOB & \\
\hline PBLK\&B & \\
\hline PBLKBB & \\
\hline PBLK8B & \\
\hline PBLK15 & \\
\hline PBLK15 & \\
\hline PQLK15 & \\
\hline PQLK15 & \\
\hline PELX15 & . \\
\hline PBLK15 & \\
\hline PBLKK17 & \\
\hline PBLK17 & \\
\hline PELK17 & \\
\hline PQLK17 & \\
\hline PBLK17 & \\
\hline PBLK17 & \\
\hline
\end{tabular}




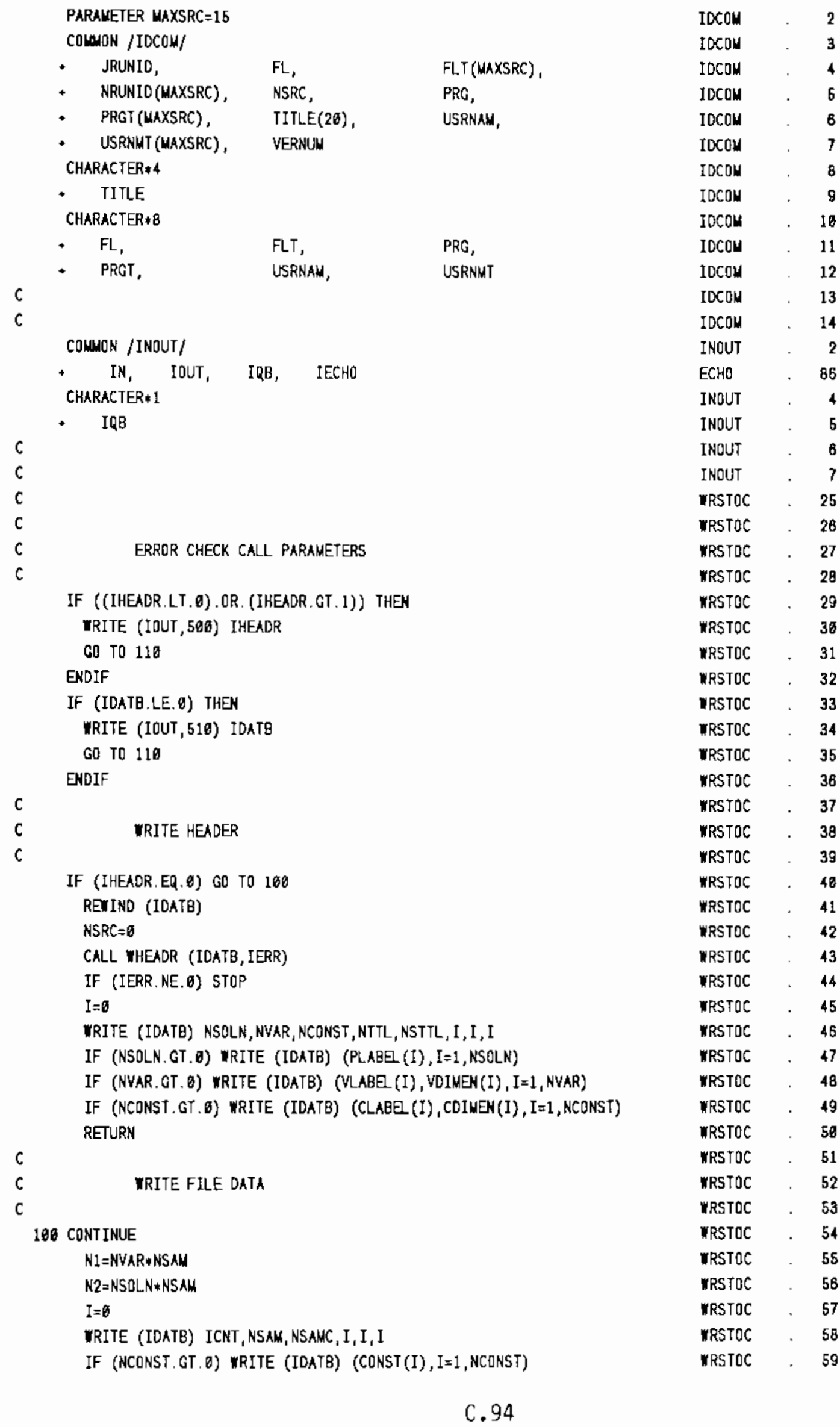




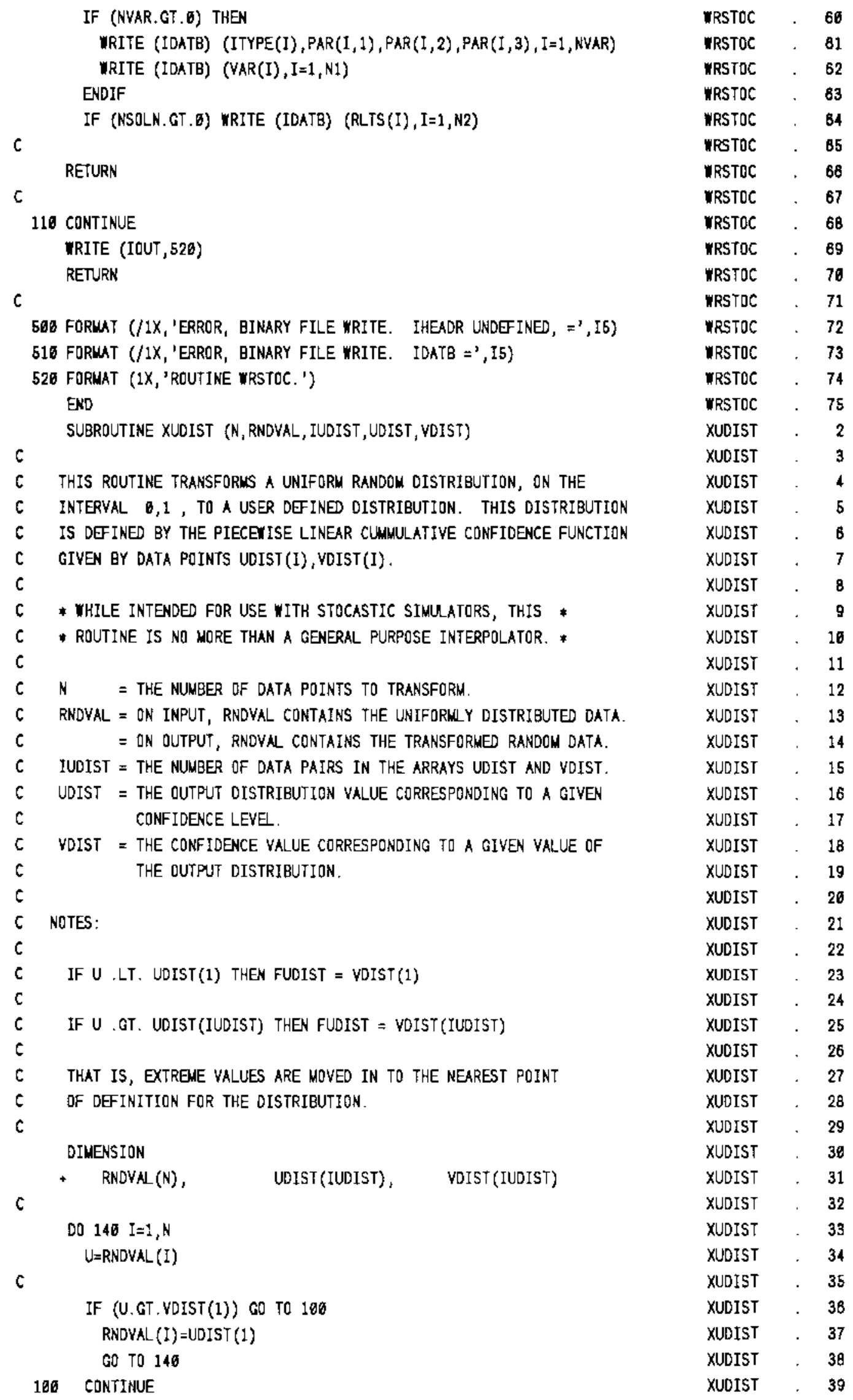




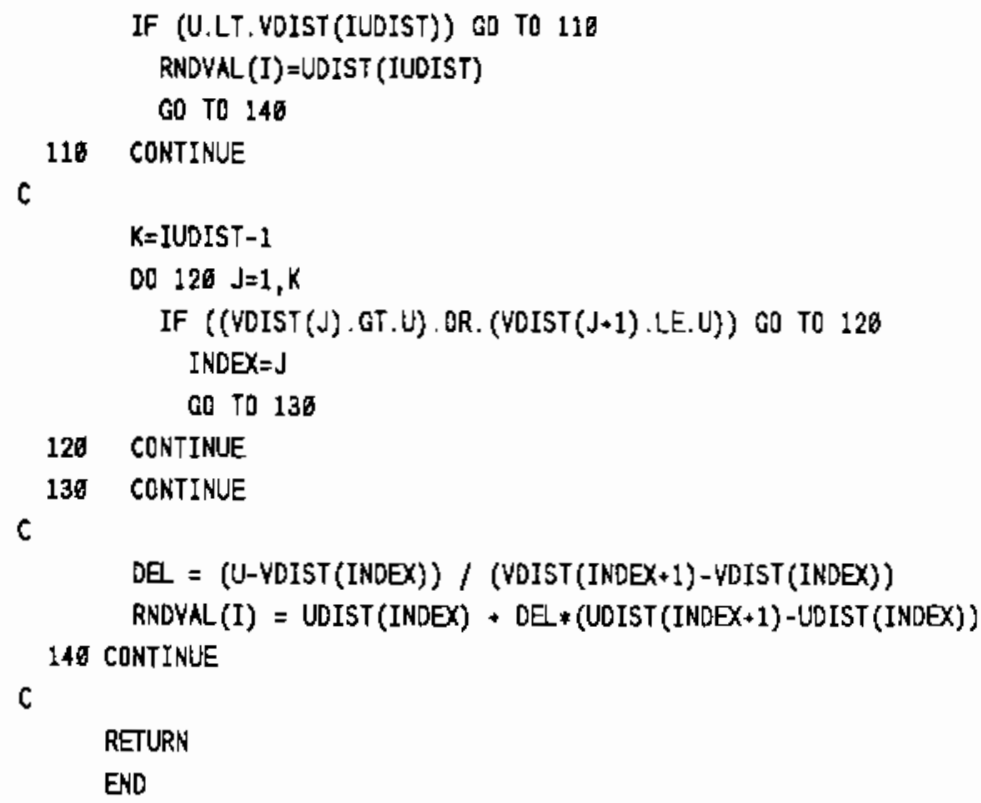

$\begin{array}{lll}\text { XUDIST } & \cdot & 46 \\ \text { XUDIST } & \cdot & 41 \\ \text { XUDIST } & \cdot & 42 \\ \text { XUDIST } & \cdot & 43 \\ \text { XUDIST } & \cdot & 44 \\ \text { XUDIST } & \cdot & 45 \\ \text { XUDIST } & \cdot & 46 \\ \text { XUDIST } & \cdot & 47 \\ \text { XUDIST } & \cdot & 48 \\ \text { XUDIST } & \cdot & 49 \\ \text { XUDIST } & \cdot & 56 \\ \text { XUDIST } & \cdot & 51 \\ \text { XUDIST } & \cdot & 52 \\ \text { XUDIST } & \cdot & 53 \\ \text { XUDIST } & \cdot & 54 \\ \text { XUDIST } & \cdot & 55 \\ \text { XUDIST } & \cdot & 56 \\ \text { XUDIST } & \cdot & 57 \\ \text { XUDIST } & \cdot & 58\end{array}$


APPENDIX D

PACSTAT UPDATE FILES 
PACSTAT_AO.UPD

0.1 


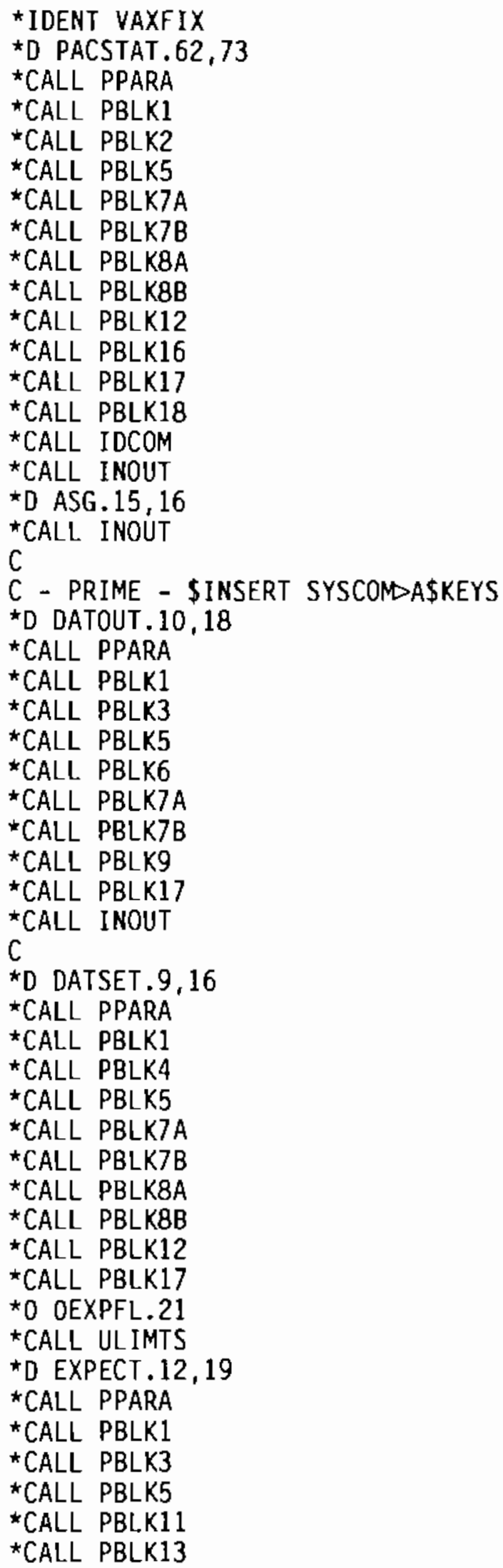

PACSTAT_AO.UPD.1 


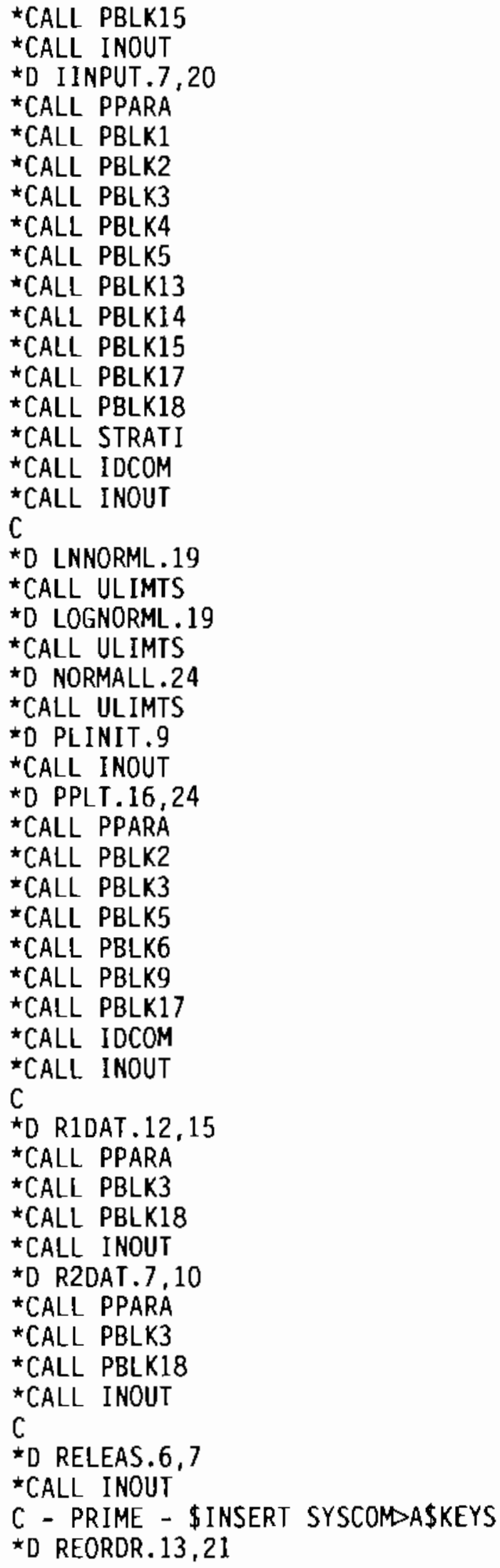

PACSTAT_AO.UPD.2

0.3 
*CAll pPara

*CALL PBLK1

*CALL PBLK5

*CALL PBLK6

*CALL PBLK7A

*CALL PBLK7B

*CALL PBLKBA

*CALL PBLKBB

*CALL PBLK12

^CALL PBLK17

*CALL INOUT

*D RNGS.16,22

*CAll PPARA

${ }^{\star}$ CALL PBLK13

*CALL PBLK15

*CALL PBLK16

*CALL PBLK18

*CALl STRATI

*CALL ULIMTS

*CALL INOUT

*D SOLN.29,32

*CALL PPARA

*CALL PBLK4

*CALL PBLK5

*CALL INOUT

*D STATOT.19,30

*CALL PPARA

*CALL PBLK1

*CALL PBLK3

${ }^{\star}$ CALL PBLK5

*CALL PBLK6

*CALL PBLK7A

*CALL PBLK7B

*CALl PBLK8A

*CALL PBLK8B

*CALL PBLK10

*CALL PBLK13

*CALL PBLK15

*CALL PBLKI7

*CALL INOUT

*D U01S.30

*CALl STRATI

*D VARSET $.13,17$

*CALL PPARA

*CALL PBLK1

*CALL PBLK6

*CALL PBLKBA

*CALL PBLK8B

*CALL PBLK17

*D WHEADR. 30,31

*CALL PPARA

*CALL IDCOM

PACSTAT_A0.UPD. 3

D. 4 
*CALL INOUT

$\star D$ WRSTOC. 11,22

*CALL PPARA

*CALL PBLK1

*CALL PBLK2

*CALL PBLK3

*CALL PBLK4

*CALL PBLK5

*CALL PBLK7A

*CALL PBLK7B

*CALL PBLK8A

*CALL PBLK8B

*CALL PBLK15

*CALL PBLK17

*CALL IDCOM

${ }^{\star}$ CALL INOUT

$\mathrm{C}$

*PURDECK JCLPRIM

*PURDECK MANUAL

*PURDECK BLOCK

*PURDECK SOLN2

«PURDECK OUTPUT

\#PURDECK BLOCK2

$\star D$ PPARA. 2, 10

PARAMETER ISIZE $=50000$

PARAMETER MAXCNT $=10$

PARAMETER MAXDST $=100$

PARAMETER MAXLVS $=100$

PARAMETER MAXNS $=10000$

PARAMETER MAXSOL $=10$

PARAMETER MAXVAR $=25$

PARAMETER NMLEN $=10$

PARAMETER NTYPE $=11$

${ }^{\circ}$ W WRSTOC. 73,74

*D BLKDAT. 14,18

*CALL PPARA

*CALL PBLK1

*CALL PBLK2

*CALL PBLK3

*CALL IDCDM

$C$ - PRIME - \$INSERT SYSTEMS $>$ UTIL $>$ PACSTAT $>$ COMMON>PPARA

C - PRIME - \$INSERT SYSTEMS>UTIL $>P A C S T A T>C O M M O N>P B L K 1$

$C$ - PRIME - \$INSERT SYSTEMS $>U T I L>P A C S T A T>C O M M O N>P B L K 2$

$C$ - PRIME - \$INSERT SYSTEMS>UTIL>PACSTAT>COMMON>PBLK3

$C$ - PRIME - \$INSERT SYSTEMS>UTIL>PACSTAT>COMMDN>IDCOM

$\star$ D EXPECT. 40,44

*I EXPECT.23

C

c

OUTPUT FILE UNIT

C

$$
\begin{aligned}
& \text { II I = IDAT } \\
& \text { IF (IDAT.EQ.0) II I = IOUT }
\end{aligned}
$$

PACSTAT_AO.UPD. 4

D. 5 
*D ALNORM. 20

$$
T=\operatorname{ABS}(X)
$$

$Z=\operatorname{DBLE}(T)$

*D ALNORM. 22

IF (T.LT.UTZERO) GO TO 100

*D ALNORM.27

IF (T.GT.CON) GO TO 110

*D PACSTAT.124,126

MAXSAM = ISIZE/NVAR

$I=I S I Z E$

IF (NSOLN .GT. O) I=ISIZE/NSOLN

IF (I .LT. MAXSAM) MAXSAM $=$ I

*D ASG. 52

C LOGIC = EXST\$A (INF, INTS (NML4))

*D ASG.75

C LOGIC = OPEN\$A(A\$READ+A\$SAMF, INF, INTS(NML4), INTS(IFUNIT))

*D ASG.77

C LOGIC = OPEN\$A(A\$RDWR+A\$SAMF, INF, INTS(NML4), INTS(IFUNIT))

*D ASG.104

C LOGIC = DELE\$A(INF, INTS(NML4))

${ }^{\star} D$ ASG.111

C LOGIC = OPEN\$A(A\$WRIT+ASAMF, INF, INTS(NML4), INTS(IFUNIT))

${ }^{\star} D$ RELEAS. 15

C LOGIC $=$ CLOS\$A(INTS(IFUNIT))

C CLOSING VAX OPEN STATEMENTS

C

CLOSE (UNIT $=12)$

CLOSE (UNIT=13)

LOGIC $=$.TRUE.

*D PACSTAT. 158

C CALL IDNUM (JRUNID, USRNAM)

C

C VAX EQUIVALENT CALL TO IDNUM

C

*D IDNUM. 42,44

C CALL TIMDAT (LOCAL(1), INTS(16))

C CALL DATE\$A (DATE)

C CALL TIME\$A (TIME)

*D PACSTAT.94,98

$\mathrm{IN}=5$

IOUT $=6$

IDAT $=12$

IDATB $=13$

INFIL $=35$

CALL ASGVAX

C

C PRINT BANNER PAGE

C

CALL VERSION

*D PACSTAT.137

PACSTAT_A0.UPD. 5

D. 6 


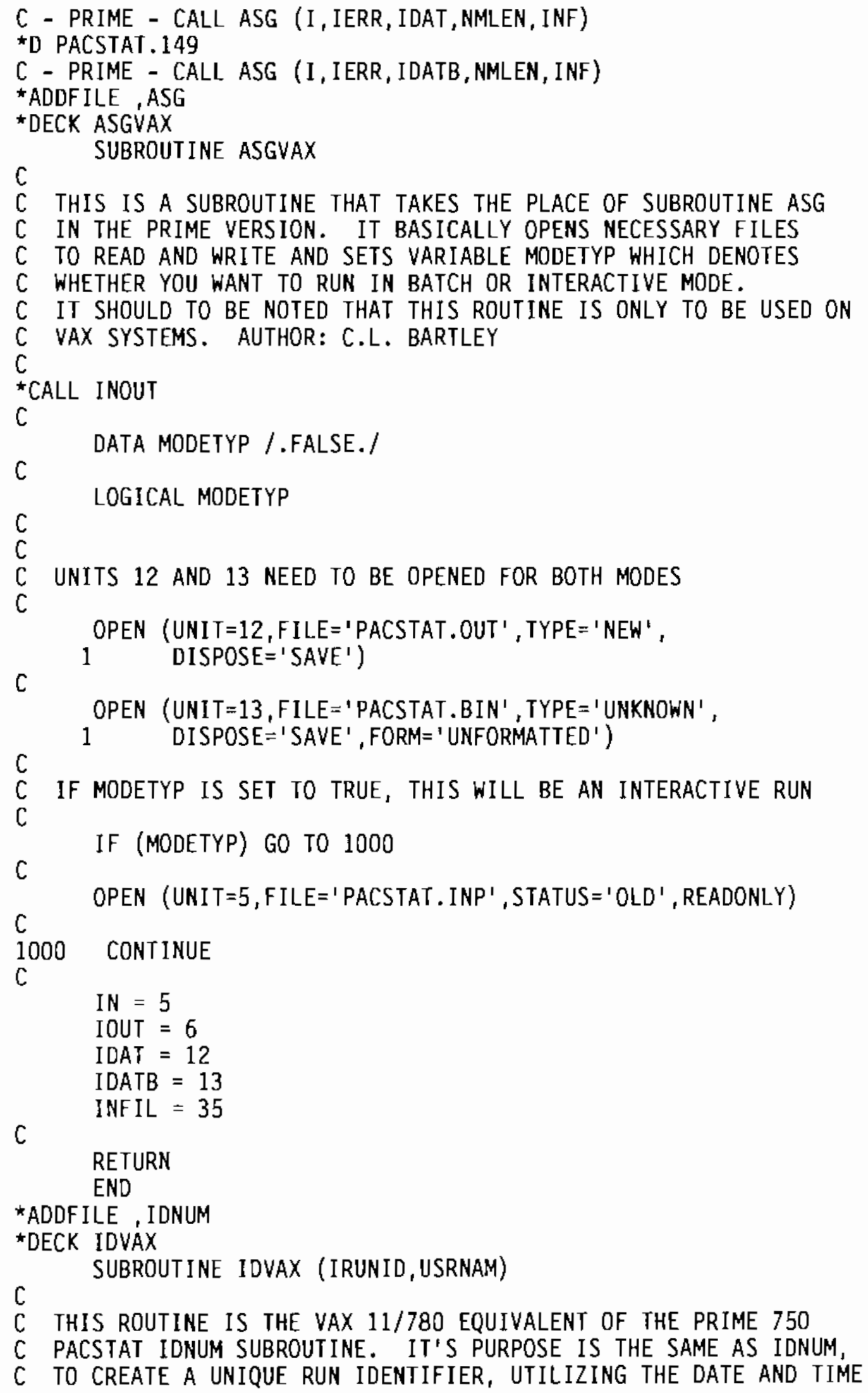

PACSTAT_AO.UPD.6 
C SYSTEM'S LIBRARIES AT THE TIME THIS ROUTINE IS CALLED. THE

C SYSTEM USER NAME IS ALSO PLACED INTO A CHARACTER VARIABLE. THIS

C IFORMATION IS USED TO INSURE TRACEABILITY AND ACCOUNTABILITY FOR

C DATA FILES FROM SIMULATION RUNS. AUTHOR: C.L. BARTLEY

C

C

C

$$
\begin{aligned}
\text { IRUNID }= & \text { RUN IDENTIFIER, AS FOLLOWS: } \\
& \text { DIGITS } 9-10=\text { MONTH OF CURRENT DATE. }
\end{aligned}
$$$$
\text { DIGITS 7-8 = DAY OF THE MONTH (1 TO 31). }
$$$$
\text { DIGITS } 5-6=\text { CURRENT YEAR. }
$$$$
\text { DIGITS } 3-4=\text { CURRENT HOUR (0 TO 23). }
$$

C

$$
\begin{aligned}
& \text { DIGITS } 1-2=\text { CURRENT MINUTE }\left(\begin{array}{lll}
0 & \text { TO } & 59
\end{array}\right) . \\
& \text { USRNAM }=8 \text { CHARACTER NAME OF THE CURRENT SYSTEM USER. }
\end{aligned}
$$

\section{INTEGER*4}

+ IRUNID,

+ JHR,

JMM, JMIN

JDD，

JYY,

$$
\begin{aligned}
& \text { INTEGER }{ }^{\star 2} \\
& +\quad \text { LOCAL (15) } \\
& + \text { CHARACTER } \\
& +
\end{aligned}
$$

LOCAL ARRAY FOR READING DATE AND TIME

+ TIM,

CHARACTER ${ }^{\star} 16$

USRN, USRNAM

+ DATE

EQUIVALENCE

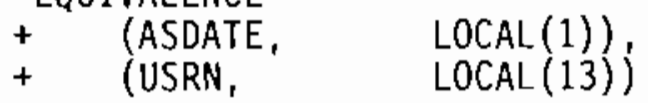

$c$
$C$
$C$
$C$
$C$
$c$
$C$
$C$
$C$
$C$
$C$

C

CALL IDATE (LOCAL(1), LOCAL(2), LOCAL(3))

GET CURRENT TIME AND DATE IDATE RETURNING:

LOCAL (1) =MM (MONTH)

$\operatorname{LOCAL}(2)=D D \quad$ (DAY)

TIME RETURNING:

$$
\text { LOCAL }(3)=Y Y \quad(\text { YEAR) }
$$

c

$$
\begin{aligned}
& \text { JMM }=\operatorname{LOCAL(1)} \\
& \text { JDD }=\operatorname{LOCAL(2)} \\
& \text { JYY }=\operatorname{LOCAL(3)} \\
& \text { CALL TIME (TIM) }
\end{aligned}
$$

C

READ (TIM, 500) JHR, JMIN

FORMAT $(2(12,1 X))$

$J M M=J M M \star 100000000$

$J D 0=J D D * 1000000$

$J Y Y=J Y Y * 10000$

$J H R=J H R^{\star} 100$ 


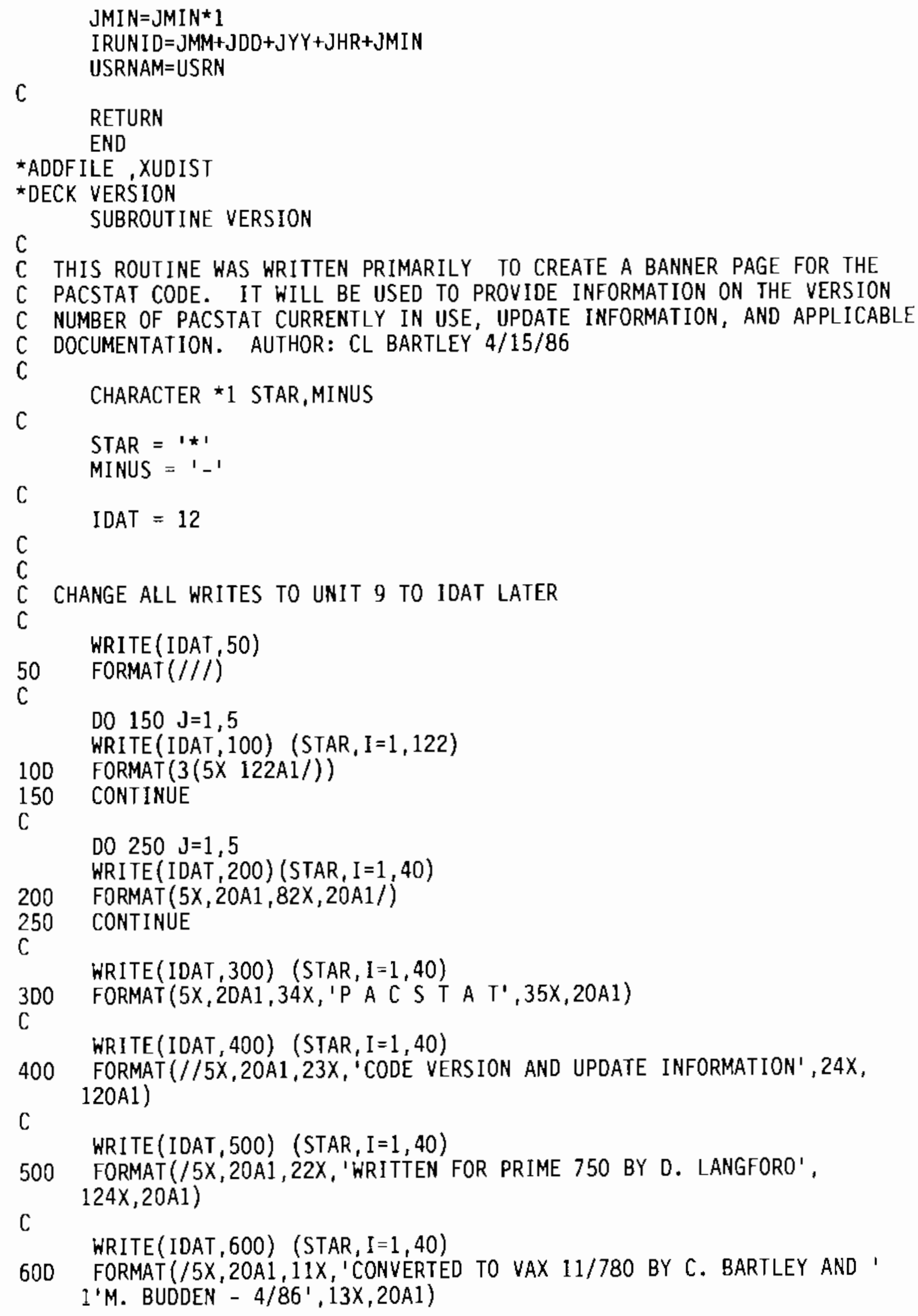

PACSTAT_AO.UPD.8 


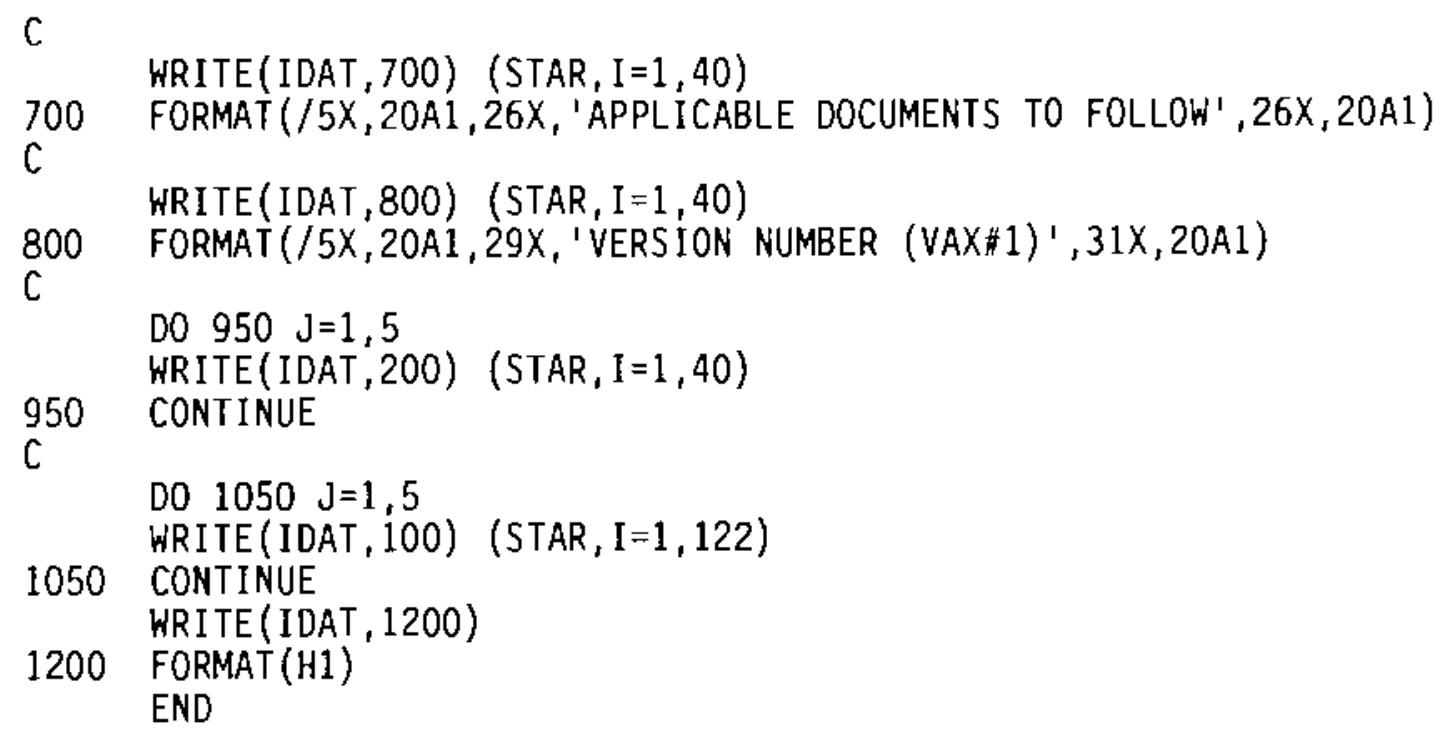


PACSTAT_AL.UPD

0.11 
* IDENT DUMMY

* I PACSTAT.2

EXTERNAL BLXDAT.

PACSTAT_A1.UPD. 1

D. 12 
PACSTAT_A2.UPD

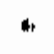

D. 13 


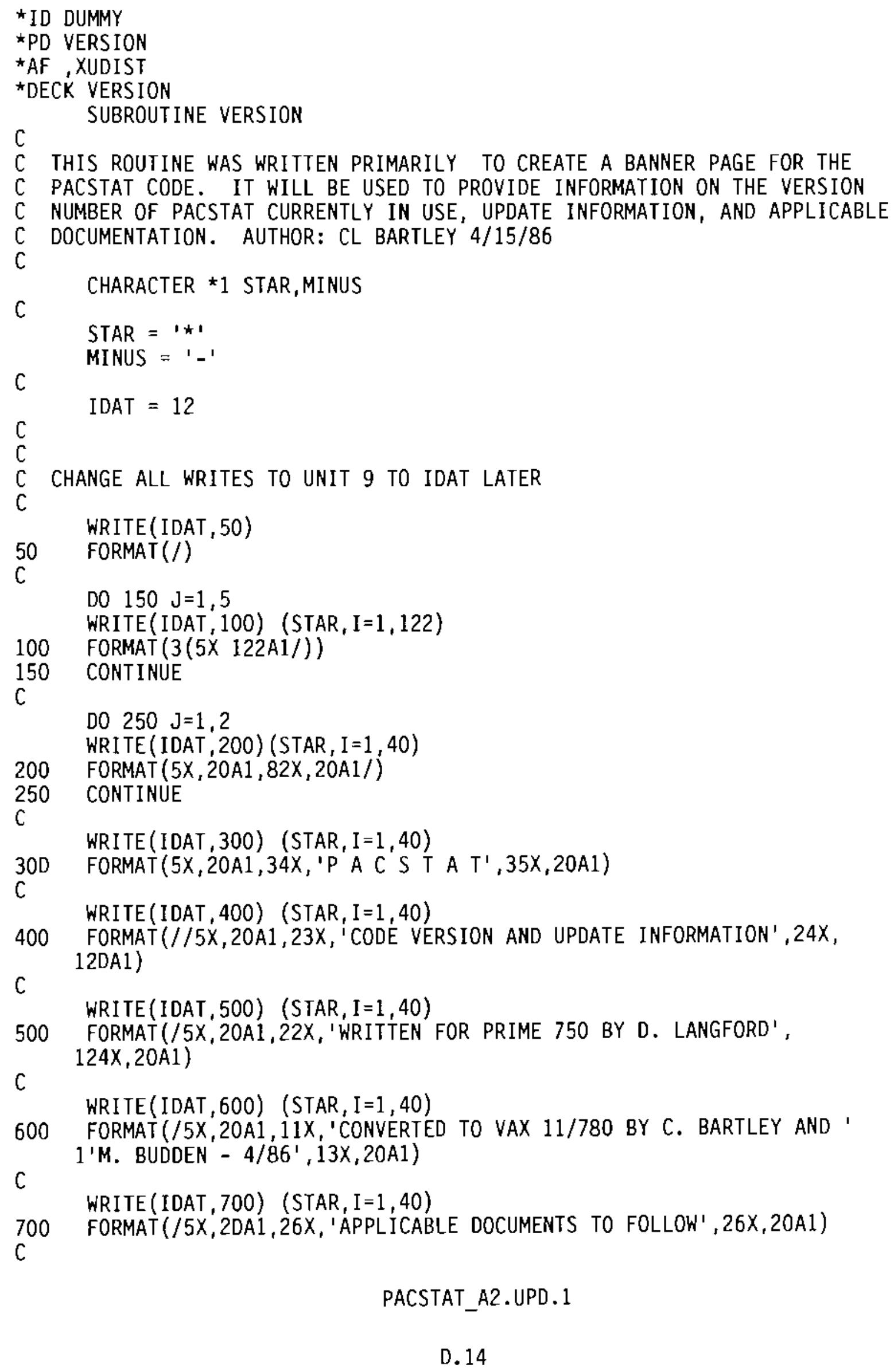




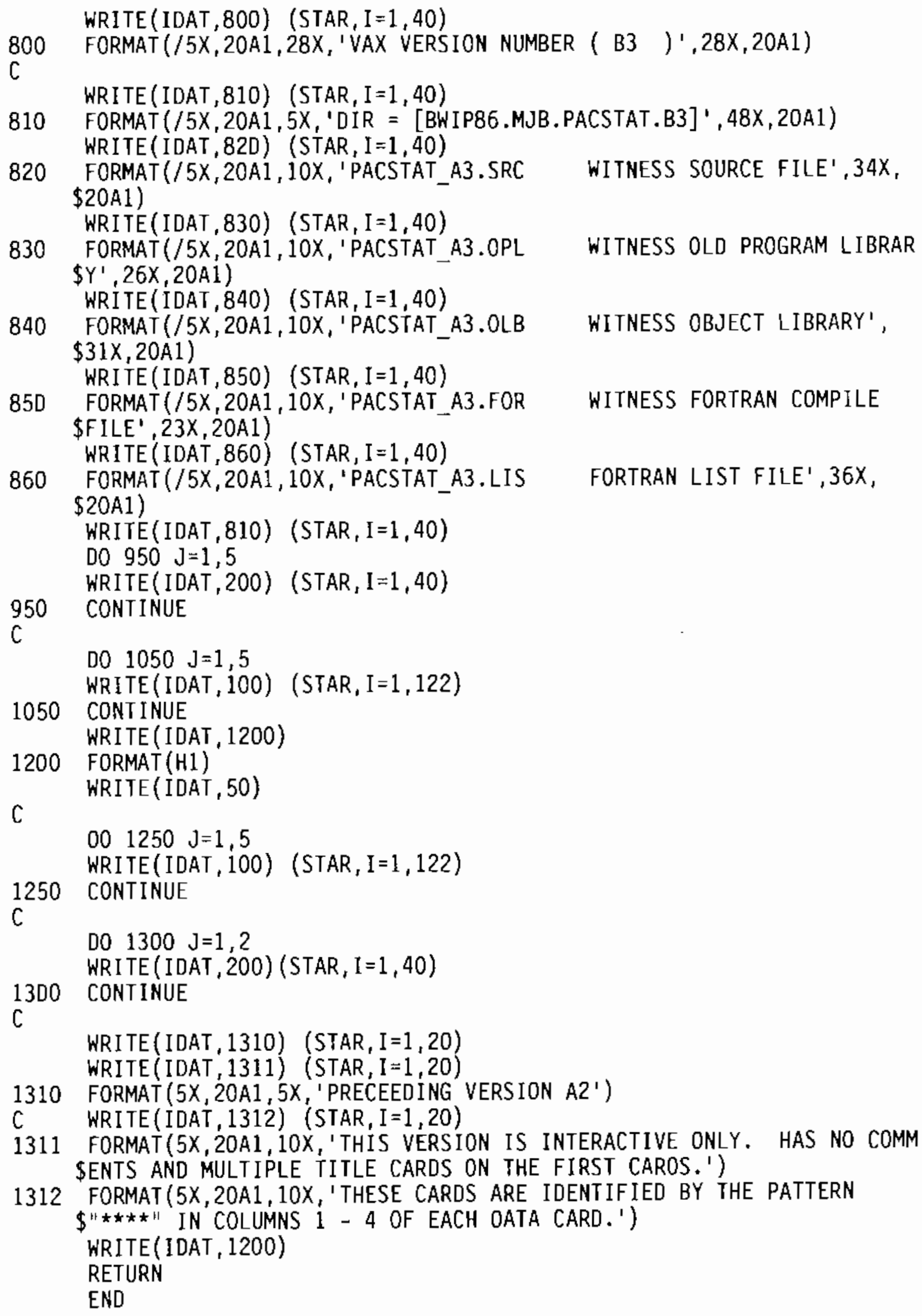

PACSTAT_A2.UPD.2 
PACSTAT_A3.UPD

,

D. 16 


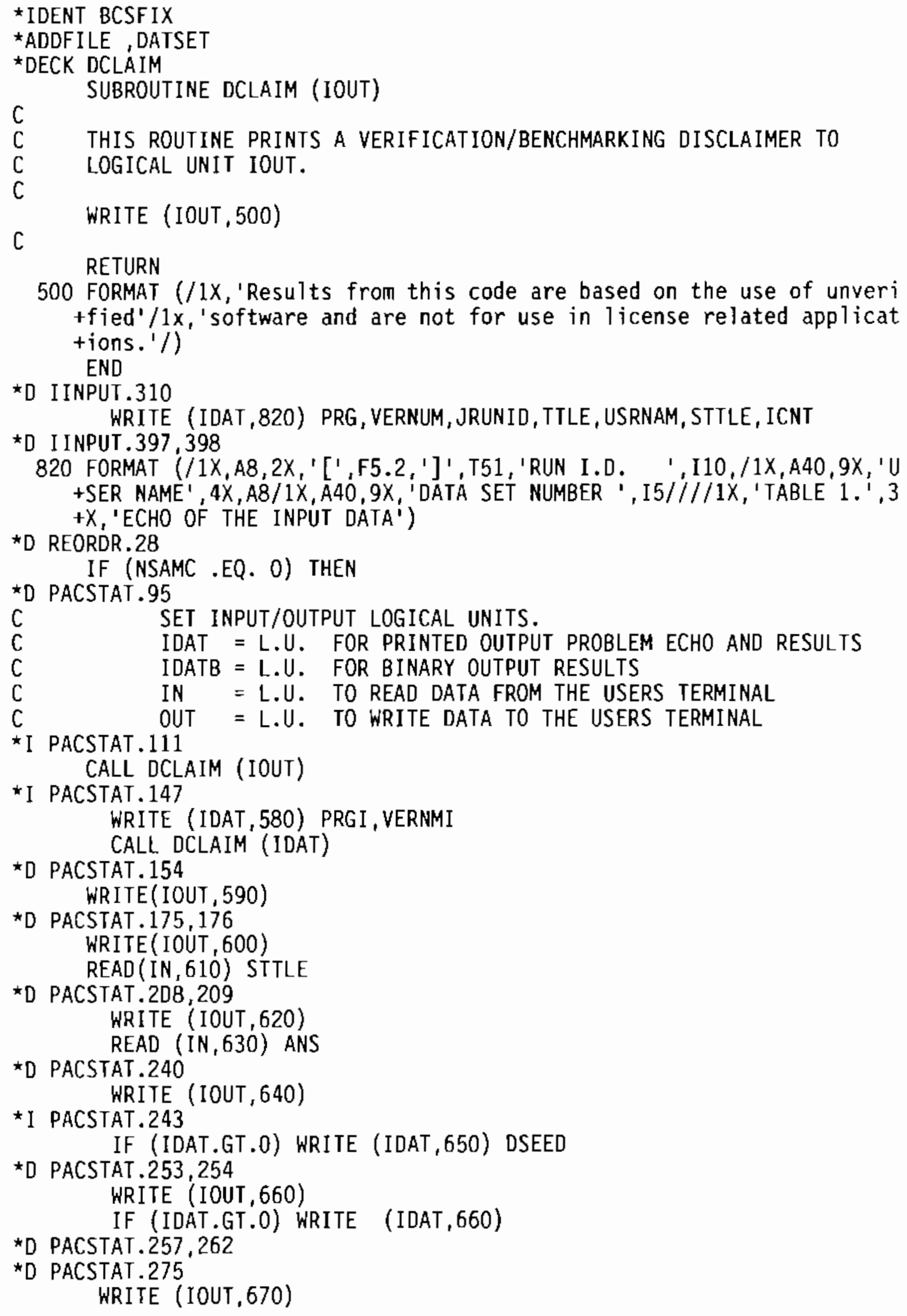


$\star D$ PACSTAT.296,297

WRITE (IOUT, 680) INDEX

IF (IDAT.GT.0) WRITE (IDAT,680) INDEX

*D PACSTAT.299, 300

WRITE (IOUT, 690)

IF (IDAT.GT.0) WRITE (IDAT,690)

*D PACSTAT. 331,332

WRITE (IOUT, 700) IQB

READ (IN,630) ANS

$\star D$ PACSTAT. 360,379

580 FORMAT (1H1, 1X, ' (', A8, 1X,F5.2,' ')')

590 FORMAT (/1X,' 'ENTER THE NAME FOR THE BINARY OUTPUT DATA FILE.' $/ 1 X, '$ $+($ ' 'NONE' ' TO OMIT)' $)$

595 FORMAT (/1X, 'ENTER THE NAME FOR THE BINARY OUTPUT DATA FILE, '/1X,'

+ (OR A CARRIAGE RETURN TO DEFAULT TO DATA FILE PACSTAT.BIN) ${ }^{\prime}$ )

600 FORMAT (/1X, 'ENTER LABEL FOR THIS RUN (MAX 40 CHARACTERS)')

610 FORMAT (A40)

620 FORMAT (/1X, 'WOULD YOU LIKE TO CONTINUE OR QUIT IF AN ERROR'/1X, 'I $+S$ ENCOUNTERED IN THE SOLUTION ROUTINE $(\mathrm{C} / \mathrm{Q})$ ? ')

630 FORMAT (A1)

640 FORMAT (/1X,'ENTER AN INITIAL SEED NUMBER FOR THE RANDOM NUMBER GE +NERATOR.' $\left./ 1 X^{\prime}, '(\text { A REAL NUMBER BETWEEN } 1.0 \text { AND }(2 \wedge 31)-2 .)^{\prime}\right)$

650 FORMAT (/5X,' INITIAL RANDOM SEED NUMBER IS ',1PD14.7)

660 FORMAT (/1X, $1 \star \star$ ERROR COMPUTING INPUT DISTRIBUTION DATA. THIS ITE +RATION WAS'/4X, 'ABORTED DUE TO AN INABILITY TO COMPUTE AND/OR READ + THE DESIRED'/4X, 'RANDOM SAMPLES.' $)$

670 FORMAT (/1X, $1 \star \star \star$ ERROR, NO SUCCESSFUL SAMPLES WERE COMPUTED.' $/ 5 X, '$ + THE INPUT VARIABLE STATISTICS WILL BE EXAMINED, '/5X,'BUT THE OUTPU $+T$ DATA WILL BE IGNORED, AND THE BINARY'/5X, 'OUTPUT FILE RECORD WIL $+L$ NOT BE WRITTEN.')

680 FORMAT $(/ / / 5 X, 1--$ ERROR ABORT, SAMPLE NUMBER $1, I 5)$

690 FORMAT ( $5 X, \cdots$ PROCEDURE WILL CONTINUE WITH REDUCED SAMPLE SPACE. $+1)$

700 FORMAT (/1X,'CREATE ANOTHER DATA SET $(\mathrm{Y} / \mathrm{N})$ ? ' 'A1)

* I STATOT. 45

C

C

TABLE HEADER AND INPUT VARIABLE STATISTICS

WRITE (III, 500)

CALL EXPECT

*D STATOT.114,115

WRITE（I I I , 510)

WRITE (III, 520)

*D STATOT.117

WRITE (III, 530) $\operatorname{VLABEL}(\mathbf{N}), \operatorname{VMIN}(\mathrm{N}), \operatorname{VMaX}(\mathrm{N}), \operatorname{VaVG}(\mathrm{N}), \operatorname{VMED}(\mathbf{N})$,

*D STATOT. 126

WRITE (I I I , 540)

$\star D$ STATOT.223

WRITE (I I I , 550) VLABEL (N), ANS, RD (1), RD (2) , $\operatorname{RM}(1), \operatorname{RM}(2)$

*D STATOT.225

WRITE (II I, 560) VLABEL(N),ANS, RD(1), RM(1)

${ }^{\star}$ D STATOT. 228

PACSTAT_A3.UPD.2 
${ }^{*}$ D STATOT.230,306

C

C

PRINT NUMBER OF RANDOM SAMPLES REJECTED DUE TO LIMTS

$\mathrm{C}$

IF (NVAR.LT.1) GO TO 400

DO $360 \quad N=1$, NVAR

IF (ILIM(N).EQ.0) GO TO 360

IF ((NLIM(N,1).EQ.0).AND. $(\operatorname{NLIM}(N, 2) \cdot E Q \cdot 0))$ GO TO 360 WRITE (IOUT, 580) VLABEL(N)

IF (IDAT.NE.0) WRITE (IDAT,580) VLABEL(N)

IF $(\operatorname{NLIM}(N, 1)$.EQ.0) GO TO 340 WRITE (IOUT, 590) $\operatorname{NLIM}(\mathrm{N}, 1)$

IF (IDAT.NE.0) WRITE (IDAT,590) NLIM(N,1)

340 CONTINUE

$\operatorname{IF}(\operatorname{NLIM}(N, 2) . E Q .0)$ GO TO 350

WRITE (IOUT, 600) NLIM $(N, 2)$

350 CONTINUE

IF (IDAT.NE.0) WRITE (IDAT,600) $\operatorname{NLIM}(N, 2)$

360 CONTINUE

C

C

STRATIFIED SAMPLING DIAGNOSTICS

IF ((NLEVEL.LT.2) .OR. (IDAT. EQ.0)) GO TO 390

WRITE (IDAT, 610)

DO $380 \mathrm{~N}=1$, NVAR

IF (ITYPE (N).EQ.NTYPE) GO TO 380

IF (ITYPE(N).EQ.10) GO TO 380

$K=N L E V E L$

IF (K.GT.10) K=10

WRITE (IDAT, 620) VLABEL (N), (NPTSPL $(I, N), I=1, K$ )

370

IF (K.EQ.NLEVEL) GO TO 380

CONTINUE

$\mathrm{J}=\mathrm{K}+1$

$\mathrm{K}=\mathrm{K}+10$

IF (K.GT.NLEVEL) $K=$ NLEVEL

WRITE (IDAT, 630) (NPTSPL(I, N) , I=J, K)

380 CONTINUE

IF (K.LT.NLEVEL) GO TO 370

C

390 CONTINUE

400 CONTINUE

C

C

C

IF (NSOLN.LT.1) GO TO 412

WRITE (III, 640)

WRITE (II I, 520)

DO $410 \mathrm{~N}=1, \mathrm{NSOLN}$

WRITE (III,650) PLABEL (N)

WRITE (III,660) FMIN(N), FMAX (N), FAvG(N), FMED(N), FSTD(N), FSKW(N)

410 CONTINUE

PACSTAT_A3.UPD.3

0.19 
C

\section{CONTINUE}

\section{RETURN}

500 FORMAT $(1 H 1,1 X$, 'TABLE 2 . INPUT VARIABLE STATISTICS')

510 FORMAT ( $/ / 1 X_{,}^{\prime} * \star---$ SAMPLE DIAGNOSTICS, INPUT DISTRIBUTIONS')

520 FORMAT (/T19, 'MIN', T30, 'MAX', T40, 'MEAN', T51, 'MEDIAN', T61, 'STND DEV $+{ }^{1}$, T72, 'SKEWNESS')

530 FORMAT (1X,A12,1X,1PE1D.3,5(1X,E10.3))

540 FORMAT ( $/ / 1 X,{ }^{\prime} *$ - - MAXIMUM LIKELIHOOD ESTIMATORS FOR INPUT DISTRI +BUTIONS'/T24, 'COMPUTED VALUES', T52, 'EXPECTED VALUES'/3X, 'VARIABLE' + , T20, 'PARAMETER 1',T33, 'PARAMETER 2', T48, 'PARameter 1', T61, 'PARame +TER 2')

550 FORMAT (1X,A12,3X,A1, T20, 1PE10.3, 3X, E10.3, T48, E10.3,T61, E10.3)

560 FORMAT (1X,A12,3X,A1, T20, 1PE10.3, T48, E10.3)

570 FORMAT (T33,' * INDICATES ENOPOINT LIMITING MAY MOVE THE '/T35,'DIS +TRIBUTION AWAY FROM THE EXPECTED VALUES.')

580 FORMAT (//3X,'FOR THE DISTRIBUTION IN ',A12)

590 FORMAT (3X, I6,' RANDOM SAMPLES WERE BELOW THE LOWER LIMIT, AND SET + TO THAT LIMIT. ')

600 FORMAT (3X, I6,' RANOOM SAMPLES WERE ABOVE THE UPPER LIMIT, AND SET + TO THAT LIMIT.')

610 FORMAT ( $/ / / 7 X$, 'STRATIFIED SAMPLING DIAGNOSTICS' $/ / 1 X$, 'VARIABLE' $17 X$ ,+ 'NUMBER OF SAMPLES CHOSEN FROM EACH LEVEL')

620 FORMAT $(1 X, A 12,2 X, 10(1 X, 15))$

630 FORMAT $(15 X, 10(1 X, I 5))$

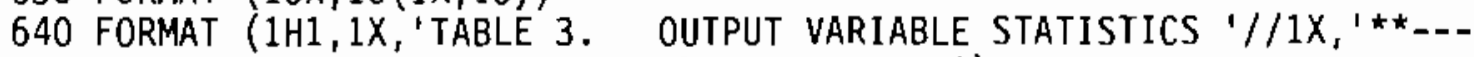
+ SAMPLE DIAGNOSTICS, OUTPUT DISTRIBUTIONS')

650 FORMAT $(1 X, A 32)$

660 FORMAT $(1 X, 13 X, 1$ PE10.3,5(1X,E10.3))

END

*0 I INPUT. 399,424

830 FORMAT (/5X,A12,' (',A12, ')'/15X,'DISTRIBUTION IS ',A20)

840 FORMAT $(22 X$, 'MEAN VALUE $=1$, T35, 1PE12.4/17X, 'STND DEVIATION $=1$, T3 $+5, E 12.4)$

850 FORMAT (22X, 'CONSTANTS FOR THE UNDERLYING NORMAL DISTRIBUTION ARE' $+/ 17 X$, 'MEAN VALUE $=1$, T35, IPE12.4/17X, 'STND DEVIATION $=', T 35$, E12.4)

860 FORMAT (22X, 'MIN, MAX EXPONENTS FOR THE UNDERLYING' $/ 17 X$, 'UNIFORM D +ISTRIBUITON ARE' $/ 17 X,{ }^{\prime} M I N=1,1$ PE12.4,', $M A X=1, E 12.4$ )

870 FORMAT (22X, 'MEAN VALUE IS', T35, 1PE12.4)

880 FORMAT (22X,' 'LOWER ENDPOINT $=1$ ' T35, 1PE12.4/17X, 'UPPER ENOPOINT =', $+\mathrm{T} 35, \mathrm{E} 12.4)$

890 FORMAT (22X, 'LOWER VALUE $=1$, T35, 1PE12.4/17X, 'UPPER VALUE $=$ ', T35, E1 $+2.4 / 17 X,{ }^{\prime}$ 'LOW VALUE PROB $\left.=1, T 35, E 12.4\right)$

900 FORMAT (22X,' THE CONFIDENCE FUNCTION WILL BE READ AS A PIECE WISE' $+/ 17 X, '$ CONTINUOUS DATA FUNCTION FROM THE EXTERNAL FILE: ' $/ 18 X, 20 A 4)$

910 FORMAT (22X,' THE RANDOM DATA POINTS WILL BE READ'/17X,' FROM THE EX +TERNAL FILE: ' $/ 18 X, 20 A 4)$

920 FORMAT (22X,' CONSTANT VALUE $=1, T 35,1 \mathrm{PE} 12.4)$

930 FORMAT (22X, 'LOWER BOUND $='$, T35, 1PE12.4/17X, 'UPPER BOUND $=$ ', T35, E1 $+2.4)$

940 FORMAT (/)

950 FORMAT (5X, 'VALUE FOR ',A12,2X,' (',A12,') IS ',1PE12.4)

PACSTAT_A3.UPD. 4 
960 FORMAT (//5X, 'NUMBER OF SAMPLES IS ' I8/1X, 'PRINT INTERVAL IS', $4 X$, $+I 8)$

970 FORMAT (5X,'STRATIFIED SAMPLING CHOSEN, USING ' I5,' LEVELS. ')

980 FORMAT (5X,'STRATIFIED SAMPLING NOT CHOSEN. ')

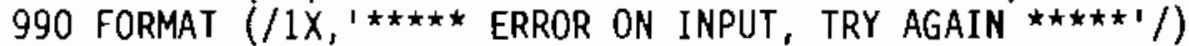

*ID ECHO

*I RELEAS. 18

CLOSE (UNIT=7)

*I PACSTAT. 143

CALL ASGVAX (NMLEN, INF, IFLAG)

WRITE (IECH0,570) (INF (I), I =1, NMLEN)

*B PACSTAT. 156

75 CONTINUE

WRITE (IOUT, 595)

WRITE (IECHO, 570) (INF(I), I=1, NMLEN)

*B PACSTAT.210

WRITE (IECHO,630) ANS

*I PACSTAT.241

WRITE (IECHO ${ }^{\star}{ }^{*}$ ) WD

*B PACSTAT. 333

*I ASG.63 WRITE (IECHO,630) ANS

*I ASG.69 WRITE $($ IECHO, 540) ANS

*I ASG.90

WRITE (IECHO, 560) (INF (I), I =1, NLEN)

WRITE (IECHO, 540) ANS

*I ASG.97

WRITE (IECHO,540) ANS

*I ASG. 108

WRITE (IECHO, 560) (INF (I), I=1, NLEN)

^I ASG.120

WRITE (IECHO, 540)

*I ASG.126

WRITE (IECH0,560) (INF(I), I=1, NLEN)

*I DATOUT. 50

WRITE (IECHO, *) ISOLN

*I DATOUT. 56 WRITE (IECHO, *) NPRT

*I DATOUT. 66 WRITE (IECHO ${ }^{*}$ ) ICCD

*I DATOUT. 144 WRITE (IECH0,620) ANS

*I IINPUT. 66 WRITE (IECHO,510) ANS

*I I INPUT. 92 WRITE (IECHO, *) IT

*I IINPUT. 102 WRITE (IECHO, *) PAR(N,1)

* I IINPUT. 105 WRITE (IECHO, *) PAR $(\mathbb{N}, 2)$

$\star$ I IINPUT. 118

PACSTAT_A3.UPD. 5 
*I IINPUT.127

WRITE (IECHO,$\left.^{*}\right) \operatorname{PAR}(\mathrm{N}, 1)$

*I IINPUT.139

WRITE (IECHO $\left.{ }^{\star}\right) \operatorname{PAR}(\mathrm{N}, 2)$

WRITE $\left(\operatorname{IECHO},{ }^{\star}\right) \operatorname{PAR}(N, 1), \operatorname{PAR}(N, 2)$

*I IINPUT.153

WRITE (IECHO, *) PAR $(\mathrm{N}, 1)$

*I IINPUT.166

WRITE (IECHO $\left.{ }^{*}\right) \operatorname{PAR}(N, 1), \operatorname{PAR}(N, 2)$

*I IINPUT.179

WRITE (IECHO, $\left.{ }^{\star}\right) \operatorname{PAR}(N, 1), \operatorname{PAR}(N, 2)$

*I IINPUT.187

WRITE (IECHO, *) PAR $(\mathrm{N}, 3)$

*I IINPUT.197

WRI TE (IECHO ,690) (JJNF ( I , N) , I =1, NMLEN)

*I IINPUT.205

WRITE (IECHO, *) PAR $(N, 1)$

*I IINPUT. 212

WRITE (IECHO,510) ANS

*I IINPUT.219

WRITE $\left(\operatorname{IECHO}{ }^{*}\right) \operatorname{VLIM}(\mathrm{N}, 1), \operatorname{VLIM}(\mathrm{N}, 2)$

^I IINPUT.240 WRITE (IECHO, 510) ANS

*I I INPUT.257 WRITE (IECHO $\left.{ }^{\star}, \mathrm{ERR}=410\right) \operatorname{CONST}(\mathrm{I})$

*I IINPUT.266 WRITE (IECHO, *) NSAM

*I I INPUT. 278 WRITE (IECHO * *) NPRT

*I I INPUT.292 WRITE (IECHO, *) NLEVEL

*I PLINIT.19 WRITE (IECHO ${ }^{\star}$ ) KOEV

*I PLINIT.28 WRITE (IECHO, 520) ANS

*I PLINIT.35 HRITE (IECHO $\left.{ }^{\star}\right) \times L 2, Y L 2$

*I PPLT. 54 WRITE (IECHO,520) ANS

*I PPLT.61 WRITE $\left(\right.$ IECHO $\left.{ }^{\star}\right) \mathrm{C} 1, \mathrm{C} 2$

*I PPLT. 100 WRITE (IECHO,520) ANS

*I PPLT.125 WRITE (IECHO,520) ANS

*B PACSTAT. 177 WRITE (IECHO,610) STTLE

*D PACSTAT. 101, 102 IFLAG $=0$ CALL ASGVAX (NMLEN, INF, IFLAG)

*D ASGVAX.2

SUBROUTINE ASGVAX (NLEN, INF, IFLAG)

PACSTAT_A3.UPD. 6 


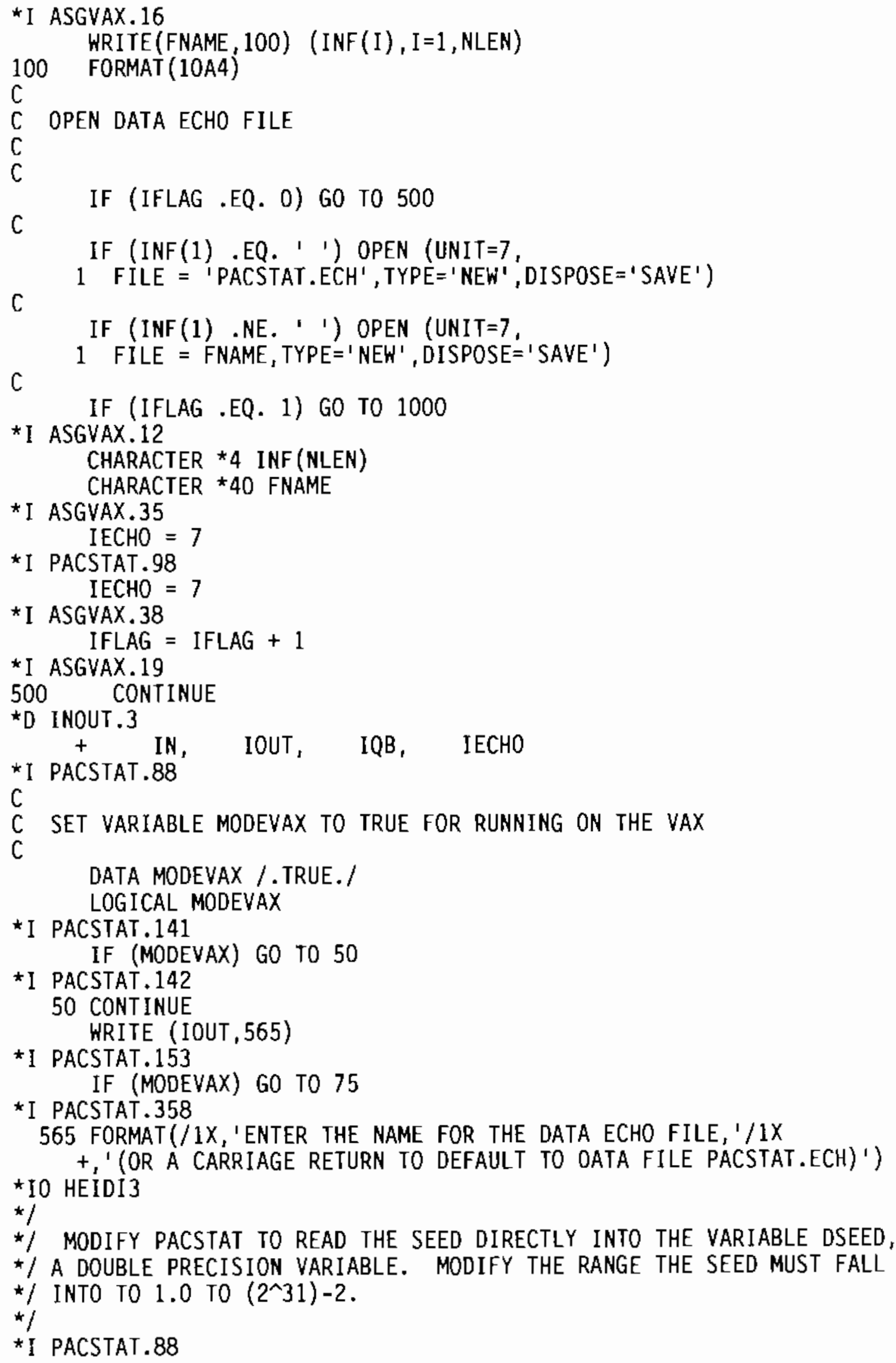




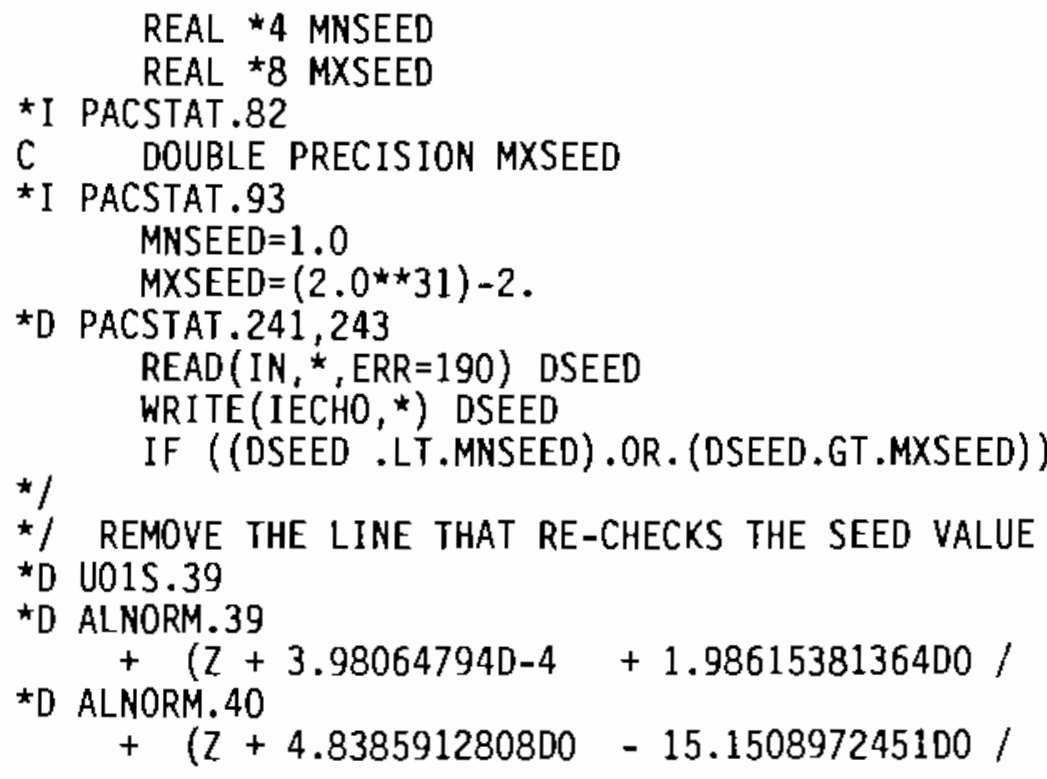


PACSTAT_B3.UPD

D.25 


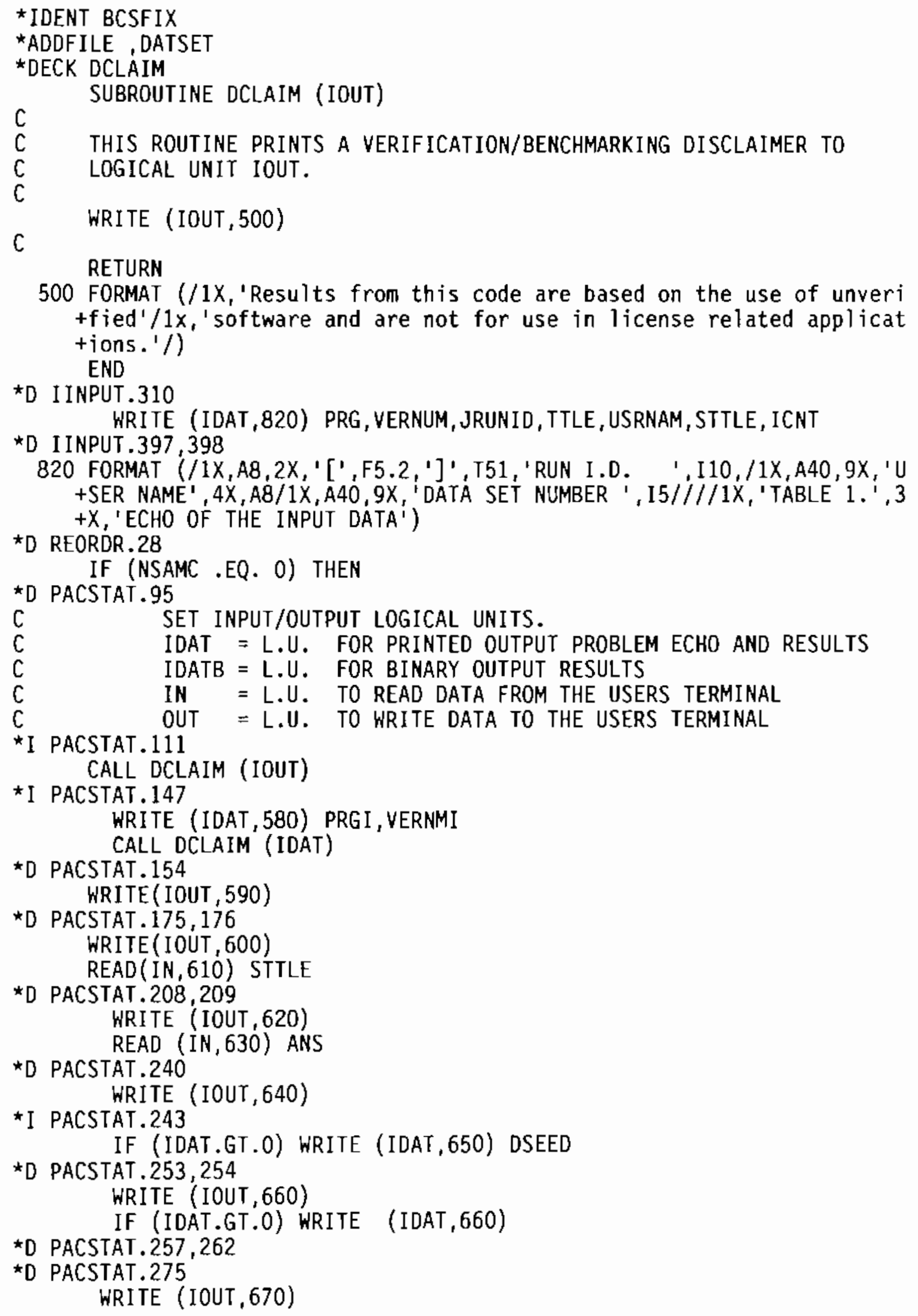

PACSTAT . B3.UPD. 1 


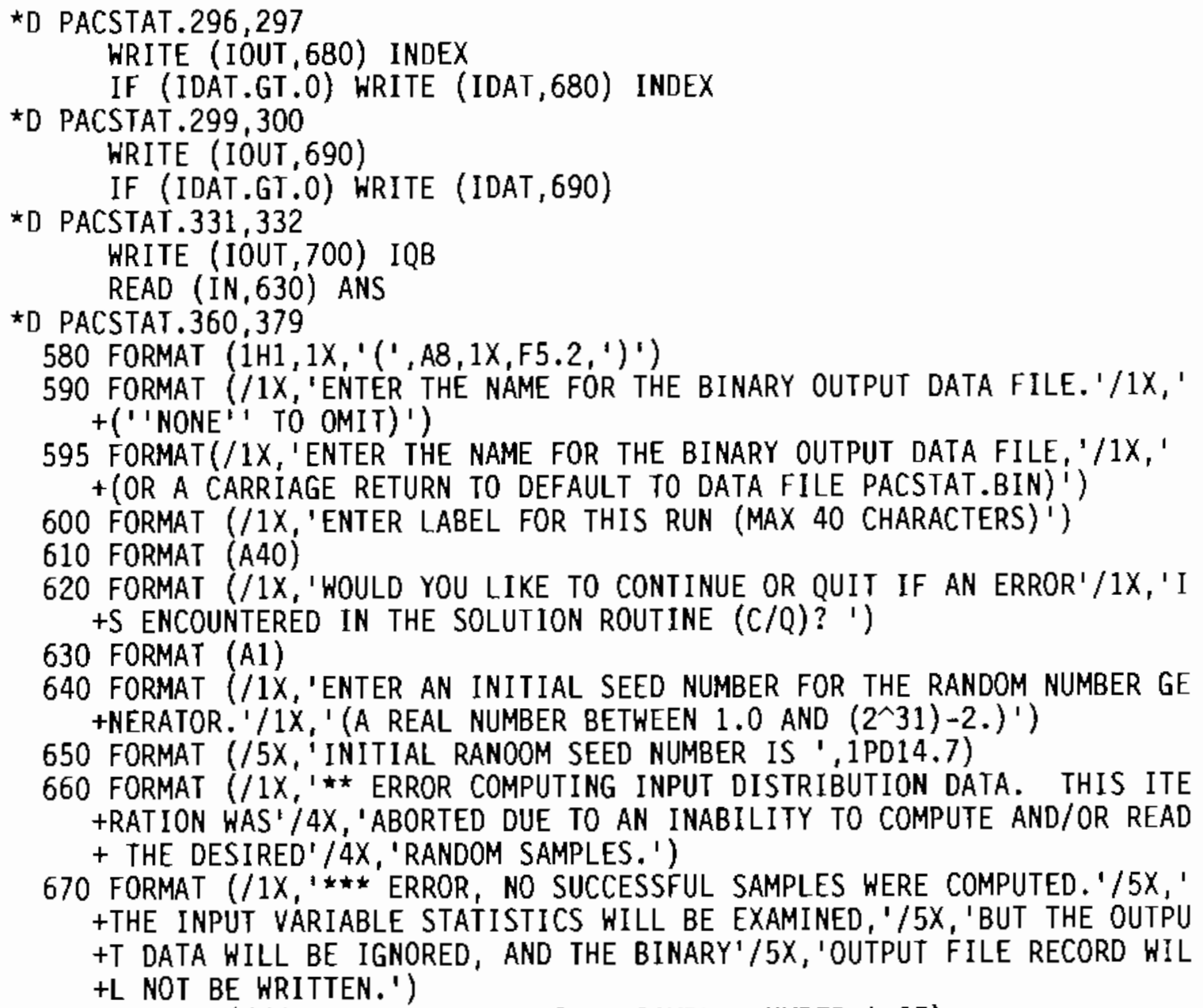




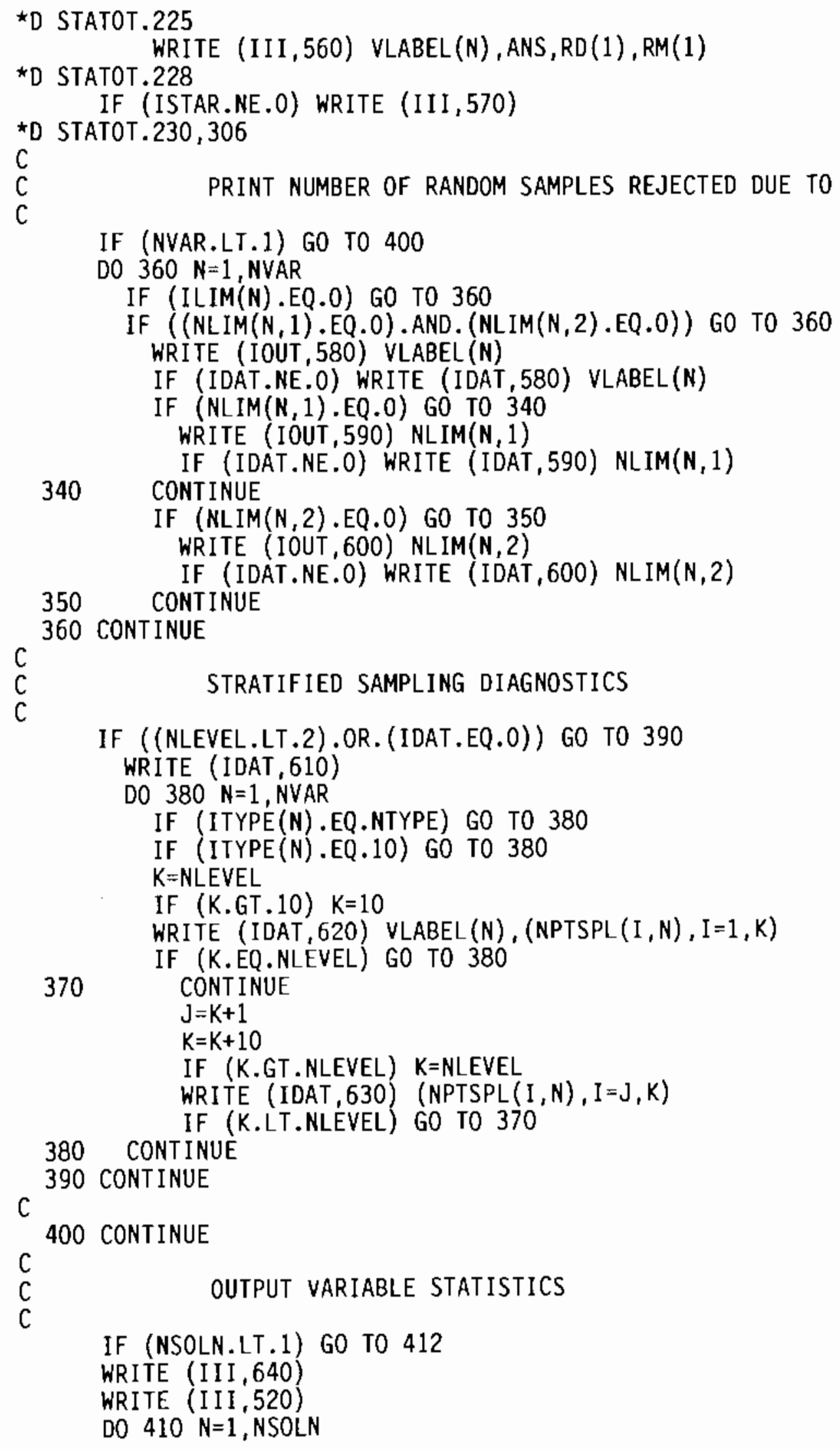

PACSTAT.B3.UPD. 3 


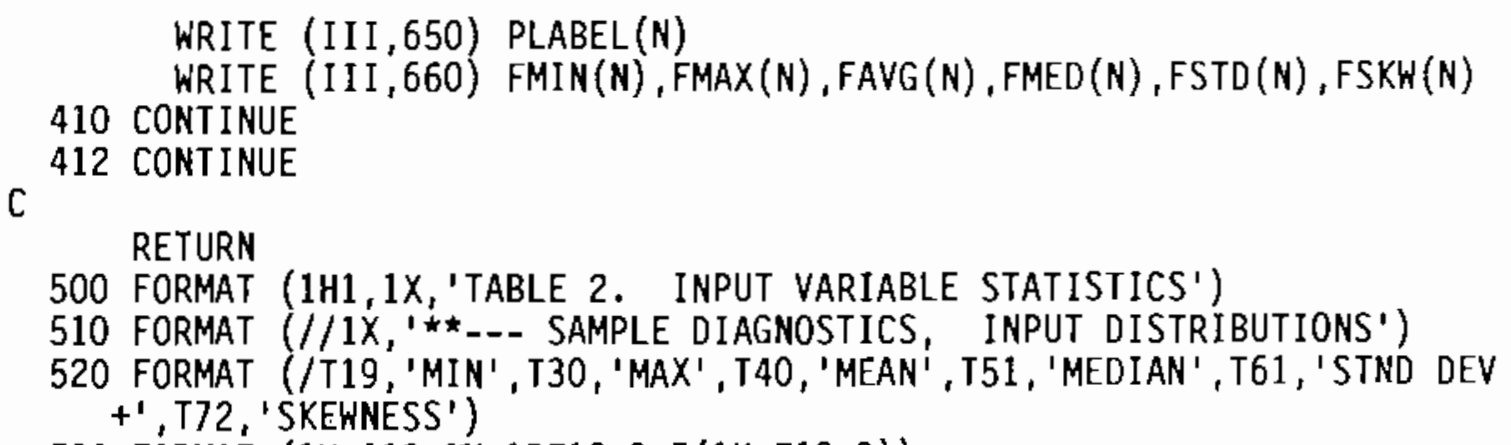

590 FORMAT ( $3 X, I 6, '$ RANDOM SAMPLES WERE BELOW THE LOWER LIMIT, AND SET + TO THAT LIMIT.' $)$

600 FORMAT $(3 X, 16, '$ RANDOM SAMPLES WERE ABOVE THE UPPER LIMIT, AND SET + TO THAT LIMIT. ')

610 FORMAT $(/ / / 7 X$, 'STRATIFIED SAMPLING DIAGNOSTICS' $/ / 1 X$, 'VARIABLE' $17 \mathrm{X}$ ,+ 'NUMBER OF SAMPLES CHOSEN FROM EACH LEVEL')

620 FORMAT $(1 X, A 12,2 X, 10(1 X, I 5))$

630 FORMAT $(15 X, 10(1 X, I 5))$

640 FORMAT ( $1 H 1,1 X$, 'TABLE 3 . OUTPUT VARIABLE STATISTICS $1 / 11 X_{1}, * \star---$ + SAMPLE DIAGNOSTICS, OUTPUT DISTRIBUTIONS')

650 FORMAT $(1 X, A 32)$

660 FORMAT $(1 X, 13 X, 1$ PE10.3,5(1X,E10.3))

END

$\star D$ I INPUT . 399,424

830 FORMAT (/5X,A12,' (',A12,')'/15X,'DISTRIBUTION IS ',A20)

840 FORMAT $(22 X, '$ MEAN VALUE $=1$, T35, 1 PE12.4/17X,' STND DEVIATION $=$ ', T3 $+5, \mathrm{E} 12.4)$

850 FORMAT (22X, 'CONSTANTS FOR THE UNDERLYING NORMAL DISTRIBUTION ARE' $+/ 17 X$, 'MEAN VALUE $=1$, T35, 1PE12.4/17X, 'STND DEVIATION $=1$, T35, E12.4)

860 FORMAT (22X, 'MIN, MAX EXPONENTS FOR THE UNDERLYING' $/ 17 X$, ' UNIFORM D +ISTRIBUITON ARE' $/ 17 X,{ }^{\prime} M I N=1,1$ PE12.4,', $M A X=1, E 12.4$ )

870 FORMAT (22X, 'MEAN VALUE IS ' , T35, 1 PE 12.4)

880 FORMAT (22X, 'LOWER ENDPOINT $=1, T 35,1$ PE12.4/17X, ' UPPER ENDPOINT $='$, + T35, E12.4)

890 FORMAT (22X, 'LOWER VALUE $=1, T 35,1$ PE $12.4 / 17 X$, 'UPPER VALUE $=1, T 35, E 1$ $+2.4 / 17 X, '$ 'LOW VALUE PROB $=', T 35, E 12.4)$

900 FORMAT (22X, 'THE CONFIDENCE FUNCTION WILL BE READ AS A PIECE WISE' $+/ 17 X, '$ CONTINUOUS DATA FUNCTION FRDM THE EXTERNAL FILE: '/18X, 20A4)

910 FORMAT (22X,' THE RANDOM DATA POINTS WILL BE READ'/17X, 'FROM THE EX +TERNAL FILE:' $/ 18 X, 20 A 4)$

920 FORMAT (22X, 'CONSTANT VALUE $=1, T 35,1$ PE 12.4 )

930 FORMAT (22X, 'LOWER BOUND $=1$, T35, lPE12,4/17X,' UPPER BOUND = ', T35, E1 


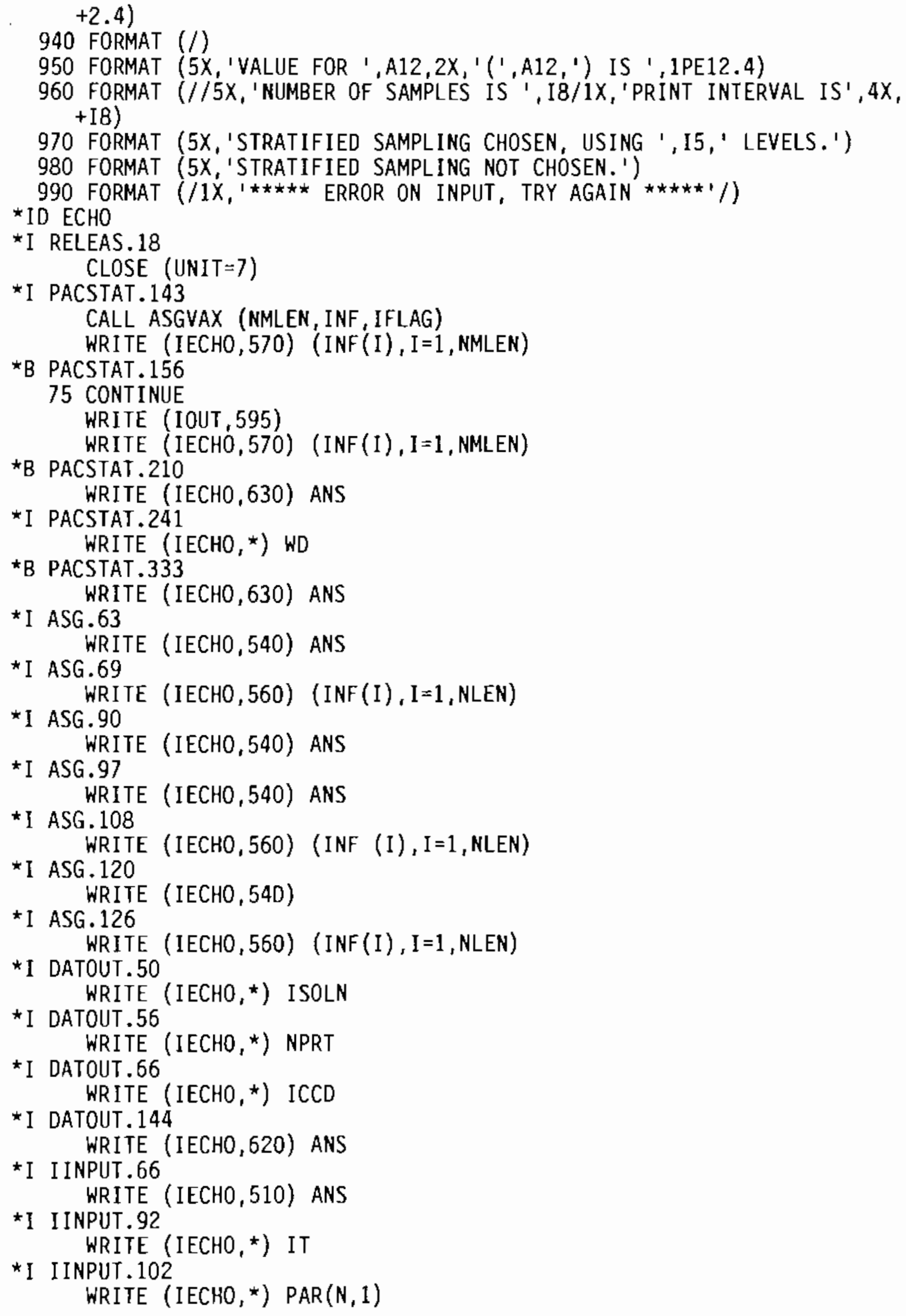

*I I INPUT. 66 WRITE (IECH0,510) ANS

*I IINPUT.92 WRITE (IECHO, *) IT

*I IINPUT. 102 WRITE (IECHO, *) PAR $(N, 1)$

PACSTAT.B3.UPD. 5 
*I IINPUT. 105

$$
\text { WRITE (IECHO, } \left.{ }^{\star}\right) \operatorname{PAR}(\mathrm{N}, 2)
$$

*I I INPUT.118 WRITE (IECHO, $\left.{ }^{\star}\right) \operatorname{PAR}(\mathrm{N}, 1)$

*I IINPUT.127 WRITE (IECHO $\left.{ }^{\star}\right) \operatorname{PAR}(\mathrm{N}, 2)$

*I IINPUT. 139 WRITE (IECHO, *) $\operatorname{PAR}(\mathbf{N}, 1), \operatorname{PAR}(\mathbf{N}, 2)$

*I IINPUT.153

*I I INPUT . 166

$$
\text { WRITE (IECHO, *) } \operatorname{PAR}(N, 1)
$$

* I IINPUT. 179

$$
\text { WRITE (IECH0, } \left.{ }^{\star}\right) \operatorname{PAR}(\mathbf{N}, 1), \operatorname{PAR}(\mathbf{N}, 2)
$$

WRITE $\left(\operatorname{IECHO}{ }^{*}\right) \operatorname{PAR}(\mathbf{N}, 1), \operatorname{PAR}(\mathbf{N}, 2)$

*I I INPUT. 187 WRITE $\left(\right.$ IECHO $\left.{ }^{\star}\right) \operatorname{PAR}(N, 3)$

*I IINPUT.197 WRITE (IECH0,690) (JJNF(I,N), I=1, NMLEN)

*I IINPUT.205 WRITE (IECHO, *) PAR(N,1)

*I IINPUT.212 WRITE (IECH0,510) ANS

*I I INPUT.219 WRITE (IECH0, $\left.{ }^{\star}\right) \operatorname{VLIM}(\mathbf{N}, 1), \operatorname{VLIM}(\mathbf{N}, 2)$

* I IINPUT.240 WRITE (IECHO,510) ANS

*I IINPUT. 257 WRITE (IECHO * * ERR=410) CONST(I)

*I I INPUT.266 WRITE (IECHO , *) NSAM

*I IINPUT. 278 WRITE (IECHO, *) NPRT

*I IINPUT.292 WRITE $\left(\right.$ IECHO $\left.{ }^{*}\right)$ NLEVEL

*I PLINIT.19 WRITE (IECHO, *) KDEV

*I PLINIT.28 WRITE (IECH0,520) ANS

*I PLINIT.35

*I PPLT.54 WRITE (IECH0, *) XL2, YL2

ॠ I PPLT.61 WRITE (IECHO, 520) ANS

*I PPLT. 100 WRITE (IECHO, *) C1,C2 WRITE (IECH0,520) ANS

*I PPLT.125 WRITE (IECH0,520) ANS

*B PACSTAT. 177 WRITE (IECH0,610) STTLE

$\star D$ PACSTAT. 101, 102 IFLAG $=0$ 
CALL ASGVAX (NMLEN, INF, IFLAG)

*D ASGVAX. 2

SUBROUTINE ASGVAX (NLEN, INF, IFLAG)

*I ASGVAX.16

100 WORMAT (10A4)

C

C OPEN DATA ECHO FILE

c

c

C

IF (IFLAG .EQ. 0) GO TO 500

C

IF (INF(1) .EQ. ' ' ') OPEN (UNIT=7,

1 FILE = 'PACSTAT.ECH', TYPE='NEW', DISPOSE='SAVE')

C IF (INF(1) .NE. ' ' ') OPEN (UNIT=7,

1 FILE = FNAME, TYPE $=$ ' NEW', DISPOSE $={ }^{\prime}$ SAVE' $)$

IF (IFLAG .EQ. 1) GO TO 1000

\# I ASGVAX.12

CHARACTER *4 INF (NLEN)

CHARACTER * 40 FNAME

*I ASGVAX. 35

$\mathrm{IECHO}=7$

*I PACSTAT.98

IECHO $=7$

*I ASGVAX. 38

IFLAG = IFLAG + 1

*I ASGVAX.19

500 CONTINUE

*D INOUT. 3

*I PACSTAT. 88

IN, IOUT, IQB, IECHO

C

C SET VARIABLE MODEVAX TO TRUE FOR RUNNING ON THE VAX

C

DATA MODEVAX /.FALSE./ LOGICAL MODEVAX

*I PACSTAT. 141

IF (MODEVAX) GO TO 65

*I PACSTAT.142

65 CONTINUE

WRITE (IOUT, 565)

*I PACSTAT. 153

IF (MODEVAX) GO TO 75

*I PACSTAT. 358

565 FORMAT(/1X, 'ENTER THE NAME FOR THE DATA ECHO FILE, $1 / 1 X$

$+, '$ (OR A CARRIAGE RETURN TO DEFAULT TO DATA FILE PACSTAT.ECH)' $)$

* I UPDATE TO WRITE COMMENT CARDS TO THE DATA ECHO FILE FOR QA

* PURPOSES

$\star /$

PACSTAT.B3.UPD.7

D. 32 
C WRITE（IECHO, 11000） INREC, KARD

*ID HEIDI3

$\star /$

* MODIFY PACSTAT TO READ THE SEED DIRECTLY INTO THE VARIABLE DSEED,

* A DOUBLE PRECISION VARIABLE. MODIFY THE RANGE THE SEED MUST FALL

* I INTO TO 1.0 TO (2^31) -2.

$\star /$

* I PACSTAT. 88

REAL * 4 MNSEED

REAL *B MXSEED

* I PACSTAT.82

C DOUBLE PRECISION MXSEED

*I PACSTAT.93

MNSEED $=1.0$

$M \times S E E D=(2.0 * \star 31)-2$.

*D PACSTAT.241,243

READ (IN * , ERR=190) DSEED

WRITE(IECHO, *) DSEED

$* 1$

IF ((DSEED .LT.MNSEED) .OR. (DSEED.GT.MXSEED)) GO TO 190

* REMOVE the LINE that RE-CHECKS the SEed VALUE

$\star D$ U01S.39

*D ALNORM.39

$+(Z+3.980647940-4+1.9861538136400 /$

$D$ ALNORM. 40

¿PD VERSION

*AF ,XUDIST

*DECK VERSION

SUBROUTINE VERSION

C

C THIS ROUTINE WAS WRITTEN PRIMARILY TO CREATE A BANNER PAGE FOR THE

C PACSTAT CODE. IT WILL BE USED TO PROVIDE INFORMATION ON THE VERSION

C NUMBER OF PACSTAT CURRENTLY IN USE, UPDATE INFORMATION, AND APPLICABLE

C DOCUMENTATION. AUTHOR: CL BARTLEY $4 / 15 / 86$

C

C

CHARACTER * 1 STAR, MINUS

$\begin{array}{ll}\text { C STAR }=1 * 1 \\ \text { C } & \text { MINUS }=1-1 \\ C & \text { IDAT }=12 \\ C & \\ C & \\ 50 & \text { WRITE }(\text { IDAT , 50) } \\ C & \text { FORMAT }(/)\end{array}$

C

DO $150 \mathrm{~J}=1,5$

WRITE(IDAT, 100) (STAR, I=1,122)

PACSTAT.B3.UPD.8 


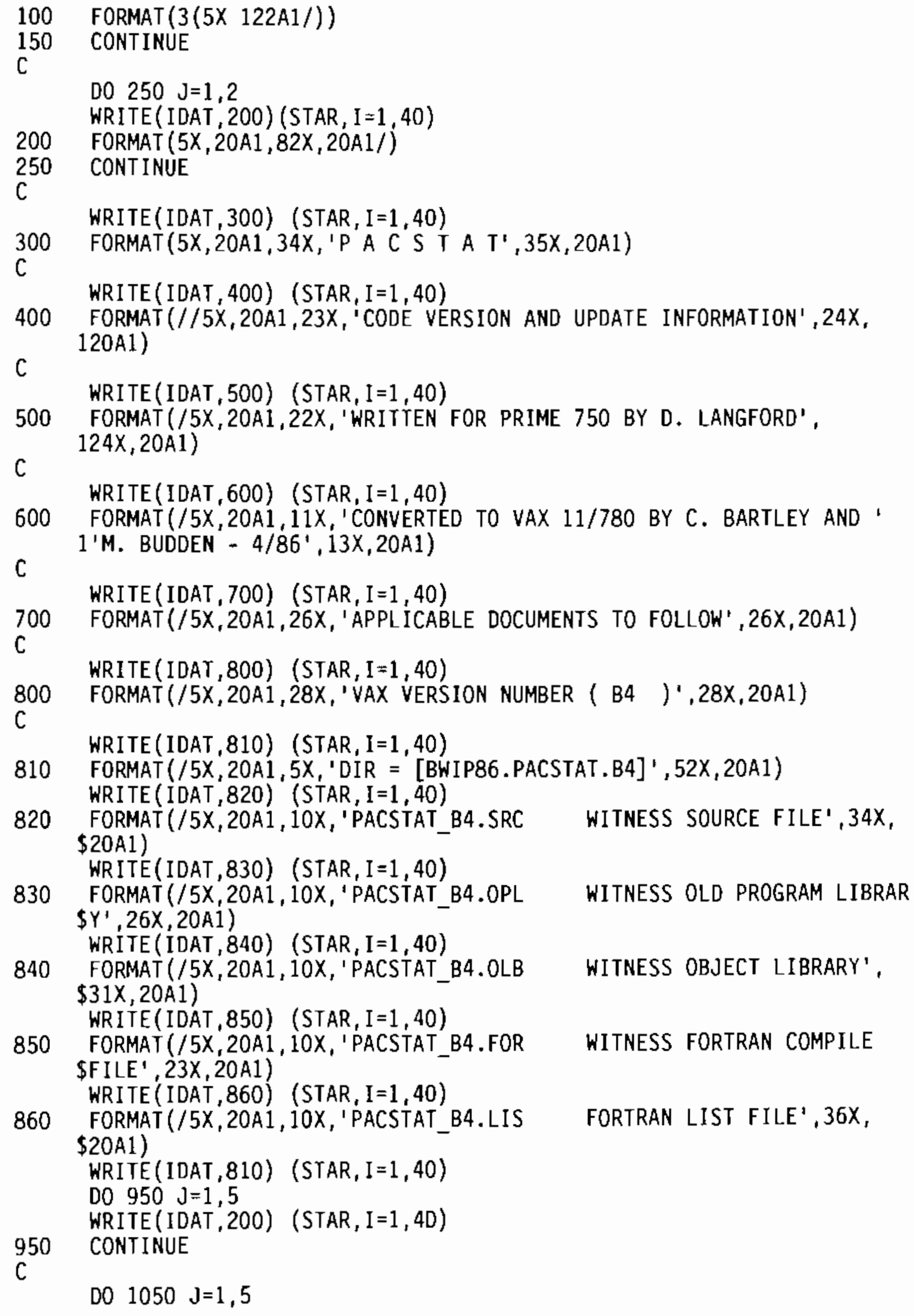

PACSTAT.B3.UPD. 9 


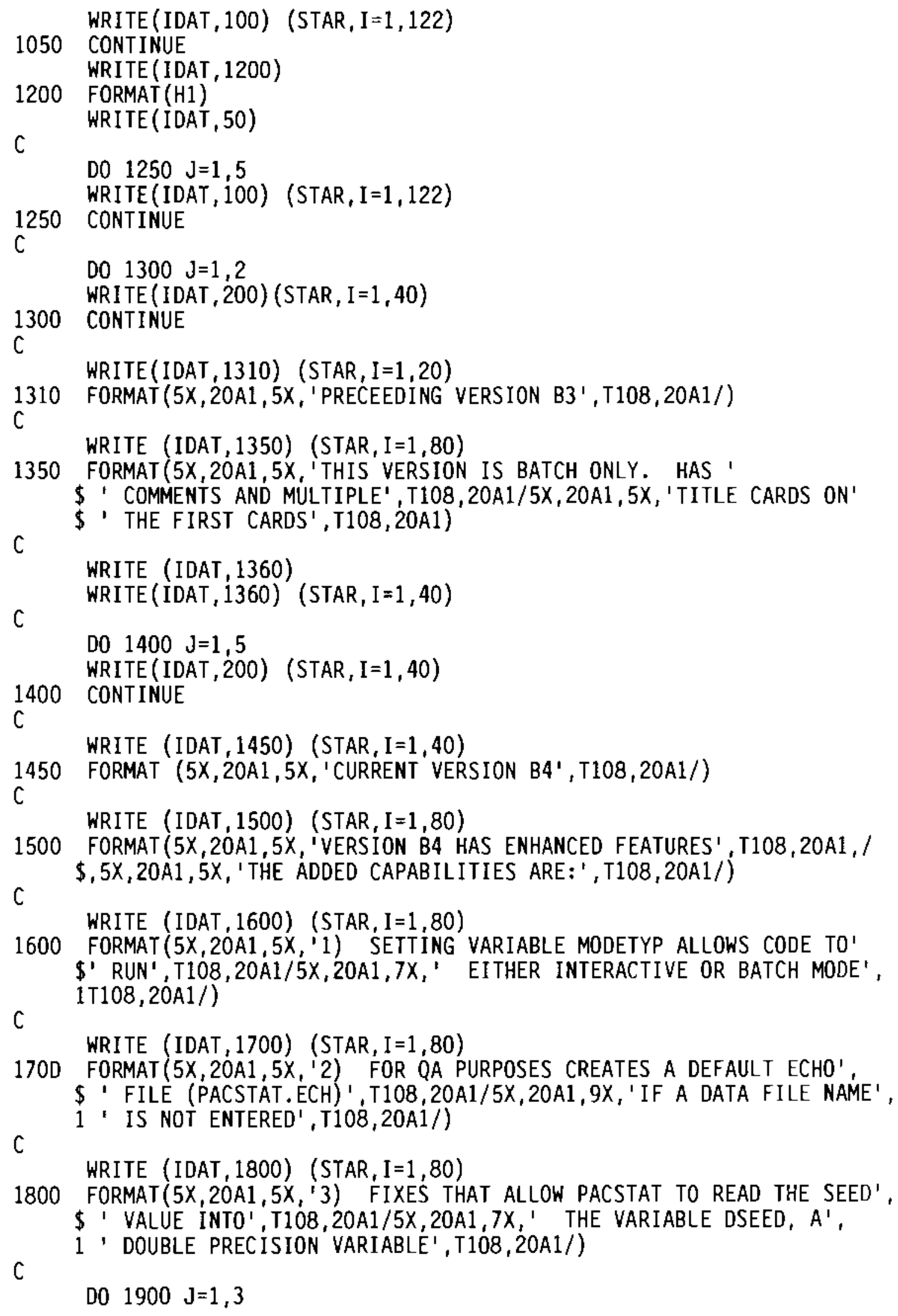




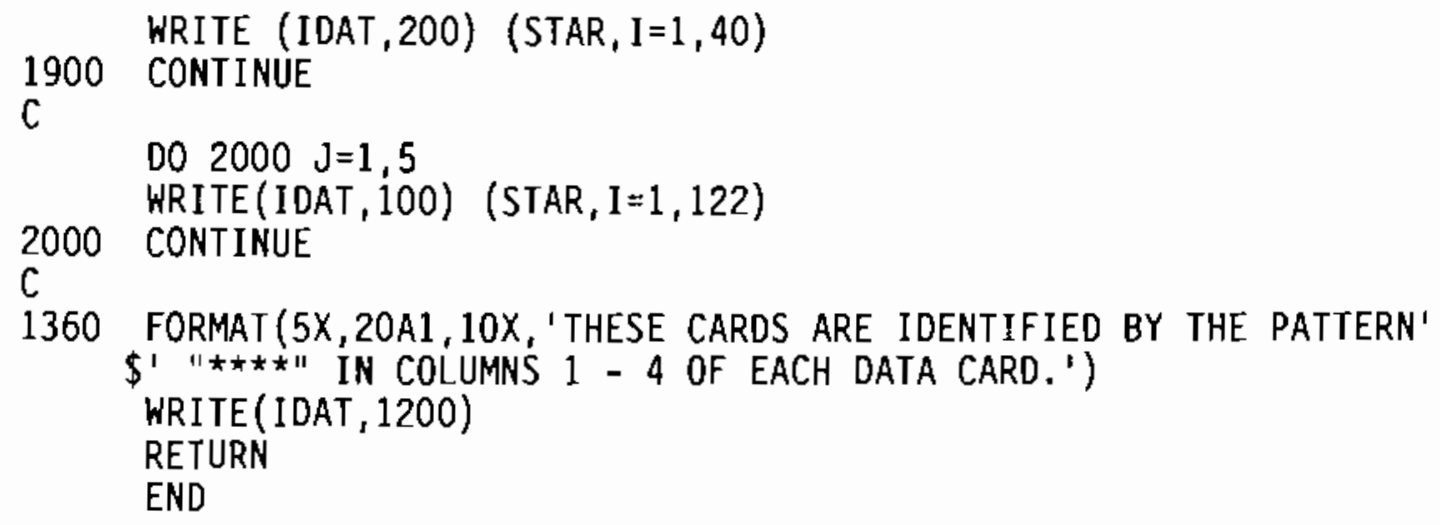

PACSTAT.B3.UPD. 11 
APPENDIX E

PACSTAT EXAMPLE INPUT 


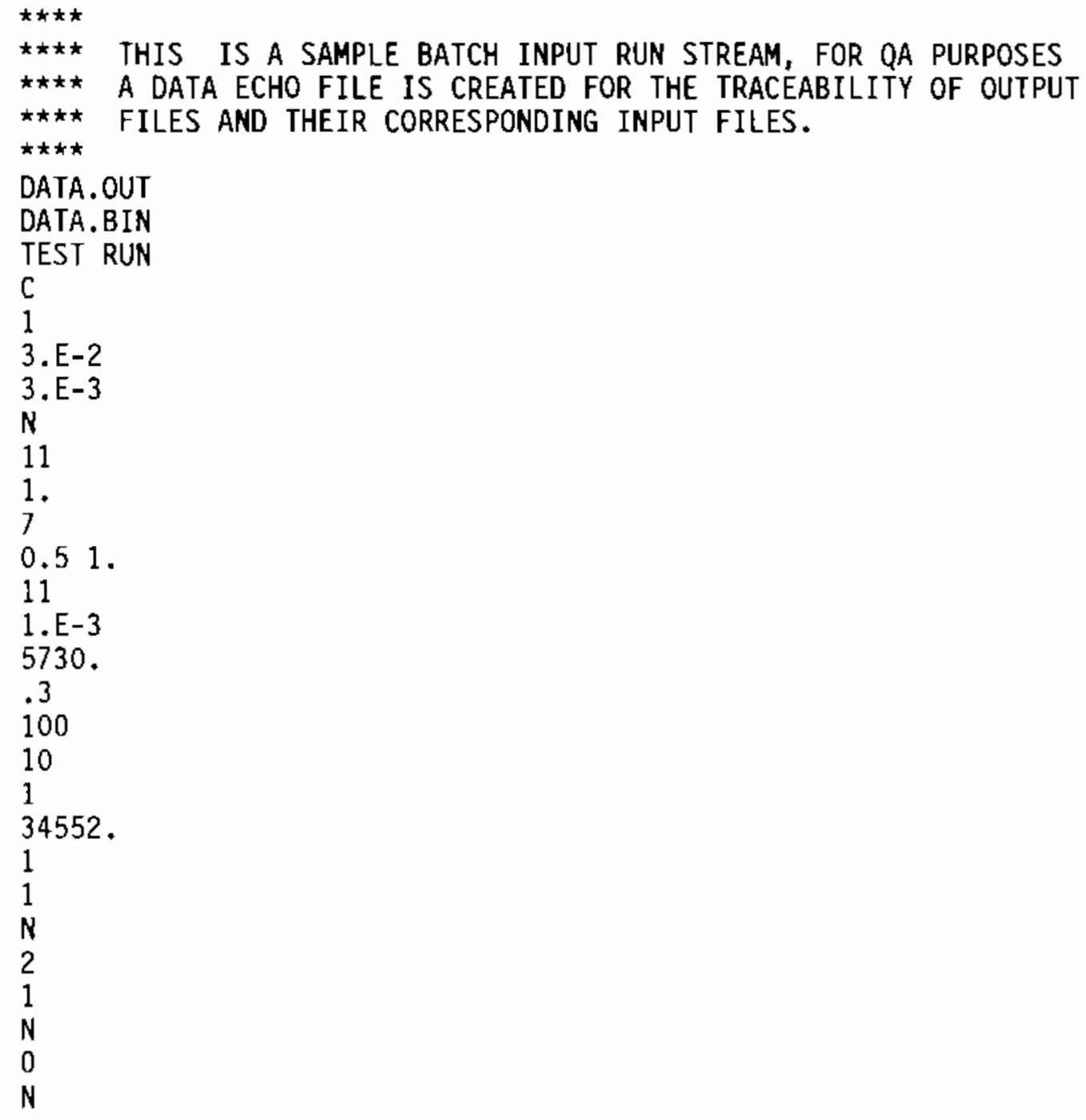




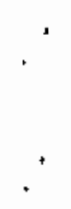


APPENDIX F

PACSTAT EXAMPLE OUTPUT 


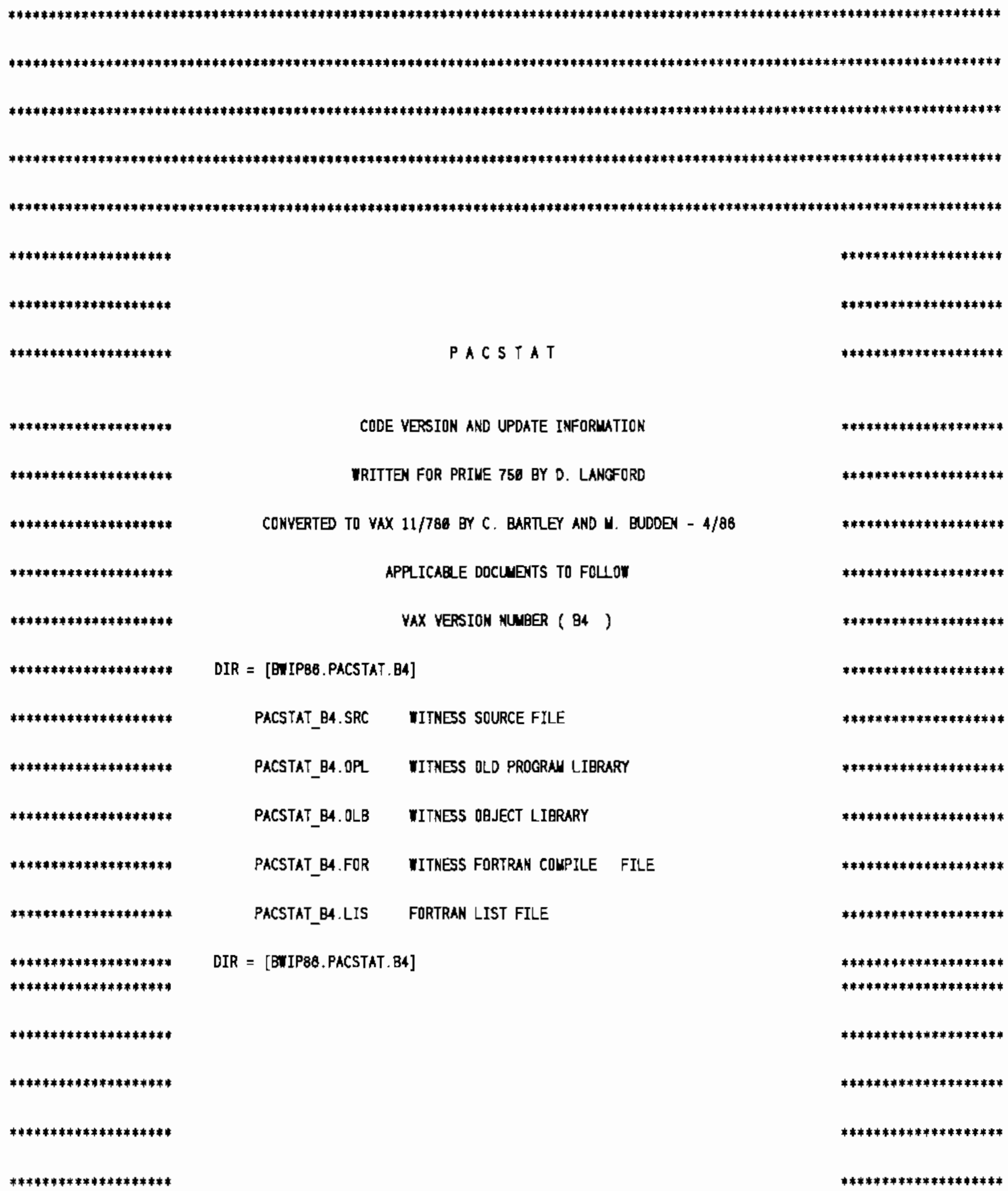

(1)




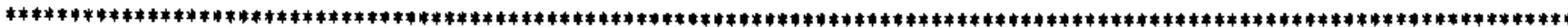

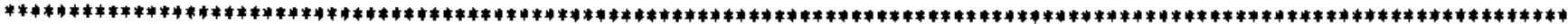

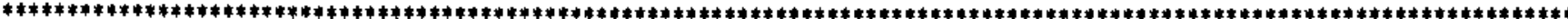

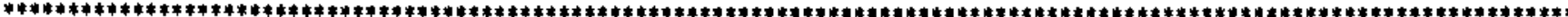

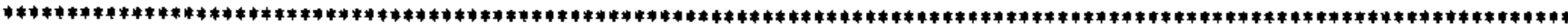

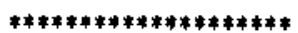

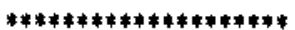

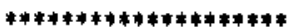

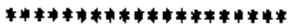
$* * * * * * * * * * * * * * * * * * * *$

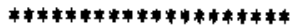

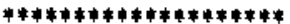

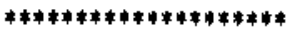

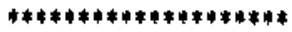
$\neq * * * * * * * * * * * * * * * * * * *$

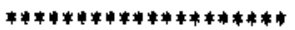

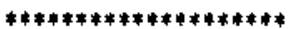

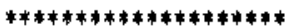

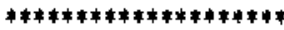

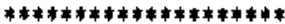

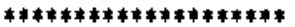

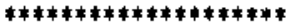

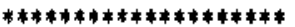

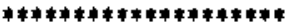

PRECEEDING VERSION B3

THIS VERSION IS BATCH ONLY. HAS COMENTS AND MLTIPLE TITLE CARDS ON THE FIRST CARDS

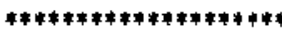

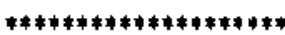

THESE CARDS ARE IOENTIFIED BY THE PATTERN '****' IN COLUMNS 1 - 4 OF EACH DATA CARD. THESE CARDS ARE IDENTIFIED BY THE PATTERN 1****' IN COLUNNS 1 - 4 OF EACH DATA CARD.

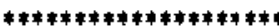

$* * * * * * * * * * * * * * * * * * * * *$

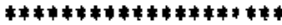
$* * * * * * * * * * * * * * * * * * * *$ $* * * * * * * * 4 * 4 * * * * * * * * *$ CURRENT VERSION B4

$* * * * * * * * * * * * * * * * * * * *$ VERSION B4 HAS ENHANCED FEATURES THE ADDED CAPABILITIES ARE:

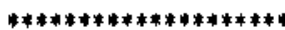

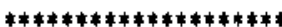

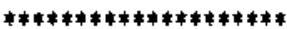

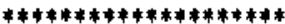
EITHER INTERACTIVE DR BATCH MDDE

2) FOR QA PURPOSES CREATES A DEALUT ECHD FILE (PACSTAT. ECH) IF A DATA FILE NALE IS NOT ENTERED

3) FIXES THAT ALLON PACSTAT TO READ THE SEED YALUE INTO THE VARIABLE DSEED, A DOUBLE PRECISION VARIABLE

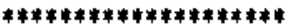
$* * * * * * * * * * * * * * * * * * * *$

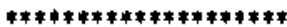
$\forall * * * * * * * * * * * * * * * * \cdots * * * *$ 
**** THIS IS A SAUPLE BATCH INPUT RUN STREA, FOR QA PURPOSES

**** A DATA ECHO FILE IS CREATED FOR THE TRACEABILITY DF DUTPUT

**** FILES AND THEIR CORRESPBNDING INPUT FILES.

$\$ \#$ \$*

DATA. OUT

1 (PACSTAT 1.06)

Results from this code are based on the use of unverified sof tware and are not for use in license related applications.

DIF-STAT [ 1. DO]

MASS STORAGE AND REEEASE RATE INTO LAYER TEST RUN
RUN I.D. 1621861254

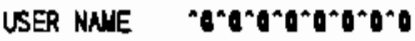
DATA SET MUBER 1

TABLE 1. ECHO OF THE INPUT DATA

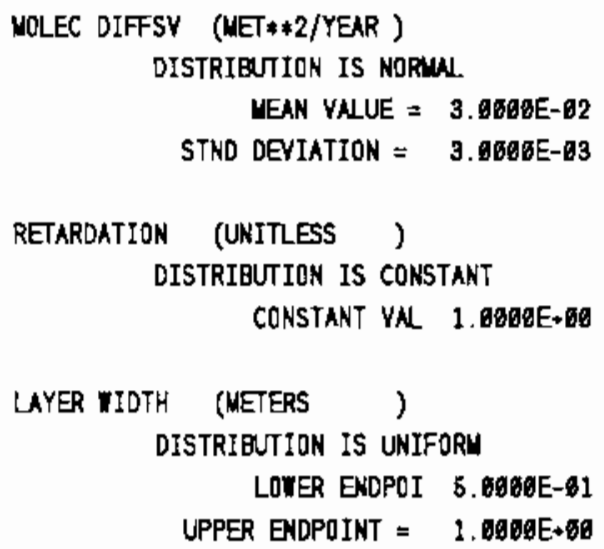


CONSTANT VAL 1.0000E-03

\begin{tabular}{|c|c|c|c|}
\hline IR HALF LIFE & (YEARS & ) IS & $5.7360 E+83$ \\
\hline VALUE FOR POROSITY & 〔UNITLESS & ) IS & 3.0896E- \\
\hline
\end{tabular}

NUMBER OF SAMPLES IS 190

PRINT INTERVAL IS 10

STRATIFIED SAMPLING NDT CHDSEN.

INITIAL RANDOM SEED NUMBER IS $3.45520000+94$

DNE DIMENSIONAL DIFFUSIONAL RELEASE INTO A BOUNDARY

LAYER OVER A SOLUBILITY LIMITED SOURCE ZONE.

1 TAELE 2. INPUT VARIABLE STATISTICS

**-- EXPECTED INPUT VARIABLE STATISTICS

VARIABLE MODE MEAN WEDIAN STND OEY

MULEC DIFFSV 3. $06 \mathrm{E}-62 \quad 3.06 \mathrm{E}-92 \quad 3.69 \mathrm{E}-62 \quad 3.60 \mathrm{E}-63$

LAYER WIDTH $\quad 7.50 \mathrm{E}-61 \quad 7.56 \mathrm{E}-81 \quad 7.59 \mathrm{E}-61 \quad 1.44 \mathrm{E}-61$

**--- SAMPLE DIAGNOSTICS, INPUT DISTRIBUTIONS

\begin{tabular}{|c|c|c|c|c|c|c|}
\hline & MIN & WAX & MEAN & WEDIAN & STND DEV & SKENNESS \\
\hline WOLEC DIFFSV & $2.175 \mathrm{E}-62$ & $3.752 \mathrm{E}-62$ & $3.014 \mathrm{E}-62$ & $2.998 \mathrm{E}-02$ & $2.876 \mathrm{E}-63$ & $-1.559 E-63$ \\
\hline ION & $1.696 \mathrm{E}+96$ & $1.008 E+06$ & $1.608 \mathrm{E}+68$ & $1.090 \mathrm{E} \rightarrow 00$ & $0.600 \mathrm{E}+60$ & 0.00 \\
\hline & 5.007E-61 & $9.991 \mathrm{E}-81$ & 7.625E-91 & 7. $688 \mathrm{E}-81$ & $1.497 \mathrm{E}-01$ & 7. \\
\hline & 009E- 03 & $.009 \mathrm{E}-63$ & 1. $060 \mathrm{E}-63$ & $1.800 \mathrm{E}-03$ & $0.000 \mathrm{E}+06$ & $E+\infty$ \\
\hline
\end{tabular}

**-.- MAXIUUN LIKELIHODD ESTIMATORS FOR INPUT DISTRIBUTIONS

\begin{tabular}{|c|c|c|c|c|}
\hline & \multicolumn{2}{|c|}{ COMPUTED VALUES } & \multicolumn{2}{|c|}{ EXPECTED VALUES } \\
\hline VARIABLE & PARAMETER 1 & PARAMETER 2 & PARAHETER 1 & PARALETER 2 \\
\hline MQLEC DIFFSV & 3. $014 \mathrm{E}-62$ & $2.876 \mathrm{E}-03$ & $3.000 \mathrm{E}-62$ & $3.606 \mathrm{E}-93$ \\
\hline RETARDATION & $1.606 \mathrm{E}+60$ & & 1. $.000 E+08$ & \\
\hline LAYER NIDTY & $5.607 \mathrm{E}-61$ & $9.991 E-61$ & $5.000 \mathrm{E}-01$ & $1.660 \mathrm{E}+60$ \\
\hline SOLUBILITY & $1.000 E-03$ & & $1.000 \mathrm{E}-83$ & \\
\hline
\end{tabular}

1 TABLE 3. OUTPUT VARIABLE STATISTICS

**--- SAHPLE DIAGNOSTICS, OUTPUT DISTRIBUTIONS

MASS STORAGE IN LAYER, GH/M**2

WIN MAX MEAN MEDIAN STND DEV SKEINESS

8. $697 \mathrm{E}-62 \quad 2.779 \mathrm{E}-61 \quad 3.316 \mathrm{E}-91 \quad 1.141 \mathrm{E}-01 \quad 5.737 \mathrm{E}-92 \quad-5.417 \mathrm{E}-62$

RE RATE (S.S.), GK/M**3-YR

$6.275 \mathrm{E}-66 \quad 1.954 \mathrm{E}-6 \mathrm{~S} \quad 8.583 \mathrm{E}-66 \quad 8.527 \mathrm{E}-66 \quad 7.843 \mathrm{E}-87 \quad-2.972 \mathrm{E}-67$ 
**--- DUTPUT CUMUATIVE PROBABILITIES FQR STOCASTIC VARIABLE,

MASS STORAGE IN LAYER, CM/U*:2

\begin{tabular}{|c|c|c|}
\hline SAMPLE & VARIABLE & CUMUUATIVE \\
\hline NUMBER & VALUE & CONFIDENCE \\
\hline 1 & $0.6607 E-61$ & 0.01000 \\
\hline 16 & $9.7641 E-91$ & 0.16060 \\
\hline 20 & $0.7 B 59 \mathrm{E}-61$ & 0.20060 \\
\hline 30 & 6. $0068 \mathrm{E}-91$ & ๑. 36060 \\
\hline 40 & $6.9848 \mathrm{E}-61$ & 0.46060 \\
\hline 50 & 0.1140 & 6.50860 \\
\hline 68 & 0.1281 & 0.80890 \\
\hline 70 & 0.1553 & 0.70000 \\
\hline 80 & 0.1811 & 7. 80800 \\
\hline 90 & 6.2149 & 0.89000 \\
\hline 108 & - .2770 & 1. .0000 \\
\hline
\end{tabular}

**--- OUTPUT CUMRLATIVE PROBABILITIES FOR STOCASTIC VARIAQE,

RE RATE (S.S.), GW/ $4 * 3$-YR

\begin{tabular}{|c|c|c|}
\hline SAMPLE & VARIABLE & CUAMLATIVE \\
\hline NULBER & YALUE & CONFIDENICE \\
\hline 1 & $0.6275 \mathrm{E}-66$ & 0.61600 \\
\hline 10 & 9.7626E-65 & 6.10600 \\
\hline 28 & $0.7851 E-65$ & 0.26000 \\
\hline 38 & 6. $8118 \mathrm{E}-65$ & 0.39306 \\
\hline 40 & $6.8368 \mathrm{E}-65$ & 0.48000 \\
\hline 50 & $6.8498 \mathrm{E}-65$ & 0.58090 \\
\hline 89 & 9. $8898 \mathrm{E}-65$ & 0.68900 \\
\hline 76 & 6. $8936 \mathrm{E}-95$ & 0.70060 \\
\hline 80 & $8.9304 \mathrm{E}-65$ & 0.80080 \\
\hline 96 & $6.9752 \mathrm{E}-65$ & 0.96969 \\
\hline 180 & $6.1654 \mathrm{E}-84$ & 1.80000 \\
\hline
\end{tabular}


, 
APPENDIX G

SOLN AND BLKDAT ROUTINES 
QOCK DATA QKDAT

C

THIS QOCK DATA CONTAIHS THE PROBLE DEFINITION FOR A PACSTAT MDNTE CARLD SIMLTAION RUN.

This problea definition is for the duany problem constructed in in SOLN to allow us to renove strings of numbers directly from the randon number generator.

PARAMETER ISIZE $=50000$ ! WAX DATA SET SIZE

PARAMETER MAXCNT $=10$ ! WXX NUMBER OF CONSTANTS

PARAMETER MAXOST $=100 \quad !$ MXX NUMBER OF INPUT DATA POINTS

PARAMETER WAXVS $=100 \quad !$ WX LEYES FOR STRATIFIED SNMPLING

PARANETER MAXNS $=10900$ ! MXX NUMGER OF SNIPLES

PARANETER MAXSOL $=10$ ! MAX NUMBER OF SOLUTIONS

PARAMETER MAXYR $=25$ ! MAX NUMBER OF INPUT VARIAELES

PARAMETER NILEN $=10$ I FILE NAME LENGTH

PARAMETER NTYPE $=11$ ! MUMER OF BISTRIBUTIOK TYPES

$c$

COMHON /PBLK1/

- NCONST.

C

COMON /PBL2/

+ NSTTL,

NTTL, STLE,

CHARACTER $* 40$

c

- STLE, TLE

CDLANGN /PBLKG/

- CDIMEN(WNXCNT),

CLABE (WAXCNT),

PUBR,(WXSOL),

- VDIMEN(MAXYAR), VABE (WAXVAR)

NYAR

CHARACTER 12

- CDIMEH,

CLABEZ,

STRE

CHARACTER $\$ 32$

$+\quad$ PLABE

$\stackrel{c}{c}$

PARAMETER WAXSRC $=15$

CDMLON /IDCON/

- JRLNID.

- NRLNID(NAXSRC),

- PRGT (MXXSRC)

- USRNHT (MAXSRC),

CHARACTER $* 4$

- TITLE

CHARACTER $* 8$

+ Fl,

c

+ PRGT,

Fl,

TITLE (26),

FLT (WXXSR),

VERANY

PRG,

USRNAM,

VDINES,

\begin{tabular}{|c|c|c|}
\hline QKDAT & . & 2 \\
\hline QLKDAT & . & 3 \\
\hline BLKDAF & . & 4 \\
\hline BUKDAT & . & 5 \\
\hline BLKDAT & . & 6 \\
\hline HEIDII & . & 2 \\
\hline HEIDI1 & . & 3 \\
\hline HEIOII & . & 4 \\
\hline BLKDAT & . & 13 \\
\hline PPARA & . & 2 \\
\hline PPARA & . & \\
\hline PPARA & . & 1 \\
\hline PPARA & . & 5 \\
\hline PPARA & . & $\theta$ \\
\hline PPARA & . & 7 \\
\hline PPARA & . & 8 \\
\hline PPARA & . & 8 \\
\hline PPARA & . & 18 \\
\hline PPARA & . & 11 \\
\hline PPARA & . & 12 \\
\hline PBLKL & . & 2 \\
\hline PBLK1 & . & 3 \\
\hline PBLKI & . & 4 \\
\hline PQLKI & . & 5 \\
\hline PBLK2 & . & 2 \\
\hline PBLK2 & . & 3 \\
\hline PBLK2 & . & 4 \\
\hline PQXX2 & . & 5 \\
\hline PQLK2 & . & 6 \\
\hline PBK2 & . & 7 \\
\hline PBLK2 & . & 8 \\
\hline PBLK3 & . & 2 \\
\hline PBLK3 & . & 3 \\
\hline PQK3 & . & 4 \\
\hline PBLK3 & . & 5 \\
\hline PQKK3 & . & 6 \\
\hline PBLK3 & . & 7 \\
\hline PQK3 & . & $\theta$ \\
\hline PQLK3 & . & 0 \\
\hline PQLK3 & . & 18 \\
\hline PBLK3 & . & 11 \\
\hline IDCDN & . & 2 \\
\hline IDCDN & . & 3 \\
\hline IDCOM & . & 4 \\
\hline IDCOM & . & 5 \\
\hline IDCON & . & 6 \\
\hline IDCON & . & 7 \\
\hline IDCOH & . & $\theta$ \\
\hline IDCOM & . & 9 \\
\hline IDCoH & . & 10 \\
\hline IDCOM & . & 11 \\
\hline IDCON & . & 12 \\
\hline IDCON & . & 13 \\
\hline IDCON & . & 1 \\
\hline BLKDAT & . & 19 \\
\hline BLKDAT & . & 28 \\
\hline BLKDAT & . & 21 \\
\hline BLKOAT & . & 22 \\
\hline BLXOAT & & 23 \\
\hline BQKDAT & & 24 \\
\hline BLKDAT & & $2 \mathrm{E}$ \\
\hline BLKDAT & . & 26 \\
\hline HEIDII & 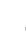 & 5 \\
\hline HEIDII & . & 6 \\
\hline HEIDII & . & 7 \\
\hline HEIDII & the & $\varepsilon$ \\
\hline HEIOII & . & 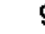 \\
\hline HEIDII & & 10 \\
\hline HEIDII & . & 11 \\
\hline HEIDI1 & - & 18 \\
\hline HEIDII & 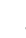 & 13 \\
\hline REIDII & 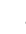 & 27 \\
\hline HEIDI1 & & 15 \\
\hline
\end{tabular}

G. 1 
Set number of solutions and the plotting label

for the output variable for each solution

DATA

NSDLN/1/

- RlA咟(1)/'Dunny solution'/

C Set the names of input variables and their nanes

DATA

WNAR/1/

DATA

+ Mabel (1)/'Input'/

Set the units for the input variables

DATA

+ VOLEN(1)/'No units'/

C Set the number of problen constants and the nane and units

for each constant.

DATA

$+\quad$ NCONST/E/

c

END

END

HEIDI1 * 18

HEIDI -17

HEIDI! , 18

HEIDI1 - 18

HEIDI: - 28

HEIDII - 21

HEIDI1 22

HEIDI1 - 23

HEIOI1 24

HEDI1 - 25

HEIOI1 - 26

HEIDI1 - 27

HEIDI $\quad 28$

HEIDI1 . 29

HEIOI1 - 30

HEIDI! - 31

HEIDII - 32

HEIDII - 33

HEIDI1 . 34

HEIDI1 - 35

HEIDII \& 36

HEIDI1 $\quad 37$

HEIDI1 - 38

HEIDI1 - 39

HEIOI1 - 48

HEIDI1 , 4!

HEIDI1 , 42

BLKDAT - 70

QKDAT 71

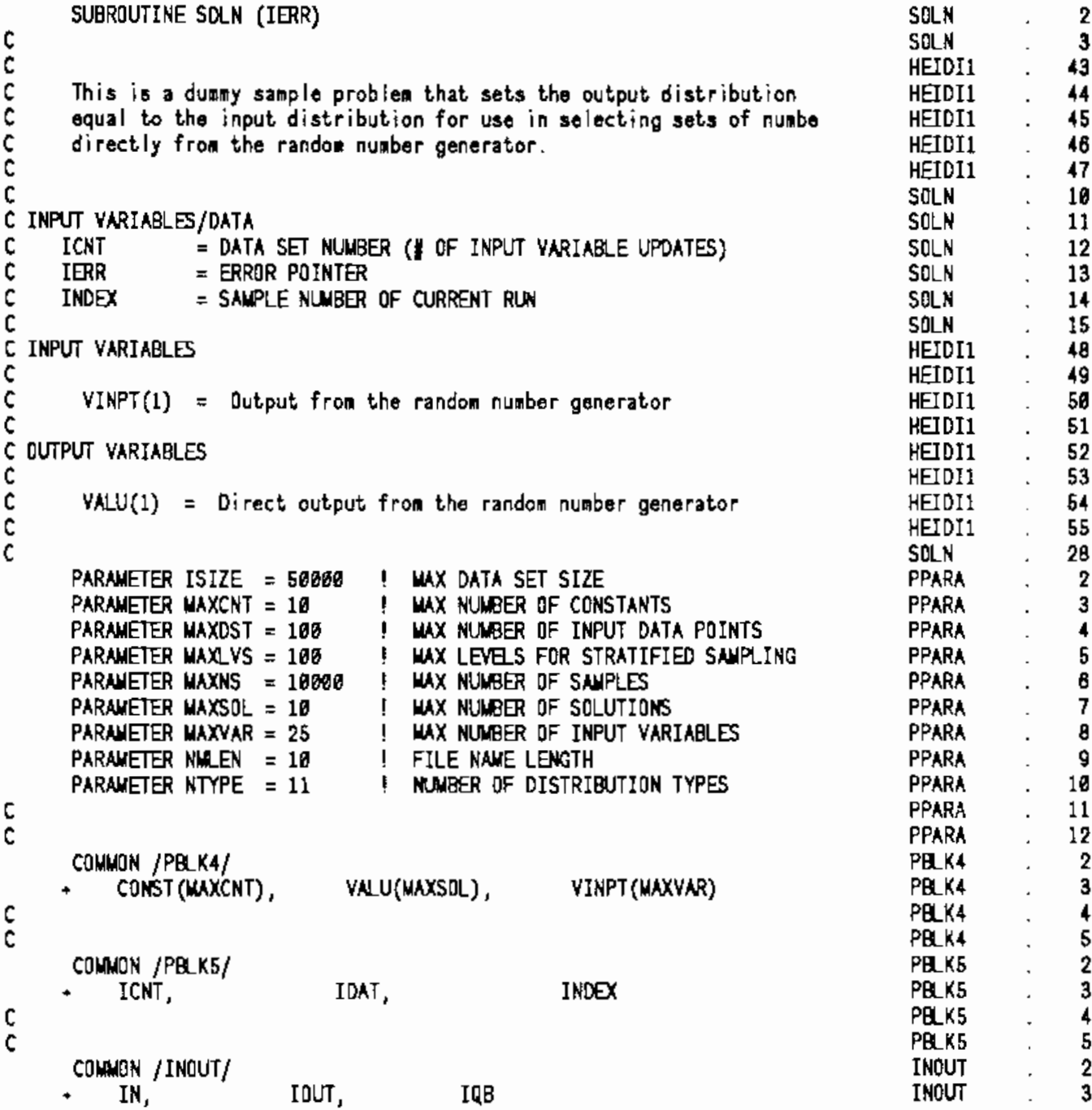




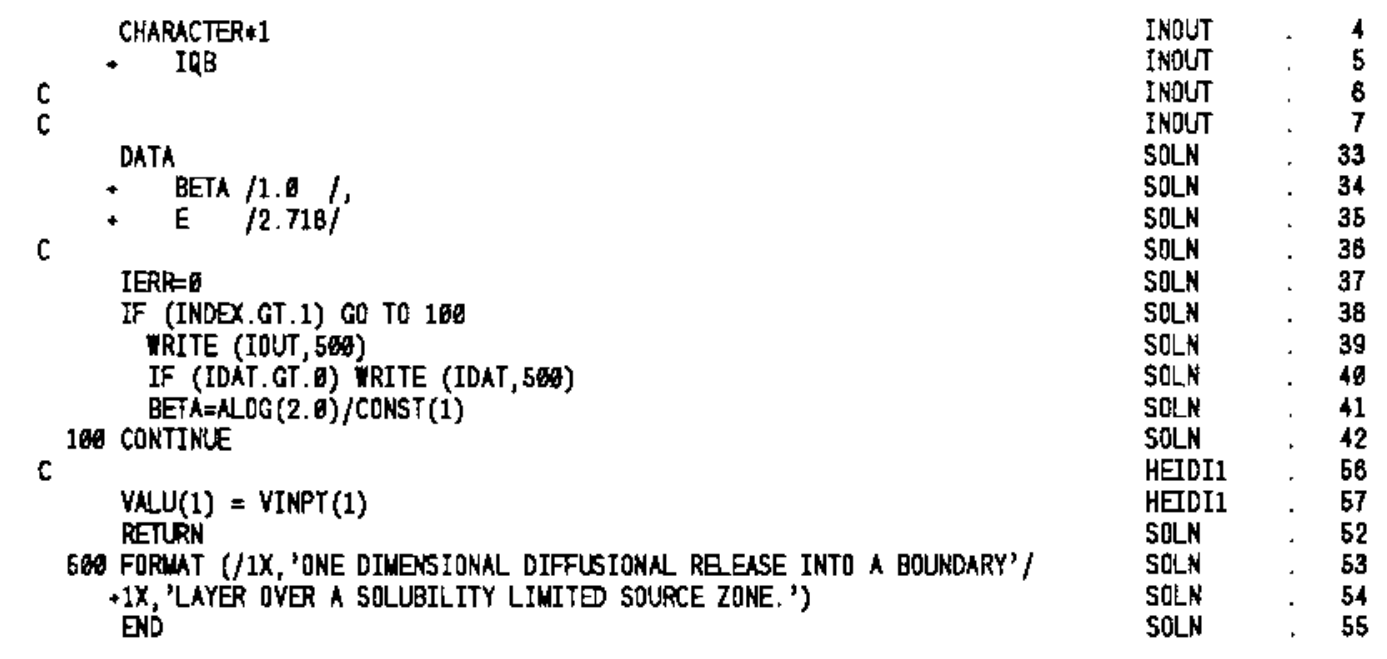




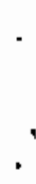

1 
APPENDIX H

EXAMPLE VAX/VMS COMMAND FILE USED TO

OBTAIN 100 SETS OF 10,000 UNIFORM $(0,1)$

VARIATES FROM PACSTAT 
$\$$ SD.HH

$\$$ !

\$! PACSTAT A4 TEST RUN: 2

$\$ !$

\$ RUN PACSTAT A4

NONE.

FILE.BIN

PACSTAT A4 TEST RUN: 2

C

7

$0 ., 1$.

10000

1

1

1499244925

1

1

N

0

N

\$ APP PACSTAT.BIN BINARY.OUTPUT4

$\$$ DEL PACSTAT.BIN; *
! Set subdirectory.

! File name of diagnostic data echo file

! File name of binary output file

! Documentation label for run

! Continue if error encountered in solution

! routine

! Generate data from uniform distribution

! Endpoints of uniform interval

! Number of variates in sample

1 Print interval for output

! Number of intervals for stratified sampling

! Initial seed for random number generator

! Variable to solve for

! Cumulative probability description on

! output

! No plotting desired

! Exit variable solution menu

! Another data set not desired

! Combine output file from this run with

! previous output generated for this

! distribution

! Delete remaining file 
APPENDIX I

PROGRAM REAO USED TO PREPARE PACSTAT OUTPUT FOR USE BY SAS FOR STATISTICAL TESTING PURPOSES 
PROGRAM READ USED TO PREPARE PACSTAT OUTPUT FOR USE BY SAS FOR STATISTICAL TESTING PURPOSES

\section{PROGRAM READ}

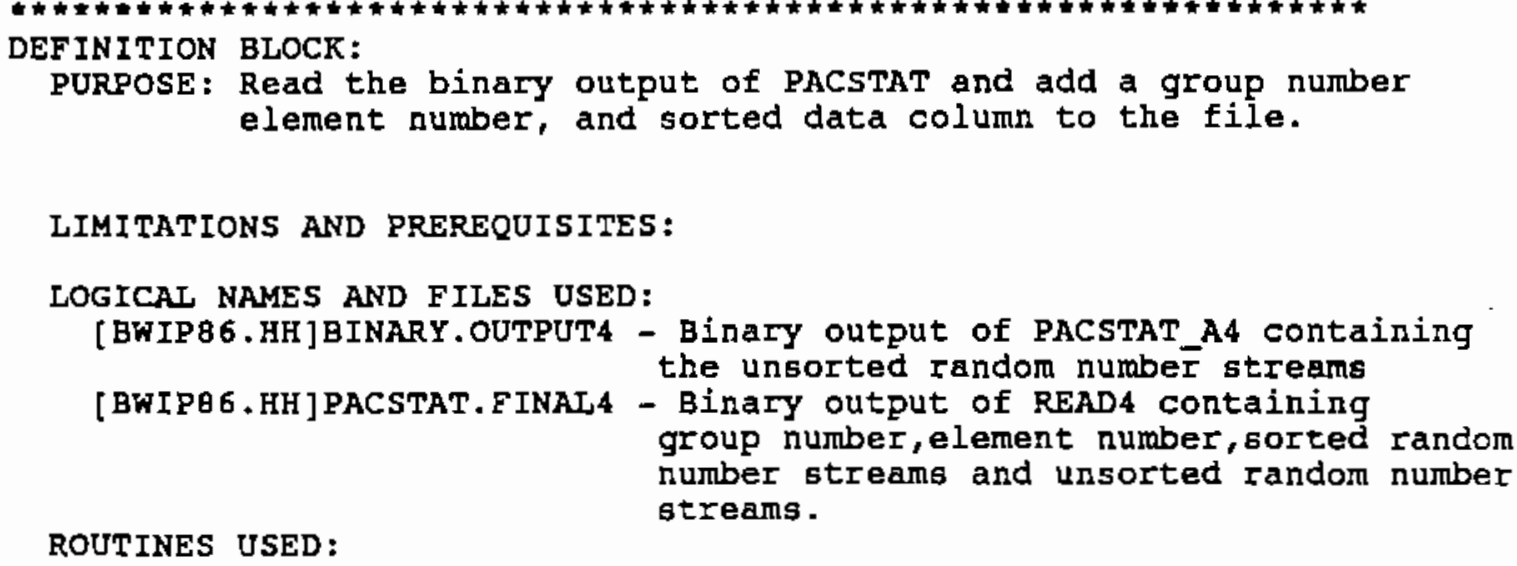
group number, element number, sorted random number streams and unsorted random number

ROUTINES USED: streams.

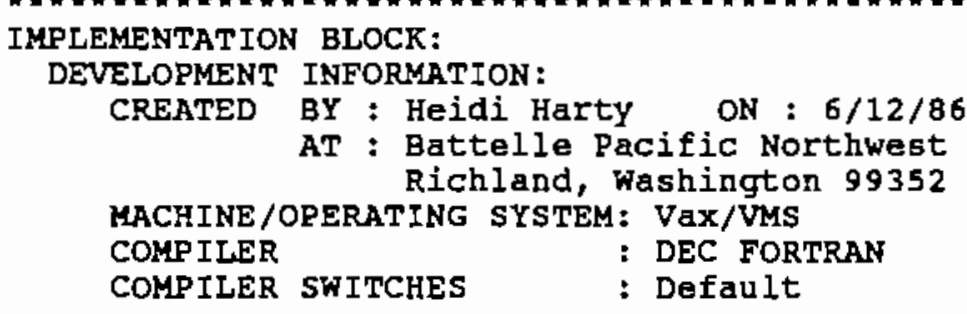

ALGORITHM: Read each line of the log file until the appropriate data is reached. Write data to output file along with identification numbers.

CONSTANTS (PARAMETER STATEMENTS):

PARAMETER IDIM=I0000

C

COMMON BLOCKS :

VAR IABLES :

DIMENSION VALUE1(IDIM), VALUE2(IDIM)

VALUEl: Sanple value of sorted data

VAULE2: Sample value from unsorted file

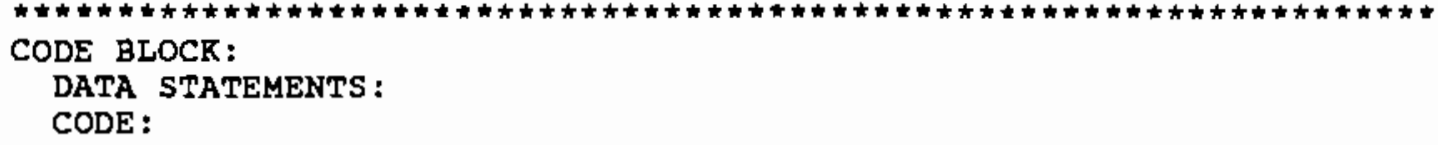




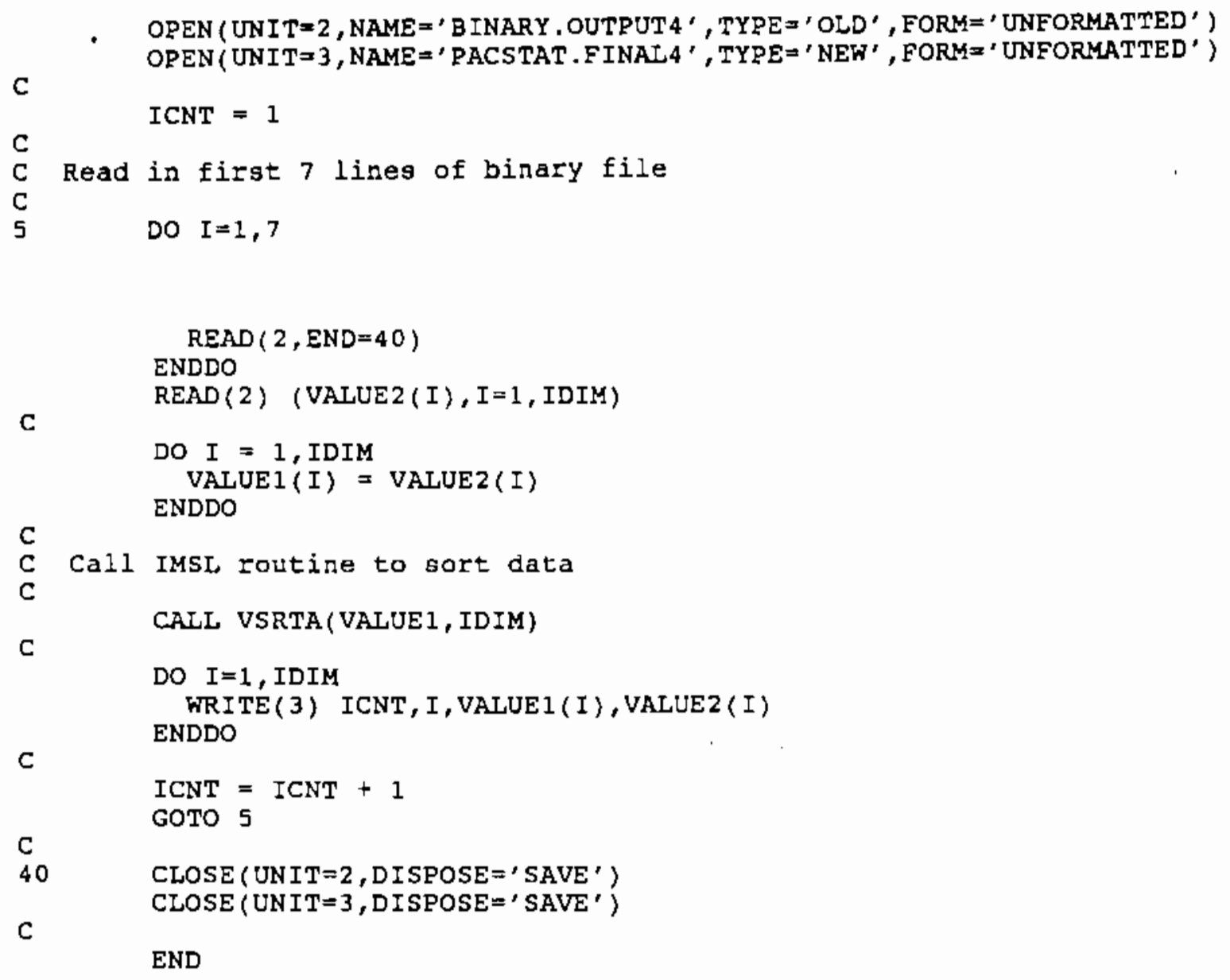




\section{DISTRIBUTION}

No. of

Copies

OFFSITE

10 DOE Technical Information Center

9 DOE Office of Civilian Radioactive Waste Management

Forrestal Building

Washington, DC 20585

ATTN: D. H. Alexander, RW-232

L. H. Barrett, $\mathrm{RW}-33$

A. Berusch, RW-231

C. R. Cooley, RW-4

N. Eisenberg, $R W-232$

J. R. Hilley, RW-30

S. Kale, $R W-20$

D. E. Shelor, RW-32

R. Stein, RW-23

3 DOE Office of Defense Waste \& GTN Transportation Management

Washington, DC 20545

ATTN: T. C. Chee, DP-123

G. H. Daly, DP-124

J. E. Lytle, DP-12

4 DOE Office of Remedial Action \& GTN Waste Technology

Washington, DC 20545

ATTN: J. A. Coleman, NE-24

T. W. Mcintosh, NE-24

W. R. Voight, NE-20

H. F. Walter, NE-24

A. T. Clark

Division of Fuel Material Safety Nuclear Regulatory Commission Washington, DC 20555
No. of

Copies

V. Stello

Office of the Executive Director for Operations

Nuclear Regulatory Commission

Mail Station 6209

Washington, DC 20555

G. L. Sjobiom

Environmental Protection Agency

Office of Radiation Programs

401 M Street, S.W.

Washington, DC 2D460

J. M. McGough

DOE Albuquerque Operations Office

P.0. Box 5400

Albuquerque, NM 87185

P. G. Hagan

Joint Integration office

Bldg. 3, 2nd Floor

2201 San Pedro, N.E.

Albuquerque, NM 87110

E. Maestas

DOE West Valley Operations Office

P.0. Box 191

West Valley, NY 14171

2 DOE Idaho Operations Office

550 Second Street

Idaho Falls, ID 83401

ATTN: S. T. Hinschberger

J. P. Hamric 
No. of

Copies

F. T. Fong

DOE San Francisco 0perations

1333 Broadway

0akland, CA 94612

M. R. Jugan

DOE Oak Ridge Operations office

P.0. Box E

Oak Ridge, TN 37830

W. J. Brumley

DOE Savannah River Operations Office

P.0. Box A

Aiken, SC 29801

M. J. Steindler

Argonne National Laboratory

9700 South Cass Avenue

Argonne, IL 60439

C. S. Abrams

Argonne National Laboratory

P.0. Box 2528

Idaho Falls, ID 83401

D. Schweitzer

Brookhaven National Laboratory Upton, NY 11973

K. Pruess

Lawrence Berkeley Laboratory

Berkeley, CA 84720

2 Battelle Memorial Institute

Project Management Division

$505 \mathrm{King}$ Avenue

Columbus, $\mathrm{OH} \quad 43201$

ATTN: J. F. Kircher

Technical Library
No. of

Copies

L. D. Ramspott

Lawrence Livermore National Laboratory

University of California

P.0. Box 808

Livermore, CA 94550

D. T. Oakley, MS 619

Los Alamos Scientific Laboratory

P.0. Box 1663

Los Alamos, NM 87544

4 Oak Ridge National Laboratory

P.0. BoX Y

Oak Ridge, TN 37830

ATTN: J. 0. Blomeke

W. D. Burch

R. T. Jubin

L. J. Mezga

3 Sandia Laboratories

P.0. Box 5800

Albuquerque, NM 87185

ATTN: R. W. Lynch

T. 0. Hunter

Technical Library

J. R. Berreth

Westinghouse Idaho Nuclear

Co., Inc.

P.0. Box 4000

Idaho Falls, ID 83401

6 E. I. du Pont de Nemours Company

Savannah River Laboratory

Aiken, SC 29801

ATTN: R. G. Baxter

M. D. Boersma

J. G. Glasscock

J. R. Knight

M. J. Plodinec

C. T. Randall 
No, of

Copies

E. A. Jennrich

EG\&G Idaho

P.0. Box 1625

Idaho Falls, ID 83415

R. Shaw

Electric Power Research Institute

3412 Hillview Avenue

P.0. Box 10412

Palo Alto, CA 94304

West Valley Nuclear Services

Company

P.0. Box 191

West Valley, NY 14171

ATTN: J. M. Pope

J. L. White, Chairman

Energy Research \& Oevelopment Authority

Empire State Plaza

Albany, NY 12223

F. B. Cheung

Department of Mechanical Engineering

The Pennsylvania State University

University Park, PA 16802

\section{ONSITE}

5 DOE Richland Operations Office

N. T. Karagianes

P. E. Lamont

D. H. Dahlem

J. L. Rhoades

M. W. Shupe
No, of

Copies

8 Westinghouse Hanford Company

R. C. Arnett

T. A. Curran

H. J. Dahlke

E. A. Fredenburg

J. Graham

S. B. Sonnichsen

J. A. Thies

S. C. Yung

40 Pacific Northwest Laboratory

C. R. Allen

M. K. Altenhofen

W. W. Ballard, Jr.

C. L. Bartley

S. H. Bian

D. J. Bradley

M. J. Budden (10)

J. R. Burnell

T. T. Claudson

T. E. Gates

G. W. Gee

R. W. Goles

H. Harty

W. L. Kuhn

L. T. Lakey

A. M. Liebetrau

J. E. Mendel/M. R. Kreiter

M. E. Peterson

G. F. Piepel

P. F. Salter

G. J. Sevigny

C. W. Stewart

M. R. Toland

A. P. Toste

Publishing Coordination (2)

Jechnical Report Files (5) 
. 